\title{
Direct Access to Chiral Secondary Amides by Copper-Catalyzed Borylative Carboxamidation of Vinylarenes with Isocyanates
}

\author{
Daniele Fiorito, ${ }^{\dagger}$ Yangbin Liu,${ }^{\dagger}$ Céline Besnard,${ }^{\ddagger}$ Clément Mazet $^{\star}, \dagger$ \\ † Department of Organic Chemistry, University of Geneva, \\ 30 quai Ernest Ansermet, 1211 Geneva, Switzerland. \\ ₹ Laboratory of Crystallography, University of Geneva, \\ 24 quai Ernest Ansermet, 1211 Geneva, Switzerland. \\ E-mail: clement.mazet@unige.ch
}

\section{Table of Contents}

1. General information $\quad \mathrm{S2}$

2. Racemic copper-catalyzed borylative carboxamidation of alkenes S3

2.1. Reaction optimization $\quad$ S3

$\begin{array}{ll}\text { 2.2. Scope of the reaction } & \text { S9 }\end{array}$

3. Enantioselective copper-catalyzed borylative carboxamidation $\begin{array}{lr}\text { of vinylarenes } & \mathbf{S 4 7}\end{array}$

$\begin{array}{ll}\text { 3.1. Chiral ligand screening } & \mathbf{S 4 7}\end{array}$

$\begin{array}{ll}\text { 3.2. Reaction optimization } & \mathbf{S 5 0}\end{array}$

3.3. Synthesis of phosphanamine $(R, R)-6 \mathbf{k} \quad \mathbf{S 5 2}$

3.4. Hammett study $\$ 53$

3.5. Enantiomeric ratio monitoring $\quad \mathrm{S57}$

3.6. Scope of the reaction $\quad$ S58

4. Copper-catalyzed borylative carboxamidation of alkynes $\quad \mathbf{S 1 2 1}$

5. Derivatization of amides $\quad \mathbf{S 1 2 5}$

$\begin{array}{lll}\text { 6. X-Ray analyses } & \$ 137\end{array}$

\begin{tabular}{ll} 
7. & References \\
\hline
\end{tabular}

8. NMR spectra of new compounds $\quad \mathbf{S 1 4 2}$ 


\section{General information}

Unless otherwise noted, all reactions were carried out under an inert atmosphere of nitrogen using either two-manifold vacuum/inert gas lines or a M. Braun glove-box. Solvents were dried over activated alumina columns and further degassed by three successive "freeze-pumpthaw" cycles. NMR spectra were recorded on AMX-300, AMX-400 and AMX-500 Bruker Avance spectrometers at $298 \mathrm{~K} .{ }^{1} \mathrm{H}$ and ${ }^{13} \mathrm{C}\left\{{ }^{1} \mathrm{H}\right\}$ NMR chemical shifts are given in ppm relative to $\mathrm{SiMe}_{4}$, with the solvent resonance used as internal reference. ${ }^{1} \mathrm{H}$ NMR spectra were referenced to $\mathrm{CDCl}_{3}(7.26 \mathrm{ppm}), \mathrm{CD}_{2} \mathrm{Cl}_{2}(5.32 \mathrm{ppm})$ and ${ }^{13} \mathrm{C}\left\{{ }^{1} \mathrm{H}\right\}$ NMR spectra were referenced to $\mathrm{CDCl}_{3}(77.16 \mathrm{ppm}), \mathrm{CD}_{2} \mathrm{Cl}_{2}$ (53.84 ppm). ${ }^{31} \mathrm{P}\left\{{ }^{1} \mathrm{H}\right\}$ NMR chemical shifts are reported in ppm relative to $\mathrm{H}_{3} \mathrm{PO}_{4} \cdot{ }^{19} \mathrm{~F}\left\{{ }^{1} \mathrm{H}\right\} \mathrm{NMR}$ chemical shifts are reported in ppm with absolute reference relative to ${ }^{1} \mathrm{H}$. ${ }^{11} \mathrm{~B}$ NMR chemical shifts are reported in ppm with absolute reference relative to ${ }^{1} \mathrm{H}$. Infrared spectra were obtained on a Perkin-Elmer $1650 \mathrm{FT}-\mathrm{IR}$ spectrometer using neat samples on a diamond ATR Golden Gate sampler. HRMS were obtained on a Xevo G2 Tof spectrometer (Ionization mode: ESI positive polarity; Mobile phase: $\mathrm{MeOH} 100 \mu \mathrm{l} / \mathrm{min}$ ). Mass spectrum is calibrated by the use of the MS lockspray system (LeuEnk calibration solution). The enantiomeric ratios (er) were determined by HPLC or SFC analyses. HPLC analysis were performed on a Shimadzu CTO-20AA with column DAICEL OD-H, OJ-H, AD-H and IC. SFC analyses were performed on a Waters Acquity UPC2 with columns OD-3, OJ-3, OZ-3, OB-H, $A Z-3, A D, A S-3, A Y-H$. Retention times $\left(t_{R}\right)$ are given in minutes. Thin layer chromatography (TLC) was performed on plates of silica precoated with $0.25 \mathrm{~mm}$ Kieselgel $60 \mathrm{~F}_{254}$ from Merck. Flash chromatography was performed using silica gel SiliaFlash ${ }^{\circledR}$ P60 (230-400 mesh) from Silicycle. Liquid reagents were transferred with stainless steel syringes or cannula. Commercial precatalysts, ligands, olefins $\mathbf{1}$, isocyanates 2 and alkynes 4 were purchased from Aldrich, Fluka, Acros or Strem and used without purification unless otherwise noted. NHCcopper precatalysts [(SIMes)CuCl], [(IMes)CuCl], [(SIPr)CuCl], [(IPr)CuCl], [(ICy)CuCl] and $[(\mathrm{ItBu}) \mathrm{CuBr}]$ were prepared following procedures reported in the literature. ${ }^{1}$ 


\section{Racemic copper-catalyzed borylative carboxamidation of alkenes}

\subsection{Reaction optimization}

Table S1. Precatalyst screening ${ }^{a}$

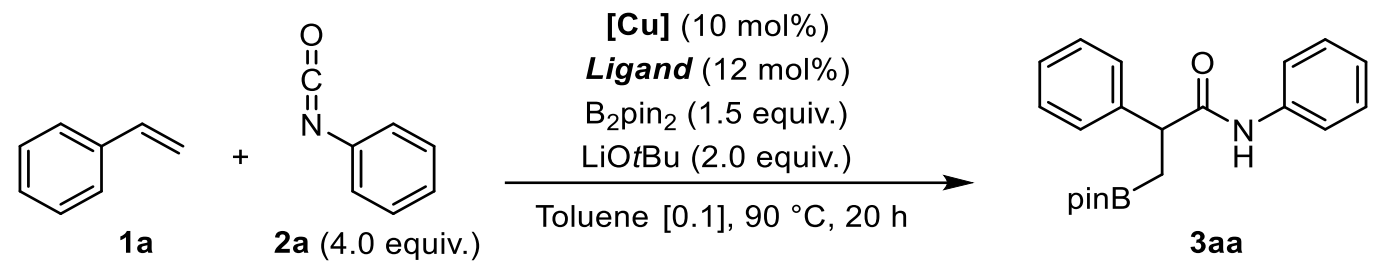

$\begin{array}{llll}\text { entry } & {[\mathrm{Cu}]} & \text { Ligand } & \text { conv. 3aa }(\%)^{b} \\ 1 & \text { none } & \text { none } & 0 \\ 2 & \mathrm{CuCl} & \text { none } & 0 \\ 3 & \mathrm{CuCl} & \mathrm{PPh}_{3} & 24 \\ 4 & \mathrm{CuCl} & \mathrm{PCy}_{2} \mathrm{Ph} & 21 \\ 5 & \mathrm{CuCl} & \mathrm{PCy}_{3} & 54(25) \\ 6^{c} & \mathrm{CuCl} & \mathrm{PCy}_{3} & 61(36) \\ 7 & \mathrm{CuCl} & \mathrm{PtBu}_{3} & 65 \\ 8 & \mathrm{CuCl} & \text { CyJohnPhos } & 80 \\ 9 & \mathrm{CuCl} & \text { DavePhos } & 31 \\ 10 & {[(\mathrm{IPr}) \mathrm{CuCl}]} & \text { none } & 25(19) \\ 11 & {[(\mathrm{SIPr}) \mathrm{CuCl}]} & \text { none } & 19 \\ 12 & {[(\mathrm{IMes}) \mathrm{CuCl}]} & \text { none } & 91(68) \\ 13 & {[(\mathrm{SIMes}) \mathrm{CuCl}]} & \text { none } & 90(82) \\ 14 & \mathrm{CuCl} & \text { SIMes } & 89 \\ 15 & {[(\mathrm{ItBu}) \mathrm{CuBr}]} & \text { none } & 67 \\ 16 & {[(\mathrm{ICy}) \mathrm{CuCl}]} & \text { none } & 39\end{array}$

${ }^{a}$ Reaction conditions: $1 \mathrm{a}(0.15 \mathrm{mmol}), \mathbf{2 a}(0.6 \mathrm{mmol}), \mathrm{B}_{2} \mathrm{pin}_{2}(0.23 \mathrm{mmol})$, LiOtBu $(0.3 \mathrm{mmol})$, [Cu] $(0.015 \mathrm{mmol})$, Ligand $(0.018 \mathrm{mmol})$ in toluene $(1.5 \mathrm{~mL}, 0.1 \mathrm{M})$ at $90{ }^{\circ} \mathrm{C}$ for $20 \mathrm{~h} .{ }^{b}$ Determined by ${ }^{1} \mathrm{H}$ NMR of the crude reaction mixture in presence of an internal standard. In parenthesis, isolated yield of 3 aa after purification by column chromatography. ${ }^{c} \mathrm{PCy}_{3}(21$ mol\%). 


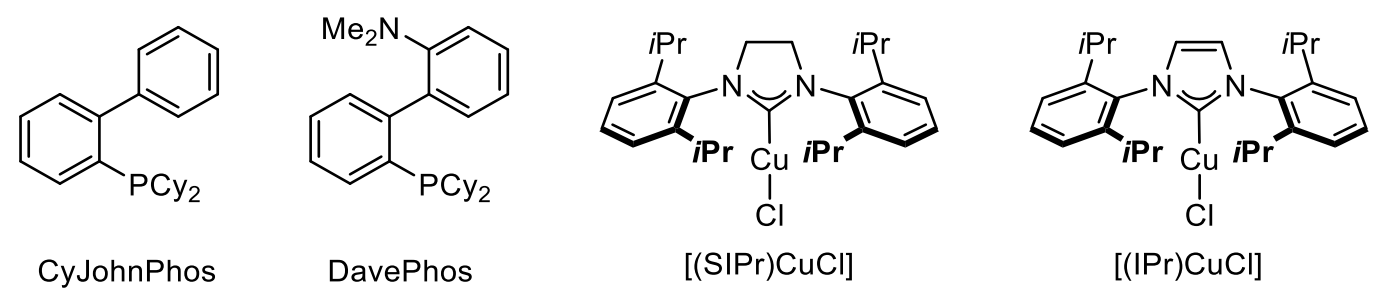

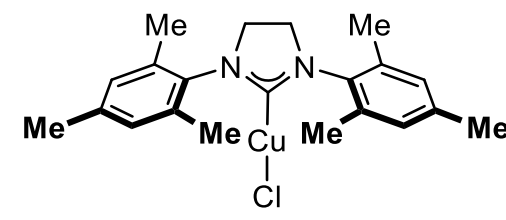

[(SIMes)CuCl]

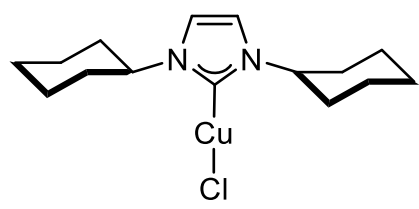

$[(\mathrm{ICy}) \mathrm{CuCl}]$

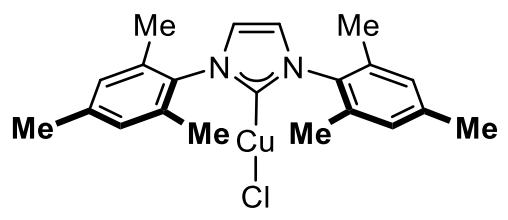

[(IMes)CuCl]

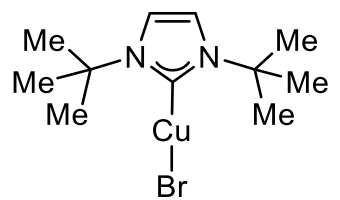

[(ItBu)CuBr]

Figure S1. Ligands and Cu precatalysts tested in Table S1

\section{General procedure for precatalyst screening}

In a $10 \mathrm{~mL}$ Schlenk tube, the appropriate $\mathrm{Cu}$ precursor $(0.015 \mathrm{mmol}, 10.0 \mathrm{~mol} \%)$, the appropriate ligand ( $0.018 \mathrm{mmol}, 12 \mathrm{~mol} \%$ ), LiOtBu (24 mg, $0.3 \mathrm{mmol}, 2.0$ equiv.) and $\mathrm{B}_{2} \mathrm{pin}_{2}$ (57 mg, $0.23 \mathrm{mmol}, 1.5$ equiv.) were dissolved in $1.5 \mathrm{~mL}$ of toluene $(0.1 \mathrm{M})$. The resulting dark solution was stirred for $10 \mathrm{~min}$ at $23^{\circ} \mathrm{C}$, then styrene $1 \mathrm{a}(0.018 \mathrm{~mL}, 0.15 \mathrm{mmol}, 1.0$ equiv.) was added neat with a micro-syringe. The resulting mixture was stirred for $5 \mathrm{~min}$ at $23^{\circ} \mathrm{C}$, then isocyanatobenzene $2 \mathrm{a}(0.066 \mathrm{~mL}, 0.6 \mathrm{mmol}, 4$ equiv.) was added neat with a microsyringe. The sealed tube was stirred at $90{ }^{\circ} \mathrm{C}$ for $20 \mathrm{~h}$. After $20 \mathrm{~h}$, the reaction mixture was cooled to $23^{\circ} \mathrm{C}$ and quenched by addition of a saturated aqueous $\mathrm{NH}_{4} \mathrm{Cl}$ solution $(1.0 \mathrm{~mL})$. The reaction mixture was diluted with ethyl acetate $(5 \mathrm{~mL})$ and water $(5 \mathrm{~mL})$ and extracted with ethyl acetate $(3 \times 5 \mathrm{~mL})$. The combined organic phases were dried over sodium sulfate and the solvent was removed under reduced pressure. Conversion into borylated secondary amide 3aa was assessed by ${ }^{1} \mathrm{H}$ NMR analysis of the crude residue in presence of an internal standard. Purification by flash chromatography on silica gel afforded the pure borylated secondary amide 3 aa. 
Table S2. Screening of bases ${ }^{a}$

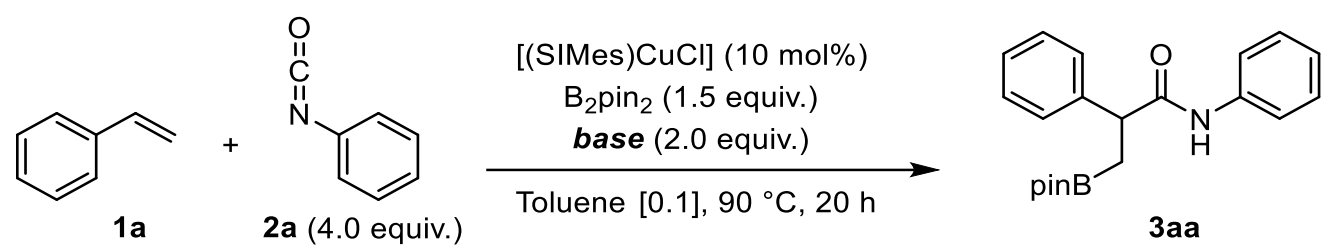

$\begin{array}{lll}\text { entry } & \text { base } & \text { conv. 3aa }(\%)^{b} \\ 1 & \mathrm{LiOtBu} & 90(82) \\ 2 & \mathrm{KOtBu} & 51 \\ 3 & \mathrm{NaOtBu} & 27 \\ 4 & \mathrm{NaOMe} & 31 \\ 5^{c} & \mathrm{NaOTMS} & 46 \\ 6 & \mathrm{CsF} & 16 \\ 7 & \mathrm{Cs}_{2} \mathrm{CO}_{3} & 20\end{array}$

${ }^{a}$ Reaction conditions: 1a $(0.15 \mathrm{mmol}), 2 \mathrm{a}(0.6 \mathrm{mmol}), \mathrm{B}_{2} \mathrm{pin}_{2}(0.23 \mathrm{mmol})$, base $(0.3 \mathrm{mmol})$ and [(SIMes) $\mathrm{CuCl}](0.015 \mathrm{mmol})$ in toluene $(1.5 \mathrm{~mL}, 0.1 \mathrm{M})$ at $90{ }^{\circ} \mathrm{C}$ for $20 \mathrm{~h} .{ }^{b}$ Determined by ${ }^{1} \mathrm{H}$ NMR of the crude reaction mixture in presence of an internal standard. In parenthesis, isolated yield of 3 aa after purification by column chromatography. ${ }^{c} \mathrm{NaOTMS}$ in THF solution. Reaction solvent: Toluene/THF 4:1, 0.1 M. NaOTMS = sodium trimethylsilanolate. 
Table S3. Solvent and temperature screening ${ }^{a}$

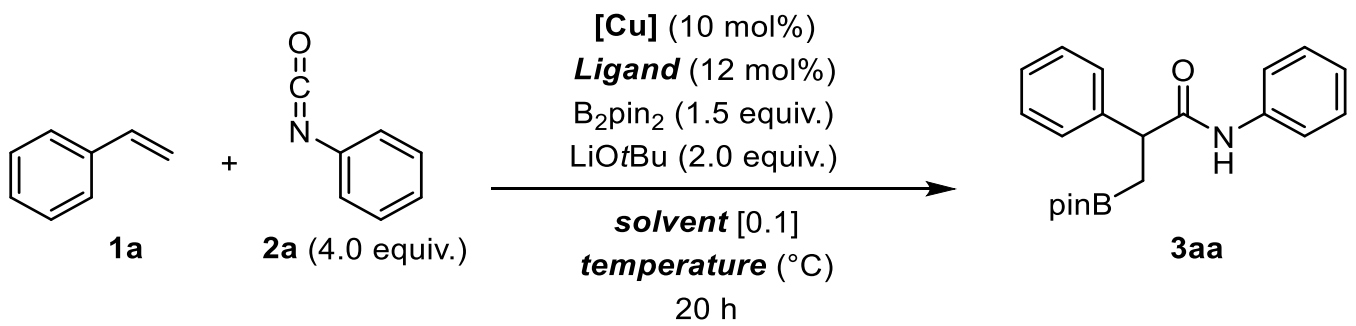

$\begin{array}{llllll}\text { entry } & {[\mathrm{Cu}]} & \text { ligand } & \text { solvent } & \text { temp. }\left({ }^{\circ} \mathrm{C}\right) & \text { conv. 3aa }(\%)^{b} \\ 1 & {[(\mathrm{SIMes}) \mathrm{CuCl}]} & \text { none } & \text { toluene } & 90 & 90(82) \\ 2 & {[(\mathrm{SIMes}) \mathrm{CuCl}]} & \text { none } & \text { toluene } & 120 & 85 \\ 3 & {[(\mathrm{SIMes}) \mathrm{CuCl}]} & \text { none } & \text { toluene } & 50 & 90 \\ 4 & {[(\mathrm{SIMes}) \mathrm{CuCl}]} & \text { none } & \text { THF } & 90 & 30 \\ 5 & {[(\mathrm{SIMes}) \mathrm{CuCl}]} & \text { none } & 1,4-\text { dioxane } & 90 & 37 \\ 6 & {[(\mathrm{SIMes}) \mathrm{CuCl}]} & \text { none } & \text { DMF } & 90 & <5 \\ 7 & \mathrm{CuCl} & \mathrm{PCy}_{3} & \text { toluene } & 90 & 54(25) \\ 8 & \mathrm{CuCl} & \mathrm{PCy}_{3} & \text { THF } & 90 & 67 \\ 9 & \mathrm{CuCl} & \mathrm{PCy}_{3} & \text { THF } & 50 & 74 \\ 10 & \mathrm{CuCl} & \mathrm{PtBu}_{3} & \text { toluene } & 90 & 65 \\ 11 & \mathrm{CuCl} & \mathrm{PtBu}_{3} & \text { THF } & 50 & 74\end{array}$

${ }^{a}$ Reaction conditions: $1 \mathrm{a}(0.15 \mathrm{mmol}), \mathbf{2 a}(0.6 \mathrm{mmol}), \mathrm{B}_{2} \mathrm{pin}_{2}(0.23 \mathrm{mmol})$, LiOtBu $(0.3 \mathrm{mmol})$ and [(SIMes)CuCl] $(0.015 \mathrm{mmol})$ in the selected solvent $(1.5 \mathrm{~mL}, 0.1 \mathrm{M})$ at the selected temperature for $20 \mathrm{~h} .{ }^{b}$ Determined by ${ }^{1} \mathrm{H}$ NMR of the crude reaction mixture in presence of an internal standard. In parenthesis, isolated yield of 3aa after purification by column chromatography. 
Table S4. Condition refinement for isocyanate $2 \mathrm{a}^{a}$<smiles>C=Cc1ccccc1</smiles>

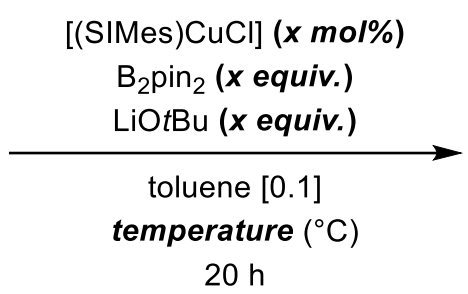<smiles>O=C(Nc1ccccc1)C(C[Pb])c1ccccc1</smiles>

$20 \mathrm{~h}$

$\begin{array}{lllllll}\text { entry } & \begin{array}{l}2 \mathrm{a} \\ \text { (equiv.) }\end{array} & \begin{array}{l}\mathrm{B}_{2} \mathrm{Pin}_{2} \\ \text { (equiv.) }\end{array} & \begin{array}{l}\mathrm{LiOtBu} \\ \text { (equiv.) }\end{array} & \begin{array}{l}\text { [(SIMes)CuCl }] \\ (\text { mol\%) }\end{array} & \begin{array}{l}\text { temp. } \\ \left({ }^{\circ} \mathrm{C}\right)\end{array} & \begin{array}{l}\text { conv. 3aa } \\ (\%)^{b}\end{array} \\ 1 & 4.0 & 1.5 & 2.0 & 10 & 90 & 90(82) \\ 2 & 4.0 & 1.5 & 2.0 & 10 & 50 & 90 \\ 3 & 4.0 & 1.5 & 2.0 & 5 & 90 & 90(70) \\ 4 & 4.0 & 1.5 & 2.0 & 5 & 50 & 68 \\ 5 & 2.0 & 1.5 & 2.0 & 10 & 90 & 90 \\ 6 & 2.0 & 1.2 & 1.5 & 5 & 90 & 81(62) \\ 7 & 3.0 & 1.2 & 1.5 & 5 & 90 & 91(75)\end{array}$

${ }^{a}$ Reaction conditions: $1 \mathrm{a}(0.15 \mathrm{mmol})$ in toluene $(1.5 \mathrm{~mL}, 0.1 \mathrm{M})$ for $20 \mathrm{~h} .{ }^{b}$ Determined by ${ }^{1} \mathrm{H}$ NMR of the crude reaction mixture in presence of an internal standard. In parenthesis, isolated yield of 3aa after purification by column chromatography. 
Table S5. Condition refinement for isocyanate $\mathbf{2} \mathbf{b}^{a}$

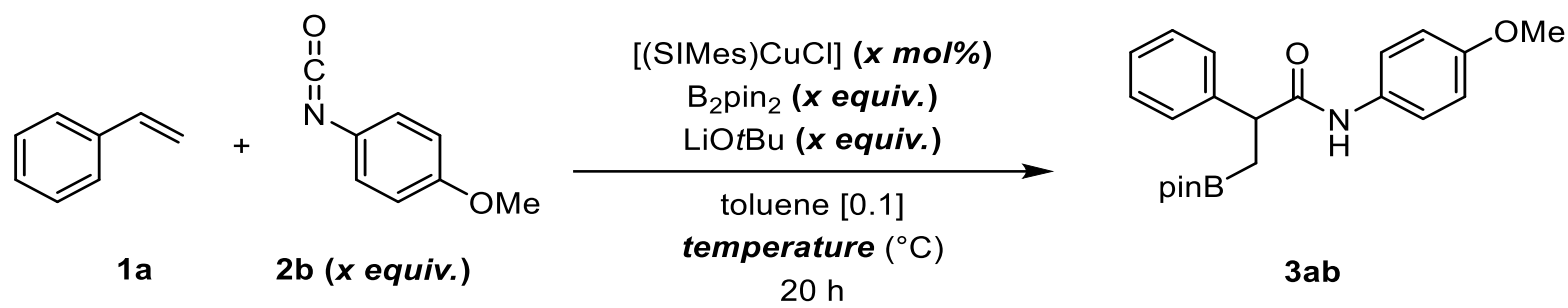

\begin{tabular}{|c|c|c|c|c|c|c|c|}
\hline entry & $\begin{array}{l}\mathbf{2 b} \\
\text { (equiv.) }\end{array}$ & $\begin{array}{l}\mathrm{B}_{2} \mathrm{Pin}_{2} \\
\text { (equiv.) }\end{array}$ & $\begin{array}{l}\text { LiOtBu } \\
\text { (equiv.) }\end{array}$ & $\begin{array}{l}\text { [(SIMes)CuCl] } \\
(\mathrm{mol} \%)\end{array}$ & $\begin{array}{l}\text { temp. } \\
\left({ }^{\circ} \mathrm{C}\right)\end{array}$ & $\begin{array}{l}\text { time } \\
\text { (h) }\end{array}$ & $\begin{array}{l}\text { conv. } \\
\text { 3ab }(\%)^{b}\end{array}$ \\
\hline 1 & 4.0 & 1.5 & 2.0 & 10 & 90 & $1 \mathrm{~h}$ & $>99$ \\
\hline $2^{c}$ & 4.0 & 1.5 & 2.0 & 10 & 120 & $1 \mathrm{~h}$ & 57 \\
\hline $3^{c}$ & 4.0 & 1.5 & 2.0 & 10 & 120 & $5 \mathrm{~h}$ & 61 \\
\hline 4 & 4.0 & 1.5 & 2.0 & 5 & 90 & $1 \mathrm{~h}$ & 89 \\
\hline 5 & 3.0 & 1.2 & 1.5 & 5 & 90 & $1 \mathrm{~h}$ & 56 \\
\hline 6 & 3.0 & 1.2 & 1.5 & 5 & 90 & $5 \mathrm{~h}$ & $>99$ \\
\hline 7 & 3.0 & 1.2 & 1.5 & 5 & 90 & $20 \mathrm{~h}$ & >99 (70) \\
\hline 8 & 3.0 & 1.2 & 1.5 & 2.5 & 90 & $1 \mathrm{~h}$ & 20 \\
\hline 9 & 3.0 & 1.2 & 1.5 & 2.5 & 90 & $20 \mathrm{~h}$ & 80 \\
\hline
\end{tabular}

${ }^{a}$ Reaction conditions: $1 \mathrm{a}(0.15 \mathrm{mmol})$ in toluene $(1.5 \mathrm{~mL}, 0.1 \mathrm{M})$ for $20 \mathrm{~h} .{ }^{b}$ Determined by ${ }^{1} \mathrm{H}$ NMR of the crude reaction mixture in presence of an internal standard. In parenthesis, isolated yield of 3ab after purification by column chromatography. ${ }^{c}$ Reaction run under microwave irradiation. 


\subsection{Scope of the reaction}

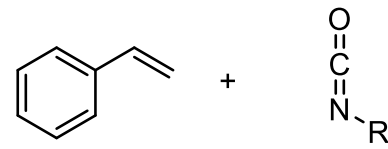

$1 \mathrm{a}$

2a-I (3.0 equiv.)

[(SIMes)CuCl] (5 mol\%) $\mathrm{B}_{2} \mathrm{pin}_{2}$ (1.2 equiv.), LiOtBu (1.5 equiv.)

Toluene [0.1], $90{ }^{\circ} \mathrm{C}, 20 \mathrm{~h}$<smiles>[R]NC(=O)C(C[Pb])c1ccccc1</smiles>

Isocyanates<smiles>O=C(Nc1ccccc1)C(C[18OH])c1ccccc1</smiles>

$75 \%$<smiles>COc1ccc(NC(=O)C(C[Pb])c2ccccc2)cc1</smiles>

$70 \%$<smiles>COc1ccccc1NC(=O)C(C[Pb])c1ccccc1</smiles>

$3 \mathrm{ac}^{a}$

$63 \%$<smiles>CN(C)c1ccc(NC(=O)C(C[Pb])c2ccccc2)cc1</smiles>

$60 \%$<smiles>O=C(Nc1ccc2c(c1)OCO2)C(C[Pb])c1ccccc1</smiles>

$3 a$ e

$61 \%$<smiles>Cc1cc(NC(=O)C(C[Pb])c2ccccc2)cc([N+](=O)[O-])c1</smiles><smiles>O=C(Nc1ccc(Br)cc1)C(CP)c1ccccc1</smiles><smiles>O=C(Nc1ccc(Cl)cc1)C(C[PbH])c1ccccc1</smiles><smiles>O=C(Nc1ccc(F)cc1)C(C[Pb])c1ccccc1</smiles><smiles>O=C(NC1CCCCC1)C(C[18OH])c1ccccc1</smiles>
$>5 \%$ conv.<smiles>CC(C)(C)NC(=O)C(C[Pb])c1ccccc1</smiles>

$n r$<smiles>CCCCCCCNC(=O)C(C[18OH])c1ccccc1</smiles>

$n r$

Figure S2. Effect of the structure of the isocyanate on the Cu-catalyzed borylative carboxamidation of styrene $(0.3 \mathrm{mmol})$ scale. ${ }^{a}[(\mathrm{SIMes}) \mathrm{CuCl}] 10 \mathrm{~mol} \%{ }^{b}$ Determined by ${ }^{1} \mathrm{H}$ NMR using an internal standard. 


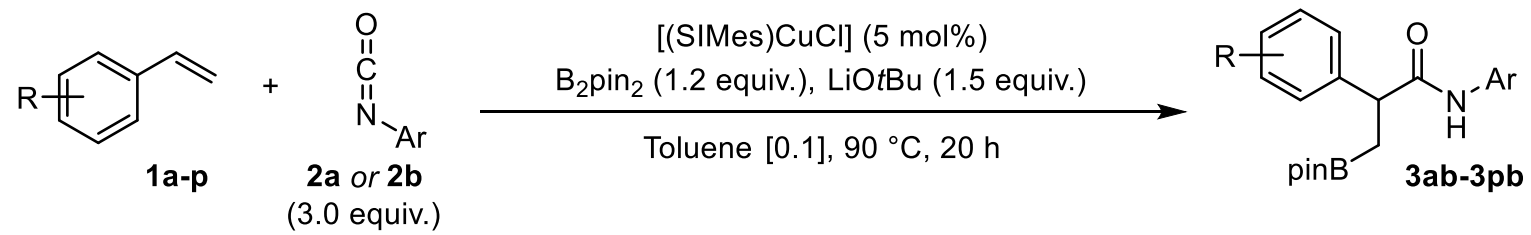

Vinylarenes<smiles>COc1ccc(NC(=O)C(C[Pb])c2ccc(OC)cc2)cc1</smiles><smiles>COc1ccc(NC(=O)C(C[Pb])c2ccc(F)cc2)cc1</smiles><smiles>COc1ccc(NC(=O)C(C[Pb])c2cccc(Br)c2)cc1</smiles><smiles>COc1ccc(NC(=O)C(C[18OH])c2ccc(C([18OH])([18OH])C[Pb])cc2)cc1</smiles><smiles>COc1ccc(NC(=O)C(C[Pb])c2ccccc2NC(=O)C(C[Pb])c2ccc(N3CCOCC3)cc2)cc1</smiles><smiles>O=C(Nc1ccccc1)C(C[18OH])c1ccc(-c2ccccn2)cc1</smiles><smiles>COc1ccc(NC(=O)C(C[Pb])c2ccc3c(ccn3C(=O)c3ccccc3)c2)cc1</smiles><smiles>COc1ccc(NC(=O)C(C[Pb])c2ccccc2)cc1</smiles><smiles>COc1ccc(NC(=O)C(C[Pb])c2ccc(B3OC(C)(C)C(C)(C)O3)cc2)cc1</smiles><smiles>COc1ccc(NC(=O)C(C[Pb])c2ccc(P(c3ccccc3)c3ccccc3)cc2)cc1</smiles>

TBSO $\mathrm{Me}$

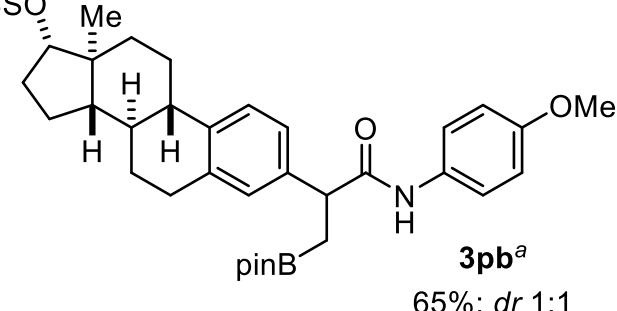

Figure S3. Cu-catalyzed borylative carboxamidation of vinylarenes $(0.3 \mathrm{mmol}$ scale). Yields of isolated product after purification. ${ }^{a}[(\mathrm{SIMes}) \mathrm{CuCl}] 10 \mathrm{~mol} \%{ }^{b} 48 \mathrm{~h}$. 


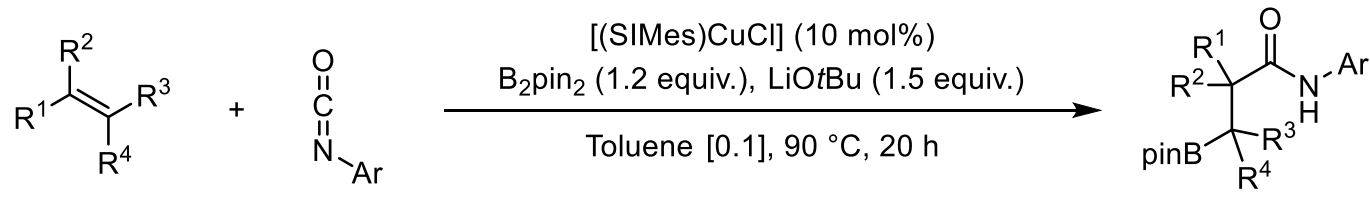

1q-x

2a-b

(3.0 equiv.)

3

Styrene derivatives<smiles>CC(CCc1ccccc1)(C(=O)Nc1ccccc1)c1ccccc1</smiles>

3qa

$24 \%$<smiles>COc1ccc(NC(=O)C(c2ccccc2)[C@H](Cc2ccccc2)c2ccccc2)cc1</smiles>

from 2-propenylbenzofuran:

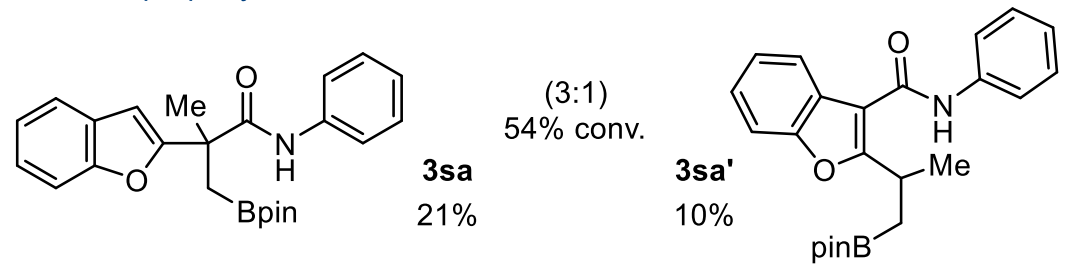

Conjugated 1,3-dienes<smiles>C=C(CCC=C(C)C)C(CP)C(=O)Nc1ccc(OC)cc1</smiles><smiles>C=C(C1CCCCC1)C(Cc1ccccc1)C(=O)Nc1ccc(OC)cc1</smiles>

$\mathrm{MeO}$ $57 \%$

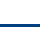




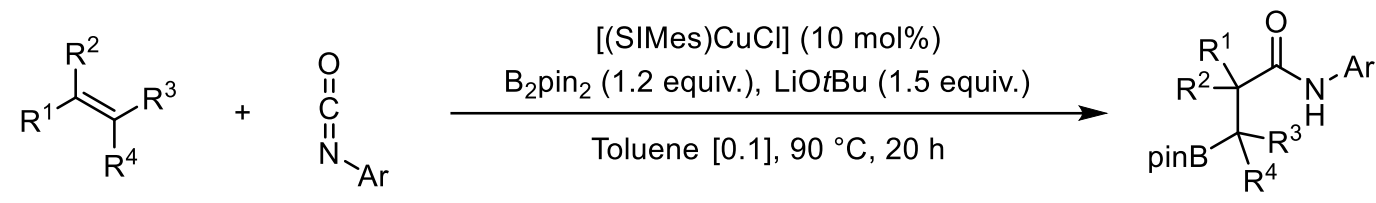

$2 b$

3

(3.0 equiv.)

Limitations (<5\% conv.)<smiles>C=Cc1ccc(C(F)(F)F)cc1</smiles><smiles>C=Cc1ccc(N)cc1</smiles><smiles>C=Cc1ccc(OC)nc1</smiles><smiles>C=Cc1cnc2ccccc2c1</smiles><smiles>C=Cc1coc2ccccc12</smiles><smiles>C=Cc1cc2ccccc2o1</smiles><smiles>C=C[14CH2][Te]</smiles><smiles>C1=CCCCC1</smiles><smiles>CC=Cc1ccccc1</smiles><smiles>C=Cn1c2ccccc2c2ccccc21</smiles><smiles>CCOC(=O)/C=C/c1ccccc1</smiles><smiles>C=CC=Cc1ccccc1</smiles><smiles>C=CC(=C)c1ccccc1</smiles><smiles>C=C(C)C(=C)C</smiles><smiles>C=CC#Cc1ccccc1</smiles><smiles>C=CCC1CCCCC1</smiles>

Figure S5. Limitations of the Cu-catalyzed borylative carboxamidation of alkenes (0.2-0.3 mmol scale). 
General procedure for the copper-catalyzed borylative carboxamidation of olefins with (SIMes)CuCl

In a $10 \mathrm{~mL}$ Schlenk tube, [(SIMes)CuCl] $(6.1 \mathrm{mg}, 0.015 \mathrm{mmol}, 5.0 \mathrm{~mol} \%)$, LiOtBu (36 mg, 0.45 mmol, 1.5 equiv.) and $B_{2} p_{i n}(91 \mathrm{mg}, 0.36 \mathrm{mmol}, 1.2$ equiv.) were dissolved in $3.0 \mathrm{~mL}$ of anhydrous and degassed toluene $(0.1 \mathrm{M})$. The resulting dark solution was stirred for $10 \mathrm{~min}$ at $23^{\circ} \mathrm{C}$, then olefin $1(0.3 \mathrm{mmol}, 1.0$ equiv.) was added neat with a micro-syringe, if liquid, or dissolved in $0.5 \mathrm{~mL}$ of toluene, if solid. The resulting mixture was stirred for $5 \mathrm{~min}$ at $23^{\circ} \mathrm{C}$, then the isocyanate $2(0.9 \mathrm{mmol}, 3.0$ equiv.) was added neat with a micro-syringe, if liquid, or dissolved in $0.5 \mathrm{~mL}$ of toluene, if solid. The sealed tube was stirred at $90^{\circ} \mathrm{C}$ for $20 \mathrm{~h}$. After 20 $\mathrm{h}$, the reaction mixture was cooled to $23^{\circ} \mathrm{C}$ and quenched by addition of a saturated aqueous $\mathrm{NH}_{4} \mathrm{Cl}$ solution $(1.0 \mathrm{~mL})$. The reaction mixture was diluted with ethyl acetate $(5 \mathrm{~mL})$ and water $(5 \mathrm{~mL})$ and extracted with ethyl acetate $(3 \times 10 \mathrm{~mL})$. The combined organic phases were dried over sodium sulfate and the solvent was removed under reduced pressure. Conversion into the borylated secondary amide 3 was assessed by ${ }^{1} \mathrm{H}$ NMR analysis of the crude residue in presence of an internal standard. Purification by flash chromatography on silica gel afforded the pure borylated secondary amide 3 . 
N,2-diphenyl-3-(4,4,5,5-tetramethyl-1,3,2-dioxaborolan-2-yl)propanamide 3aa

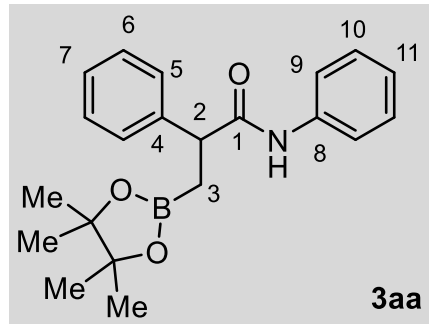

This product was prepared according to the general procedure for the copper-catalyzed borylative carboxamidation of olefins using styrene $1 \mathrm{a}$ (31 mg, $0.3 \mathrm{mmol}, 1.0$ equiv.), [(SIMes)CuCl] $(6.1 \mathrm{mg}$, $0.015 \mathrm{mmol}, 5.0 \mathrm{~mol} \%$ ), LiOtBu (36 mg, $0.45 \mathrm{mmol}, 1.5$ equiv.), $\mathrm{B}_{2} \mathrm{pin}_{2}(91 \mathrm{mg}, 0.36 \mathrm{mmol}, 1.2$ equiv.) and isocyanatobenzene $\mathbf{2 a}$ $(0.098 \mathrm{~mL}, 0.9 \mathrm{mmol}, 3.0$ equiv.) in toluene $(3.0 \mathrm{~mL}, 0.1 \mathrm{M})$. Reaction time: $20 \mathrm{~h} .91 \%$ conversion into 3aa determined by ${ }^{1} \mathrm{H}$ NMR analysis of the crude reaction mixture in presence of an internal standard. Purification by flash chromatography (pentane/ethyl acetate 9:1) gave pure 3aa as a white solid ( $81 \mathrm{mg}, 0.23 \mathrm{mmol}, 75 \%$ yield).

TLC: pentane/ethyl acetate $4: 1, \mathrm{R}_{\mathrm{f}}=0.3$.

${ }^{1} \mathrm{H}$ NMR $\left(400 \mathrm{MHz}, \mathrm{CDCl}_{3}\right) \delta(\mathrm{ppm})=7.40(\mathrm{~m}, 2 \mathrm{H}, \mathrm{H}-9), 7.36(\mathrm{~m}, 4 \mathrm{H}), 7.26(\mathrm{~m}, 3 \mathrm{H}), 7.09$ (bs, $1 \mathrm{H}, \mathrm{N}-H), 7.05\left(\mathrm{t},{ }^{3} \mathrm{~J}_{\mathrm{HH}}=6.9 \mathrm{~Hz}, 1 \mathrm{H}, H-11\right), 3.87$ (dd, $\left.{ }^{3} \mathrm{~J}_{\mathrm{HH}}=9.6,6.5 \mathrm{~Hz}, 1 \mathrm{H}, H-2\right), 1.67$ (dd, $\left.{ }^{2} J_{\mathrm{HH}}=15.8,{ }^{3} J_{\mathrm{HH}}=9.6 \mathrm{~Hz}, 1 \mathrm{H}, H-3\right), 1.30\left(\mathrm{dd},{ }^{2} J_{\mathrm{HH}}=15.8,{ }^{3} J_{\mathrm{HH}}=6.5 \mathrm{~Hz}, 1 \mathrm{H}, H-3\right.$ ), $1.21(\mathrm{~s}$, 6H, $H$-MeBpin), 1.19 (s, 6H, H-MeBpin).

${ }^{13} \mathrm{C}\left\{{ }^{1} \mathrm{H}\right\}$ NMR $\left(101 \mathrm{MHz}, \mathrm{CDCl}_{3}\right) \delta(\mathrm{ppm})=172.6(C-1), 141.7(C-4), 138.2(C-8), 129.2(\mathrm{CH})$, $129.0(\mathrm{CH}), 128.1(\mathrm{CH}), 127.5(\mathrm{CH}-7), 124.1(\mathrm{CH}-11), 119.7(\mathrm{CH}-9), 83.5$ (CO-Bpin), 50.1 (CH-2), 24.9 ( $\left.\mathrm{CH}_{3}-\mathrm{MeBpin}\right), 24.8$ ( $\left.\mathrm{CH}_{3}-\mathrm{MeBpin}\right), 17.0$ (bs, $\left.\mathrm{CH}_{2}-3\right)$.

${ }^{11} \mathbf{B}$ NMR $\left(128 \mathrm{MHz} \mathrm{CDCl}_{3}\right) \delta(\mathrm{ppm})=33.9$.

HRMS (ESI +): calculated for $\mathrm{C}_{21} \mathrm{H}_{26} \mathrm{BNO}_{3}[\mathrm{M}+\mathrm{H}]^{+}:$352.2083; found: 352.2074.

IR (neat): $v\left(\mathrm{~cm}^{-1}\right)=3309,1661,1600,1539,1496,1440,1369,1320,1142,967,846,753$, 695.

m.p.: $128-130^{\circ} \mathrm{C}$ 
$N$-(4-methoxyphenyl)-2-phenyl-3-(4,4,5,5-tetramethyl-1,3,2-dioxaborolan-2-yl)propanamide

$3 a b$

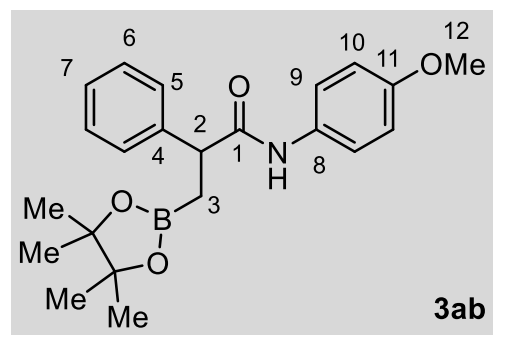

This product was prepared according to the general procedure for the copper-catalyzed borylative carboxamidation of olefins using styrene $1 \mathrm{a}$ (31 $\mathrm{mg}, 0.3 \mathrm{mmol}, 1.0$ equiv.), [(SIMes)CuCl] (6.1 mg, $0.015 \mathrm{mmol}, 5.0 \mathrm{~mol} \%$ ), LiOtBu (36 mg, $0.45 \mathrm{mmol}$, 1.5 equiv.), $B_{2}$ pin $_{2}$ (91 mg, $0.36 \mathrm{mmol}, 1.2$ equiv.) and 1 isocyanato-4-methoxybenzene $\mathbf{2 b}(0.117 \mathrm{~mL}, 0.9 \mathrm{mmol}, 3.0$ equiv.) in toluene ( $3.0 \mathrm{~mL}, 0.1 \mathrm{M})$. Reaction time: 20 h. $>99 \%$ conversion into 3ab determined by ${ }^{1} \mathrm{H}$ NMR analysis of the crude reaction mixture in presence of an internal standard. Purification by flash chromatography (pentane/ethyl acetate $4: 1$ ) gave pure $3 \mathrm{ab}$ as a white solid ( $80 \mathrm{mg}, 0.21 \mathrm{mmol}, 70 \%$ yield).

TLC: pentane/ethyl acetate $4: 1, \mathrm{R}_{\mathrm{f}}=0.2$.

${ }^{1} \mathrm{H}$ NMR $\left(500 \mathrm{MHz}, \mathrm{CDCl}_{3}\right) \delta(\mathrm{ppm})=7.35(\mathrm{~m}, 4 \mathrm{H}, \mathrm{H}-5+\mathrm{H}-6), 7.31\left(\mathrm{~d},{ }^{3} \mathrm{JHH}_{\mathrm{H}}=9.0 \mathrm{~Hz}, 2 \mathrm{H}, \mathrm{H}-\right.$ 9), $7.28(\mathrm{~m}, 1 \mathrm{H}, H-7), 6.99(\mathrm{bs}, 1 \mathrm{H}, \mathrm{N}-H), 6.79\left(\mathrm{~d},{ }^{3} \mathrm{~J}_{\mathrm{HH}}=9.0 \mathrm{~Hz}, 2 \mathrm{H}, H-10\right), 3.85\left(\mathrm{dd},{ }^{3} J_{\mathrm{HH}}=\right.$ 9.6, $6.6 \mathrm{~Hz}, 1 \mathrm{H}, H-2), 3.75(\mathrm{~s}, 3 \mathrm{H}, H-12), 1.66\left(\mathrm{dd},{ }^{2} J_{\mathrm{HH}}=15.7,{ }^{3} J_{\mathrm{HH}}=9.6 \mathrm{~Hz}, 1 \mathrm{H}, H-3\right), 1.29$ (dd, ${ }^{2} J_{H H}=15.7,{ }^{3} J_{H H}=6.6 \mathrm{~Hz}, 1 \mathrm{H}, H-3$ '), 1.20 (s, 6H, H-MeBpin), 1.19 (s, 6H, H-MeBpin).

${ }^{13} \mathrm{C}\left\{{ }^{1} \mathrm{H}\right\}$ NMR $\left(126 \mathrm{MHz}, \mathrm{CDCl}_{3}\right) \delta(\mathrm{ppm})=172.4(C-1), 156.3(C-11), 141.8(C-4), 131.4(C-$ 8), 129.1 ( $\mathrm{CH}-6), 128.1$ ( $\mathrm{CH}-5), 127.5(\mathrm{CH}-7), 121.5$ ( $\mathrm{CH}-9), 114.1(\mathrm{CH}-10), 83.4$ (CO-Bpin), 55.6 ( $\left.\mathrm{CH}_{3}-12\right)$, 49.9 ( $\left.\mathrm{CH}-2\right), 24.9$ ( $\left.\mathrm{CH}_{3}-\mathrm{MeBpin}\right), 24.8\left(\mathrm{CH}_{3}-\mathrm{MeBpin}\right), 16.9$ (bs, $\left.\mathrm{CH}_{2}-3\right)$.

${ }^{11} \mathbf{B}$ NMR $\left(96 \mathrm{MHz}, \mathrm{CDCl}_{3}\right) \delta(\mathrm{ppm})=33.3$.

HRMS (ESI +): calculated for $\mathrm{C}_{22} \mathrm{H}_{28} \mathrm{BNO}_{4}[\mathrm{M}+\mathrm{H}]^{+}:$382.2189; found: 382.2187 .

IR (neat): $v\left(\mathrm{~cm}^{-1}\right)=3300,1657,1603,1511,1370,1322,1241,1143,829,699$.

m.p.: $145-146^{\circ} \mathrm{C}$ 
$N$-(2-methoxyphenyl)-2-phenyl-3-(4,4,5,5-tetramethyl-1,3,2-dioxaborolan-2-yl)propanamide

3ac

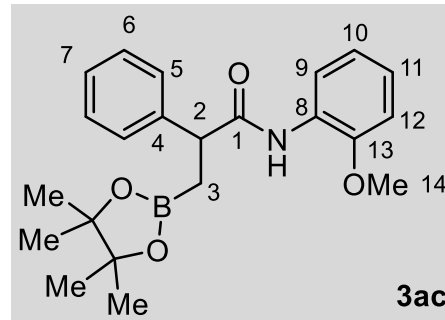

This product was prepared according to the general procedure for the copper-catalyzed borylative carboxamidation of olefins using styrene 1a (31 mg, $0.3 \mathrm{mmol}, 1.0$ equiv.), [(SIMes)CuCl] (12.2 mg, $0.030 \mathrm{mmol}, 10.0 \mathrm{~mol} \%$ ), LiOtBu (36 mg, $0.45 \mathrm{mmol}$, 3ac 1.5 equiv.), $B_{2} p_{i n}$ (91 mg, $0.36 \mathrm{mmol}, 1.2$ equiv.) and 1isocyanato-2-methoxybenzene $2 \mathrm{c}(0.120 \mathrm{~mL}, 0.9 \mathrm{mmol}, 3.0$ equiv.) in toluene $(3.0 \mathrm{~mL}, 0.1 \mathrm{M})$. Reaction time: 20 h. $77 \%$ conversion into 3ac determined by ${ }^{1} \mathrm{H}$ NMR analysis of the crude reaction mixture in presence of an internal standard. Purification by flash chromatography (pentane with acetone $5 \%$ ) gave pure 3 ac as a white solid (70 mg, $0.19 \mathrm{mmol}, 63 \%$ yield).

TLC: pentane with acetone $5 \%, \mathrm{R}_{\mathrm{f}}=0.3$.

${ }^{1} \mathrm{H}$ NMR $\left(400 \mathrm{MHz}, \mathrm{CDCl}_{3}\right) \delta(\mathrm{ppm})=8.35\left(\mathrm{dd},{ }^{3} \mathrm{~J}_{\mathrm{HH}}=7.9 \mathrm{~Hz},{ }^{4} \mathrm{~J}_{\mathrm{HH}} 1.5 \mathrm{~Hz}, 1 \mathrm{H}, H-9\right), 7.76$ (bs, $1 \mathrm{H}, \mathrm{N}-H), 7.42-7.31(\mathrm{~m}, 4 \mathrm{H}, \mathrm{H}-5+\mathrm{H}-6), 7.26\left(\mathrm{t},{ }^{3} \mathrm{JHH}_{\mathrm{HH}}=7.7 \mathrm{~Hz}, 1 \mathrm{H}, \mathrm{H}-7\right), 6.97\left(\mathrm{td},{ }^{3} J_{\mathrm{HH}}=7.8\right.$ $\left.\mathrm{Hz},{ }^{4} J_{\mathrm{HH}}=1.8 \mathrm{~Hz}, 1 \mathrm{H}, \mathrm{H}-11\right), 6.90\left(\mathrm{td},{ }^{3} J_{\mathrm{HH}}=7.7 \mathrm{~Hz},{ }^{4} J_{\mathrm{HH}}=1.4 \mathrm{~Hz}, 1 \mathrm{H}, \mathrm{H}-10\right), 6.77\left(\mathrm{dd},{ }^{3} J_{\mathrm{HH}}=\right.$ $\left.8.0 \mathrm{~Hz},{ }^{4} \mathrm{~J}_{\mathrm{HH}}=1.4 \mathrm{~Hz}, 1 \mathrm{H}, H-12\right), 3.90\left(\mathrm{dd},{ }^{3} \mathrm{~J}_{\mathrm{HH}}=9.0,7.5 \mathrm{~Hz}, 1 \mathrm{H}, H-2\right), 3.70(\mathrm{~s}, 3 \mathrm{H}, H-14)$, $1.66\left(\mathrm{dd},{ }^{2} J_{\mathrm{HH}}=15.6,{ }^{3} J_{\mathrm{HH}}=9.0 \mathrm{~Hz}, 1 \mathrm{H}, \mathrm{H}-3\right), 1.35\left(\mathrm{dd},{ }^{2} J_{\mathrm{HH}}=15.6,{ }^{3} J_{\mathrm{HH}}=7.5 \mathrm{~Hz}, 1 \mathrm{H}, H-3\right.$ '), 1.19 (s, 6H, H-MeBpin), 1.17 (s, 6H, H-MeBpin).

${ }^{13} \mathrm{C}\left\{{ }^{1} \mathrm{H}\right\}$ NMR $\left(101 \mathrm{MHz}, \mathrm{CDCl}_{3}\right) \delta(\mathrm{ppm})=172.4(C-1), 147.9(C-13), 141.7(C-4), 129.0(\mathrm{CH}-$ 6), $128.2(\mathrm{CH}-5), 128.1(\mathrm{C}-8) 127.4(\mathrm{CH}-7), 123.4(\mathrm{CH}-11), 121.2(\mathrm{CH}-10), 119.6(\mathrm{CH}-9)$, 110.0 (CH-12), 83.4 (CO-Bpin), $55.8\left(\mathrm{CH}_{3}-14\right), 50.4(\mathrm{CH}-2), 24.9\left(\mathrm{CH}_{3}-\mathrm{MeBpin}\right), 24.8\left(\mathrm{CH}_{3^{-}}\right.$ MeBpin), 16.6 (bs, $\left.\mathrm{CH}_{2}-3\right)$.

${ }^{11} \mathbf{B}$ NMR $\left(96 \mathrm{MHz}, \mathrm{CDCl}_{3}\right) \delta(\mathrm{ppm})=34.2$.

HRMS $(E S I+)$ : calculated for $\mathrm{C}_{22} \mathrm{H}_{28} \mathrm{BNO}_{4}[\mathrm{M}+\mathrm{H}]^{+}:$382.2189; found: 382.2187.

IR (neat): $v\left(\mathrm{~cm}^{-1}\right)=3396,1977,1682,1601,1520,1484,1459,1433,1369,1321,1250$, 1218, 1165, 1142, 1115, 1027, 968, 846, 746, 700.

m.p.: $120-123^{\circ} \mathrm{C}$ 
$N$-(4-(dimethylamino)phenyl)-2-phenyl-3-(4,4,5,5-tetramethyl-1,3,2-dioxaborolan-2-

yl)propanamide $3 \mathrm{ad}$

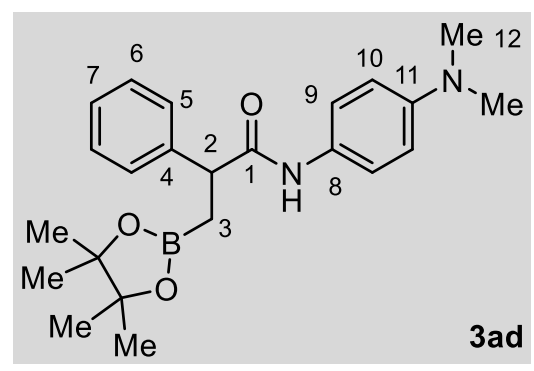

This product was prepared according to the general procedure for the copper-catalyzed borylative carboxamidation of olefins using styrene $1 \mathrm{a}(16 \mathrm{mg}, 0.15$ mmol, 1.0 equiv), [(SIMes)CuCl] (6.1 mg, $0.015 \mathrm{mmol}, 10.0$ mol\%), LiOtBu (18 mg, $0.23 \mathrm{mmol}, 1.5$ equiv), $\mathrm{B}_{2} \mathrm{pin}_{2}(46 \mathrm{mg}$, $0.18 \mathrm{mmol}, 1.2$ equiv) and 4 -isocyanato- $N, N$-dimethylaniline 2d $(0.73 \mathrm{mg}, 0.45 \mathrm{mmol}, 3.0$ equiv) in toluene (1.5 mL, $0.1 \mathrm{M})$. Reaction time: $20 \mathrm{~h} .67 \%$ conversion into 3ad determined by ${ }^{1} \mathrm{H}$ NMR analysis of the crude reaction mixture in presence of an internal standard. Purification by flash chromatography $\left(\mathrm{CH}_{2} \mathrm{Cl}_{2}\right.$ with $\left.\mathrm{MeOH} 2 \%\right)$ gave pure $3 \mathrm{ad}$ as a brown solid ( $35 \mathrm{mg}, 0.09 \mathrm{mmol}, 60 \%$ yield).

TLC: $\mathrm{CH}_{2} \mathrm{Cl}_{2}$ with $\mathrm{MeOH} 2 \%, \mathrm{R}_{\mathrm{f}}=0.3$.

${ }^{1} \mathrm{H}$ NMR $\left(400 \mathrm{MHz}, \mathrm{CDCl}_{3}\right) \delta(\mathrm{ppm})=7.26(\mathrm{~m}, 3 \mathrm{H}), 7.16(\mathrm{~m}, 4 \mathrm{H}), 6.81(\mathrm{bs}, 1 \mathrm{H}, \mathrm{N}-H), 6.55(\mathrm{~m}$, $2 \mathrm{H}$ ), $3.75\left(\mathrm{dd},{ }^{3} \mathrm{JHH}_{\mathrm{HH}}=9.6,6.6 \mathrm{~Hz}, 1 \mathrm{H}, \mathrm{H}-2\right), 2.79(\mathrm{~s}, 6 \mathrm{H}, \mathrm{H}-12), 1.56\left(\mathrm{dd},{ }^{2} J_{\mathrm{HH}}=15.7,{ }^{3} J_{\mathrm{HH}}=9.6\right.$ $\mathrm{Hz}, 1 \mathrm{H}, H-3), 1.20\left(\mathrm{dd},{ }^{2} J_{\mathrm{HH}}=15.7,{ }^{3} \mathrm{~J}_{\mathrm{HH}}=6.6 \mathrm{~Hz}, 1 \mathrm{H}, H-3\right), 1.11$ (s, 6H, H-MeBpin), 1.10 (s, $6 \mathrm{H}, \mathrm{H}$-MeBpin).

${ }^{13} \mathrm{C}\left\{{ }^{1} \mathrm{H}\right\}$ NMR $\left(126 \mathrm{MHz}, \mathrm{CDCl}_{3}\right) \delta(\mathrm{ppm})=172.2(\mathrm{C}-1), 148.0(\mathrm{C}-11), 142.0(\mathrm{C}-4), 129.1(\mathrm{CH}-$ 6), 128.2 ( $\mathrm{CH}-5), 127.4(\mathrm{CH}-7), 121.4(\mathrm{CH}-9), 113.2$ ( $\mathrm{CH}-10)$, 83.4 (CO-Bpin), 49.8 (CH-2), $41.1\left(\mathrm{CH}_{3}-12\right), 24.9$ ( $\left.\mathrm{CH}_{3}-\mathrm{MeBpin}\right), 24.8$ ( $\mathrm{CH}_{3}$-MeBpin), 17.0 (bs, $\left.\mathrm{CH}_{2}-3\right)$.

${ }^{11} \mathbf{B}$ NMR $\left(96 \mathrm{MHz}, \mathrm{CDCl}_{3}\right) \delta(\mathrm{ppm})=32.5$.

HRMS (ESI +): calculated for $\mathrm{C}_{17} \mathrm{H}_{20} \mathrm{BN}_{2} \mathrm{O}_{2}[\mathrm{M}+\mathrm{H}]^{+}:$: 295.1616; found: 295.1622 .

IR (neat): $v\left(\mathrm{~cm}^{-1}\right)=3307,2978,1654,1600,1518,1368,1321,1256,1142,698$.

m.p.: $164-167^{\circ} \mathrm{C}$ 
$N$-(benzo[d][1,3]dioxol-5-yl)-2-phenyl-3-(4,4,5,5-tetramethyl-1,3,2-dioxaborolan-2yl)propanamide 3ae

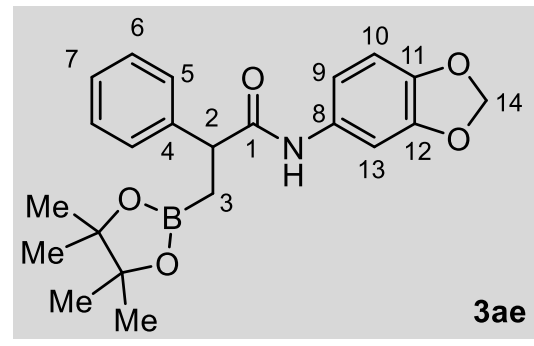

This product was prepared according to the general procedure for the copper-catalyzed borylative carboxamidation of olefins using styrene 1a $(31 \mathrm{mg}, 0.3$ mmol, 1.0 equiv.), [(SIMes)CuCl] $(6.1 \mathrm{mg}, 0.015 \mathrm{mmol}, 5.0$ mol\%), LiOtBu (36 mg, 0.45 mmol, 1.5 equiv.), B Bin $_{2}$ (91 mg, $\quad 0.36$ mmol, 1.2 equiv.) and 5isocyanatobenzo[d][1,3]dioxole $\mathbf{2 e}(147 \mathrm{mg}, 0.9 \mathrm{mmol}, 3.0$ equiv.) in toluene $(3.0 \mathrm{~mL}, 0.1 \mathrm{M})$. Reaction time: 20 h. $84 \%$ conversion into 3ae determined by ${ }^{1} \mathrm{H}$ NMR analysis of the crude reaction mixture in presence of an internal standard. Purification by flash chromatography (pentane/diethyl ether $4: 1$ to 3:1) gave pure 3ae as a white solid (72 mg, $0.18 \mathrm{mmol}, 61 \%$ yield).

TLC: pentane/diethyl ether 3:2, $R_{f}=0.3$.

${ }^{1} \mathrm{H}$ NMR $\left(400 \mathrm{MHz}, \mathrm{CDCl}_{3}\right) \delta(\mathrm{ppm})=7.37-7.33(\mathrm{~m}, 4 \mathrm{H}, \mathrm{H}-5+\mathrm{H}-6), 7.28(\mathrm{~m}, 1 \mathrm{H}, \mathrm{H}-7), 7.18$ (s, $1 \mathrm{H}, H-13), 6.99$ (bs, $1 \mathrm{H}, \mathrm{N}-H), 6.66\left(\mathrm{~d},{ }^{3} \mathrm{~J}_{\mathrm{HH}}=8.3 \mathrm{~Hz}, 1 \mathrm{H}, \mathrm{H}-10\right), 6.62\left(\mathrm{dd},{ }^{3} \mathrm{JHH}_{\mathrm{HH}}=8.3,{ }^{4} \mathrm{JHH}_{\mathrm{HH}}\right.$ $=2.0 \mathrm{~Hz}, 1 \mathrm{H}, H-9), 5.90(\mathrm{~s}, 2 \mathrm{H}, H-13), 3.84\left(\mathrm{dd},{ }^{3} J_{\mathrm{HH}}=9.6,6.5 \mathrm{~Hz}, 1 \mathrm{H}, \mathrm{H}-2\right), 1.65\left(\mathrm{dd},{ }^{2} J_{\mathrm{HH}}=\right.$ $\left.15.8,{ }^{3} J_{H H}=9.6 \mathrm{~Hz}, 1 \mathrm{H}, H-3\right), 1.27\left(\mathrm{dd},{ }^{2} J_{H H}=15.8,{ }^{3} J_{H H}=6.5 \mathrm{~Hz}, 1 \mathrm{H}, H-3\right.$ '), $1.21(\mathrm{~s}, 6 \mathrm{H}, H-$ MeBpin), 1.19 (s, 6H, H-MeBpin).

${ }^{13} \mathrm{C}\left\{{ }^{1} \mathrm{H}\right\}$ NMR $\left.(101 \mathrm{MHz}, \mathrm{CDCl})_{3}\right) \delta(\mathrm{ppm})=172.4(C-1), 147.8(C-12), 144.2(C-11), 141.7(C-$ 4), $132.5(\mathrm{C}-8), 129.1(\mathrm{CH}-6), 128.1(\mathrm{CH}-5), 127.5(\mathrm{CH}-7), 112.7(\mathrm{CH}-9), 108.0(\mathrm{CH}-10), 102.7$ (CH-13), $101.3\left(\mathrm{CH}_{2}-14\right), 83.5$ (CO-Bpin), $49.9(\mathrm{CH}-2), 24.9\left(\mathrm{CH}_{3}-\mathrm{MeBpin}\right), 24.8\left(\mathrm{CH}_{3^{-}}\right.$ MeBpin), 17.0 (bs, $\mathrm{CH}_{2}-3$ ).

${ }^{11}$ B NMR $\left(96 \mathrm{MHz}, \mathrm{CDCl}_{3}\right) \delta(\mathrm{ppm})=32.9$.

HRMS $(\mathrm{ESI}+)$ : calculated for $\mathrm{C}_{22} \mathrm{H}_{26} \mathrm{BNO}_{5}[\mathrm{M}+\mathrm{H}]^{+}:$: 396.1986; found: 396.1974.

IR (neat): $v\left(\mathrm{~cm}^{-1}\right)=3296,2978,1657,1616,1541,1502,1489,1370,1321,1216,1190$, 1143, 1039, 968, 901, 846, 805, 699.

m.p.: $154-156^{\circ} \mathrm{C}$ 
$N$-(3,5-dimethylphenyl)-2-phenyl-3-(4,4,5,5-tetramethyl-1,3,2-dioxaborolan-2-yl)propanamide 3af

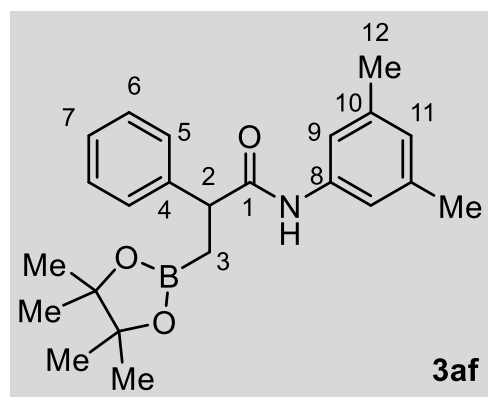
This product was prepared according to the general procedure for the copper-catalyzed borylative carboxamidation of olefins using styrene $1 \mathrm{a}$ (31 $\mathrm{mg}, 0.3 \mathrm{mmol}, 1.0$ equiv.), [(SIMes)CuCl] (12.2 mg, $0.015 \mathrm{mmol}, 5.0 \mathrm{~mol} \%$ ), LiOtBu (36 mg, $0.45 \mathrm{mmol}$, 1.5 equiv.), $B_{2} p_{1}$ (91 mg, 0.36 mmol, 1.2 equiv.) and $1-$ isocyanato-3,5-dimethylbenzene $2 \mathrm{f}(0.127 \mathrm{~mL}, 0.9 \mathrm{mmol}, 3.0$ equiv.) in toluene $(3.0 \mathrm{~mL}, 0.1 \mathrm{M})$. Reaction time: $20 \mathrm{~h}$. $75 \%$ conversion into 3af determined by ${ }^{1} \mathrm{H}$ NMR analysis of the crude reaction mixture in presence of an internal standard. Purification by flash chromatography (pentane/acetone 10:1) gave pure 3af as a white solid (60 mg, $0.16 \mathrm{mmol}, 53 \%$ yield).

TLC: pentane/acetone 9:1, $R_{f}=0.3$.

${ }^{1} \mathbf{H}$ NMR $\left(400 \mathrm{MHz}, \mathrm{CDCl}_{3}\right) \delta(\mathrm{ppm})=7.36-7.30(\mathrm{~m}, 4 \mathrm{H}, \mathrm{H}-5+\mathrm{H}-6), 7.28(\mathrm{~m}, 1 \mathrm{H}, \mathrm{H}-7), 7.05$ (s, 2H, H-9), 7.00 (bs, $1 \mathrm{H}, \mathrm{N}-H), 6.69$ (s, $1 \mathrm{H}, H-11$ ), 3.84 (dd, $\left.{ }^{3} J_{\mathrm{HH}}=9.3,6.8 \mathrm{~Hz}, 1 \mathrm{H}, H-2\right), 2.24$ (s, 6H, H-12), $1.66\left(\mathrm{dd},{ }^{2} J_{\mathrm{HH}}=15.8,{ }^{3} J_{\mathrm{HH}}=9.3 \mathrm{~Hz}, 1 \mathrm{H}, H-3\right), 1.30\left(\mathrm{dd},{ }^{2} J_{\mathrm{HH}}=15.8,{ }^{3} J_{\mathrm{HH}}=6.8\right.$ Hz, 1H, H-3'), 1.20 (s, 6H, H-MeBpin), 1.18 (s, 6H, H-MeBpin).

${ }^{13} \mathrm{C}\left\{{ }^{1} \mathrm{H}\right\}$ NMR $\left(101 \mathrm{MHz}, \mathrm{CDCl}_{3}\right) \delta(\mathrm{ppm})=172.5(C-1), 141.7(C-4), 138.7(C-10), 138.1(C-$ 8), 129.1 ( $\mathrm{CH}-6), 128.1$ ( $\mathrm{CH}-5), 127.5$ ( $\mathrm{CH}-7), 125.8$ ( $\mathrm{CH}-11), 117.4$ (CH-9), 83.5 (CO-Bpin), 50.1 ( $\mathrm{CH}-2)$, 24.9 ( $\left.\mathrm{CH}_{3}-\mathrm{MeBpin}\right), 24.8\left(\mathrm{CH}_{3}-\mathrm{MeBpin}\right), 21.4\left(\mathrm{CH}_{3}-12\right), 16.8$ (bs, $\left.\mathrm{CH}_{2}-3\right)$.

${ }^{11} \mathrm{~B}$ NMR $\left(128 \mathrm{MHz}, \mathrm{CDCl}_{3}\right) \delta(\mathrm{ppm})=33.9$.

HRMS (ESI +): calculated for $\mathrm{C}_{22} \mathrm{H}_{30} \mathrm{BNO}_{3}[\mathrm{M}+\mathrm{H}]^{+}:$380.2396; found: 380.2377.

IR (neat): $v\left(\mathrm{~cm}^{-1}\right)=3310,2978,1660,1616,1553,1454,1370,1321,1143,968,845,698$.

m.p.: $134-136^{\circ} \mathrm{C}$ 
$N$-(4-bromophenyl)-2-phenyl-3-(4,4,5,5-tetramethyl-1,3,2-dioxaborolan-2-yl)propanamide

3ag

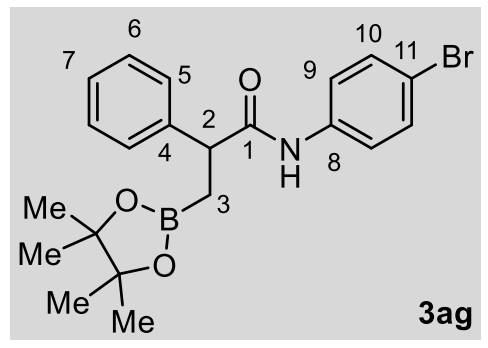

This product was prepared according to the general procedure for the copper-catalyzed borylative carboxamidation of olefins using styrene 1a (31 mg, $0.3 \mathrm{mmol}, 1.0$ equiv.), [(SIMes)CuCl] (6.1 mg, $0.015 \mathrm{mmol}, 5.0 \mathrm{~mol} \%$ ), LiOtBu (36 mg, $0.45 \mathrm{mmol}$, 1.5 equiv.), $B_{2} \operatorname{pin}_{2}(91 \mathrm{mg}, 0.36 \mathrm{mmol}, 1.2$ equiv.) and 1 -bromo4-isocyanatobenzene $\mathbf{2 g}$ (178 $\mathrm{mg}, 0.9 \mathrm{mmol}, 3.0$ equiv.) in toluene (3.0 mL, $0.1 \mathrm{M})$. Reaction time: $20 \mathrm{~h}$. 77\% conversion into 3ag determined by ${ }^{1} \mathrm{H}$ NMR analysis of the crude reaction mixture in presence of an internal standard. Purification by flash chromatography (pentane/diethyl ether $4: 1$ ) gave pure 3 ag as a white solid ( $80 \mathrm{mg}, 0.19 \mathrm{mmol}$, $62 \%$ yield).

TLC: pentane/ diethyl ether $4: 1, \mathrm{R}_{\mathrm{f}}=0.2$.

${ }^{1} \mathrm{H}$ NMR $\left(300 \mathrm{MHz}, \mathrm{CDCl}_{3}\right) \delta(\mathrm{ppm})=7.42-7.27$ (m, 9H, $\mathrm{H}$-Ar), 7.08 (bs, 1H, N-H), 3.86 (dd, $\left.{ }^{3} J_{\mathrm{HH}}=9.7,6.4 \mathrm{~Hz}, 1 \mathrm{H}, \mathrm{H}-2\right), 1.66\left(\mathrm{dd},{ }^{2} J_{\mathrm{HH}}=15.8,{ }^{3} J_{\mathrm{HH}}=9.7 \mathrm{~Hz}, 1 \mathrm{H}, H-3\right), 1.28\left(\mathrm{dd},{ }^{2} J_{\mathrm{HH}}=\right.$ $\left.15.8,{ }^{3} \mathrm{JHH}_{\mathrm{H}}=6.4 \mathrm{~Hz}, 1 \mathrm{H}, H-3^{\prime}\right), 1.21$ (s, 6H, H-MeBpin), 1.18 (s, 6H, H-MeBpin).

${ }^{13} \mathrm{C}\left\{{ }^{1} \mathrm{H}\right\}$ NMR $\left(75 \mathrm{MHz}, \mathrm{CDCl}_{3}\right) \delta(\mathrm{ppm})=172.6(C-1), 141.4(C-4), 137.3(C-8), 131.9(\mathrm{CH}-$ 10), 129.2 (CH-6), 128.1 (CH-5), 127.7 (CH-7), 121.2 (CH-9), 116.7 (C-11), 83.5 (CO-Bpin), 50.1 ( $\mathrm{CH}-2)$, 25.0 ( $\left.\mathrm{CH}_{3}-\mathrm{MeBpin}\right), 24.8$ ( $\left.\mathrm{CH}_{3}-\mathrm{MeBpin}\right)$. The boron-bound carbon $\mathrm{CH}_{2}-3$ was not detected due to quadrupolar relaxation.

${ }^{11} \mathbf{B}$ NMR $\left(96 \mathrm{MHz}, \mathrm{CDCl}_{3}\right) \delta(\mathrm{ppm})=32.2$.

HRMS $(\mathrm{ESI}+)$ : calculated for $\mathrm{C}_{21} \mathrm{H}_{25} \mathrm{BBrNO}_{3}[\mathrm{M}+\mathrm{H}]^{+}: 432.1170$; found: 432.1174 .

IR (neat): $v\left(\mathrm{~cm}^{-1}\right)=3350,2985,1681,1590,1523,1490,1391,1370,1332,1243,1141,827$, 699.

m.p.: $212-214^{\circ} \mathrm{C}$ 
$N$-(4-Chlorophenyl)-2-phenyl-3-(4,4,5,5-tetramethyl-1,3,2-dioxaborolan-2-yl)propanamide

$3 a h$

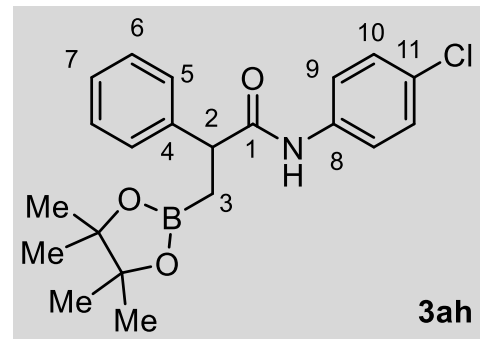

This product was prepared according to the general procedure for the copper-catalyzed borylative carboxamidation of olefins using styrene 1a (31 mg, $0.3 \mathrm{mmol}, 1.0$ equiv), [(SIMes)CuCl] (6.1 mg, $0.015 \mathrm{mmol}, 5.0 \mathrm{~mol} \%$ ), LiOtBu (36 mg, $0.45 \mathrm{mmol}$, 1.5 equiv), $B_{2}$ pin $_{2}$ (91 mg, $0.36 \mathrm{mmol}, 1.2$ equiv) and 1-chloro4-isocyanatobenzene $2 \mathrm{~h}(0.120 \mathrm{~mL}, 0.9 \mathrm{mmol}, 3.0$ equiv) in toluene (3.0 mL, $0.1 \mathrm{M})$. Reaction time: $20 \mathrm{~h} .84 \%$ conversion into 3ah determined by ${ }^{1} \mathrm{H}$ NMR analysis of the crude reaction mixture in presence of an internal standard. Purification by flash chromatography (pentane/diethyl ether $4: 1$ ) gave pure 3 ah as a white solid $(70 \mathrm{mg}, 0.18 \mathrm{mmol}$, $61 \%$ yield).

TLC: pentane/diethyl ether $4: 1, R_{f}=0.2$.

${ }^{1} \mathrm{H}$ NMR $\left(500 \mathrm{MHz}, \mathrm{CDCl}_{3}\right) \delta(\mathrm{ppm})=7.37\left(\mathrm{~d},{ }^{3} \mathrm{JHH}_{\mathrm{HH}}=8.9 \mathrm{~Hz}, 2 \mathrm{H}, \mathrm{H}-9\right), 7.34(\mathrm{~s}, 4 \mathrm{H}, \mathrm{H}-5+\mathrm{H}-6)$, $7.28(\mathrm{~m}, 1 \mathrm{H}, H-7), 7.21\left(\mathrm{~d},{ }^{3} \mathrm{~J}_{\mathrm{HH}}=8.9 \mathrm{~Hz}, 2 \mathrm{H}, \mathrm{H}-10\right), 7.09$ (bs, $\left.1 \mathrm{H}, \mathrm{N}-H\right), 3.86$ (dd, ${ }^{3} \mathrm{~J}_{\mathrm{HH}}=9.7$, $6.4 \mathrm{~Hz}, 1 \mathrm{H}, \mathrm{H}-2), 1.66\left(\mathrm{dd},{ }^{2} J_{\mathrm{HH}}=15.8,{ }^{3} J_{\mathrm{HH}}=9.7 \mathrm{~Hz}, 1 \mathrm{H}, H-3\right.$ '), $1.28\left(\mathrm{dd},{ }^{2} J_{H H}=15.8,{ }^{3} J_{H H}=\right.$ $6.4 \mathrm{~Hz}, 1 \mathrm{H}, H$-3'), 1.21 (s, 6H, $H$-MeBpin), 1.19 (s, 6H, $H$-MeBpin).

${ }^{13} \mathrm{C}\left\{{ }^{1} \mathrm{H}\right\}$ NMR $\left(126 \mathrm{MHz}, \mathrm{CDCl}_{3}\right) \delta(\mathrm{ppm})=172.6(C-1), 141.4(C-4), 136.8(C-8), 129.2(\mathrm{CH}-$ 10), 129.1 (C-11), 129.0 ( $\mathrm{CH}-6), 128.1$ ( $\mathrm{CH}-5), 127.7$ (CH-7), 120.9 (CH-9), 83.5 (CO-Bpin), 50.1 ( $\mathrm{CH}-2)$, 25.0 ( $\mathrm{CH}_{3}$-MeBpin), 24.8 ( $\mathrm{CH}_{3}$-MeBpin), 16.9 (bs, $\left.\mathrm{CH}_{2}-3\right)$.

${ }^{11} \mathbf{B}$ NMR $\left(96 \mathrm{MHz}, \mathrm{CDCl}_{3}\right) \delta(\mathrm{ppm})=32.2$

HRMS (ESI +): calculated for $\mathrm{C}_{21} \mathrm{H}_{25} \mathrm{BCINO}_{3}[\mathrm{M}+\mathrm{Na}]^{+}:$:08.1513; found: 408.1501 .

IR (neat): $v\left(\mathrm{~cm}^{-1}\right)=3343,2983,1680,1595,1521,1492,1394,1368,1329,1242,1140,970$, $829,699$.

m.p.: $190-191^{\circ} \mathrm{C}$ 
$N$-(4-fluorophenyl)-2-phenyl-3-(4,4,5,5-tetramethyl-1,3,2-dioxaborolan-2-yl)propanamide 3ai<smiles>CC1(C)OB(C[C@H](C(=O)Nc2ccc(F)cc2)c2ccccc2)OC1(C)C</smiles>

This product was prepared according to the general procedure for the copper-catalyzed borylative carboxamidation of olefins using styrene $1 \mathrm{a}$ (31 $\mathrm{mg}, 0.3 \mathrm{mmol}, 1.0$ equiv.), [(SIMes)CuCl] (6.1 mg, $0.015 \mathrm{mmol}, 5.0 \mathrm{~mol} \%$ ), LiOtBu (36 mg, $0.45 \mathrm{mmol}, 1.5$ equiv.), $B_{2}$ pin 2 (91 mg, $0.36 \mathrm{mmol}, 1.2$ equiv.) and 1-fluoro-4isocyanatobenzene $\mathbf{2 i}(0.117 \mathrm{~mL}, 0.9 \mathrm{mmol}, 3.0$ equiv.) in toluene (3.0 mL, $0.1 \mathrm{M}$ ). Reaction time: $20 \mathrm{~h} .92 \%$ conversion into 3ai determined by ${ }^{1} \mathrm{H}$ NMR analysis of the crude reaction mixture in presence of an internal standard. Purification by flash chromatography (pentane/diethyl ether 4:1 to 7:3) gave pure 3ai as a white solid (91 mg, 0.25 $\mathrm{mmol}, 83 \%$ yield).

TLC: pentane/diethyl ether $4: 1, R_{f}=0.2$.

${ }^{1} \mathrm{H}$ NMR $\left(300 \mathrm{MHz}, \mathrm{CDCl}_{3}\right) \delta(\mathrm{ppm})=7.41-7.24(\mathrm{~m}, 7 \mathrm{H}, \mathrm{H}-\mathrm{Ar}), 7.13(\mathrm{bs}, 1 \mathrm{H}, \mathrm{N}-H), 6.92(\mathrm{~m}$, $2 \mathrm{H}, H$-Ar), $3.86\left(\mathrm{dd},{ }^{3} J_{\mathrm{HH}}=9.6,6.5 \mathrm{~Hz}, 1 \mathrm{H}, \mathrm{H}-2\right), 1.66\left(\mathrm{dd},{ }^{2} J_{\mathrm{HH}}=15.8,{ }^{3} J_{\mathrm{HH}}=9.6 \mathrm{~Hz}, 1 \mathrm{H}, \mathrm{H}-\right.$ 3), $1.29\left(\mathrm{dd},{ }^{2} \mathrm{JHH}_{\mathrm{HH}}=15.8,{ }^{3} \mathrm{~J}_{\mathrm{HH}}=6.5 \mathrm{~Hz}, 1 \mathrm{H}, \mathrm{H}-3\right.$ '), 1.20 (s, 6H, H-MeBpin), 1.18 (s, 6H, HMeBpin).

${ }^{13} \mathrm{C}\left\{{ }^{1} \mathrm{H}\right\} \operatorname{NMR}\left(75 \mathrm{MHz}, \mathrm{CDCl}_{3}\right) \delta(\mathrm{ppm})=172.6(C-1), 159.3\left(\mathrm{~d},{ }^{1} \mathrm{~J}_{\mathrm{CF}}=243.3 \mathrm{~Hz}, C-11\right), 141.6$ $(C-4), 134.2\left(\mathrm{~d},{ }^{4} \mathrm{~J}_{\mathrm{CF}}=2.8 \mathrm{~Hz}, \mathrm{C}-8\right), 129.2(\mathrm{CH}-6), 128.1(\mathrm{CH}-5), 127.6(\mathrm{CH}-7), 121.5\left(\mathrm{~d},{ }^{2} \mathrm{~J}_{\mathrm{CF}}\right.$ $=7.9 \mathrm{~Hz}, \mathrm{CH}-10), 115.6$ (d, $\left.{ }^{3} \mathrm{~J}_{\mathrm{CF}}=22.4 \mathrm{~Hz}, \mathrm{CH}-9\right), 83.5$ (CO-Bpin), $49.9(\mathrm{CH}-2), 24.9\left(\mathrm{CH}_{3^{-}}\right.$ MeBpin), 24.8 ( $\left.\mathrm{CH}_{3}-\mathrm{MeBpin}\right), 16.9$ (bs, $\left.\mathrm{CH}_{2}-3\right)$.

${ }^{11} \mathbf{B}$ NMR $\left(96 \mathrm{MHz}, \mathrm{CDCl}_{3}\right) \delta(\mathrm{ppm})=32.8$

${ }^{19} \mathrm{~F}\left\{{ }^{1} \mathrm{H}\right\}$ NMR $\left(282 \mathrm{MHz}, \mathrm{CDCl}_{3}\right) \delta(\mathrm{ppm})=-118.5$

HRMS (ESI +): calculated for $\mathrm{C}_{21} \mathrm{H}_{25} \mathrm{BFNO}_{3}[\mathrm{M}+\mathrm{H}]^{+}:$:370.1989; found: 370.1976 .

IR (neat): $v\left(\mathrm{~cm}^{-1}\right)=3301,2979,1660,1614,1542,1508,1406,1370,1322,1213,1142,968$, 833, 733, 699.

m.p.: $148-150{ }^{\circ} \mathrm{C}$ 
N,2-bis(4-methoxyphenyl)-3-(4,4,5,5-tetramethyl-1,3,2-dioxaborolan-2-yl)propanamide 3bb

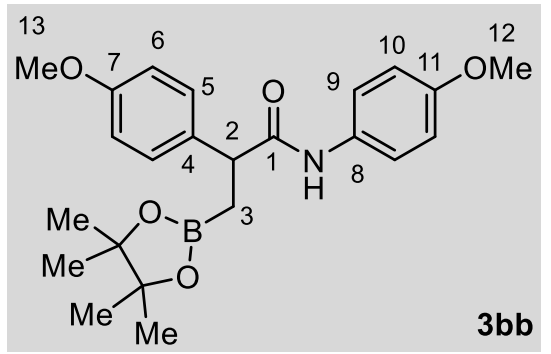

This product was prepared according to the general procedure for the copper-catalyzed borylative carboxamidation of olefins using 4-methoxy styrene $\mathbf{1 b}$ (40 $\mathrm{mg}, 0.3 \mathrm{mmol}, 1.0$ equiv.), [(SIMes)CuCl] $(6.1 \mathrm{mg}, 0.015$ mmol, $5.0 \mathrm{~mol} \%$ ), LiOtBu (36 mg, $0.45 \mathrm{mmol}, 1.5$ equiv.), $\mathrm{B}_{2} \mathrm{pin}_{2}$ (91 mg, $0.36 \mathrm{mmol}, 1.2$ equiv.) and 1-isocyanato-4methoxybenzene $2 \mathbf{b}(0.117 \mathrm{~mL}, 0.9 \mathrm{mmol}, 3.0$ equiv. $)$ in toluene ( $3.0 \mathrm{~mL}, 0.1 \mathrm{M})$. Reaction time: 20 h. $93 \%$ conversion into $3 \mathbf{b b}$ determined by ${ }^{1} \mathrm{H}$ NMR analysis of the crude reaction mixture in presence of an internal standard. Purification by flash chromatography (pentane/ethyl acetate $4: 1$ ) gave pure $3 \mathrm{bb}$ as a white solid (97 $\mathrm{mg}, 0.24 \mathrm{mmol}, 80 \%$ yield).

TLC: pentane/ethyl acetate $4: 1, \mathrm{R}_{\mathrm{f}}=0.1$.

${ }^{1} \mathrm{H}$ NMR $\left(400 \mathrm{MHz}, \mathrm{CDCl}_{3}\right) \delta(\mathrm{ppm})=7.31\left(\mathrm{~d},{ }^{3} \mathrm{~J}_{\mathrm{HH}}=9.0 \mathrm{~Hz}, 2 \mathrm{H}, \mathrm{H}-9\right), 7.27\left(\mathrm{~d},{ }^{3} \mathrm{JHH}_{\mathrm{HH}}=9.0 \mathrm{~Hz}\right.$, $2 \mathrm{H}, \mathrm{H}-5), 6.97$ (bs, $1 \mathrm{H}, \mathrm{N}-H), 6.88\left(\mathrm{~d},{ }^{3} \mathrm{~J}_{\mathrm{HH}}=9.0 \mathrm{~Hz}, 2 \mathrm{H}, \mathrm{H}-6\right), 6.79\left(\mathrm{~d},{ }^{3} \mathrm{~J}_{\mathrm{HH}}=9.0 \mathrm{~Hz}, 2 \mathrm{H}, \mathrm{H}-\right.$ 10), $3.81\left(\mathrm{dd},{ }^{3} \mathrm{~J}_{\mathrm{HH}}=9.5,6.7 \mathrm{~Hz}, 1 \mathrm{H}, H-2\right), 3.80(\mathrm{~s}, 3 \mathrm{H}, H-13), 3.76(\mathrm{~s}, 3 \mathrm{H}, H-12), 1.63$ (dd, $\left.{ }^{2} J_{\mathrm{HH}}=15.7,{ }^{3} J_{\mathrm{HH}}=9.5 \mathrm{~Hz}, 1 \mathrm{H}, H-3\right), 1.24\left(\mathrm{dd},{ }^{2} J_{\mathrm{HH}}=15.7,{ }^{3} \mathrm{JHH}_{\mathrm{HH}}=6.7 \mathrm{~Hz}, 1 \mathrm{H}, H-3^{\prime}\right), 1.21(\mathrm{~s}$, $6 \mathrm{H}, \mathrm{H}$-MeBpin), 1.19 (s, 6H, H-MeBpin).

${ }^{13} \mathrm{C}\left\{{ }^{1} \mathrm{H}\right\}$ NMR $\left(101 \mathrm{MHz}, \mathrm{CDCl}_{3}\right) \delta(\mathrm{ppm})=172.8(C-1), 159.0(C-7), 156.3(C-11), 133.9(C-$ 4), 131.5 (C-8), 129.2 (CH-5), $121.4(\mathrm{CH}-9), 114.5$ (CH-6), 114.1 (CH-10), 83.4 (CO-Bpin), 55.6 ( $\left.\mathrm{CH}_{3}-12\right)$, $55.4\left(\mathrm{CH}_{3}-13\right), 49.1(\mathrm{CH}-2), 24.9$ ( $\left.\mathrm{CH}_{3}-\mathrm{MeBpin}\right), 24.9\left(\mathrm{CH}_{3}-\mathrm{MeBpin}\right), 17.0$ (bs, $\left.\mathrm{CH}_{2}-3\right)$.

${ }^{11} \mathbf{B}$ NMR $\left(96 \mathrm{MHz}, \mathrm{CDCl}_{3}\right) \delta(\mathrm{ppm})=32.8$

HRMS $(\mathrm{ESI}+)$ : calculated for $\mathrm{C}_{23} \mathrm{H}_{30} \mathrm{BNO}_{5}[\mathrm{M}+\mathrm{H}]^{+}:$: 412.2294 ; found: 412.2308 .

IR (neat): $v\left(\mathrm{~cm}^{-1}\right)=3310,2978,1657,1608,1510,1370,1322,1243,1143,1034,829$.

m.p.: $138-140^{\circ} \mathrm{C}$ 
2-(4-fluorophenyl)- $N$-(4-methoxyphenyl)-3-(4,4,5,5-tetramethyl-1,3,2-dioxaborolan-2yl)propanamide $\mathbf{3 c b}$

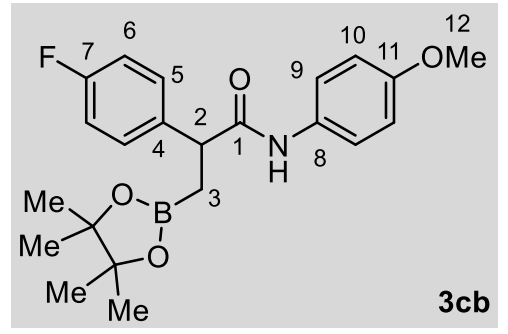

This product was prepared according to the general procedure for the copper-catalyzed borylative carboxamidation of olefins using 4-fluoro styrene 1c (37 mg, $0.3 \mathrm{mmol}, 1.0$ equiv.), [(SIMes)CuCl] (6.1 mg, $0.015 \mathrm{mmol}, 5.0 \mathrm{~mol} \%)$, LiOtBu (36 $\mathrm{mg}, 0.45 \mathrm{mmol}, 1.5$ equiv.), $B_{2}$ pin $_{2}(91 \mathrm{mg}, 0.36 \mathrm{mmol}, 1.2$ equiv.) and 1-isocyanato-4-methoxybenzene $2 \mathbf{b}(0.117 \mathrm{~mL}, 0.9 \mathrm{mmol}, 3.0$ equiv.) in toluene (3.0 mL, $0.1 \mathrm{M})$. Reaction time: $20 \mathrm{~h} .86 \%$ conversion into $3 \mathrm{cb}$ determined by ${ }^{1} \mathrm{H}$ NMR analysis of the crude reaction mixture in presence of an internal standard. Purification by flash chromatography (pentane/ethyl acetate $4: 1$ ) gave pure $3 \mathrm{cb}$ as a white solid (84 $\mathrm{mg}, 0.21$ mmol, $70 \%$ yield).

TLC: pentane/ethyl acetate $4: 1, R_{f}=0.3$.

${ }^{1} \mathrm{H}$ NMR $\left(400 \mathrm{MHz}, \mathrm{CDCl}_{3}\right) \delta(\mathrm{ppm})=7.35(\mathrm{~m}, 4 \mathrm{H}, \mathrm{H}-9+\mathrm{H}-6), 7.04(\mathrm{~m}, 2 \mathrm{H}, \mathrm{H}-5), 6.80\left(\mathrm{~d},{ }^{3} \mathrm{JHH}_{\mathrm{H}}\right.$ $=9.0 \mathrm{~Hz}, 2 \mathrm{H}, H-10), 3.82\left(\mathrm{dd},{ }^{3} J_{\mathrm{HH}}=9.2,6.8 \mathrm{~Hz}, 1 \mathrm{H}, H-2\right), 3.76(\mathrm{~s}, 3 \mathrm{H}, H-13), 1.63\left(\mathrm{dd},{ }^{2} J_{\mathrm{HH}}\right.$ $\left.=15.8,{ }^{3} J_{H H}=9.2 \mathrm{~Hz}, 1 \mathrm{H}, H-3\right), 1.29\left(\mathrm{dd},{ }^{2} J_{H H}=15.8,{ }^{3} J_{H H}=6.8 \mathrm{~Hz}, 1 \mathrm{H}, H-3\right.$ '), $1.19(\mathrm{~s}, 6 \mathrm{H}, H-$ MeBpin), 1.18 (s, 6H, H-MeBpin).

${ }^{13} \mathrm{C}\left\{{ }^{1} \mathrm{H}\right\}$ NMR $\left(101 \mathrm{MHz}, \mathrm{CDCl}_{3}\right) \delta(\mathrm{ppm})=172.2(C-1), 162.2\left(\mathrm{~d},{ }^{1} J_{\mathrm{CF}}=245.8 \mathrm{~Hz}, C-7\right), 156.4$ $(C-11), 137.6\left(d,{ }^{4} J_{\mathrm{CF}}=3.3 \mathrm{~Hz}, C-4\right), 131.3(C-8), 129.7$ (d, $\left.{ }^{2} J_{\mathrm{CF}}=8.0 \mathrm{~Hz}, \mathrm{CH}-6\right), 121.5(\mathrm{CH}-$ 9), $115.8\left(\mathrm{~d},{ }^{3} \mathrm{~J}_{\mathrm{CF}}=21.3 \mathrm{~Hz}, \mathrm{CH}-5\right), 114.2(\mathrm{CH}-10)$, 83.5 (CO-Bpin), $55.6\left(\mathrm{CH}_{3}-12\right), 49.0(\mathrm{CH}-$ 2), 24.9 ( $\left.\mathrm{CH}_{3}-\mathrm{MeBpin}\right), 24.8$ ( $\left.\mathrm{CH}_{3}-\mathrm{MeBpin}\right), 17.1$ (bs, $\left.\mathrm{CH}_{2}-3\right)$.

${ }^{11}$ B NMR $\left(128 \mathrm{MHz}, \mathrm{CDCl}_{3}\right) \delta(\mathrm{ppm})=33.3$

${ }^{19} \mathrm{~F}\left\{{ }^{1} \mathrm{H}\right\}$ NMR $\left(282 \mathrm{MHz}, \mathrm{CDCl}_{3}\right) \delta(\mathrm{ppm})=-115.3$

HRMS (ESI +): calculated for $\mathrm{C}_{22} \mathrm{H}_{27} \mathrm{BFNO}_{4}[\mathrm{M}+\mathrm{H}]^{+}:$: 400.2094; found: 400.2093.

IR (neat): $v\left(\mathrm{~cm}^{-1}\right)=3289,2978,1656,1604,1509,1370,1323,1235,1142,1034,830$.

m.p.: $146-151^{\circ} \mathrm{C}$ 
2-(3-bromophenyl)- N-(4-methoxyphenyl)-3-(4,4,5,5-tetramethyl-1,3,2-dioxaborolan-2yl)propanamide $\mathbf{3 d b}$

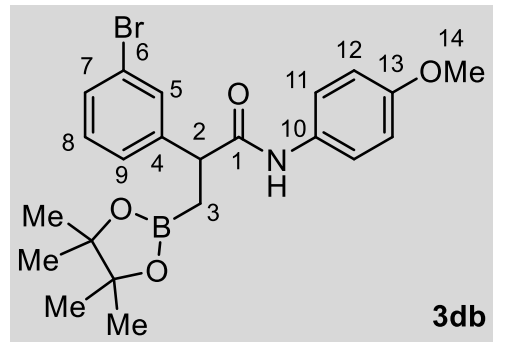

This product was prepared according to the general procedure for the copper-catalyzed borylative carboxamidation of olefins using 3-bromo styrene 1d (55 $\mathrm{mg}, 0.3 \mathrm{mmol}, 1.0$ equiv.), [(SIMes)CuCl] (12.2 mg, $0.030 \mathrm{mmol}, 10.0 \mathrm{~mol} \%)$, LiOtBu (36 $\mathrm{mg}, 0.45 \mathrm{mmol}, 1.5$ equiv.), $\mathrm{B}_{2} \mathrm{pin}_{2}(91 \mathrm{mg}, 0.36 \mathrm{mmol}, 1.2$ equiv.) and 1-isocyanato-4-methoxybenzene $2 \mathbf{b}(0.117 \mathrm{~mL}, 0.9 \mathrm{mmol}, 3.0$ equiv.) in toluene (3.0 mL, $0.1 \mathrm{M})$. Reaction time: $20 \mathrm{~h} .83 \%$ conversion into $3 \mathrm{db}$ determined by ${ }^{1} \mathrm{H}$ NMR analysis of the crude reaction mixture in presence of an internal standard. Purification by flash chromatography (pentane/ethyl acetate $4: 1$ ) gave pure $3 \mathbf{d b}$ as a yellow solid (90 $\mathrm{mg}, 0.20$ mmol, $67 \%$ yield).

TLC: pentane/ethyl acetate $4: 1, \mathrm{R}_{\mathrm{f}}=0.2$.

${ }^{1} \mathrm{H}$ NMR $\left(400 \mathrm{MHz}, \mathrm{CDCl}_{3}\right) \delta(\mathrm{ppm})=7.52\left(\mathrm{t},{ }^{4} \mathrm{~J}_{\mathrm{HH}}=1.6 \mathrm{~Hz}, 1 \mathrm{H}, \mathrm{H}-5\right), 7.40\left(\mathrm{~d},{ }^{3} \mathrm{JHH}_{\mathrm{HH}}=10.6 \mathrm{~Hz}\right.$, $1 \mathrm{H}, H-7), 7.34\left(\mathrm{~d},{ }^{3} \mathrm{~J}_{\mathrm{HH}}=9.0 \mathrm{~Hz}, 2 \mathrm{H}, H-11\right), 7.31\left(\mathrm{~d},{ }^{3} \mathrm{~J}_{\mathrm{HH}}=7.9 \mathrm{~Hz}, 1 \mathrm{H}, H-9\right), 7.21\left(\mathrm{t},{ }^{3} \mathrm{JHH}_{\mathrm{HH}}=7.9\right.$ $\mathrm{Hz}, 1 \mathrm{H}, H-8), 7.05$ (bs, $1 \mathrm{H}, \mathrm{N}-H), 6.81\left(\mathrm{~d},{ }^{3} \mathrm{JHH}_{\mathrm{HH}}=9.0 \mathrm{~Hz}, 2 \mathrm{H}, H-12\right), 3.78(\mathrm{~m}, 1 \mathrm{H}, H-2), 3.77(\mathrm{~s}$, $3 \mathrm{H}, H-14), 1.63\left(\mathrm{dd},{ }^{2} J_{\mathrm{HH}}=15.9,{ }^{3} \mathrm{~J}_{\mathrm{HH}}=9.2 \mathrm{~Hz}, 1 \mathrm{H}, H-3\right), 1.30\left(\mathrm{dd},{ }^{2} J_{\mathrm{HH}}=15.7,{ }^{3} J_{\mathrm{HH}}=6.7 \mathrm{~Hz}\right.$, 1H, $H$-3'), 1.20 (s, 6H, H-MeBpin), 1.18 (s, 6H, H-MeBpin).

${ }^{13} \mathrm{C}\left\{{ }^{1} \mathrm{H}\right\}$ NMR $(101 \mathrm{MHz}, \mathrm{CDCl})_{3} \delta(\mathrm{ppm})=171.6(C-1), 156.5(C-13), 144.1(C-4), 131.3(\mathrm{CH}-$ 5), $131.2(\mathrm{C}-10), 130.6(\mathrm{CH}-8), 130.6(\mathrm{CH}-7), 126.6(\mathrm{CH}-9), 122.9(\mathrm{C}-6), 121.6(\mathrm{CH}-11), 114.2$ ( $\mathrm{CH}-12)$, 83.6 (CO-Bpin), 55.6 ( $\left.\mathrm{CH}_{3}-14\right)$, 49.5 ( $\left.\mathrm{CH}-2\right)$, 24.9 ( $\left.\mathrm{CH}_{3}-\mathrm{MeBpin}\right), 24.9$ ( $\left.\mathrm{CH}_{3}-\mathrm{MeBpin}\right)$. The boron-bound carbons $\mathrm{CH}_{2}-3$ was not detected due to quadrupolar relaxation.

${ }^{11}$ B NMR $\left(128 \mathrm{MHz}, \mathrm{CDCl}_{3}\right) \delta(\mathrm{ppm})=33.4$

HRMS (ESI +): calculated for $\mathrm{C}_{22} \mathrm{H}_{27} \mathrm{BBrNO}_{4}[\mathrm{M}+\mathrm{H}]^{+}: 460.1294$; found: 460.1277 .

IR (neat): $v\left(\mathrm{~cm}^{-1}\right)=3300,2978,1656,1603,1510,1369,1322,1239,1141,1035,828$.

m.p.: $42-44^{\circ} \mathrm{C}$ 
2-(3-chlorophenyl)- $N$-(4-methoxyphenyl)-3-(4,4,5,5-tetramethyl-1,3,2-dioxaborolan-2yl)propanamide $\mathbf{3 e b}$

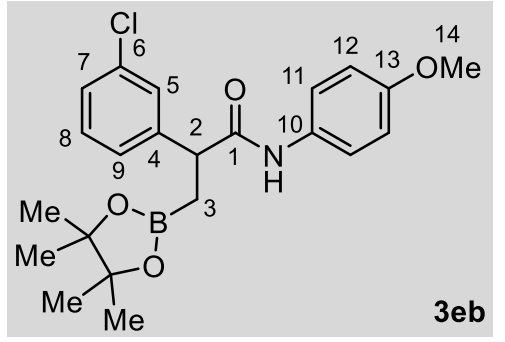

This product was prepared according to the general procedure for the copper-catalyzed borylative carboxamidation of olefins using 3-chloro styrene 1e (42 mg, $0.3 \mathrm{mmol}, 1.0$ equiv.), [(SIMes)CuCl] (12.2 mg, $0.030 \mathrm{mmol}, 10.0 \mathrm{~mol} \%)$, LiOtBu (36 $\mathrm{mg}, 0.45 \mathrm{mmol}, 1.5$ equiv.), $\mathrm{B}_{2} \mathrm{pin}_{2}(91 \mathrm{mg}, 0.36 \mathrm{mmol}, 1.2$ equiv.) and 1-isocyanato-4-methoxybenzene $2 \mathbf{b}(0.117 \mathrm{~mL}$, $0.9 \mathrm{mmol}, 3.0$ equiv.) in toluene $(3.0 \mathrm{~mL}, 0.1 \mathrm{M})$. Reaction time: $20 \mathrm{~h} .71 \%$ conversion into 3eb determined by ${ }^{1} \mathrm{H}$ NMR analysis of the crude reaction mixture in presence of an internal standard. Purification by flash chromatography (pentane/ethyl acetate $4: 1$ ) gave pure 3eb as a white solid ( $70 \mathrm{mg}, 0.17 \mathrm{mmol}, 56 \%$ yield).

TLC: pentane/ethyl acetate $4: 1, \mathrm{R}_{\mathrm{f}}=0.2$.

${ }^{1} \mathrm{H}$ NMR $\left(300 \mathrm{MHz}, \mathrm{CDCl}_{3}\right) \delta(\mathrm{ppm})=7.37(\mathrm{~s}, 1 \mathrm{H}, \mathrm{H}-5), 7.33\left(\mathrm{~d},{ }^{3} \mathrm{~J}_{\mathrm{HH}}=9.0 \mathrm{~Hz}, 2 \mathrm{H}, H-11\right), 7.30$ - 7.20 (m, 3H, H-7, H-8, H-9), 7.06 (bs, $1 \mathrm{H}, \mathrm{N}-H$ ), 6.81 (d, ${ }^{3} \mathrm{JHH}_{\mathrm{HH}}=9.0 \mathrm{~Hz}, 2 \mathrm{H}, H-12$ ), 3.79 (dd, $\left.{ }^{3} J_{H H}=9.3,6.8 \mathrm{~Hz}, 1 \mathrm{H}, H-2\right), 3.76(\mathrm{~s}, 3 \mathrm{H}, H-14), 1.64\left(\mathrm{dd},{ }^{2} J_{\mathrm{HH}}=15.9,{ }^{3} J_{\mathrm{HH}}=9.2 \mathrm{~Hz}, 1 \mathrm{H}, H-3\right)$, $1.30\left(\mathrm{dd},{ }^{2} \mathrm{~J}_{\mathrm{HH}}=15.9,{ }^{3} \mathrm{~J}_{\mathrm{HH}}=6.8 \mathrm{~Hz}, 1 \mathrm{H}, H-3\right.$ '), 1.20 (s, 6H, H-MeBpin), 1.19 (s, 6H, H-MeBpin). ${ }^{13} \mathrm{C}\left\{{ }^{1} \mathrm{H}\right\}$ NMR $\left(75 \mathrm{MHz}, \mathrm{CDCl}_{3}\right) \delta(\mathrm{ppm})=171.6(C-1), 156.5(C-13), 143.8(C-4), 134.7(C-6)$, $131.2(\mathrm{C}-10), 130.3(\mathrm{CH}-8), 128.4(\mathrm{CH}-5), 127.6(\mathrm{CH}-7), 126.2(\mathrm{CH}-9), 121.6(\mathrm{CH}-11), 114.2$ (CH-12), 83.6 (CO-Bpin), 55.6 ( $\left.\mathrm{CH}_{3}-14\right)$, 49.5 ( $\left.\mathrm{CH}-2\right)$, 25.0 ( $\left.\mathrm{CH}_{3}-\mathrm{MeBpin}\right), 24.9$ ( $\left.\mathrm{CH}_{3}-\mathrm{MeBpin}\right)$. The boron-bound carbons $\mathrm{CH}_{2}-3$ was not detected due to quadrupolar relaxation.

${ }^{11} \mathrm{~B}$ NMR $\left(128 \mathrm{MHz}, \mathrm{CDCl}_{3}\right) \delta(\mathrm{ppm})=34.2$.

HRMS (ESI +): calculated for $\mathrm{C}_{22} \mathrm{H}_{27} \mathrm{BCINO}_{4}[\mathrm{M}+\mathrm{H}]^{+}: 416.1799$; found: 416.1815 .

IR (neat): $v\left(\mathrm{~cm}^{-1}\right)=3301,2979,1656,1589,1511,1370,1325,1243,1141,967,830$.

m.p.: $117-121^{\circ} \mathrm{C}$ 
2-([1,1'-biphenyl]-4-yl)- $N$-(4-methoxyphenyl)-3-(4,4,5,5-tetramethyl-1,3,2-dioxaborolan-2yl)propanamide $\mathbf{3 f b}$

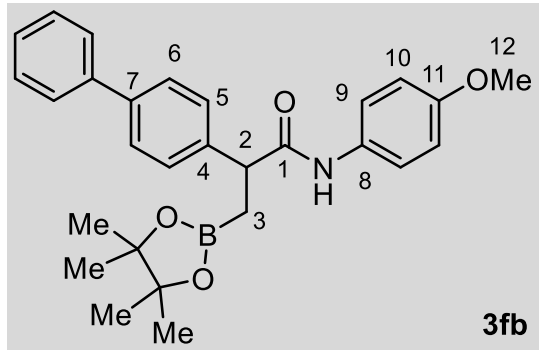

This product was prepared according to the general procedure for the copper-catalyzed borylative carboxamidation of olefins using 4-phenyl styrene 1f $(54 \mathrm{mg}$, $0.3 \mathrm{mmol}, 1.0$ equiv.), [(SIMes)CuCl] $(6.1 \mathrm{mg}, 0.015 \mathrm{mmol}$, $5.0 \mathrm{~mol} \%$ ), LiOtBu (36 mg, $0.45 \mathrm{mmol}, 1.5$ equiv.), $\mathrm{B}_{2} \mathrm{pin}_{2}$ (91 mg, $0.36 \mathrm{mmol}, 1.2$ equiv.) and 1-isocyanato-4methoxybenzene $\mathbf{2 b}(0.117 \mathrm{~mL}, 0.9 \mathrm{mmol}, 3.0$ equiv.) in toluene $(3.0 \mathrm{~mL}, 0.1 \mathrm{M})$. Reaction time: 20 h. $85 \%$ conversion into $3 \mathrm{fb}$ determined by ${ }^{1} \mathrm{H}$ NMR analysis of the crude reaction mixture in presence of an internal standard. Purification by flash chromatography (pentane/ethyl acetate 4:1) gave pure $3 \mathrm{fb}$ as a white solid (103 mg, $0.23 \mathrm{mmol}, 75 \%$ yield).

TLC: pentane/ethyl acetate $4: 1, \mathrm{R}_{\mathrm{f}}=0.2$.

${ }^{1} \mathrm{H}$ NMR $\left(400 \mathrm{MHz}, \mathrm{CDCl}_{3}\right) \delta(\mathrm{ppm})=7.58(\mathrm{~m}, 4 \mathrm{H}, \mathrm{H}-\mathrm{Ar}), 7.44(\mathrm{~m}, 4 \mathrm{H}, \mathrm{H}-\mathrm{Ar}), 7.34(\mathrm{~m}, 3 \mathrm{H}, \mathrm{H}-$ Ar), $7.06(\mathrm{bs}, 1 \mathrm{H}, \mathrm{N}-H), 6.80\left(\mathrm{~d},{ }^{3} J_{\mathrm{HH}}=9.0 \mathrm{~Hz}, 2 \mathrm{H}, H-10\right), 3.90\left(\mathrm{dd},{ }^{3} J_{\mathrm{HH}}=9.6,6.5 \mathrm{~Hz}, 1 \mathrm{H}, \mathrm{H}-\right.$ 2), $3.76(\mathrm{~s}, 3 \mathrm{H}, \mathrm{H}-12), 1.70\left(\mathrm{dd},{ }^{2} J_{\mathrm{HH}}=15.8,{ }^{3} \mathrm{JHH}_{\mathrm{HH}}=9.6 \mathrm{~Hz}, 1 \mathrm{H}, \mathrm{H}-3\right), 1.33\left(\mathrm{dd},{ }^{2} J_{\mathrm{HH}}=15.7,{ }^{3} J_{\mathrm{HH}}\right.$ $=6.5 \mathrm{~Hz}, 1 \mathrm{H}, H$-3'), 1.22 (s, 6H, H-MeBpin), 1.20 (s, 6H, H-MeBpin).

${ }^{13} \mathrm{C}\left\{{ }^{1} \mathrm{H}\right\}$ NMR $\left(101 \mathrm{MHz}, \mathrm{CDCl}_{3}\right) \delta(\mathrm{ppm})=172.3(C-1), 156.4(C-11), 140.9(C-4), 140.8(C-$ $\mathrm{Ar}), 140.4$ (C-Ar), $131.4(\mathrm{C}-8), 128.9$ ( $\mathrm{CH}-\mathrm{Ar}), 128.6$ ( $\mathrm{CH}-\mathrm{Ar}), 127.8(\mathrm{CH}-\mathrm{Ar}), 127.5(\mathrm{CH}-\mathrm{Ar})$, $127.2(\mathrm{CH}-\mathrm{Ar}), 121.5(\mathrm{CH}-9), 114.2(\mathrm{CH}-10), 83.5$ (CO-Bpin), $55.6\left(\mathrm{CH}_{3}-12\right), 49.6(\mathrm{CH}-2)$, $25.0\left(\mathrm{CH}_{3}-\mathrm{MeBpin}\right), 24.9\left(\mathrm{CH}_{3}-\mathrm{MeBpin}\right)$. The boron-bound carbons $\mathrm{CH}_{2}-3$ was not detected due to quadrupolar relaxation.

${ }^{11} \mathbf{B}$ NMR $\left(96 \mathrm{MHz}, \mathrm{CDCl}_{3}\right) \delta(\mathrm{ppm})=33.3$.

HRMS (ESI +): calculated for $\mathrm{C}_{28} \mathrm{H}_{32} \mathrm{BNO}_{4}[\mathrm{M}+\mathrm{H}]^{+}: 458.2503$; found: 458.2517 .

IR (neat): $v\left(\mathrm{~cm}^{-1}\right)=3294,2977,1656,1603,1510,1367,1321,1241,1142,1035,968,829$, 760.

m.p.: $162-164^{\circ} \mathrm{C}$ 
2-(4-(tert-butyl)phenyl)- $N$-(4-methoxyphenyl)-3-(4,4,5,5-tetramethyl-1,3,2-dioxaborolan-2yl)propanamide $\mathbf{3 g b}$

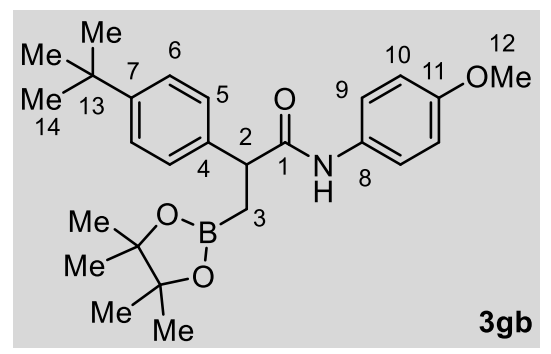

This product was prepared according to the general procedure for the copper-catalyzed borylative carboxamidation of olefins using 4- tert-butyl styrene $\mathbf{1 g}$ (51 mg, $0.3 \mathrm{mmol}, 1.0$ equiv.), [(SIMes)CuCl] $(6.1 \mathrm{mg}, 0.015$ mmol, $5.0 \mathrm{~mol} \%$ ), LiOtBu (36 mg, $0.45 \mathrm{mmol}, 1.5$ equiv.), $\mathrm{B}_{2} \mathrm{pin}_{2}$ (91 mg, $0.36 \mathrm{mmol}, 1.2$ equiv.) and 1-isocyanato-4methoxybenzene $\mathbf{2 b}(0.117 \mathrm{~mL}, 0.9 \mathrm{mmol}, 3.0$ equiv.) in toluene (3.0 mL, $0.1 \mathrm{M})$. Reaction time: 20 h. $90 \%$ conversion into $\mathbf{3 g b}$ determined by ${ }^{1} \mathrm{H}$ NMR analysis of the crude reaction mixture in presence of an internal standard. Purification by flash chromatography (pentane/ethyl acetate 4:1) gave pure $3 \mathrm{gb}$ as a white solid (100 mg, $0.23 \mathrm{mmol}, 77 \%$ yield).

TLC: pentane/ethyl acetate $4: 1, \mathrm{R}_{\mathrm{f}}=0.2$.

${ }^{1} \mathbf{H}$ NMR $\left(400 \mathrm{MHz}, \mathrm{CDCl}_{3}\right) \delta(\mathrm{ppm})=7.35\left(\mathrm{~d},{ }^{3} \mathrm{~J}_{\mathrm{HH}}=8.7 \mathrm{~Hz}, 2 \mathrm{H}, \mathrm{H}-5\right), 7.32\left(\mathrm{~d},{ }^{3} \mathrm{~J}_{\mathrm{HH}}=8.7 \mathrm{~Hz}\right.$, $2 \mathrm{H}, H-9), 7.27\left(\mathrm{~d},{ }^{3} \mathrm{~J}_{\mathrm{HH}}=8.7 \mathrm{~Hz}, 2 \mathrm{H}, H-6\right), 7.01(\mathrm{bs}, 1 \mathrm{H}, \mathrm{N}-H), 6.79\left(\mathrm{~d},{ }^{3} J_{\mathrm{HH}}=8.7 \mathrm{~Hz}, 2 \mathrm{H}, \mathrm{H}-\right.$ 10), $3.83\left(\mathrm{dd},{ }^{3} J_{\mathrm{HH}}=9.6,6.5 \mathrm{~Hz}, 1 \mathrm{H}, \mathrm{H}-2\right), 3.75(\mathrm{~s}, 3 \mathrm{H}, H-13), 1.64\left(\mathrm{dd},{ }^{2} J_{\mathrm{HH}}=15.5,{ }^{3} J_{\mathrm{HH}}=9.6\right.$ $\mathrm{Hz}, 1 \mathrm{H}, H-3$ ), 1.31 (s, 9H, H-14), 1.26 (m, 1H, H-3'), 1.20 (s, 6H, $H$-MeBpin), 1.18 (s, 6H, $H$ MeBpin).

${ }^{13} \mathrm{C}\left\{{ }^{1} \mathrm{H}\right\}$ NMR $\left(101 \mathrm{MHz}, \mathrm{CDCl}_{3}\right) \delta(\mathrm{ppm})=172.6(C-1), 156.3(C-11), 150.3(C-7), 138.7(C-$ 4), 131.5 (C-8), 127.8 (CH-5), 126.0 ( $\mathrm{CH}-6), 121.5$ ( $\mathrm{CH}-9), 114.1$ (CH-10), 83.4 (CO-Bpin), $55.6\left(\mathrm{CH}_{3}-12\right), 49.4(\mathrm{CH}-2), 34.6(\mathrm{C}-13), 31.5\left(\mathrm{CH}_{3}-14\right), 25.0\left(\mathrm{CH}_{3}-\mathrm{MeBpin}\right), 24.8\left(\mathrm{CH}_{3^{-}}\right.$ MeBpin), 16.9 (bs, $\mathrm{CH}_{2}-3$ ).

${ }^{11} \mathbf{B}$ NMR $\left(96 \mathrm{MHz}, \mathrm{CDCl}_{3}\right) \delta(\mathrm{ppm})=34.6$

HRMS $(\mathrm{ESI}+)$ : calculated for $\mathrm{C}_{26} \mathrm{H}_{36} \mathrm{BNO}_{4}[\mathrm{M}+\mathrm{H}]^{+}:$: 438.2815; found: 438.2835 .

IR (neat): $v\left(\mathrm{~cm}^{-1}\right)=3350,2949,1711,1679,1595,1514,1269,1167,1032,829$.

m.p.: $140-141^{\circ} \mathrm{C}$ 
$N$-(4-methoxyphenyl)-3-(4,4,5,5-tetramethyl-1,3,2-dioxaborolan-2-yl)-2-(o-tolyl)propanamide 3hb

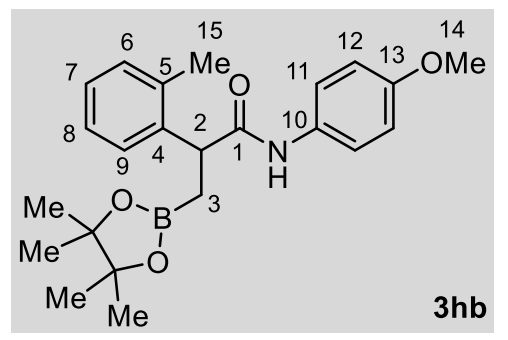

This product was prepared according to the general procedure for the copper-catalyzed borylative carboxamidation of olefins using 2-methyl styrene $1 \mathrm{~h}$ (25 $\mathrm{mg}, 0.2 \mathrm{mmol}, 1.0$ equiv.), [(SIMes)CuCl] (8.1 mg, $0.02 \mathrm{mmol}, 10.0 \mathrm{~mol} \%)$, LiOtBu (24 $\mathrm{mg}, 0.3 \mathrm{mmol}, 1.5$ equiv.), B $\mathrm{pin}_{2}(61 \mathrm{mg}, 0.24 \mathrm{mmol}, 1.2$ equiv.) and 1-isocyanato-4-methoxybenzene $\mathbf{2 b}(0.078 \mathrm{~mL}$, $0.6 \mathrm{mmol}, 3.0$ equiv.) in toluene $(2.0 \mathrm{~mL}, 0.1 \mathrm{M})$. Reaction time: $20 \mathrm{~h} .78 \%$ conversion into 3hb determined by ${ }^{1} \mathrm{H}$ NMR analysis of the crude reaction mixture in presence of an internal standard. Purification by flash chromatography (pentane/ethyl acetate 9:1) gave pure 3hb as a yellow solid (50 mg, $0.13 \mathrm{mmol}, 65 \%$ yield).

TLC: pentane/ethyl acetate $4: 1, \mathrm{R}_{\mathrm{f}}=0.3$.

${ }^{1} \mathbf{H}$ NMR $\left(400 \mathrm{MHz}, \mathrm{CDCl}_{3}\right) \delta(\mathrm{ppm})=7.33\left(\mathrm{~d},{ }^{3} \mathrm{~J}_{\mathrm{HH}}=6.9 \mathrm{~Hz}, 1 \mathrm{H}, \mathrm{H}-9\right), 7.29\left(\mathrm{~d},{ }^{3} \mathrm{~J}_{\mathrm{HH}}=9.0 \mathrm{~Hz}\right.$, $2 \mathrm{H}, \mathrm{H}-11), 7.24-7.15(\mathrm{~m}, 3 \mathrm{H}, \mathrm{H}-6+\mathrm{H}-7+\mathrm{H}-8), 6.88(\mathrm{bs}, 1 \mathrm{H}, \mathrm{N}-\mathrm{H}), 6.79\left(\mathrm{~d},{ }^{3} \mathrm{~J}_{\mathrm{HH}}=9.0 \mathrm{~Hz}\right.$, $2 \mathrm{H}, H-12), 4.08\left(\mathrm{dd},{ }^{3} \mathrm{~J}_{\mathrm{HH}}=9.0,7.3 \mathrm{~Hz}, 1 \mathrm{H}, \mathrm{H}-2\right), 3.75(\mathrm{~s}, 3 \mathrm{H}, \mathrm{H}-14), 2.40(\mathrm{~s}, 3 \mathrm{H}, H-15), 1.65$ $\left(\mathrm{dd},{ }^{2} J_{\mathrm{HH}}=15.7,{ }^{3} J_{\mathrm{HH}}=9.1 \mathrm{~Hz}, 1 \mathrm{H}, H-3\right), 1.28\left(\mathrm{dd},{ }^{2} J_{\mathrm{HH}}=15.7,{ }^{3} J_{\mathrm{HH}}=7.3 \mathrm{~Hz}, 1 \mathrm{H}, H-3\right.$ '), 1.18 (s, 12H, H-MeBpin).

${ }^{13} \mathrm{C}\left\{{ }^{1} \mathrm{H}\right\}$ NMR $\left.(101 \mathrm{MHz}, \mathrm{CDCl})_{3}\right) \delta(\mathrm{ppm})=172.7(C-1), 156.4(C-13), 139.8(C-4), 136.5(C-$ 5), 131.4 (C-10), 131.1 ( $\mathrm{CH}-6), 128.1$ ( $\mathrm{CH}-\mathrm{Ar}), 127.5$ ( $\mathrm{CH}-\mathrm{Ar}), 126.9(\mathrm{CH}-\mathrm{Ar}), 121.6(\mathrm{CH}-11)$, 114.1 ( $\mathrm{CH}-12), 83.4$ (CO-Bpin), $55.6\left(\mathrm{CH}_{3}-14\right)$, 46.4 (CH-2), $24.9\left(\mathrm{CH}_{3}-\mathrm{MeBpin}\right), 24.8\left(\mathrm{CH}_{3}-\right.$ MeBpin), 19.9 ( $\left.\mathrm{CH}_{3}-15\right), 15.9$ (bs, $\left.\mathrm{CH}_{2}-3\right)$.

${ }^{11} \mathbf{B}$ NMR $\left(96 \mathrm{MHz}, \mathrm{CDCl}_{3}\right) \delta(\mathrm{ppm})=33.2$

HRMS $(E S I+)$ : calculated for $\mathrm{C}_{23} \mathrm{H}_{30} \mathrm{BNO}_{4}[\mathrm{M}+\mathrm{H}]^{+}:$:396.2345; found: 396.2376.

IR (neat): $v\left(\mathrm{~cm}^{-1}\right)=3304,2977,1659,1602,1511,1369,1320,1242,1168,1143,1036,968$, 829.

m.p.: $136-141^{\circ} \mathrm{C}$ 
$N$-(4-methoxyphenyl)-2-(naphthalen-2-yl)-3-(4,4,5,5-tetramethyl-1,3,2-dioxaborolan-2-

yl)propanamide 3ib

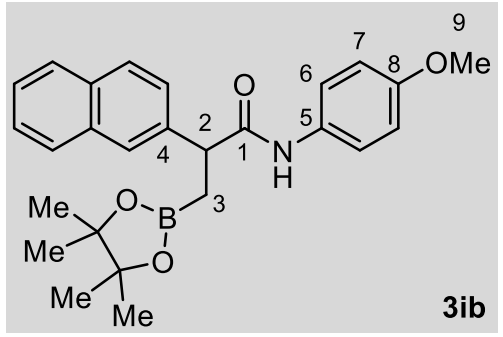

This product was prepared according to the general procedure for the copper-catalyzed borylative carboxamidation of olefins using 2-vinyl naphthalene $1 \mathbf{i}$ (46 mg, $0.3 \mathrm{mmol}, 1.0$ equiv.), [(SIMes)CuCl] $(6.1 \mathrm{mg}, 0.015 \mathrm{mmol}, 5.0 \mathrm{~mol} \%)$, LiOtBu (36 $\mathrm{mg}, 0.45 \mathrm{mmol}, 1.5$ equiv.), $B_{2} \mathrm{pin}_{2}(91 \mathrm{mg}, 0.36 \mathrm{mmol}, 1.2$ equiv.) and 1-isocyanato-4-methoxybenzene $2 \mathrm{~b}(0.117 \mathrm{~mL}$, $0.9 \mathrm{mmol}, 3.0$ equiv.) in toluene (3.0 mL, $0.1 \mathrm{M}$ ). Reaction time: $20 \mathrm{~h}$. $75 \%$ conversion into 3ib determined by ${ }^{1} \mathrm{H}$ NMR analysis of the crude reaction mixture in presence of an internal standard. Purification by flash chromatography (pentane/ethyl acetate $4: 1$ ) gave pure 3ib as a yellow solid ( $85 \mathrm{mg}, 0.20 \mathrm{mmol}, 67 \%$ yield).

TLC: pentane/ethyl acetate $4: 1, \mathrm{R}_{\mathrm{f}}=0.3$.

${ }^{1} \mathbf{H}$ NMR $\left(400 \mathrm{MHz}, \mathrm{CDCl}_{3}\right) \delta(\mathrm{ppm})=7.83$ (m, 4H, H-Napht), 7.48 (m, 3H, H-Napht), 7.30 (d, $\left.{ }^{3} J_{\mathrm{HH}}=9.0 \mathrm{~Hz}, 2 \mathrm{H}, H-6\right), 7.02(\mathrm{bs}, 1 \mathrm{H}, \mathrm{N}-H), 6.78\left(\mathrm{~d},{ }^{3} \mathrm{~J}_{\mathrm{HH}}=9.0 \mathrm{~Hz}, 2 \mathrm{H}, H-7\right), 4.04\left(\mathrm{dd},{ }^{3} \mathrm{~J}_{\mathrm{HH}}=\right.$ 9.5, $6.5 \mathrm{~Hz}, 1 \mathrm{H}, \mathrm{H}-2), 3.75(\mathrm{~s}, 3 \mathrm{H}, H-9), 1.76\left(\mathrm{dd},{ }^{2} J_{\mathrm{HH}}=15.7,{ }^{3} J_{\mathrm{HH}}=9.5 \mathrm{~Hz}, 1 \mathrm{H}, H-3\right), 1.36$ (dd, ${ }^{2} J_{H H}=15.7,{ }^{3} J_{H H}=6.7 \mathrm{~Hz}, 1 \mathrm{H}, H$-3'), 1.20 (s, 6H, H-MeBpin), 1.19 (s, 6H, H-MeBpin).

${ }^{13} \mathrm{C}\left\{{ }^{1} \mathrm{H}\right\}$ NMR $(101 \mathrm{MHz}, \mathrm{CDCl}) \delta(\mathrm{ppm})=172.3(C-1), 156.4(C-8), 139.3(C-4), 133.7(C-$ Napht), 132.8 (C-Napht), 131.4 (C-5), 129.1 (CH-Napht), 127.9 (CH-Napht), 127.9 (CHNapht), 127.0 (CH-Napht), 126.5 (CH-Napht), 126.1 (CH-Napht), 126.0 (CH-Napht), 121.5 (CH-6), 114.1 ( $\mathrm{CH}-7), 83.5$ (CO-Bpin), $55.5\left(\mathrm{CH}_{3}-9\right), 50.1$ ( $\left.\mathrm{CH}-2\right), 25.0$ ( $\left.\mathrm{CH}_{3}-\mathrm{MeBpin}\right), 24.9$ $\left(\mathrm{CH}_{3}\right.$-MeBpin). The boron-bound carbons $\mathrm{CH}_{2}-3$ was not detected due to quadrupolar relaxation.

11B NMR $\left(96 \mathrm{MHz}, \mathrm{CDCl}_{3}\right) \delta(\mathrm{ppm})=33.3$.

HRMS $(\mathrm{ESI}+)$ : calculated for $\mathrm{C}_{26} \mathrm{H}_{30} \mathrm{BNO}_{4}[\mathrm{M}+\mathrm{H}]^{+}:$432.2346; found: 432.2365 .

IR (neat): $v\left(\mathrm{~cm}^{-1}\right)=3300,2977,1656,1602,1510,1412,1369,1323,1241,1142,1035,968$, 827.

m.p.: $58-60^{\circ} \mathrm{C}$ 
$N$-(4-methoxyphenyl)-2-(4-morpholinophenyl)-3-(4,4,5,5-tetramethyl-1,3,2-dioxaborolan-2yl)propanamide $\mathbf{3 j b}$

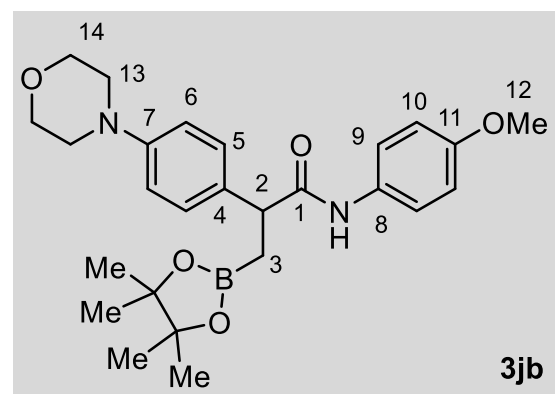

This product was prepared according to the general procedure for the copper-catalyzed borylative carboxamidation of olefins using styrene $1 \mathbf{j}^{2}(57 \mathrm{mg}, 0.3$ mmol, 1.0 equiv.), [(SIMes)CuCl] $(6.1 \mathrm{mg}, 0.015 \mathrm{mmol}, 5.0$ mol\%), LiOtBu (36 mg, $0.45 \mathrm{mmol}, 1.5$ equiv.), $\mathrm{B}_{2} \mathrm{pin}_{2}(91$ 3jb $\mathrm{mg}, \quad 0.36 \mathrm{mmol}, 1.2$ equiv.) and 1-isocyanato-4methoxybenzene $\mathbf{2 b}(0.117 \mathrm{~mL}, 0.9 \mathrm{mmol}, 3.0$ equiv.) in toluene (3.0 mL, $0.1 \mathrm{M})$. Reaction time: $20 \mathrm{~h}$. $88 \%$ conversion into $3 \mathbf{j b}$ determined by ${ }^{1} \mathrm{H}$ NMR analysis of the crude reaction mixture in presence of an internal standard. Purification by flash chromatography (dichloromethane with $1 \%$ to $2 \%$ methanol) gave pure $3 \mathrm{jb}$ as a white solid $(90 \mathrm{mg}, 0.19 \mathrm{mmol}$, $64 \%$ yield).

TLC: dichloromethane with $2 \%$ methanol, $\mathrm{R}_{\mathrm{f}}=0.4$.

${ }^{1} \mathrm{H}$ NMR $\left(400 \mathrm{MHz}, \mathrm{CDCl}_{3}\right) \delta(\mathrm{ppm})=7.30\left(\mathrm{~d},{ }^{3} \mathrm{~J}_{\mathrm{HH}}=8.9 \mathrm{~Hz}, 2 \mathrm{H}, \mathrm{H}-9\right), 7.25\left(\mathrm{~d},{ }^{3} \mathrm{JHH}_{\mathrm{HH}}=8.2 \mathrm{~Hz}\right.$, $2 \mathrm{H}, H-5), 6.98(\mathrm{bs}, 1 \mathrm{H}, \mathrm{N}-\mathrm{H}), 6.88\left(\mathrm{~d},{ }^{3} \mathrm{~J}_{\mathrm{HH}}=8.2 \mathrm{~Hz}, 2 \mathrm{H}, \mathrm{H}-6\right), 6.78\left(\mathrm{~d},{ }^{3} \mathrm{~J}_{\mathrm{HH}}=8.9 \mathrm{~Hz}, 2 \mathrm{H}, \mathrm{H}-\right.$ 10), 3.85 (m, 4H, H-14), 3.80 (m, 1H, H-2), 3.75 (s, 3H, H-12), 3.14 (m, 4H, H-13), 1.62 (dd, $\left.{ }^{2} J_{\mathrm{HH}}=15.6,{ }^{3} J_{\mathrm{HH}}=9.7 \mathrm{~Hz}, 1 \mathrm{H}, H-3\right), 1.25(\mathrm{~m}, 1 \mathrm{H}, H-3$ '), 1.21 (s, 6H, H-MeBpin), 1.19 (s, 6H, H-MeBpin).

${ }^{13} \mathrm{C}\left\{{ }^{1} \mathrm{H}\right\}$ NMR $\left(101 \mathrm{MHz}, \mathrm{CDCl}_{3}\right) \delta(\mathrm{ppm})=172.8(C-1), 156.3(C-11), 150.6(C-7), 133.1(C-$ 4), 131.5 (C-8), 128.9 (CH-5), $121.4(\mathrm{CH}-9), 116.2$ ( $\mathrm{CH}-6), 114.1$ ( $\mathrm{CH}-10), 83.4$ (CO-Bpin), $67.0\left(\mathrm{CH}_{2}-14\right), 55.6\left(\mathrm{CH}_{3}-12\right), 49.4\left(\mathrm{CH}_{2}-13\right), 49.1(\mathrm{CH}-2), 25.0\left(\mathrm{CH}_{3}-\mathrm{MeBpin}\right), 24.9\left(\mathrm{CH}_{3}{ }^{-}\right.$ MeBpin), 16.9 (bs, $\left.\mathrm{CH}_{2}-3\right)$.

${ }^{11} \mathbf{B}$ NMR $\left(96 \mathrm{MHz}, \mathrm{CDCl}_{3}\right) \delta(\mathrm{ppm})=33.5$

HRMS (ESI +): calculated for $\mathrm{C}_{23} \mathrm{H}_{35} \mathrm{BN}_{2} \mathrm{O}_{5}[\mathrm{M}+\mathrm{H}]^{+}: 467.2717$; found: 467.2721

IR (neat): $v\left(\mathrm{~cm}^{-1}\right)=3309,2975,1659,1610,1510,1451,1369,1321,1231,1142,1119$, 1034, 968, 927, 827.

m.p.: $174-179^{\circ} \mathrm{C}$ 
N-phenyl-2-(4-(pyridin-2-yl)phenyl)-3-(4,4,5,5-tetramethyl-1,3,2-dioxaborolan-2yl)propanamide $\mathbf{3 k a}$

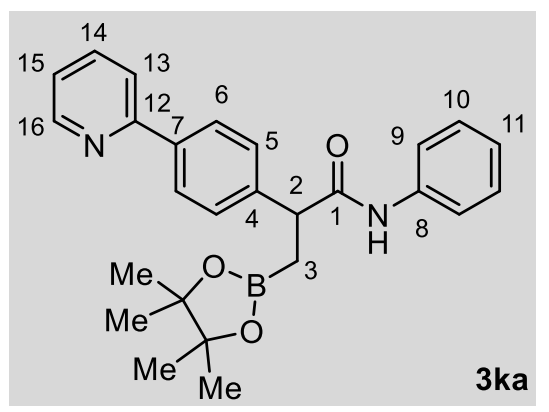

This product was prepared according to the general procedure for the copper-catalyzed borylative carboxamidation of olefins using 2-(4-vinylphenyl)pyridine 1k (27 mg, $0.15 \mathrm{mmol}, 1.0$ equiv), ${ }^{3}$ [(SIMes)CuCl] $(6.1 \mathrm{mg}$, $0.015 \mathrm{mmol}, 10.0 \mathrm{~mol} \%$ ), LiOtBu (18 mg, $0.23 \mathrm{mmol}, 1.5$ equiv), $\mathrm{B}_{2} \mathrm{pin}_{2}$ (46 mg, $0.18 \mathrm{mmol}, 1.2$ equiv) and 1 isocyanatobenzene $2 \mathrm{a}(0.049 \mathrm{~mL}, 0.45 \mathrm{mmol}, 3.0$ equiv) in toluene $(1.5 \mathrm{~mL}, 0.1 \mathrm{M})$. Reaction time: $20 \mathrm{~h} .71 \%$ conversion into 3 ka determined by ${ }^{1} \mathrm{H} \mathrm{NMR}$ analysis of the crude reaction mixture in presence of an internal standard. Purification by flash chromatography (pentane/ethyl acetate 4:1) gave pure 3ka as a white solid (32 mg, 0.075 $\mathrm{mmol}, 50 \%$ yield).

TLC: pentane/ethyl acetate $4: 1, \mathrm{R}_{\mathrm{f}}=0.3$.

${ }^{1} \mathrm{H}$ NMR $\left(500 \mathrm{MHz}, \mathrm{CDCl}_{3}\right) \delta(\mathrm{ppm})=8.69(\mathrm{~m}, 1 \mathrm{H}, \mathrm{H}-16), 7.99\left(\mathrm{~d},{ }^{3} \mathrm{JHH}=8.0 \mathrm{~Hz}, 2 \mathrm{H}, H-6\right)$, $7.74(\mathrm{~m}, 2 \mathrm{H}, H-13+H-14), 7.47\left(\mathrm{~d},{ }^{3} \mathrm{JHH}_{\mathrm{HH}}=8.0 \mathrm{~Hz}, 2 \mathrm{H}, H-5\right), 7.41\left(\mathrm{~d},{ }^{3} \mathrm{~J}_{\mathrm{HH}}=7.9 \mathrm{~Hz}, 2 \mathrm{H}, H-9\right)$, $7.29-7.21(\mathrm{~m}, 3 \mathrm{H}, H-10+H-15), 7.12(\mathrm{bs}, 1 \mathrm{H}, \mathrm{N}-H), 7.05\left(\mathrm{t},{ }^{3} \mathrm{JHH}_{\mathrm{HH}}=7.4 \mathrm{~Hz}, 1 \mathrm{H}, H-11\right), 3.94$ $\left(\mathrm{dd},{ }^{3} \mathrm{JHH}_{\mathrm{HH}}=9.5,6.5 \mathrm{~Hz}, 1 \mathrm{H}, \mathrm{H}-2\right), 1.71\left(\mathrm{dd},{ }^{2} \mathrm{~J}_{\mathrm{HH}}=15.8,{ }^{3} \mathrm{JHH}_{\mathrm{HH}}=9.6 \mathrm{~Hz}, 1 \mathrm{H}, \mathrm{H}-3\right), 1.32\left(\mathrm{dd},{ }^{2} \mathrm{~J}_{\mathrm{HH}}\right.$ $\left.=15.8,{ }^{3} \mathrm{JHH}_{\mathrm{HH}}=6.4 \mathrm{~Hz}, 1 \mathrm{H}, H-3^{\prime}\right), 1.22$ (s, 6H, H-MeBpin), 1.20 (s, 6H, H-MeBpin).

${ }^{13} \mathrm{C}\left\{{ }^{1} \mathrm{H}\right\}$ NMR $\left(126 \mathrm{MHz}, \mathrm{CDCl}_{3}\right) \delta(\mathrm{ppm})=172.3(C-1), 157.1(C-12), 149.8(C-16), 142.5(C-$ 4), $138.7(\mathrm{C}-7), 138.2(\mathrm{C}-8), 137.0(\mathrm{CH}-14), 129.0(\mathrm{CH}-10), 128.6(\mathrm{CH}-5), 127.7(\mathrm{CH}-6), 124.2$ (CH-11), $122.3(\mathrm{CH}-15), 120.6(\mathrm{CH}-13), 119.7$ (CH-9), 83.5 (CO-Bpin), $49.9(\mathrm{CH}-2), 25.0$ ( $\mathrm{CH}_{3}$-MeBpin), $24.9\left(\mathrm{CH}_{3}\right.$-MeBpin), 16.9 (bs, $\left.\mathrm{CH}_{2}-3\right)$.

${ }^{11} \mathrm{~B}$ NMR $\left(96 \mathrm{MHz}, \mathrm{CDCl}_{3}\right) \delta(\mathrm{ppm})=32.7$.

HRMS (ESI +): calculated for $\mathrm{C}_{26} \mathrm{H}_{29} \mathrm{BN}_{2} \mathrm{O}_{3}[\mathrm{M}+\mathrm{H}]^{+}: 429.2349$; found: 429.2351 .

IR (neat): $v\left(\mathrm{~cm}^{-1}\right)=3339,2977,1681,1600,1534,1438,1368,1315,1249,1142,969,849$, 789, 756, 695.

m.p.: $189-190^{\circ} \mathrm{C}$ 
tert-butyl 5-(1-((4-methoxyphenyl)amino)-1-oxo-3-(4,4,5,5-tetramethyl-1,3,2-dioxaborolan-2yl)propan-2-yl)-1H-indole-1-carboxylate 3lb

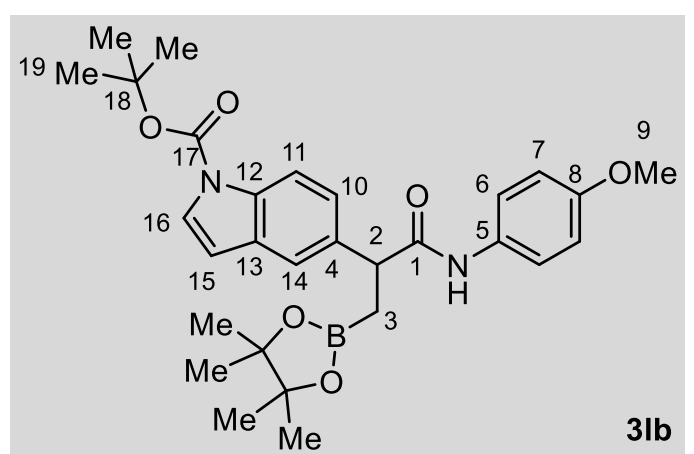

This product was prepared according to the general procedure for the copper-catalyzed borylative carboxamidation of olefins using 5-vinyl $\mathrm{N}$-Boc indole 1 ( $73 \mathrm{mg}, 0.3 \mathrm{mmol}, 1.0$ equiv.), ${ }^{4}$ [(SIMes)CuCl] $(6.1 \mathrm{mg}, 0.015 \mathrm{mmol}, 5.0 \mathrm{~mol} \%)$, LiOtBu (36 mg, 0.45 mmol, 1.5 equiv.), $B_{2} p$ in $_{2}$ (91 $\mathrm{mg}, 0.36 \mathrm{mmol}, 1.2$ equiv.) and 1-isocyanato-4methoxybenzene $\mathbf{2 b}(0.117 \mathrm{~mL}, 0.9 \mathrm{mmol}, 3.0$ equiv.) in toluene $(3.0 \mathrm{~mL}, 0.1 \mathrm{M})$. Reaction time: 20 h. $83 \%$ conversion into 3lb determined by ${ }^{1} \mathrm{H}$ NMR analysis of the crude reaction mixture in presence of an internal standard. Purification by flash chromatography (pentane/ethyl acetate $4: 1$ ) gave pure 3lb as a yellow solid (60 mg, $0.12 \mathrm{mmol}, 40 \%$ yield).

TLC: pentane/ethyl acetate $4: 1, R_{f}=0.3$.

${ }^{1} \mathrm{H}$ NMR $\left(500 \mathrm{MHz}, \mathrm{CDCl}_{3}\right) \delta(\mathrm{ppm})=8.10\left(\mathrm{~d},{ }^{3} \mathrm{JHH}_{\mathrm{HH}}=7.9 \mathrm{~Hz}, 1 \mathrm{H}, \mathrm{H}-11\right), 7.60\left(\mathrm{~d},{ }^{3} \mathrm{JHH}_{\mathrm{HH}}=3.4 \mathrm{~Hz}\right.$, $1 \mathrm{H}, H-16), 7.54(\mathrm{~s}, 1 \mathrm{H}, H-14), 7.28(\mathrm{~m}, 2 \mathrm{H}, H-6+H-10), 6.98$ (bs, $1 \mathrm{H}, \mathrm{N}-H), 6.77$ (d, ${ }^{3} \mathrm{JHH}_{\mathrm{H}}=$ $9.0 \mathrm{~Hz}, 2 \mathrm{H}, H-7), 6.54\left(\mathrm{~d},{ }^{3} \mathrm{~J}_{\mathrm{HH}}=3.4 \mathrm{~Hz}, 1 \mathrm{H}, H-15\right), 3.96\left(\mathrm{dd},{ }^{3} \mathrm{~J}_{\mathrm{HH}}=9.5,6.6 \mathrm{~Hz}, 1 \mathrm{H}, H-2\right), 3.74$ (s, 3H, $H-9$ ), 1.71 (dd, $\left.{ }^{2} J_{H H}=15.7,{ }^{3} J_{H H}=9.5 \mathrm{~Hz}, 1 \mathrm{H}, H-3\right), 1.67$ (s, 9H, $\left.H-19\right), 1.31$ (dd, ${ }^{2} J_{H H}$ $\left.=15.7,{ }^{3} J_{\mathrm{HH}}=6.6 \mathrm{~Hz}, 1 \mathrm{H}, H-3^{\prime}\right), 1.20$ (s, 6H, H-MeBpin), 1.19 (s, 6H, H-MeBpin).

${ }^{13} \mathrm{C}\left\{{ }^{1} \mathrm{H}\right\}$ NMR $\left(126 \mathrm{MHz}, \mathrm{CDCl}_{3}\right) \delta(\mathrm{ppm})=172.8(C-1), 156.3(C-8), 149.8(C-17), 136.2(C-$ 4), $134.5(C-12), 131.5(C-8), 131.2(C-13), 126.7$ ( $C H-16), 124.4(C H-10), 121.4(C H-6), 120.4$ ( $\mathrm{CH}-14), 115.8(\mathrm{CH}-11), 114.1(\mathrm{CH}-7), 107.4(\mathrm{CH}-15), 84.0$ (C-18), 83.4 (CO-Bpin), $55.6\left(\mathrm{CH}_{3^{-}}\right.$ 9), 49.8 ( $\mathrm{CH}-2)$, 28.3 ( $\left.\mathrm{CH}_{3}-19\right), 24.9$ ( $\mathrm{CH}_{3}$-MeBpin), 24.9 ( $\left.\mathrm{CH}_{3}-\mathrm{MeBpin}\right), 17.3$ (bs, $\left.\mathrm{CH}_{2}-3\right)$.

${ }^{11} \mathbf{B}$ NMR $\left(96 \mathrm{MHz}, \mathrm{CDCl}_{3}\right) \delta(\mathrm{ppm})=33.7$.

HRMS (ESI +): calculated for $\mathrm{C}_{29} \mathrm{H}_{37} \mathrm{BN}_{2} \mathrm{O}_{6}[\mathrm{M}+\mathrm{H}]^{+}$: 521.2823; found: 521.2825.

IR (neat): $v\left(\mathrm{~cm}^{-1}\right)=3310,2978,1733,1658,1603,1511,1371,1329,1245,1165,1143$, 1025, 829.

m.p.: $168-173^{\circ} \mathrm{C}$ 
Ferrocenyl amide $3 \mathrm{mb}$

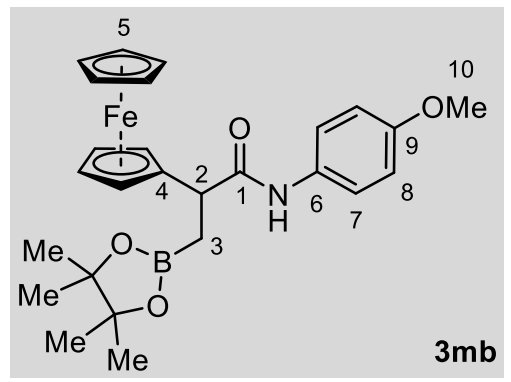

This product was prepared according to the general procedure for the copper-catalyzed borylative carboxamidation of olefins using vinyl ferrocene $1 \mathrm{~m}$ (64 $\mathrm{mg}, 0.3 \mathrm{mmol}, 1.0$ equiv.), [(SIMes)CuCl] (12.2 mg, $0.030 \mathrm{mmol}, 10.0 \mathrm{~mol} \%)$, LiOtBu (36 $\mathrm{mg}, 0.45 \mathrm{mmol}, 1.5$ equiv.), $B_{2} \mathrm{pin}_{2}$ (91 mg, $0.36 \mathrm{mmol}, 1.2$ equiv.) and 1-isocyanato-4-methoxybenzene $2 \mathbf{b}(0.117 \mathrm{~mL}$, $0.9 \mathrm{mmol}, 3.0$ equiv.) in toluene $(3.0 \mathrm{~mL}, 0.1 \mathrm{M})$. Reaction time: $48 \mathrm{~h} .77 \%$ conversion into $3 \mathrm{mb}$ determined by ${ }^{1} \mathrm{H}$ NMR analysis of the crude reaction mixture in presence of an internal standard. Purification by flash chromatography (pentane/ethyl acetate $4: 1$ ) gave pure $3 \mathrm{mb}$ as a yellow solid (90 mg, $0.18 \mathrm{mmol}, 61 \%$ yield).

TLC: pentane/ethyl acetate $4: 1, \mathrm{R}_{\mathrm{f}}=0.3$.

${ }^{1} \mathrm{H}$ NMR $\left(400 \mathrm{MHz}, \mathrm{CDCl}_{3}\right) \delta(\mathrm{ppm})=7.51(\mathrm{bs}, 1 \mathrm{H}, \mathrm{N}-H), 7.36\left(\mathrm{~d},{ }^{3} J_{\mathrm{HH}}=8.8 \mathrm{~Hz}, 2 \mathrm{H}, H-7\right), 6.81$ (d, $\left.{ }^{3} J_{\mathrm{HH}}=8.8 \mathrm{~Hz}, 2 \mathrm{H}, H-8\right), 4.24(\mathrm{~m}, 1 \mathrm{H}, H-\mathrm{Fc}), 4.18(\mathrm{~m}, 8 \mathrm{H}, H-\mathrm{Fc}), 3.77$ (s, 3H, $\left.H-10\right), 3.60$ (dd, $\left.{ }^{3} J_{H H}=9.9,5.3 \mathrm{~Hz}, 1 \mathrm{H}, H-2\right), 1.44(\mathrm{~s}, 2 \mathrm{H}, H-3), 1.28$ (s, 6H, H-MeBpin), 1.26 (s, 6H, HMeBpin).

${ }^{13} \mathrm{C}\left\{{ }^{1} \mathrm{H}\right\}$ NMR $\left(101 \mathrm{MHz}, \mathrm{CDCl}_{3}\right) \delta(\mathrm{ppm})=172.2(C-1), 156.3(C-9), 131.4(C-6), 121.5(\mathrm{CH}-$ 7), 114.2 (CH-8), 90.1 (C-4), 83.5 (CO-Bpin), 69.0 (CH-5), $68.2(\mathrm{CH}-\mathrm{Fc}), 68.1$ (CH-Fc), 68.0 ( $\mathrm{CH}-\mathrm{Fc}), 67.0$ ( $\mathrm{CH}-\mathrm{Fc}), 55.6$ ( $\left.\mathrm{CH}_{3}-10\right)$, 43.9 ( $\left.\mathrm{CH}-2\right), 25.1$ ( $\left.\mathrm{CH}_{3}-\mathrm{MeBpin}\right), 25.0\left(\mathrm{CH}_{3}-\mathrm{MeBpin}\right)$. The boron-bound carbon $\mathrm{CH}_{2}-3$ was not detected due to quadrupolar relaxation.

${ }^{11} \mathbf{B}$ NMR $\left(96 \mathrm{MHz}, \mathrm{CDCl}_{3}\right) \delta(\mathrm{ppm})=32.5$

HRMS (ESI +): calculated for $\mathrm{C}_{26} \mathrm{H}_{32} \mathrm{BFeNO}_{4}[\mathrm{M}+\mathrm{H}]+:$ : 490.1852; found: 521.1852.

IR (neat): $v\left(\mathrm{~cm}^{-1}\right)=3304,2978,1657,1603,1511,1368,1322,1242,1144,1036,968,828$. m.p.: $136-141^{\circ} \mathrm{C}$ 
N-(4-methoxyphenyl)-3-(4,4,5,5-tetramethyl-1,3,2-dioxaborolan-2-yl)-2-(4-(4,4,5,5-

tetramethyl-1,3,2-dioxaborolan-2-yl)phenyl)propanamide 3nb

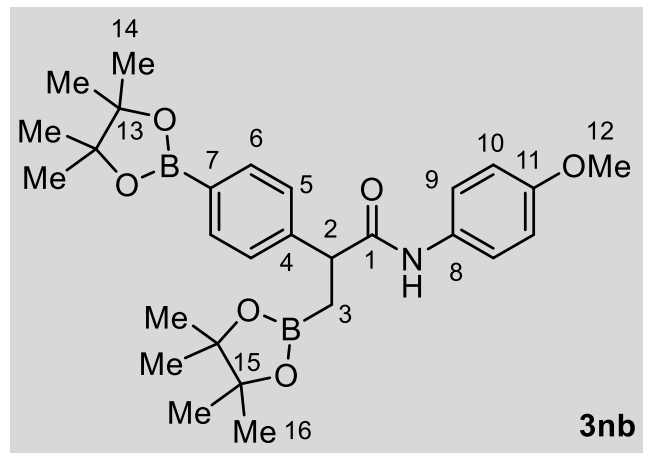

This product was prepared according to the general procedure for the copper-catalyzed borylative carboxamidation of olefins using styrene 1n (69 mg, $0.3 \mathrm{mmol}, 1.0$ equiv. $),{ }^{5}[(\mathrm{SIMes}) \mathrm{CuCl}](6.1 \mathrm{mg}, 0.015$ mmol, 5.0 mol\%), LiOtBu (36 mg, $0.45 \mathrm{mmol}, 1.5$ equiv.), $B_{2}$ pin $_{2}$ (91 mg, $0.36 \mathrm{mmol}, 1.2$ equiv.) and 1mmol, 3.0 equiv.) in toluene $(3.0 \mathrm{~mL}, 0.1 \mathrm{M})$. Reaction time: $20 \mathrm{~h}$. $82 \%$ conversion into $3 \mathrm{nb}$ determined by ${ }^{1} \mathrm{H}$ NMR analysis of the crude reaction mixture in presence of an internal standard. Purification by flash chromatography (pentane/ethyl acetate $4: 1$ ) gave pure $3 \mathrm{nb}$ as a white solid (100 mg, $0.20 \mathrm{mmol}, 66 \%$ yield).

TLC: pentane/ethyl acetate $4: 1, R_{f}=0.2$.

${ }^{1} \mathrm{H}$ NMR $\left(400 \mathrm{MHz}, \mathrm{CDCl}_{3}\right) \delta(\mathrm{ppm})=7.79\left(\mathrm{~d},{ }^{3} \mathrm{~J}_{\mathrm{HH}}=7.9 \mathrm{~Hz}, 2 \mathrm{H}, \mathrm{H}-6\right), 7.36\left(\mathrm{~d},{ }^{3} \mathrm{JHH}_{\mathrm{HH}}=7.9 \mathrm{~Hz}\right.$, $2 \mathrm{H}, H-5), 7.28\left(\mathrm{~d},{ }^{3} \mathrm{~J}_{\mathrm{HH}}=9.0 \mathrm{~Hz}, 2 \mathrm{H}, H-9\right), 6.94(\mathrm{bs}, 1 \mathrm{H}, \mathrm{N}-H), 6.78\left(\mathrm{~d},{ }^{3} \mathrm{~J}_{\mathrm{HH}}=9.0 \mathrm{~Hz}, 2 \mathrm{H}, \mathrm{H}-\right.$ 10), $3.87\left(\mathrm{dd},{ }^{3} J_{\mathrm{HH}}=9.6,6.4 \mathrm{~Hz}, 1 \mathrm{H}, \mathrm{H}-2\right), 3.75(\mathrm{~s}, 3 \mathrm{H}, \mathrm{H}-12), 1.66\left(\mathrm{dd},{ }^{2} J_{\mathrm{HH}}=15.8,{ }^{3} J_{\mathrm{HH}}=9.7\right.$ $\mathrm{Hz}, 1 \mathrm{H}, H-3), 1.34$ (s, 12H, $H-4), 1.28$ (m, 1H, $H-3$ ), 1.21 (s, 6H, $H-16), 1.19$ (s, 6H, $H-16)$. ${ }^{13} \mathrm{C}\left\{{ }^{1} \mathrm{H}\right\}$ NMR $\left(101 \mathrm{MHz}, \mathrm{CDCl}_{3}\right) \delta(\mathrm{ppm})=172.1(C-1), 156.3(C-11), 145.0(C-7), 135.6(\mathrm{CH}-$ 6), $131.4(\mathrm{C}-8), 127.5(\mathrm{CH}-5), 121.5(\mathrm{CH}-9), 114.1(\mathrm{CH}-10), 84.0(\mathrm{C}-13), 83.4(\mathrm{C}-15), 55.6$ ( $\left.\mathrm{CH}_{3}-12\right), 50.1(\mathrm{CH}-2), 25.0\left(\mathrm{CH}_{3}-14\right), 25.0\left(\mathrm{CH}_{3}-14\right), 24.9\left(\mathrm{CH}_{3}-16\right), 24.8\left(\mathrm{CH}_{3}-16\right), 16.8$ (bs, $\left.\mathrm{CH}_{2}-3\right)$.

${ }^{11}$ B NMR $\left(128 \mathrm{MHz}, \mathrm{CDCl}_{3}\right) \delta(\mathrm{ppm})=32.5$

HRMS (ESI +): calculated for $\mathrm{C}_{28} \mathrm{H}_{39} \mathrm{~B}_{2} \mathrm{NO}_{6}[\mathrm{M}+\mathrm{H}]^{+}:$: 508.3046; found: 508.3063 .

IR (neat): $v\left(\mathrm{~cm}^{-1}\right)=3363,2979,1678,1610,1534,1512,1358,1322,1236,1143,1091,966$, 831.

m.p.: $218-222^{\circ} \mathrm{C}$ 
2-(4-(diphenylphosphanyl)phenyl)-N-(4-methoxyphenyl)-3-(4,4,5,5-tetramethyl-1,3,2dioxaborolan-2-yl)propanamide 3ob

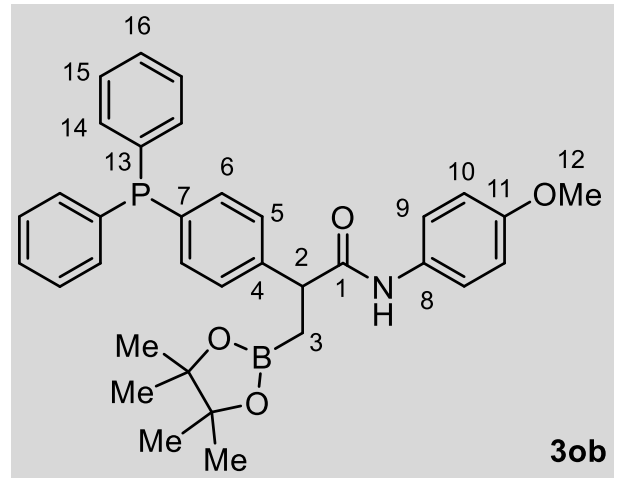

This product was prepared according to the general procedure for the copper-catalyzed borylative carboxamidation of olefins using 4-diphenylphosphine styrene 10 (87 mg, $0.3 \mathrm{mmol}, 1.0$ equiv.), [(SIMes)CuCl] (6.1 mg, $0.015 \mathrm{mmol}, 5.0 \mathrm{~mol} \%$ ), LiOtBu (36 mg, 0.45 mmol, 1.5 equiv.), $B_{2} \operatorname{pin}_{2}$ (91 mg, 0.36 mmol, 1.2 equiv.) and 1-isocyanato-4-methoxybenzene $2 \mathrm{~b}(0.117 \mathrm{~mL}$, $0.9 \mathrm{mmol}, 3.0$ equiv.) in toluene $(3.0 \mathrm{~mL}, 0.1 \mathrm{M})$.

Reaction time: 20 h. $63 \%$ conversion into 3ob determined by ${ }^{1} \mathrm{H}$ NMR analysis of the crude reaction mixture in presence of an internal standard. Purification by flash chromatography (pentane/ethyl acetate $4: 1$ ) gave pure $30 \mathrm{~b}$ as a white solid ( $80 \mathrm{mg}, 0.14 \mathrm{mmol}, 47 \%$ yield).

TLC: pentane/ethyl acetate $4: 1, R_{f}=0.2$.

${ }^{1} \mathrm{H}$ NMR $\left(300 \mathrm{MHz}, \mathrm{CDCl}_{3}\right) \delta(\mathrm{ppm})=7.33(\mathrm{~m}, 16 \mathrm{H}, \mathrm{H}-\mathrm{Ar}+\mathrm{H}-9), 7.10(\mathrm{bs}, 1 \mathrm{H}, \mathrm{N}-\mathrm{H}), 6.81(\mathrm{~d}$, $\left.{ }^{3} J_{\mathrm{HH}}=9.0 \mathrm{~Hz}, 2 \mathrm{H}, H-10\right), 3.84\left(\mathrm{dd},{ }^{3} J_{\mathrm{HH}}=9.2,6.8 \mathrm{~Hz}, 1 \mathrm{H}, H-2\right), 3.76(\mathrm{~s}, 3 \mathrm{H}, H-12), 1.66$ (dd, $\left.{ }^{2} J_{H H}=15.8,{ }^{3} J_{H H}=9.2 \mathrm{~Hz}, 1 \mathrm{H}, H-3\right), 1.36\left(\mathrm{dd},{ }^{2} J_{H H}=15.8,{ }^{3} J_{H H}=6.8 \mathrm{~Hz}, 1 \mathrm{H}, H-3\right.$ '), 1.19 (s, 6H, H-MeBpin), 1.18 (s, 6H, H-MeBpin).

${ }^{13} \mathrm{C}\left\{{ }^{1} \mathrm{H}\right\}$ NMR $(75 \mathrm{MHz}, \mathrm{CDCl}) \delta(\mathrm{ppm})=172.0(C-1), 156.4(C-11), 142.3(C-4), 137.2\left(\mathrm{~d},{ }^{1} \mathrm{JPC}\right.$ $=10.7 \mathrm{~Hz}, C-13), 137.1\left(\mathrm{~d},{ }^{1} J_{\mathrm{PC}}=10.6 \mathrm{~Hz}, C-13\right.$ '), $136.3\left(\mathrm{~d},{ }^{1} J_{\mathrm{PC}}=11.0 \mathrm{~Hz}, C-7\right), 134.4(\mathrm{~d}$, $\left.{ }^{2} J_{\mathrm{PC}}=19.6 \mathrm{~Hz}, \mathrm{CH}-6\right), 133.8\left(\mathrm{~d},{ }^{2} J_{\mathrm{PC}}=19.6 \mathrm{~Hz}, \mathrm{CH}-14\right), 133.8\left(\mathrm{~d},{ }^{2} J_{\mathrm{PC}}=19.6 \mathrm{~Hz}, \mathrm{CH}-14\right.$ ') $131.3(C-8), 128.6$ (d, ${ }^{3} J_{P C}=6.9 \mathrm{~Hz}, C H-15+C H-15$ ) $, 128.3\left(d,{ }^{3} J_{P C}=7.0 \mathrm{~Hz}, C H-5\right), 121.5$ (CH-9), 114.1 ( $\mathrm{CH}-10), 83.5$ (CO-Bpin), $55.6\left(\mathrm{CH}_{3}-12\right)$, 49.6 ( $\left.\mathrm{CH}-2\right), 24.9$ ( $\left.\mathrm{CH}_{3}-\mathrm{MeBpin}\right), 248$ ( $\left.\mathrm{CH}_{3}-\mathrm{MeBpin}\right), 16.8$ (bs, $\left.\mathrm{CH}_{2}-3\right)$.

${ }^{11} \mathbf{B}$ NMR $\left(96 \mathrm{MHz}, \mathrm{CDCl}_{3}\right) \delta(\mathrm{ppm})=34.6$.

${ }^{31} \mathbf{P}\left\{{ }^{1} \mathrm{H}\right\}$ NMR $\left(121 \mathrm{MHz}, \mathrm{CDCl}_{3}\right) \delta(\mathrm{ppm})=-6.0$.

HRMS (ESI +): calculated for $\mathrm{C}_{34} \mathrm{H}_{37} \mathrm{BNO}_{4} \mathrm{P}[\mathrm{M}+\mathrm{H}]^{+}:$566.2632; found: 566.2625.

IR (neat): $v\left(\mathrm{~cm}^{-1}\right)=3301,2977,2925,1656,1602,1510,1369,1321,1240,1142,1035,968$, $827,742$.

m.p.: $66-75^{\circ} \mathrm{C}$ 
steroid $\mathbf{3 p b}$

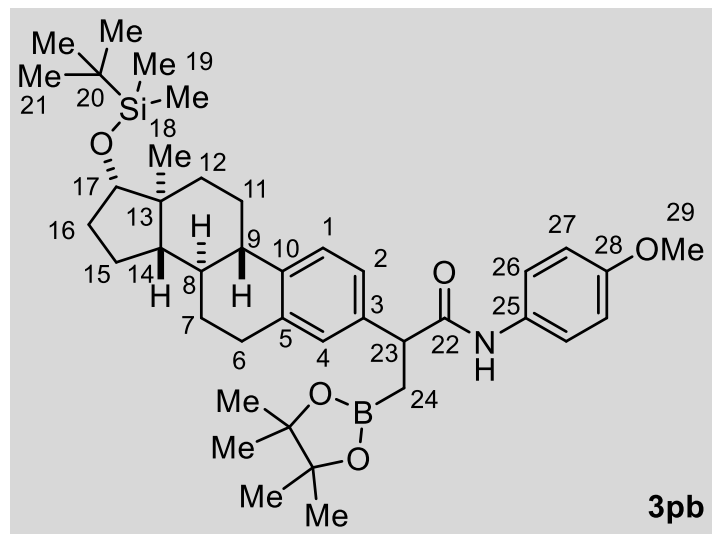

This product was prepared according to the general procedure for the copper-catalyzed borylative carboxamidation of olefins using $\beta$ estradiol derived styrene 1p (60 mg, $0.15 \mathrm{mmol}$, 1.0 equiv.), ${ }^{6}$ [(SIMes)CuCl] $(6.1 \mathrm{mg}, 0.015 \mathrm{mmol}$, $10.0 \mathrm{~mol} \%$ ), LiOtBu (18 mg, $0.23 \mathrm{mmol}, 1.5$ equiv.), $B_{2}$ pin $_{2}$ (46 mg, $0.18 \mathrm{mmol}, 1.2$ equiv.) and 1-isocyanato-4-methoxybenzene $\mathbf{2 b}(0.060 \mathrm{~mL}$, $0.45 \mathrm{mmol}, 3.0$ equiv.) in toluene (1.5 mL, $0.1 \mathrm{M})$. Reaction time: $20 \mathrm{~h} .80 \%$ conversion into $3 \mathrm{pb}$ determined by ${ }^{1} \mathrm{H}$ NMR analysis of the crude reaction mixture in presence of an internal standard. Purification by flash chromatography (pentane/ethyl acetate 9:1 to 85:15) gave pure $3 \mathrm{pb}$ as a white solid (65 mg, $0.097 \mathrm{mmol}, 65 \%$ yield).

TLC: pentane/ethyl acetate $4: 1, R_{f}=0.4$.

${ }^{1} \mathrm{H}$ NMR $\left(500 \mathrm{MHz}, \mathrm{CDCl}_{3}\right) \delta(\mathrm{ppm})=7.33\left(\mathrm{~d},{ }^{3} \mathrm{~J}_{\mathrm{HH}}=9.0 \mathrm{~Hz}, 2 \mathrm{H}, \mathrm{H}-26\right), 7.25\left(\mathrm{~d},{ }^{3} \mathrm{JHH}_{\mathrm{HH}}=8.0 \mathrm{~Hz}\right.$, $1 \mathrm{H}, H-1), 7.10\left(\mathrm{~d},{ }^{3} J_{\mathrm{HH}}=8.0 \mathrm{~Hz}, 1 \mathrm{H}, H-2\right), 7.04(\mathrm{~s}, 1 \mathrm{H}, H-4), 7.03(\mathrm{~s}, 1 \mathrm{H}, \mathrm{N}-H), 6.79\left(\mathrm{~d},{ }^{3} J_{\mathrm{HH}}=\right.$ $9.0 \mathrm{~Hz}, 2 \mathrm{H}, H-27), 3.79\left(\mathrm{dd},{ }^{3} \mathrm{~J}_{\mathrm{HH}}=9.7,{ }^{3} \mathrm{JHH}_{\mathrm{H}}=5.9 \mathrm{~Hz}, 1 \mathrm{H}, H-23\right), 3.76(\mathrm{~s}, 3 \mathrm{H}, H-29), 3.65$ (t, $\left.{ }^{3} J_{H H}=8.3 \mathrm{~Hz}, 1 \mathrm{H}, H-17\right), 2.84(\mathrm{~m}, 2 \mathrm{H}, H-6), 2.29(\mathrm{~m}, 1 \mathrm{H}, H-11), 2.20(\mathrm{~m}, 1 \mathrm{H}, H-9), 1.94(\mathrm{~m}$, $1 \mathrm{H}, H-7), 1.89(\mathrm{~m}, 2 \mathrm{H}, H-16+H-12), 1.67(\mathrm{~m}, 1 \mathrm{H}, H-15), 1.62\left(\mathrm{dd},{ }^{2} J_{\mathrm{HH}}=15.7 \mathrm{~Hz},{ }^{3} J_{\mathrm{HH}}=9.7\right.$ $\mathrm{Hz}, 1 \mathrm{H}, H-24), 1.47\left(\mathrm{~m}, 3 \mathrm{H}, H-8+H-11+H-16^{\prime}\right), 1.33\left(\mathrm{~m}, 3 \mathrm{H}, H-7^{\prime}+H-12^{\prime}+H-15^{\prime}\right), 1.27$ (m, 1H, $H$-24'), 1.23 (s, 6H, H-MeBpin), 1.20 (s, 6H, H-MeBpin), 1.15 (m, 1H, $H$-14), 0.90 (s, 9H, $H-21), 0.75$ (s, 3H, H-18), 0.04 (s, 3H, H-19), 0.03 (s, 3H, H-19).

${ }^{13} \mathrm{C}\left\{{ }^{1} \mathrm{H}\right\}$ NMR $(126 \mathrm{MHz}, \mathrm{CDCl}) \delta(\mathrm{ppm})=172.6(C-22), 156.2(C-28), 139.8(C-10), 139.0(C-$ 3), $137.5(C-5), 131.5(C-25), 128.6(\mathrm{CH}-4), 126.1(\mathrm{CH}-1), 125.2(\mathrm{CH}-2), 121.5(\mathrm{CH}-26), 114.1$ (CH-27), 83.3 (CO-Bpin), $81.9(\mathrm{CH}-17), 55.6\left(\mathrm{CH}_{3}-29\right), 49.9(\mathrm{CH}-14), 49.5(\mathrm{CH}-23), 44.6(\mathrm{CH}-$ 9), 43.7 (C-13), $38.8(\mathrm{CH}-8), 37.3\left(\mathrm{CH}_{2}-12\right), 31.1\left(\mathrm{CH}_{2}-16\right), 29.7\left(\mathrm{CH}_{2}-6\right), 27.3\left(\mathrm{CH}_{2}-7\right), 26.3$ ( $\left.\mathrm{CH}_{2}-11\right)$, $26.0\left(\mathrm{CH}_{3}-21\right)$, 25.0 ( $\left.\mathrm{CH}_{3}-\mathrm{MeBpin}\right), 24.8$ ( $\mathrm{CH}_{3}$-MeBpin), $23.4\left(\mathrm{CH}_{2}-15\right), 18.3(\mathrm{C}-20)$, 17.1 (bs, $\left.\mathrm{CH}_{2}-24\right), 11.5\left(\mathrm{CH}_{3}-18\right),-4.3\left(\mathrm{CH}_{3}-19\right),-4.7\left(\mathrm{CH}_{3}-19\right)$.

${ }^{11} \mathbf{B}$ NMR $\left(96 \mathrm{MHz}, \mathrm{CDCl}_{3}\right) \delta(\mathrm{ppm})=34.6$.

HRMS (ESI +): calculated for $\mathrm{C}_{40} \mathrm{H}_{60} \mathrm{BNO}_{5} \mathrm{Si}[\mathrm{M}+\mathrm{H}]^{+}: 674.4415$; found: 674.4425

IR (neat): $v\left(\mathrm{~cm}^{-1}\right)=3314,2929,2856,1659,1603,1511,1464,1369,1321,1246,1142$, 1095, 968, 909, 885, 833, 775, 733.

m.p.: $158-160^{\circ} \mathrm{C}$

The diastereoisomeric ratio $d r$ 1:1 was determined after boronate oxidation to alcohol 15 and derivatization to the (S)-Mosher (MTPA) ester using ( $R$ )-MTPA-Cl. Details can be found in Section 5. 
2-methyl-N,2-diphenyl-3-(4,4,5,5-tetramethyl-1,3,2-dioxaborolan-2-yl)propanamide 3qa

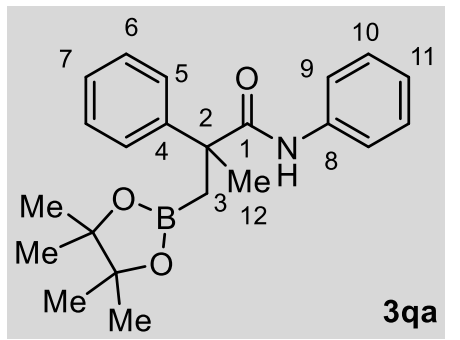

This product was prepared according to the general procedure for the copper-catalyzed borylative carboxamidation of olefins using $\alpha$-methyl styrene 1q (36 $\mathrm{mg}, 0.3 \mathrm{mmol}, 1.0$ equiv.), [(SIMes)CuCl] (12.2 mg, $0.030 \mathrm{mmol}, 10.0 \mathrm{~mol} \%)$, LiOtBu (36 mg, $0.45 \mathrm{mmol}, 1.5$ equiv.), $B_{2}$ pin $_{2}(91 \mathrm{mg}, 0.36 \mathrm{mmol}, 1.2$ equiv.) and 1-isocyanatobenzene $2 \mathrm{a}(0.098 \mathrm{~mL}, 0.9 \mathrm{mmol}, 3.0$ equiv.) in toluene (3.0 mL, $0.1 \mathrm{M}$ ). Reaction time: $20 \mathrm{~h}$. 33\% conversion into $3 q$ a determined by ${ }^{1} \mathrm{H}$ NMR analysis of the crude reaction mixture in presence of an internal standard. Purification by flash chromatography (pentane/ethyl acetate 10:1 to 9:1) gave pure 3qa as a colorless oil (26 mg, $0.072 \mathrm{mmol}, 24 \%$ yield).

TLC: pentane/ethyl acetate $4: 1, R_{f}=0.3$.

${ }^{1} \mathbf{H}$ NMR $\left(400 \mathrm{MHz}, \mathrm{CDCl}_{3}\right) \delta(\mathrm{ppm})=7.62(\mathrm{bs}, 1 \mathrm{H}, \mathrm{N}-\mathrm{H}), 7.47-7.37(\mathrm{~m}, 4 \mathrm{H}, \mathrm{H}-\mathrm{Ar}), 7.34(\mathrm{t}$, $\left.{ }^{3} J_{H H}=7.6 \mathrm{~Hz}, 2 \mathrm{H}, \mathrm{H}-\mathrm{Ar}\right), 7.27(\mathrm{~m}, 3 \mathrm{H}, \mathrm{H}-\mathrm{Ar}), 7.05\left(\mathrm{t},{ }^{3} \mathrm{~J}_{\mathrm{HH}}=7.4 \mathrm{~Hz}, 1 \mathrm{H}, \mathrm{H}-11\right), 1.73(\mathrm{~s}, 3 \mathrm{H}, \mathrm{H}-$ 12), $1.69\left(\mathrm{~d},{ }^{2} J_{\mathrm{HH}}=15.8 \mathrm{~Hz}, 1 \mathrm{H}, H-3\right), 1.51\left(\mathrm{~d},{ }^{2} \mathrm{~J}_{\mathrm{HH}}=15.8 \mathrm{~Hz}, 1 \mathrm{H}, H-3\right), 1.17$ (s, 6H, H-MeBpin), 1.12 (s, 6H, H-MeBpin).

${ }^{13} \mathrm{C}\left\{{ }^{1} \mathrm{H}\right\}$ NMR $\left(75 \mathrm{MHz}, \mathrm{CDCl}_{3}\right) \delta(\mathrm{ppm})=175.8(C-1), 145.9(\mathrm{C}-4), 138.7(C-8), 129.0(\mathrm{CH}-$ $\mathrm{Ar}), 128.8$ (CH-Ar), $127.0(\mathrm{CH}-7), 126.6(\mathrm{CH}-\mathrm{Ar}), 123.9(\mathrm{CH}-11), 119.6(\mathrm{CH}-9), 83.6$ (COBpin), 50.2 (C-2), 28.3 ( $\left.\mathrm{CH}_{3}-12\right)$, 25.0 ( $\left.\mathrm{CH}_{3}-\mathrm{MeBpin}\right), 24.7\left(\mathrm{CH}_{3}-\mathrm{MeBpin}\right)$. The boron-bound carbon $\mathrm{CH}-3$ was not detected due to quadrupolar relaxation.

${ }^{11} \mathbf{B}$ NMR $\left(96 \mathrm{MHz}, \mathrm{CDCl}_{3}\right) \delta(\mathrm{ppm})=32.5$

HRMS (ESI +): calculated for $\mathrm{C}_{22} \mathrm{H}_{28} \mathrm{BNO}_{3} \mathrm{Na}[\mathrm{M}+\mathrm{Na}]^{+}:$389.2137; found: 389.2047.

IR (neat): $v\left(\mathrm{~cm}^{-1}\right)=3341,2978,1672,1599,1521,1498,1439,1357,1313,1241,1142,969$, 847. 
anti-N-(4-methoxyphenyl)-2,3-diphenyl-3-(4,4,5,5-tetramethyl-1,3,2-dioxaborolan-2-

yl)propanamide 3rb

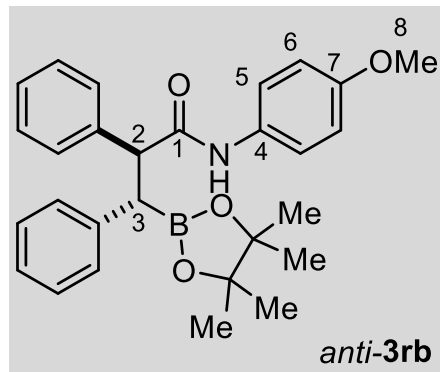

This product was prepared according to the general procedure for the copper-catalyzed borylative carboxamidation of olefins using trans-stilbene $1 \mathrm{r}$ (38 mg, $0.2 \mathrm{mmol}, 1.0$ equiv.), [(SIMes)CuCl] (8.1 mg, $0.02 \mathrm{mmol}, 10.0 \mathrm{~mol} \%$ ), LiOtBu (24 mg, $0.3 \mathrm{mmol}, 1.5$ equiv.), $B_{2}$ pin $_{2}$ (61 mg, $0.24 \mathrm{mmol}, 1.2$ equiv.) and 1-isocyanato4-methoxybenzene $2 \mathrm{~b}(0.078 \mathrm{~mL}, 0.6 \mathrm{mmol}, 3.0$ equiv.) in toluene $(2.0 \mathrm{~mL}, 0.1 \mathrm{M})$. Reaction time: $20 \mathrm{~h} .23 \%$ conversion into anti-3rb $(d r>20: 1)$ determined by ${ }^{1} \mathrm{H}$ NMR analysis of the crude reaction mixture in presence of an internal standard. Purification by flash chromatography (pentane/ethyl acetate 85:15) gave pure anti3rb $(d r>20: 1)$ as a white solid (15 mg, $0.033 \mathrm{mmol}, 17 \%$ yield).

TLC: pentane/ethyl acetate $4: 1, R_{f}=0.3$.

${ }^{1} \mathrm{H}$ NMR $\left(400 \mathrm{MHz}, \mathrm{CDCl}_{3}\right) \delta(\mathrm{ppm})=7.32\left(\mathrm{~d},{ }^{3} \mathrm{~J}_{\mathrm{HH}}=8.6 \mathrm{~Hz}, 2 \mathrm{H}, \mathrm{H}-5\right), 7.17(\mathrm{~m}, 3 \mathrm{H}), 7.12-$ $7.03(\mathrm{~m}, 6 \mathrm{H}), 7.01(\mathrm{~m}, 1 \mathrm{H}), 6.85(\mathrm{bs}, 1 \mathrm{H}, \mathrm{N}-\mathrm{H}), 6.80\left(\mathrm{~d},{ }^{3} \mathrm{~J}_{\mathrm{HH}}=8.6 \mathrm{~Hz}, 2 \mathrm{H}, \mathrm{H}-6\right), 4.11\left(\mathrm{~d},{ }^{3} J_{\mathrm{HH}}\right.$ $=12.2 \mathrm{~Hz}, 1 \mathrm{H}, H-2), 3.76(\mathrm{~s}, 3 \mathrm{H}, H-8), 2.95\left(\mathrm{~d},{ }^{3} \mathrm{~J}_{\mathrm{HH}}=12.2 \mathrm{~Hz}, 1 \mathrm{H}, H-3\right), 1.24(\mathrm{~s}, 6 \mathrm{H}, H-$ MeBpin), 1.17 (s, 6H, H-MeBpin).

${ }^{13} \mathrm{C}\left\{{ }^{1} \mathrm{H}\right\}$ NMR $\left(101 \mathrm{MHz}, \mathrm{CDCl}_{3}\right) \delta(\mathrm{ppm})=172.5(C-1), 156.4(C-7), 139.8(C-\mathrm{Ar}), 139.3(C-$ Ar), 131.2 (C-4), 129.2 (CH-Ar), 129.0 (CH-Ar), 128.6 (CH-Ar), 128.1 ( $\mathrm{CH}-\mathrm{Ar}), 127.4$ (CH-Ar), 125.4 (CH-Ar), 121.5 ( $\mathrm{CH}-\mathrm{Ar}), 114.2$ ( $\mathrm{CH}-\mathrm{Ar}), 83.4$ (CO-Bpin), $57.7(\mathrm{CH}-2), 55.6\left(\mathrm{CH}_{3}-8\right), 37.7$ (bs, $\mathrm{CH}-3), 24.8$ ( $\left.\mathrm{CH}_{3}-\mathrm{MeBpin}\right), 24.7\left(\mathrm{CH}_{3}-\mathrm{MeBpin}\right)$.

${ }^{11} \mathbf{B}$ NMR $\left(128 \mathrm{MHz}, \mathrm{CDCl}_{3}\right) \delta(\mathrm{ppm})=31.7$

HRMS (ESI +): calculated for $\mathrm{C}_{28} \mathrm{H}_{32} \mathrm{BNO}_{4}[\mathrm{M}+\mathrm{H}]^{+}:$: 458.2503; found: 458.2517.

IR (neat): $v\left(\mathrm{~cm}^{-1}\right)=3324,2978,1672,1530,1511,1357,1321,1237,1138,1033,829,735$, 700.

Relative stereochemistry for anti-3rb was assigned after oxidation to the corresponding antialcohol by ${ }^{1} \mathrm{H}$ NMR correlation with a known compound for which relative stereochemistry was unambiguously assigned. ${ }^{7}$ 
2-(benzofuran-2-yl)-2-methyl-N-phenyl-3-(4,4,5,5-tetramethyl-1,3,2-dioxaborolan-2yl)propanamide 3sa

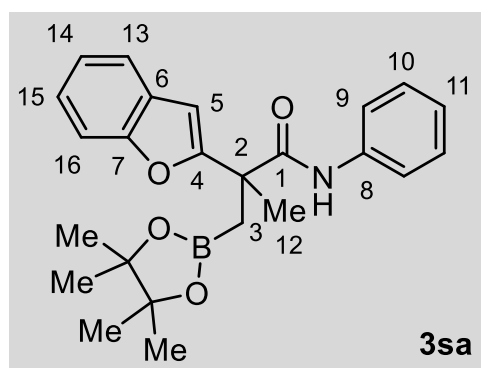

This product was prepared according to the general procedure for the copper-catalyzed borylative carboxamidation of olefins using 2-(prop-1-en-2-yl)benzofuran $1 \mathrm{~s}$ (24 mg, $0.15 \mathrm{mmol}, 1.0$ equiv.), [(SIMes)CuCl] (6.1 $\mathrm{mg}, 0.015 \mathrm{mmol}, 10.0 \mathrm{~mol} \%)$, LiOtBu (18 mg, $0.23 \mathrm{mmol}, 1.5$ equiv.), $\mathrm{B}_{2} \mathrm{pin}_{2}$ (46 mg, 0.18 3sa mmol, 1.2 equiv.) and 1-isocyanatobenzene $2 \mathbf{a}(0.098 \mathrm{~mL}, 0.9$ mmol, 3.0 equiv.) in toluene $(1.5 \mathrm{~mL}, 0.1 \mathrm{M})$. Reaction time: $20 \mathrm{~h} .54 \%$ conversion into a mixture 3sa/3sa' = 3:1 mixture determined by ${ }^{1} \mathrm{H}$ NMR analysis of the crude reaction mixture in presence of an internal standard. Purification by flash chromatography (pentane/ethyl acetate 9:1) allowed to separate pure $3 \mathbf{s a}$ (13 mg, $0.03 \mathrm{mmol}, 21 \%$ yield) and 3sa' (6 mg, $0.015 \mathrm{mmol}, 10 \%$ yield) as colorless oils.

Full characterization for 3 sa:

TLC: pentane/ethyl acetate $4: 1, \mathrm{R}_{\mathrm{f}}=0.2$.

${ }^{1} \mathrm{H}$ NMR $\left(300 \mathrm{MHz}, \mathrm{CD}_{2} \mathrm{Cl}_{2}\right) \delta(\mathrm{ppm})=7.78(\mathrm{bs}, 1 \mathrm{H}, \mathrm{N}-\mathrm{H}), 7.58(\mathrm{~m}, 1 \mathrm{H}), 7.45(\mathrm{~m}, 3 \mathrm{H}), 7.22(\mathrm{~m}$, $4 \mathrm{H}), 7.07\left(\mathrm{t},{ }^{3} \mathrm{~J}_{\mathrm{HH}}=7.4 \mathrm{~Hz}, 1 \mathrm{H}, H-11\right), 6.71\left(\mathrm{~d},{ }^{4} \mathrm{~J}_{\mathrm{HH}}=0.9 \mathrm{~Hz}, 1 \mathrm{H}, H-5\right), 1.80(\mathrm{~s}, 3 \mathrm{H}, H-12), 1.71$ (d, $\left.{ }^{2} J_{H H}=15.5 \mathrm{~Hz}, 1 \mathrm{H}, H-3\right), 1.45\left(\mathrm{~d},{ }^{2} J_{H H}=15.5 \mathrm{~Hz}, 1 \mathrm{H}, H-3\right.$ '), 1.19 (s, 6H, H-MeBpin), 1.18 (s, $6 \mathrm{H}, H$-MeBpin).

${ }^{13} \mathrm{C}\left\{{ }^{1} \mathrm{H}\right\}$ NMR $\left(75 \mathrm{MHz}, \mathrm{CD}_{2} \mathrm{Cl}_{2}\right) \delta(\mathrm{ppm})=172.6(C-1), 161.7(C-4), 155.3(C-7), 138.7(C-8)$, 129.2 (CH-Ar), 128.8 (C-6), 124.6 (CH-Ar), 124.4 (CH-Ar), 123.2 ( $\mathrm{CH}-\mathrm{Ar}), 121.3$ (CH-Ar), 120.3 (CH-9), 111.4 (CH-16), 103.8 (CH-5), 83.7 (CO-Bpin), 47.5 (C-2), 25.1 ( $\left.\mathrm{CH}_{3}-\mathrm{MeBpin}\right)$, $24.9\left(\mathrm{CH}_{3}-\mathrm{MeBpin}\right)$. The boron-bound carbon $\mathrm{CH}_{2}-3$ was not detected due to quadrupolar relaxation.

${ }^{11} \mathbf{B}$ NMR $\left(96 \mathrm{MHz}, \mathrm{CD}_{2} \mathrm{Cl}_{2}\right) \delta(\mathrm{ppm})=32.7$

HRMS (ESI +): calculated for $\mathrm{C}_{24} \mathrm{H}_{28} \mathrm{BNO}_{4} \mathrm{Na}[\mathrm{M}+\mathrm{Na}]^{+}:$: 429.2087; found: 429.2012.

IR (neat): $v\left(\mathrm{~cm}^{-1}\right)=3341,2978,1686,1599,1525,1454,1440,1360,1316,1254,1143$, $970,848,751,692$. 
N-phenyl-2-(1-(4,4,5,5-tetramethyl-1,3,2-dioxaborolan-2-yl)propan-2-yl)benzofuran-3carboxamide 3sa'

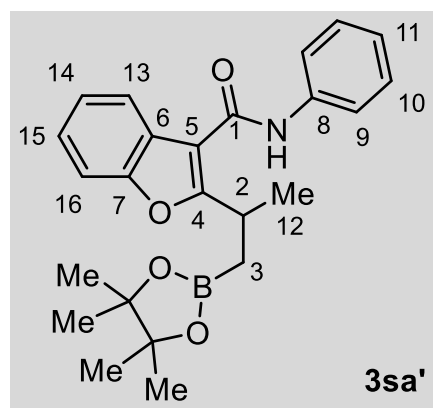

This product was prepared according to the general procedure for the copper-catalyzed borylative carboxamidation of olefins using 2-(prop-1-en-2-yl)benzofuran $1 \mathrm{~s}$ (24 mg, $0.15 \mathrm{mmol}, 1.0$ equiv.), [(SIMes)CuCl] (6.1 mg, $0.015 \mathrm{mmol}, 10.0 \mathrm{~mol} \%)$, LiOtBu (18 mg, $0.23 \mathrm{mmol}, 1.5$ equiv.), $\mathrm{B}_{2} \operatorname{pin}_{2}(46 \mathrm{mg}, 0.18 \mathrm{mmol}, 1.2$ equiv.) and 1-isocyanatobenzene $2 a(0.098 \mathrm{~mL}, 0.9 \mathrm{mmol}, 3.0$ equiv.) in toluene $(1.5 \mathrm{~mL}, 0.1 \mathrm{M})$. Reaction time: $20 \mathrm{~h} .54 \%$ conversion into a mixture 3sa/3sa' = 3:1 mixture determined by ${ }^{1} \mathrm{H}$ NMR analysis of the crude reaction mixture in presence of an internal standard. Purification by flash chromatography (pentane/ethyl acetate 9:1) allowed to separate pure 3sa (13 mg, $0.03 \mathrm{mmol}, 21 \%$ yield) and 3sa' (6 mg, $0.015 \mathrm{mmol}, 10 \%$ yield) as colorless oils.

Full characterization for 3sa':

TLC: pentane/ethyl acetate $4: 1, R_{f}=0.3$.

${ }^{1} \mathrm{H}$ NMR $\left(400 \mathrm{MHz}, \mathrm{CDCl}_{3}\right) \delta(\mathrm{ppm})=9.31(\mathrm{bs}, 1 \mathrm{H}, \mathrm{N}-H), 7.90(\mathrm{~m}, 1 \mathrm{H}, H-13), 7.80\left(\mathrm{~d},{ }^{3} \mathrm{JHH}_{\mathrm{HH}}=\right.$ $9.5 \mathrm{~Hz}, 2 \mathrm{H}, \mathrm{H}-10), 7.45(\mathrm{~m}, 1 \mathrm{H}, \mathrm{H}-16), 7.39$ (m, 2H, H-9), $7.28(\mathrm{~m}, 2 \mathrm{H}, \mathrm{H}-14+\mathrm{H}-15), 7.15$ (t, $\left.{ }^{3} J_{\mathrm{HH}}=7.9 \mathrm{~Hz}, 1 \mathrm{H}, H-11\right), 3.71(\mathrm{~m}, 1 \mathrm{H}, H-2), 1.61\left(\mathrm{dd},{ }^{2} J_{\mathrm{HH}}=16.6 \mathrm{~Hz},{ }^{3} J_{\mathrm{HH}}=11.9 \mathrm{~Hz}, 1 \mathrm{H}, \mathrm{H}-\right.$ 3), $1.40\left(\mathrm{~d},{ }^{3} \mathrm{~J}_{\mathrm{HH}}=6.8 \mathrm{~Hz}, 3 \mathrm{H}, H-12\right), 1.35\left(\mathrm{dd},{ }^{2} J_{\mathrm{HH}}=16.6 \mathrm{~Hz},{ }^{3} J_{\mathrm{HH}}=4.8 \mathrm{~Hz}, 1 \mathrm{H}, H-3\right.$ '), 1.12 (s, 6H, H-MeBpin), 1.05 (s, 6H, H-MeBpin).

${ }^{13} \mathrm{C}\left\{{ }^{1} \mathrm{H}\right\}$ NMR $\left(101 \mathrm{MHz}, \mathrm{CDCl}_{3}\right) \delta(\mathrm{ppm})=163.3(C-1), 162.5(C-4), 153.9(C-7), 138.9(C-8)$, 129.2 ( $\mathrm{CH}-10), 127.0$ (C-6), 124.5 ( $\mathrm{CH}-\mathrm{Ar}), 124.2(\mathrm{CH}-\mathrm{Ar}), 123.5(\mathrm{CH}-\mathrm{Ar}), 121.2(\mathrm{CH}-13)$, 119.8 (CH-9), 112.5 (C-5), $111.0(\mathrm{CH}-16), 84.2$ (CO-Bpin), $29.2\left(\mathrm{CH}_{2}-2\right), 24.9\left(\mathrm{CH}_{3}-\mathrm{MeBpin}\right)$, 24.5 ( $\left.\mathrm{CH}_{3}-\mathrm{MeBpin}\right), 23.3\left(\mathrm{CH}_{3}-12\right), 18.5$ (bs, $\left.\mathrm{CH}_{2}-3\right)$.

${ }^{11} \mathbf{B}$ NMR $\left(96 \mathrm{MHz}, \mathrm{CDCl}_{3}\right) \delta(\mathrm{ppm})=32.1$

HRMS (ESI +): calculated for $\mathrm{C}_{24} \mathrm{H}_{28} \mathrm{BNO}_{4}[\mathrm{M}+\mathrm{H}]^{+}:$406.2189; found: 406.2184 .

IR (neat): $v\left(\mathrm{~cm}^{-1}\right)=3316,2977,2928,1672,1598,1539,1442,1371,1309,1142,1058,847$, 752. 
$N$-(4-methoxyphenyl)-7-methyl-3-methylene-2-((4,4,5,5-tetramethyl-1,3,2-dioxaborolan-2yl)methyl)oct-6-enamide 3tb

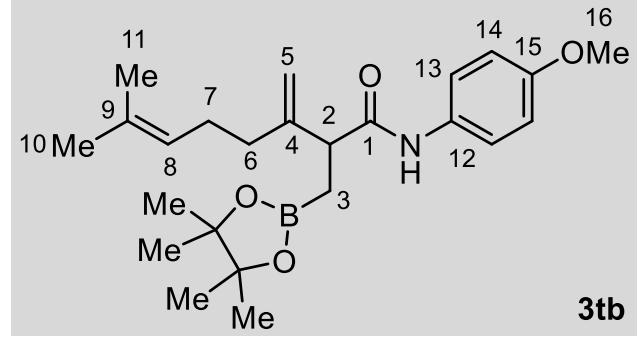

This product was prepared according to the general procedure for the copper-catalyzed borylative carboxamidation of olefins using myrcene 1t $(41 \mathrm{mg}$, 0.3 mmol, 1.0 equiv.), [(SIMes) $\mathrm{CuCl}]$ (12.2 mg, 0.030 mmol,10.0 mol\%), LiOtBu (36 mg, $0.45 \mathrm{mmol}, 1.5$ equiv.), $B_{2}$ pin $_{2}$ (91 mg, $0.36 \mathrm{mmol}, 1.2$ equiv.) and 1 isocyanato-4-methoxybenzene $\mathbf{2 b}(0.117 \mathrm{~mL}, 0.9 \mathrm{mmol}, 3.0$ equiv.) in toluene $(3.0 \mathrm{~mL}, 0.1 \mathrm{M})$. Reaction time: 20 h. $71 \%$ conversion into $3 \mathrm{tb},>20: 1$ chemoselectivity determined by ${ }^{1} \mathrm{H}$ NMR analysis of the crude reaction mixture in presence of an internal standard. Purification by flash chromatography (pentane/ethyl acetate 10:1 to 9:1) gave pure 3tb as a white solid (70 mg, $0.17 \mathrm{mmol}, 57 \%$ yield).

TLC: pentane/ethyl acetate $4: 1, R_{f}=0.3$.

${ }^{1} \mathbf{H}$ NMR $\left(300 \mathrm{MHz}, \mathrm{CDCl}_{3}\right) \delta(\mathrm{ppm})=7.39$ (bs, $\left.1 \mathrm{H}, \mathrm{N}-H\right), 7.37\left(\mathrm{~d},{ }^{3} \mathrm{JHH}_{\mathrm{HH}}=9.0 \mathrm{~Hz}, 2 \mathrm{H}, \mathrm{H}-13\right)$, $6.82\left(\mathrm{~d},{ }^{3} \mathrm{~J}_{\mathrm{HH}}=9.0 \mathrm{~Hz}, 2 \mathrm{H}, H-14\right), 5.11(\mathrm{~s}, 1 \mathrm{H}, H-5), 5.08(\mathrm{~m}, 1 \mathrm{H}, H-8), 5.03(\mathrm{~s}, 1 \mathrm{H}, H-5$ ) $), 3.77$ (s, 3H, H-16), 3.37 (dd, $\left.{ }^{3} \mathrm{JHH}_{\mathrm{H}}=9.3,6.7 \mathrm{~Hz}, 1 \mathrm{H}, \mathrm{H}-2\right), 2.14(\mathrm{~m}, 4 \mathrm{H}, \mathrm{H}-6+\mathrm{H}-7), 1.64(\mathrm{~s}, 3 \mathrm{H}, \mathrm{H}-$ 10), $1.56(\mathrm{~s}, 3 \mathrm{H}, H-11), 1.39\left(\mathrm{dd},{ }^{2} J_{H H}=15.6 \mathrm{~Hz},{ }^{3} J_{H H}=9.3 \mathrm{~Hz}, 1 \mathrm{H}, H-3\right), 1.24(\mathrm{~s}, 6 \mathrm{H}, H-$ MeBpin), 1.23 (s, 6H, H-MeBpin), 1.03 (dd, ${ }^{2} J_{H H}=15.6,{ }^{3} J_{H H}=6.7 \mathrm{~Hz}, 1 \mathrm{H}, H-3$ ').

${ }^{13} \mathrm{C}\left\{{ }^{1} \mathrm{H}\right\}$ NMR $\left.(75 \mathrm{MHz}, \mathrm{CDCl})_{3}\right) \delta(\mathrm{ppm})=171.7(C-1), 156.2(C-15), 150.2(C-4), 132.3(C-9)$, 131.5 (C-12), $123.8(\mathrm{CH}-8), 121.3(\mathrm{CH}-13), 114.1(\mathrm{CH}-14), 112.9\left(\mathrm{CH}_{2}-5\right), 83.3$ (CO-Bpin), $55.6\left(\mathrm{CH}_{3}-16\right), 51.3(\mathrm{CH}-2), 33.6\left(\mathrm{CH}_{2}-7\right), 26.4\left(\mathrm{CH}_{2}-6\right), 25.8\left(\mathrm{CH}_{3}-10\right), 25.0\left(\mathrm{CH}_{3}-\mathrm{MeBpin}\right)$, $24.8\left(\mathrm{CH}_{3}-\mathrm{MeBpin}\right), 17.9\left(\mathrm{CH}_{3}-11\right)$. The boron-bound carbons $\mathrm{CH}_{2}-3$ was not detected due to quadrupolar relaxation.

${ }^{11} \mathbf{B}$ NMR $\left(96 \mathrm{MHz}, \mathrm{CDCl}_{3}\right) \delta(\mathrm{ppm})=32.8$

HRMS (ESI +): calculated for $\mathrm{C}_{22} \mathrm{H}_{28} \mathrm{BNO}_{4}[\mathrm{M}+\mathrm{H}]^{+}:$414.2815; found: 414.2808.

IR (neat): $v\left(\mathrm{~cm}^{-1}\right)=3297,2977$ 2927, 1658, 1602, 1511, 1369, 1320, 1237, 1143, 1036, 968, $888,828$.

m.p.: $90-92^{\circ} \mathrm{C}$ 
3-cyclohexyl-N-(4-methoxyphenyl)-2-((4,4,5,5-tetramethyl-1,3,2-dioxaborolan-2yl)methyl)but-3-enamide 3ub

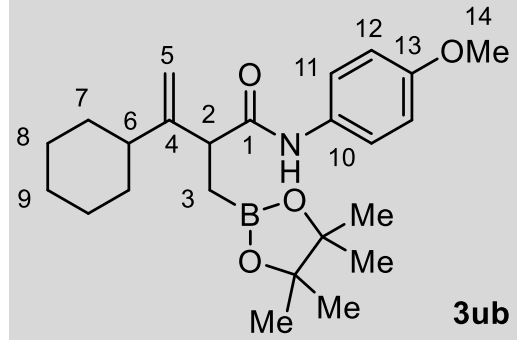

This product was prepared according to the general procedure for the copper-catalyzed borylative carboxamidation of olefins using 2-cyclohexyl 1,3-butadiene 1 u (41 mg, $0.3 \mathrm{mmol}, 1.0$ equiv. $)^{8}$, [(SIMes)CuCl] $(12.2 \mathrm{mg}$, $0.030 \mathrm{mmol}, 10.0 \mathrm{~mol} \%$ ), LiOtBu (36 mg, $0.45 \mathrm{mmol}, 1.5$ equiv.), $B_{2}$ pin $_{2}$ (91 mg, $0.36 \mathrm{mmol}, 1.2$ equiv.) and 1 isocyanato-4-methoxybenzene $\mathbf{2 b}(0.117 \mathrm{~mL}, 0.9 \mathrm{mmol}, 3.0$ equiv.) in toluene $(3.0 \mathrm{~mL}, 0.1 \mathrm{M})$. Reaction time: 20 h. $67 \%$ conversion into $3 \mathbf{u b},>20: 1$ chemoselectivity determined by ${ }^{1} \mathrm{H}$ NMR analysis of the crude reaction mixture in presence of an internal standard. Purification by flash chromatography (pentane/ethyl acetate 9:1) gave pure $3 \mathrm{ub}$ as a white solid (70 mg, 0.17 $\mathrm{mmol}, 56 \%$ yield).

TLC: pentane/ethyl acetate $4: 1, R_{f}=0.3$.

${ }^{1} \mathrm{H}$ NMR $\left(400 \mathrm{MHz}, \mathrm{CDCl}_{3}\right) \delta(\mathrm{ppm})=7.40(\mathrm{bs}, 1 \mathrm{H}, \mathrm{N}-H), 7.37\left(\mathrm{~d},{ }^{3} \mathrm{~J}_{\mathrm{HH}}=9.0 \mathrm{~Hz}, 2 \mathrm{H}, \mathrm{H}-11\right)$, $6.83\left(\mathrm{~d},{ }^{3} \mathrm{~J}_{\mathrm{HH}}=9.0 \mathrm{~Hz}, 2 \mathrm{H}, H-12\right), 5.10(\mathrm{~s}, 1 \mathrm{H}, H-5), 5.06$ (s, $\left.1 \mathrm{H}, H-5^{\prime}\right), 3.77$ (s, 3H, H-16), 3.38 $\left(\mathrm{t},{ }^{3} \mathrm{~J}_{\mathrm{HH}}=8.1 \mathrm{~Hz}, 1 \mathrm{H}, \mathrm{H}-2\right), 1.93\left(\mathrm{t},{ }^{3} \mathrm{~J}_{\mathrm{HH}}=11.1 \mathrm{~Hz}, 1 \mathrm{H}, \mathrm{H}-6\right), 1.74(\mathrm{~m}, 5 \mathrm{H}), 1.41\left(\mathrm{dd},{ }^{2} J_{\mathrm{HH}}=15.5\right.$ $\left.\mathrm{Hz},{ }^{3} \mathrm{~J}_{\mathrm{HH}}=8.8 \mathrm{~Hz}, 1 \mathrm{H}, H-3\right), 1.24$ (s, 6H, H-MeBpin), 1.23 (s, 6H, H-MeBpin), 1.17 (m, 3H), $1.07\left(\mathrm{dd},{ }^{2} \mathrm{~J}_{\mathrm{HH}}=15.5,{ }^{3} \mathrm{~J}_{\mathrm{HH}}=7.6 \mathrm{~Hz}, 1 \mathrm{H}, \mathrm{H}-3^{\prime}\right)$.

${ }^{13} \mathrm{C}\left\{{ }^{1} \mathrm{H}\right\}$ NMR $\left(101 \mathrm{MHz}, \mathrm{CDCl}_{3}\right) \delta(\mathrm{ppm})=172.2(C-1), 156.7(C-13), 156.3(C-4), 131.4(C-$ 10), $121.4(\mathrm{CH}-11), 114.3(\mathrm{CH}-14), 111.4\left(\mathrm{CH}_{2}-5\right)$, 83.4 (CO-Bpin), $55.6\left(\mathrm{CH}_{3}-14\right), 50.9(\mathrm{CH}-$ 2), 43.1 ( $\mathrm{CH}-6)$, $33.6\left(\mathrm{CH}_{2}-7\right), 26.9\left(\mathrm{CH}_{2}-8\right), 26.3\left(\mathrm{CH}_{2}-9\right), 25.1\left(\mathrm{CH}_{3}-\mathrm{MeBpin}\right), 24.9\left(\mathrm{CH}_{3^{-}}\right.$ MeBpin), 14.4 (bs, $\mathrm{CH}_{2}-3$ ).

${ }^{11} \mathbf{B}$ NMR $\left(96 \mathrm{MHz}, \mathrm{CDCl}_{3}\right) \delta(\mathrm{ppm})=33.7$

HRMS (ESI +): calculated for $\mathrm{C}_{22} \mathrm{H}_{28} \mathrm{BNO}_{4}[\mathrm{M}+\mathrm{H}]^{+}:$: 414.2815; found: 414.2808.

IR (neat): $v\left(\mathrm{~cm}^{-1}\right)=3308,2928,2857,1660,1599,1513,1369,1320,1238,1143,1038,966$.

m.p.: $132-137^{\circ} \mathrm{C}$ 
(4R)-4-(6-methoxynaphthalen-2-yl)- $N$-(4-methoxyphenyl)-3-methylene-2-((4,4,5,5tetramethyl-1,3,2-dioxaborolan-2-yl)methyl)pentanamide 3vb

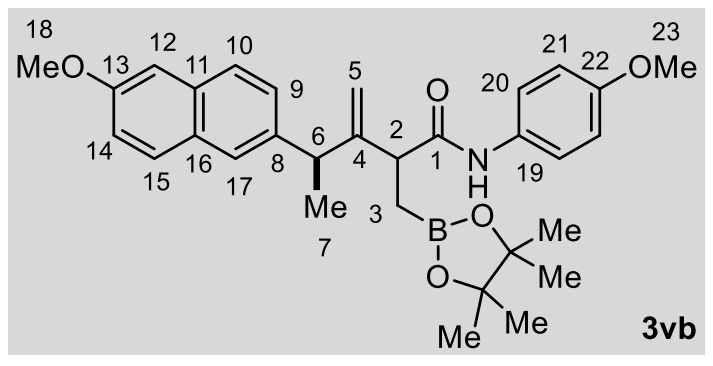

This product was prepared according to the general procedure for the copper-catalyzed borylative carboxamidation of olefins using diene $(R)-1 \mathrm{v} \quad(48 \mathrm{mg}, \quad 0.2 \mathrm{mmol}, 1.0 \text { equiv. })^{8}$, [(SIMes)CuCl] (12.2 mg, $0.030 \mathrm{mmol}, 15.0 \mathrm{~mol} \%)$, vb LiOtBu (24 mg, 0.3 mmol, 1.5 equiv.), $B_{2} \operatorname{pin}_{2}(61$

mg, 0.24 mmol, 1.2 equiv. $)$ and 1 -isocyanato-4-methoxybenzene $2 \mathbf{b}(0.078 \mathrm{~mL}, 0.6 \mathrm{mmol}, 3.0$ equiv.) in toluene $(2.0 \mathrm{~mL}, 0.1 \mathrm{M})$. Reaction time: $20 \mathrm{~h} .71 \%$ conversion into 3vb, diastereoselectivity 5:1 determined by ${ }^{1} \mathrm{H}$ NMR analysis of the crude reaction mixture in presence of an internal standard. Purification by flash chromatography (pentane/ethyl acetate 4:1) gave pure $3 \mathbf{v b}$ as a white solid $(60 \mathrm{mg}, 0.12 \mathrm{mmol}, 60 \%$ yield, $d r$ 5:1). Relative stereochemistry for $\mathbf{3 v b}$ was not assigned.

TLC: pentane/ethyl acetate $4: 1, \mathrm{R}_{\mathrm{f}}=0.3$.

${ }^{1} \mathrm{H}$ NMR $\left(400 \mathrm{MHz}, \mathrm{CDCl}_{3}\right.$, only major diastereoisomer described) $\delta(\mathrm{ppm})=7.67-7.58(\mathrm{~m}$, $3 \mathrm{H}, \mathrm{H}$-Ar), $7.33\left(\mathrm{dd},{ }^{3} \mathrm{~J}_{\mathrm{HH}}=8.5 \mathrm{~Hz},{ }^{4} \mathrm{~J}_{\mathrm{HH}}=1.7 \mathrm{~Hz}, 1 \mathrm{H}, \mathrm{H}-\mathrm{Ar}\right), 7.12\left(\mathrm{dd},{ }^{3} \mathrm{~J}_{\mathrm{HH}}=9.0 \mathrm{~Hz},{ }^{4} \mathrm{~J}_{\mathrm{HH}}=2.6\right.$ $\mathrm{Hz}, 1 \mathrm{H}, H-\mathrm{Ar}$ ), 7.07 (m, 1H, H-Ar), $7.06\left(\mathrm{~d},{ }^{3} \mathrm{~J}_{\mathrm{HH}}=9.1 \mathrm{~Hz}, 2 \mathrm{H}, H-20\right), 6.73$ (bs, $\left.1 \mathrm{H}, \mathrm{N}-H\right), 6.70$ (d, $\left.{ }^{3} J_{\mathrm{HH}}=9.1 \mathrm{~Hz}, 2 \mathrm{H}, H-21\right), 5.32(\mathrm{~s}, 1 \mathrm{H}, H-5), 5.28$ (s, 1H, H-5'), 3.91 (s, 3H, H-18), 3.75 (s, 3H, H-23), $3.69(\mathrm{~m}, 1 \mathrm{H}, \mathrm{H}-6), 3.15(\mathrm{~m}, 1 \mathrm{H}, \mathrm{H}-2), 1.50$ (d, $\left.{ }^{3} \mathrm{JHH}_{\mathrm{H}}=7.0 \mathrm{~Hz}, 3 \mathrm{H}, H-7\right), 1.41$ (dd, $\left.{ }^{2} J_{\mathrm{HH}}=15.8 \mathrm{~Hz},{ }^{3} J_{\mathrm{HH}}=9.0 \mathrm{~Hz}, 1 \mathrm{H}, H-3\right), 1.19$ (s, 6H, H-MeBpin), 1.16 (s, 6H, H-MeBpin), 1.14 (m, $\left.1 \mathrm{H}, H-3^{\prime}\right)$.

${ }^{13} \mathrm{C}\left\{{ }^{1} \mathrm{H}\right\}$ NMR $\left(101 \mathrm{MHz}, \mathrm{CDCl}_{3}\right.$, only major diastereoisomer described) $\delta(\mathrm{ppm})=172.0(C-1)$, 157.6 (C-13), 156.0 (C-22), 153.6 (C-4), 139.9 (C-11), $133.6(C-8), 131.4(C-19), 129.3(\mathrm{CH}-$ $\mathrm{Ar}), 129.1$ (C-16), 127.2 (CH-Ar), 127.0 ( $\mathrm{CH}-\mathrm{Ar}), 125.8$ ( $\mathrm{CH}-\mathrm{Ar}), 120.9(\mathrm{CH}-20), 118.9(\mathrm{CH}-9)$, 113.9 (CH-21), $112.4\left(\mathrm{CH}_{2}-5\right), 105.7(\mathrm{CH}-12)$, 83.3 (CO-Bpin), $55.6\left(\mathrm{CH}_{3}-18\right), 55.5\left(\mathrm{CH}_{3}-23\right)$, 48.4 ( $\mathrm{CH}-2), 44.8$ ( $\mathrm{CH}-6), 25.0$ ( $\left.\mathrm{CH}_{3}-\mathrm{MeBpin}\right), 24.7\left(\mathrm{CH}_{3}-\mathrm{MeBpin}\right), 21.1\left(\mathrm{CH}_{3}-7\right), 15.0$ (bs, $\left.\mathrm{CH}_{2}-3\right)$.

${ }^{11} \mathbf{B}$ NMR $\left(128 \mathrm{MHz}, \mathrm{CDCl}_{3}\right) \delta(\mathrm{ppm})=33.1$

HRMS (ESI +): calculated for $\mathrm{C}_{31} \mathrm{H}_{38} \mathrm{BNO}_{5}[\mathrm{M}+\mathrm{H}]^{+}: 516.2922$; found: 516.2927.

IR (neat): $v\left(\mathrm{~cm}^{-1}\right)=3322,2975,1678,1605,1511,1369,1322,1266,1233,1144,1034,849$. m.p.: $149^{\circ} \mathrm{C}$ 
N-phenyl-3-(4,4,5,5-tetramethyl-1,3,2-dioxaborolan-2-yl)bicyclo[2.2.1]heptane-2carboxamide 3wa

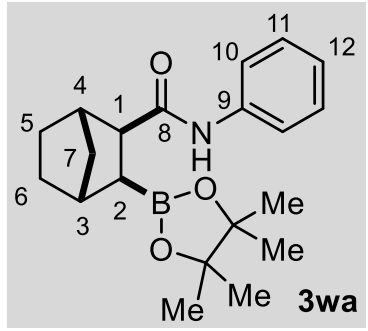

This product was prepared according to the general procedure for the copper-catalyzed borylative carboxamidation of olefins using norbornene 1w (28 mg, $0.3 \mathrm{mmol}, 1.0$ equiv.), [(SIMes)CuCl] (12.2 $\mathrm{mg}, 0.030 \mathrm{mmol}, 10.0 \mathrm{~mol} \%$ ), LiOtBu (36 mg, $0.45 \mathrm{mmol}, 1.5$ equiv.), $B_{2} \operatorname{pin}_{2}$ (91 mg, $0.36 \mathrm{mmol}, 1.2$ equiv.) and isocyanatobenzene $2 \mathrm{a}$ (0.098 mL, $0.9 \mathrm{mmol}, 3.0$ equiv.) in toluene (3.0 mL, $0.1 \mathrm{M})$. Reaction time: 20 h. $94 \%$ conversion into 3 wa, $>25: 1$ diastereoselectivity determined by ${ }^{1} \mathrm{H}$ NMR analysis of the crude reaction mixture in presence of an internal standard. Purification by flash chromatography (pentane/ethyl acetate 4:1) gave pure 3wa as a white solid (50 mg, 0.147 $\mathrm{mmol}, 49 \%$ yield, $d r>25: 1)$. Relative stereochemistry of 3wa ( $d r>25: 1)$ was determined by Xray analysis of suitable crystals obtained layering with $n$-hexane a concentrated solution of 3wa in benzene at $23^{\circ} \mathrm{C}$.

TLC: pentane/ethyl acetate $4: 1, \mathrm{R}_{\mathrm{f}}=0.2$.

${ }^{1} \mathrm{H}$ NMR $\left(300 \mathrm{MHz}, \mathrm{CDCl}_{3}\right) \delta(\mathrm{ppm})=7.49\left(\mathrm{~d},{ }^{3} \mathrm{~J}_{\mathrm{HH}}=7.9 \mathrm{~Hz}, 2 \mathrm{H}, H-10\right), 7.38(\mathrm{bs}, 1 \mathrm{H}, \mathrm{N}-H)$, $7.30\left(\mathrm{~d},{ }^{3} J_{\mathrm{HH}}=7.6 \mathrm{~Hz}, 2 \mathrm{H}, H-11\right), 7.07\left(\mathrm{t},{ }^{3} \mathrm{~J}_{\mathrm{HH}}=7.6 \mathrm{~Hz}, 1 \mathrm{H}, H-12\right), 2.58\left(\mathrm{~d},{ }^{3} \mathrm{~J}_{\mathrm{HH}}=9.6 \mathrm{~Hz}, 1 \mathrm{H}\right.$, $H-1), 2.43(\mathrm{~m}, 2 \mathrm{H}, H-3+H-4), 1.78\left(\mathrm{~d},{ }^{2} J_{\mathrm{HH}}=9.5 \mathrm{~Hz}, 1 \mathrm{H}, H-7\right), 1.59(\mathrm{~m}, 2 \mathrm{H}, H-5+H-6), 1.24$ (s, 6H, H-MeBpin), 1.21 (s, 6H, H-MeBpin), 1.17 (m, 3H, $H-5+H-6+H-7), 1.13\left(\mathrm{~d},{ }^{3} J_{H H}=9.5\right.$ $\mathrm{Hz}, 1 \mathrm{H}, \mathrm{H}-2)$.

${ }^{13} \mathrm{C}\left\{{ }^{1} \mathrm{H}\right\}$ NMR $\left(75 \mathrm{MHz}, \mathrm{CDCl}_{3}\right) \delta(\mathrm{ppm})=174.8(\mathrm{C}-8), 138.2(\mathrm{C}-9), 129.0(\mathrm{CH}-11), 124.2(\mathrm{CH}-$ 12), 119.8 ( $\mathrm{CH}-10), 82.6$ (CO-Bpin), 53.7 ( $\mathrm{CH}-1), 42.4$ ( $\mathrm{CH}-4), 38.3(\mathrm{CH}-3), 36.5\left(\mathrm{CH}_{2}-7\right), 31.2$ $\left(\mathrm{CH}_{2}-6\right), 29.7\left(\mathrm{CH}_{2}-5\right), 25.3\left(\mathrm{CH}_{3}-\mathrm{MeBpin}\right), 25.1\left(\mathrm{CH}_{3}-\mathrm{MeBpin}\right)$. The boron-bound carbons $\mathrm{CH}-$ 2 was not detected due to quadrupolar relaxation.

${ }^{11}$ B NMR $\left(96 \mathrm{MHz}, \mathrm{CDCl}_{3}\right) \delta(\mathrm{ppm})=29.7$.

HRMS (ESI +): calculated for $\mathrm{C}_{20} \mathrm{H}_{28} \mathrm{BNO}_{3}[\mathrm{M}+\mathrm{H}]^{+}:$342.2239; found: 342.2252 .

IR (neat): $v\left(\mathrm{~cm}^{-1}\right)=3197,3069,2954,1608,1587,1562,1451,1361,1238,1149,1123,971$, 757.

m.p.: $175-180^{\circ} \mathrm{C}$ 
N-phenyl-3-(4,4,5,5-tetramethyl-1,3,2-dioxaborolan-2-yl)bicyclo[2.2.1]hept-5-ene-2carboxamide $3 \times a$

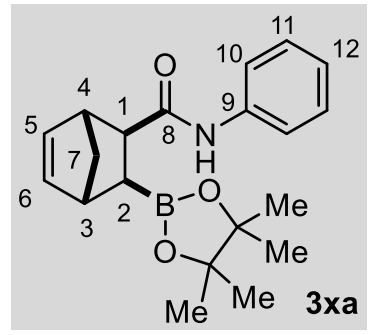

This product was prepared according to the general procedure for the copper-catalyzed borylative carboxamidation of olefins using norbonadiene 1x (28 mg, $0.3 \mathrm{mmol}, 1$ equiv.), [(SIMes)CuCl] (12.2 mg, $0.030 \mathrm{mmol}, 10.0 \mathrm{~mol} \%$ ), LiOtBu (36 mg, $0.45 \mathrm{mmol}, 1.5$ equiv.), $\mathrm{B}_{2} \mathrm{pin}_{2}(84 \mathrm{mg}, 0.36 \mathrm{mmol}, 1.1$ equiv.) and isocyanatobenzene $\mathbf{2 a}$ (0.098 mL, $0.9 \mathrm{mmol}, 3.0$ equiv.) in toluene (3.0 mL, $0.1 \mathrm{M})$. Reaction time: 20 h. $70 \%$ conversion into $3 \times 2$ a, $>25: 1$ diastereoselectivity determined by ${ }^{1} \mathrm{H}$ NMR analysis of the crude reaction mixture in presence of an internal standard. Purification by flash chromatography (pentane/ethyl acetate 3:2) gave pure $3 \times a$ as a white solid (50 mg, 0.15 $\mathrm{mmol}, 50 \%$ yield, $d r>25: 1)$. The relative stereochemistry of $3 \times a(d r>25: 1)$ was assigned by analogy with 3wa.

TLC: pentane/ethyl acetate 9:1, $R_{f}=0.2$.

${ }^{1} \mathrm{H}$ NMR $\left(300 \mathrm{MHz}, \mathrm{CDCl}_{3}\right) \delta(\mathrm{ppm})=7.52\left(\mathrm{~d},{ }^{3} \mathrm{~J}_{\mathrm{HH}}=7.9 \mathrm{~Hz}, 2 \mathrm{H}, H-10\right), 7.30(\mathrm{~m}, 2 \mathrm{H}, H-11)$, 7.27 (bs, $1 \mathrm{H}, \mathrm{N}-H), 7.07$ (t, $\left.{ }^{3} \mathrm{JHH}_{\mathrm{H}}=7.4 \mathrm{~Hz}, 1 \mathrm{H}, H-12\right), 6.25(\mathrm{~m}, 1 \mathrm{H}, H-6), 6.08(\mathrm{~m}, 1 \mathrm{H}, H-5)$, $3.01(\mathrm{~m}, 2 \mathrm{H}, H-3+H-4), 2.39\left(\mathrm{~d},{ }^{3} \mathrm{~J}_{\mathrm{HH}}=9.7 \mathrm{~Hz}, 1 \mathrm{H}, H-1\right), 1.93\left(\mathrm{~d},{ }^{2} \mathrm{~J}_{\mathrm{HH}}=8.6 \mathrm{~Hz}, 1 \mathrm{H}, H-7\right), 1.35$ (d, $\left.{ }^{2} J_{H H}=8.6 \mathrm{~Hz}, 1 \mathrm{H}, H-7\right), 1.23$ (s, 6H, H-MeBpin), 1.19 (s, 6H, H-MeBpin), 1.06 (d, ${ }^{3} J_{H H}=$ $9.7 \mathrm{~Hz}, 1 \mathrm{H}, \mathrm{H}-2)$.

${ }^{13} \mathrm{C}\left\{{ }^{1} \mathrm{H}\right\}$ NMR $\left(75 \mathrm{MHz}, \mathrm{CDCl}_{3}\right) \delta(\mathrm{ppm})=174.5(\mathrm{C}-8), 140.6(\mathrm{CH}-6), 138.4(\mathrm{C}-9), 134.9(\mathrm{CH}-$ 5), 129.0 ( $\mathrm{CH}-11), 124.1$ ( $\mathrm{CH}-12), 119.7$ ( $\mathrm{CH}-10), 83.1$ (CO-Bpin), $50.5(\mathrm{CH}-1), 48.2(\mathrm{CH}-4)$, $46.1\left(\mathrm{CH}_{2}-7\right)$, 43.9 ( $\left.\mathrm{CH}-3\right), 27.6$ (bs, $\left.\mathrm{CH}_{2}-2\right)$, 25.2 ( $\mathrm{CH}_{3}-$ MeBpin), 25.1 ( $\left.\mathrm{CH}_{3}-\mathrm{MeBpin}\right)$.

${ }^{11} \mathbf{B}$ NMR $\left(128 \mathrm{MHz}, \mathrm{CDCl}_{3}\right) \delta(\mathrm{ppm})=33.3$

HRMS $(E S I+)$ : calculated for $\mathrm{C}_{20} \mathrm{H}_{26} \mathrm{BNO}_{3}[\mathrm{M}+\mathrm{H}]^{+}:$:340.2083; found: 340.2113 .

IR (neat): $v\left(\mathrm{~cm}^{-1}\right)=3266,2975,1659,1599,1582,1543,1500,1248,1143,967,852,755$.

m.p.: $165-169{ }^{\circ} \mathrm{C}$ 


\section{Enantioselective copper-catalyzed borylative carboxamidation of vinylarenes}

\subsection{Chiral ligand screening}

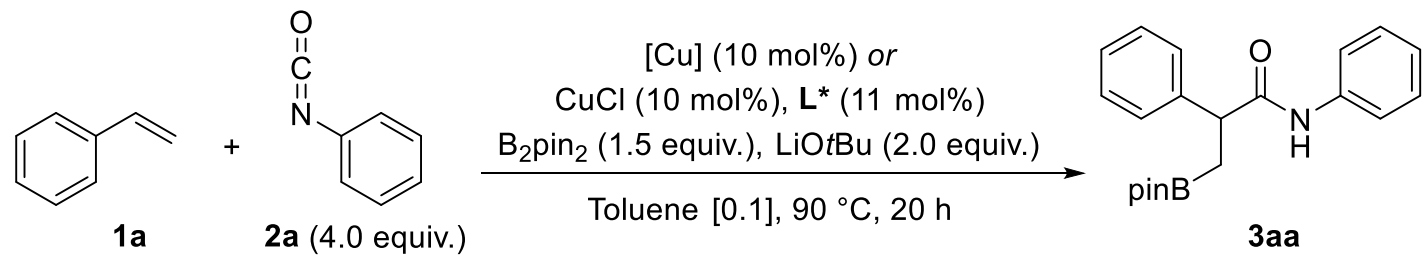

Catalysts and ligands survey<smiles></smiles>

$(R, R)-6 \mathbf{a}$

$<5 \%$ conv., nd<smiles>CC(C)CC(C)N1CCN(C(C)CC(C)C)C1Cl</smiles>

$(\mathrm{S}, \mathrm{S})-[(\mathrm{NHC}-1) \mathrm{CuCl}]$ $30 \%$ conv., $74.5: 26.5$ er<smiles></smiles>

6d, $(R)$-Binap

$<5 \%$ conv., nd

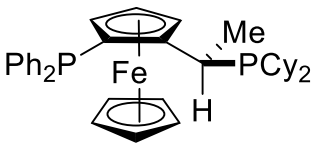

$(R, R)$-Josiphos $<5 \%$ conv., $n d$<smiles>ClC1=C(c2ccccc2)N(C(c2ccccc2)c2ccccc2)C(c2ccccc2)C1c1ccccc1</smiles>

$(R, R)-\mathbf{6 b}$

$15 \%$ conv. , 57.5:42.5 er<smiles>Cc1cc(C)c(N2CCN(C(C)CO)C2=C(Cl)Cl)c(C)c1</smiles>

(S)-[(NHC-2)CuCl] $14 \%$ conv., 55:45 er<smiles>Cc1ccccc1C(CC(C)C)N1CCN([C@@H](CC(C)(C)C)c2ccccc2C)C1Cl</smiles>

$(R, R)-6 \mathrm{c}$

$14 \%$ conv., 81.5:18.5 er<smiles>Cc1cc(C)c(N2CCN(C(C)c3ccccc3O)C2=C(Cl)Cl)c(C)c1</smiles>

$(R)-[(\mathrm{NHC}-3) \mathrm{CuCl}]$ $30 \%$ conv. , 81.5:18.5 er<smiles>CC(CC(C)Pc1ccccc1)Pc1ccccc1</smiles>

6e, $(R, R)$-BDPP $12 \%$ conv. , $75: 25$ er<smiles>CC(C)(C)[C@H]1COC(c2ccccc2P)=N1</smiles>

6f, $(S)-(\mathrm{Ph}, t \mathrm{Bu}) \mathrm{Phox}$ $36 \%$ conv., $66: 34$ er<smiles>c1ccc([PH+]C2CCC(c3ccccc3)P2CCP2C(c3ccccc3)CC[C@H]2c2ccccc2)cc1</smiles>

$(R, R)$-Ph-BPE $<5 \%$ conv., nd

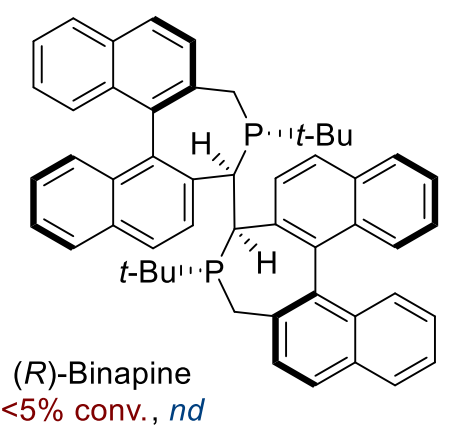

Figure S6. Enantioselective Cu-catalyzed borylative carboxamidation of styrene $(0.15 \mathrm{mmol}$ scale). Catalysts and ligands survey. Conversion determined by ${ }^{1} \mathrm{H}$ NMR using an internal standard. Er determined by HPLC. ${ }^{a}$ Reaction run at $50^{\circ} \mathrm{C}$. 


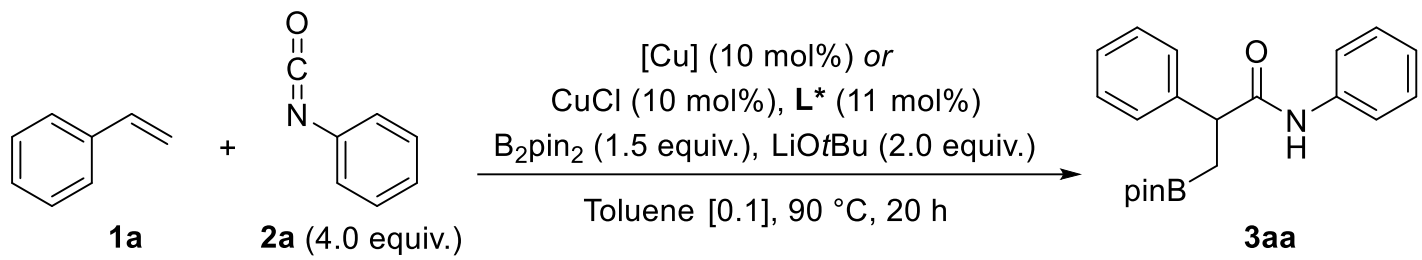

Catalysts and ligands survey<smiles>CC(C)C1COC(c2ccc3ccccc3n2)=N1</smiles>

(S)-Quinox

$<5 \%$ conv. , nd<smiles>c1ccc(C2COC(c3cccc(C4=NC(c5ccccc5)CO4)n3)=N2)cc1</smiles>

$(R, R)-$ Pybox $<5 \%$ conv., nd<smiles>c1ccc(C(Cc2ccc3ccccc3c2-c2ccc3ccccc3c2)P(Cc2ccccn2)c2ccccc2)cc1</smiles>

$(R, R, R)-\mathbf{6 g}$ $40 \%$ conv., 62.5:37.5 er<smiles>COc1ccc([SH](O)CC(C)C)c(P(O)c2ccccc2)c1OC</smiles>

6h, $(R)-(\mathrm{Ph}) \mathrm{SOP}^{a}$ $65 \%$ conv., $63: 37$ er<smiles>COc1ccc2ccccc2c1-c1c(P)ccc2ccccc12</smiles>

6i, (R)-(Cy)MOP $28 \%$ conv. , $70: 30$ er

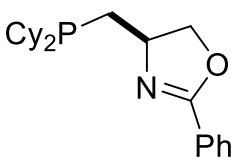
$(R)-\mathrm{L}_{3}$

$60 \%$ conv., $56.5: 43.5$ er<smiles>CC(c1ccccc1)N(C(C)c1ccccc1)P(Oc1ccccc1)Oc1ccc2ccccc2c1-c1ccccc1</smiles>

$(\mathrm{S}, \mathrm{S}, \mathrm{Sa})-\mathrm{L}_{1}$ $<5 \%$ conv., nd

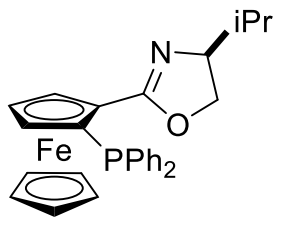

(S)- $\mathbf{L}_{\mathbf{4}}$ 13\% conv., 67.5:32.5 er<smiles>CC(c1ccccc1)N(C(C)c1ccccc1)p1oc2ccc3ccccc3c2c2c(ccc3ccccc32)o1</smiles>

(S, S,Ra)- $\mathrm{L}_{\mathbf{2}}$ $<5 \%$ conv., nd<smiles>CC(C)C1COC(c2ccccc2P)=N1</smiles>

(R)-(Ph,iPr)-Phox 25\% conv., 55:45 er<smiles>c1ccc2c3c(ccc2c1)CP(C1CCCCC1)Cc1ccc2ccccc2c1-3</smiles>

$(R)$-(Cy)-Binepine $26 \%$ conv., 68.5:31.5 er<smiles>Pc1cccc(-c2ccccc2)c1-c1nccc2ccccc12</smiles>

(R)-Quinap $13 \%$ conv., 57:43 er

Figure S6 (continued).. Enantioselective Cu-catalyzed borylative carboxamidation of styrene ( $0.15 \mathrm{mmol}$ scale). Catalysts and ligands survey. Conversion determined by ${ }^{1} \mathrm{H}$ NMR using an internal standard. Er determined by HPLC. ${ }^{a}$ Reaction run at $50{ }^{\circ} \mathrm{C}$. 


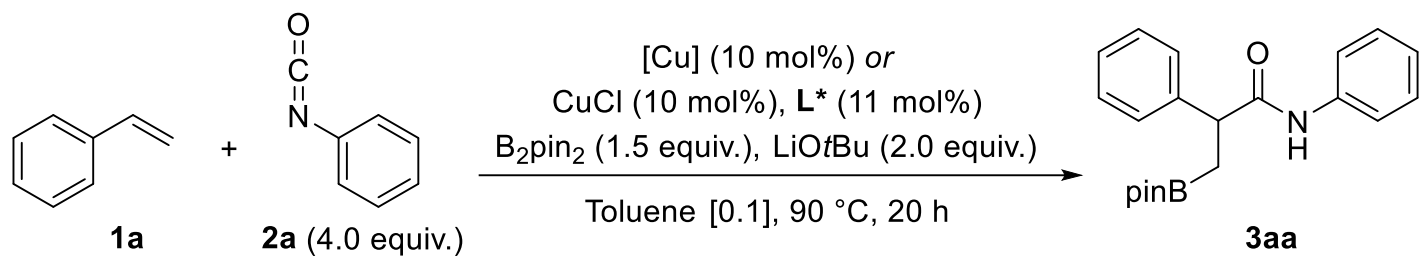

Catalysts and ligands survey<smiles>CC(c1ccc2ccccc2c1)N([C@@H](C)c1ccc2ccccc2c1)P(c1cc(C(C)(C)C)cc(C(C)(C)C)c1)c1cc(C(C)(C)C)cc(C(C)(C)C)c1</smiles>

$(R, R)-\mathbf{L}_{5}$<smiles>CC(c1ccc2ccccc2c1)N([C@@H](C)c1ccc2ccccc2c1)P(C1CCCCC1)C1CCCCC1</smiles>

$(R, R)-\mathrm{L}_{6}$ $35 \%$ conv., 81.5:18.5 er<smiles>CCCCP(CCCC)N(C(C)c1ccccc1)[C@@H](C)c1ccccc1</smiles>

$(R, R)-\mathbf{6} \mathbf{j}$

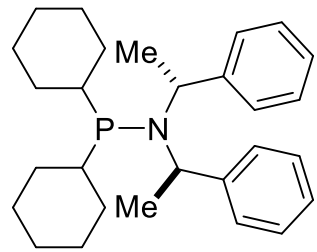

$(R, R)-6 \mathbf{k}$ $18 \%$ conv. $70: 30$ er<smiles>COc1cc(OC)cc(P(c2cc(OC)cc(OC)c2)N([C@@H](C)c2ccc3ccccc3c2)[C@@H](C)c2ccc3ccccc3c2)c1</smiles>

$(R, R)-\mathrm{L}_{7}$ $<5 \%$ conv., nd<smiles>CCCCP(CCCC)N(C(C)c1ccc2ccccc2c1)[C@@H](C)c1ccc2ccccc2c1</smiles>

$(R, R)-\mathrm{L}_{10}$

$72 \%$ conv., $62: 38$ er<smiles>CC(c1ccc2ccccc2c1)N(C(C)c1ccc2ccccc2c1)[C@H](C)c1cc(C(F)(F)F)cc(C(F)(F)F)c1</smiles>

$(R, R)-\mathrm{L}_{8}$ $18 \%$ conv. , $75: 25$ er<smiles>CCP(CC)N(C(C)c1ccc2ccccc2c1)[C@@H](C)c1ccc2ccccc2c1</smiles>

$(R, R)-\mathrm{L}_{11}$

$20 \%$ conv., $78.5: 21.5$ er<smiles>CC(c1ccc2ccccc2c1)N(C(C)c1ccc2ccccc2c1)P(c1ccc(F)cc1)c1ccc(F)cc1</smiles>

$(R, R)-\mathrm{L}_{9}$

$18 \%$ conv., $75: 25$ er<smiles>CC(c1ccc2ccccc2c1)N([C@@H](C)c1ccc2ccccc2c1)P(Cc1ccccc1)Cc1ccccc1</smiles>

$(R, R)-\mathrm{L}_{12}$ $<5 \%$ conv., nd

Figure S6 (continued). Enantioselective Cu-catalyzed borylative carboxamidation of styrene $\left(0.15 \mathrm{mmol}\right.$ scale). Catalysts and ligands survey. Conversion determined by ${ }^{1} \mathrm{H}$ NMR using an internal standard. Er determined by HPLC. ${ }^{a}$ Reaction run at $50^{\circ} \mathrm{C}$. 


\subsection{Reaction optimization}

Table S6. Solvent and temperature screening for isocyanate $\mathbf{2 b}^{a}$

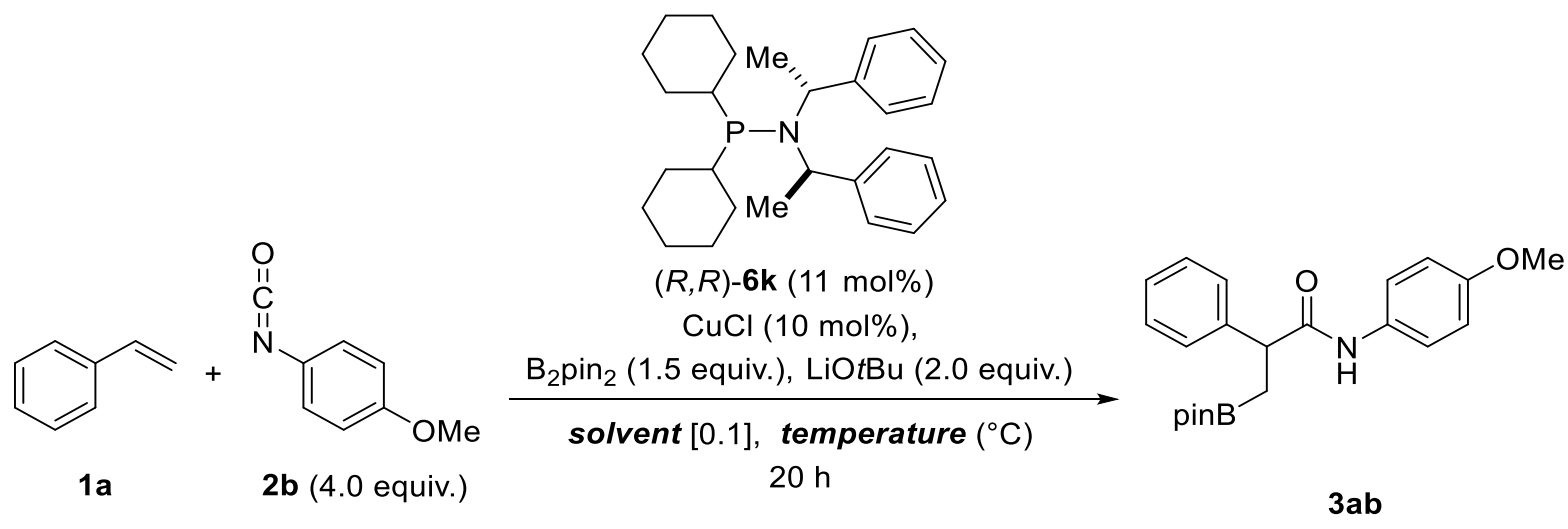

$\begin{array}{lllll}\text { Entry } & \text { Solvent } & \text { Temperature }\left({ }^{\circ} \mathrm{C}\right) & \text { conv. }^{b} & \text { er } \\ 1 & \text { toluene } & 90 & 77 \% & 82: 18 \\ 2 & \text { toluene } & 50 & 17 \% & 86: 14 \\ 3 & \text { toluene } & 25 & <5 \% & \text { nd } \\ 4 & \mathrm{DCE} & 90 & 51 \% & 66.5: 33.5 \\ 5 & \mathrm{CH}_{3} \mathrm{CN} & 90 & 30 \% & 58: 42 \\ 6 & 1,4-\text { dioxane } & 90 & <5 \% & \text { nd } \\ 7 & \mathrm{THF} & 50 & 50 \% & 67.5: 32.5 \\ 8 & 2-\mathrm{Me}-\mathrm{THF} & 50 & 51 \% & 85.5: 14.5 \\ 9 & \mathrm{Et} \mathrm{H}_{2} \mathrm{O} & 50 & 22 \% & 88: 12 \\ 10 & \mathrm{TBME} & 50 & 25 \% & 85.5: 14.5\end{array}$

${ }^{a}$ Reaction conditions: $1 \mathrm{a}(0.15 \mathrm{mmol})$ in the selected solvent $(1.5 \mathrm{~mL}, 0.1 \mathrm{M})$ for $20 \mathrm{~h} .{ }^{b}$ Determined by ${ }^{1} \mathrm{H}$ NMR of the crude reaction mixture in presence of an internal standard. In parenthesis, isolated yield of $\mathbf{3 a b}$ after purification by column chromatography. ${ }^{c}$ Er determined by HPLC 
Table S7. Solvent and temperature screening for isocyanate $2 a^{a}$

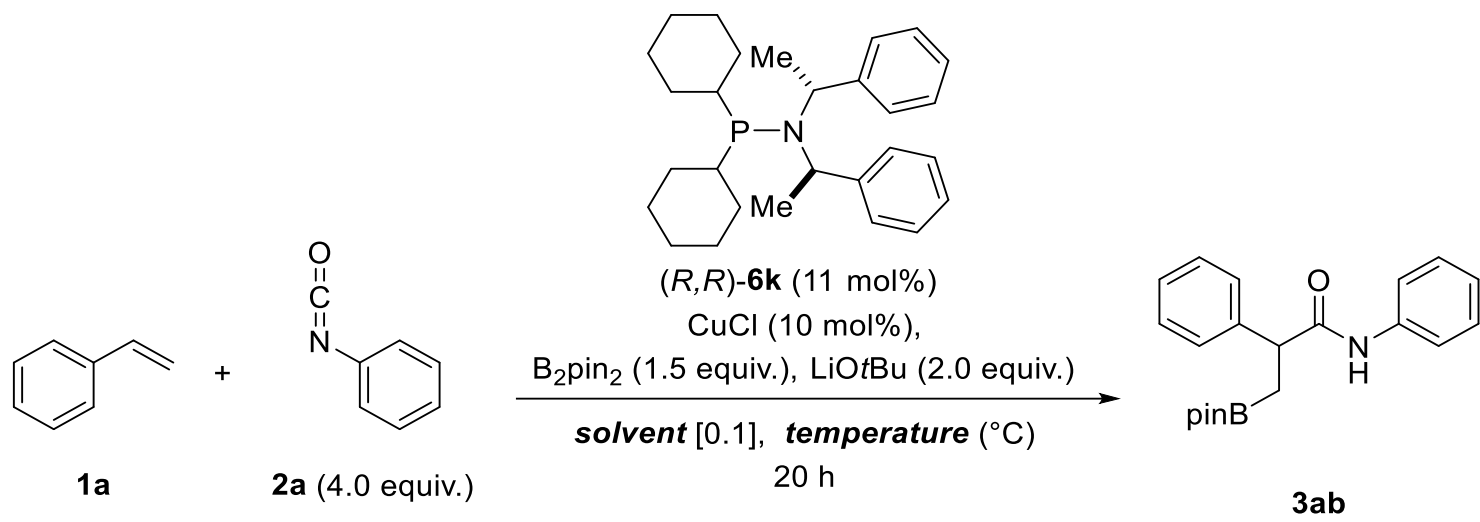

$\begin{array}{lllll}\text { Entry } & \text { Solvent } & \text { Temperature }\left({ }^{\circ} \mathrm{C}\right) & \text { conv. }^{b} & \text { er } \\ 1 & 2-\mathrm{Me}-\mathrm{THF} & 50 & 60 \% & 89: 11 \\ 2 & \mathrm{Et}_{2} \mathrm{O} & 35 & 30 \% & 91.5: 8.5 \\ 3 & 2-\mathrm{Me}-\mathrm{THF}: \mathrm{Et}_{2} \mathrm{O}(\mathrm{v}: \mathrm{v}=1: 1) & 50 & 90 \% & 89: 11 \\ 4^{d} & 2-\mathrm{Me}-\mathrm{THF}: \mathrm{Et}_{2} \mathrm{O}(\mathrm{v}: \mathrm{v}=1: 1) & 25 & 60 \% & 92.5: 7.5 \\ 5^{d} & \text { 2-Me-THF: } \mathrm{Et}_{2} \mathrm{O}(\mathrm{v}: \mathrm{v}=1: 1) & 0 & \text { trace } & -- \\ 6^{e} & \text { 2-Me-THF: } \mathrm{Et}_{2} \mathrm{O}(\mathrm{v}: \mathrm{v}=1: 1) & 30 & 90 \% & 92: 8\end{array}$

${ }^{a}$ Reaction conditions: $1 \mathrm{a}(0.15 \mathrm{mmol})$ in the selected solvent $(1.5 \mathrm{~mL}, 0.1 \mathrm{M})$ for $20 \mathrm{~h} .{ }^{b}$ Determined by ${ }^{1} \mathrm{H}$ NMR of the crude reaction mixture in presence of an internal standard. In parenthesis, isolated yield of $3 a b$ after purification by column chromatography. ${ }^{c}$ Er determined by HPLC. ${ }^{d} 48$ h. ${ }^{e} 72 \mathrm{~h}$. 


\subsection{Synthesis of phosphanamine $(R, R)-6 \mathbf{k}^{9}$}

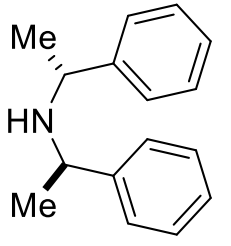

(1.1 equiv.)

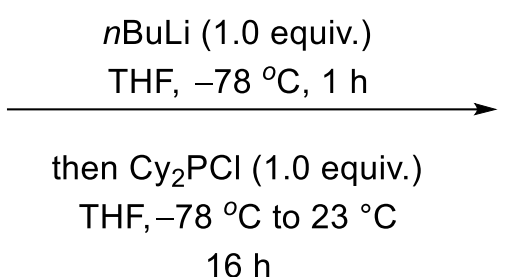

$16 \mathrm{~h}$

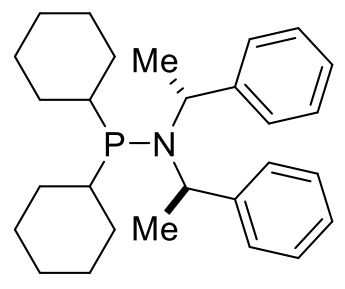

$(R, R)-6 \mathbf{k}$

To a solution of chiral amine $(0.74 \mathrm{~g}, 3.3 \mathrm{mmol}, 1.1$ equiv. $)$ in THF ( $8 \mathrm{~mL})$ was added dropwise $n B u L i\left(1.9 \mathrm{~mL}, 3.0 \mathrm{mmol}, 1.6 \mathrm{M}\right.$ in hexane, 1.0 equiv.) at $-78^{\circ} \mathrm{C}$. After $30 \mathrm{~min}, \mathrm{Cy}_{2} \mathrm{PCl}(0.66$ $\mathrm{mL}, 3.0 \mathrm{mmol}, 1.0$ equiv.) in THF ( $3 \mathrm{~mL}$ ) was added to the above solution. The reaction mixture was allowed to warm up slowly to $23^{\circ} \mathrm{C}$, stirred for $16 \mathrm{~h}$ and finally quenched with $\mathrm{MeOH}(0.2$ $\mathrm{mL}$ ). After removal of the volatiles, the crude product was purified by neutral $\mathrm{Al}_{2} \mathrm{O}_{3}$ (pentane/diethyl ether 40:1) to afford ligand $(R, R)-6 \mathbf{k}$ as a white foam (400 $\mathrm{mg}, 0.9 \mathrm{mmol}$, $30 \%)$.

1,1-dicyclohexyl-N,N-bis $((R)$-1-phenylethyl)phosphanamine $(R, R)-6 \mathbf{k}$

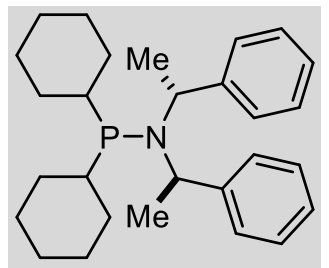

${ }^{1} \mathrm{H}$ NMR $\left(400 \mathrm{MHz}, \mathrm{CDCl}_{3}\right) \delta(\mathrm{ppm})=7.60-6.00$ (bs, 10H), $4.75-3.95$ (bs, $2 \mathrm{H}), 2.16-2.06(\mathrm{~m}, 1 \mathrm{H}), 2.00-1.93(\mathrm{~m}, 1 \mathrm{H}), 1.92-1.74(\mathrm{~m}, 4 \mathrm{H})$, $1.68-1.20(\mathrm{~m}, 17 \mathrm{H}), 1.11-0.97(\mathrm{~m}, 4 \mathrm{H}), 0.37-0.21(\mathrm{~m}, 1 \mathrm{H})$.

${ }^{13} \mathrm{C}\left\{{ }^{1} \mathrm{H}\right\}$ NMR $\left(101 \mathrm{MHz}, \mathrm{CDCl}_{3}\right) \delta(\mathrm{ppm})=144.2(\mathrm{~s}), 128.2(\mathrm{~s}), 127.5(\mathrm{~s})$, $(R, R)-6 \mathrm{k} 126.4(\mathrm{~s}), 37.2\left(\mathrm{~d}, J_{\mathrm{PC}}=20 \mathrm{~Hz}\right), 35.0\left(\mathrm{~d}, J_{\mathrm{PC}}=13 \mathrm{~Hz}\right), 30.49\left(\mathrm{~d}, J_{\mathrm{PC}}=13\right.$ $\mathrm{Hz}), 30.4\left(\mathrm{~d}, J_{\mathrm{PC}}=13 \mathrm{~Hz}\right), 29.3\left(\mathrm{~d}, J_{\mathrm{PC}}=8 \mathrm{~Hz}\right), 28.8\left(\mathrm{~d}, J_{\mathrm{PC}}=15 \mathrm{~Hz}\right), 28.6\left(\mathrm{~d}, J_{\mathrm{PC}}=15 \mathrm{~Hz}\right)$, $28.2\left(\mathrm{~d}, J_{\mathrm{PC}}=2 \mathrm{~Hz}\right), 28.1\left(\mathrm{~d}, J_{\mathrm{PC}}=2 \mathrm{~Hz}\right), 27.2\left(\mathrm{~d}, J_{\mathrm{PC}}=10 \mathrm{~Hz}\right), 26.9(\mathrm{~s}), 26.7\left(\mathrm{~d}, J_{\mathrm{PC}}=11 \mathrm{~Hz}\right)$, $26.4(\mathrm{~s})$.

${ }^{31} \mathbf{P}\left\{{ }^{1} \mathrm{H}\right\} \mathbf{N M R}\left(162 \mathrm{MHz}, \mathrm{CDCl}_{3}\right) \delta(\mathrm{ppm})=50.1$.

HRMS $(E S I+)$ : calculated for $\mathrm{C}_{21} \mathrm{H}_{40} \mathrm{NP}[\mathrm{M}+\mathrm{H}]^{+}: 422.2972$; found: 422.3047.

IR (neat): $v\left(\mathrm{~cm}^{-1}\right)=3060,3029,2968,2922,2849,1740,1601,1494,1448,1370,1274$, $1201,1123,1077,1047,1014,947,913,888,849,815,791,757,698$.

$[\alpha]^{20} \mathrm{D}=+196.6\left(c 0.28, \mathrm{CH}_{2} \mathrm{Cl}_{2}\right)$.

m.p.: $84-86^{\circ} \mathrm{C}$ 


\subsection{Hammett study}

Table S8. Carboxamidation of styrene 1a with different isocyanates ( $0.15 \mathrm{mmol}$ scale).
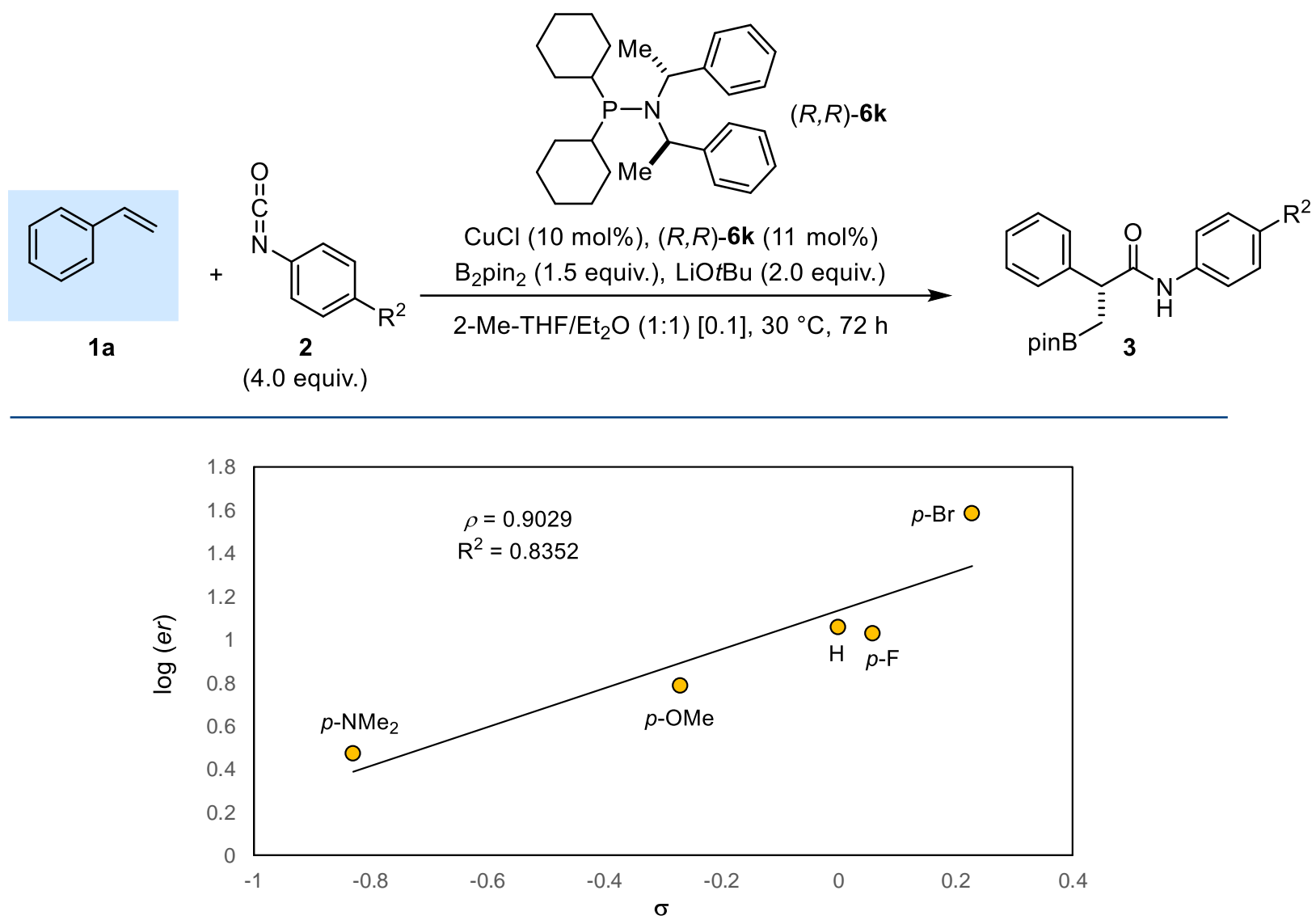

$\begin{array}{lllll}\mathrm{R}^{2} & \boldsymbol{\sigma} & \text { Conv. (\%) } & \text { er } & \log (\mathbf{e r}) \\ p-\mathrm{NMe}_{2}(\mathbf{2 d}) & -\mathbf{0 . 8 3} & 75(45) & 75: 25 & \mathbf{0 . 4 7 7} \\ p-\mathrm{OMe}(\mathbf{2 b}) & -\mathbf{0 . 2 7} & 70(53) & 86: 14 & \mathbf{0 . 7 8 8} \\ \mathrm{H}(\mathbf{2 a}) & \mathbf{0} & 90(74) & 92: 8 & \mathbf{1 . 0 6} \\ p-\mathrm{F}(\mathbf{2 i}) & \mathbf{0 . 0 6} & 90(84) & 91.5: 8.5 & \mathbf{1 . 0 3 2} \\ p-\operatorname{Br}(\mathbf{2 d}) & \mathbf{0 . 2 3} & 95(70) & 97.5: 2.5 & \mathbf{1 . 5 9}\end{array}$

a Determined by ${ }^{1} \mathrm{H}$ NMR of the crude reaction mixture in presence of an internal standard. In parenthesis, isolated yield of $\mathbf{3}$ after purification by column chromatography. ${ }^{\mathrm{b}} \mathrm{Er}$ determined by HPLC. 
Table S9. Carboxamidation of styrene $\mathbf{1 b}$ with different isocyanates $(0.15 \mathrm{mmol}$ scale $)$.
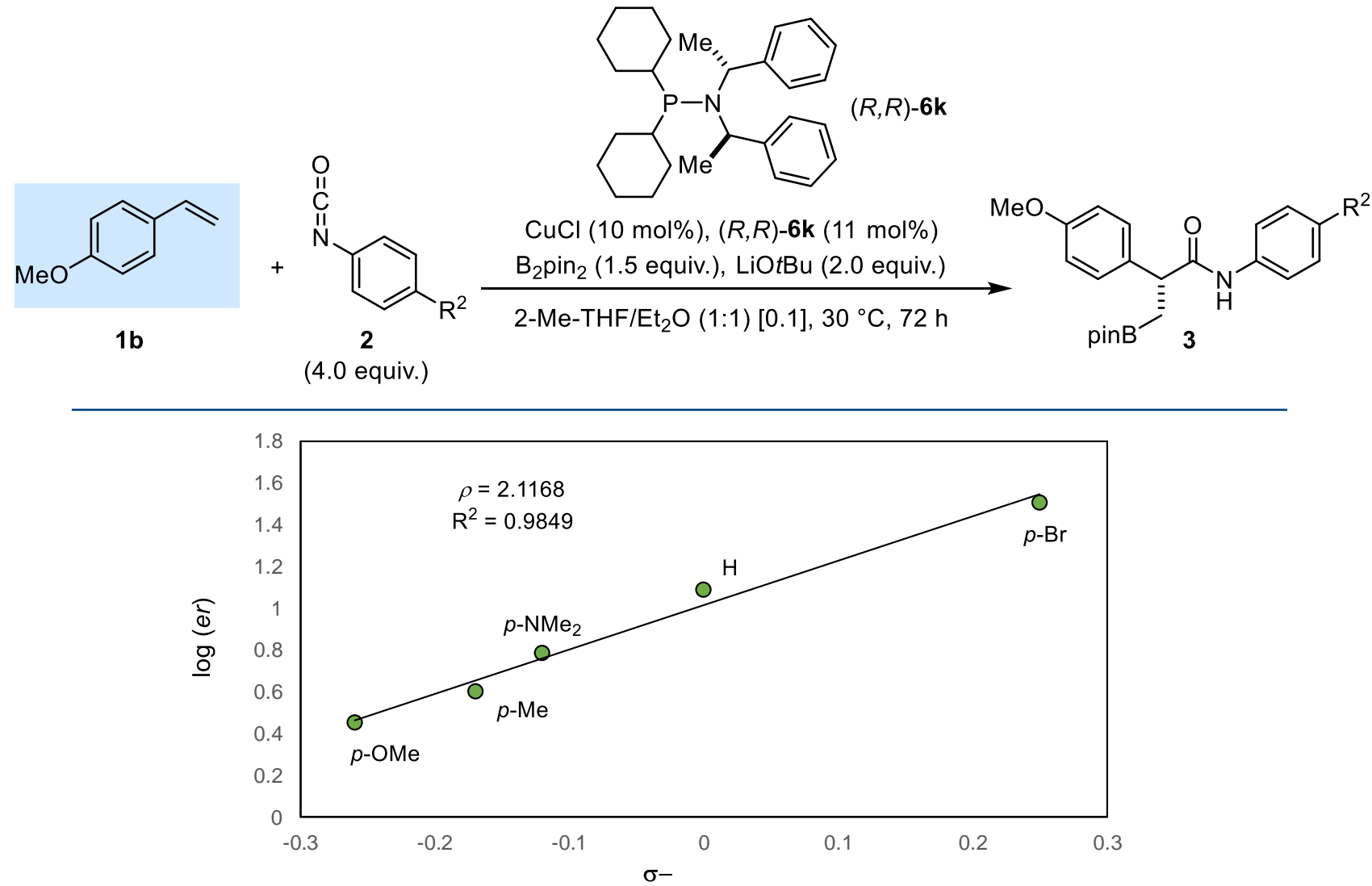

$\begin{array}{lllll}\mathrm{R}^{2} & \boldsymbol{\sigma}- & \text { Conv. }(\%)^{\mathrm{a}} & e r^{\mathrm{b}} & \log (\mathbf{e r}) \\ p-\mathrm{OMe}(\mathbf{2 b}) & -\mathbf{0 . 2 7} & 81(70) & 74: 26 & \mathbf{0 . 4 5 4} \\ p-\mathrm{Me}(\mathbf{2 m}) & -\mathbf{0 . 1 7} & 80(50) & 80: 20 & \mathbf{0 . 6 0 2} \\ p-\mathrm{NMe}_{2}(\mathbf{2 d}) & -\mathbf{0 . 1 2} & 95(83) & 86: 14 & \mathbf{0 . 7 8 8} \\ \mathrm{H}(\mathbf{2 a}) & \mathbf{0} & 80(70) & 92.5: 7.5 & \mathbf{1 . 0 9 1} \\ p-\operatorname{Br}(\mathbf{2 g}) & \mathbf{0 . 2 5} & 80(61) & 97: 3 & \mathbf{1 . 5 0 9}\end{array}$

a Determined by ${ }^{1} \mathrm{H}$ NMR of the crude reaction mixture in presence of an internal standard. In parenthesis, isolated yield of $\mathbf{3}$ after purification by column chromatography. ${ }^{\mathrm{b}} \mathrm{Er}$ determined by HPLC. 
Table S10. Carboxamidation of styrene $1 \mathbf{y}$ with different isocyanates $(0.15 \mathrm{mmol}$ scale $)$.
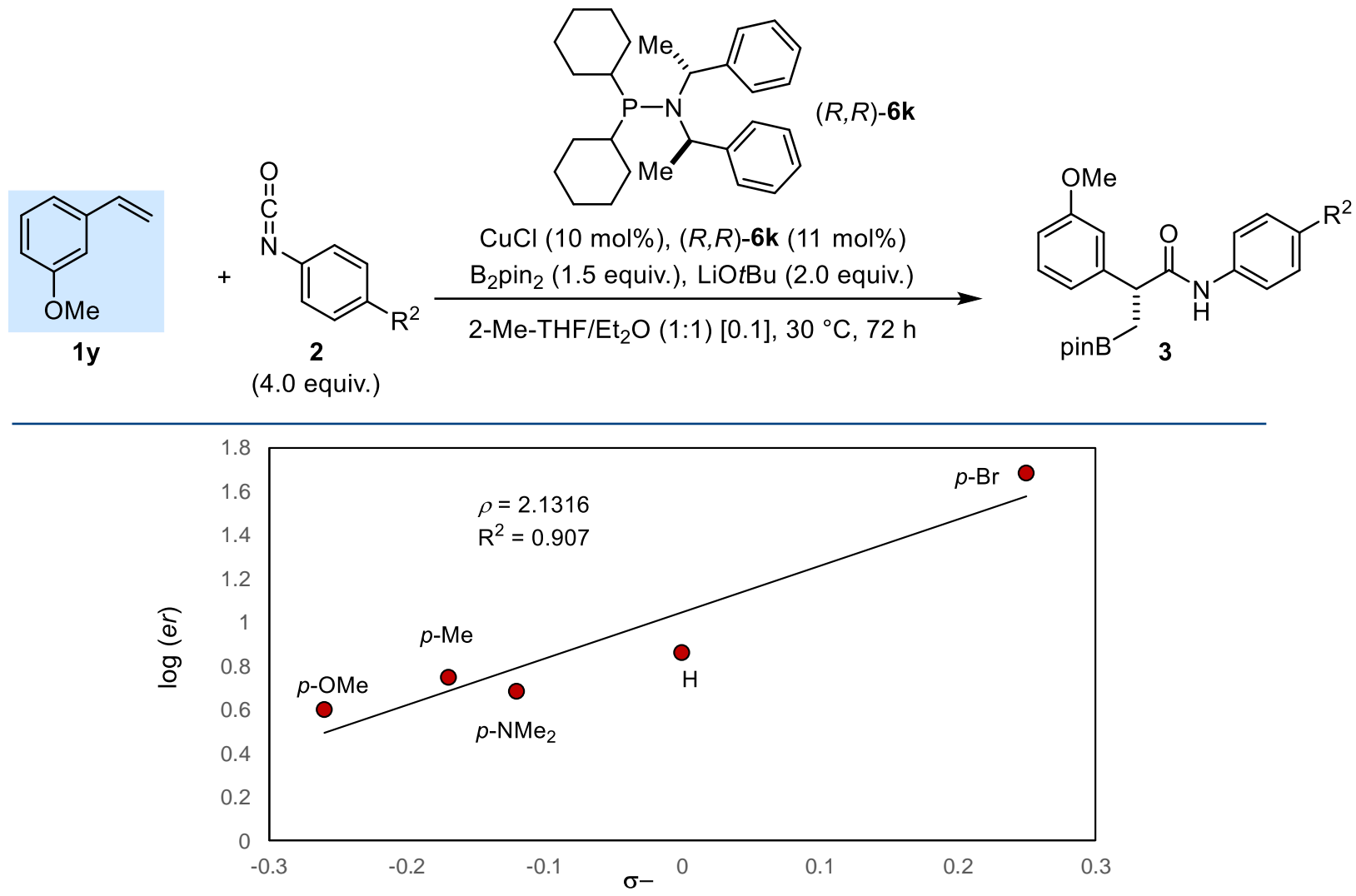

$\begin{array}{lllll}\mathrm{R}^{2} & \sigma- & \text { Conv. }(\%)^{\mathrm{a}} & e r^{\mathrm{b}} & \log (\mathbf{e r}) \\ p-\mathrm{OMe}(\mathbf{2 b}) & -\mathbf{0 . 2 7} & 90(67) & 80: 20 & \mathbf{0 . 6 0 2} \\ p-\mathrm{Me}(\mathbf{2 m}) & -\mathbf{0 . 1 7} & 95(75) & 85: 15 & \mathbf{0 . 7 5 3} \\ p-\mathrm{NMe}_{2}(\mathbf{2 d}) & -\mathbf{0 . 1 2} & 95(73) & 83: 17 & \mathbf{0 . 6 8 9} \\ \mathrm{H}(\mathbf{2 a}) & \mathbf{0} & 90(61) & 88: 12 & \mathbf{0 . 8 6 5} \\ p-\mathrm{Br}(\mathbf{2 g}) & \mathbf{0 . 2 5} & 99(87) & 98: 2 & \mathbf{1 . 6 9 0}\end{array}$

a Determined by ${ }^{1} \mathrm{H}$ NMR of the crude reaction mixture in presence of an internal standard. In parenthesis, isolated yield of $\mathbf{3}$ after purification by column chromatography. ${ }^{\mathrm{b}} \mathrm{Er}$ determined by HPLC. 
Table S11. Carboxamidation of different styrenes $\mathbf{1 y}$ with isocyanate $\mathbf{2 g}(0.15-0.3 \mathrm{mmol}$ scale).<smiles>C=Cc1cc[R1]cc1</smiles>

1

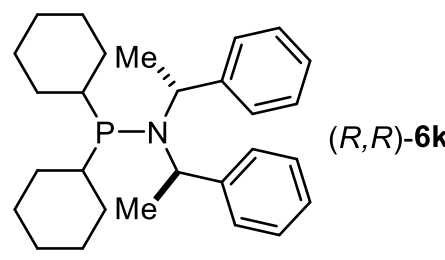

$\mathrm{CuCl}(10 \mathrm{~mol} \%),(R, R)-6 \mathbf{k}(11 \mathrm{~mol} \%)$ $\mathrm{B}_{2} \mathrm{pin}_{2}$ (1.5 equiv.), LiOtBu (2.0 equiv.)

2-Me-THF/Et ${ }_{2} \mathrm{O}(1: 1)[0.1], 30^{\circ} \mathrm{C}, 72 \mathrm{~h}$

$2 \mathrm{~g}$

(4.0 equiv.)<smiles>O=C(Nc1ccc(Br)cc1)[C@H](C[Pb])c1ccccc1</smiles>

3

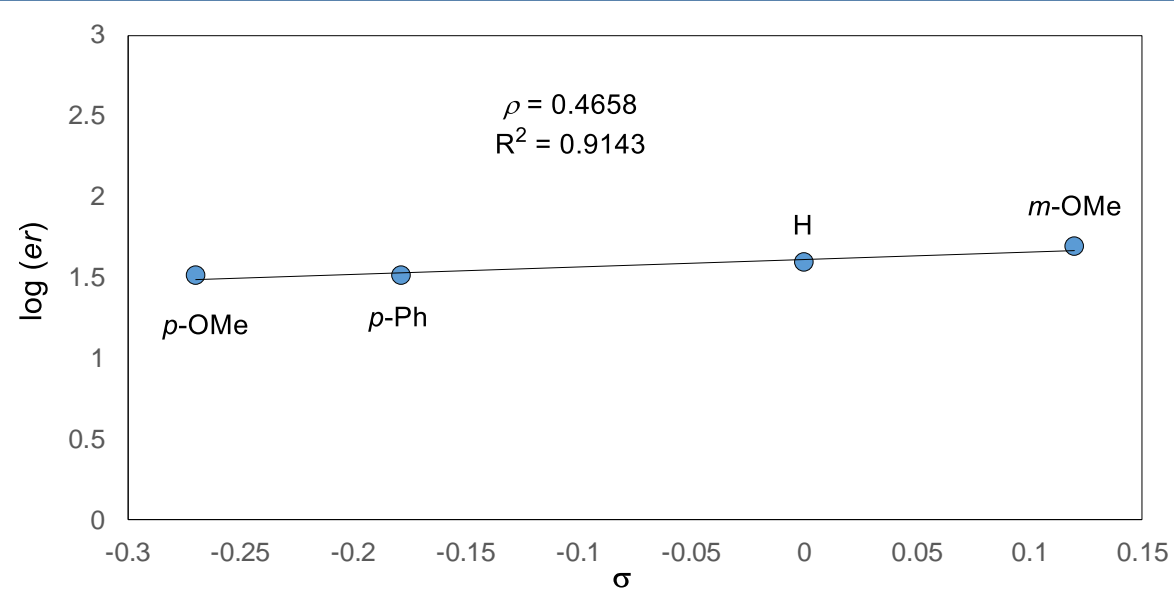

$\begin{array}{lllll}\mathrm{R}^{2} & \sigma & \text { Conv. }(\%)^{\mathrm{a}} & e r^{\mathrm{b}} & \log (\mathbf{e r}) \\ \text { p-OMe (1b) } & -\mathbf{0 . 2 7} & 80(61) & 97: 3 & \mathbf{1 . 5 0 9} \\ p \text {-Ph (1f) } & -\mathbf{0 . 1 8} & 95(73) & 97: 3 & \mathbf{1 . 5 0 9} \\ \mathrm{H}(\mathbf{1 a}) & \mathbf{0} & 95(70) & 97.5: 2.5 & \mathbf{1 . 5 9 0} \\ m-O M e(\mathbf{1 y}) & \mathbf{0 . 1 2} & 99(87) & 98: 2 & 1.690\end{array}$

a Determined by ${ }^{1} \mathrm{H}$ NMR of the crude reaction mixture in presence of an internal standard. In parenthesis, isolated yield of $\mathbf{3}$ after purification by column chromatography. ${ }^{\mathrm{b}} \mathrm{Er}$ determined by HPLC. 


\subsection{Enantiomeric ratio monitoring}

Table S12. Enantiomeric ratio monitoring ( $0.3 \mathrm{mmol}$ scale).

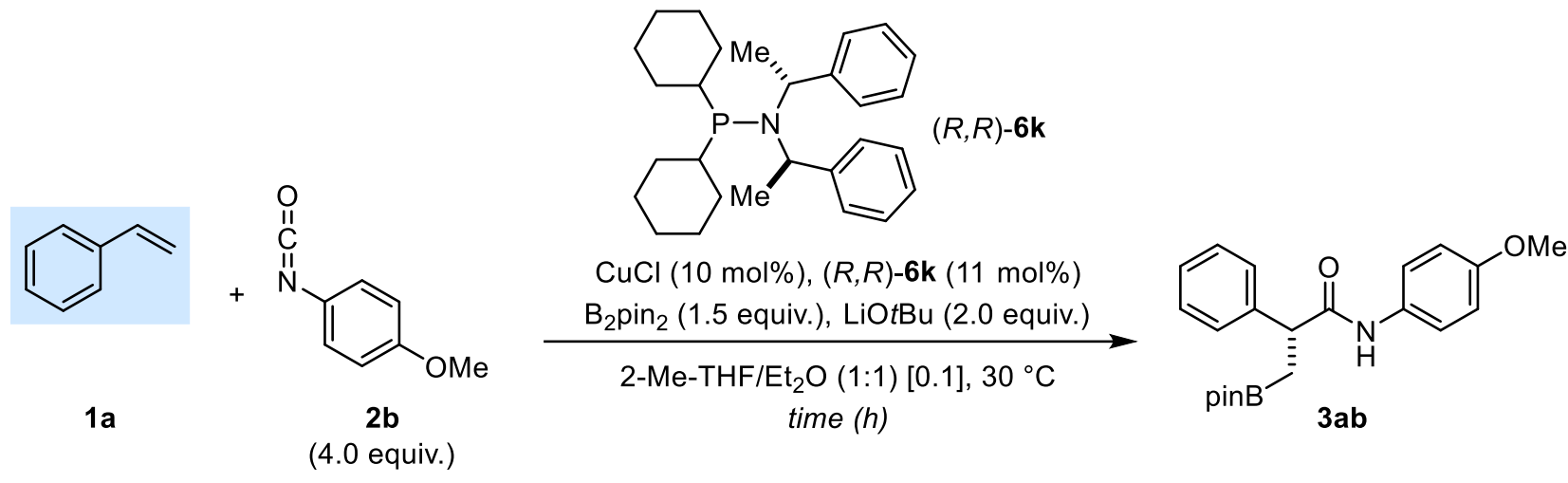

$\begin{array}{llll}\text { Time }(\mathrm{h}) & \text { Cons. 1a (\%) } & \text { Conv. 3ab (\%) } & \mathrm{er}^{\mathrm{b}} \\ 2 & 19 & 6 & n d \\ 6 & 46 & 25 & 87: 13 \\ 12 & 73 & 55 & 87: 13 \\ 24 & 95 & 71 & 86.5: 13.5 \\ 72 & >99 & 73 & 86: 14 \\ 96 & >99 & 73 & 85: 15 \\ 120 & >99 & 73 & 85: 15 \\ 168 & >99 & 73 & 84: 16\end{array}$

a Determined by ${ }^{1} \mathrm{H}$ NMR of the crude reaction mixture in presence of an internal standard. In parenthesis, isolated yield of $\mathbf{3}$ after purification by column chromatography. ${ }^{\mathrm{b}} \mathrm{Er}$ determined by HPLC. 


\subsection{Reaction scope}
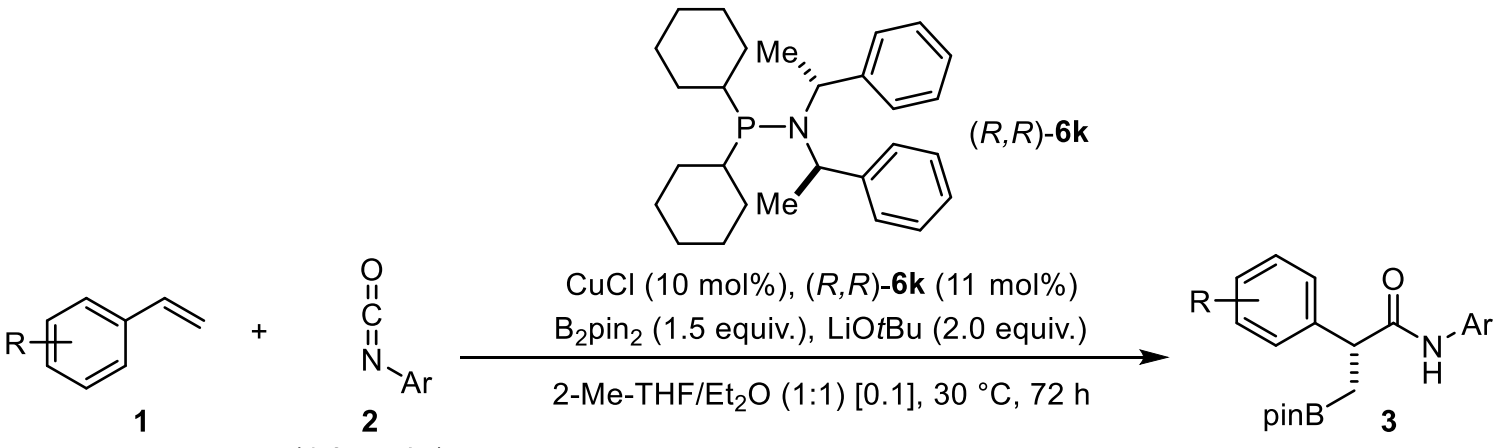

(4.0 equiv.)<smiles>O=C(Nc1ccccc1)[C@H](CBP)c1ccccc1</smiles>

$74 \%$ yield, $92: 8$ er (70\%, 99:1 er)<smiles>O=C(Nc1ccc(Br)cc1)[C@H](C[B]O)c1ccccc1</smiles>

$70 \%$ yield, 97.5:2.5 er<smiles>COc1ccc(NC(=O)[C@H](C[PbH])c2ccccc2)cc1</smiles>

$53 \%$ yield, $86: 14$ er<smiles>O=BC[C@@H](C(=O)Nc1ccc(F)cc1)c1ccccc1</smiles>

$84 \%$ yield, $91.5: 8.5$ er (67\%, 95:5 er)<smiles>CN(C)c1ccc(NC(=O)[C@H](CBP)c2ccccc2)cc1</smiles>

$45 \%$ yield, $75: 25$ er<smiles>COc1ccc([C@H](C[Pb])C(=O)Nc2ccccc2)cc1</smiles>
(76\%, 99:1 er)<smiles>COc1ccc(NC(=O)[C@H](C[Pb])c2ccc(OC)cc2)cc1</smiles>

$\mathrm{MeO}$<smiles>Cc1ccc(NC(=O)[C@H](C[PbH])c2ccc(F)cc2)cc1</smiles><smiles>COc1cccc([C@H](CB=O)C(=O)Nc2ccc(N(C)C)cc2)c1</smiles>

$73 \%$ yield, $83: 17$ er<smiles>COc1ccc(C(C[Pb])C(=O)Nc2ccc(N(C)C)cc2)cc1</smiles><smiles>COc1cccc([C@H](CB=O)C(=O)Nc2ccccc2)c1</smiles>
$61 \%$ yield, $88: 12$ er<smiles>COc1cccc([C@H](CBP)C(=O)Nc2ccc(Br)cc2)c1</smiles>

$87 \%$ yield, $98: 2$ er<smiles>COc1ccc([C@H](C[PbH])C(=O)Nc2ccc(Br)cc2)cc1</smiles>

$61 \%$ yield, 97:3 er<smiles>COc1ccc(NC(=O)[C@H](CPc2ccccc2)c2cccc(OC)c2)cc1</smiles>

$67 \%$ yield, $80: 20$ er<smiles>COc1cccc(C(CPc2ccccc2)C(=O)Nc2ccc(C)cc2)c1</smiles>

$75 \%$ yield, $85: 15$ er

Figure S7. Enantioselective Cu-catalyzed borylative carboxamidation of vinylarenes (0.15-0.3 mmol scale). Yield of isolated product after purification. Er determined by HPLC or SFC. $D r$ determined by ${ }^{1} \mathrm{H}$ and ${ }^{13} \mathrm{C}$ NMR analysis. In parenthesis, yield and er refer to the result of a single recrystallization. 

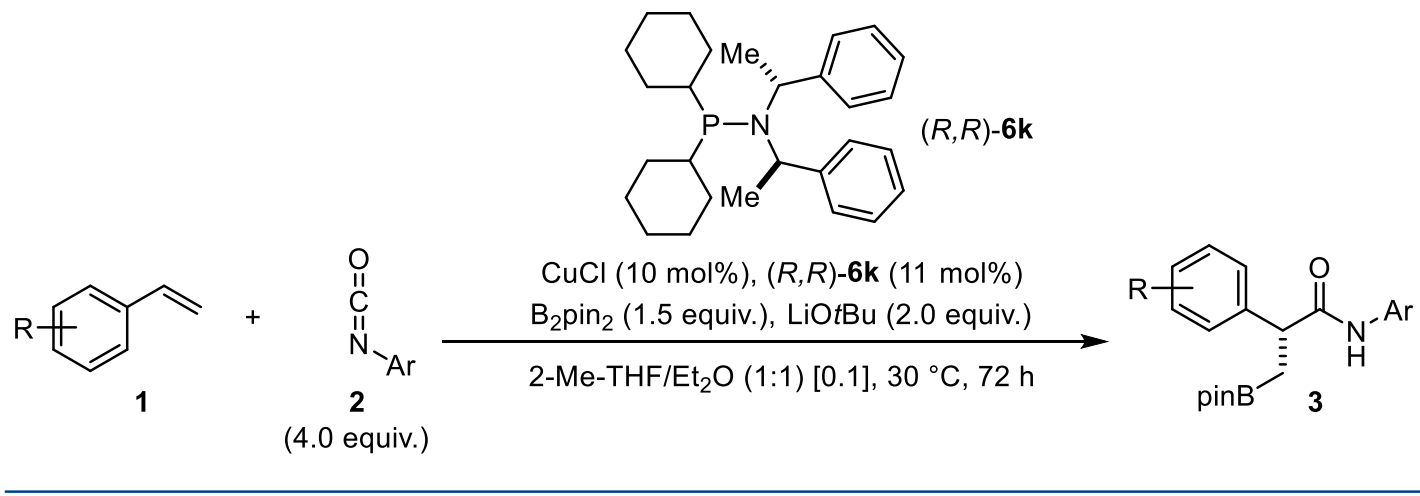<smiles>O=C(Nc1ccccc1)[C@H](C[18OH])c1ccccc1[N+](=O)[O-]</smiles>

$60 \%$ yield, $85: 15$ er<smiles>Cc1ccccc1[C@H](C[18OH])C(=O)Nc1ccc(Br)cc1</smiles>

$72 \%$ yield, $93: 7$ er<smiles>O=C(Nc1ccccc1)[C@H](C[18OH])c1ccc(F)cc1</smiles>

$77 \%$ yield, 83.5:16.5 er<smiles>O=C(Nc1ccccc1)[C@H](C[Pb])c1ccc(-c2ccccc2)cc1</smiles>

$75 \%$ yield, 88.5:11.5 er<smiles>O=C(Nc1ccc(Br)cc1)C(C[18OH])c1ccc(-c2ccccc2)cc1</smiles><smiles>O=C(Nc1ccccc1)[C@H](C[18OH])c1ccc2ccccc2c1</smiles>

$68 \%$ yield, $85.5: 14.5$ er (50\%, 98.5:1.5 er)<smiles>O=C(Nc1ccccc1)[C@H](C[18OH])c1ccc(N2CCOCC2)cc1</smiles>

$84 \%$ yield, $92: 8$ er

$(61 \%, 98.5: 1.5 \mathrm{er})$<smiles>O=CCN(CCO)c1ccc([C@@H](C[18OH])C(=O)Nc2ccc(Br)cc2)cc1</smiles><smiles>COCCN(CCO)c1ccc([C@@H](C[18OH])C(=O)Nc2ccc(Br)cc2)cn1</smiles><smiles>O=C(Nc1ccccc1)[C@H](C[18O])c1ccc2c(ccn2C(=O)c2ccccc2)c1</smiles>
$61 \%$ yield, $92: 8$ er (79\%, 96:4 er)<smiles>CC1CCC(C(C)C)C(OCc2ccc(C(CCc3ccccc3)C(=O)Nc3ccccc3)cc2)C1</smiles>
$3 b_{2} a$

$84 \%$ yield, $d r 10: 1$ (innate bias using $P C y_{3}: d r$ 1.5:1)<smiles>O=C(Nc1ccc(Br)cc1)C(C[18O])c1ccc2c(ccn2C(=O)OCc2ccccc2)c1</smiles>

$84 \%$ yield, $93: 7$ er<smiles>O=C(Nc1ccc(Br)cc1)C(C[18OH])c1ccc2sccc2c1</smiles>

$89 \%$ yield, $87: 13$ er

$$
\text { Limitations }
$$<smiles>C=C(CCC=C(C)C)C(CP)C(=O)Nc1ccc(OC)cc1</smiles>

3tb

$12 \%$ conv, $62: 38$ er<smiles>O=C(Nc1ccccc1)C1C2CCC(C2)C1Cc1ccccc1</smiles>

3wa

$11 \%$ conv, $62.5: 37.5$ er

Figure S7 (continued). Enantioselective Cu-catalyzed borylative carboxamidation of vinylarenes (0.15-0.3 mmol scale). Yield of isolated product after purification. Er determined by HPLC or SFC. Dr determined by ${ }^{1} \mathrm{H}$ and ${ }^{13} \mathrm{C}$ NMR analysis. In parenthesis, yield and er refer to the result of a single recrystallization. 


\section{General procedure for the enantioselective copper-catalyzed borylative carboxamidation of vinylarenes}

In a dry $5 \mathrm{~mL}$ vial, $\mathrm{B}_{2}$ pin 2 (114 mg, $0.45 \mathrm{mmol}, 1.5$ equiv.) and LiOtBu (48 mg, $0.6 \mathrm{mmol}, 2.0$ equiv.) were dissolved in $2.0 \mathrm{~mL}$ of anhydrous and degassed 2-Me-THF and $\mathrm{Et}_{2} \mathrm{O}(\mathrm{v}: \mathrm{v}=1: 1)$. The mixture was stirred for 30 min at $23^{\circ} \mathrm{C}$ to get a white suspension.

In a $10 \mathrm{~mL}$ Schlenk tube, $\mathrm{CuCl}(3.0 \mathrm{mg}, 0.03 \mathrm{mmol}, 10 \mathrm{~mol} \%)$ and ligand $(R, R)-6 \mathrm{k}(14 \mathrm{mg}$, $0.033 \mathrm{mmol}, 11 \mathrm{~mol} \%$ ) were dissolved in $1.0 \mathrm{~mL}$ of anhydrous and degassed 2-Me-THF and $\mathrm{Et}_{2} \mathrm{O}(\mathrm{v}: \mathrm{v}=1: 1)$. The solution was stirred for $30 \mathrm{~min}$ at $23^{\circ} \mathrm{C}$, then styrene $1(0.3 \mathrm{mmol}, 1.0$ equiv.) and isocyanate 2 (1.2 mmol, 4.0 equiv.) were added. After $5 \mathrm{~min}$, the above prepared suspension of $B_{2}$ pin ${ }_{2}$. LiOtBu was transferred into the $10 \mathrm{~mL}$ Schlenk. The reaction was stirred for $72 \mathrm{~h}$ at $30{ }^{\circ} \mathrm{C}$ before quenching with a saturated aqueous $\mathrm{NH}_{4} \mathrm{Cl}$ solution $(1.0 \mathrm{~mL})$. The reaction mixture was diluted with ethyl acetate $(5 \mathrm{~mL})$ and water $(5 \mathrm{~mL})$ and extracted with ethyl acetate $(3 \times 10 \mathrm{~mL})$. The combined organic phases were dried over sodium sulfate and the solvent was removed under reduced pressure. Purification by flash chromatography on silica gel afforded the pure borylated secondary amide 3. Enantiomeric excess was determined by HPLC or SFC analysis of boronate 3. If separation methods for HPLC or SFC analysis could not be found for racemic boronate 3 , the latter was oxidized to the corresponding alcohol following the general procedure.

\section{General procedure for the oxidation of boronate 3 to alcohol for the determination of enantiomeric excess by HPLC or SFC analysis}

To a solution of pure $3(0.1 \mathrm{mmol})$ in THF $(1 \mathrm{~mL})$ and $\mathrm{H}_{2} \mathrm{O}(1 \mathrm{~mL})$ was added $\mathrm{NaBO}_{3} \cdot 4 \mathrm{H}_{2} \mathrm{O}$ (56 mg, $0.8 \mathrm{mmol}, 8$ equiv.) at $23^{\circ} \mathrm{C}$. The reaction mixture was stirred for $3 \mathrm{~h}$ and quenched by addition of saturated aqueous $\mathrm{Na}_{2} \mathrm{~S}_{2} \mathrm{O}_{3}(2 \mathrm{~mL})$. The mixture was extracted with ethyl acetate and the combined organic layers were washed with water, brine, dried over anhydrous sodium sulfate and concentrated in vacuum. The alcohol was obtained as a white solid and analyzed by HPLC or SFC equipped with chiral columns in order to determine the enantiomeric ratio. 
(R)-N,2-diphenyl-3-(4,4,5,5-tetramethyl-1,3,2-dioxaborolan-2-yl)propanamide 3aa<smiles>CC1(C)OB(C[C@@H](C(=O)Nc2ccccc2)c2ccccc2)OC1(C)C</smiles>

According to the general procedure for enantioselective coppercatalyzed borylative carboxamidation using styrene $\mathbf{1 a}(31 \mathrm{mg}, 0.3$ mmol, 1.0 equiv.), $\mathrm{CuCl}(3.0 \mathrm{mg}, 0.03 \mathrm{mmol}, 10 \mathrm{~mol} \%)$, ligand $(R, R)$ 6k (14 mg, $0.033 \mathrm{mmol}, 11 \mathrm{~mol} \%$ ), LiOtBu (48 mg, $0.6 \mathrm{mmol}, 2.0$ equiv.), $B_{2} p_{2}(114 \mathrm{mg}, \quad 0.45 \mathrm{mmol}, 1.5$ equiv.) and isocyanatobenzene $2 \mathrm{a}(0.132 \mathrm{~mL}, 1.2 \mathrm{mmol}, 4.0$ equiv. $)$ in 2-Me-THF and $\mathrm{Et}_{2} \mathrm{O}(3.0 \mathrm{~mL}, \mathrm{v}: \mathrm{v}=$ $1: 1,0.1 \mathrm{M}$ ). $90 \%$ conversion into 3aa determined by ${ }^{1} \mathrm{H}$ NMR analysis of the crude reaction mixture in presence of an internal standard. Purification by flash chromatography (pentane/diethyl ether 3:1) gave pure 3aa as a white solid (78 mg, $0.22 \mathrm{mmol}, 74 \%$ yield).

HPLC analysis of 3aa after oxidation to the corresponding alcohol 7 following the general procedure indicated 92:8 er, chiral stationary column: OD-H, mobile phase: $n$-hexane/iPrOH $=95: 5,1.0 \mathrm{~mL} / \mathrm{min}, 254 \mathrm{~nm}, 30^{\circ} \mathrm{C}, \mathrm{t}_{\mathrm{R}}$ (major) = $21.9 \mathrm{~min}, \mathrm{t}_{\mathrm{R}}$ (minor) $=12.6 \mathrm{~min}$.

Alcohol $\mathbf{7}$ is a known compound and all spectroscopic and spectrometric analyses were in agreement with the literature. ${ }^{10}$ NMR spectra are reported.

$[\alpha]^{20} \mathrm{D}=-79.7\left(\mathrm{c} 0.33, \mathrm{CH}_{2} \mathrm{Cl}_{2}\right)$ for the corresponding alcohol 7.

Recrystallization: 3aa (320 mg, 92:8 er) was dissolved in $\mathrm{CH}_{2} \mathrm{Cl}_{2} / \mathrm{Et}_{2} \mathrm{O}(1: 20,10 \mathrm{~mL})$ and the solution was concentrated by slow evaporation at $23^{\circ} \mathrm{C}$ until a white crystalline precipitate appeared. The precipitate was filtered, washed with $n$-hexane and dried under vacuum (226 mg, 70\% yield, 99:1 er). 
$<$ Chromatogram $>$

mAU

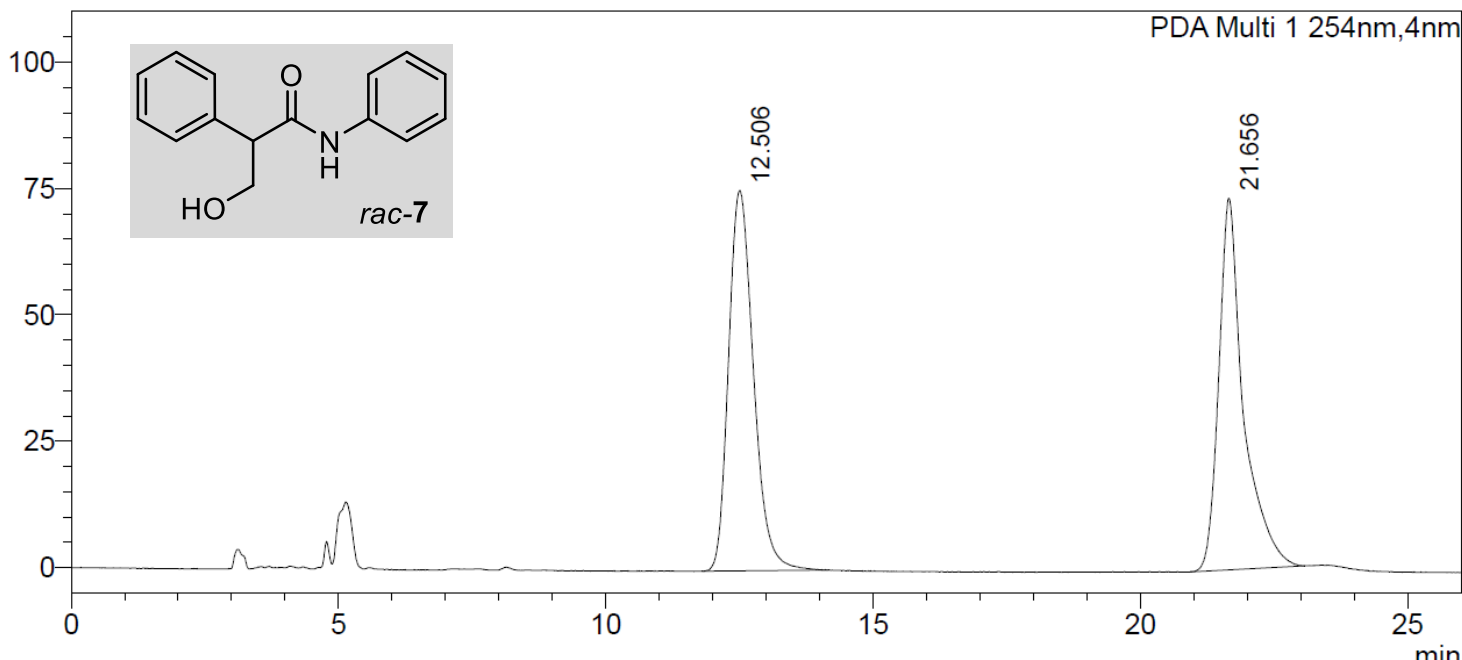

<Peak Table>

PDA Ch1 254nm

\begin{tabular}{|r|r|l|r|}
\hline Peak\# & Ret. Time & Area & Area\% \\
\hline 1 & 12.506 & 2442790 & 50.759 \\
\hline 2 & 21.656 & 2369748 & 49.241 \\
\hline Total & & 4812539 & 100.000 \\
\hline
\end{tabular}

\section{<Chromatogram>}

$\mathrm{mAU}$

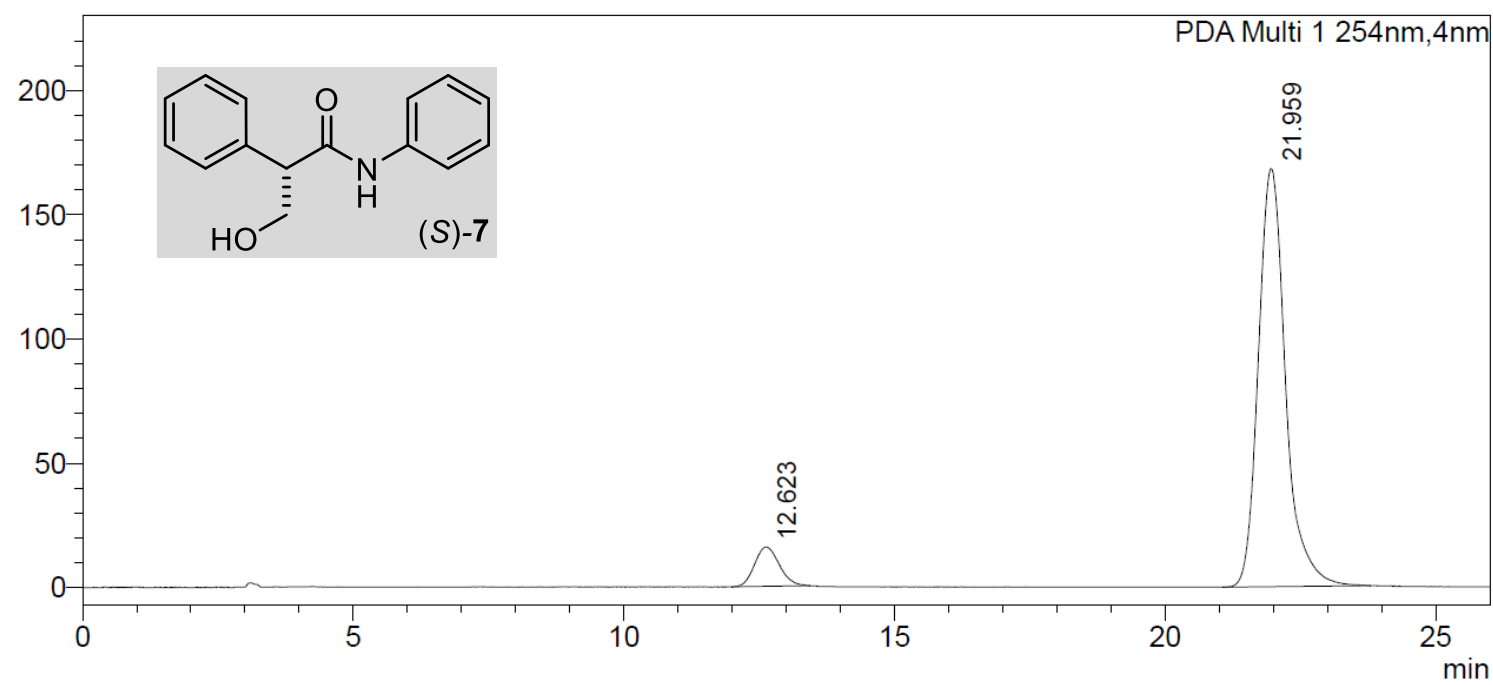

<Peak Table>

PDA Ch1 254nm

\begin{tabular}{|r|r} 
Peak\# & Ret. Time \\
\hline 1 & 12.623
\end{tabular}

\begin{tabular}{|c|c|c|c|}
\hline Peak\# & Ret. IIme & Area & Area\% \\
\hline 1 & 12.623 & 504056 & 7.896 \\
\hline 2 & 21.959 & 5879619 & 92.104 \\
\hline Total & & 6383675 & 100.000 \\
\hline
\end{tabular}




\section{<Chromatogram>}

mAU

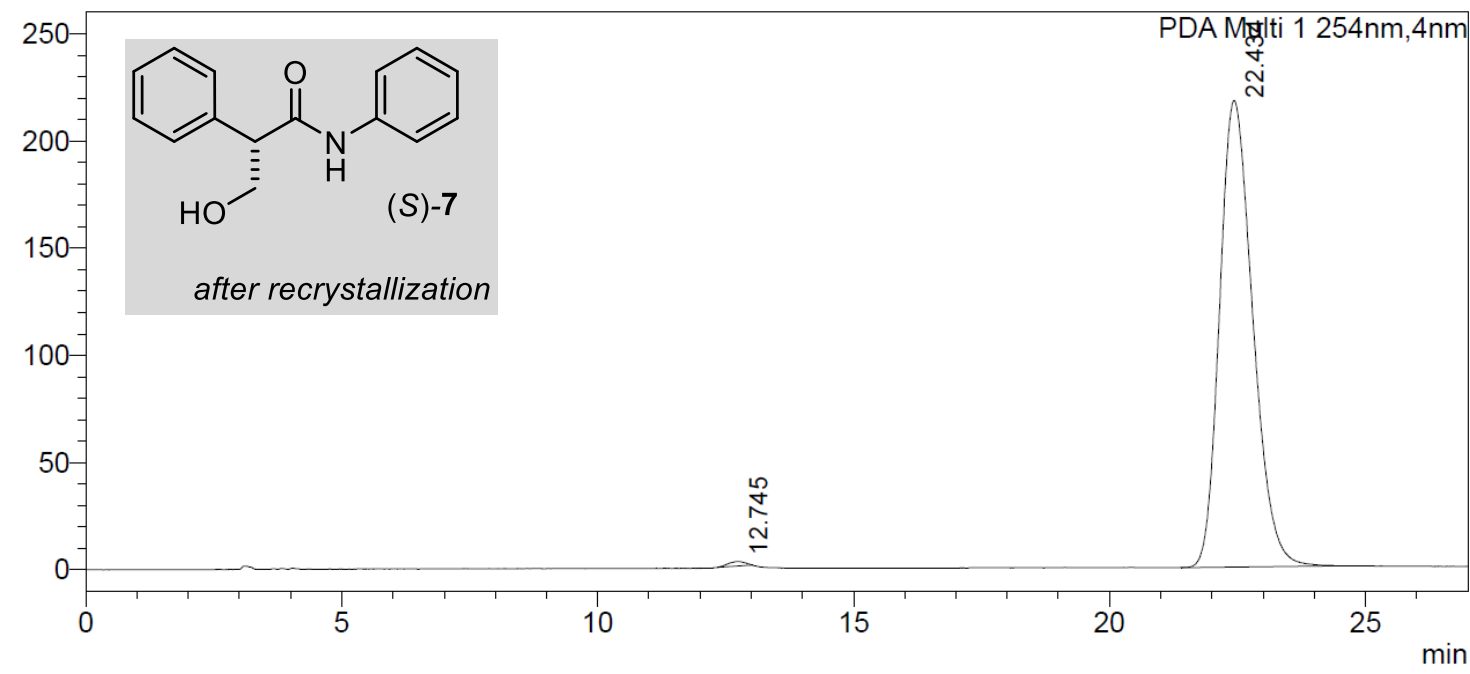

<Peak Table>

PDA Ch1 254nm

\begin{tabular}{|r|r|r|r|}
\hline Peak\# & Ret. Time & \multicolumn{1}{|c|}{ Area } & \multicolumn{1}{|c|}{ Area\% } \\
\hline 1 & 12.745 & 48057 & 0.487 \\
\hline 2 & 22.434 & 9813183 & 99.513 \\
\hline Total & & 9861240 & 100.000 \\
\hline
\end{tabular}


(R)-N-(4-methoxyphenyl)-2-phenyl-3-(4,4,5,5-tetramethyl-1,3,2-dioxaborolan-2yl)propanamide $3 \mathbf{a b}$<smiles>COc1ccc(NC(=O)[C@H](CB2OC(C)(C)C(C)(C)O2)c2ccccc2)cc1</smiles>

According to the general procedure for enantioselective copper-catalyzed borylative carboxamidation using styrene 1a (31 mg, $0.3 \mathrm{mmol}, 1.0$ equiv.), $\mathrm{CuCl}$ (3.0 mg, $0.03 \mathrm{mmol}, 10$ mol\%), ligand $(R, R)-6 \mathbf{k}(14 \mathrm{mg}, 0.033 \mathrm{mmol}, 11 \mathrm{~mol} \%)$, LiOtBu (48 mg, $0.6 \mathrm{mmol}, 2.0$ equiv.), $\mathrm{B}_{2} \mathrm{pin}_{2}(114 \mathrm{mg}, 0.45 \mathrm{mmol}, 1.5$ equiv.) and 1-isocyanato 4-methoxybenzene $2 b(0.156 \mathrm{~mL}, 1.2 \mathrm{mmol}, 4.0$ equiv.) in 2-MeTHF and $\mathrm{Et}_{2} \mathrm{O}(3.0 \mathrm{~mL}, \mathrm{v}: \mathrm{v}=1: 1,0.1 \mathrm{M}) .70 \%$ conversion into $3 \mathrm{ab}$ determined by ${ }^{1} \mathrm{H} \mathrm{NMR}$ analysis of the crude reaction mixture in presence of an internal standard. Purification by flash chromatography (pentane/diethyl ether 2:1) gave pure $3 \mathrm{ab}$ as a white solid $(60 \mathrm{mg}, 0.16 \mathrm{mmol}$, $53 \%$ yield).

HPLC analysis of 3ab indicated 86:14 er, chiral stationary column: OD-H, mobile phase: $n$ hexane $/ \mathrm{PrOH}=95: 5,1.0 \mathrm{~mL} / \mathrm{min}, 254 \mathrm{~nm}, 30^{\circ} \mathrm{C}, \mathrm{t}_{\mathrm{R}}$ (major) $=15.9 \mathrm{~min}, \mathrm{t}_{\mathrm{R}}$ (minor) $=18.4 \mathrm{~min}$. $[\alpha]^{20} \mathrm{D}=-42.1\left(\mathrm{c} 0.58, \mathrm{CH}_{2} \mathrm{Cl}_{2}\right)$. 


\section{$<$ Chromatogram $>$}

mAU

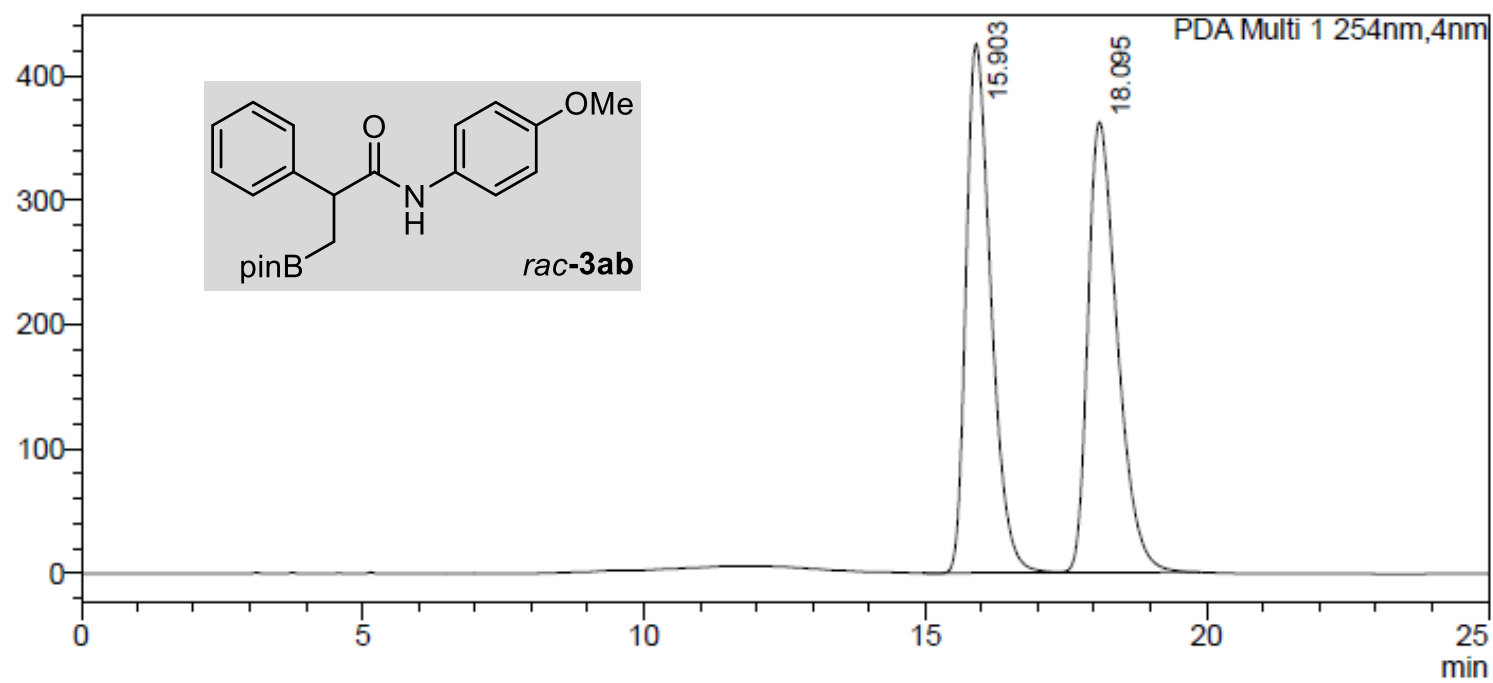

<Peak Table>

PDA Ch1 254nm

\begin{tabular}{|r|r|r|r|}
\hline Peak\# & Ret. Time & Area & \multicolumn{1}{c|}{ Area\% } \\
\hline 1 & 15.903 & 13108246 & 49.816 \\
\hline 2 & 18.095 & 13204958 & 50.184 \\
\hline Total & & 26313204 & 100.000 \\
\hline
\end{tabular}

\section{$<$ Chromatogram $>$}

mAU

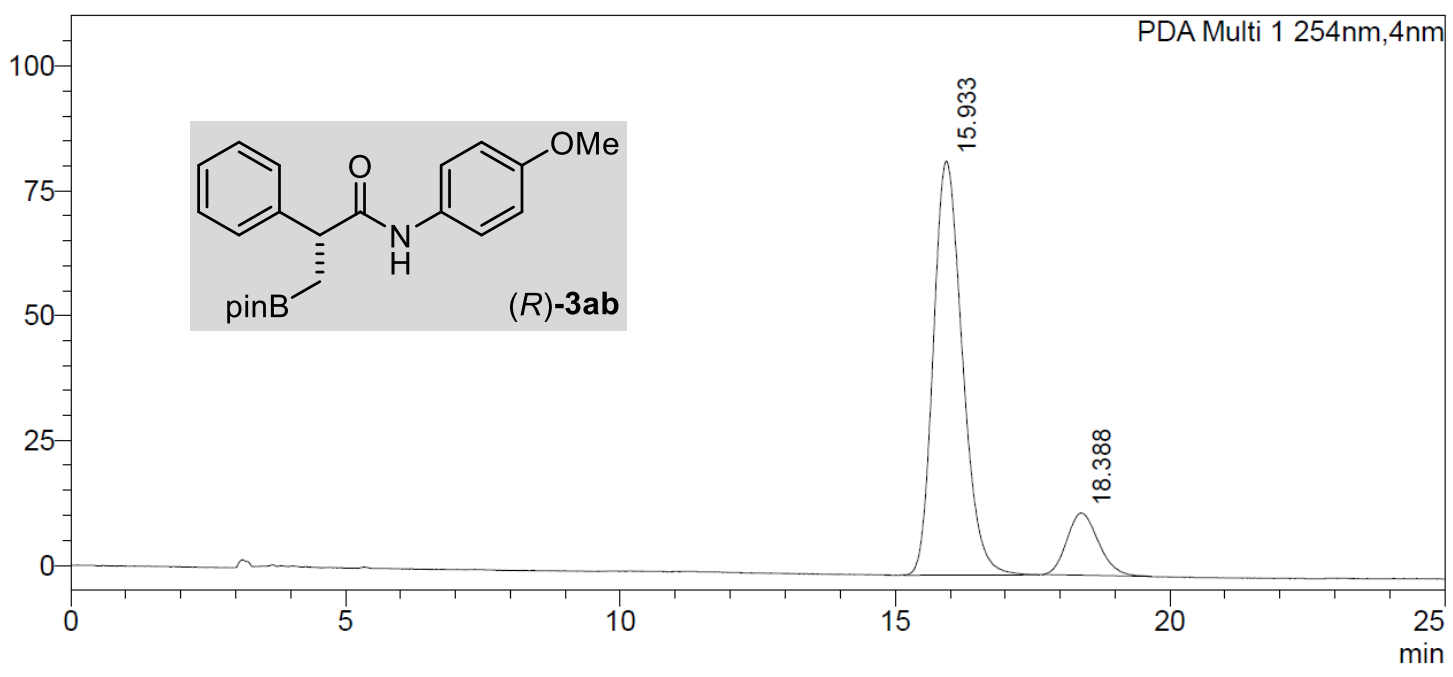

<Peak Table>

PDA Ch1 254nm

\begin{tabular}{|r|r|r|r|}
\hline Peak\# & Ret. Time & \multicolumn{1}{|c|}{ Area } & \multicolumn{1}{|c|}{ Area\% } \\
\hline 1 & 15.933 & 3042250 & 85.938 \\
\hline 2 & 18.388 & 497789 & 14.062 \\
\hline Total & & 3540039 & 100.000 \\
\hline
\end{tabular}


(R)-N-(4-(dimethylamino)phenyl)-2-phenyl-3-(4,4,5,5-tetramethyl-1,3,2-dioxaborolan-2yl)propanamide $3 \mathrm{ad}$<smiles>CN(C)c1ccc(NC(=O)[C@H](CB2OC(C)(C)C(C)(C)O2)c2ccccc2)cc1</smiles>

According to the general procedure for enantioselective copper-catalyzed borylative carboxamidation using styrene $1 \mathrm{a}$ (16 mg, $0.15 \mathrm{mmol}, 1.0$ equiv.), $\mathrm{CuCl}$ (1.5 mg, 0.015 $\mathrm{mmol}, 10 \mathrm{~mol} \%)$, ligand $(R, R)-6 \mathrm{k}(7.0 \mathrm{mg}, 0.017 \mathrm{mmol}, 11$ 3ad mol\%), LiOtBu (24 mg, 0.3 mmol, 2.0 equiv.), B 2pin $_{2}(57 \mathrm{mg}$,

$0.23 \mathrm{mmol}, 1.5$ equiv. $)$ and 4-isocyanato- $N, N$-dimethylaniline $2 \mathrm{~d}(0.97 \mathrm{mg}, 0.60 \mathrm{mmol}, 4.0$ equiv.) in 2-Me-THF and $\mathrm{Et}_{2} \mathrm{O}$ (1.5 mL, v:v = 1:1, $\left.0.1 \mathrm{M}\right) .75 \%$ conversion into 3ad determined by ${ }^{1} \mathrm{H}$ NMR analysis of the crude reaction mixture in presence of an internal standard. Purification by flash chromatography $\left(\mathrm{CH}_{2} \mathrm{Cl}_{2}\right.$ with $\left.\mathrm{MeOH} 2 \%\right)$ gave pure 3ad as a brown solid (27 mg, $0.07 \mathrm{mmol}, 45 \%$ yield).

HPLC analysis of 3ad indicated 75:25 er, chiral stationary column: AS-H, mobile phase: $n$ hexane $/ \mathrm{PrOH}=90: 10,1.0 \mathrm{~mL} / \mathrm{min}, 276 \mathrm{~nm}, 30^{\circ} \mathrm{C}, \mathrm{t}_{\mathrm{R}}=8.9 \mathrm{~min}$ (major), $\mathrm{t}_{\mathrm{R}}=14.2 \mathrm{~min}$ (minor). $[\alpha]^{20}=-17.8\left(c 0.325, \mathrm{CH}_{2} \mathrm{Cl}_{2}\right)$. 


\section{<Chromatogram>}

mAU

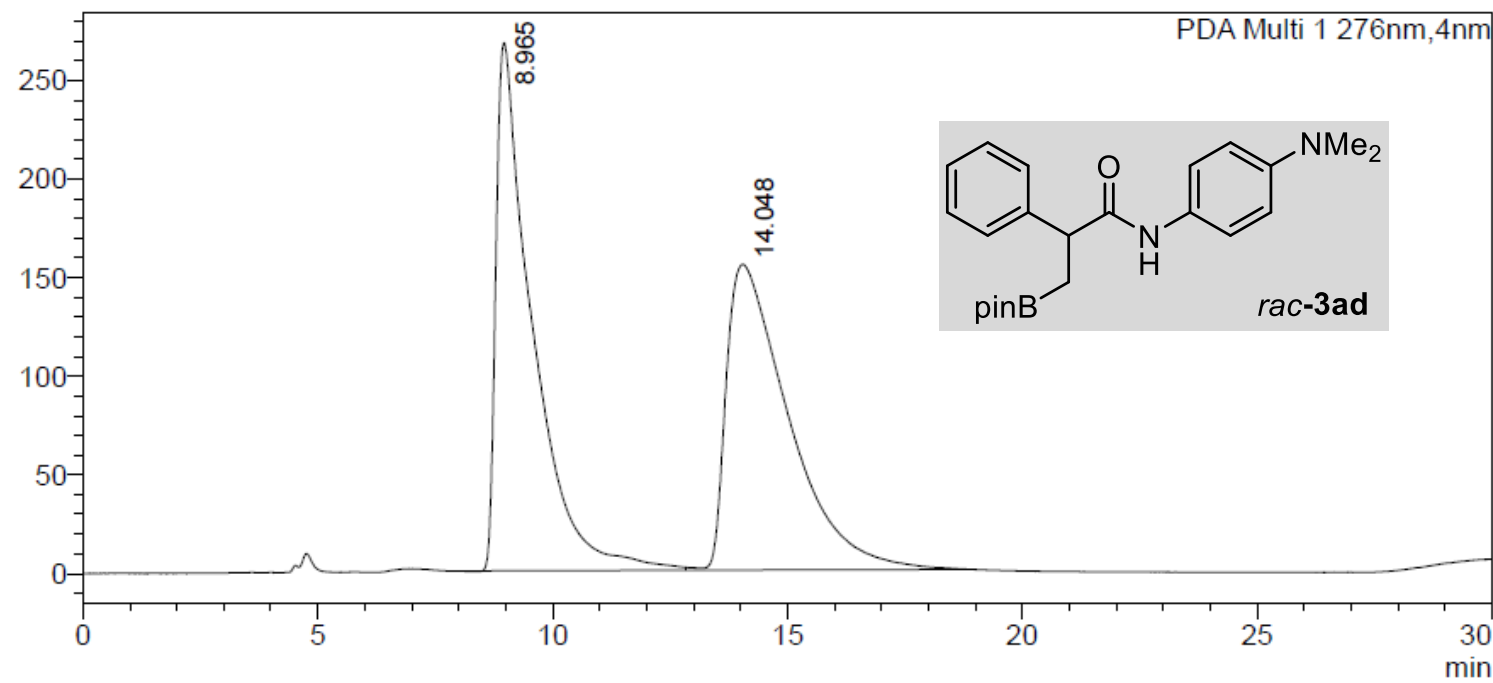

<Peak Table>

PDA Ch1 276nm

\begin{tabular}{|r|r|r|r|}
\hline Peak\# & Ret. Time & Area & \multicolumn{1}{c|}{ Area $\%$} \\
\hline 1 & 8.965 & 14427966 & 50.193 \\
\hline 2 & 14.048 & 14316918 & 49.807 \\
\hline Total & & 28744883 & 100.000 \\
\hline
\end{tabular}

<Chromatogram>

$\mathrm{mAU}$

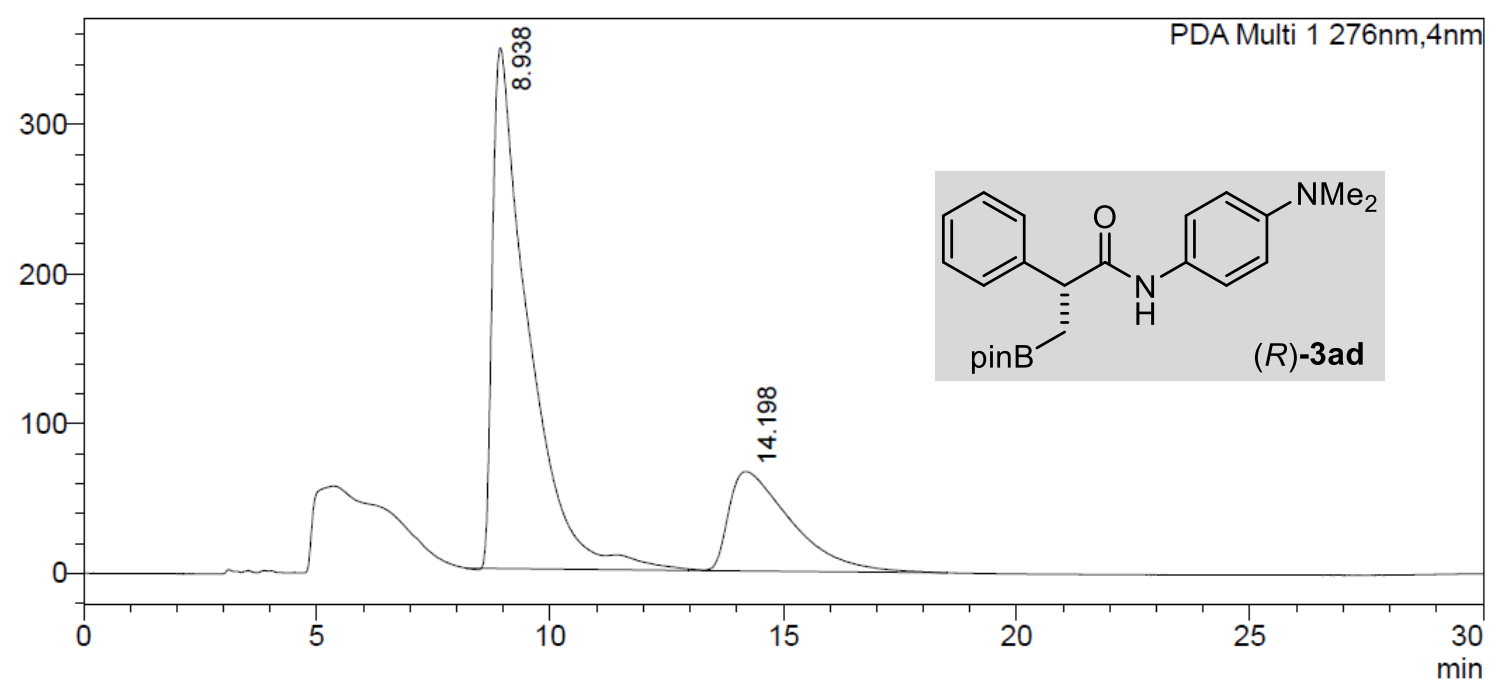

<Peak Table>

PDA Ch1 276nm

\begin{tabular}{|r|r|r|r|}
\hline Peak\# & Ret. Time & \multicolumn{1}{|c|}{ Area } & \multicolumn{1}{c|}{ Area\% } \\
\hline 1 & 8.938 & 18600736 & 75.092 \\
\hline 2 & 14.198 & 6169718 & 24.908 \\
\hline Total & & 24770454 & 100.000 \\
\hline
\end{tabular}


(R)-N-(4-Bromophenyl)-2-phenyl-3-(4,4,5,5-tetramethyl-1,3,2-dioxaborolan-2-yl)propanamide 3ag<smiles>CC1(C)OB(C[C@H](C(=O)Nc2ccc(Br)cc2)c2ccccc2)OC1(C)C</smiles>

According to the general procedure for enantioselective coppercatalyzed borylative carboxamidation using styrene 1a (16 mg, $0.15 \mathrm{mmol}, 1.0$ equiv), $\mathrm{CuCl}$ (1.5 mg, $0.015 \mathrm{mmol}, 10 \mathrm{~mol} \%)$, ligand $(R, R)-6 \mathbf{k}$ (7.0 mg, $0.017 \mathrm{mmol}, 11 \mathrm{~mol} \%$ ), LiOtBu (24 mg, 3ag $0.3 \mathrm{mmol}, 2.0$ equiv), $\mathrm{B}_{2} \mathrm{pin}_{2}(57 \mathrm{mg}, 0.23 \mathrm{mmol}, 1.5$ equiv) and 4-isocyanato-1-bromobenzene $\mathbf{2 g}\left(0.119 \mathrm{mg}, 0.60 \mathrm{mmol}, 4.0\right.$ equiv) in 2-Me-THF and $\mathrm{Et}_{2} \mathrm{O}$ $(1.5 \mathrm{~mL}, \mathrm{v}: \mathrm{v}=1: 1,0.1 \mathrm{M}) .95 \%$ conversion into 3ag determined by ${ }^{1} \mathrm{H}$ NMR analysis of the crude reaction mixture in presence of an internal standard. Purification by flash chromatography (pentane/diethyl ether $4: 1$ ) gave pure 3 ag as a white solid ( $45 \mathrm{mg}, 0.11 \mathrm{mmol}$, $70 \%$ yield).

HPLC analysis of 3ag indicated 97.5:2.5 er, chiral stationary column: IC, mobile phase: $n$ hexane $/ \mathrm{PrOH}=95: 5,1.0 \mathrm{~mL} / \mathrm{min}, 253 \mathrm{~nm}, 30^{\circ} \mathrm{C}, \mathrm{t}_{\mathrm{R}}=14.9 \mathrm{~min}$ (major), $\mathrm{t}_{\mathrm{R}}=31.0 \mathrm{~min}$ (minor). $[\alpha]^{20} \mathrm{D}=-36.6\left(c 0.30, \mathrm{CH}_{2} \mathrm{Cl}_{2}\right)$. 


\section{$<$ Chromatogram>}

$\mathrm{mAU}$

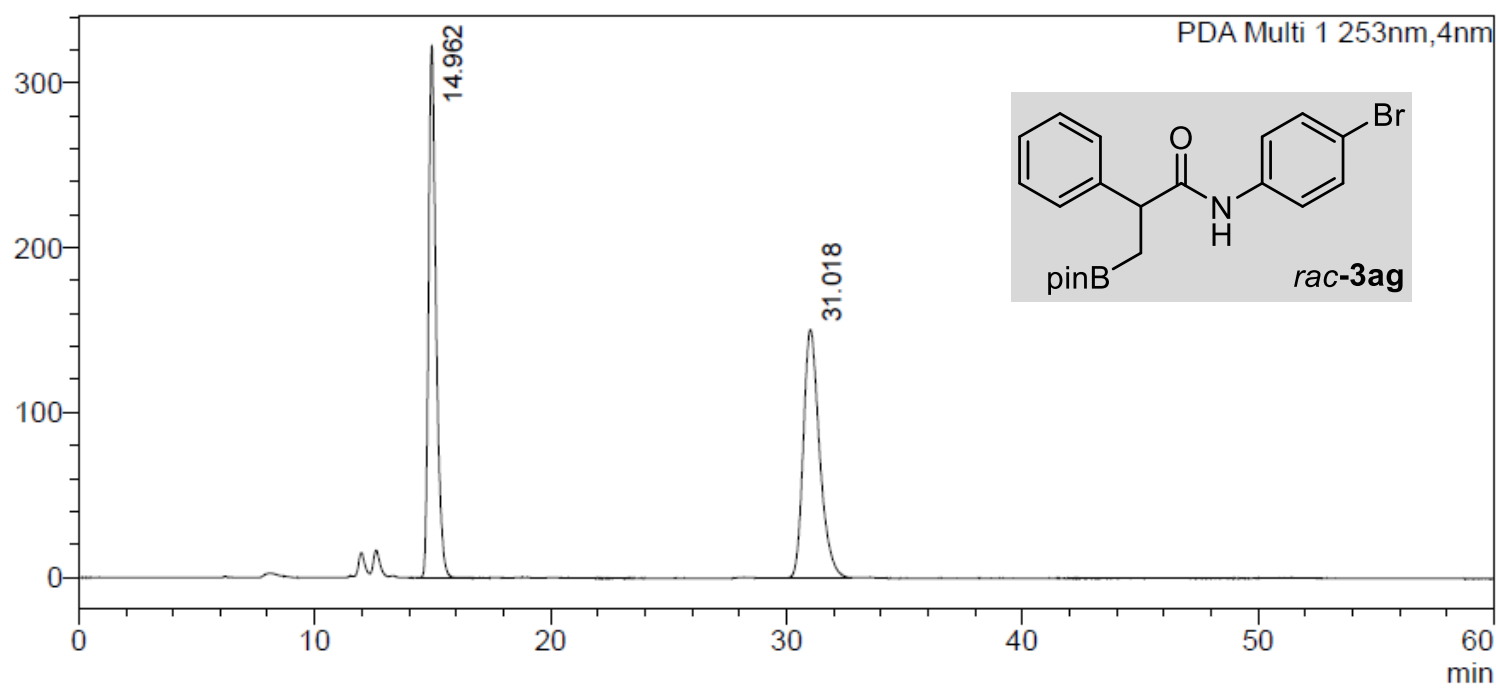

\section{<Peak Table>}

PDA Ch1 253nm

\begin{tabular}{|r|r|r|r|}
\hline Peak\# & Ret. Time & \multicolumn{1}{|c|}{ Area } & \multicolumn{1}{c|}{ Area\% } \\
\hline 1 & 14.962 & 7195722 & 49.936 \\
\hline 2 & 31.018 & 7214265 & 50.064 \\
\hline Total & & 14409988 & 100.000 \\
\hline
\end{tabular}

\section{$<$ Chromatogram $>$}

$\mathrm{mAU}$

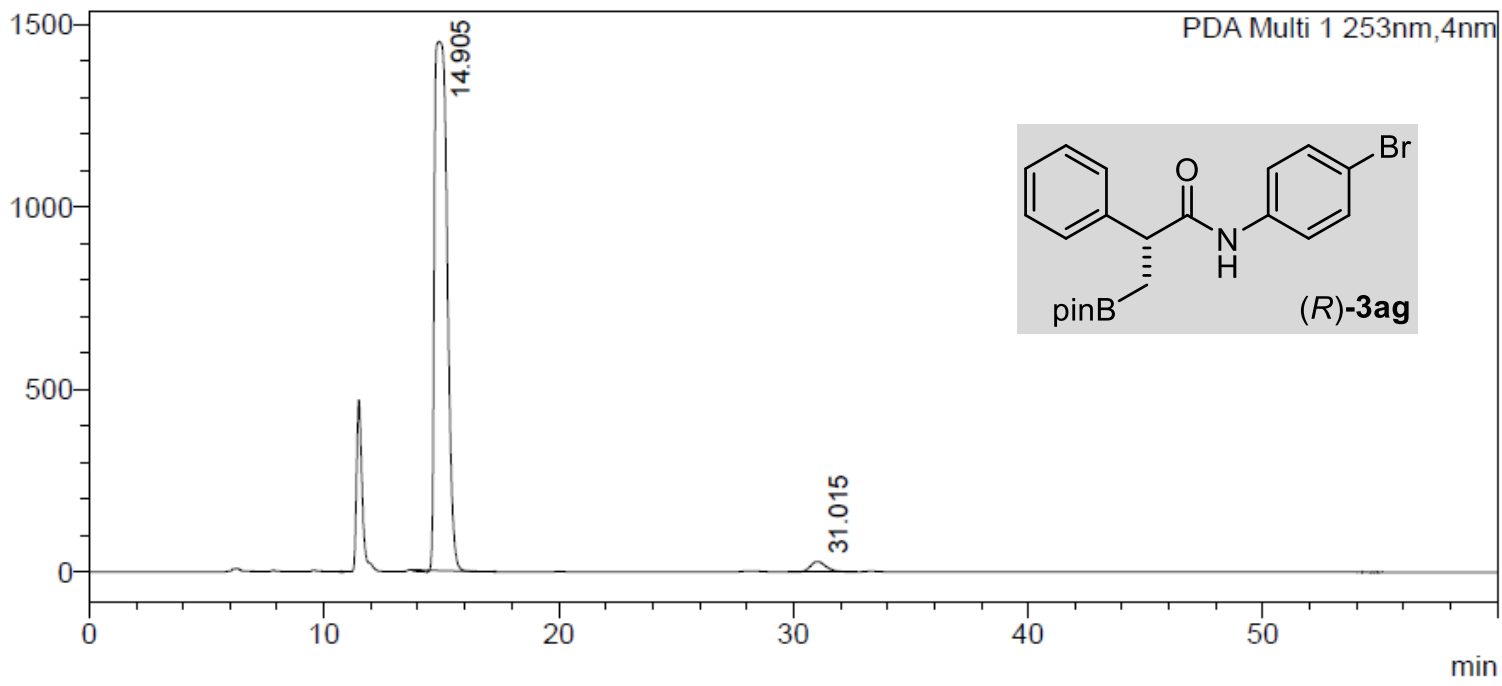

<Peak Table>

PDA Ch1 $253 \mathrm{~nm}$
\begin{tabular}{|r|r|r|r|}
\hline Peak\# & Ret. Time & \multicolumn{1}{c|}{ Area } & \multicolumn{1}{c|}{ Area $\%$} \\
\hline 1 & 14.905 & 54115822 & 97.489 \\
\hline 2 & 31.015 & 1393961 & 2.511 \\
\hline Total & & 55509783 & 100.000 \\
\hline
\end{tabular}


(R)-N-(4-fluorophenyl)-2-phenyl-3-(4,4,5,5-tetramethyl-1,3,2-dioxaborolan-2-yl)propanamide 3ai<smiles>CC1(C)OB(C[C@@H](C(=O)Nc2ccc(F)cc2)c2ccccc2)OC1(C)C</smiles>

According to the general procedure for enantioselective coppercatalyzed borylative carboxamidation using styrene 1a (31 mg, $0.3 \mathrm{mmol}, 1.0$ equiv.), $\mathrm{CuCl}$ (3.0 $\mathrm{mg}, 0.03 \mathrm{mmol}, 10 \mathrm{~mol} \%$ ), ligand $(R, R)-6 \mathrm{k}(14 \mathrm{mg}, 0.033 \mathrm{mmol}, 11 \mathrm{~mol} \%)$, LiOtBu (48 mg, 0.6 mmol, 2.0 equiv.), $B_{2} \operatorname{pin}_{2}(114 \mathrm{mg}, 0.45 \mathrm{mmol}, 1.5$ equiv.) and 1-isocyanate 4-fluorobenzene $2 \mathbf{i}(0.148 \mathrm{~mL}, 1.2 \mathrm{mmol}, 4.0$ equiv. $)$ in 2-Me-THF and $\mathrm{Et}_{2} \mathrm{O}$ $(3.0 \mathrm{~mL}, \mathrm{v}: \mathrm{v}=1: 1,0.1 \mathrm{M}) .90 \%$ conversion into 3ai determined by ${ }^{1} \mathrm{H}$ NMR analysis of the crude reaction mixture in presence of an internal standard. Purification by flash chromatography (pentane/diethyl ether 3:1) gave pure 3ai as a white solid (93 mg, $0.25 \mathrm{mmol}$, $84 \%$ yield).

HPLC analysis of 3ai after oxidation to the corresponding alcohol following the general procedure indicated 91.5:8.5 er, chiral stationary column: OD-H, mobile phase: $n$ hexane $/ \mathrm{PrOH}=95: 5,1.0 \mathrm{~mL} / \mathrm{min}, 254 \mathrm{~nm}, 30^{\circ} \mathrm{C}, \mathrm{t}_{\mathrm{R}}$ (major) $=15.1 \mathrm{~min}, \mathrm{t}_{\mathrm{R}}$ (minor) $=10.3 \mathrm{~min}$. $[\alpha]^{20} \mathrm{D}=-42.8\left(c 0.95, \mathrm{CH}_{2} \mathrm{Cl}_{2}\right)$.

Recrystallization: 3ai (61 mg, 91.5:8.5 er) was dissolved in $\mathrm{CH}_{2} \mathrm{Cl}_{2} / \mathrm{Et}_{2} \mathrm{O}$ (1: 20, $6 \mathrm{~mL}$ ) and the solution was concentrated by slow evaporation at $23^{\circ} \mathrm{C}$ until a white crystalline precipitate appeared. The precipitate was filtered, washed with $n$-hexane and dried under vacuum (41 mg, 67\% yield, 95:5 er).

SFC analysis of recrystallized 3ai after oxidation to the corresponding alcohol following the general procedure indicated 95:5 er, chiral stationary column: OB, mobile phase: $2 \%$ to $30 \%$ $\mathrm{MeOH}, 3.0 \mathrm{~mL} / \mathrm{min}, 210 \mathrm{~nm}, 30^{\circ} \mathrm{C}, \mathrm{t}_{\mathrm{R}}$ (major) $=5.5 \mathrm{~min}, \mathrm{t}_{\mathrm{R}}($ minor $)=5.1 \mathrm{~min}$. 


\section{$<$ Chromatogram $>$}

mAU

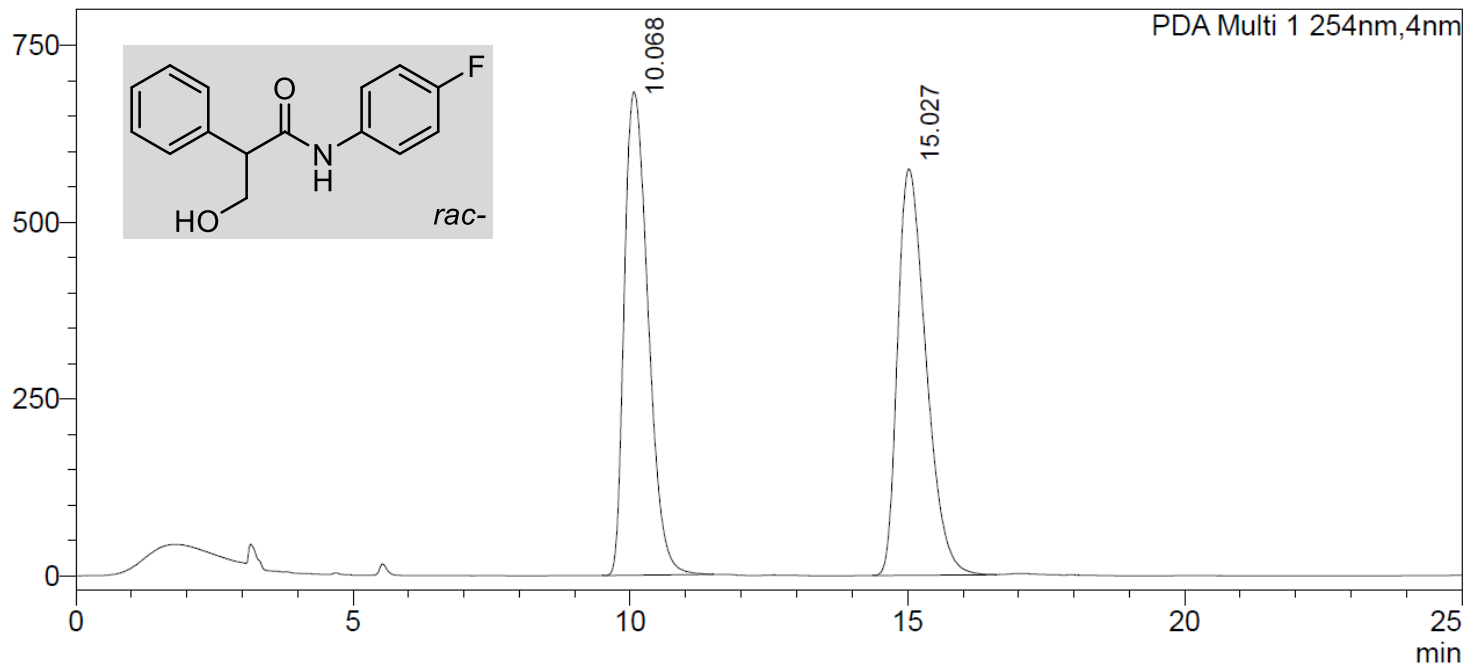

<Peak Table>

PDA Ch1 254nm

\begin{tabular}{|r|c|c|r|}
\hline Peak\# & Ret. Time & Area & \multicolumn{1}{|c|}{ Area\% } \\
\hline 1 & 10.068 & 20412921 & 49.890 \\
\hline 2 & 15.027 & 20502831 & 50.110 \\
\hline Total & & 40915752 & 100.000 \\
\hline
\end{tabular}

\section{<Chromatogram>}

mAU

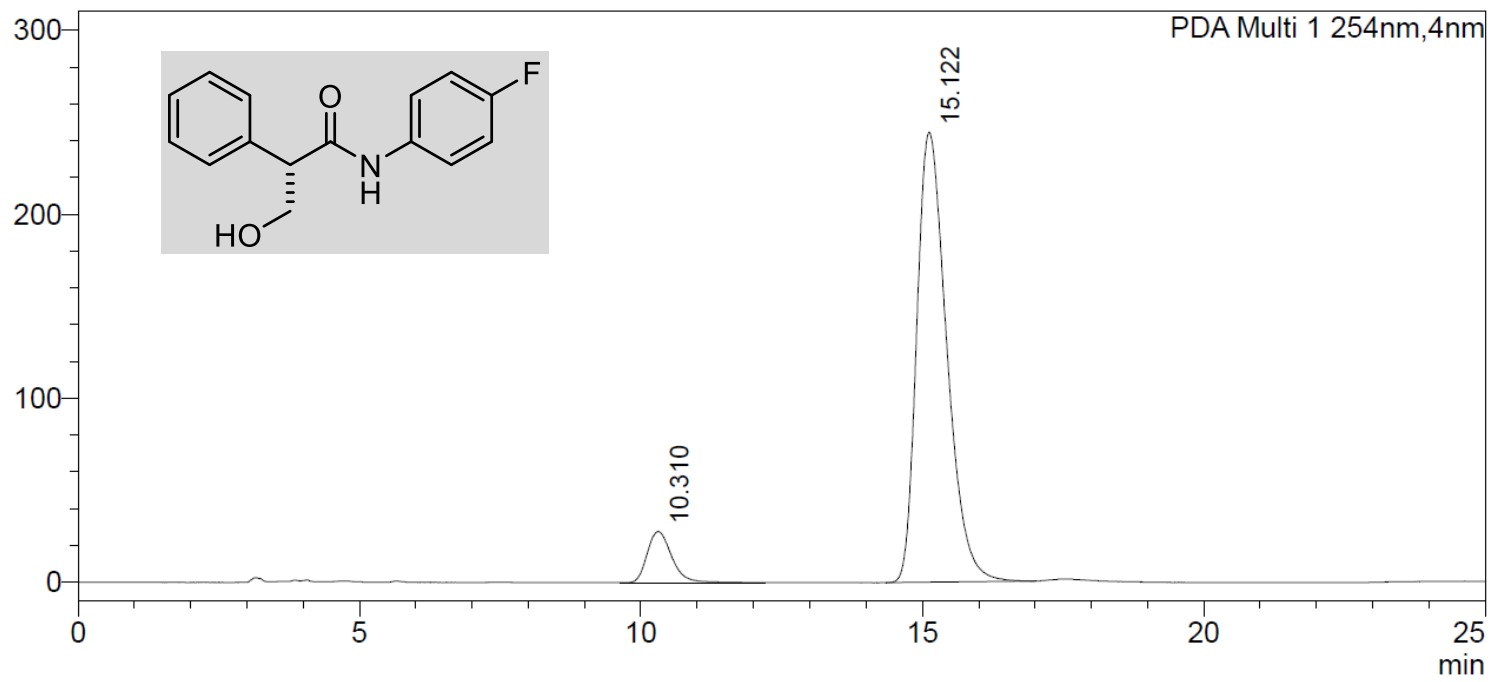

\section{<Peak Table>}

PDA Ch1 254nm

\begin{tabular}{|r|r|r|r|}
\hline Peak\# & Ret. Time & \multicolumn{1}{|c|}{ Area } & Area\% \\
\hline 1 & 10.310 & 848709 & 8.565 \\
\hline 2 & 15.122 & 9059751 & 91.435 \\
\hline Total & & 9908460 & 100.000 \\
\hline
\end{tabular}




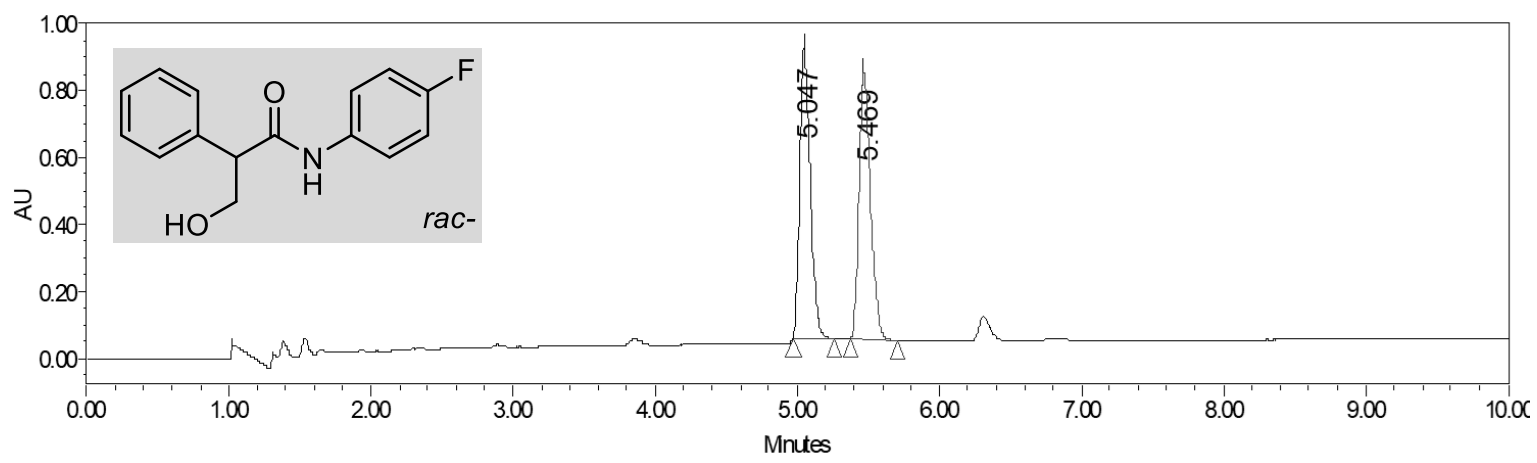

\begin{tabular}{|c|c|c|c|}
\hline & Retention Time & Area & \% Area \\
\hline 1 & 5.047 & 4454648 & 49.57 \\
\hline 2 & 5.469 & 4532450 & 50.43 \\
\hline
\end{tabular}

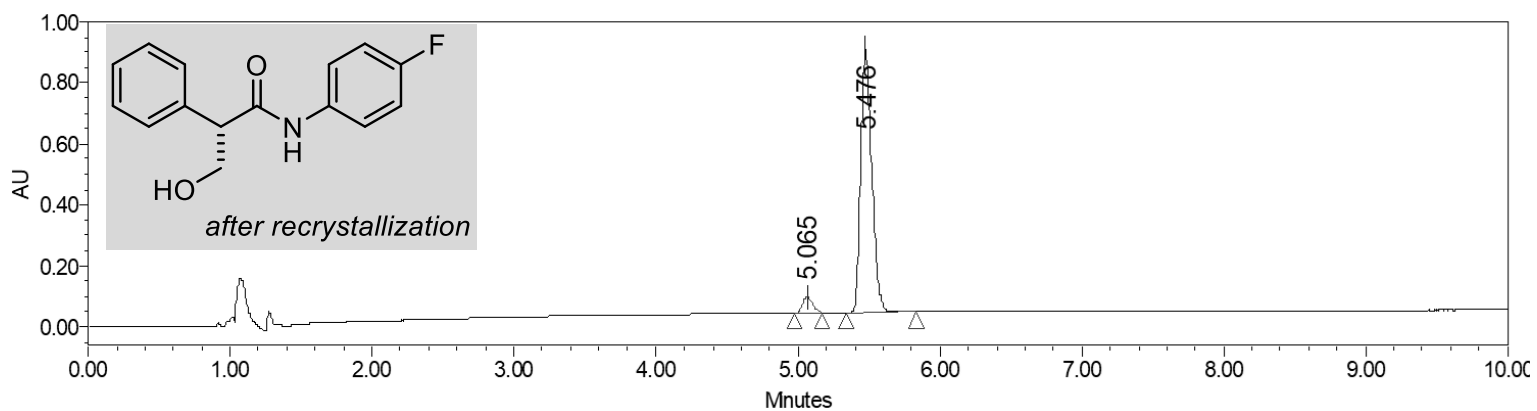

\begin{tabular}{|c|c|c|c|}
\hline & Retention Time & Area & \% Area \\
\hline 1 & 5.065 & 250734 & 5.06 \\
\hline 2 & 5.476 & 4706076 & 94.94 \\
\hline
\end{tabular}


(R)-2-(4-methoxyphenyl)-N-phenyl-3-(4,4,5,5-tetramethyl-1,3,2-dioxaborolan-2yl)propanamide 3 ba

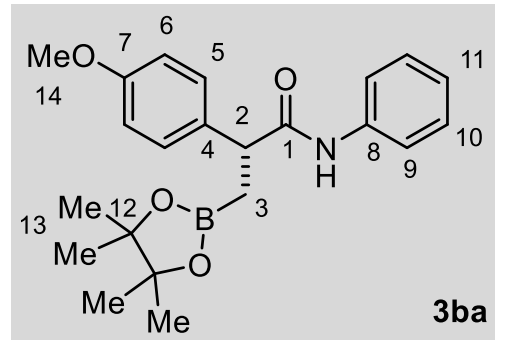

According to the general procedure for enantioselective copper-catalyzed borylative carboxamidation using styrene $\mathbf{1 b}$ (40.2 mg, 0.3 mmol, 1.0 equiv.), $\mathrm{CuCl}$ (3.0 mg, $0.03 \mathrm{mmol}, 10$ mol\%), ligand $(R, R)-6 \mathbf{k}(14 \mathrm{mg}, 0.033 \mathrm{mmol}, 11 \mathrm{~mol} \%)$, LiOtBu (48 mg, $0.6 \mathrm{mmol}, 2.0$ equiv.), $\mathrm{B}_{2} \mathrm{pin}_{2}(114 \mathrm{mg}, 0.45 \mathrm{mmol}, 1.5$ equiv.) and isocyanatobenzene $2 \mathbf{a}(0.132 \mathrm{~mL}, 1.2 \mathrm{mmol}, 4.0$ equiv.) in 2-Me-THF and $\mathrm{Et}_{2} \mathrm{O}$ (3.0 mL, v:v = 1:1, $\left.0.1 \mathrm{M}\right) .80 \%$ conversion into 3 ba determined by ${ }^{1} \mathrm{H}$ NMR analysis of the crude reaction mixture in presence of an internal standard. Purification by flash chromatography (pentane/diethyl ether 3:1) gave pure 3 ba as a white solid (80 mg, $0.21 \mathrm{mmol}, 70 \%$ yield).

TLC: pentane/ethyl acetate $4: 1, R_{f}=0.2$.

${ }^{1} \mathrm{H}$ NMR $\left(400 \mathrm{MHz}, \mathrm{CDCl}_{3}\right) \delta(\mathrm{ppm})=7.40\left(\mathrm{~d},{ }^{3} \mathrm{~J}_{\mathrm{HH}}=7.9 \mathrm{~Hz}, 2 \mathrm{H}\right), 7.28(\mathrm{~m}, 1 \mathrm{H}), 7.23(\mathrm{~m}, 2 \mathrm{H})$, $7.09(\mathrm{~s}, 1 \mathrm{H}, N-H), 7.03(\mathrm{~m}, 1 \mathrm{H}), 6.93-6.82(\mathrm{~m}, 2 \mathrm{H}), 3.86-3.80(\mathrm{~m}, 1 \mathrm{H}, H-2), 3.79(\mathrm{~s}, 1 \mathrm{H}, H-$ 14), $1.63\left(\mathrm{dd},{ }^{2} \mathrm{~J}_{\mathrm{HH}}=15.8,{ }^{3} \mathrm{~J}_{\mathrm{HH}}=9.4 \mathrm{~Hz}, 1 \mathrm{H}, H-3\right), 1.29-1.24(\mathrm{~m}, 1 \mathrm{H}, H-3), 1.20(\mathrm{~s}, 6 \mathrm{H}, H-$ 13), $1.20(\mathrm{~s}, 6 \mathrm{H}, \mathrm{H}-13)$.

${ }^{13} \mathrm{C}\left\{{ }^{1} \mathrm{H}\right\}$ NMR $\left(75 \mathrm{MHz}, \mathrm{CDCl}_{3}\right) \delta(\mathrm{ppm})=172.9(C-1), 158.9(C-7), 138.1(C-8), 133.5(C-4)$, $129.1(C-6), 128.9$ (C-10), 124.0 (C-11), 119.5 (C-9), $114.4(C-5), 83.3(C-12), 55.3(C-14)$, 49.1 (C-2), 24.8 (C-13), $24.7(C-13)$.

${ }^{11} \mathrm{~B}$ NMR $\left(128 \mathrm{MHz}, \mathrm{CDCl}_{3}\right) \delta(\mathrm{ppm})=33.9$.

HRMS (ESI +): calculated for $\mathrm{C}_{22} \mathrm{H}_{28} \mathrm{BNO}_{4}[\mathrm{M}+\mathrm{H}]^{+}:$: 382.2189; found: 382.2147.

IR (neat): $v\left(\mathrm{~cm}^{-1}\right)=3461,3310,3001,1971,1738,1663,1600,1537,1500,1510,1441$, 1366, 1229, 1217, 1143, 1104, 10.34, 968, 889, 847, 756, 693

m.p.: $135-137^{\circ} \mathrm{C}$

HPLC analysis of 3 ba after oxidation to the corresponding alcohol following the general procedure indicated 92.5:7.5 er, chiral stationary column: OD-H, mobile phase: $n$ hexane $/ \mathrm{PrOH}=95: 5,1.0 \mathrm{~mL} / \mathrm{min}, 254 \mathrm{~nm}, 30^{\circ} \mathrm{C}, \mathrm{t}_{\mathrm{R}}$ (major) $=32.7 \mathrm{~min}, \mathrm{t}_{\mathrm{R}}$ (minor) $=18.4 \mathrm{~min}$. $[\alpha]^{20} \mathrm{D}=-49.8\left(c 0.54, \mathrm{CH}_{2} \mathrm{Cl}_{2}\right)$.

Recrystallization: 3ba (60 mg, 92.5:7.5 er) was dissolved in $\mathrm{CH}_{2} \mathrm{Cl}_{2} / \mathrm{Et}_{2} \mathrm{O}(1: 20,6 \mathrm{~mL}$ ) and the solution was concentrated by slow evaporation at $23^{\circ} \mathrm{C}$ until a white crystalline precipitate appeared. The precipitate was filtered, washed with $n$-hexane and dried under vacuum (46 mg, $76 \%$ yield, $99: 1$ er).

SFC analysis of recrystallized $\mathbf{3 b a}$ after oxidation to the corresponding alcohol following the general procedure indicated 99:1 er, chiral stationary column: OB, mobile phase: $2 \%$ to $30 \%$ $\mathrm{MeOH}, 3.0 \mathrm{~mL} / \mathrm{min}, 210 \mathrm{~nm}, 30^{\circ} \mathrm{C}, \mathrm{t}_{\mathrm{R}}$ (major) $=8.0 \mathrm{~min}, \mathrm{t}_{\mathrm{R}}$ (minor) $=6.6 \mathrm{~min}$. 


\section{<Chromatogram>}

mAU

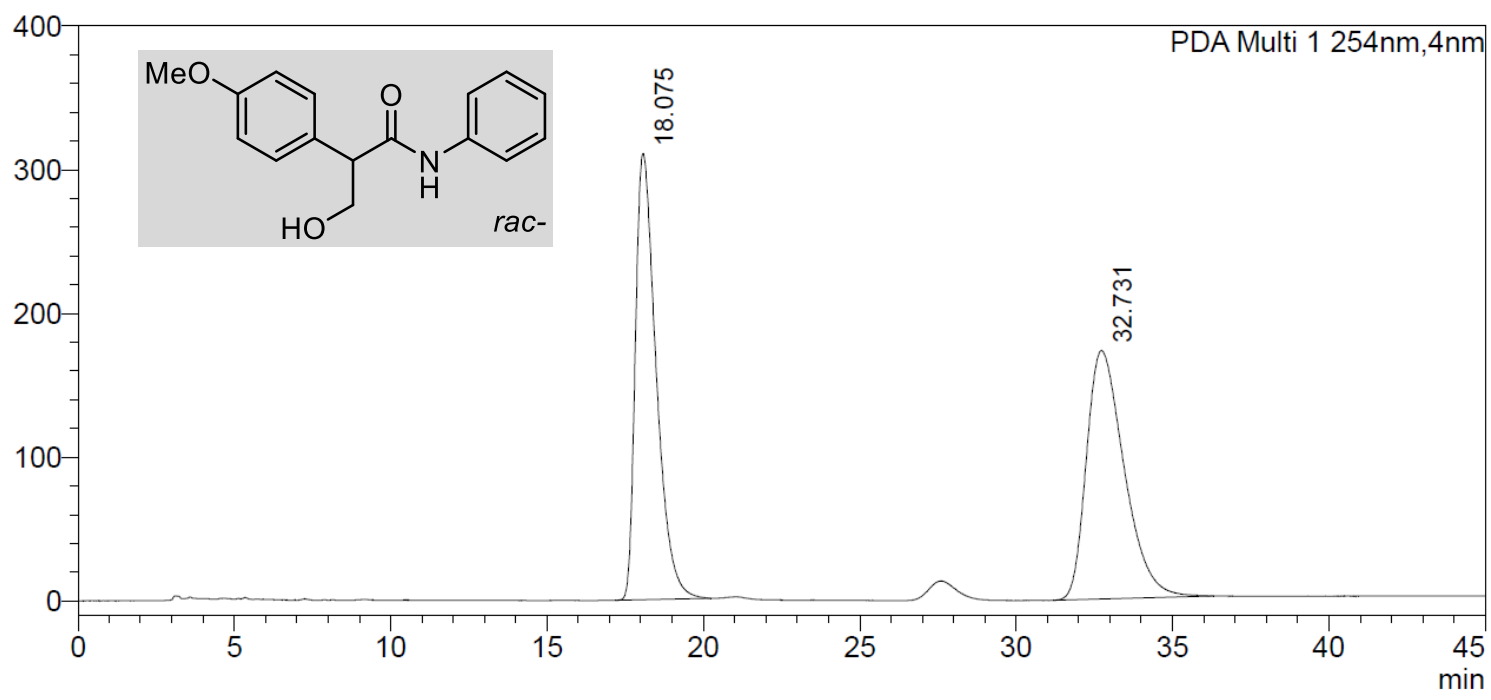

<Peak Table>

PDA Ch1 254nm

\begin{tabular}{|r|r|r|r|}
\hline Peak\# & Ret. Time & \multicolumn{1}{c|}{ Area } & Area\% \\
\hline 1 & 18.075 & 14360320 & 49.687 \\
\hline 2 & 32.731 & 14541434 & 50.313 \\
\hline Total & & 28901754 & 100.000 \\
\hline
\end{tabular}

\section{<Chromatogram>}

mAU

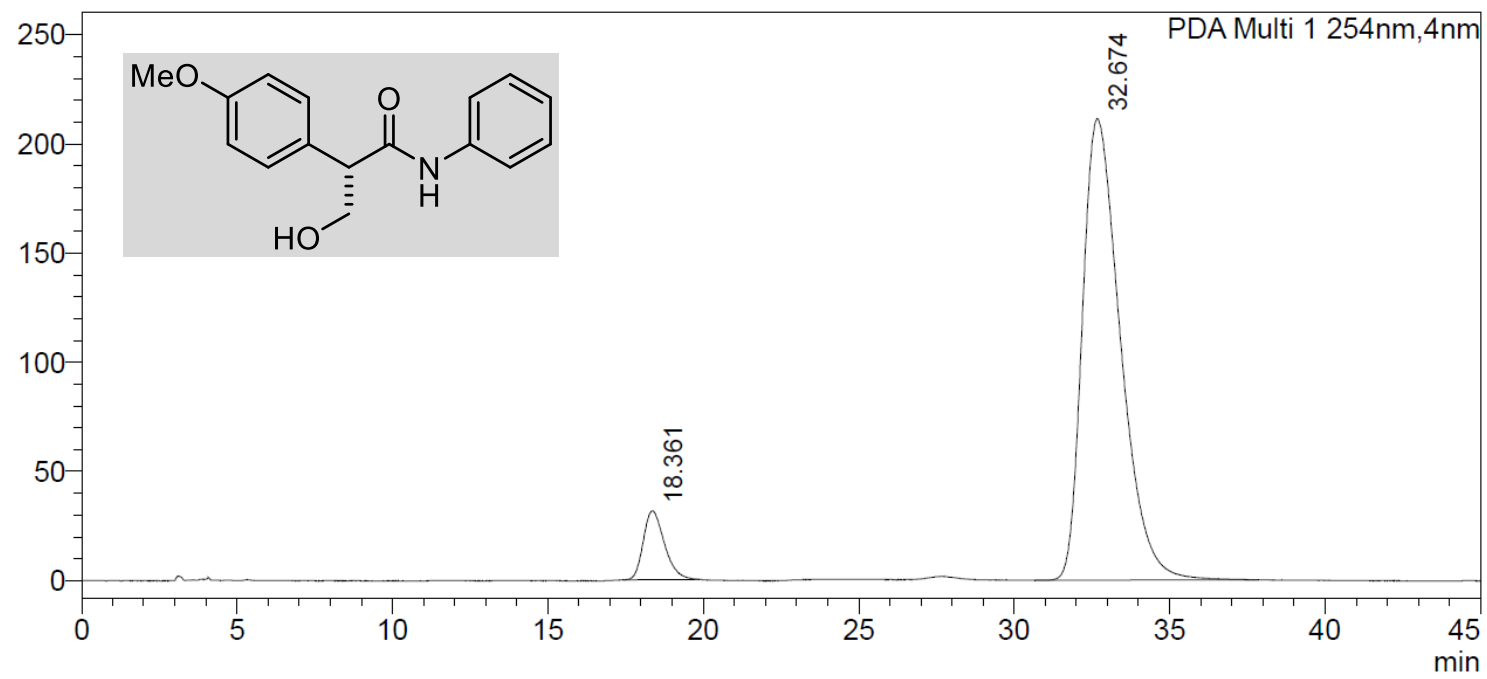

<Peak Table>

PDA Ch1 254nm

\begin{tabular}{|r|r|r|r|}
\hline Peak\# & Ret. Time & \multicolumn{1}{|c|}{ Area } & \multicolumn{1}{|c|}{ Area\% } \\
\hline 1 & 18.361 & 1489497 & 7.655 \\
\hline 2 & 32.674 & 17968343 & 92.345 \\
\hline Total & & 19457840 & 100.000 \\
\hline
\end{tabular}




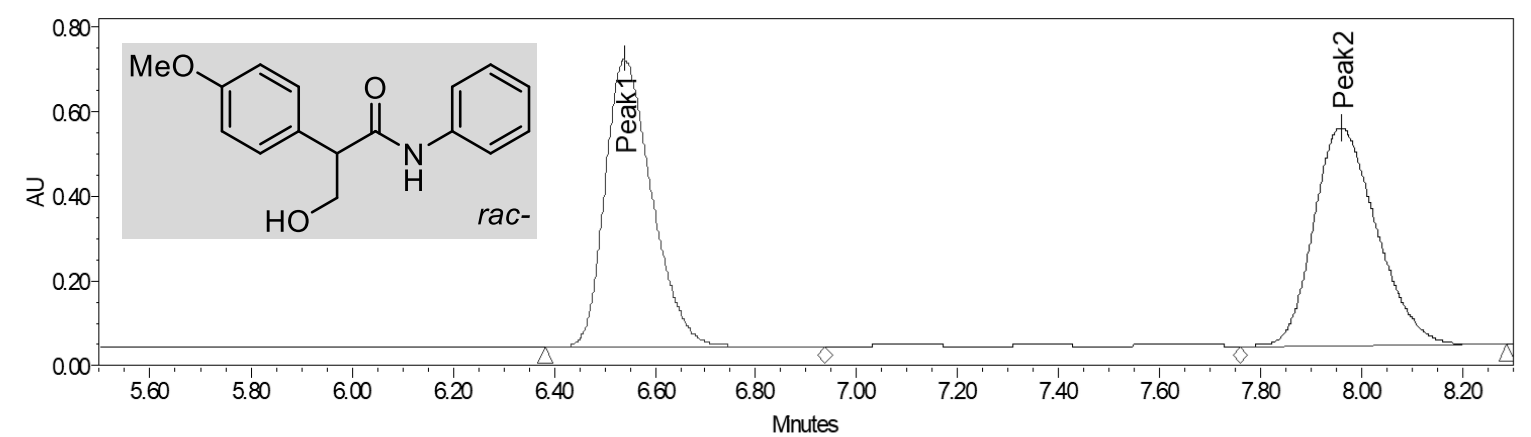

\begin{tabular}{|c|c|c|c|}
\hline & Retention Time & Area & \% Area \\
\hline 1 & 6.540 & 4372833 & 49.59 \\
\hline 2 & 7.960 & 4444739 & 50.41 \\
\hline
\end{tabular}

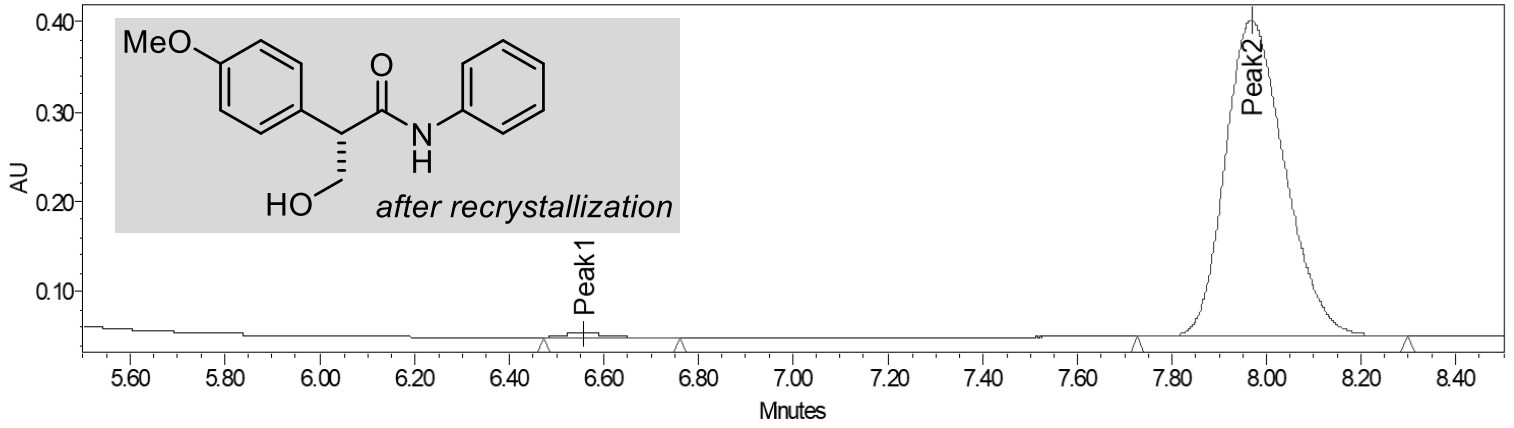

\begin{tabular}{|c|c|c|c|}
\hline & Retention Time & Area & \% Area \\
\hline 1 & 6.557 & 22762 & 0.74 \\
\hline 2 & 7.968 & 3063222 & 99.26 \\
\hline
\end{tabular}


(R)-N,2-bis(4-methoxyphenyl)-3-(4,4,5,5-tetramethyl-1,3,2-dioxaborolan-2-yl)propanamide $3 b b$<smiles>COc1ccc(NC(=O)[C@H](CB2OC(C)(C)C(C)(C)O2)c2ccc(OC)cc2)cc1</smiles>
According to the general procedure for enantioselective copper-catalyzed borylative carboxamidation using 4methoxystyrene $\mathbf{1 b}$ (20 mg, $0.15 \mathrm{mmol}, 1.0$ equiv), $\mathrm{CuCl}$ $(1.5 \mathrm{mg}, 0.015 \mathrm{mmol}, 10 \mathrm{~mol} \%)$, ligand $(R, R)-6 \mathrm{k}(7.0 \mathrm{mg}$, 3bb $0.017 \mathrm{mmol}, 11 \mathrm{~mol} \%$ ), LiOtBu (24 mg, $0.3 \mathrm{mmol}, 2.0$ equiv), $\mathrm{B}_{2}$ pin $_{2}$ (57 mg, $0.23 \mathrm{mmol}, 1.5$ equiv) and 1-isocyanato 4-methoxybenzene $\mathbf{2 b}$ ( 0.80 $\mathrm{mL}, 0.6 \mathrm{mmol}, 4.0$ equiv) in 2-Me-THF and $\mathrm{Et}_{2} \mathrm{O}(1.5 \mathrm{~mL}, \mathrm{v}: \mathrm{v}=1: 1,0.1 \mathrm{M}) .81 \%$ conversion into $3 \mathbf{b b}$ determined by ${ }^{1} \mathrm{H}$ NMR analysis of the crude reaction mixture in presence of an internal standard. Purification by flash chromatography (pentane/diethyl ether 2:1) gave pure 3bb as a white solid ( $43 \mathrm{mg}, 0.11 \mathrm{mmol}, 70 \%$ yield).

HPLC analysis of $\mathbf{3 b b}$ indicated 74:26 er, chiral stationary column: OD-H, mobile phase: $n$ hexane $/ \mathrm{PrOH}=95: 5,1.0 \mathrm{~mL} / \mathrm{min}, 254 \mathrm{~nm}, 30^{\circ} \mathrm{C}, \mathrm{t}_{\mathrm{R}}$ (major) $=26.4 \mathrm{~min}, \mathrm{t}_{\mathrm{R}}$ (minor) $=30.8 \mathrm{~min}$. $[\alpha]^{20} \mathrm{D}=-26.1\left(\mathrm{c} 0.25, \mathrm{CH}_{2} \mathrm{Cl}_{2}\right)$. 


\section{<Chromatogram>}

mAU

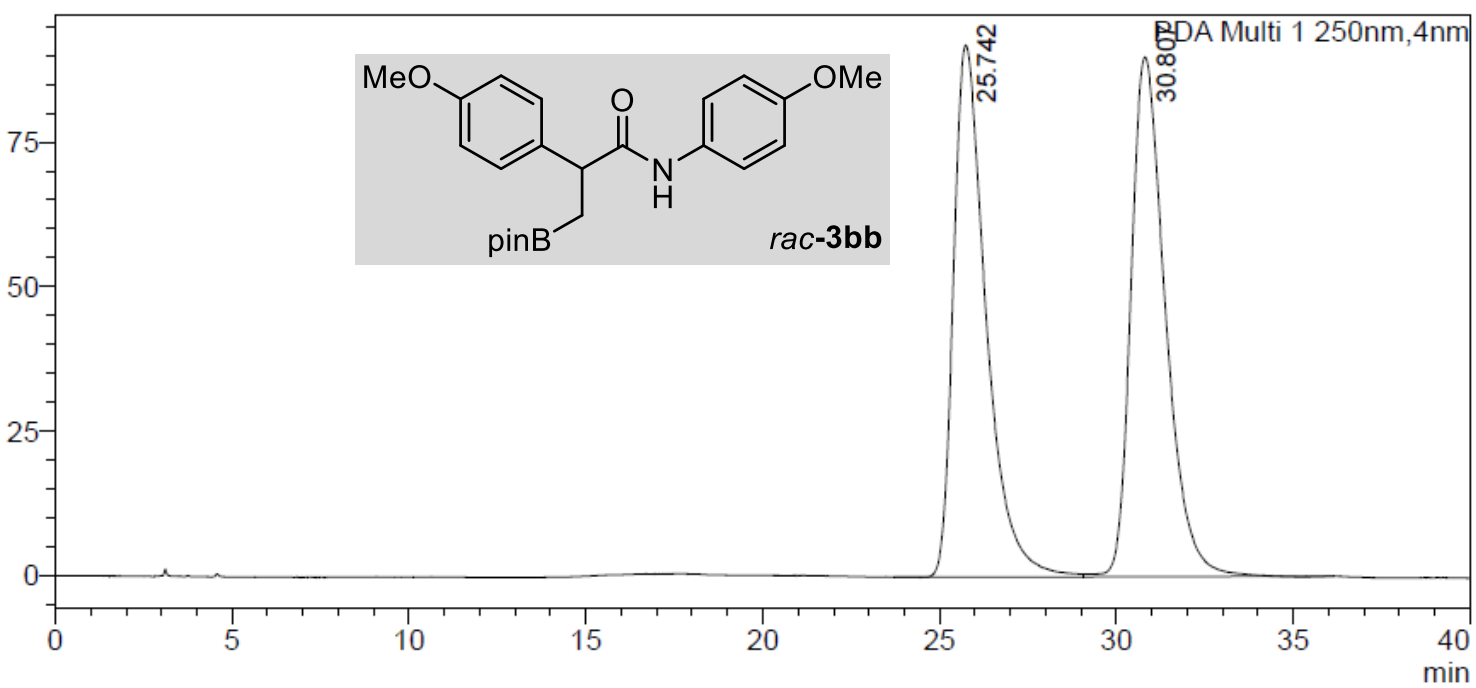

<Peak Table>

\begin{tabular}{|c|c|c|c|}
\hline & \multicolumn{2}{|c|}{$\begin{array}{l}\text { PDA Ch1 250nm } \\
\text { Peak\# Ret. Time }\end{array}$} \\
\hline 1 & 25.742 & 5903779 & 49.262 \\
\hline 2 & 30.807 & 6080788 & 50.738 \\
\hline Total & & 11984567 & 100.000 \\
\hline
\end{tabular}

\section{<Chromatogram>}

mAU

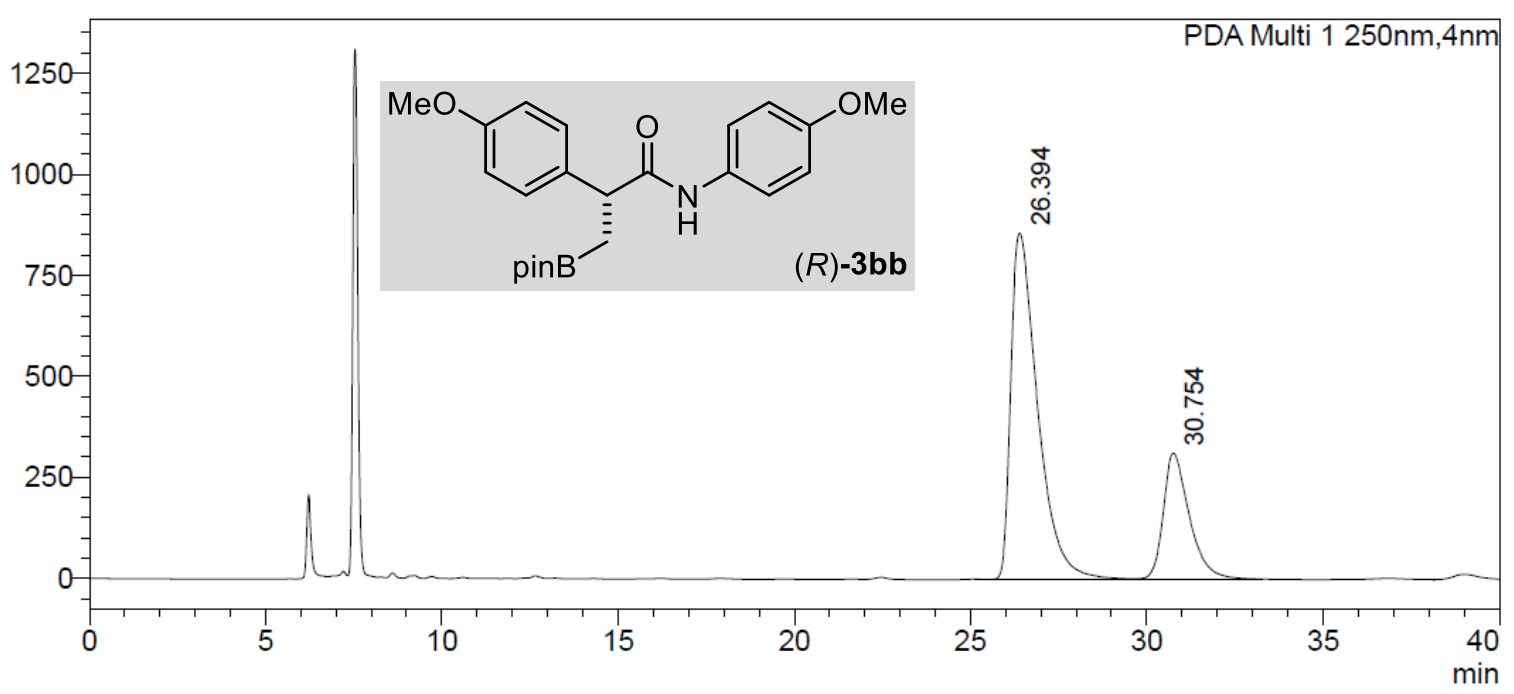

<Peak Table>

PDA Ch1 250nm

\begin{tabular}{|r|r|r|r|}
\hline Peak\# & Ret. Time & \multicolumn{1}{c|}{ Area } & \multicolumn{1}{c|}{ Area\% } \\
\hline 1 & 26.394 & 45264288 & 73.825 \\
\hline 2 & 30.754 & 16048716 & 26.175 \\
\hline Total & & 61313003 & 100.000 \\
\hline
\end{tabular}


(R)-N-(4-(dimethylamino)phenyl)-2-(4-methoxyphenyl)-3-(4,4,5,5-tetramethyl-1,3,2dioxaborolan-2-yl)propanamide 3 bd

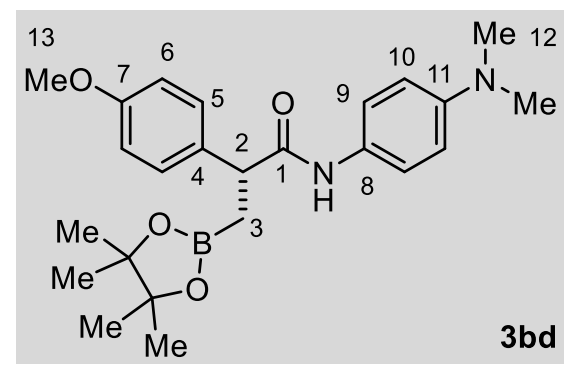

According to the general procedure for enantioselective copper-catalyzed borylative carboxamidation using 4methoxystyrene $1 \mathrm{~b}$ (20 $\mathrm{mg}, 0.15 \mathrm{mmol}, 1.0$ equiv.), $\mathrm{CuCl}$ $(1.5 \mathrm{mg}, 0.015 \mathrm{mmol}, 10 \mathrm{~mol} \%)$, ligand $(R, R)-6 \mathrm{k}(7.0 \mathrm{mg}$, $0.017 \mathrm{mmol}, 11 \mathrm{~mol} \%$ ), LiOtBu (24 mg, $0.3 \mathrm{mmol}, 2.0$ 3bd equiv.), $\mathrm{B}_{2} \mathrm{pin}_{2}$ (57 mg, $0.23 \mathrm{mmol}, 1.5$ equiv.) and 4isocyanato- $\mathrm{N}, \mathrm{N}$-dimethylaniline $\mathbf{2 d}\left(0.97 \mathrm{mg}, 0.60 \mathrm{mmol}, 4.0\right.$ equiv.) in 2-Me-THF and $\mathrm{Et}_{2} \mathrm{O}$ $(1.5 \mathrm{~mL}, \mathrm{v}: \mathrm{v}=1: 1,0.1 \mathrm{M}) .95 \%$ conversion into $3 \mathrm{bd}$ determined by ${ }^{1} \mathrm{H}$ NMR analysis of the crude reaction mixture in presence of an internal standard. Purification by flash chromatography (pentane/ethyl acetate 3:2) gave pure 3bd as a light brown solid (53 mg, 0.12 $\mathrm{mmol}, 83 \%$ yield).

TLC: pentane/ethyl acetate $3: 2, R_{f}=0.4$.

${ }^{1} \mathrm{H}$ NMR $\left(500 \mathrm{MHz}, \mathrm{CDCl}_{3}\right) \delta(\mathrm{ppm})=7.28\left(\mathrm{~d},{ }^{3} \mathrm{~J}_{\mathrm{HH}}=8.7 \mathrm{~Hz}, 2 \mathrm{H}, \mathrm{H}-5\right), 7.25\left(\mathrm{~d},{ }^{3} \mathrm{JHH}_{\mathrm{HH}}=9.1 \mathrm{~Hz}\right.$, $2 \mathrm{H}, H-9), 6.90(\mathrm{bs}, 1 \mathrm{H}, \mathrm{N}-H), 6.87\left(\mathrm{~d},{ }^{3} \mathrm{~J}_{\mathrm{HH}}=8.7 \mathrm{~Hz}, 2 \mathrm{H}, H-6\right), 6.64\left(\mathrm{~d},{ }^{3} \mathrm{~J}_{\mathrm{HH}}=9.1 \mathrm{~Hz}, 2 \mathrm{H}, \mathrm{H}-\right.$ 10), $3.80(\mathrm{~m}, 1 \mathrm{H}, H-2), 3.80(\mathrm{~s}, 3 \mathrm{H}, H-13), 2.88(\mathrm{~s}, 6 \mathrm{H}, H-12), 1.62\left(\mathrm{dd},{ }^{2} J_{H H}=15.7,{ }^{3} J_{H H}=9.5\right.$ $\mathrm{Hz}, 1 \mathrm{H}, H-3), 1.26$ (m, 1H, H-3'), 1.20 (s, 6H, H-MeBpin), 1.19 (s, 6H, H-MeBpin).

${ }^{13} \mathrm{C}\left\{{ }^{1} \mathrm{H}\right\}$ NMR $\left.(126 \mathrm{MHz}, \mathrm{CDCl})_{3}\right) \delta(\mathrm{ppm})=172.6(C-1), 158.9(C-7), 148.0(C-11), 134.1(C-$ 4), 129.2 (CH-5), 128.3 (C-8), 121.4 (CH-9), 114.4 (CH-6), 113.2 (CH-10), 83.3 (CO-Bpin), $55.4\left(\mathrm{CH}_{3}-13\right), 49.0(\mathrm{CH}-2), 41.1\left(\mathrm{CH}_{3}-12\right), 24.9$ ( $\mathrm{CH}_{3}-$ MeBpin), $24.9\left(\mathrm{CH}_{3}-\mathrm{MeBpin}\right), 17.1$ (bs, $\left.\mathrm{CH}_{2}-3\right)$.

${ }^{11}$ B NMR $\left(128 \mathrm{MHz}, \mathrm{CDCl}_{3}\right) \delta(\mathrm{ppm})=32.5$

HRMS (ESI +): calculated for $\mathrm{C}_{21} \mathrm{H}_{33} \mathrm{BN}_{2} \mathrm{O}_{4}[\mathrm{M}+\mathrm{H}]^{+}:$: 425.2611 ; found: 425.2607 .

IR (neat): $v\left(\mathrm{~cm}^{-1}\right)=3411,2979,1658,1610,1511,1370,1320,1247,1140,1104,1034,948$, $845,817$.

m.p.: $160-162^{\circ} \mathrm{C}$

HPLC analysis of $\mathbf{3 b d}$ indicated 86:14 er, chiral stationary column: AS- $\mathrm{H}$, mobile phase: $n$ hexane $/ \mathrm{PrOH}=90: 10,1.0 \mathrm{~mL} / \mathrm{min}, 276 \mathrm{~nm}, 30^{\circ} \mathrm{C}, \mathrm{t}_{\mathrm{R}}=15.7 \mathrm{~min}, \mathrm{t}_{\mathrm{R}}=20.9 \mathrm{~min}$.

$[\alpha]^{20} \mathrm{D}=-36.9\left(c 0.25, \mathrm{CH}_{2} \mathrm{Cl}_{2}\right)$. 


\section{<Chromatogram>}

mAU

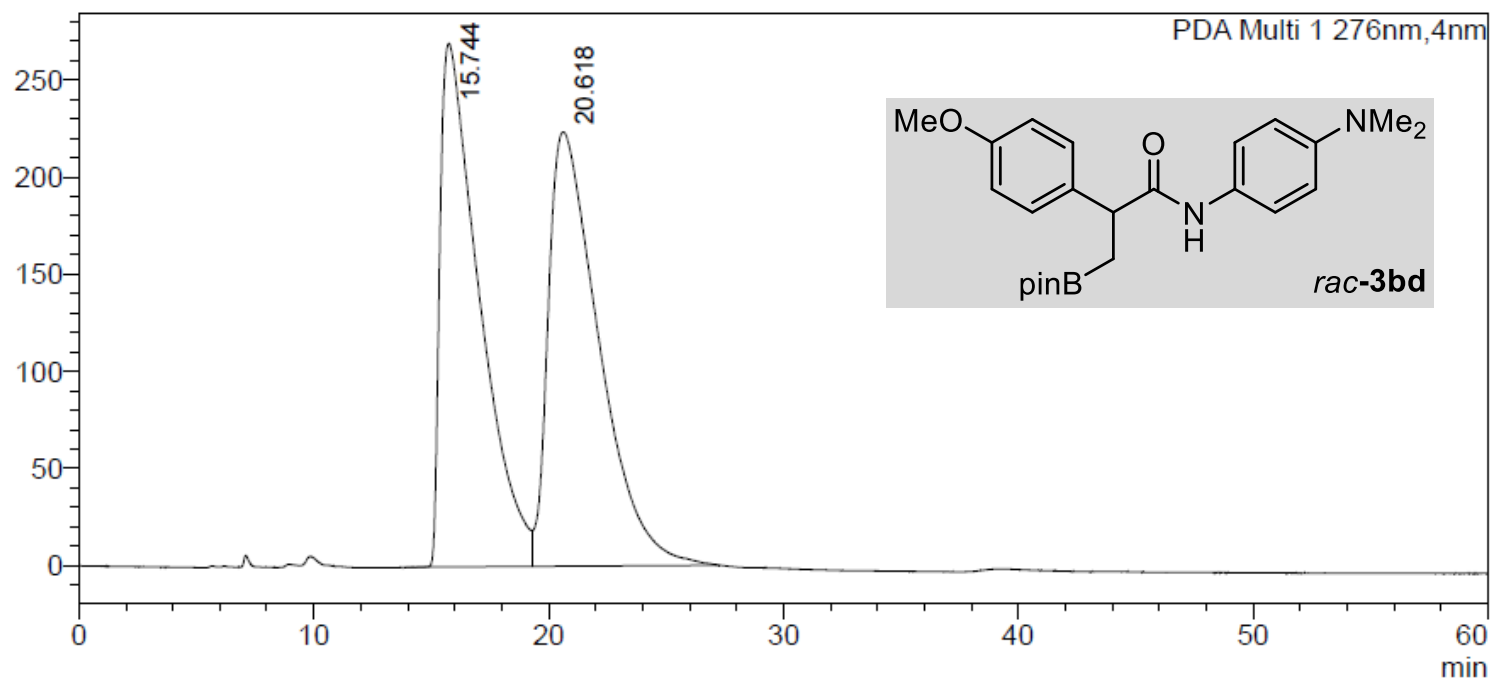

<Peak Table>

PDA Ch1 276nm

\begin{tabular}{|r|r|r|r|}
\hline Peak\# & Ret. Time & Area & \multicolumn{1}{|c|}{ Area $\%$} \\
\hline 1 & 15.744 & 31385761 & 48.315 \\
\hline 2 & 20.618 & 33575218 & 51.685 \\
\hline Total & & 64960979 & 100.000 \\
\hline
\end{tabular}

\section{<Chromatogram>}

$\mathrm{mAU}$

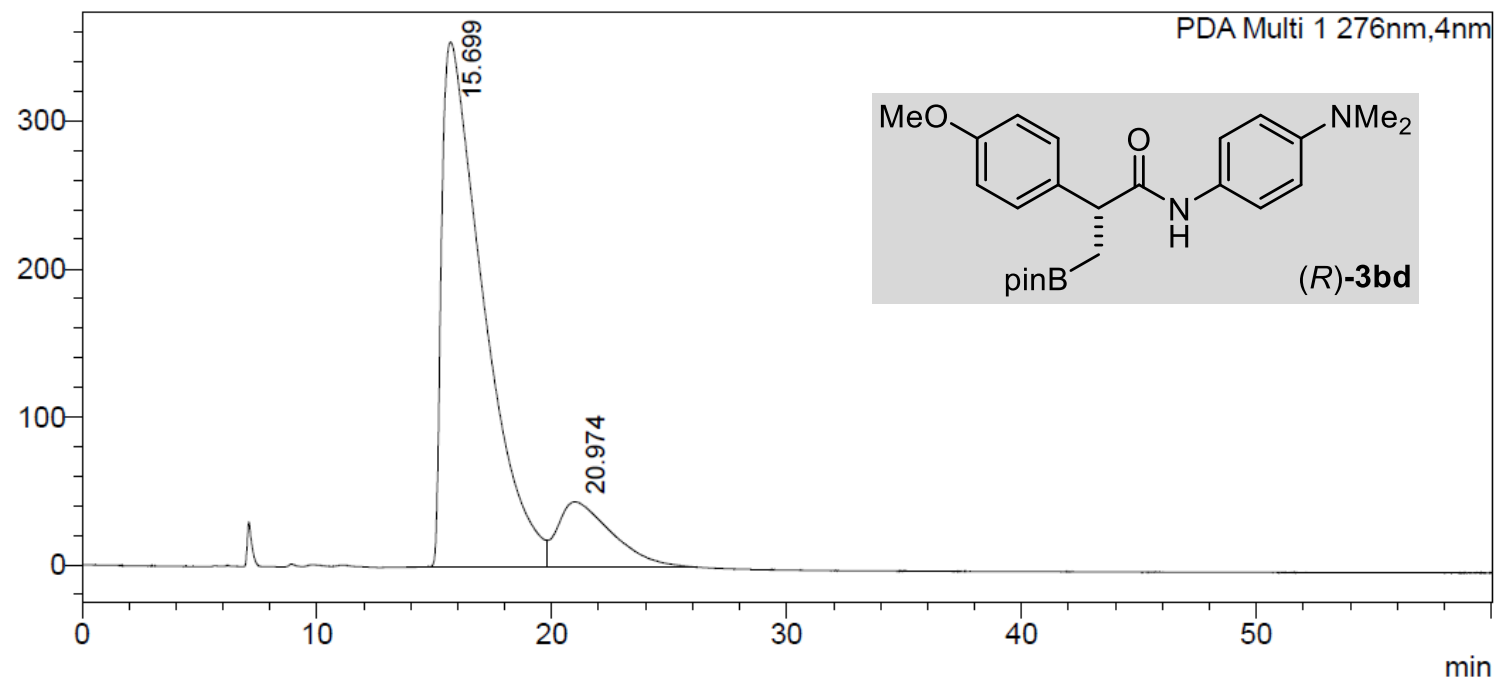

<Peak Table>

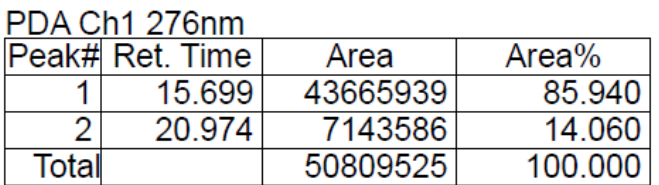


(R)-N-(4-bromophenyl)-2-(4-methoxyphenyl)-3-(4,4,5,5-tetramethyl-1,3,2-dioxaborolan-2yl)propanamide $\mathbf{3 b g}$

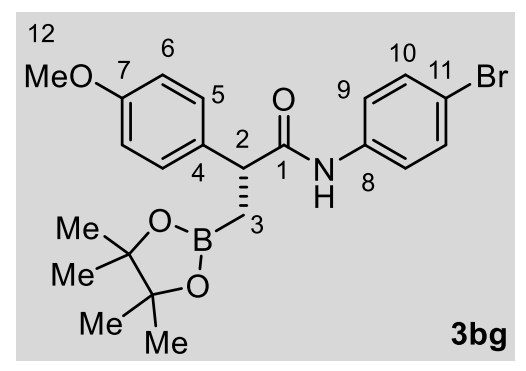

According to the general procedure for enantioselective copper-catalyzed borylative carboxamidation using 4methoxystyrene $1 \mathbf{b}(20 \mathrm{mg}, 0.15 \mathrm{mmol}, 1.0$ equiv.), $\mathrm{CuCl}(1.5$ $\mathrm{mg}, 0.015 \mathrm{mmol}, 10 \mathrm{~mol} \%)$, ligand $(R, R)-6 \mathrm{k}(7.0 \mathrm{mg}, 0.017$ mmol, $11 \mathrm{~mol} \%$ ), LiOtBu (24 mg, 0.3 mmol, 2.0 equiv.), $\mathrm{B}_{2} \mathrm{pin}_{2}$ (57 mg, $0.23 \mathrm{mmol}, 1.5$ equiv.) and 4-isocyanato-1bromobenzene $\mathbf{2 g}\left(0.119 \mathrm{mg}, 0.60 \mathrm{mmol}, 4.0\right.$ equiv.) in 2-Me-THF and $\mathrm{Et}_{2} \mathrm{O}(1.5 \mathrm{~mL}, \mathrm{v}: \mathrm{v}=$ $1: 1,0.1 \mathrm{M}) .80 \%$ conversion into $3 \mathrm{bg}$ determined by ${ }^{1} \mathrm{H}$ NMR analysis of the crude reaction mixture in presence of an internal standard. Purification by flash chromatography (pentane/diethyl ether 7:3) gave pure $\mathbf{3 b g}$ as a white solid (42 $\mathrm{mg}, 0.092 \mathrm{mmol}, 61 \%$ yield).

TLC: pentane/diethyl ether $7: 3, \mathrm{R}_{\mathrm{f}}=0.2$.

${ }^{1} \mathbf{H}$ NMR $\left(400 \mathrm{MHz}, \mathrm{CDCl}_{3}\right) \delta(\mathrm{ppm})=7.36\left(\mathrm{~d},{ }^{3} \mathrm{~J}_{\mathrm{HH}}=9.0 \mathrm{~Hz}, 2 \mathrm{H}, \mathrm{H}-9\right), 7.31\left(\mathrm{~d},{ }^{3} \mathrm{~J}_{\mathrm{HH}}=9.0 \mathrm{~Hz}\right.$, $2 \mathrm{H}, H-10), 7.25\left(\mathrm{~d},{ }^{3} \mathrm{~J}_{\mathrm{HH}}=8.6 \mathrm{~Hz}, 2 \mathrm{H}, H-5\right), 7.07(\mathrm{bs}, 1 \mathrm{H}, \mathrm{N}-H), 6.89\left(\mathrm{~d},{ }^{3} \mathrm{~J}_{\mathrm{HH}}=8.7 \mathrm{~Hz}, 2 \mathrm{H}, \mathrm{H}-\right.$ 6), $3.83(\mathrm{~m}, 1 \mathrm{H}, H-2), 3.80(\mathrm{~s}, 3 \mathrm{H}, H-12), 1.62\left(\mathrm{dd},{ }^{2} J_{H H}=15.7,{ }^{3} J_{H H}=9.7 \mathrm{~Hz}, 1 \mathrm{H}, H-3\right), 1.25$ (m, 1H, H-3'), 1.21 (s, 6H, H-MeBpin), 1.19 (s, 6H, H-MeBpin).

${ }^{13} \mathrm{C}\left\{{ }^{1} \mathrm{H}\right\}$ NMR $\left(101 \mathrm{MHz}, \mathrm{CDCl}_{3}\right) \delta(\mathrm{ppm})=173.0(C-1), 159.1(C-7), 137.3(C-8), 133.4(C-4)$, $131.9(\mathrm{CH}-9), 129.2(\mathrm{CH}-5), 121.1(\mathrm{CH}-10), 116.6$ (C-11), 114.6 (CH-6), 83.5 (CO-Bpin), 55.5 ( $\left.\mathrm{CH}_{3}-12\right)$, 49.2 ( $\left.\mathrm{CH}-2\right), 24.9$ ( $\left.\mathrm{CH}_{3}-\mathrm{MeBpin}\right), 24.8$ ( $\left.\mathrm{CH}_{3}-\mathrm{MeBpin}\right), 16.8$ (bs, $\left.\mathrm{CH}_{2}-3\right)$.

${ }^{11} \mathrm{~B}$ NMR $\left(128 \mathrm{MHz}, \mathrm{CDCl}_{3}\right) \delta(\mathrm{ppm})=33.5$

HRMS (ESI +): calculated for $\mathrm{C}_{22} \mathrm{H}_{27} \mathrm{BN}_{2} \mathrm{O}_{2}[\mathrm{M}+\mathrm{H}]^{+}:$: 460.1294; found: 460.1277 .

IR (neat): $v\left(\mathrm{~cm}^{-1}\right)=3342,2922,1719,1688,1597,1528,1510,1488,1393,1372,1319$, $1254,1138,824$.

m.p.: $177-178^{\circ} \mathrm{C}$

HPLC analysis of $\mathbf{3 b g}$ indicated 97:3 er, chiral stationary column: OD- $\mathrm{H}$, mobile phase: $n$ hexane/iPrOH = 95:5, $1.0 \mathrm{~mL} / \mathrm{min}, 207 \mathrm{~nm}, 30^{\circ} \mathrm{C}, \mathrm{t}_{\mathrm{R}}=19.2 \mathrm{~min}, \mathrm{t}_{\mathrm{R}}=21.2 \mathrm{~min}$.

$[\alpha]^{20} \mathrm{D}=-25.0\left(c 0.35, \mathrm{CH}_{2} \mathrm{Cl}_{2}\right)$. 


\section{<Chromatogram>}

mAU

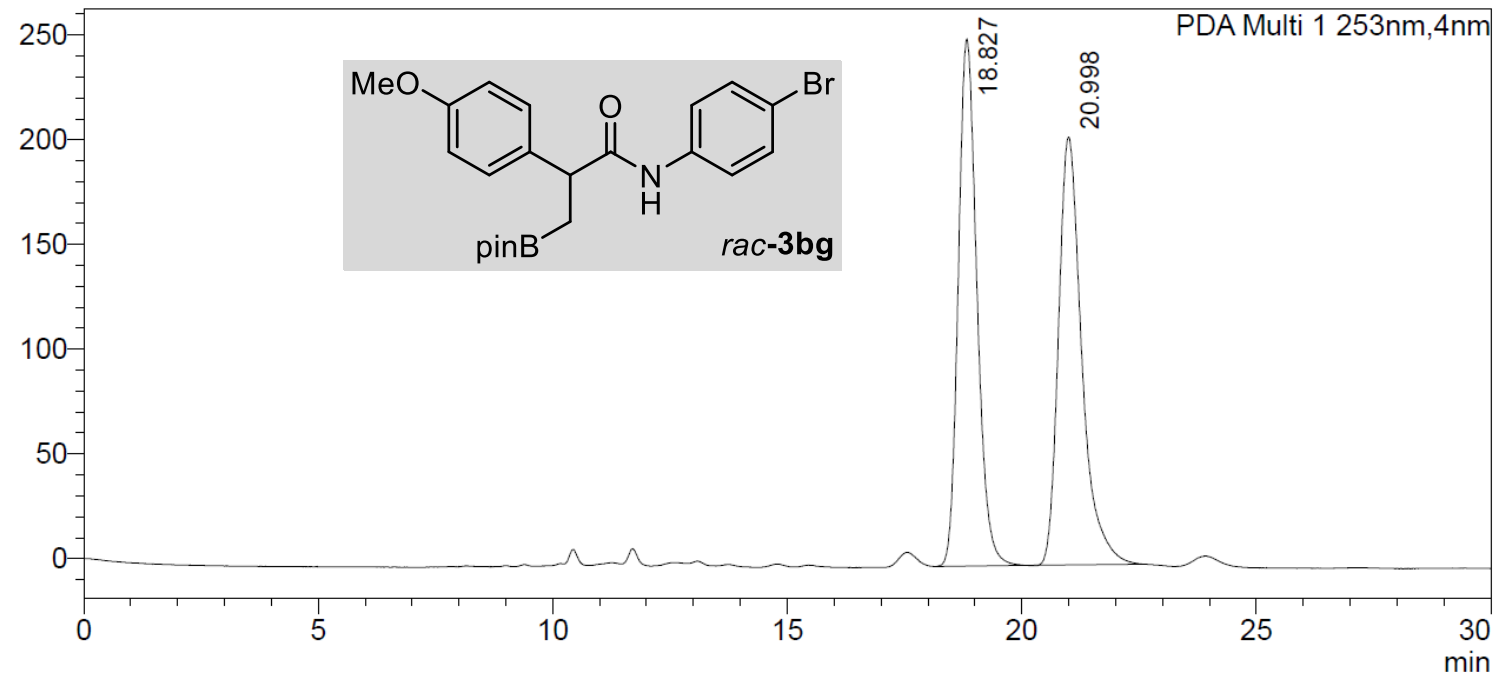

<Peak Table>

PDA Ch1 253nm

\begin{tabular}{|c|c|c|c|}
\hline Peak\# & Ret. Time & Area & Area\% \\
\hline 1 & 18.827 & 6951745 & 49.632 \\
\hline 2 & 20.998 & 7054816 & 50.368 \\
\hline Total & & 14006561 & 100.000 \\
\hline
\end{tabular}

\section{<Chromatogram>}

mAU

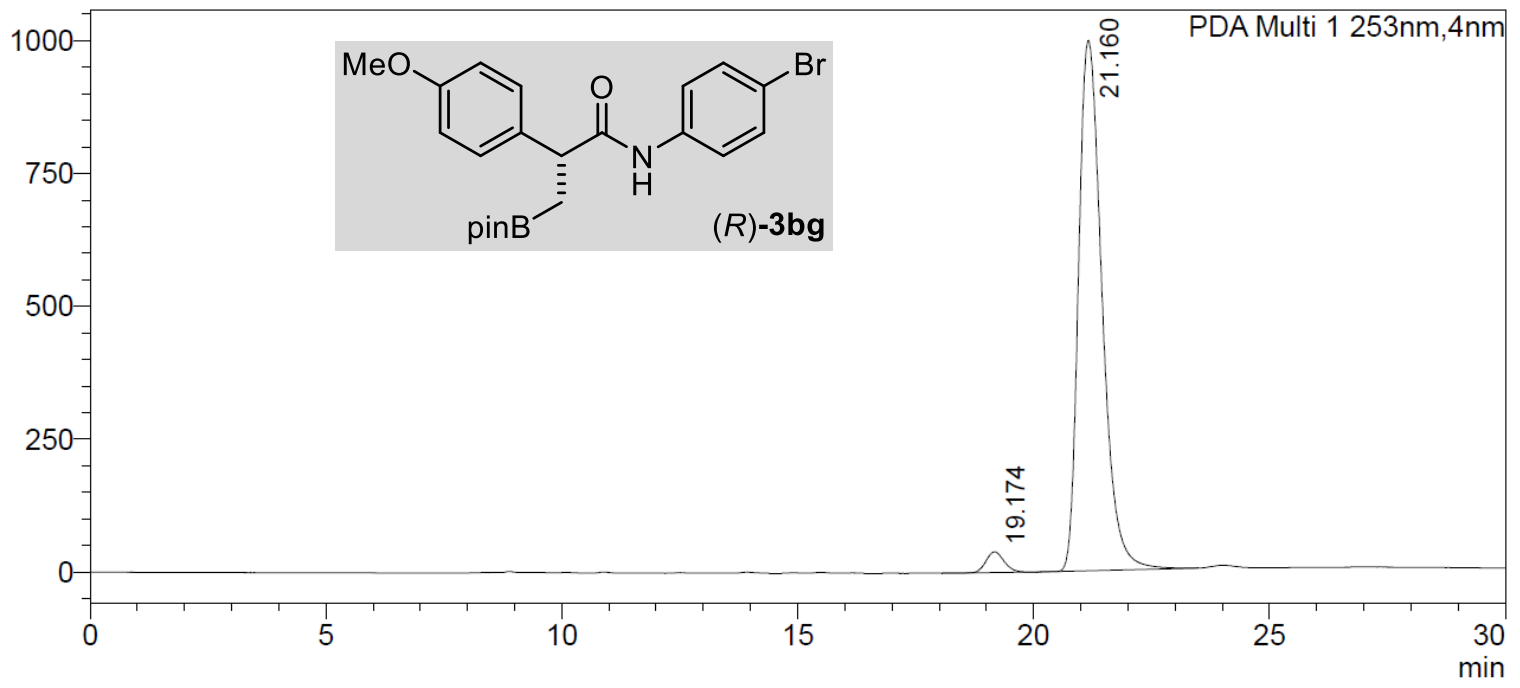

<Peak Table>

\begin{tabular}{|c|c|c|c|}
\hline \multicolumn{4}{|c|}{ PDA Ch1 253nm } \\
\hline Peak\# & Ret. Time & Area & Area\% \\
\hline 1 & 19.174 & 1028717 & 2.893 \\
\hline 2 & 21.160 & 34527201 & 97.107 \\
\hline Total & & 35555919 & 100.000 \\
\hline
\end{tabular}


(R)-2-(4-Methoxyphenyl)-3-(4,4,5,5-tetramethyl-1,3,2-dioxaborolan-2-yl)-N-(ptolyl)propanamide $\mathbf{3 b m}$

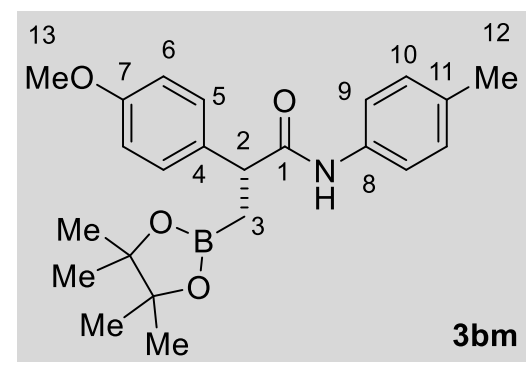

According to the general procedure for enantioselective copper-catalyzed borylative carboxamidation using 4methoxystyrene $\mathbf{1 b}$ (20 mg, $0.15 \mathrm{mmol}, 1.0$ equiv.), $\mathrm{CuCl}(1.5$ $\mathrm{mg}, 0.015 \mathrm{mmol}, 10 \mathrm{~mol} \%)$, ligand $(R, R)-6 \mathrm{k}(7.0 \mathrm{mg}, 0.017$ mmol, $11 \mathrm{~mol} \%$ ), LiOtBu (24 mg, $0.3 \mathrm{mmol}, 2.0$ equiv.), $B_{2}$ pin $_{2}$ (57 mg, $0.23 \mathrm{mmol}, 1.5$ equiv.) and 1-isocyanato-4methylbenzene $2 \mathrm{~m}(0.076 \mathrm{~mL}, 0.60 \mathrm{mmol}, 4.0$ equiv. $)$ in 2-Me-THF and $\mathrm{Et}_{2} \mathrm{O}(1.5 \mathrm{~mL}, \mathrm{v}: \mathrm{v}=$ $1: 1,0.1 \mathrm{M}) .80 \%$ conversion into $3 \mathrm{bm}$ determined by ${ }^{1} \mathrm{H}$ NMR analysis of the crude reaction mixture in presence of an internal standard. Purification by flash chromatography (pentane/diethyl ether $4: 1$ ) gave pure $3 \mathrm{bm}$ as a white solid (30 mg, $0.075 \mathrm{mmol}, 50 \%$ yield).

TLC: pentane/diethyl ether $4: 1, \mathrm{R}_{\mathrm{f}}=0.2$.

${ }^{1} \mathbf{H}$ NMR $\left(400 \mathrm{MHz}, \mathrm{CDCl}_{3}\right) \delta(\mathrm{ppm})=7.29\left(\mathrm{~d},{ }^{3} \mathrm{~J}_{\mathrm{HH}}=8.3 \mathrm{~Hz}, 2 \mathrm{H}, \mathrm{H}-9\right), 7.26\left(\mathrm{~d},{ }^{3} \mathrm{~J}_{\mathrm{HH}}=8.7 \mathrm{~Hz}\right.$, $2 \mathrm{H}, H-5), 7.05\left(\mathrm{~d},{ }^{3} \mathrm{~J}_{\mathrm{HH}}=8.3 \mathrm{~Hz}, 2 \mathrm{H}, \mathrm{H}-10\right), 7.01(\mathrm{bs}, 1 \mathrm{H}, \mathrm{N}-\mathrm{H}), 6.88\left(\mathrm{~d},{ }^{3} \mathrm{~J}_{\mathrm{HH}}=8.6 \mathrm{~Hz}, 2 \mathrm{H}, \mathrm{H}-\right.$ 6), $3.81(\mathrm{~m}, 1 \mathrm{H}, H-2), 3.80(\mathrm{~s}, 3 \mathrm{H}, H-13), 2.27(\mathrm{~s}, 3 \mathrm{H}, H-12), 1.63\left(\mathrm{dd},{ }^{2} J_{\mathrm{HH}}=15.7,{ }^{3} J_{\mathrm{HH}}=9.5\right.$ $\mathrm{Hz}, 1 \mathrm{H}, H-3), 1.26$ (m, 1H, $H$-3'), 1.21 (v), 1.19 (s, 6H, H-MeBpin).

${ }^{13} \mathrm{C}\left\{{ }^{1} \mathrm{H}\right\}$ NMR $\left(101 \mathrm{MHz}, \mathrm{CDCl}_{3}\right) \delta(\mathrm{ppm})=172.8(C-1), 159.0(C-7), 135.7(C-8), 133.8(C-4)$, $129.5(\mathrm{CH}-10), 129.2(\mathrm{CH}-5), 119.7$ ( $\mathrm{CH}-9), 114.5$ ( $\mathrm{CH}-6), 83.4$ (CO-Bpin), $55.4\left(\mathrm{CH}_{3}-13\right)$, 49.2 ( $\mathrm{CH}-2)$, 25.0 ( $\mathrm{CH}_{3}$-MeBpin), $24.9\left(\mathrm{CH}_{3}-\mathrm{MeBpin}\right), 21.0\left(\mathrm{CH}_{3}-12\right)$. The boron-bound carbon $\mathrm{CH}_{2}-3$ was not detected due to quadrupolar relaxation.

${ }^{11}$ B NMR $\left(128 \mathrm{MHz}, \mathrm{CDCl}_{3}\right) \delta(\mathrm{ppm})=33.8$

HRMS (ESI +): calculated for $\mathrm{C}_{23} \mathrm{H}_{30} \mathrm{~B}_{2} \mathrm{NO}_{4}[\mathrm{M}+\mathrm{H}]^{+}$: 396.2345; found: 396.2336.

IR (neat): $v\left(\mathrm{~cm}^{-1}\right)=3343,2923,1684,1600,1510,1463,1371,1319,1251,1179,1138$, 1036, 965, 844, 812.

m.p.: $170-172^{\circ} \mathrm{C}$

HPLC analysis of $\mathbf{3 b m}$ indicated 80:20 er, chiral stationary column: OD-H, mobile phase: $n$ hexane $/ \mathrm{PrOH}=95: 5,1.0 \mathrm{~mL} / \mathrm{min}, 245 \mathrm{~nm}, 30^{\circ} \mathrm{C}, \mathrm{t}_{\mathrm{R}}=15.3 \mathrm{~min}$ (minor), $\mathrm{t}_{\mathrm{R}}=16.9 \mathrm{~min}$ (major). $[\alpha]^{20} \mathrm{D}=-39.8\left(c 0.30, \mathrm{CH}_{2} \mathrm{Cl}_{2}\right)$. 


\section{<Chromatogram>}

mAU

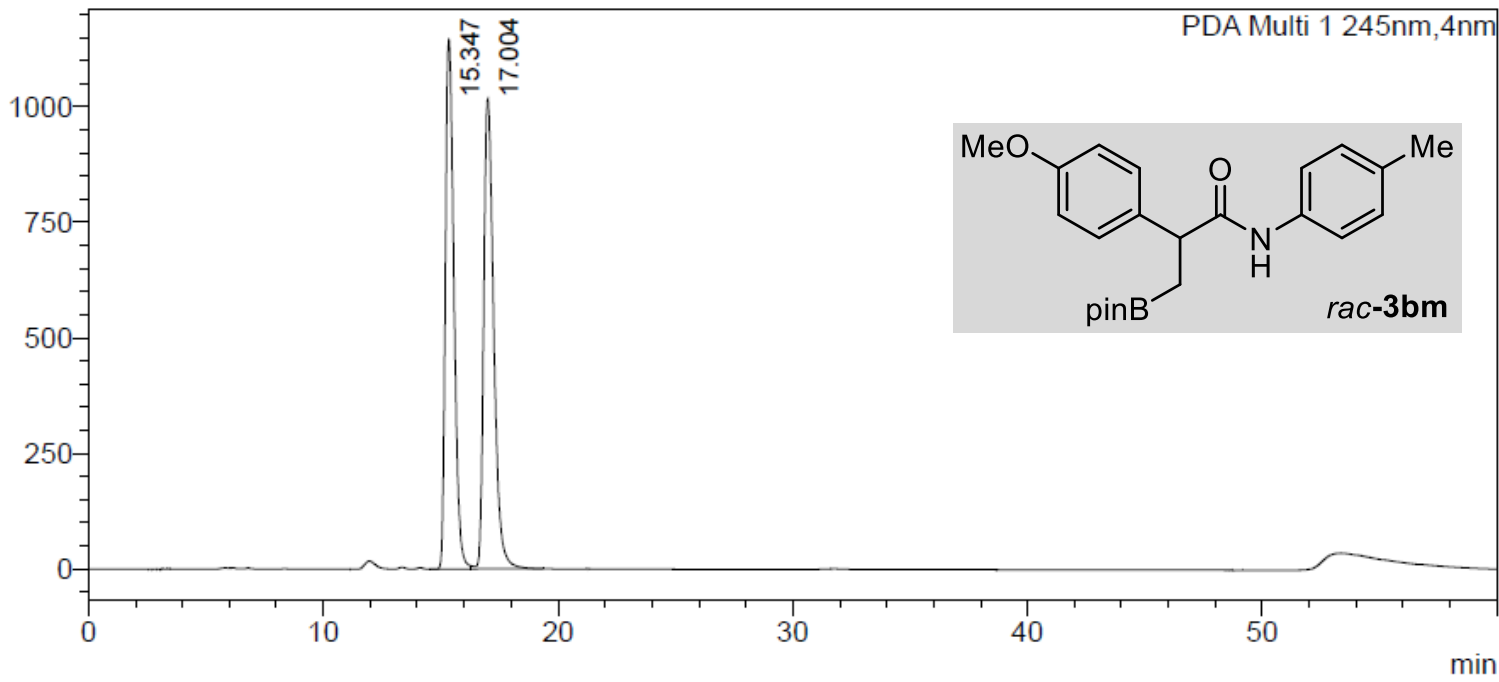

<Peak Table>

PDA Ch1 245nm

\begin{tabular}{|r|r|r|r|}
\hline Peak\# & Ret. Time & Area & Area $\%$ \\
\hline 1 & 15.347 & 29115485 & 49.144 \\
\hline 2 & 17.004 & 30129498 & 50.856 \\
\hline Total & & 59244983 & 100.000 \\
\hline
\end{tabular}

<Chromatogram>

mAU

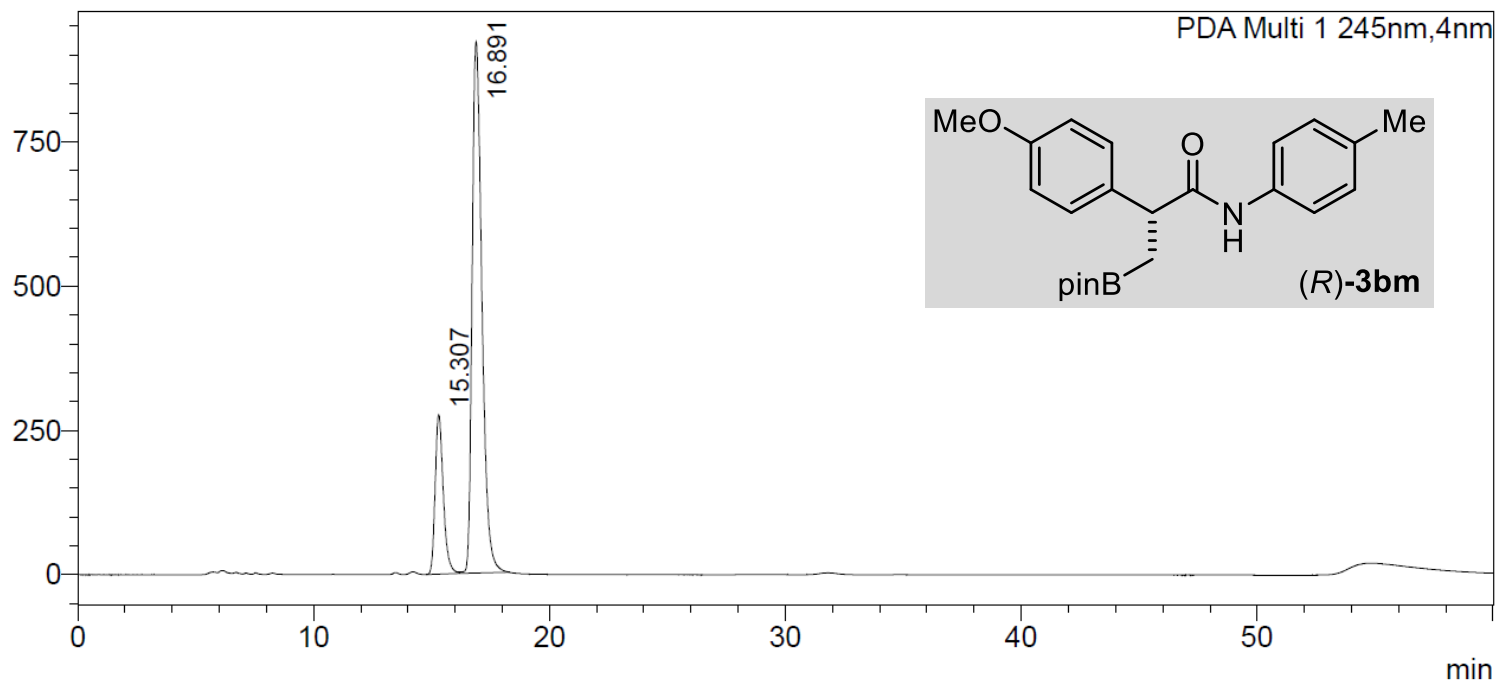

<Peak Table>

PDA Ch1 245nm

\begin{tabular}{|r|r|r|r|}
\hline Peak\# & Ret. Time & \multicolumn{1}{|c|}{ Area } & \multicolumn{1}{c|}{ Area\% } \\
\hline 1 & 15.307 & 6656751 & 20.022 \\
\hline 2 & 16.891 & 26590947 & 79.978 \\
\hline Total & & 33247697 & 100.000 \\
\hline
\end{tabular}


(R)-2-(4-fluorophenyl)-N-phenyl-3-(4,4,5,5-tetramethyl-1,3,2-dioxaborolan-2-yl)propanamide $3 \mathrm{ca}$

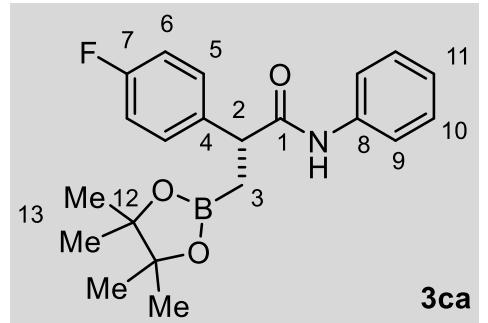

According to the general procedure for enantioselective copper-catalyzed borylative carboxamidation using styrene 1c (36.6 mg, $0.3 \mathrm{mmol}, 1.0$ equiv.), $\mathrm{CuCl}$ ( $3.0 \mathrm{mg}, 0.03 \mathrm{mmol}, 10$ mol\%), ligand $(R, R)-6 \mathbf{k}(14 \mathrm{mg}, 0.033 \mathrm{mmol}, 11 \mathrm{~mol} \%)$, LiOtBu (48 mg, 0.6 mmol, 2.0 equiv.), $B_{2} p i_{2}(114 \mathrm{mg}, 0.45 \mathrm{mmol}, 1.5$ equiv.) and isocyanatobenzene $2 \mathrm{a}(0.132 \mathrm{~mL}, 1.2 \mathrm{mmol}, 4.0$ equiv. $)$ in 2-Me-THF and $\mathrm{Et}_{2} \mathrm{O}$ (3.0 mL, v:v = 1:1, 0.1 M). 90\% conversion into 3ca determined by ${ }^{1} \mathrm{H}$ NMR analysis of the crude reaction mixture in presence of an internal standard. Purification by flash chromatography (pentane/diethyl ether 3:1) gave pure 3ca as a white solid ( $86 \mathrm{mg}, 0.23 \mathrm{mmol}$, $77 \%$ yield).

TLC: pentane/ethyl acetate $4: 1, \mathrm{R}_{\mathrm{f}}=0.4$.

${ }^{1} \mathrm{H}$ NMR $\left(400 \mathrm{MHz}, \mathrm{CDCl}_{3}\right) \delta(\mathrm{ppm})=7.44\left(\mathrm{~d},{ }^{3} \mathrm{JHH}_{\mathrm{HH}}=8.0 \mathrm{~Hz}, 2 \mathrm{H}, \mathrm{H}-10\right), 7.36(\mathrm{~m}, 2 \mathrm{H}), 7.30(\mathrm{~m}$, $2 \mathrm{H}, H-9), 7.18(\mathrm{~s}, 1 \mathrm{H}, \mathrm{N}-H), 7.06(\mathrm{~m}, 3 \mathrm{H}), 3.87\left(\mathrm{dd},{ }^{3} J_{\mathrm{HH}}=9.3,6.7 \mathrm{~Hz}, 1 \mathrm{H}, H-2\right), 1.67\left(\mathrm{dd},{ }^{2} J_{\mathrm{HH}}\right.$ $\left.=16.0,{ }^{3} J_{H H}=9.3 \mathrm{~Hz}, 1 \mathrm{H}, H-3\right), 1.32\left(\mathrm{dd},{ }^{2} J_{\mathrm{HH}}=16.0,{ }^{3} J_{\mathrm{HH}}=6.7 \mathrm{~Hz}, 1 \mathrm{H}, \mathrm{H}-3\right), 1.23(\mathrm{~s}, 6 \mathrm{H}, \mathrm{H}-$ 13), $1.21(\mathrm{~s}, 6 \mathrm{H}, \mathrm{H}-13)$.

${ }^{13} \mathrm{C}\left\{{ }^{1} \mathrm{H}\right\}$ NMR $\left(101 \mathrm{MHz}, \mathrm{CDCl}_{3}\right) \delta(\mathrm{ppm})=172.2(C-1), 162.1\left({ }^{1} \mathrm{JFC}_{\mathrm{FC}}=250 \mathrm{~Hz}, C-7\right), 138.0(C-$ 8), $137.3\left({ }^{4} J_{\mathrm{FC}}=4 \mathrm{~Hz}, C-4\right), 129.6\left({ }^{2} J_{\mathrm{FC}}=10 \mathrm{~Hz}, C-6\right), 128.9(C-10), 124.1(C-11), 119.5(C-$ 9), $115.8\left({ }^{3} J_{\mathrm{FC}}=20 \mathrm{~Hz}, C-5\right), 83.4(C-12), 49.1(C-2), 24.8(C-13), 24.7(C-13), 17.0(C-3)$.

${ }^{11} \mathbf{B}$ NMR $\left(128 \mathrm{MHz}, \mathrm{CDCl}_{3}\right) \delta(\mathrm{ppm})=33.7$.

${ }^{19} \mathrm{~F}$ NMR $\left(282 \mathrm{MHz}, \mathrm{CDCl}_{3}\right) \delta(\mathrm{ppm})=-115.2$.

HRMS (ESI +): calculated for $\mathrm{C}_{21} \mathrm{H}_{25} \mathrm{BFNO}_{3}[\mathrm{M}+\mathrm{H}]^{+}: 370.1989$; found: 370.1976 .

IR (neat): $v\left(\mathrm{~cm}^{-1}\right)=3299,2979,2933,1659,1601,1541,1507,1442,1370.1322,1225$, 1142, 1109, 1093, 1016, 968, 889, 846, 755, 693.

m.p.: $139-141^{\circ} \mathrm{C}$

HPLC analysis of 3ca after oxidation to the corresponding alcohol following the general procedure indicated 83.5:16.5 er, chiral stationary column: OD-H, mobile phase: $n$ hexane $/ \mathrm{PrOH}=95 / 5,1.0 \mathrm{~mL} / \mathrm{min}, 254 \mathrm{~nm}, 30^{\circ} \mathrm{C}, \mathrm{t}_{\mathrm{R}}$ (major) $=23.2 \mathrm{~min}, \mathrm{t}_{\mathrm{R}}$ (minor) $=13.1 \mathrm{~min}$. $[\alpha]^{20} \mathrm{D}=-50.5\left(c 0.17, \mathrm{CH}_{2} \mathrm{Cl}_{2}\right)$ for the corresponding alcohol. 


\section{$<$ Chromatogram $>$}

mAU

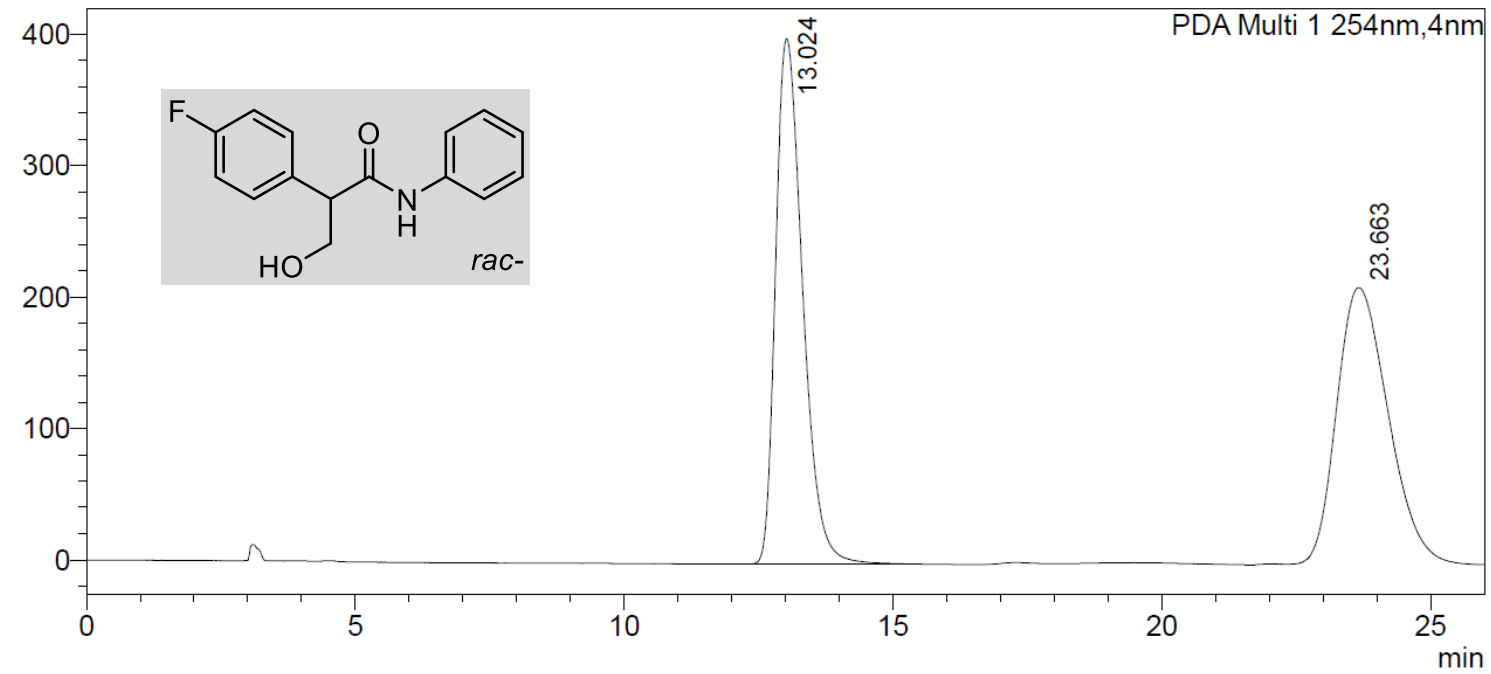

<Peak Table>

PDA Ch1 254nm

\begin{tabular}{|r|r|r|r|}
\hline Peak\# & Ret. Time & Area & \multicolumn{1}{c|}{ Area\% } \\
\hline 1 & 13.024 & 13998354 & 49.873 \\
\hline 2 & 23.663 & 14069520 & 50.127 \\
\hline Total & & 28067874 & 100.000 \\
\hline
\end{tabular}

<Chromatogram>

mAU

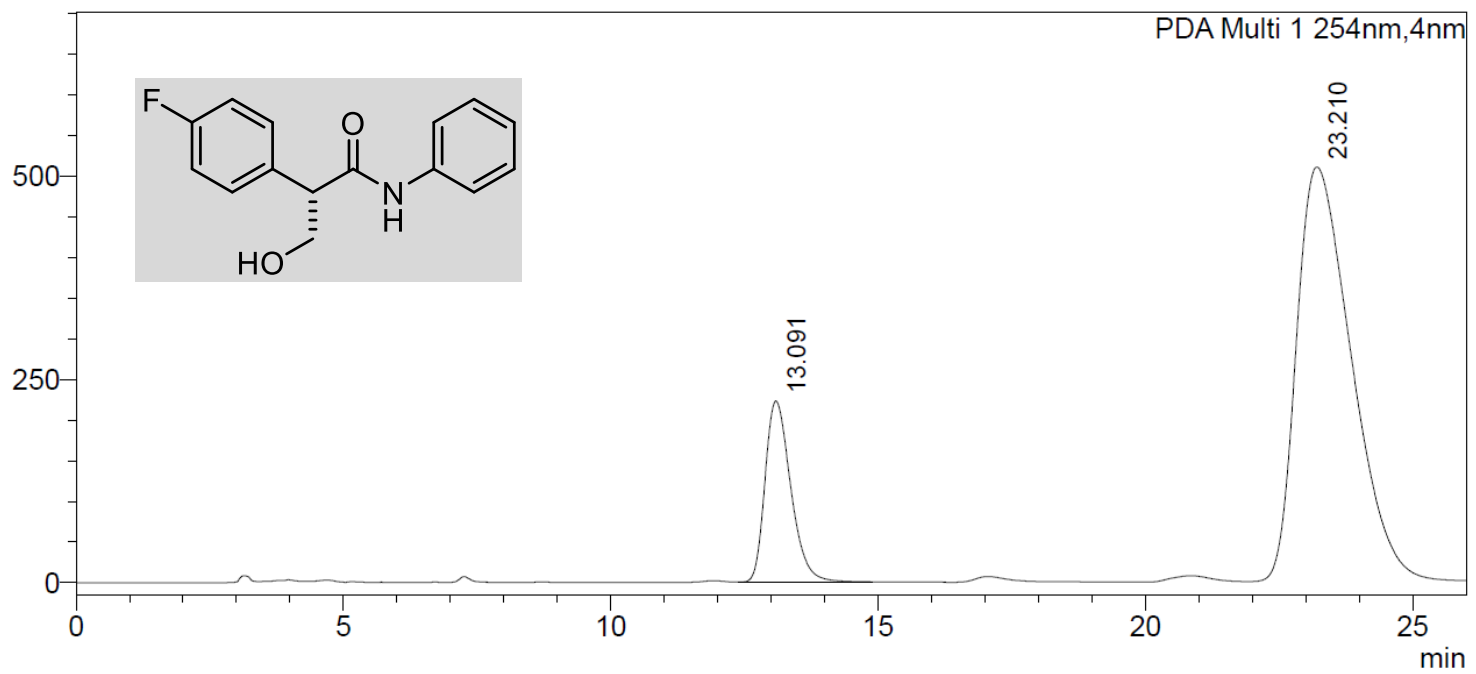

<Peak Table>

PDA Ch1 254nm

\begin{tabular}{|r|r|r|r|}
\hline Peak\# & Ret. Time & \multicolumn{1}{c|}{ Area } & \multicolumn{1}{c|}{ Area\% } \\
\hline 1 & 13.091 & 7450378 & 17.081 \\
\hline 2 & 23.210 & 36166905 & 82.919 \\
\hline Total & & 43617283 & 100.000 \\
\hline
\end{tabular}


(R)-N-phenyl-3-(4,4,5,5-tetramethyl-1,3,2-dioxaborolan-2-yl)-2-(o-tolyl)propanamide 3ha

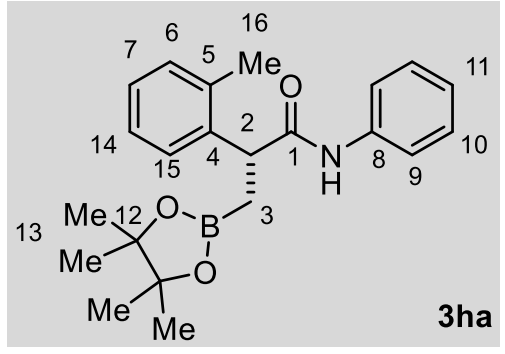
According to the general procedure for enantioselective copper-catalyzed borylative carboxamidation using styrene $\mathbf{1 h}$ (36 mg, $0.3 \mathrm{mmol}, 1.0$ equiv.), $\mathrm{CuCl}$ (3.0 mg, $0.03 \mathrm{mmol}, 10$ mol\%), ligand $(R, R)-6 \mathrm{k}(14 \mathrm{mg}, 0.033 \mathrm{mmol}, 11 \mathrm{~mol} \%)$, LiOtBu (48 mg, $0.6 \mathrm{mmol}, 2.0$ equiv.), $\mathrm{B}_{2} \mathrm{pin}_{2}(114 \mathrm{mg}, 0.45 \mathrm{mmol}, 1.5$ equiv.) and isocyanatobenzene $2 \mathrm{a}(0.132 \mathrm{~mL}, 1.2 \mathrm{mmol}, 4.0$ equiv.) in 2-Me-THF and $\mathrm{Et}_{2} \mathrm{O}(3.0 \mathrm{~mL}, \mathrm{v}: \mathrm{v}=1: 1,0.1 \mathrm{M}) .80 \%$ conversion into 3ha determined by ${ }^{1} \mathrm{H}$ NMR analysis of the crude reaction mixture in presence of an internal standard. Purification by flash chromatography (pentane/diethyl ether 3:1) gave pure 3ha as a white solid (65 mg, $0.18 \mathrm{mmol}, 60 \%$ yield).

TLC: pentane/ethyl acetate $4: 1, R_{f}=0.5$.

${ }^{1} \mathrm{H}$ NMR $\left(400 \mathrm{MHz}, \mathrm{CDCl}_{3}\right) \delta(\mathrm{ppm})=7.43(\mathrm{~m}, 2 \mathrm{H}), 7.36(\mathrm{~m}, 1 \mathrm{H}), 7.26(\mathrm{~m}, 5 \mathrm{H}), 7.07(\mathrm{~m}, 1 \mathrm{H})$, $7.02(\mathrm{~s}, 1 \mathrm{H}, \mathrm{N}-H), 4.13\left(\mathrm{dd},{ }^{3} \mathrm{~J}_{\mathrm{HH}}=9.1,7.0 \mathrm{~Hz}, 1 \mathrm{H}, H-2\right), 2.44(\mathrm{~s}, 3 \mathrm{H}, H-16), 1.69$ (dd, ${ }^{2} J_{\mathrm{HH}}=$ $\left.15.7,{ }^{3} J_{H H}=9.1 \mathrm{~Hz}, 1 \mathrm{H}, H-3\right), 1.31\left(\mathrm{dd},{ }^{2} J_{\mathrm{HH}}=15.7,{ }^{3} J_{\mathrm{HH}}=7.0 \mathrm{~Hz}, 1 \mathrm{H}, H-3\right), 1.21(\mathrm{~s}, 12 \mathrm{H}, H-$ 13).

${ }^{13} \mathrm{C}\left\{{ }^{1} \mathrm{H}\right\}$ NMR $(101 \mathrm{MHz}, \mathrm{CDCl})_{3} \delta(\mathrm{ppm})=172.9(C-1), 139.7(C-4), 138.2(C-8), 136.4(C-5)$, $131.1(C-6), 129.0$ (C-10), 128.0 (C-15), 127.5 (C-7), 127.0 (C-14), 124.1 (C-11), $119.7(C-9)$, 83.4 (C-12), 46.6 (C-2), 24.9 (C-13), 24.8 (C-13), 19.9 (C-16), $15.9(C-3)$.

${ }^{11} \mathbf{B}$ NMR $\left(128 \mathrm{MHz}, \mathrm{CDCl}_{3}\right) \delta(\mathrm{ppm})=34.0$.

HRMS (ESI +): calculated for $\mathrm{C}_{22} \mathrm{H}_{28} \mathrm{BNO}_{3}[\mathrm{M}+\mathrm{H}]^{+}: 366.2240$; found: 366.2254.

IR (neat): $v\left(\mathrm{~cm}^{-1}\right)=3463,3323,3017,3001,2971,2947,1739,1599,1524,1499,1440$, 1366, 1229, 1217, 1143, 1093, 968, 889, 847, 754, 693.

m.p.: $133-135^{\circ} \mathrm{C}$

HPLC analysis of 3 ha after being oxidized to the corresponding alcohol by $\mathrm{H}_{2} \mathrm{O}_{2} / \mathrm{NaOH}$ indicated 85:15 er, chiral stationary column: AD-H, mobile phase: $n$-hexane/iPrOH $=95: 5,1.0$ $\mathrm{mL} / \mathrm{min}, 254 \mathrm{~nm}, 30^{\circ} \mathrm{C}, \mathrm{t}_{\mathrm{R}}$ (major) $=24.4 \mathrm{~min}, \mathrm{t}_{\mathrm{R}}($ minor $)=20.0 \mathrm{~min}$.

$[\alpha]^{20} \mathrm{D}=-29.7\left(c 0.20, \mathrm{CH}_{2} \mathrm{Cl}_{2}\right)$. 


\section{$<$ Chromatogram $>$}

$\mathrm{mAU}$

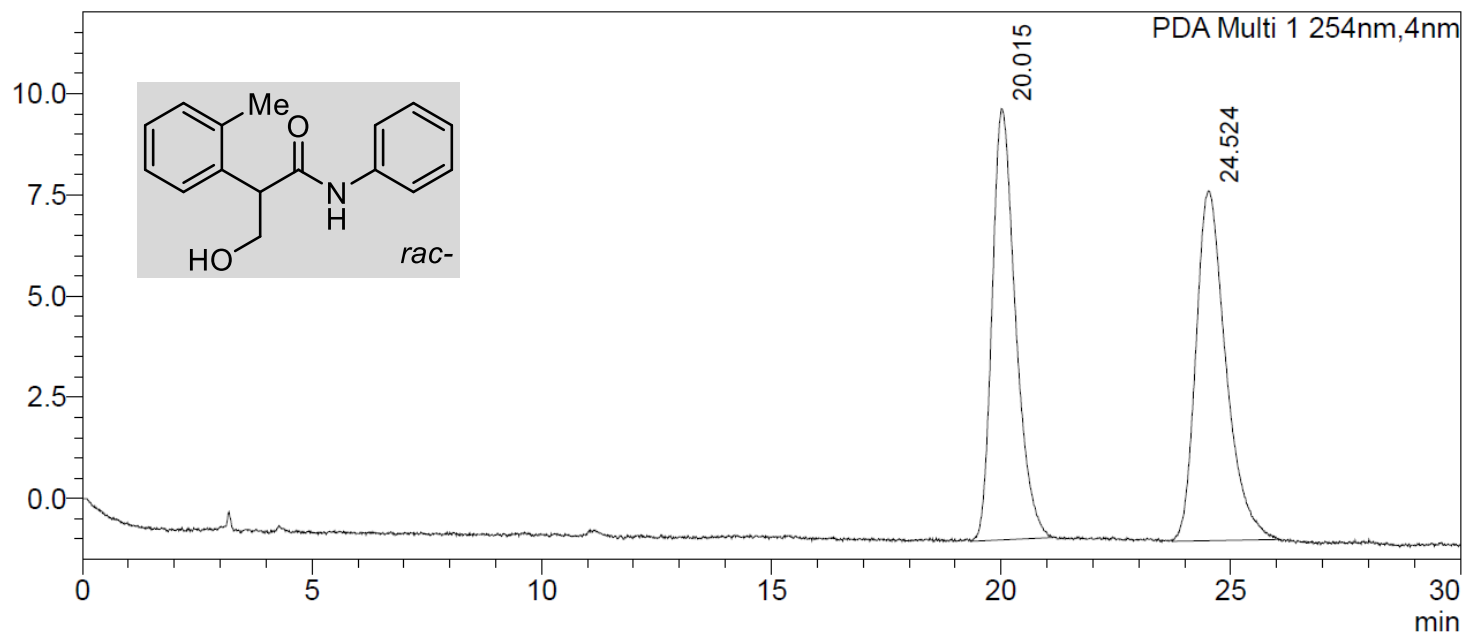

<Peak Table>

PDA Ch1 254nm

\begin{tabular}{|r|r|r|r|}
\hline Peak\# & Ret. Time & \multicolumn{1}{|c|}{ Area } & \multicolumn{1}{|c|}{ Area\% } \\
\hline 1 & 20.015 & 376488 & 49.026 \\
\hline 2 & 24.524 & 391451 & 50.974 \\
\hline Total & & 767939 & 100.000 \\
\hline
\end{tabular}

\section{<Chromatogram>}

mAU

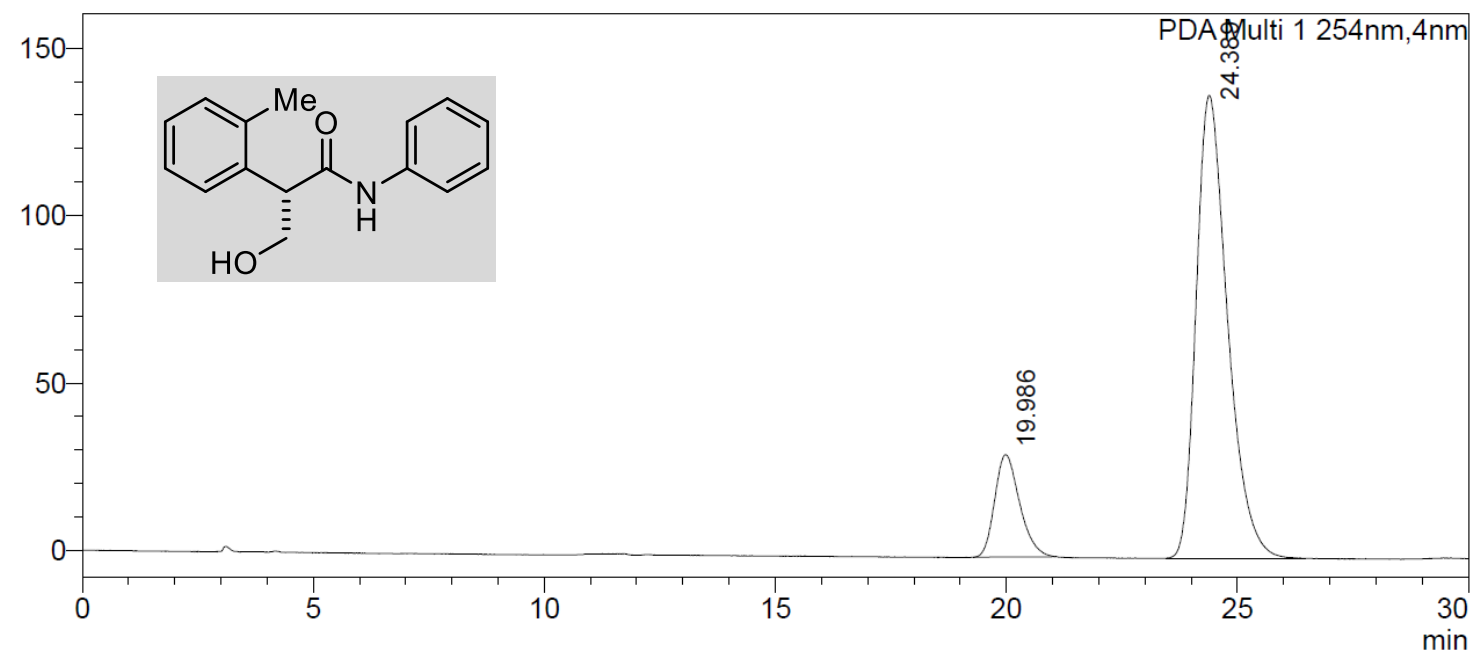

<Peak Table>

PDA Ch1 254nm

\begin{tabular}{|r|r|r|r|}
\hline Peak\# & Ret. Time & \multicolumn{1}{|c|}{ Area } & \multicolumn{1}{c|}{ Area\% } \\
\hline 1 & 19.986 & 1149762 & 14.958 \\
\hline 2 & 24.389 & 6536659 & 85.042 \\
\hline Total & & 7686421 & 100.000 \\
\hline
\end{tabular}


(R)-N-(4-bromophenyl)-3-(4,4,5,5-tetramethyl-1,3,2-dioxaborolan-2-yl)-2-(otolyl)propanamide $\mathbf{3 h g}$

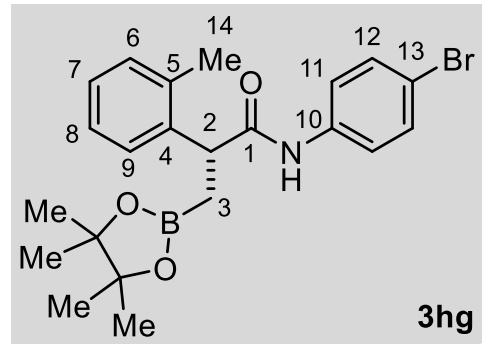

According to the general procedure for enantioselective copper-catalyzed borylative carboxamidation using styrene $\mathbf{1 h}$ (18 mg, $0.15 \mathrm{mmol}, 1.0$ equiv.), $\mathrm{CuCl}(1.5 \mathrm{mg}, 0.015 \mathrm{mmol}, 10$ mol\%), ligand $(R, R)-6 \mathbf{k}(7 \mathrm{mg}, 0.017 \mathrm{mmol}, 11 \mathrm{~mol} \%)$, LiOtBu (24 mg, $0.3 \mathrm{mmol}, 2.0$ equiv.), B $\mathrm{B}_{2} \mathrm{pin}_{2}(57 \mathrm{mg}, 0.23 \mathrm{mmol}, 1.5$ equiv.) and 4-isocyanato-1-bromobenzene $2 \mathrm{~g}(0.119 \mathrm{mg}, 0.60$ mmol, 4.0 equiv.) in 2-Me-THF and $\mathrm{Et}_{2} \mathrm{O}(1.5 \mathrm{~mL}, \mathrm{v}: \mathrm{v}=1: 1,0.1 \mathrm{M}) .90 \%$ conversion into $3 \mathrm{hg}$ determined by ${ }^{1} \mathrm{H}$ NMR analysis of the crude reaction mixture in presence of an internal standard. Purification by flash chromatography (pentane/diethyl ether 3:1) gave pure $\mathbf{3 h g}$ as a white solid (48 mg, $0.11 \mathrm{mmol}, 72 \%$ yield).

TLC: pentane/diethyl ether $4: 1, R_{f}=0.2$.

${ }^{1} \mathrm{H}$ NMR $\left(300 \mathrm{MHz}, \mathrm{CDCl}_{3}\right) \delta(\mathrm{ppm})=7.44-7.33$ (m, 8H, H-Ar), 7.04 (bs, 1H, N-H), 4.15 (dd, $\left.{ }^{3} J_{\mathrm{HH}}=9.1,7.0 \mathrm{~Hz}, 1 \mathrm{H}, \mathrm{H}-2\right), 2.46(\mathrm{~s}, 3 \mathrm{H}, H-14), 1.70\left(\mathrm{dd},{ }^{2} J_{\mathrm{HH}}=15.7,{ }^{3} J_{\mathrm{HH}}=9.1 \mathrm{~Hz}, 1 \mathrm{H}, H-3\right)$, $1.33\left(\mathrm{dd},{ }^{2} \mathrm{~J}_{\mathrm{HH}}=15.7,{ }^{3} \mathrm{JHH}_{\mathrm{HH}}=7.0 \mathrm{~Hz}, 1 \mathrm{H}, H-3\right.$ '), 1.25 (s, 12H, H-MeBpin).

${ }^{13} \mathrm{C}\left\{{ }^{1} \mathrm{H}\right\}$ NMR $(75 \mathrm{MHz}, \mathrm{CDCl}) \delta(\mathrm{ppm})=173.0(C-1), 139.4(C-4), 137.2(C-10), 136.4(C-5)$, $131.9(\mathrm{CH}-11), 131.2(\mathrm{CH}-6), 128.0(\mathrm{CH}-\mathrm{Ar}), 127.6(\mathrm{CH}-\mathrm{Ar}), 127.1(\mathrm{CH}-\mathrm{Ar}), 121.3(\mathrm{CH}-12)$, 116.7 (C-13), 83.5 (CO-Bpin), 46.6 (CH-2), 24.9 ( $\mathrm{CH}_{3}$-MeBpin), 24.8 ( $\left.\mathrm{CH}_{3}-\mathrm{MeBpin}\right), 19.9$ $\left(\mathrm{CH}_{3}-14\right)$. The boron-bound $\mathrm{CH}_{2}-3$ was not detected due to quadrupolar relaxation.

${ }^{11} \mathbf{B}$ NMR $\left(128 \mathrm{MHz}, \mathrm{CDCl}_{3}\right) \delta(\mathrm{ppm})=33.4$.

HRMS (ESI +): calculated for $\mathrm{C}_{22} \mathrm{H}_{27} \mathrm{BBrNO}_{3}[\mathrm{M}+\mathrm{H}]^{+}:$:444.1344; found: 444.1354 .

IR (neat): $v\left(\mathrm{~cm}^{-1}\right)=3362,2924,2854,1682,1591,1518,1490,1390,1368,1329,1141,825$. m.p.: $192-195^{\circ} \mathrm{C}$

HPLC analysis of $\mathbf{3 h g}$ after being oxidized to the corresponding alcohol following the general procedure indicated 97:3 er, chiral stationary column: OD-H, mobile phase: $n$-hexane/iPrOH $=95: 5,1.0 \mathrm{~mL} / \mathrm{min}, 254 \mathrm{~nm}, 30^{\circ} \mathrm{C}, \mathrm{t}_{\mathrm{R}}$ (major) $=15.7 \mathrm{~min}, \mathrm{t}_{\mathrm{R}}$ (minor) $=10.9 \mathrm{~min}$.

$[\alpha]^{20} \mathrm{D}=-39.0\left(c 0.3, \mathrm{CH}_{2} \mathrm{Cl}_{2}\right)$. 


\section{$<$ Chromatogram>}

mAU

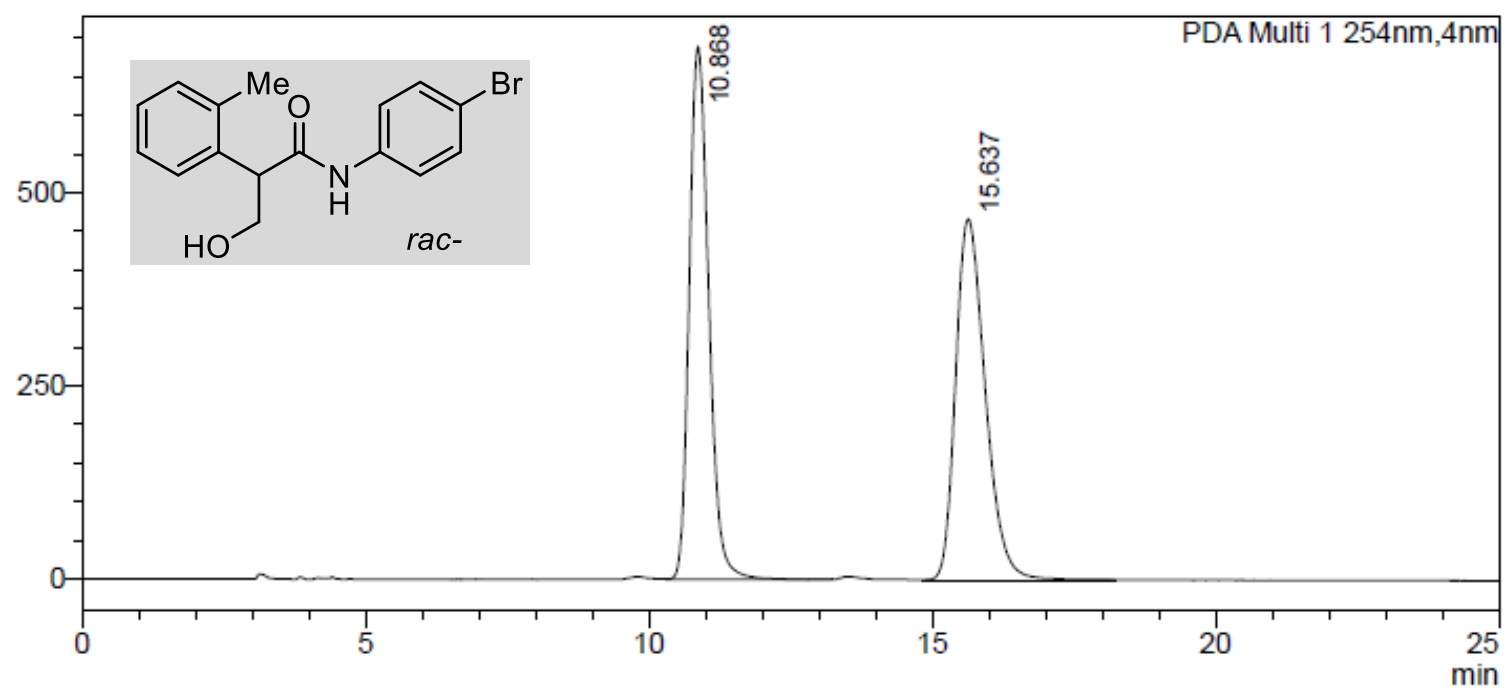

<Peak Table>

PDA Ch1 254nm

\begin{tabular}{|r|r|r|r|}
\hline Peak\# & Ret. Time & \multicolumn{1}{c|}{ Area } & \multicolumn{1}{c|}{ Area\% } \\
\hline 1 & 10.868 & 16491164 & 49.240 \\
\hline 2 & 15.637 & 17000466 & 50.760 \\
\hline Total & & 33491630 & 100.000 \\
\hline
\end{tabular}

<Chromatogram> mAU

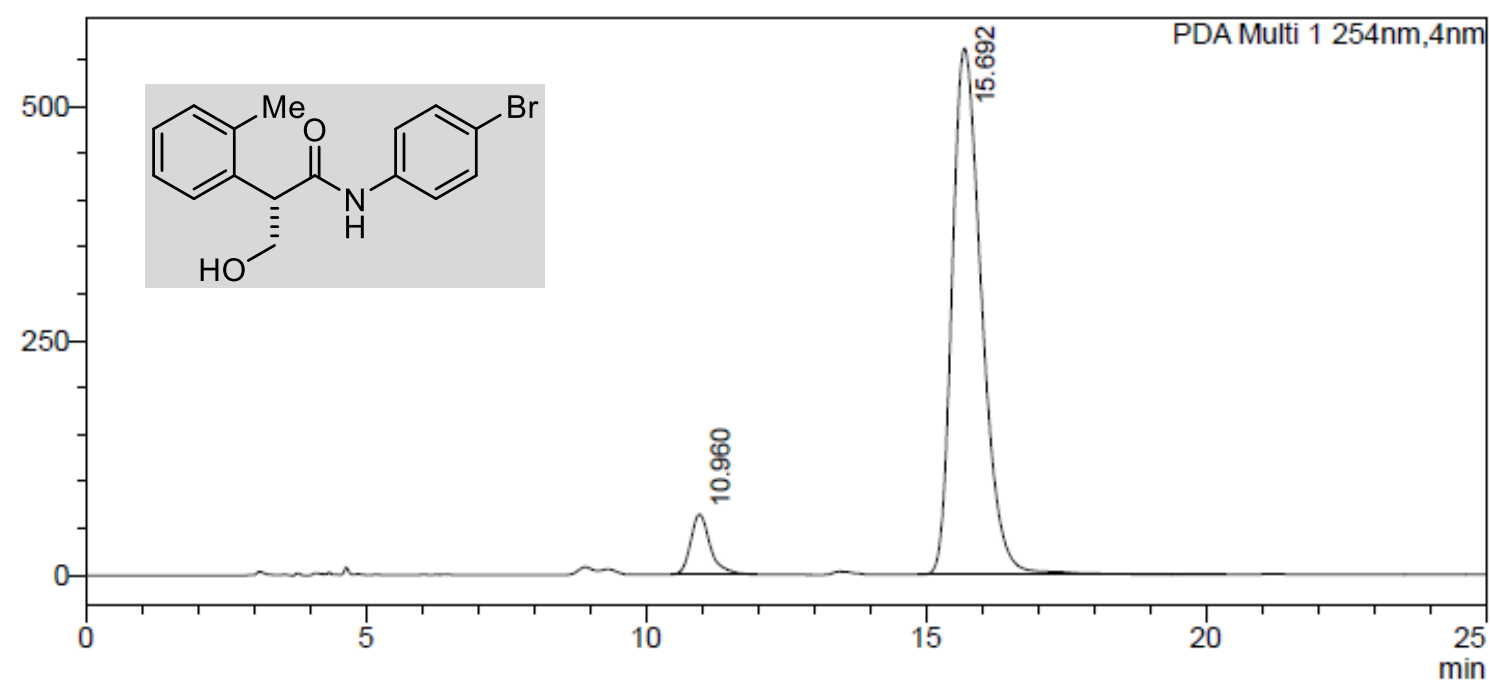

<Peak Table>

PDA Ch1 254nm

\begin{tabular}{|r|r|r|r|}
\hline Peak\# & Ret. Time & \multicolumn{1}{c|}{ Area } & \multicolumn{1}{c|}{ Area\% } \\
\hline 1 & 10.960 & 1557909 & 7.043 \\
\hline 2 & 15.692 & 20561372 & 92.957 \\
\hline Total & & 22119281 & 100.000 \\
\hline
\end{tabular}


(R)-2-([1,1'-biphenyl]-4-yl)-N-phenyl-3-(4,4,5,5-tetramethyl-1,3,2-dioxaborolan-2yl)propanamide $3 f a$

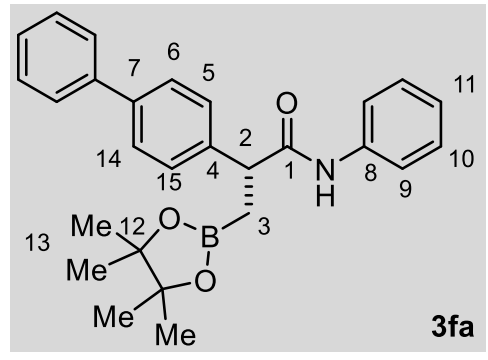

According to the general procedure for enantioselective copper-catalyzed borylative carboxamidation using styrene $\mathbf{1 f}$ (54 mg, $0.3 \mathrm{mmol}, 1.0$ equiv.), $\mathrm{CuCl}(3.0 \mathrm{mg}, 0.03 \mathrm{mmol}, 10$ mol\%), ligand $(R, R)-6 \mathbf{k}(14 \mathrm{mg}, 0.033 \mathrm{mmol}, 11 \mathrm{~mol} \%)$, LiOtBu (48 mg, 0.6 mmol, 2.0 equiv.), $B_{2}$ pin $_{2}(114 \mathrm{mg}, 0.45 \mathrm{mmol}, 1.5$ equiv.) and isocyanatobenzene $2 \mathrm{a}(0.132 \mathrm{~mL}, 1.2 \mathrm{mmol}, 4.0$ equiv.) in 2-Me-THF and $\mathrm{Et}_{2} \mathrm{O}(3.0 \mathrm{~mL}, \mathrm{v}: \mathrm{v}=1: 1,0.1 \mathrm{M}) .85 \%$ conversion into 3 fa determined by ${ }^{1} \mathrm{H}$ NMR analysis of the crude reaction mixture in presence of an internal standard. Purification by flash chromatography (pentane/diethyl ether 3:1) gave pure 3fa as a white solid (96 mg, $0.22 \mathrm{mmol}, 75 \%$ yield).

TLC: pentane/ethyl acetate $4: 1, \mathrm{R}_{\mathrm{f}}=0.3$.

${ }^{1} \mathrm{H}$ NMR $\left(400 \mathrm{MHz}, \mathrm{CDCl}_{3}\right) \delta(\mathrm{ppm})=7.59(\mathrm{~m}, 4 \mathrm{H}), 7.44(\mathrm{~m}, 6 \mathrm{H}), 7.35(\mathrm{~m}, 1 \mathrm{H}), 7.26(\mathrm{~m}, 2 \mathrm{H})$, $7.16(\mathrm{~s}, 1 \mathrm{H}, \mathrm{N}-H), 7.05(\mathrm{~m}, 1 \mathrm{H}), 3.92\left(\mathrm{dd},{ }^{3} J_{\mathrm{HH}}=9.6,6.4 \mathrm{~Hz}, 1 \mathrm{H}, H-2\right), 1.71\left(\mathrm{dd},{ }^{2} J_{H H}=15.8\right.$, $\left.{ }^{3} J_{\mathrm{HH}}=9.6 \mathrm{~Hz}, 1 \mathrm{H}, H-3\right), 1.34\left(\mathrm{dd},{ }^{2} J_{\mathrm{HH}}=15.8,{ }^{3} J_{\mathrm{HH}}=6.4 \mathrm{~Hz}, 1 \mathrm{H}, H-3\right), 1.22(\mathrm{~s}, 6 \mathrm{H}, H-13), 1.20$ (s, 6H, H-13).

${ }^{13} \mathrm{C}\left\{{ }^{1} \mathrm{H}\right\}$ NMR $\left(101 \mathrm{MHz}, \mathrm{CDCl}_{3}\right) \delta(\mathrm{ppm})=172.4(C-1), 140.6(C-\mathrm{Ar}), 140.6(C-\mathrm{Ar}), 140.3(C-$ 4), 138.1 (C-8), 128.9 (C-10), 128.8 (C-Ar), 128.4 (C-Ar), 127.7 (C-Ar), 127.4 (C-Ar), 127.1 (C-Ar), 124.0 (C-11), 119.5 (C-9), 83.4 (C-12), 49.6 (C-2), 24.8 (C-13), $24.7(C-13), 16.8$ (C3).

${ }^{11} \mathbf{B}$ NMR $\left(128 \mathrm{MHz}, \mathrm{CDCl}_{3}\right) \delta(\mathrm{ppm})=33.3$.

HRMS (ESI +): calculated for $\mathrm{C}_{27} \mathrm{H}_{30} \mathrm{BNO}_{3}[\mathrm{M}+\mathrm{H}]^{+}: 428.2397$; found: 428.2397.

IR (neat): $v\left(\mathrm{~cm}^{-1}\right)=3311,1662,1600,1540,1441,1370,1322,1143,846,755,695$.

m.p.: $168-170^{\circ} \mathrm{C}$

HPLC analysis of $\mathbf{3 f a}$ after oxidation to the corresponding alcohol following the general procedure indicated $88.5: 11.5$ er, chiral stationary column: OD-H, mobile phase: $n$ hexane $/ \mathrm{PrOH}=95: 5,1.0 \mathrm{~mL} / \mathrm{min}, 254 \mathrm{~nm}, 30^{\circ} \mathrm{C}, \mathrm{t}_{\mathrm{R}}$ (major) $=47.8 \mathrm{~min}, \mathrm{t}_{\mathrm{R}}$ (minor) $=22.2 \mathrm{~min}$. $[\alpha]^{20} \mathrm{D}=-47.5\left(c 0.65, \mathrm{CH}_{2} \mathrm{Cl}_{2}\right)$. 


\section{<Chromatogram>}

mAU

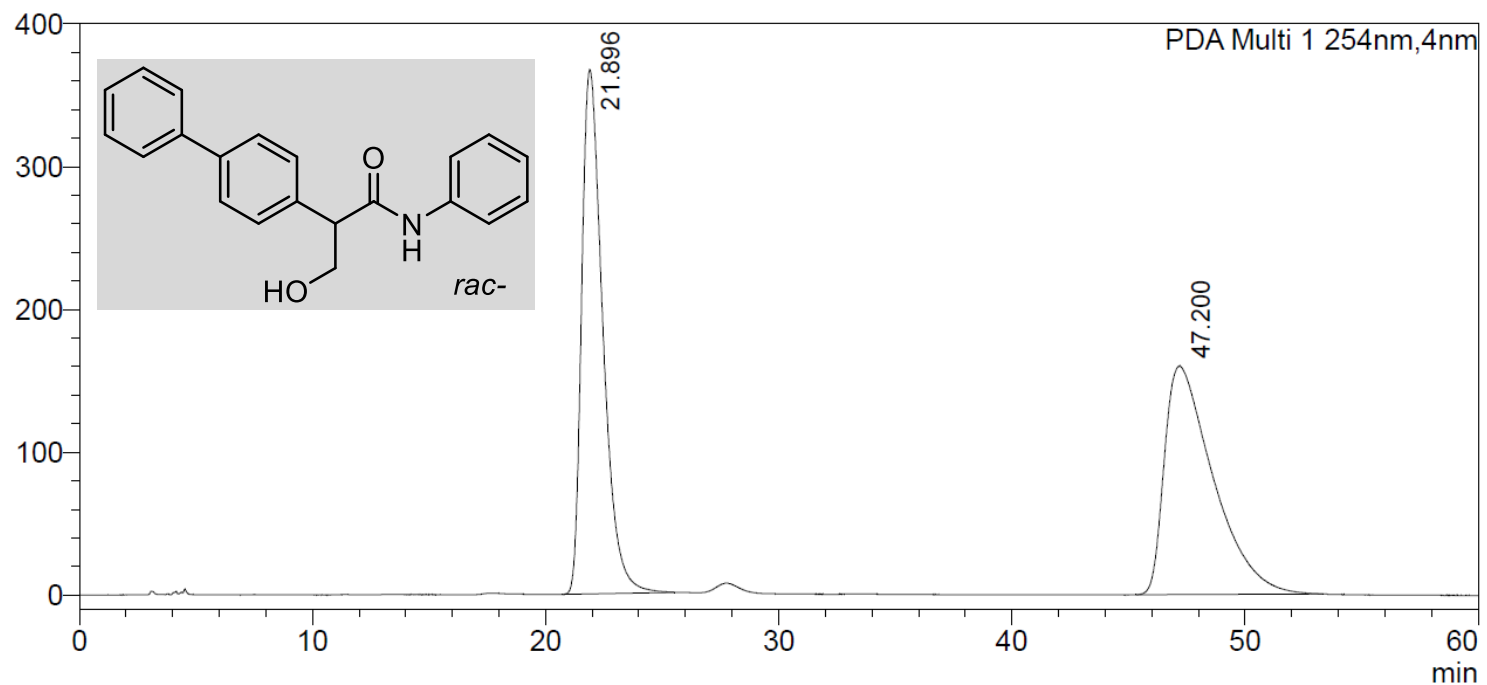

\section{<Peak Table>}

PDA Ch1 254nm

\begin{tabular}{|r|r|r|r|}
\hline Peak\# & Ret. Time & Area & \multicolumn{1}{c|}{ Area\% } \\
\hline 1 & 21.896 & 23181955 & 49.834 \\
\hline 2 & 47.200 & 23336343 & 50.166 \\
\hline Total & & 46518298 & 100.000 \\
\hline
\end{tabular}

\section{<Chromatogram>}

$\mathrm{mAU}$

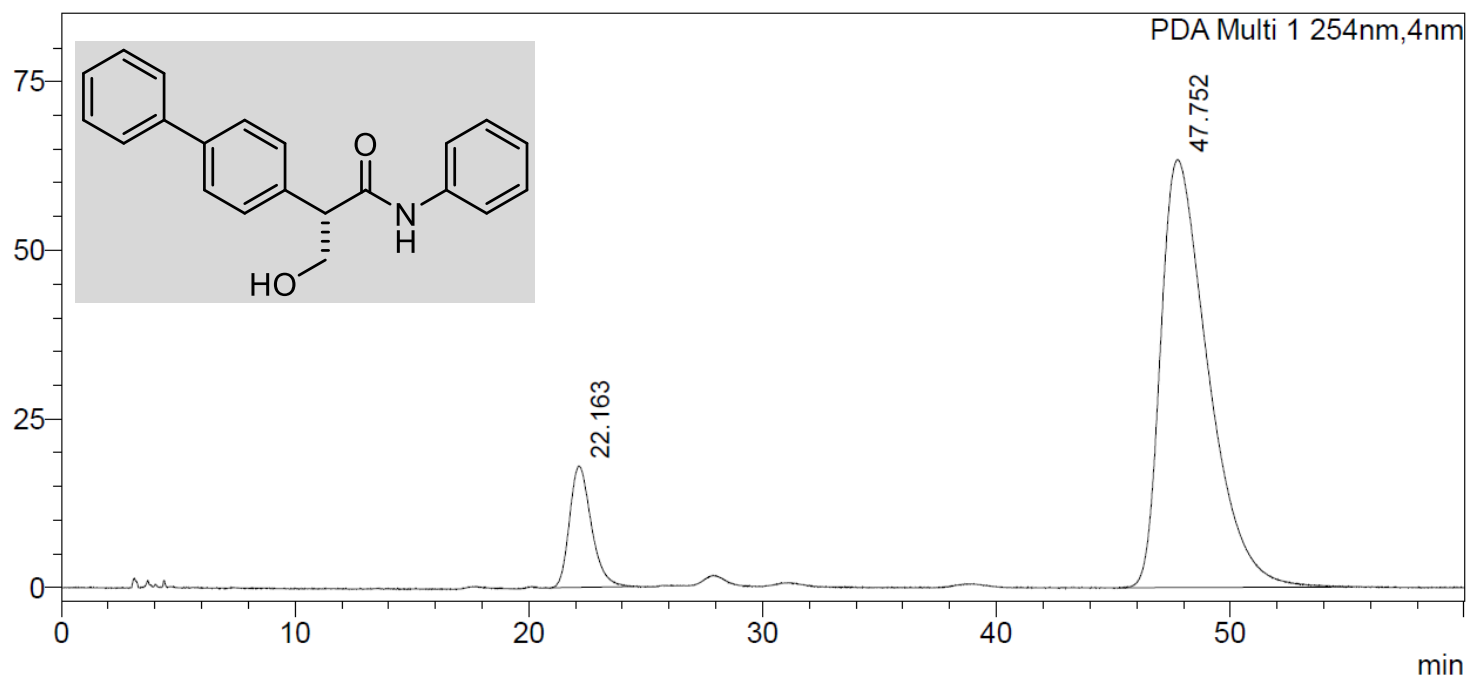

<Peak Table>

PDA Ch1 254nm

\begin{tabular}{|r|r|r|r|}
\hline Peak\# & Ret. Time & \multicolumn{1}{|c|}{ Area } & \multicolumn{1}{|c|}{ Area\% } \\
\hline 1 & 22.163 & 1164803 & 11.538 \\
\hline 2 & 47.752 & 8930701 & 88.462 \\
\hline Total & & 10095505 & 100.000 \\
\hline
\end{tabular}


(R)-2-([1,1'-biphenyl]-4-yl)-N-(4-bromophenyl)-3-(4,4,5,5-tetramethyl-1,3,2-dioxaborolan-2yl)propanamide $\mathbf{3 f g}$

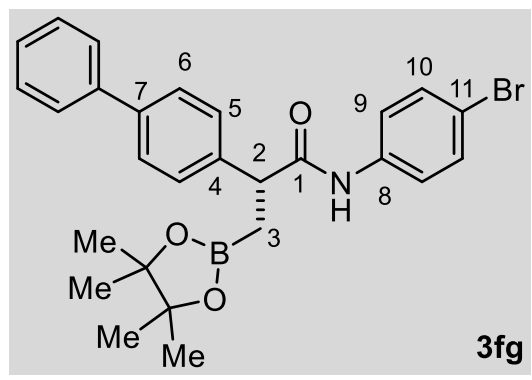

According to the general procedure for enantioselective copper-catalyzed borylative carboxamidation using styrene $1 f$ (27 mg, $0.15 \mathrm{mmol}, 1.0$ equiv.), $\mathrm{CuCl}$ (1.5 mg, 0.015 $\mathrm{mmol}, 10 \mathrm{~mol} \%)$, ligand $(R, R)-6 \mathrm{k}(7 \mathrm{mg}, 0.017 \mathrm{mmol}, 11$ mol\%), LiOtBu (24 mg, $0.3 \mathrm{mmol}, 2.0$ equiv.), $\mathrm{B}_{2} \mathrm{pin}_{2}(57 \mathrm{mg}$,

$3 \mathrm{fg} \quad 0.23 \mathrm{mmol}, 1.5$ equiv.) and 4-isocyanato-1-bromobenzene $2 \mathrm{~g}(0.119 \mathrm{mg}, 0.60 \mathrm{mmol}, 4.0$ equiv. $)$ in 2-Me-THF and $\mathrm{Et}_{2} \mathrm{O}(1.5 \mathrm{~mL}, \mathrm{v}: \mathrm{v}=1: 1,0.1 \mathrm{M}) .90 \%$ conversion into $\mathbf{3 f g}$ determined by ${ }^{1} \mathrm{H}$ NMR analysis of the crude reaction mixture in presence of an internal standard. Purification by flash chromatography (pentane/diethyl ether 3:1) gave pure $\mathbf{3 f g}$ as a white solid (59 $\mathrm{mg}, 0.12 \mathrm{mmol}, 77 \%$ yield).

TLC: pentane/diethyl ether $4: 1, R_{f}=0.2$.

${ }^{1} \mathrm{H}$ NMR $\left(400 \mathrm{MHz}, \mathrm{CDCl}_{3}\right) \delta(\mathrm{ppm})=7.58(\mathrm{~m}, 4 \mathrm{H}, \mathrm{H}-\mathrm{Ar}), 7.49-7.38(\mathrm{~m}, 4 \mathrm{H}, \mathrm{H}-\mathrm{Ar}), 7.39-$ $7.32(\mathrm{~m}, 5 \mathrm{H}, \mathrm{H}-\mathrm{Ar}), 7.17$ (bs, $1 \mathrm{H}, \mathrm{N}-\mathrm{H}), 3.90\left(\mathrm{dd},{ }^{3} \mathrm{~J}_{\mathrm{HH}}=9.8,6.3 \mathrm{~Hz}, 1 \mathrm{H}, \mathrm{H}-2\right), 1.69$ (dd, ${ }^{2} J_{\mathrm{HH}}=$ $\left.15.8 \mathrm{~Hz},{ }^{3} \mathrm{~J}_{\mathrm{HH}}=9.8 \mathrm{~Hz}, 1 \mathrm{H}, \mathrm{H}-3\right), 1.32\left(\mathrm{dd},{ }^{2} \mathrm{~J}_{\mathrm{HH}}=15.8 \mathrm{~Hz},{ }^{3} \mathrm{~J}_{\mathrm{HH}}=6.2 \mathrm{~Hz}, 1 \mathrm{H}, H-3\right.$ ), 1.22 (s, 6H, H-MeBpin), 1.20 (s, 6H, H-MeBpin).

${ }^{13} \mathrm{C}\left\{{ }^{1} \mathrm{H}\right\}$ NMR $\left(101 \mathrm{MHz}, \mathrm{CDCl}_{3}\right) \delta(\mathrm{ppm})=172.6(C-1), 140.6(C-\mathrm{Ar}), 140.5(C-\mathrm{Ar}), 140.4(C-$ Ar), 137.2 (C-8), 132.0 ( $\mathrm{CH}-9), 129.0$ ( $\mathrm{CH}-\mathrm{Ar}), 128.5$ ( $\mathrm{CH}-\mathrm{Ar}), 127.9$ ( $\mathrm{CH}-\mathrm{Ar}), 127.6$ (CH-Ar), 127.2 ( $\mathrm{CH}-\mathrm{Ar}), 121.2$ ( $\mathrm{CH}-10), 116.7$ (C-11), 83.6 (CO-Bpin), $49.7(\mathrm{CH}-2), 25.0\left(\mathrm{CH}_{3}-\mathrm{MeBpin}\right)$, $24.8\left(\mathrm{CH}_{3}-\mathrm{MeBpin}\right), 17.0$ (bs, $\left.\mathrm{CH}_{2}-3\right)$.

${ }^{11} \mathbf{B}$ NMR $\left(128 \mathrm{MHz}, \mathrm{CDCl}_{3}\right) \delta(\mathrm{ppm})=33.3$.

HRMS (ESI +): calculated for $\mathrm{C}_{27} \mathrm{H}_{29} \mathrm{BBrNO}_{3}[\mathrm{M}+\mathrm{H}]^{+:}: 506.1502$; found: 506.1503 .

IR (neat): $v\left(\mathrm{~cm}^{-1}\right)=3342,2922,1688,1597,1528,1509,1488,1393,1372,1319,1255$, 1180, 1138.

m.p.: $207-210^{\circ} \mathrm{C}$

HPLC analysis of $\mathbf{3 f g}$ after oxidation to the corresponding alcohol following the general procedure indicated 97:3 er, chiral stationary column: OD-H, mobile phase: $n$-hexane/iPrOH $=95 / 5,1.0 \mathrm{~mL} / \mathrm{min}, 254 \mathrm{~nm}, 30^{\circ} \mathrm{C}, \mathrm{t}_{\mathrm{R}}$ (major) $=43.4 \mathrm{~min}, \mathrm{t}_{\mathrm{R}}$ (minor) $=20.5 \mathrm{~min}$.

$[\alpha]^{20} \mathrm{D}=-48.9\left(c \quad 0.25, \mathrm{CH}_{2} \mathrm{Cl}_{2}\right)$. 


\section{$<$ Chromatogram>}

$\mathrm{mAU}$

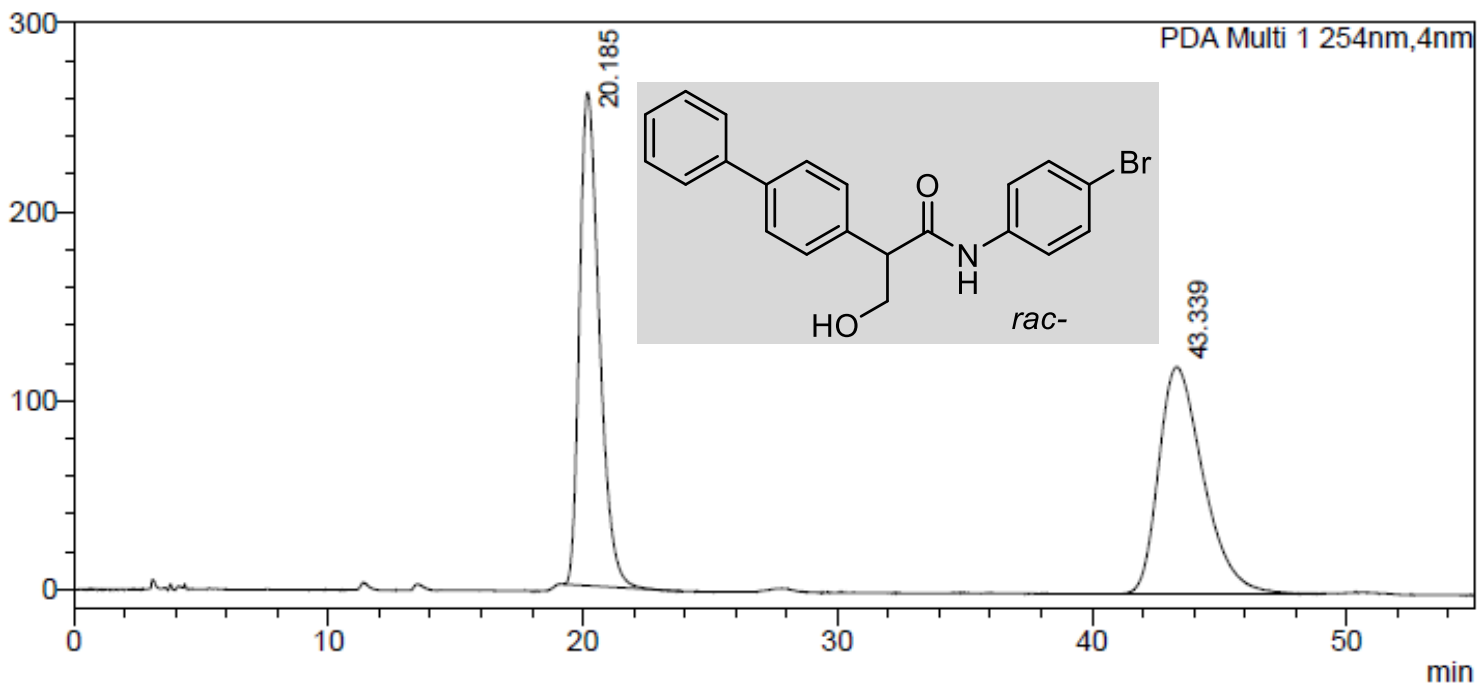

<Peak Table>

PDA Ch1 254nm

\begin{tabular}{|r|r|r|r|}
\hline Peak\# & Ret. Time & Area & \multicolumn{1}{c|}{ Area\% } \\
\hline 1 & 20.185 & 14161854 & 49.112 \\
\hline 2 & 43.339 & 14673950 & 50.888 \\
\hline Total & & 28835805 & 100.000 \\
\hline
\end{tabular}

\section{$<$ Chromatogram>}

mAU

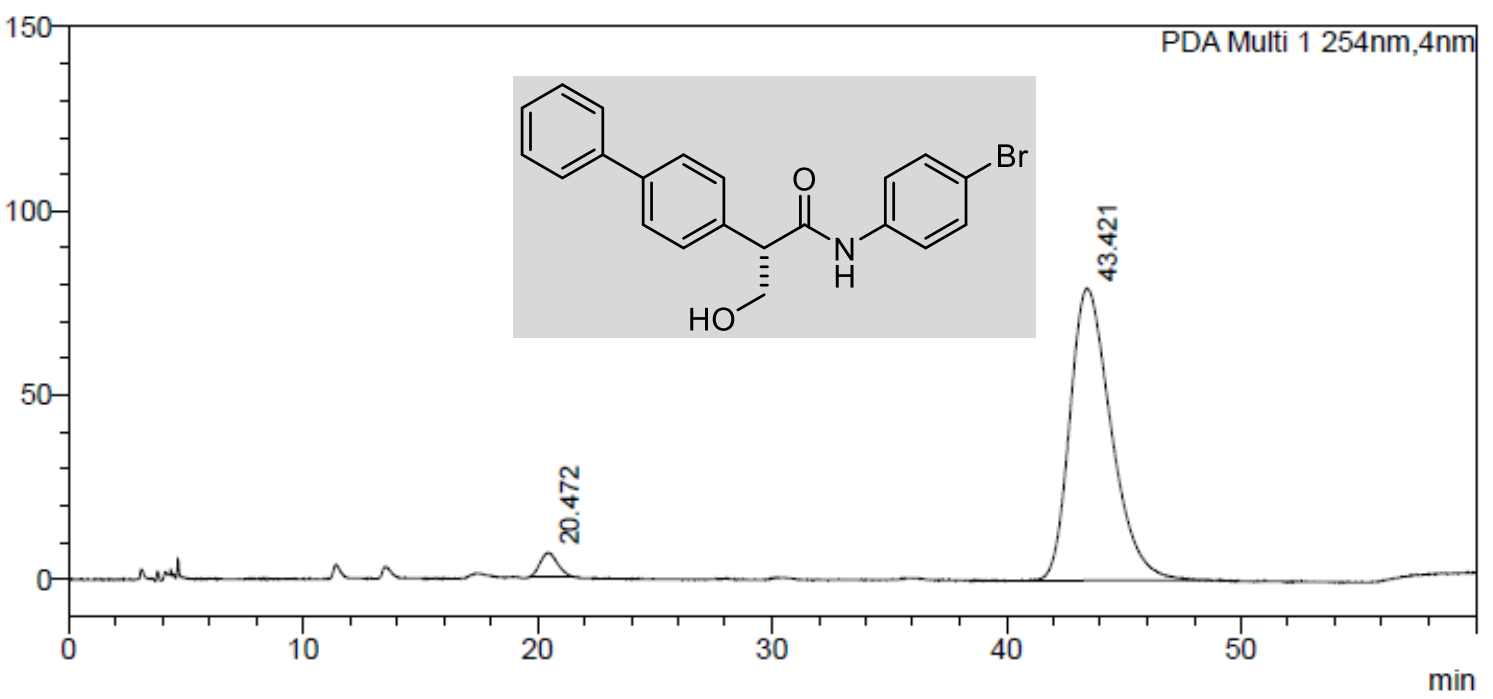

<Peak Table>

PDA Ch1 254nm

\begin{tabular}{|r|r|r|r|}
\hline Peak\# & Ret. Time & \multicolumn{1}{|c|}{ Area } & Area\% \\
\hline 1 & 20.472 & 329533 & 3.291 \\
\hline 2 & 43.421 & 9682212 & 96.709 \\
\hline Total & & 10011745 & 100.000 \\
\hline
\end{tabular}


(R)-2-(naphthalen-2-yl)- $N$-phenyl-3-(4,4,5,5-tetramethyl-1,3,2-dioxaborolan-2yl)propanamide 3ia

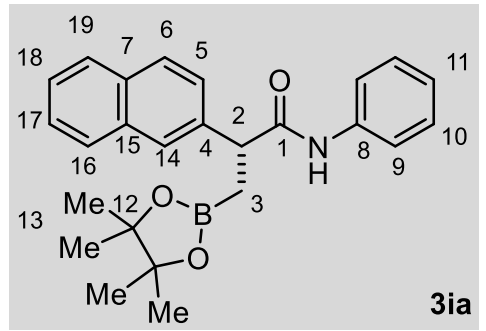

According to the general procedure for enantioselective copper-catalyzed borylative carboxamidation using styrene $\mathbf{1 i}$ (47 mg, $0.3 \mathrm{mmol}, 1.0$ equiv.), $\mathrm{CuCl}(3.0 \mathrm{mg}, 0.03 \mathrm{mmol}, 10$ mol\%), ligand $(R, R)-6 \mathbf{k}(14 \mathrm{mg}, 0.033 \mathrm{mmol}, 11 \mathrm{~mol} \%)$, LiOtBu (48 mg, $0.6 \mathrm{mmol}, 2.0$ equiv.), $B_{2}$ pin $_{2}(114 \mathrm{mg}, 0.45 \mathrm{mmol}, 1.5$ equiv.) and isocyanatobenzene $2 \mathrm{a}(0.132 \mathrm{~mL}, 1.2 \mathrm{mmol}, 4.0$ equiv. $)$ in 2-Me-THF and $\mathrm{Et}_{2} \mathrm{O}$ $\left(3.0 \mathrm{~mL}, \mathrm{v}: \mathrm{v}=1: 1,0.1 \mathrm{M}\right.$ ). $80 \%$ conversion into 3ia determined by ${ }^{1} \mathrm{H}$ NMR analysis of the crude reaction mixture in presence of an internal standard. Purification by flash chromatography (pentane/diethyl ether 3:1) gave pure 3ia as a white solid ( $82 \mathrm{mg}, 0.20 \mathrm{mmol}$, $68 \%$ yield).

TLC: pentane/ethyl acetate $4: 1, R_{f}=0.4$.

${ }^{1} \mathbf{H}$ NMR $\left(400 \mathrm{MHz}, \mathrm{CDCl}_{3}\right) \delta(\mathrm{ppm})=7.84(\mathrm{~m}, 4 \mathrm{H}), 7.48(\mathrm{~m}, 3 \mathrm{H}), 7.40\left(\mathrm{~d},{ }^{3} \mathrm{~J}_{\mathrm{HH}}=7.7 \mathrm{~Hz}, 2 \mathrm{H}\right)$, $7.26-7.20(\mathrm{~m}, 2 \mathrm{H}), 7.14(\mathrm{~s}, 1 \mathrm{H}, \mathrm{N}-\mathrm{H}), 7.08-7.00(\mathrm{~m}, 1 \mathrm{H}), 4.07\left(\mathrm{dd},{ }^{3} \mathrm{~J}_{\mathrm{HH}}=9.6,6.4 \mathrm{~Hz}, 1 \mathrm{H}\right.$, $H-2), 1.77\left(\mathrm{dd},{ }^{2} J_{H H}=15.8,{ }^{3} J_{H H}=9.6 \mathrm{~Hz}, 1 \mathrm{H}, H-3\right), 1.36\left(\mathrm{dd},{ }^{2} J_{H H}=15.8,{ }^{3} J_{H H}=6.4 \mathrm{~Hz}, 1 \mathrm{H}\right.$, $H-3), 1.21$ (s, 6H, $H-13), 1.19(\mathrm{~s}, 6 \mathrm{H}, H-13)$.

${ }^{13} \mathrm{C}\left\{{ }^{1} \mathrm{H}\right\}$ NMR $(101 \mathrm{MHz}, \mathrm{CDCl}) \delta(\mathrm{ppm})=172.3(C-1), 139.0(C-4), 138.0(C-8), 133.5(C-\mathrm{Ar})$, 132.7 (C-Ar), 128.9 (C-Ar), 128.8 (C-10), 127.7 (C-Ar), 127.6 (C-Ar), 126.8 (C-Ar), 126.3 (CAr), 126.0 (C-Ar), 125.8 (C-Ar), 124.0 (C-11), 119.5 (C-9), 83.3 (C-12), 50.1 (C-2)2, 24.8 (C13), 24.7 (C-13), $16.8(C-3)$.

${ }^{11} \mathrm{~B}$ NMR $\left(128 \mathrm{MHz}, \mathrm{CDCl}_{3}\right) \delta(\mathrm{ppm})=34.0$.

HRMS (ESI +): calculated for $\mathrm{C}_{25} \mathrm{H}_{28} \mathrm{BNO}_{3}[\mathrm{M}+\mathrm{H}]^{+}:$: 402.2240; found: 402.2211 .

IR (neat): $v\left(\mathrm{~cm}^{-1}\right)=3346,3058,2978,2926,1683,1663,1601,1535,1500,1440,1371$, 1324, 1260, 1249, 1213, 1166, 1141, 1107, 1019, 968, 888, 858, 846, 826, 750, 695.

m.p.: $159-160^{\circ} \mathrm{C}$

HPLC analysis of 3ia after oxidation to the corresponding alcohol following the general procedure indicated 88.5:14.5 er, chiral stationary column: OD-H, mobile phase: $n$ hexane $/ \mathrm{PrOH}=95: 5,1.0 \mathrm{~mL} / \mathrm{min}, 254 \mathrm{~nm}, 30^{\circ} \mathrm{C}, \mathrm{t}_{\mathrm{R}}$ (major) $=34.3 \mathrm{~min}, \mathrm{t}_{\mathrm{R}}$ (minor) $=20.0 \mathrm{~min}$.

Recrystallization: 3ia (60 mg, 85.5:14.5 er) was dissolved in $\mathrm{Et}_{2} \mathrm{O}(6 \mathrm{~mL})$ and the solution was concentrated by slow evaporation at $23^{\circ} \mathrm{C}$ until a white crystalline precipitate appeared. The precipitate was filtered, washed with $n$-hexane and dried under vacuum. (30 $\mathrm{mg}, 50 \%$ yield, 98.5:1.5 er).

SFC analysis of the recrystallized product 3ia after oxidation the corresponding alcohol following the general procedure indicated $98.5: 1.5$ er, chiral stationary column: $O B$, mobile 
phase: $2 \%$ to $30 \% \mathrm{MeOH}, 3.0 \mathrm{~mL} / \mathrm{min}, 210 \mathrm{~nm}, 30^{\circ} \mathrm{C}, \mathrm{t}_{\mathrm{R}}$ (major) $=10.9 \mathrm{~min}, \mathrm{t}_{\mathrm{R}}($ minor $)=9.1$ $\min$.

$[\alpha]^{20}{ }_{D}=-78.9\left(c 0.65, \mathrm{CH}_{2} \mathrm{Cl}_{2}\right)$.

\section{$<$ Chromatogram>}

$\mathrm{mAU}$

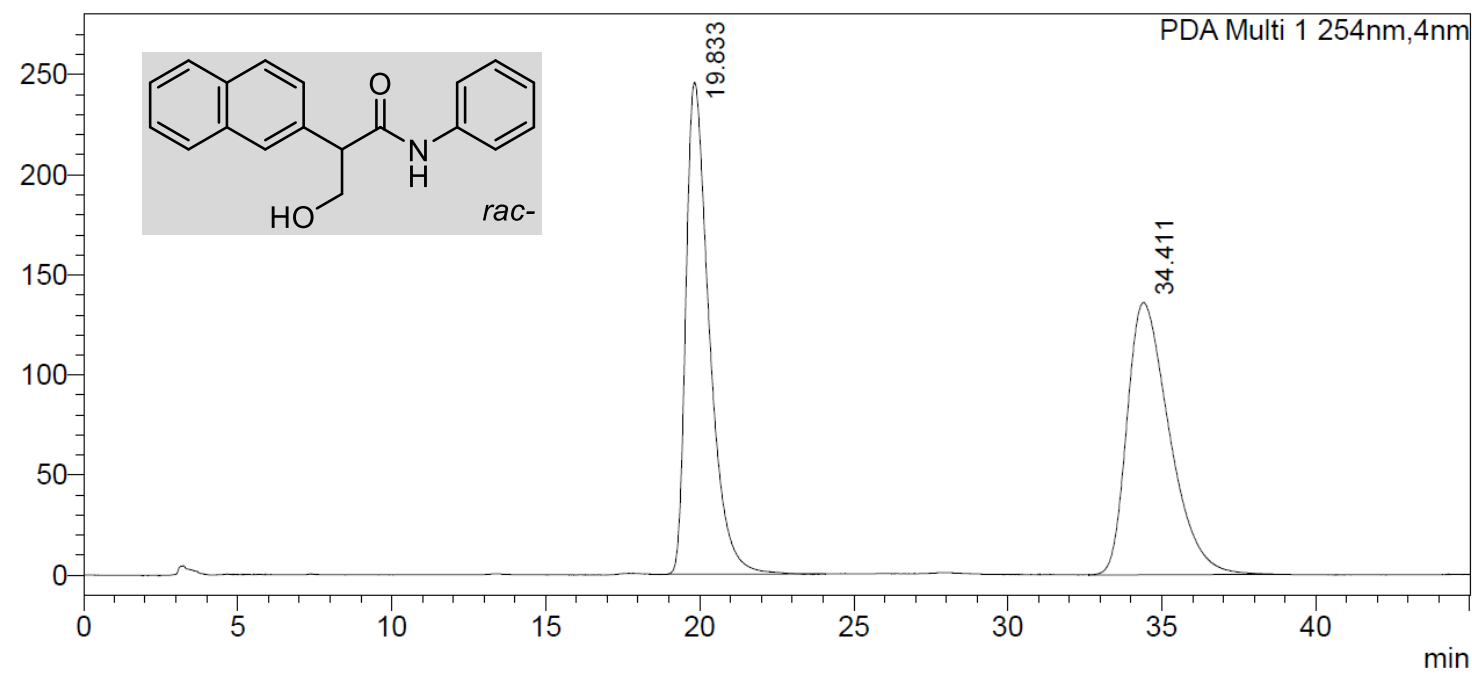

<Peak Table>

PDA Ch1 254nm

\begin{tabular}{|r|r|r|r|}
\hline Peak\# & Ret. Time & \multicolumn{1}{c|}{ Area } & \multicolumn{1}{c|}{ Area\% } \\
\hline 1 & 19.833 & 12937557 & 49.844 \\
\hline 2 & 34.411 & 13018800 & 50.156 \\
\hline Total & & 25956357 & 100.000 \\
\hline
\end{tabular}

\section{$<$ Chromatogram >}

mAU

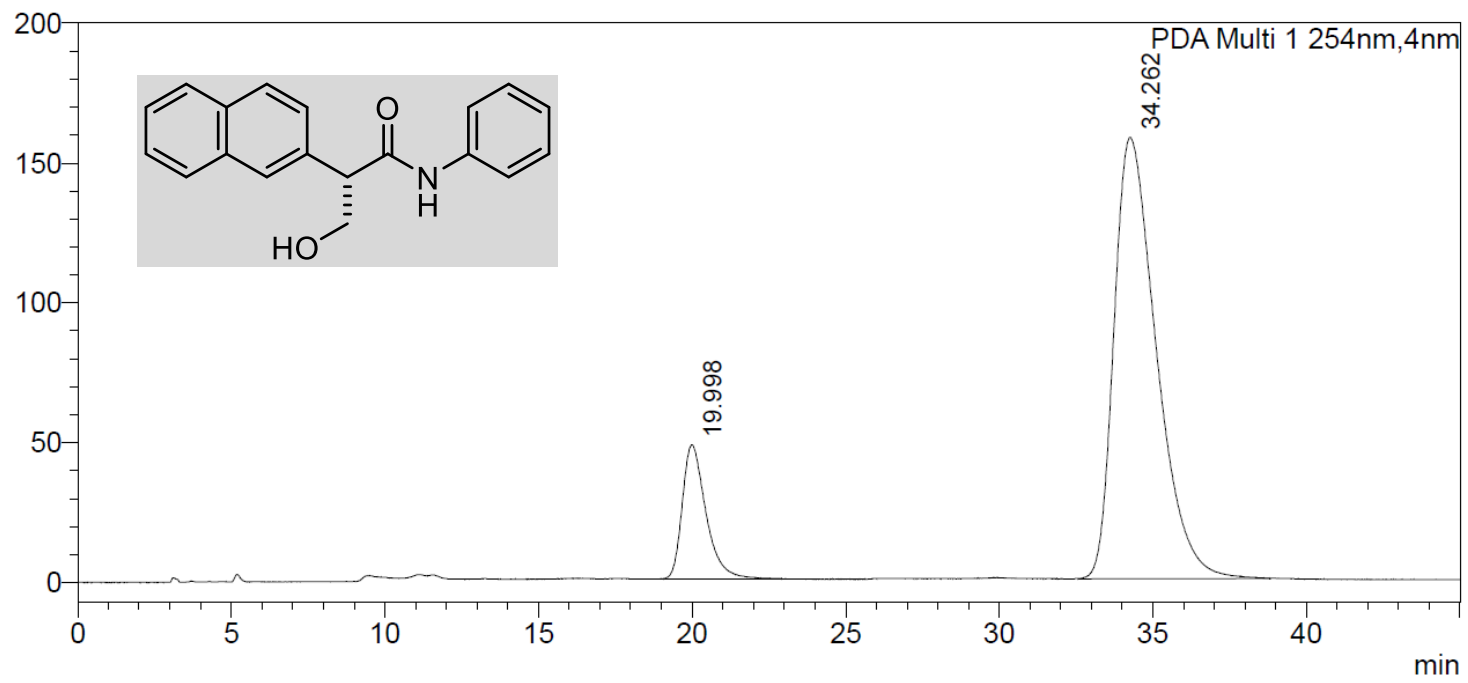

<Peak Table>

PDA Ch1 254nm

\begin{tabular}{|r|r|r|r|}
\hline Peak\# & Ret. Time & \multicolumn{1}{|c|}{ Area } & \multicolumn{1}{c|}{ Area\% } \\
\hline 1 & 19.998 & 2576438 & 14.583 \\
\hline 2 & 34.262 & 15091250 & 85.417 \\
\hline Total & & 17667688 & 100.000 \\
\hline
\end{tabular}




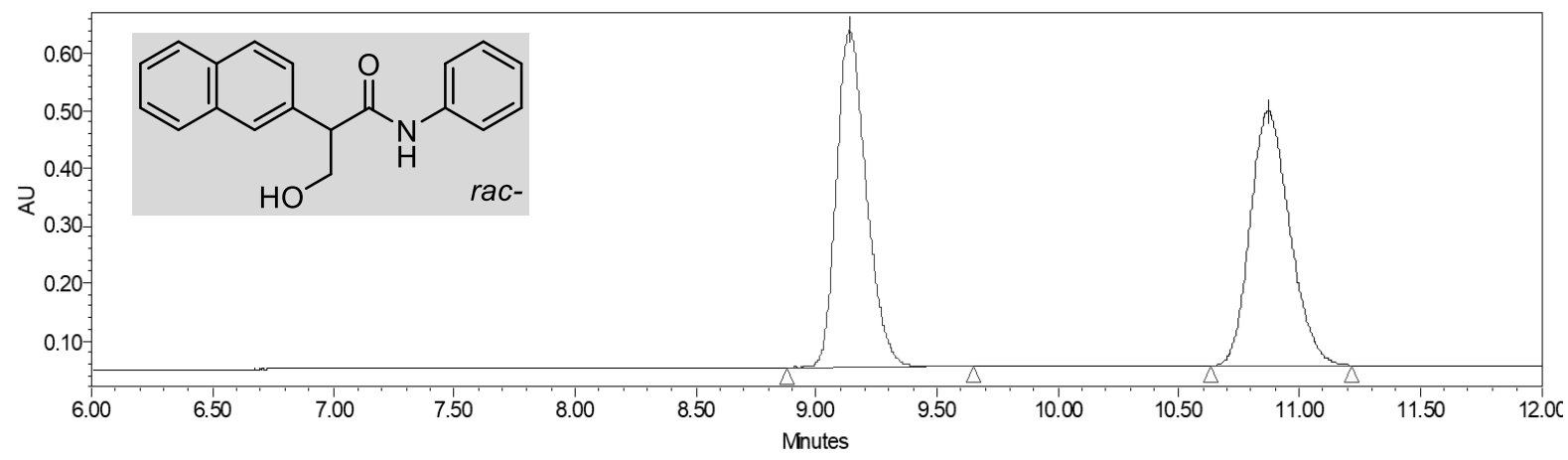

\begin{tabular}{|c|c|c|c|}
\hline & Retention Time & Area & \% Area \\
\hline 1 & 9.137 & 5027492 & 49.97 \\
\hline 2 & 10.869 & 5033739 & 50.03 \\
\hline
\end{tabular}

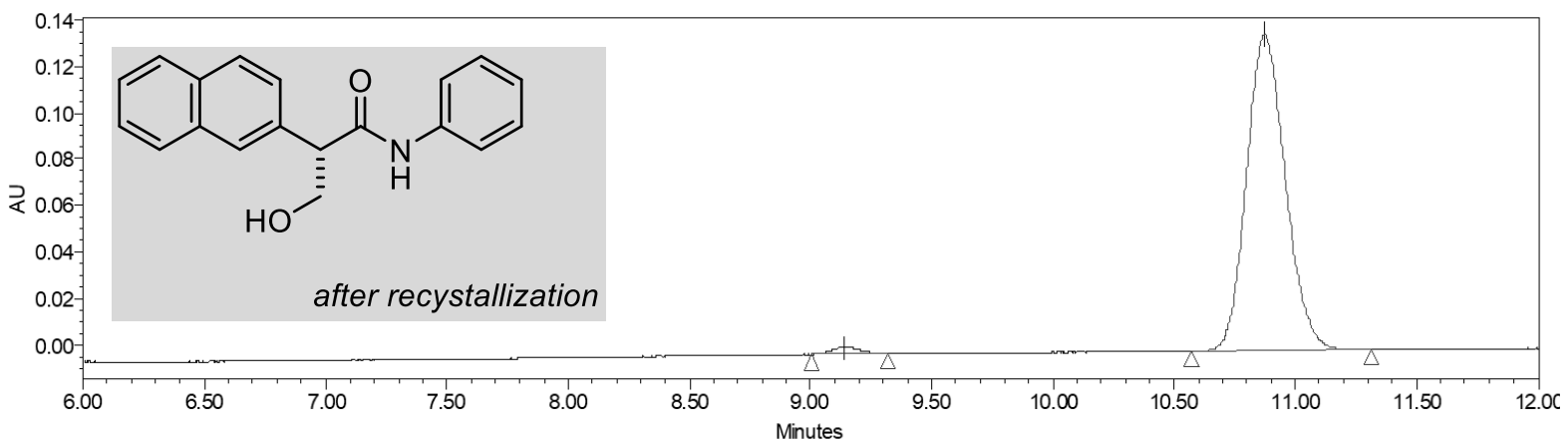

\begin{tabular}{|c|c|c|c|}
\hline & Retention Time & Area & \% Area \\
\hline 1 & 9.141 & 21535 & 1.40 \\
\hline 2 & 10.873 & 1518288 & 98.60 \\
\hline
\end{tabular}


(R)-2-(4-morpholinophenyl)- $N$-phenyl-3-(4,4,5,5-tetramethyl-1,3,2-dioxaborolan-2yl)propanamide $\mathbf{3 j a}$

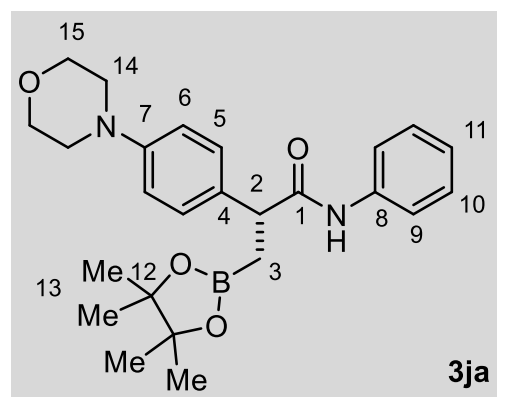

According to the general procedure for enantioselective copper-catalyzed borylative carboxamidation using styrene $\mathbf{1} \mathbf{j}$ (57 mg, 0.3 mmol, 1.0 equiv.), $\mathrm{CuCl}$ (3.0 mg, $0.03 \mathrm{mmol}, 10$ mol\%), ligand $(R, R)-6 \mathbf{k}(14 \mathrm{mg}, 0.033 \mathrm{mmol}, 11 \mathrm{~mol} \%)$, LiOtBu (48 mg, $0.6 \mathrm{mmol}, 2.0$ equiv.), $\mathrm{B}_{2} \mathrm{pin}_{2}(114 \mathrm{mg}, 0.45 \mathrm{mmol}, 1.5$ equiv.) and isocyanatobenzene $2 \mathrm{a}(0.132 \mathrm{~mL}, 1.2 \mathrm{mmol}, 4.0$ equiv.) in 2-Me-THF and $\mathrm{Et}_{2} \mathrm{O}$ (3.0 mL, v:v = 1:1, $\left.0.1 \mathrm{M}\right) .90 \%$ conversion into 3ja determined by ${ }^{1} \mathrm{H}$ NMR analysis of the crude reaction mixture in presence of an internal standard. Purification by flash chromatography $\left(\mathrm{CH}_{2} \mathrm{Cl}_{2} / \mathrm{MeOH}\right.$ 50:1) gave pure 3ja as a yellow solid (110 mg, $0.25 \mathrm{mmol}, 84 \%$ yield).

TLC: $\mathrm{CH}_{2} \mathrm{Cl}_{2} / \mathrm{MeOH} 20: 1, \mathrm{R}_{\mathrm{f}}=0.2$.

${ }^{1} \mathrm{H}$ NMR $\left(400 \mathrm{MHz}, \mathrm{CDCl}_{3}\right) \delta(\mathrm{ppm})=7.39(\mathrm{~m}, 2 \mathrm{H}), 7.25(\mathrm{~m}, 4 \mathrm{H}), 7.08(\mathrm{~s}, 1 \mathrm{H}, \mathrm{N}-H), 7.03(\mathrm{~m}$, $1 \mathrm{H}), 6.92-6.83(\mathrm{~m}, 2 \mathrm{H}), 3.87(\mathrm{~m}, 4 \mathrm{H}, H-15), 3.81\left(\mathrm{dd},{ }^{3} J_{\mathrm{HH}}=9.8,6.4 \mathrm{~Hz}, 1 \mathrm{H}, H-2\right), 3.15(\mathrm{~m}$, $4 \mathrm{H}, H-14), 1.63\left(\mathrm{dd},{ }^{2} J_{\mathrm{HH}}=15.7,{ }^{3} \mathrm{JHH}_{\mathrm{HH}}=9.8 \mathrm{~Hz}, 1 \mathrm{H}, H-3\right), 1.25(\mathrm{~m}, 1 \mathrm{H}, H-3), 1.22(\mathrm{~s}, 6 \mathrm{H}, H-$ 13), $1.20(\mathrm{~s}, 6 \mathrm{H}, \mathrm{H}-13)$.

${ }^{13} \mathrm{C}\left\{{ }^{1} \mathrm{H}\right\}$ NMR $\left(101 \mathrm{MHz}, \mathrm{CDCl}_{3}\right) \delta(\mathrm{ppm})=172.9(C-1), 150.5(C-7), 138.1(C-8), 132.8(C-4)$, 128.8 (C-10), 128.7 (C-5), 123.9 (C-11), 119.5 (C-9), $116.1(C-6), 83.3$ (C-12), 66.9 (C-15), 49.3 (C-14), 49.1 (C-2), 24.8 (C-13), $24.7(C-13), 16.7(C-3)$.

${ }^{11} \mathrm{~B}$ NMR $\left(128 \mathrm{MHz}, \mathrm{CDCl}_{3}\right) \delta(\mathrm{ppm})=34.5$.

HRMS (ESI +): calculated for $\mathrm{C}_{25} \mathrm{H}_{33} \mathrm{BN}_{2} \mathrm{O}_{4}[\mathrm{M}+\mathrm{H}]^{+}:$: 437.2611; found: 437.2606.

IR (neat): $v\left(\mathrm{~cm}^{-1}\right)=3316,2975,2858,1686,1600,1515,1500,1440,1371,1323,1234$, 1167, 1144, 1122,1051, 968, 930, 889, 847, 826, 755, 694.

m.p.: $180-182^{\circ} \mathrm{C}$

HPLC analysis of 3 ja indicated $92: 8 \mathrm{er}$, chiral stationary column: IC, mobile phase: $n$ hexane/ $\mathrm{PrOH}=85: 15,1.0 \mathrm{~mL} / \mathrm{min}, 254 \mathrm{~nm}, 30^{\circ} \mathrm{C}, \mathrm{t}_{\mathrm{R}}$ (major) $=34.8 \mathrm{~min}, \mathrm{t}_{\mathrm{R}}$ (minor) $=30.5$ $\min$.

$[\alpha]^{20} \mathrm{D}=-42.8\left(c 0.73, \mathrm{CH}_{2} \mathrm{Cl}_{2}\right)$.

Recrystallization: 3 ja (105 mg, 92:8 er) was dissolved in $\mathrm{CH}_{2} \mathrm{Cl}_{2} / \mathrm{Et}_{2} \mathrm{O}(1: 20,10 \mathrm{~mL})$ and the solution was concentrated by slow evaporation at $23{ }^{\circ} \mathrm{C}$ until a white crystalline precipitate appeared. The precipitate was filtered, washed with $n$-hexane and dried under vacuum. (64 mg, $61 \%$ yield, $98.5: 1.5 \mathrm{er}$ ).

Absolute configuration of stereochemistry of $(R)$-3ja was determined by X-ray analysis of suitable crystals obtained by slow evaporation of a concentrated solution of $(R)-3 \mathbf{j a}$ in $\mathrm{CH}_{2} \mathrm{Cl}_{2} / \mathrm{Et}_{2} \mathrm{O} 1: 20$ at $23^{\circ} \mathrm{C}$. 


\section{<Chromatogram>}

mAU

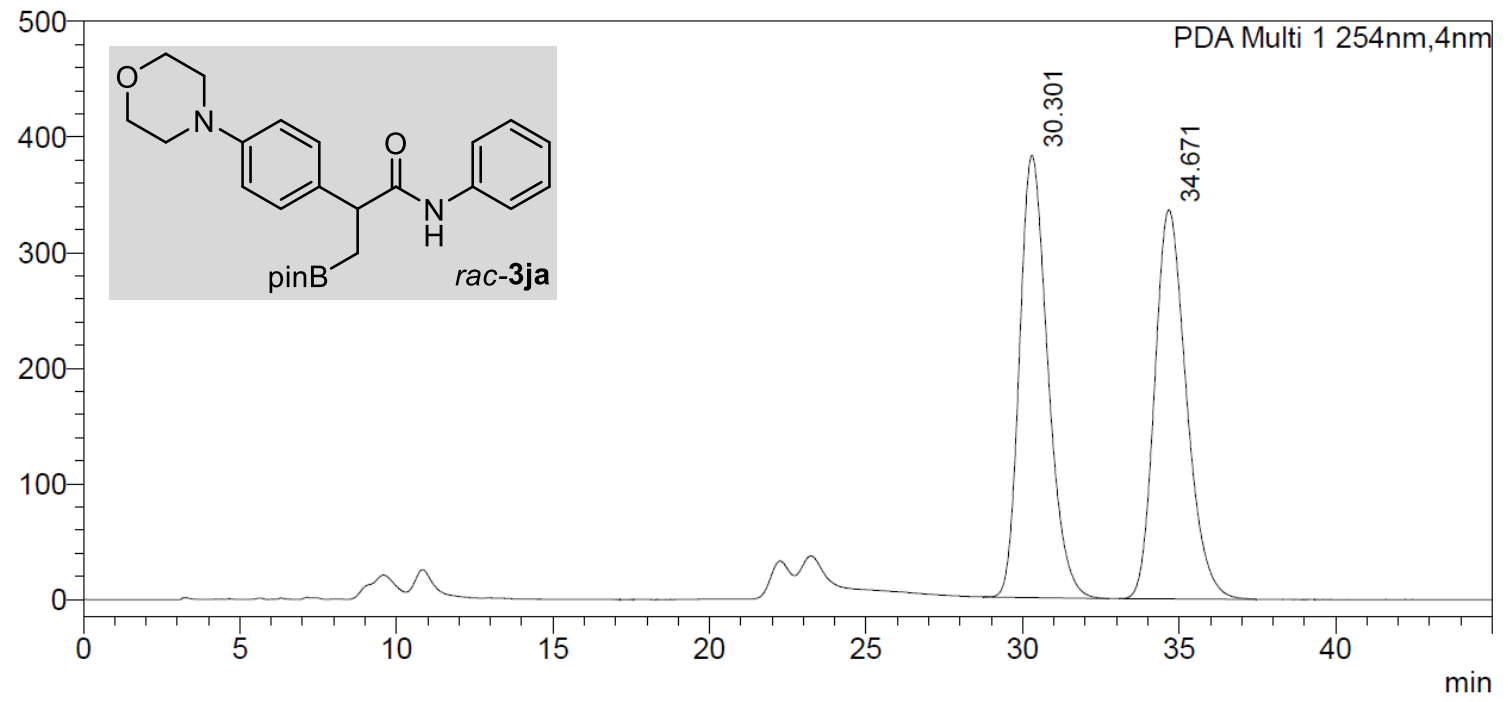

<Peak Table>

PDA Ch1 254nm

\begin{tabular}{|r|r|r|r|}
\hline Peak\# & Ret. Time & Area & \multicolumn{1}{|c|}{ Area\% } \\
\hline 1 & 30.301 & 24029332 & 50.299 \\
\hline 2 & 34.671 & 23744053 & 49.701 \\
\hline Total & & 47773385 & 100.000 \\
\hline
\end{tabular}

\section{<Chromatogram>}

$\mathrm{mAU}$

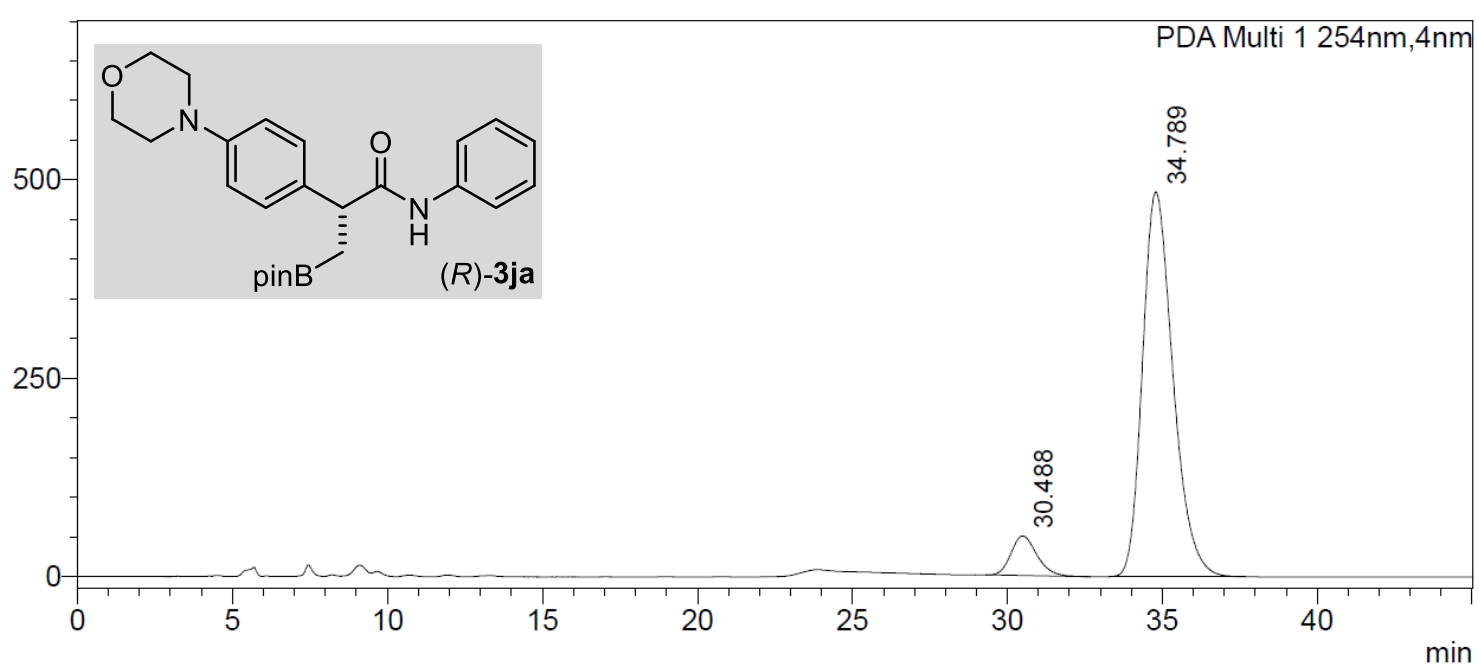

<Peak Table>

PDA Ch1 254nm
\begin{tabular}{|r|r|r|r|}
\hline Peak\# & Ret. Time & Area & Area $\%$ \\
\hline 1 & 30.488 & 2903244 & 8.040 \\
\hline 2 & 34.789 & 33208866 & 91.960 \\
\hline Total & & 36112110 & 100.000 \\
\hline
\end{tabular}




\section{<Chromatogram>}

mAU

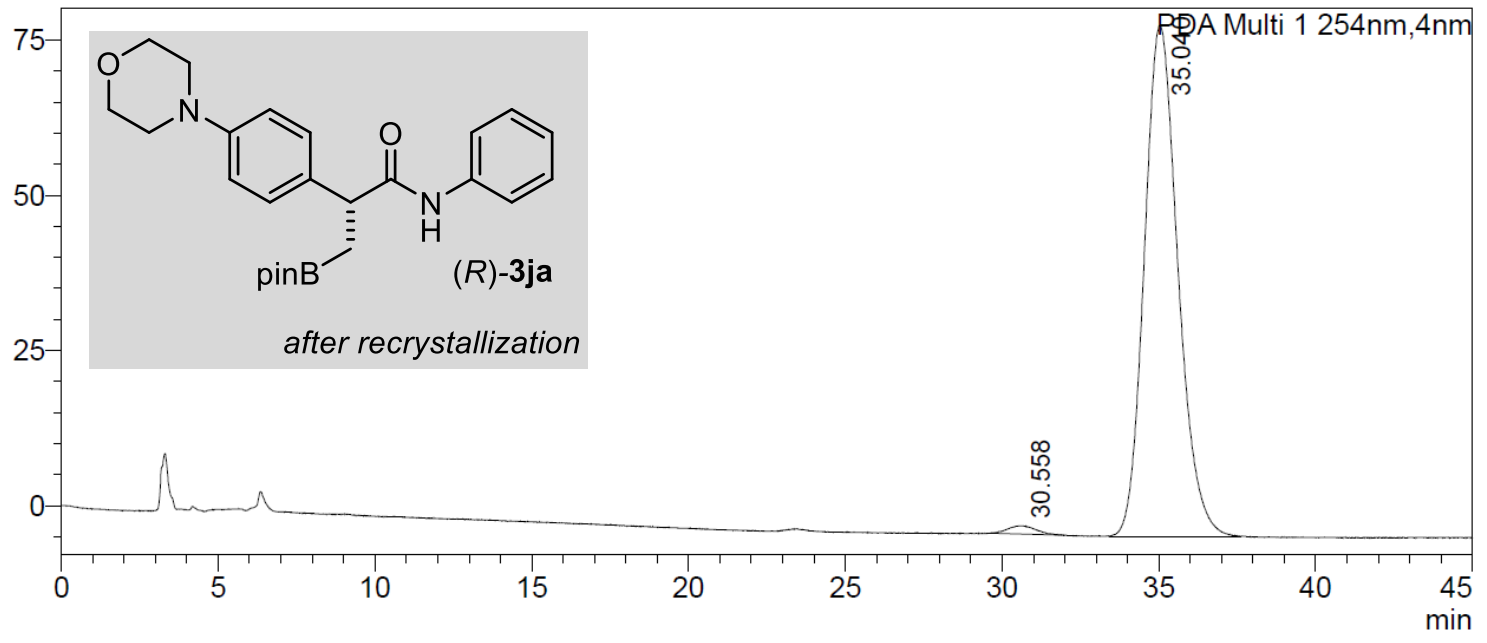

<Peak Table>

PDA Ch1 254nm

\begin{tabular}{|r|r|r|r|}
\hline Peak\# & Ret. Time & \multicolumn{1}{|c|}{ Area } & \multicolumn{1}{c|}{ Area\% } \\
\hline 1 & 30.558 & 81619 & 1.316 \\
\hline 2 & 35.040 & 6122723 & 98.684 \\
\hline Total & & 6204342 & 100.000 \\
\hline
\end{tabular}


(R)-2-(4-bromophenyl)- $N$-phenyl-3-(4,4,5,5-tetramethyl-1,3,2-dioxaborolan-2-yl)propanamide $3 j g$

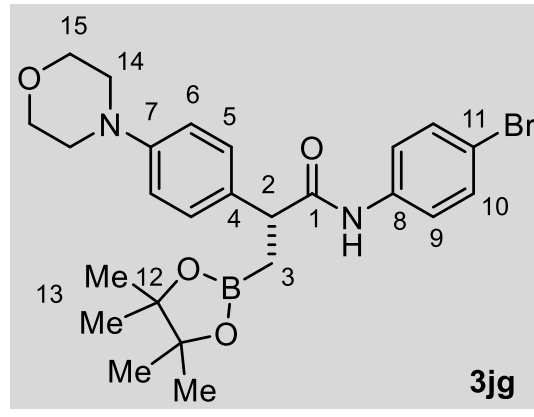

According to the general procedure for enantioselective copper-catalyzed borylative carboxamidation using styrene 1j (29 mg, $0.15 \mathrm{mmol}, 1.0$ equiv.), $\mathrm{CuCl}(1.5 \mathrm{mg}, 0.015$ mmol, $10 \mathrm{~mol} \%)$, ligand $(R, R)-6 \mathrm{k}(7 \mathrm{mg}, 0.017 \mathrm{mmol}, 11$ mol\%), LiOtBu (24 mg, 0.3 mmol, 2.0 equiv.), $B_{2} p_{i n}(57 \mathrm{mg}$, $0.23 \mathrm{mmol}, 1.5$ equiv.) and 4-isocyanato-1-bromobenzene 2g (119 mg, 0.60 mmol, 4.0 equiv.) in 2-Me-THF and $\mathrm{Et}_{2} \mathrm{O}$ $(1.5 \mathrm{~mL}, \mathrm{v}: \mathrm{v}=1: 1,0.1 \mathrm{M}) .90 \%$ conversion into $3 \mathrm{jg}$ determined by ${ }^{1} \mathrm{H}$ NMR analysis of the crude reaction mixture in presence of an internal standard. Purification by flash chromatography $\left(\mathrm{CH}_{2} \mathrm{Cl}_{2} / \mathrm{MeOH} 50: 1\right)$ gave pure 3jg as a white solid (60 mg, $0.12 \mathrm{mmol}, 78 \%$ yield).

TLC: $\mathrm{CH}_{2} \mathrm{Cl}_{2} / \mathrm{MeOH} 50: 1, \mathrm{R}_{\mathrm{f}}=0.2$.

${ }^{1} \mathrm{H}$ NMR $\left(500 \mathrm{MHz}, \mathrm{CDCl}_{3}\right) \delta(\mathrm{ppm})=7.35\left(\mathrm{~d},{ }^{3} \mathrm{JHH}_{\mathrm{HH}}=8.9 \mathrm{~Hz}, 2 \mathrm{H}, \mathrm{H}-10\right), 7.31\left(\mathrm{~d},{ }^{3} \mathrm{JHH}_{\mathrm{HH}}=8.9 \mathrm{~Hz}\right.$, $2 \mathrm{H}, H-9), 7.23\left(\mathrm{~d},{ }^{3} \mathrm{~J}_{\mathrm{HH}}=8.3 \mathrm{~Hz}, 2 \mathrm{H}, H-5\right), 7.09(\mathrm{bs}, 1 \mathrm{H}, \mathrm{N}-H), 6.89\left(\mathrm{~d},{ }^{3} \mathrm{~J}_{\mathrm{HH}}=8.3 \mathrm{~Hz}, 2 \mathrm{H}, \mathrm{H}-\right.$ 6), $3.86(\mathrm{~m}, 4 \mathrm{H}, H-13), 3.80\left(\mathrm{dd},{ }^{3} \mathrm{~J}_{\mathrm{HH}}=9.8,6.2 \mathrm{~Hz}, 1 \mathrm{H}, H-2\right), 3.15(\mathrm{~m}, 4 \mathrm{H}, H-12), 1.61$ (dd, $\left.{ }^{2} J_{H H}=15.7,{ }^{3} J_{H H}=9.9 \mathrm{~Hz}, 1 \mathrm{H}, H-3\right), 1.24\left(\mathrm{dd},{ }^{2} J_{H H}=15.7,{ }^{3} J_{H H}=6.3 \mathrm{~Hz}, 1 \mathrm{H}, H-3\right.$ '), $1.22(\mathrm{~s}$, 6H, $H$-MeBpin), 1.19 (s, 6H, H-MeBpin).

${ }^{13} \mathrm{C}\left\{{ }^{1} \mathrm{H}\right\}$ NMR $\left(126 \mathrm{MHz}, \mathrm{CDCl}_{3}\right) \delta(\mathrm{ppm})=173.1(C-1), 150.7(C-7), 137.3(C-8), 132.6(C-4)$, 131.9 ( $\mathrm{CH}-10), 128.9$ (CH-5), 121.2 (CH-9), 116.5 (C-11), 116.2 (CH-6), 83.5 (CO-Bpin), 67.0 ( $\left.\mathrm{CH}_{2}-13\right)$, $49.3\left(\mathrm{CH}_{2}-12\right)$, $49.2(\mathrm{CH}-2), 25.0\left(\mathrm{CH}_{3}-\mathrm{MeBpin}\right), 24.8\left(\mathrm{CH}_{3}-\mathrm{MeBpin}\right), 16.9$ (bs, $\mathrm{CH}_{2}-$ 3).

${ }^{11} \mathbf{B}$ NMR $\left(128 \mathrm{MHz}, \mathrm{CDCl}_{3}\right) \delta(\mathrm{ppm})=34.4$.

HRMS (ESI +): calculated for $\mathrm{C}_{25} \mathrm{H}_{32} \mathrm{BBrN}_{2} \mathrm{O}_{4}[\mathrm{M}+\mathrm{H}]^{+}: 515.1716$; found: 515.1685 .

IR (neat): $v\left(\mathrm{~cm}^{-1}\right)=3348,2981,1682,1593,1517,1370,1323,1248,1140,1118,927,845$, 824.

m.p.: $214-216^{\circ} \mathrm{C}$

HPLC analysis of $\mathbf{3 j g}$ after oxidation to the corresponding alcohol following the general procedure indicated 92:8 er, chiral stationary column: $\mathrm{AD}-\mathrm{H}$, mobile phase: $n$-hexane/ $\mathrm{PrOH}=$ $80: 20,1.0 \mathrm{~mL} / \mathrm{min}, 254 \mathrm{~nm}, 30^{\circ} \mathrm{C}, \mathrm{t}_{\mathrm{R}}$ (major) $=28.5 \mathrm{~min}, \mathrm{t}_{\mathrm{R}}($ minor $)=18.5 \mathrm{~min}$.

$[\alpha]^{20} \mathrm{D}=-30.7\left(c 0.25, \mathrm{CH}_{2} \mathrm{Cl}_{2}\right)$. 


\section{<Chromatogram>}

$\mathrm{mAU}$

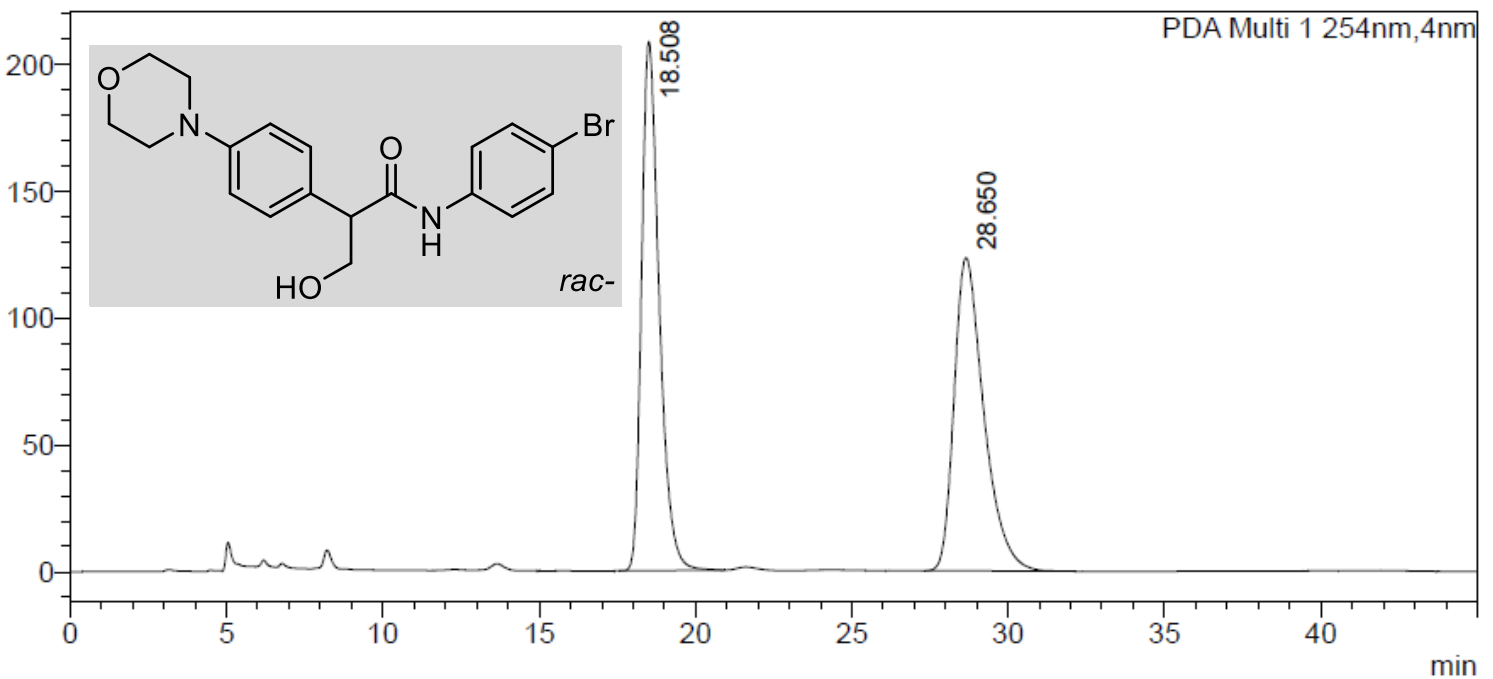

<Peak Table>

PDA Ch1 254nm

\begin{tabular}{|r|r|r|r|}
\hline Peak\# & Ret. Time & \multicolumn{1}{|c|}{ Area } & \multicolumn{1}{c|}{ Area $\%$} \\
\hline 1 & 18.508 & 8210153 & 50.121 \\
\hline 2 & 28.650 & 8170549 & 49.879 \\
\hline Total & & 16380702 & 100.000 \\
\hline
\end{tabular}

\section{$<$ Chromatogram $>$}

mAU

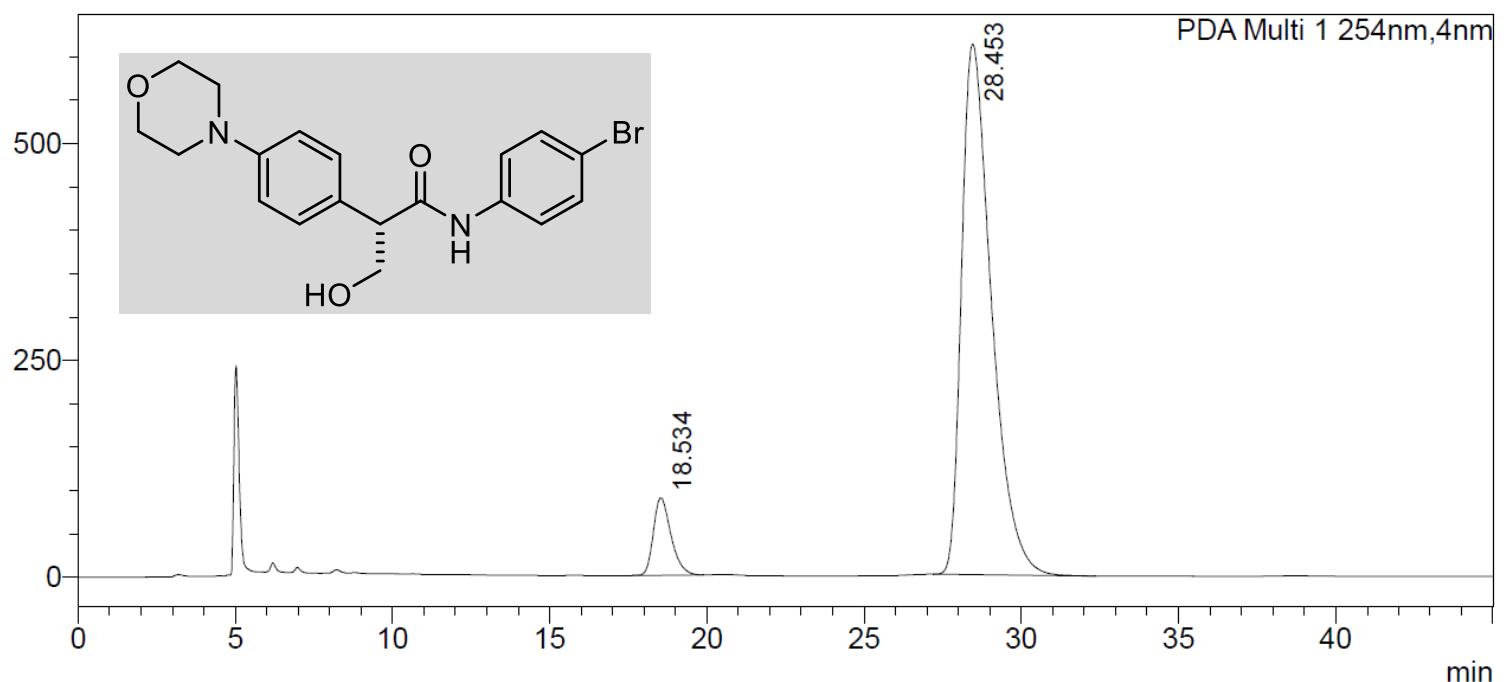

<Peak Table>

PDA Ch1 254nm

Peak\# Ret. Time

\begin{tabular}{|c|c|c|c|}
\hline Deak\# & Ret. Time & Area & Area\% \\
\hline 1 & 18.534 & 3498530 & 7.943 \\
\hline 2 & 28.453 & 40547258 & 92.057 \\
\hline Tota & & 44045788 & 100.000 \\
\hline
\end{tabular}


tert-butyl (R)-5-(1-oxo-1-(phenylamino)-3-(4,4,5,5-tetramethyl-1,3,2-dioxaborolan-2-yl) propan-2-yl)-1H-indole-1-carboxylate 3la

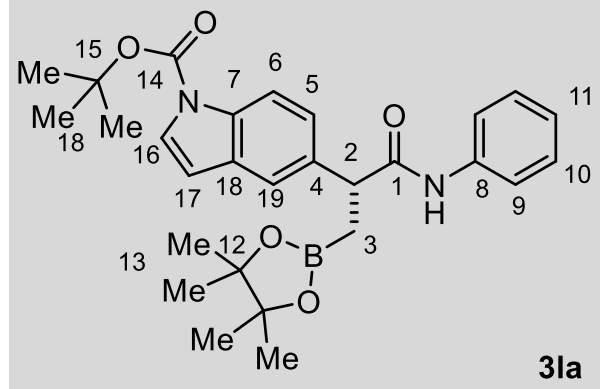

in 2-Me-THF and $\mathrm{Et}_{2} \mathrm{O}(3.0 \mathrm{~mL}, \mathrm{v}: \mathrm{v}=1: 1,0.1 \mathrm{M}) .75 \%$ conversion into 3la determined by ${ }^{1} \mathrm{H}$ NMR analysis of the crude reaction mixture in presence of an internal standard. Purification by flash chromatography (pentane/diethyl ether 3:1) gave pure 3la as a white solid (89 mg, $0.18 \mathrm{mmol}, 61 \%$ yield).

TLC: pentane/ethyl acetate $4: 1, \mathrm{R}_{\mathrm{f}}=0.3$.

${ }^{1} \mathrm{H}$ NMR $\left(400 \mathrm{MHz}, \mathrm{CDCl}_{3}\right) \delta(\mathrm{ppm})=8.13\left(\mathrm{~d},{ }^{3} \mathrm{~J}_{\mathrm{HH}}=8.5 \mathrm{~Hz}, 1 \mathrm{H}, \mathrm{H}-6\right), 7.63\left(\mathrm{~d},{ }^{3} \mathrm{~J}_{\mathrm{HH}}=3.7 \mathrm{~Hz}\right.$, $1 \mathrm{H}, H-16), 7.57\left(\mathrm{~d},{ }^{4} J_{H H}=1.8 \mathrm{~Hz}, 1 \mathrm{H}\right), 7.40(\mathrm{~m}, 2 \mathrm{H}), 7.34(\mathrm{~m}, 2 \mathrm{H}), 7.25(\mathrm{~m}, 2 \mathrm{H}), 7.10(\mathrm{~m}, 1 \mathrm{H})$, $7.05(\mathrm{~m}, 1 \mathrm{H}), 6.56\left(\mathrm{~d},{ }^{3} J_{\mathrm{HH}}=3.7 \mathrm{~Hz}, 1 \mathrm{H}, \mathrm{H}-17\right), 4.01\left(\mathrm{dd},{ }^{3} J_{\mathrm{HH}}=9.5,6.5 \mathrm{~Hz}, 1 \mathrm{H}, H-2\right), 1.74(\mathrm{dd}$, $\left.{ }^{2} J_{H H}=15.7 \mathrm{~Hz},{ }^{3} J_{H H}=9.7 \mathrm{~Hz}, 1 \mathrm{H}, H-3\right), 1.69(\mathrm{~s}, 9 \mathrm{H}), 1.35\left(\mathrm{dd},{ }^{2} J_{\mathrm{HH}}=15.7 \mathrm{~Hz},{ }^{3} J_{\mathrm{HH}}=6.6 \mathrm{~Hz}\right.$, $\left.1 \mathrm{H}, H_{-3}\right), 1.24(\mathrm{~s}, 6 \mathrm{H}), 1.22(\mathrm{~s}, 6 \mathrm{H})$.

${ }^{13} \mathrm{C}\left\{{ }^{1} \mathrm{H}\right\}$ NMR $\left(101 \mathrm{MHz}, \mathrm{CDCl}_{3}\right) \delta(\mathrm{ppm})=172.8(C-1), 149.7(C-14), 138.1(C-8), 135.9(C-$ 4), 134.5 (C-7), 131.1 (C-18), 128.8 (C-10), 126.6 (C-17), $124.2(C-5), 123.9(C-11), 120.3(C-$ 6), $119.4(C-9), 115.7(C-19), 107.2(C-16), 83.8(C-15), 83.3(C-12), 49.8(C-2), 28.2(C-18)$, $24.8(C-3), 24.7(C-13), 17.1(C-3)$.

${ }^{11} \mathbf{B}$ NMR $\left(128 \mathrm{MHz}, \mathrm{CDCl}_{3}\right) \delta(\mathrm{ppm})=33.9$.

HRMS (ESI +): calculated for $\mathrm{C}_{28} \mathrm{H}_{35} \mathrm{BN}_{2} \mathrm{O}_{5}[\mathrm{M}+\mathrm{H}]^{+}: 491.2717$; found: 491.2728 .

IR (neat): $v\left(\mathrm{~cm}^{-1}\right)=3458,3017,3003,2971,2948,1738,1686,1600,1533,1500,1438$, 1366, 1330, 1229, 1217, 1206, 1140, 1079, 1025, 967, 895, 753, 693.

m.p.: $183-185^{\circ} \mathrm{C}$

HPLC analysis of 3la indicated 92:8 er, chiral stationary column: OD-H, mobile phase: $n$ hexane $/ \mathrm{PrOH}=95: 55,1.0 \mathrm{~mL} / \mathrm{min}, 254 \mathrm{~nm}, 30^{\circ} \mathrm{C}, \mathrm{t}_{\mathrm{R}}$ (major) $=15.8 \mathrm{~min}, \mathrm{t}_{\mathrm{R}}$ (minor) $=11.1$ $\min$

$[\alpha]^{20} \mathrm{D}=-50.0\left(c 1.34, \mathrm{CH}_{2} \mathrm{Cl}_{2}\right)$.

Recrystallization: 3la (86 mg, 92:8 er) was dissolved in $\mathrm{CH}_{2} \mathrm{Cl}_{2} / \mathrm{Et}_{2} \mathrm{O}(1: 20,6 \mathrm{~mL})$ and then and the solution was concentrated by slow evaporation at $23^{\circ} \mathrm{C}$ until a white crystalline precipitate appeared. The precipitate was filtered, washed with $n$-hexane and dried under vacuum. (68 mg, 79\% yield, 96:4 er). 


\section{<Chromatogram>}

mAU

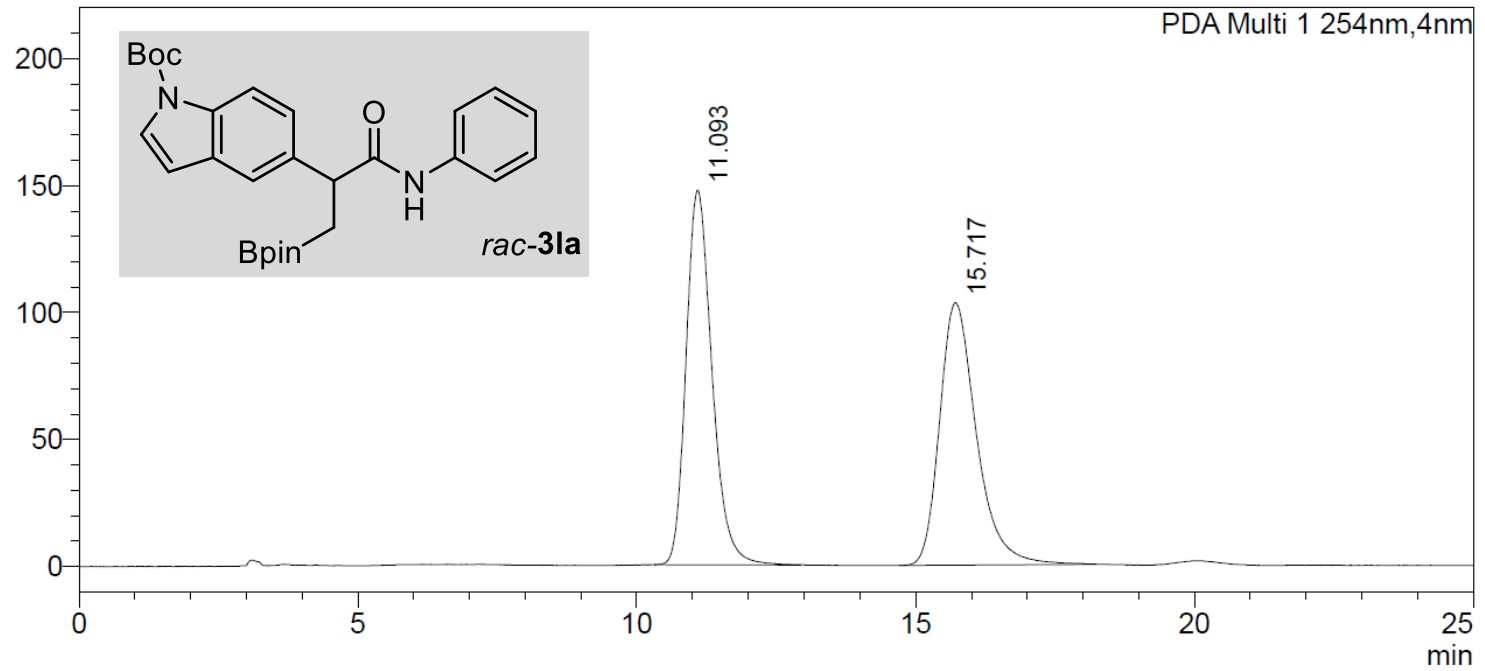

\section{<Peak Table>}

PDA Ch1 254nm

\begin{tabular}{|r|r|l|r|}
\hline Peak\# & Ret. Time & Area & \multicolumn{1}{|c|}{ Area\% } \\
\hline 1 & 11.093 & 4785642 & 50.166 \\
\hline 2 & 15.717 & 4753897 & 49.834 \\
\hline Total & & 9539539 & 100.000 \\
\hline
\end{tabular}

\section{<Chromatogram>}

mAU

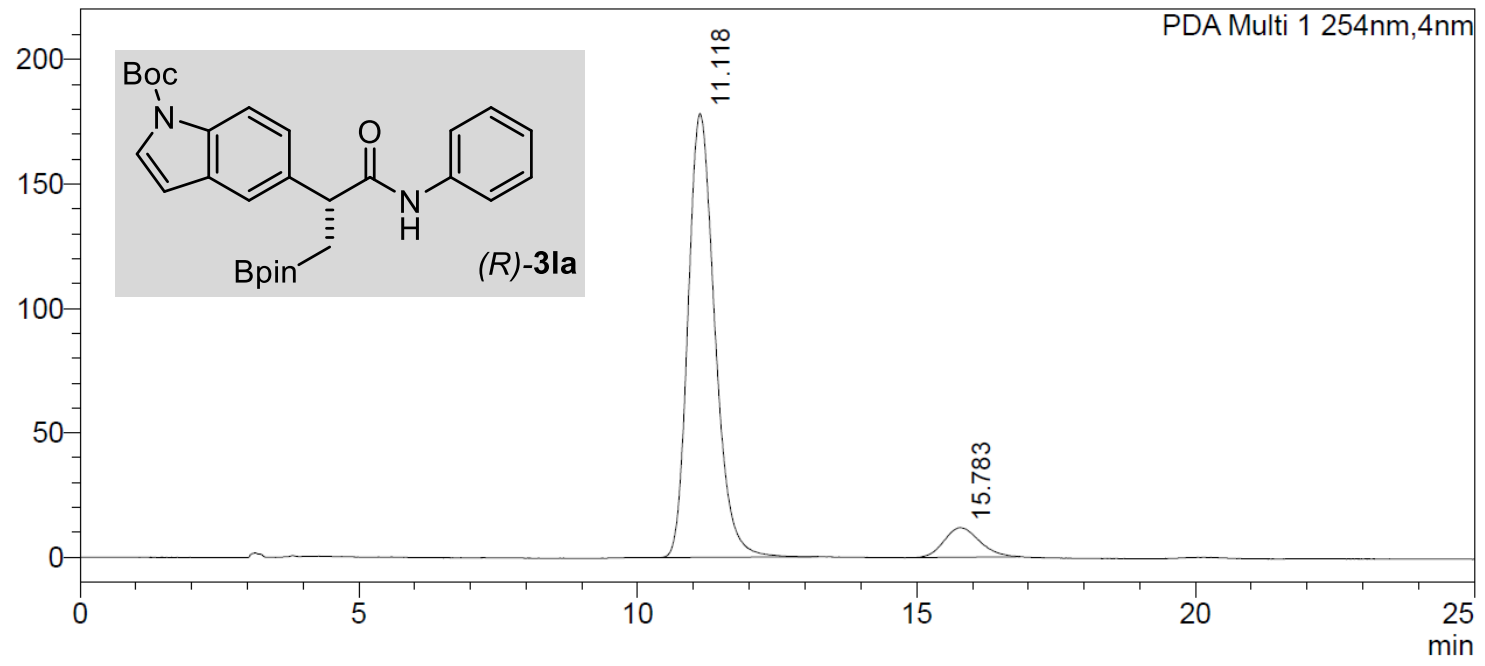

\section{<Peak Table>}

PDA Ch1 254nm

\begin{tabular}{|r|r|r|r|}
\hline Peak\# & Ret. Time & \multicolumn{1}{|c|}{ Area } & \multicolumn{1}{c|}{ Area\% } \\
\hline 1 & 11.118 & 5943525 & 91.909 \\
\hline 2 & 15.783 & 523211 & 8.091 \\
\hline Total & & 6466735 & 100.000 \\
\hline
\end{tabular}


tert-butyl $\quad(R)$-5-(1-((4-bromophenyl)amino)-1-oxo-3-(4,4,5,5-tetramethyl-1,3,2-dioxaborolan2-yl)propan-2-yl)-1H-indole-1-carboxylate 3lg

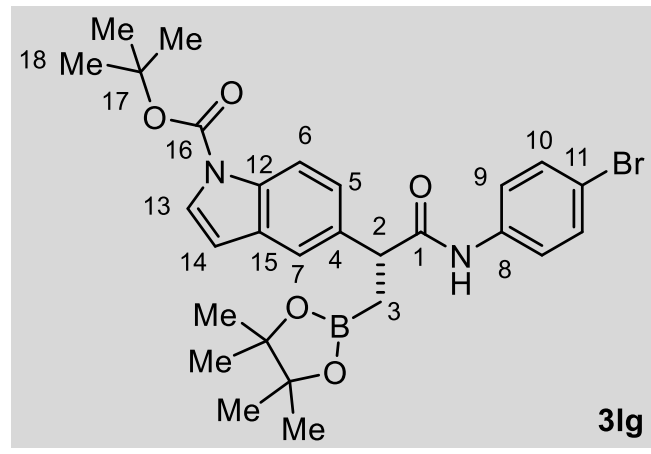

According to the general procedure for enantioselective copper-catalyzed borylative carboxamidation using styrene 1 l (37 mg, $0.15 \mathrm{mmol}$, 1.0 equiv.), $\mathrm{CuCl}(1.5 \mathrm{mg}, 0.015 \mathrm{mmol}, 10 \mathrm{~mol} \%)$, ligand $(R, R)-6 \mathbf{k}(7 \mathrm{mg}, 0.017 \mathrm{mmol}, 11 \mathrm{~mol} \%)$, LiOtBu (24 mg, 0.3 mmol, 2.0 equiv.), $B_{2} p_{i n}$ (57 mg, 0.23

$3 \mathrm{lg}$ mol, 1.5 equiv.) and 4-isocyanato-1-bromobenzene $2 \mathrm{~g}$ (119 mg, $0.60 \mathrm{mmol}, 4.0$ equiv.) in 2-Me-THF and $\mathrm{Et}_{2} \mathrm{O}$ (1.5 mL, v:v = 1:1, $\left.0.1 \mathrm{M}\right) .90 \%$ conversion into $3 \mathrm{lg}$ determined by ${ }^{1} \mathrm{H}$ NMR analysis of the crude reaction mixture in presence of an internal standard. Purification by flash chromatography (pentane/diethyl ether 3:1) gave pure 3lg as a light brown solid (50 $\mathrm{mg}, 0.105 \mathrm{mmol}, 68 \%$ yield).

TLC: pentane/diethyl ether $3: 1, \mathrm{R}_{\mathrm{f}}=0.2$.

${ }^{1} \mathrm{H}$ NMR $\left(400 \mathrm{MHz}, \mathrm{CDCl}_{3}\right) \delta(\mathrm{ppm})=8.11\left(\mathrm{~d},{ }^{3} \mathrm{~J}_{\mathrm{HH}}=8.0 \mathrm{~Hz}, 1 \mathrm{H}, \mathrm{H}-6\right), 7.61\left(\mathrm{~d},{ }^{3} \mathrm{JHH}_{\mathrm{HH}}=3.6 \mathrm{~Hz}\right.$, $1 \mathrm{H}, H-13), 7.53\left(\mathrm{~d},{ }^{4} \mathrm{~J}_{\mathrm{HH}}=1.5 \mathrm{~Hz}, 1 \mathrm{H}, H-7\right), 7.34\left(\mathrm{~d},{ }^{3} \mathrm{~J}_{\mathrm{HH}}=9.0 \mathrm{~Hz}, 2 \mathrm{H}, H-10\right), 7.30-7.26(\mathrm{~d}$, $\left.{ }^{3} J_{H H}=9.0 \mathrm{~Hz}, 2 \mathrm{H}, H-9+\mathrm{m}, 1 \mathrm{H}, H-5\right), 7.07$ (bs, $\left.1 \mathrm{H}, \mathrm{N}-H\right), 6.54\left(\mathrm{~d},{ }^{3} \mathrm{~J}_{\mathrm{HH}}=4.1 \mathrm{~Hz}, 1 \mathrm{H}, H-14\right)$, $3.97\left(\mathrm{dd},{ }^{3} J_{\mathrm{HH}}=9.7,6.4 \mathrm{~Hz}, 1 \mathrm{H}, \mathrm{H}-2\right), 1.70\left(\mathrm{dd},{ }^{2} J_{\mathrm{HH}}=15.8,{ }^{3} J_{\mathrm{HH}}=9.7 \mathrm{~Hz}, 1 \mathrm{H}, H-3\right), 1.67(\mathrm{~s}$, $9 \mathrm{H}, H-18), 1.30\left(\mathrm{dd},{ }^{2} J_{\mathrm{HH}}=15.8,{ }^{3} \mathrm{~J}_{\mathrm{HH}}=6.4 \mathrm{~Hz}, 1 \mathrm{H}, H-3\right.$ ), 1.21 (s, 6H, $H$-MeBpin), 1.19 (s, 6H, $H$-MeBpin).

${ }^{13} \mathrm{C}\left\{{ }^{1} \mathrm{H}\right\}$ NMR $\left(101 \mathrm{MHz}, \mathrm{CDCl}_{3}\right) \delta(\mathrm{ppm})=173.1(C-1), 149.8(C-16), 137.3(C-8), 135.7(C-$ 4), 134.6 (C-12), 131.9 ( $\mathrm{CH}-10), 131.2(\mathrm{C}-15), 126.8(\mathrm{CH}-13), 124.3(\mathrm{CH}-5), 121.1(\mathrm{CH}-9)$, 120.4 (CH-7), 116.5 (C-11), 115.9 ( $\mathrm{CH}-6), 107.3$ (CH-14), 84.1 (C-17), 83.5 (CO-Bpin), 50.0 ( $\mathrm{CH}-2)$, 28.3 ( $\left.\mathrm{CH}_{3}-18\right)$, 24.9 ( $\mathrm{CH}_{3}$-MeBpin), 24.8 ( $\mathrm{CH}_{3}$-MeBpin), 17.1 (bs, $\left.\mathrm{CH}_{2}-3\right)$.

${ }^{11} \mathbf{B}$ NMR $\left(128 \mathrm{MHz}, \mathrm{CDCl}_{3}\right) \delta(\mathrm{ppm})=33.7$.

HRMS (ESI +): calculated for $\mathrm{C}_{28} \mathrm{H}_{34} \mathrm{BBrN}_{2} \mathrm{O}_{5}[\mathrm{M}+\mathrm{H}]^{+}: 569.1822$; found: 504.1819 .

IR (neat): $v\left(\mathrm{~cm}^{-1}\right)=3350,2980,1735,1683,1593,1525,1469,1371,1336,1257,1167$, $1141,1023,843,826$.

m.p.: $190-191^{\circ} \mathrm{C}$

HPLC analysis of $\mathbf{3 l g}$ after oxidation to the corresponding alcohol following the general procedure indicated 93:7 er, chiral stationary column: OD-H, mobile phase: hexane/ $\mathrm{PrOH}=$ $95: 5,1.0 \mathrm{~mL} / \mathrm{min}, 254 \mathrm{~nm}, 30^{\circ} \mathrm{C}, \mathrm{t}_{\mathrm{R}}$ (major) $=27.5 \mathrm{~min}, \mathrm{t}_{\mathrm{R}}$ (minor) $=20.7 \mathrm{~min}$.

$[\alpha]^{20}{ }_{D}=-55.1\left(c 0.25, \mathrm{CH}_{2} \mathrm{Cl}_{2}\right)$. 


\section{$<$ Chromatogram>}

mAU

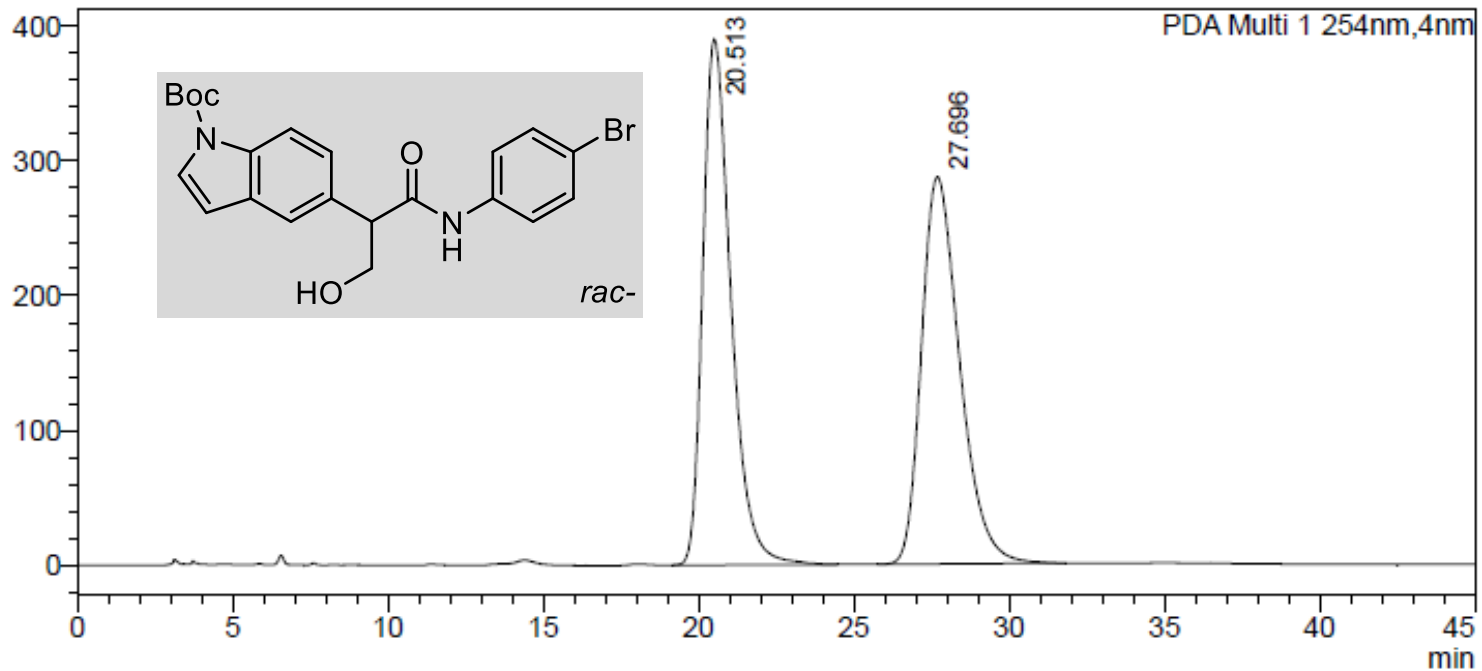

<Peak Table>

PDA Ch1 254nm

\begin{tabular}{|r|r|r|r|}
\hline Peak\# & Ret. Time & \multicolumn{1}{|c|}{ Area } & \multicolumn{1}{c|}{ Area\% } \\
\hline 1 & 20.513 & 24644804 & 50.054 \\
\hline 2 & 27.696 & 24591301 & 49.946 \\
\hline Total & & 49236105 & 100.000 \\
\hline
\end{tabular}

$<$ Chromatogram>

mAU

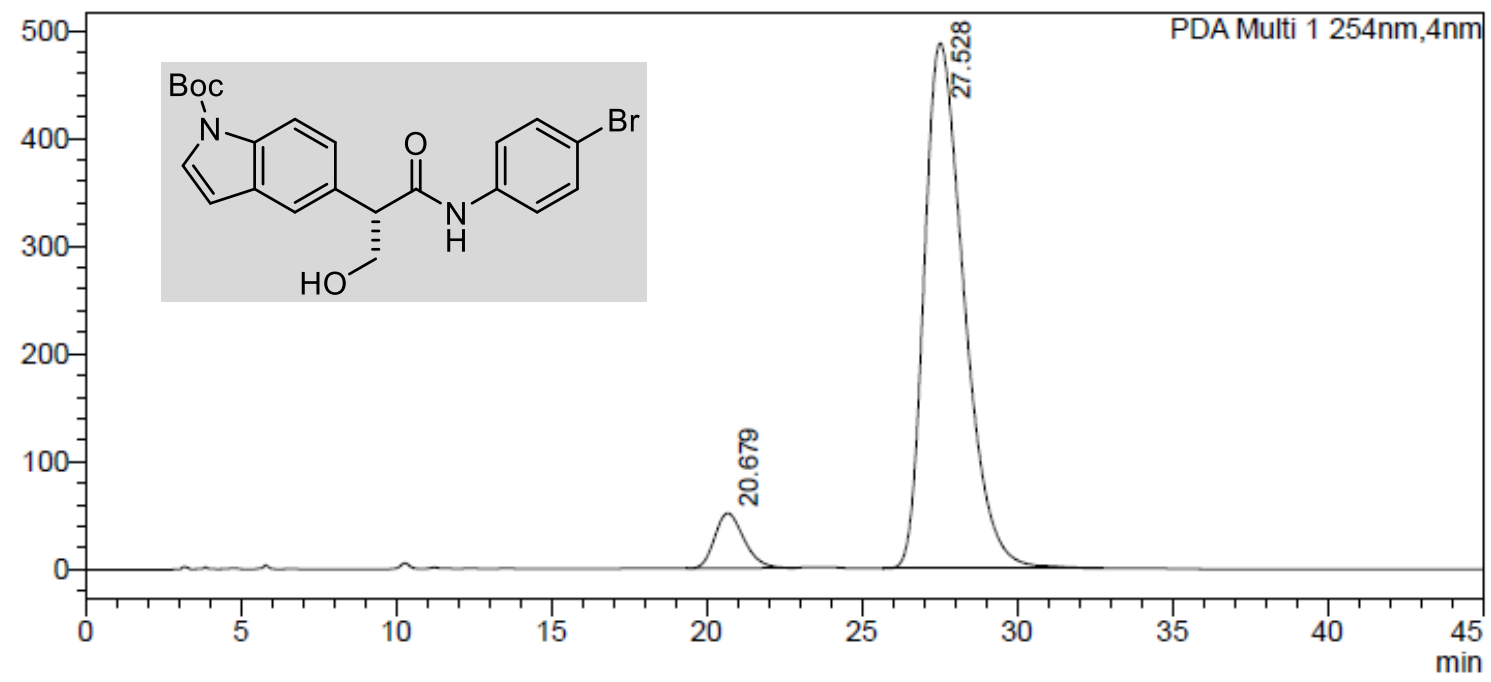

<Peak Table>

PDA Ch1 254nm

\begin{tabular}{|r|r|r|r|}
\hline Peak\# & Ret. Time & \multicolumn{1}{|c|}{ Area } & \multicolumn{1}{c|}{ Area $\%$} \\
\hline 1 & 20.679 & 3334753 & 7.122 \\
\hline 2 & 27.528 & 43489391 & 92.878 \\
\hline Total & & 46824143 & 100.000 \\
\hline
\end{tabular}


(R)-2-(3-methoxyphenyl)-N-phenyl-3-(4,4,5,5-tetramethyl-1,3,2-dioxaborolan-2yl)propanamide 3ya

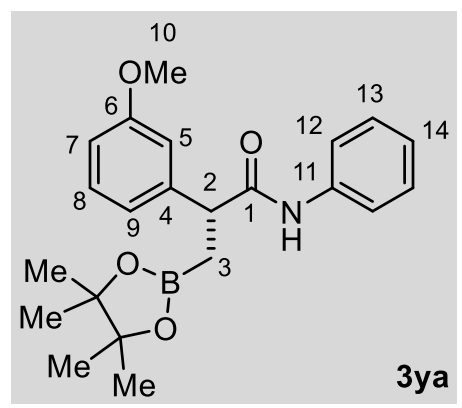

According to the general procedure for enantioselective coppercatalyzed borylative carboxamidation using 3-methoxystyrene 1y (20 mg, $0.15 \mathrm{mmol}, 1.0$ equiv.), $\mathrm{CuCl}(1.5 \mathrm{mg}, 0.015 \mathrm{mmol}, 10$ mol\%), ligand $(R, R)-6 \mathbf{k}(7.0 \mathrm{mg}, 0.017 \mathrm{mmol}, 11 \mathrm{~mol} \%)$, LiOtBu (24 mg, 0.3 mmol, 2.0 equiv.), $B_{2}$ pin $_{2}(57 \mathrm{mg}, 0.23 \mathrm{mmol}, 1.5$ equiv.) and isocyanatobenzene $2 \mathrm{a}(0.065 \mathrm{~mL}, 0.6 \mathrm{mmol}, 4.0$ equiv.) in 2-Me-THF and $\mathrm{Et}_{2} \mathrm{O}(1.5 \mathrm{~mL}, \mathrm{v}: \mathrm{v}=1: 1,0.1 \mathrm{M}) .90 \%$ conversion into 3ya determined by ${ }^{1} \mathrm{H}$ NMR analysis of the crude reaction mixture in presence of an internal standard. Purification by flash chromatography (pentane/diethyl ether 9:1) gave pure 3ya as a white solid (35 mg, $0.09 \mathrm{mmol}, 61 \%$ yield).

TLC: pentane/ethyl acetate 9:1, $R_{f}=0.2$.

${ }^{1} \mathrm{H}$ NMR $\left(300 \mathrm{MHz}, \mathrm{CDCl}_{3}\right) \delta(\mathrm{ppm})=7.41(\mathrm{~m}, 2 \mathrm{H}, \mathrm{H}-A r), 7.33-7.23(\mathrm{~m}, 3 \mathrm{H}, \mathrm{H}-A r), 7.12(\mathrm{bs}$, $1 \mathrm{H}, \mathrm{N}-H), 7.04\left(\mathrm{t},{ }^{3} \mathrm{JHH}_{\mathrm{HH}}=7.7 \mathrm{~Hz}, 1 \mathrm{H}, \mathrm{H}-14\right), 6.94\left(\mathrm{~d},{ }^{3} \mathrm{~J}_{\mathrm{HH}}=7.7 \mathrm{~Hz}, 1 \mathrm{H}, \mathrm{H}-9\right), 6.90\left(\mathrm{t},{ }^{4} \mathrm{~J}_{\mathrm{HH}}=2.0\right.$ $\mathrm{Hz}, 1 \mathrm{H}, H-5), 6.82\left(\mathrm{dd},{ }^{3} \mathrm{JHH}_{\mathrm{HH}}=8.2 \mathrm{~Hz},{ }^{4} \mathrm{~J}_{\mathrm{HH}}=2.0 \mathrm{~Hz}, 1 \mathrm{H}, \mathrm{H}-7\right), 3.86\left(\mathrm{dd},{ }^{3} \mathrm{~J}_{\mathrm{HH}}=9.6,6.3 \mathrm{~Hz}, 1 \mathrm{H}\right.$, $H-2), 3.79(\mathrm{~s}, 3 \mathrm{H}, H-10), 1.65\left(\mathrm{dd},{ }^{2} J_{H H}=15.7,{ }^{3} J_{H H}=9.8 \mathrm{~Hz}, 1 \mathrm{H}, H-3\right), 1.28\left(\mathrm{dd},{ }^{2} J_{H H}=15.7\right.$, ${ }^{3} J_{H H}=6.3 \mathrm{~Hz}, 1 \mathrm{H}, H-3$ '), 1.22 (s, 6H, H-MeBpin), 1.20 (s, 6H, H-MeBpin).

${ }^{13} \mathrm{C}\left\{{ }^{1} \mathrm{H}\right\}$ NMR $\left.(75 \mathrm{MHz}, \mathrm{CDCl})_{3}\right) \delta(\mathrm{ppm})=172.4(C-1), 160.2(C-6), 143.2(C-4), 138.2(C-11)$, $130.2(\mathrm{CH}-8), 129.0$ ( $\mathrm{CH}-13), 124.2(\mathrm{CH}-14), 120.4(\mathrm{CH}-9), 119.7(\mathrm{CH}-12), 113.6(\mathrm{CH}-5)$, 113.2 (CH-7), 83.5 (CO-Bpin), $55.4\left(\mathrm{CH}_{3}-10\right), 50.1(\mathrm{CH}-2), 25.0\left(\mathrm{CH}_{3}-\mathrm{MeBpin}\right), 24.8\left(\mathrm{CH}_{3^{-}}\right.$ MeBpin), 16.8 (bs, $\mathrm{CH}_{2}-3$ ).

${ }^{11} \mathbf{B}$ NMR $\left(96 \mathrm{MHz}, \mathrm{CDCl}_{3}\right) \delta(\mathrm{ppm})=34.2$.

HRMS (ESI +): calculated for $\mathrm{C}_{22} \mathrm{H}_{28} \mathrm{BNO}_{4}[\mathrm{M}+\mathrm{Na}]^{+}: 404.2008$; found: 404.2008.

IR (neat): $v\left(\mathrm{~cm}^{-1}\right)=3319,2978,1716,1667,1597,1520,1499,1439,1369,1318,1259$, 1165, 1142, 968, 847, 754, 692.

m.p.: $130-132{ }^{\circ} \mathrm{C}$

HPLC analysis of 3ya indicated 88:12 er, chiral stationary column: OD-H, mobile phase: $n$ hexane $/ \mathrm{PrOH}=95: 5,1.0 \mathrm{~mL} / \mathrm{min}, 246 \mathrm{~nm}, 30^{\circ} \mathrm{C}, \mathrm{t}_{\mathrm{R}}=14.1 \mathrm{~min}$ (major), $\mathrm{t}_{\mathrm{R}}=15.7 \mathrm{~min}$ (minor). $[\alpha]^{20} \mathrm{D}=-34.6\left(c 0.25, \mathrm{CH}_{2} \mathrm{Cl}_{2}\right)$. 


\section{$<$ Chromatogram >}

mAU

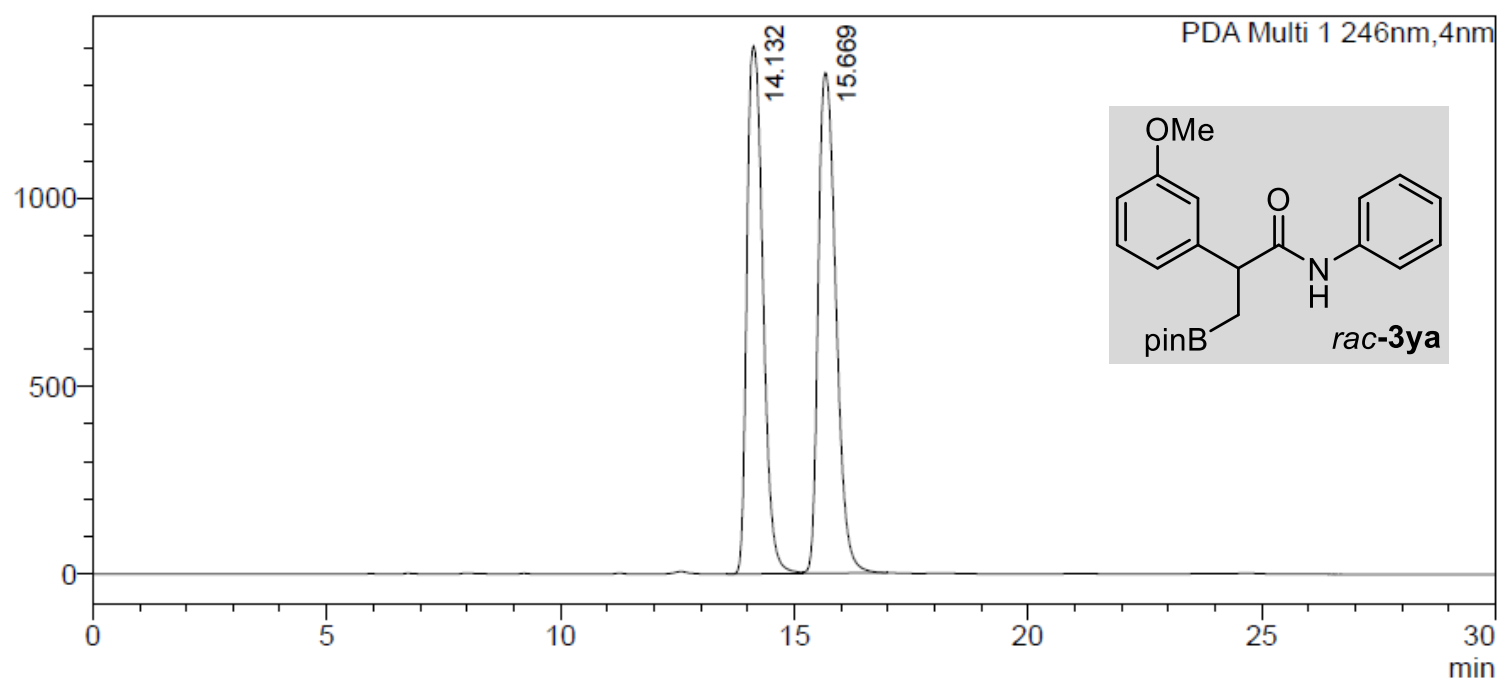

<Peak Table>

\begin{tabular}{|c|c|c|c|}
\hline & Ret. Time & Area & Area $\%$ \\
\hline 1 & 14.132 & 33663708 & 48.954 \\
\hline 2 & 15.669 & 35102258 & 51.046 \\
\hline Total & & 68765966 & 100.000 \\
\hline
\end{tabular}

<Chromatogram>

mAU

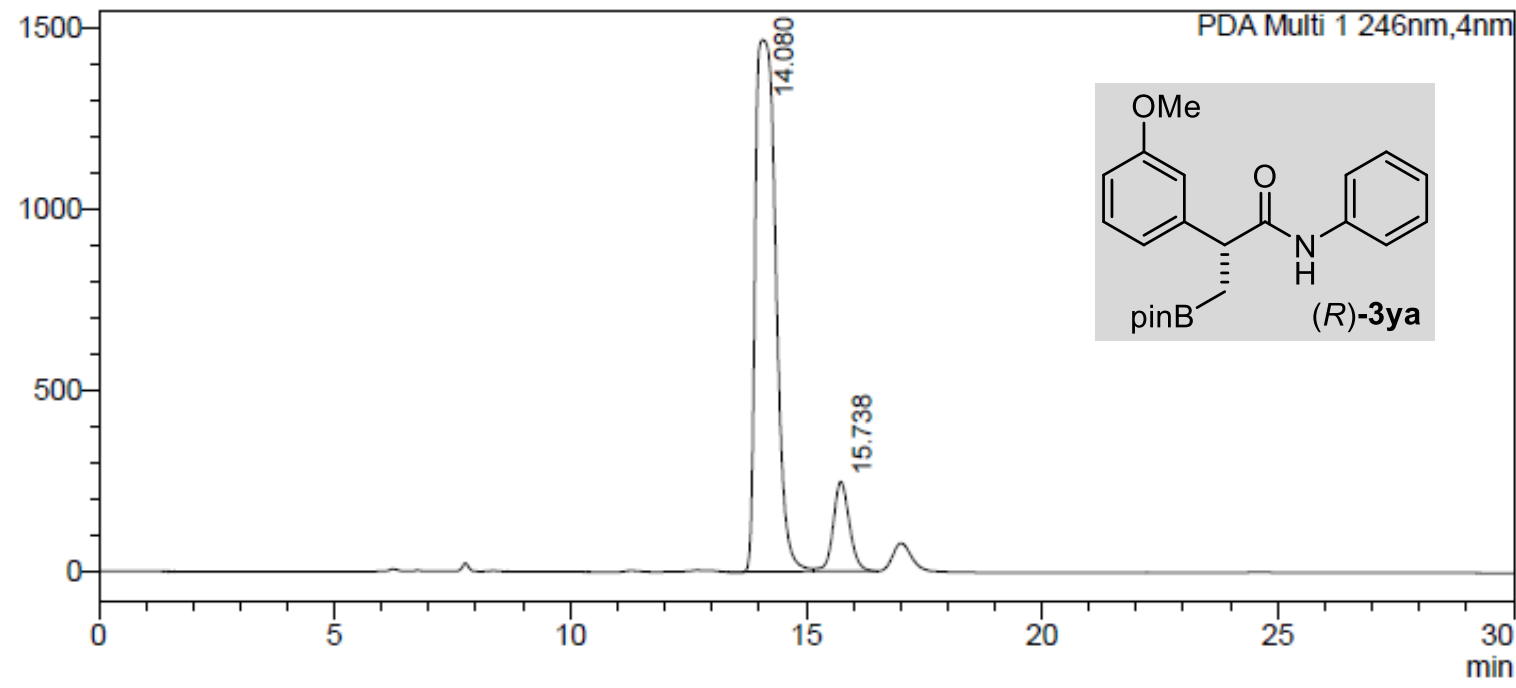

<Peak Table>

PDA Ch1 246nm

\begin{tabular}{|r|r|r|r|}
\hline Peak\# & Ret. Time & \multicolumn{1}{|c|}{ Area } & Area\% \\
\hline 1 & 14.080 & 44299254 & 88.233 \\
\hline 2 & 15.738 & 5908128 & 11.767 \\
\hline Total & & 50207382 & 100.000 \\
\hline
\end{tabular}


(R)-2-(3-methoxyphenyl)-N-(4-methoxyphenyl)-3-(4,4,5,5-tetramethyl-1,3,2-dioxaborolan-2yl)propanamide $3 y b$

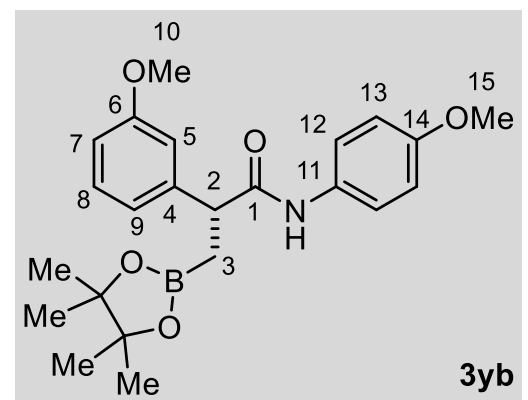

According to the general procedure for enantioselective copper-catalyzed borylative carboxamidation using 3methoxystyrene 1y (20 mg, $0.15 \mathrm{mmol}, 1.0$ equiv.), $\mathrm{CuCl}(1.5$ $\mathrm{mg}, 0.015 \mathrm{mmol}, 10 \mathrm{~mol} \%)$, ligand $(R, R)-6 \mathbf{k}(7.0 \mathrm{mg}, 0.017$ mmol, $11 \mathrm{~mol} \%$ ), LiOtBu (24 mg, 0.3 mmol, 2.0 equiv.), B $\mathrm{B}_{2} \mathrm{pin}_{2}$ (57 mg, $0.23 \mathrm{mmol}, 1.5$ equiv.) and 1-isocyanato 4methoxybenzene $\mathbf{2 b}(0.80 \mathrm{~mL}, 0.6 \mathrm{mmol}, 4.0$ equiv. $)$ in 2-Me-THF and $\mathrm{Et}_{2} \mathrm{O}(1.5 \mathrm{~mL}, \mathrm{v}: \mathrm{v}=1: 1$, $0.1 \mathrm{M}) .90 \%$ conversion into 3yb determined by ${ }^{1} \mathrm{H}$ NMR analysis of the crude reaction mixture in presence of an internal standard. Purification by flash chromatography (pentane/diethyl ether $4: 1)$ gave pure 3yb as a white solid ( $41 \mathrm{mg}, 0.10 \mathrm{mmol}, 67 \%$ yield).

TLC: pentane/ethyl acetate $7: 3, R_{f}=0.3$.

${ }^{1} \mathrm{H}$ NMR $\left(400 \mathrm{MHz}, \mathrm{CDCl}_{3}\right) \delta(\mathrm{ppm})=7.31\left(\mathrm{~d},{ }^{3} \mathrm{JHH}_{\mathrm{HH}}=9.0 \mathrm{~Hz}, 2 \mathrm{H}, \mathrm{H}-12\right), 7.25(\mathrm{~m}, 1 \mathrm{H}), 6.99(\mathrm{bs}$, $1 \mathrm{H}, \mathrm{N}-H), 6.93\left(\mathrm{~d},{ }^{3} \mathrm{JHH}_{\mathrm{HH}}=7.7 \mathrm{~Hz}, 1 \mathrm{H}, H-9\right), 6.90(\mathrm{~m}, 1 \mathrm{H}, H-5), 6.82(\mathrm{~m}, 1 \mathrm{H}, H-7), 6.79\left(\mathrm{~d},{ }^{3} J_{\mathrm{HH}}\right.$ $=9.0 \mathrm{~Hz}, 2 \mathrm{H}, H-13), 3.83\left(\mathrm{dd},{ }^{3} \mathrm{~J}_{\mathrm{HH}}=9.9,6.3 \mathrm{~Hz}, 1 \mathrm{H}, H-2\right), 3.79(\mathrm{~s}, 3 \mathrm{H}, H-10), 3.76(\mathrm{~s}, 3 \mathrm{H}, H-$ 15), $1.64\left(\mathrm{dd},{ }^{2} J_{\mathrm{HH}}=15.7,{ }^{3} \mathrm{~J}_{\mathrm{HH}}=9.7 \mathrm{~Hz}, 1 \mathrm{H}, \mathrm{H}-3\right), 1.27\left(\mathrm{dd},{ }^{2} J_{\mathrm{HH}}=15.7,{ }^{3} J_{\mathrm{HH}}=6.3 \mathrm{~Hz}, 1 \mathrm{H}, \mathrm{H}-\right.$ 3), 1.22 (s, 6H, H-MeBpin), 1.20 (s, 6H, H-MeBpin).

${ }^{13} \mathrm{C}\left\{{ }^{1} \mathrm{H}\right\}$ NMR $\left(101 \mathrm{MHz}, \mathrm{CDCl}_{3}\right) \delta(\mathrm{ppm})=172.2(C-1), 160.1(C-6), 156.3(C-14), 143.4(C-$ 4), 131.4 (C-11), $130.1(\mathrm{CH}-8), 121.5(\mathrm{CH}-12), 120.4(\mathrm{CH}-9), 114.1(\mathrm{CH}-13), 113.5(\mathrm{CH}-5)$, 113.1 (CH-7), 83.4 (CO-Bpin), $55.6\left(\mathrm{CH}_{3}-15\right), 55.4\left(\mathrm{CH}_{3}-10\right), 49.9(\mathrm{CH}-2), 25.0\left(\mathrm{CH}_{3}-\mathrm{MeBpin}\right)$, $24.8\left(\mathrm{CH}_{3}-\mathrm{MeBpin}\right), 16.8$ (bs, $\left.\mathrm{CH}_{2}-3\right)$.

${ }^{11} \mathbf{B}$ NMR $\left(96 \mathrm{MHz}, \mathrm{CDCl}_{3}\right) \delta(\mathrm{ppm})=33.4$.

HRMS $(\mathrm{ESI}+)$ : calculated for $\mathrm{C}_{23} \mathrm{H}_{30} \mathrm{BNO}_{5}[\mathrm{M}+\mathrm{H}]^{+}:$: 412.2294 ; found: 412.2266 .

IR (neat): $v\left(\mathrm{~cm}^{-1}\right)=3308,2977,1658,1600,1511,1410,1369,1319,1238,1141,1035,967$, $847,828$.

m.p.: $129^{\circ} \mathrm{C}$

HPLC analysis of 3yb indicated 80:20 er, chiral stationary column: OD-H, mobile phase: $n$ hexane $/ \mathrm{PrOH}=95: 5,1.0 \mathrm{~mL} / \mathrm{min}, 251 \mathrm{~nm}, 30^{\circ} \mathrm{C}, \mathrm{t}_{\mathrm{R}}=20.2 \mathrm{~min}$ (major), $\mathrm{t}_{\mathrm{R}}=25.5 \mathrm{~min}$ (minor). $[\alpha]^{20} \mathrm{D}=-27.6\left(c 0.30, \mathrm{CH}_{2} \mathrm{Cl}_{2}\right)$. 


\section{<Chromatogram>}

mAU

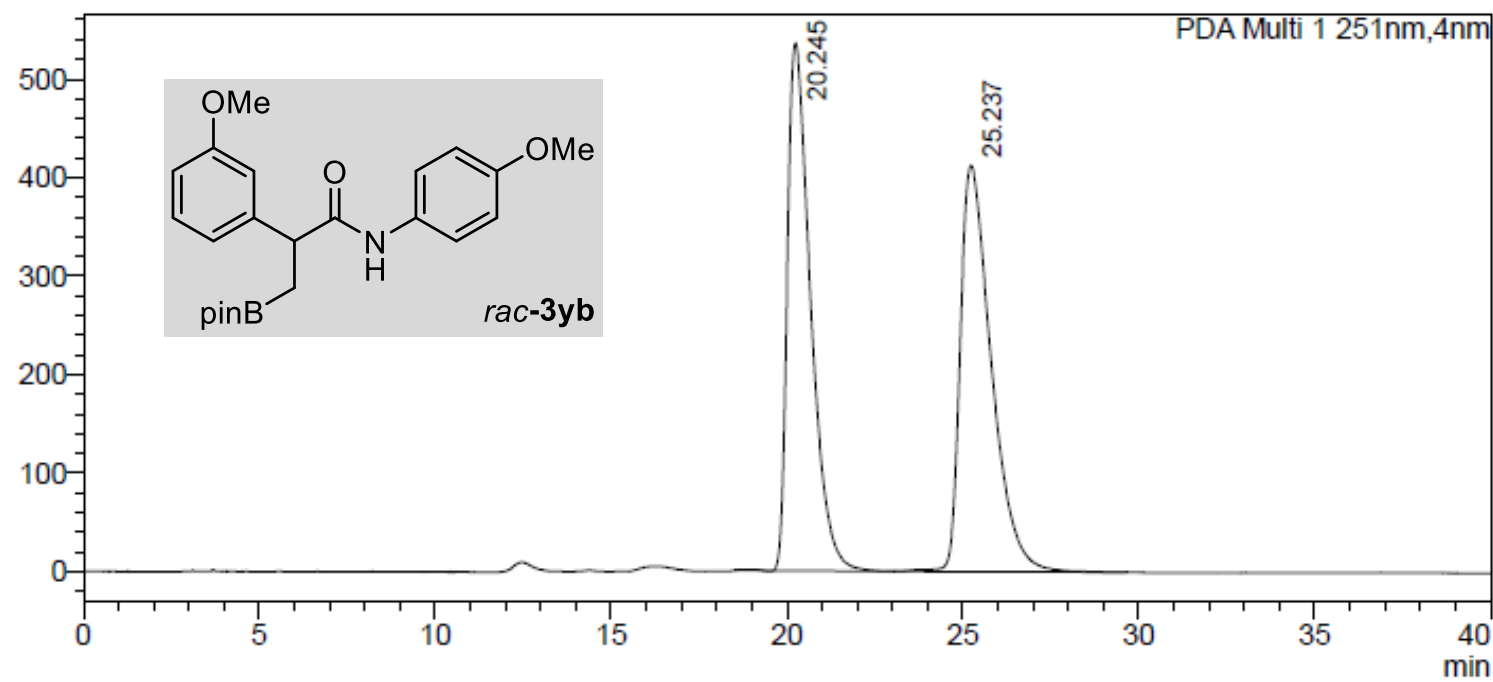

<Peak Table>

PDA Ch1 251nm

\begin{tabular}{|r|r|r|r|}
\hline Peak\# & Ret. Time & Area & \multicolumn{1}{c|}{ Area\% } \\
\hline 1 & 20.245 & 24558395 & 49.239 \\
\hline 2 & 25.237 & 25317363 & 50.761 \\
\hline Total & & 49875758 & 100.000 \\
\hline
\end{tabular}

\section{<Chromatogram>}

mAU

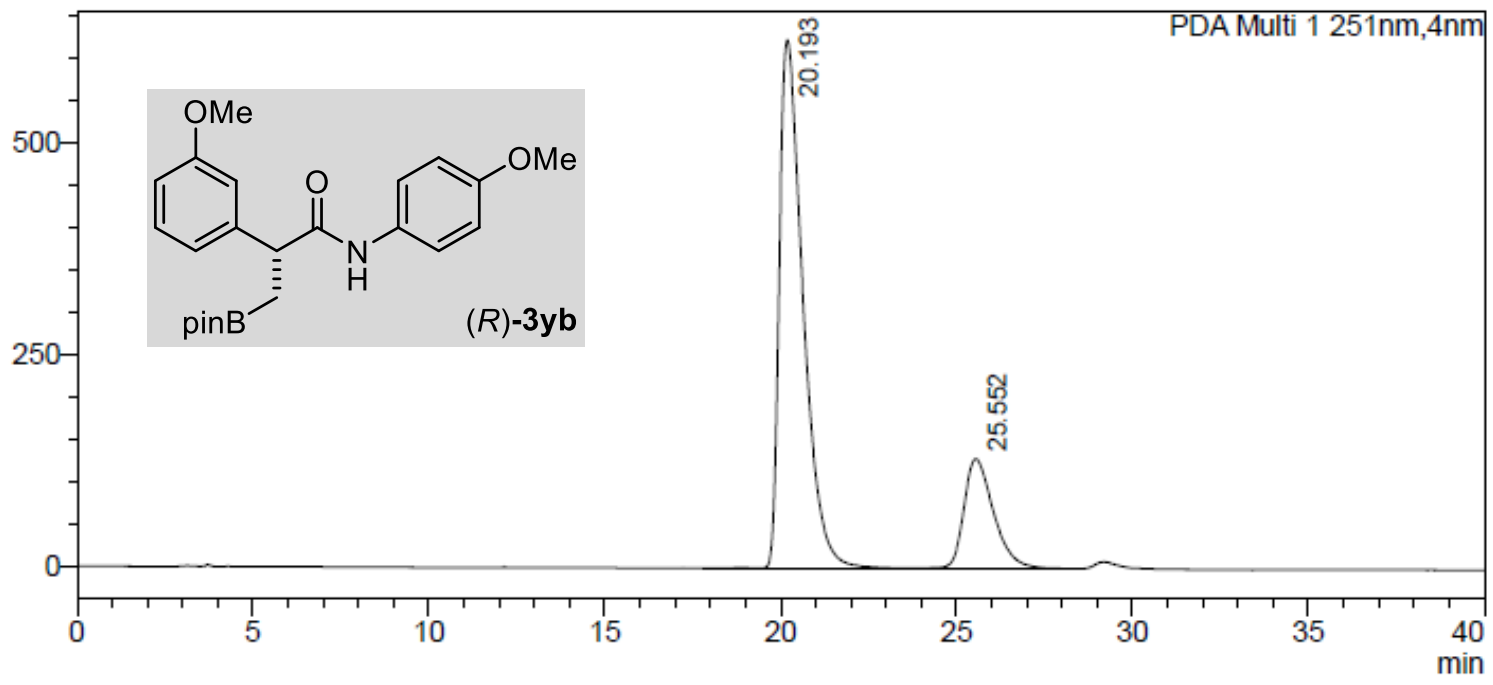

<Peak Table>

\begin{tabular}{|c|c|c|c|}
\hline Peak\# & Ret. Time & Area & Area\% \\
\hline 1 & 20.193 & 29173064 & 79.404 \\
\hline 2 & 25.552 & 7567204 & 20.596 \\
\hline Total & & 36740268 & 100.000 \\
\hline
\end{tabular}


(R)-(4-(dimethylamino)phenyl)-2-(3-methoxyphenyl)-3-(4,4,5,5-tetramethyl-1,3,2dioxaborolan-2-yl)propanamide 3yd

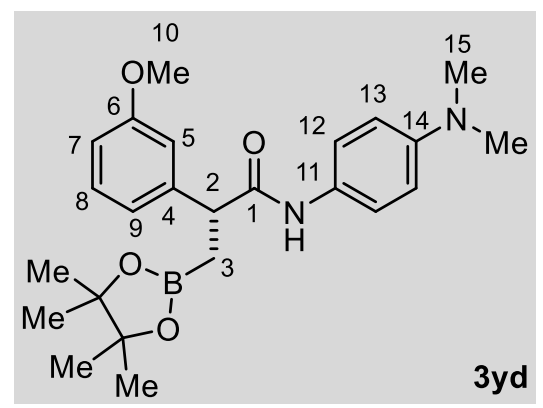

According to the general procedure for enantioselective copper-catalyzed borylative carboxamidation using 3methoxystyrene $1 \mathbf{y}(20 \mathrm{mg}, 0.15 \mathrm{mmol}, 1.0$ equiv.), $\mathrm{CuCl}(1.5$ $\mathrm{mg}, 0.015 \mathrm{mmol}, 10 \mathrm{~mol} \%)$, ligand $(R, R)-6 \mathrm{k}(7.0 \mathrm{mg}, 0.017$ mmol, 11 mol\%), LiOtBu (24 mg, 0.3 mmol, 2.0 equiv.), $B_{2}$ pin $_{2}$ (57 mg, $0.23 \mathrm{mmol}, 1.5$ equiv.) and 4-isocyanato- $\mathrm{N}, \mathrm{N}-$ dimethylaniline $\mathbf{2 d}(0.97 \mathrm{mg}, 0.60 \mathrm{mmol}, 4.0$ equiv.) in 2-Me-

THF and $\mathrm{Et}_{2} \mathrm{O}(1.5 \mathrm{~mL}, \mathrm{v}: \mathrm{v}=1: 1,0.1 \mathrm{M}) .95 \%$ conversion into 3yd determined by ${ }^{1} \mathrm{H}$ NMR analysis of the crude reaction mixture in presence of an internal standard. Purification by flash chromatography (pentane/ethyl acetate 3:2) gave pure 3yd as a white solid (45 mg, 0.11 mmol, $73 \%$ yield).

TLC: pentane/ethyl acetate $3: 2, R_{f}=0.4$.

${ }^{1} \mathrm{H}$ NMR $\left(400 \mathrm{MHz}, \mathrm{CDCl}_{3}\right) \delta(\mathrm{ppm})=7.28-7.22(\mathrm{~m}, 4 \mathrm{H}, \mathrm{H}-A r), 6.93(\mathrm{~m}, 2 \mathrm{H}, \mathrm{H}-A r+\mathrm{N}-H)$, $6.90(\mathrm{~m}, 1 \mathrm{H}, \mathrm{H}-5), 6.80\left(\mathrm{~d},{ }^{3} \mathrm{~J}_{\mathrm{HH}}=7.3 \mathrm{~Hz},{ }^{4} \mathrm{~J}_{\mathrm{HH}}=1.6 \mathrm{~Hz}, 1 \mathrm{H}, \mathrm{H}-7\right), 6.65\left(\mathrm{~d},{ }^{3} \mathrm{~J}_{\mathrm{HH}}=8.9 \mathrm{~Hz}, 2 \mathrm{H}\right.$, $H-13$ ), $3.83\left(\mathrm{dd},{ }^{3} \mathrm{~J}_{\mathrm{HH}}=9.8,6.3 \mathrm{~Hz}, 1 \mathrm{H}, H-2\right), 3.79$ (s, 3H, $\left.H-10\right), 2.88$ (s, $\left.6 \mathrm{H}, H-15\right), 1.63$ (dd, $\left.{ }^{2} J_{H H}=15.6,{ }^{3} J_{H H}=9.8 \mathrm{~Hz}, 1 \mathrm{H}, H-3\right), 1.27(\mathrm{~m}, 1 \mathrm{H}, H-3$ '), 1.22 (s, 6H, H-MeBpin), 1.20 (s, 6H, H-MeBpin).

${ }^{13} \mathrm{C}\left\{{ }^{1} \mathrm{H}\right\}$ NMR $\left(101 \mathrm{MHz}, \mathrm{CDCl}_{3}\right) \delta(\mathrm{ppm})=172.1(C-1), 160.1(C-6), 143.6(C-4), 130.1(\mathrm{CH}-8$ + C-11), $121.4(\mathrm{CH}-12), 120.5(\mathrm{CH}-9), 113.5(\mathrm{CH}-13), 113.1$ (CH-5), 83.4 (CO-Bpin), 55.4 ( $\left.\mathrm{CH}_{3}-10\right)$, $49.9(\mathrm{CH}-2), 41.3\left(\mathrm{CH}_{3}-15\right), 25.0\left(\mathrm{CH}_{3}-\mathrm{MeBpin}\right), 24.8\left(\mathrm{CH}_{3}-\mathrm{MeBpin}\right), 16.9$ (bs, $\mathrm{CH}_{2}-$ $3)$.

${ }^{11} \mathbf{B}$ NMR $\left(128 \mathrm{MHz}, \mathrm{CDCl}_{3}\right) \delta(\mathrm{ppm})=34.0$

HRMS $(E S I+)$ : calculated for $\mathrm{C}_{24} \mathrm{H}_{33} \mathrm{BN}_{2} \mathrm{O}_{4}[\mathrm{M}+\mathrm{H}]^{+}:$: 425.2611 ; found: 425.2607.

IR (neat): $v\left(\mathrm{~cm}^{-1}\right)=3309,2977,1654,1598,1520,1488,1370,1321,1258,1144,1048$.

m.p.: $159-160^{\circ} \mathrm{C}$

HPLC analysis of 3yd indicated 83:17 er, chiral stationary column: AS- $\mathrm{H}$, mobile phase: $n$ hexane $/ \mathrm{PrOH}=90: 10,1.0 \mathrm{~mL} / \mathrm{min}, 275 \mathrm{~nm}, 30^{\circ} \mathrm{C}, \mathrm{t}_{\mathrm{R}}=13.1 \mathrm{~min}$ (major), $\mathrm{t}_{\mathrm{R}}=26.1 \mathrm{~min}$ (minor). $[\alpha]^{20} \mathrm{D}=-26.1\left(c 0.25, \mathrm{CH}_{2} \mathrm{Cl}_{2}\right)$. 


\section{<Chromatogram>}

mAU

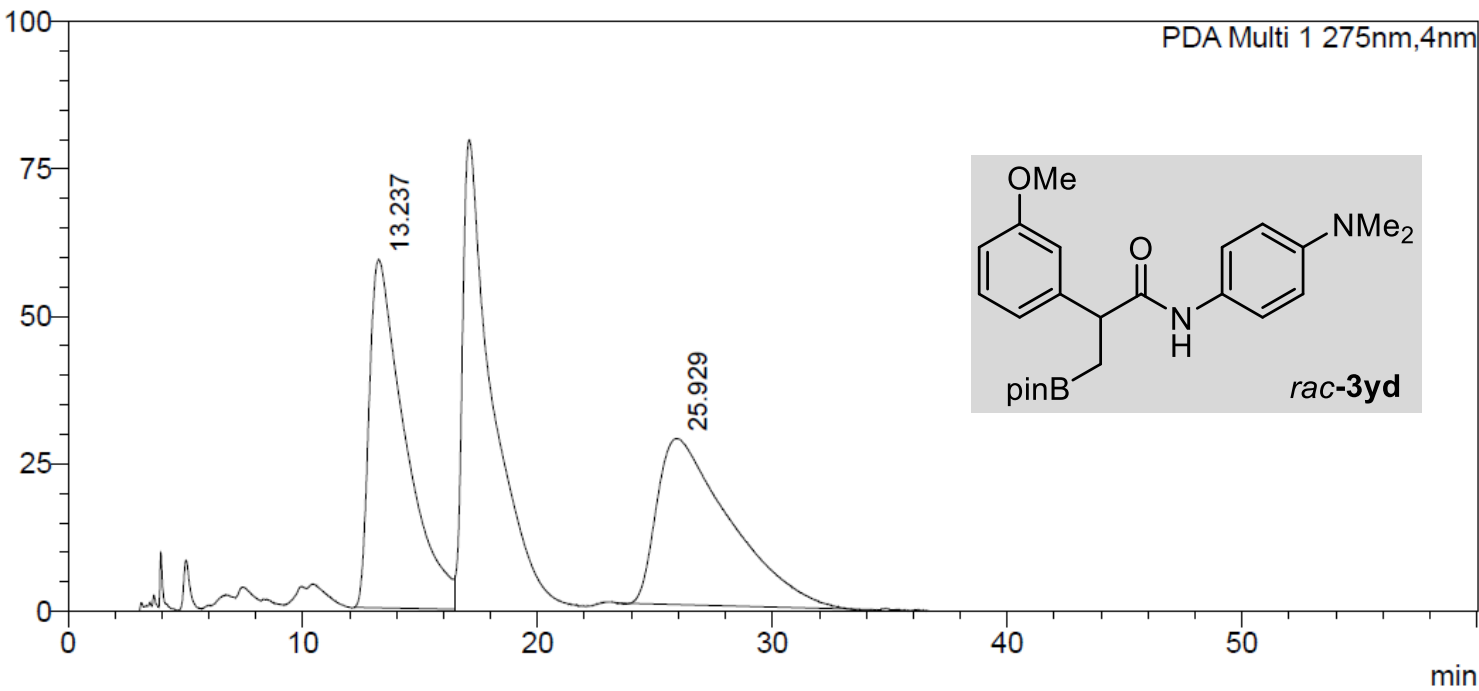

<Peak Table>

PDA Ch1 275nm

\begin{tabular}{|r|r|r|r|}
\hline Peak\# & Ret. Time & \multicolumn{1}{|c|}{ Area } & \multicolumn{1}{|c|}{ Area\% } \\
\hline 1 & 13.237 & 6299308 & 50.773 \\
\hline 2 & 25.929 & 6107404 & 49.227 \\
\hline Total & & 12406712 & 100.000 \\
\hline
\end{tabular}

\section{<Chromatogram>}

mAU

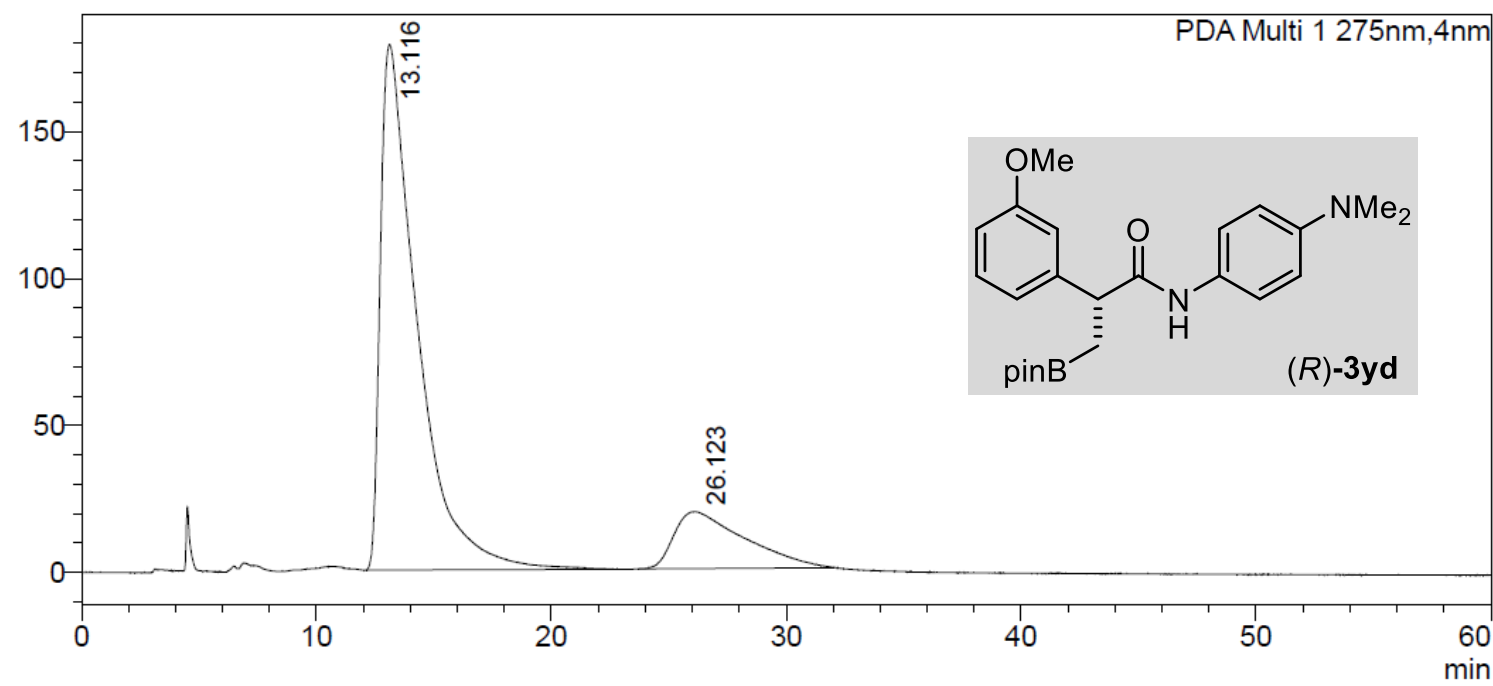

<Peak Table>

PDA Ch1 275nm

\begin{tabular}{|r|r|r|r|}
\hline Peak\# & Ret. Time & \multicolumn{1}{|c|}{ Area } & \multicolumn{1}{c|}{ Area\% } \\
\hline 1 & 13.116 & 19447227 & 82.903 \\
\hline 2 & 26.123 & 4010535 & 17.097 \\
\hline Total & & 23457762 & 100.000 \\
\hline
\end{tabular}


(R)-N-(4-bromophenyl)-2-(3-methoxyphenyl)-3-(4,4,5,5-tetramethyl-1,3,2-dioxaborolan-2yl)propanamide $\mathbf{3 y g}$

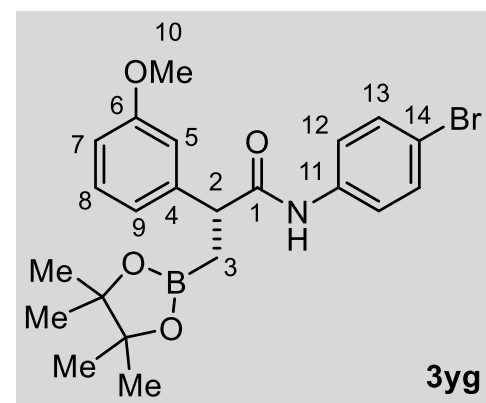

According to the general procedure for enantioselective coppercatalyzed borylative carboxamidation using 3-methoxystyrene $3 y g$ (20 mg, $0.15 \mathrm{mmol}, 1.0$ equiv.), $\mathrm{CuCl}(1.5 \mathrm{mg}, 0.015 \mathrm{mmol}$, $10 \mathrm{~mol} \%)$, ligand $(R, R)-6 \mathrm{k}(7.0 \mathrm{mg}, 0.017 \mathrm{mmol}, 11 \mathrm{~mol} \%)$, LiOtBu (24 mg, 0.3 mmol, 2.0 equiv.), $B_{2} p_{i n}(57 \mathrm{mg}, 0.23 \mathrm{mmol}$, 1.5 equiv.) and 4-isocyanato-1-bromobenzene $2 \mathrm{~g}(0.119 \mathrm{mg}$, 0.60 mmol, 4.0 equiv.) in 2-Me-THF and $\mathrm{Et}_{2} \mathrm{O}(1.5 \mathrm{~mL}, \mathrm{v}: \mathrm{v}=1: 1$, $0.1 \mathrm{M}) .99 \%$ conversion into 3yg determined by ${ }^{1} \mathrm{H}$ NMR analysis of the crude reaction mixture in presence of an internal standard. Purification by flash chromatography (pentane/diethyl ether 7:3) gave pure 3yg as a white solid (60 mg, $0.15 \mathrm{mmol}, 87 \%$ yield).

TLC: pentane/diethyl ether $7: 3, R_{f}=0.3$.

${ }^{1} \mathrm{H}$ NMR $\left(400 \mathrm{MHz}, \mathrm{CDCl}_{3}\right) \delta(\mathrm{ppm})=7.36\left(\mathrm{~d},{ }^{3} \mathrm{JHH}_{\mathrm{HH}}=9.1 \mathrm{~Hz}, 2 \mathrm{H}, \mathrm{H}-12\right), 7.31\left(\mathrm{~d},{ }^{3} \mathrm{JHH}_{\mathrm{HH}}=9.1 \mathrm{~Hz}\right.$, $2 \mathrm{H}, H-13), 7.26$ (t, J=7.9 Hz, $1 \mathrm{H}$ ), 7.11 (bs, $1 \mathrm{H}, \mathrm{N}-H), 6.91$ (d, $\left.{ }^{3} J_{\mathrm{HH}}=7.7 \mathrm{~Hz}, 1 \mathrm{H}, H-9\right), 6.88$ $(\mathrm{m}, 1 \mathrm{H}, \mathrm{H}-5), 6.82\left(\mathrm{ddd},{ }^{3} \mathrm{~J}_{\mathrm{HH}}=8.3,{ }^{4} \mathrm{~J}_{\mathrm{HH}}=2.6 \mathrm{~Hz},{ }^{4} J_{\mathrm{HH}}=0.8 \mathrm{~Hz}, 1 \mathrm{H}, H-7\right), 3.84\left(\mathrm{dd},{ }^{3} J_{\mathrm{HH}}=\right.$ 10.0, 6.2 Hz, 1H, H-2), 3.79 (s, 3H, H-10), $1.63\left(\mathrm{dd},{ }^{2} \mathrm{~J}_{\mathrm{HH}}=15.8,{ }^{3} \mathrm{~J}_{\mathrm{HH}}=10.0 \mathrm{~Hz}, 1 \mathrm{H}, H-3\right), 1.25$ (dd, ${ }^{2} J_{H H}=15.7,{ }^{3} J_{H H}=6.2 \mathrm{~Hz}, 1 \mathrm{H}, H$-3'), 1.22 (s, 6H, H-MeBpin), 1.19 (s, 6H, H-MeBpin).

${ }^{13} \mathrm{C}\left\{{ }^{1} \mathrm{H}\right\}$ NMR $\left(101 \mathrm{MHz}, \mathrm{CDCl}_{3}\right) \delta(\mathrm{ppm})=172.5(C-1), 160.2(C-6), 143.0(C-4), 137.2(C-$ 11), 131.9 ( $\mathrm{CH}-12), 130.3(\mathrm{CH}-8), 121.2(\mathrm{CH}-13), 120.3(\mathrm{CH}-9), 116.6(\mathrm{C}-14), 113.6(\mathrm{CH}-5)$, 113.1 (CH-7), 83.5 (CO-Bpin), $55.3\left(\mathrm{CH}_{3}-10\right), 50.1$ ( $\left.\mathrm{CH}-2\right), 24.9\left(\mathrm{CH}_{3}-\mathrm{MeBpin}\right), 24.8\left(\mathrm{CH}_{3}-\right.$ MeBpin), 16.8 (bs, $\mathrm{CH}_{2}-3$ ).

${ }^{11} \mathbf{B}$ NMR $\left(128 \mathrm{MHz}, \mathrm{CDCl}_{3}\right) \delta(\mathrm{ppm})=32.2$

HRMS (ESI +): calculated for $\mathrm{C}_{22} \mathrm{H}_{27} \mathrm{BBrNO}_{4}[\mathrm{M}+\mathrm{H}]^{+}: 460.1294$; found: 460.1277 .

IR (neat): $v\left(\mathrm{~cm}^{-1}\right)=3349,2977,1684,1588,1523,1487,1390,1371,1321,1259,1139$, $1044,827$.

m.p.: $153^{\circ} \mathrm{C}$

HPLC analysis of $3 \mathbf{y g}$ indicated $98: 2 \mathrm{er}$, chiral stationary column: IC, mobile phase: $n$ hexane $/ \mathrm{PrOH}=95: 5,1.0 \mathrm{~mL} / \mathrm{min}, 254 \mathrm{~nm}, 30^{\circ} \mathrm{C}, \mathrm{t}_{\mathrm{R}}=14.3 \mathrm{~min}$ (major), $\mathrm{t}_{\mathrm{R}}=16.4 \mathrm{~min}$ (minor). $[\alpha]^{20} \mathrm{D}=-42.6\left(c 0.30, \mathrm{CH}_{2} \mathrm{Cl}_{2}\right)$. 
<Chromatogram>

mAU

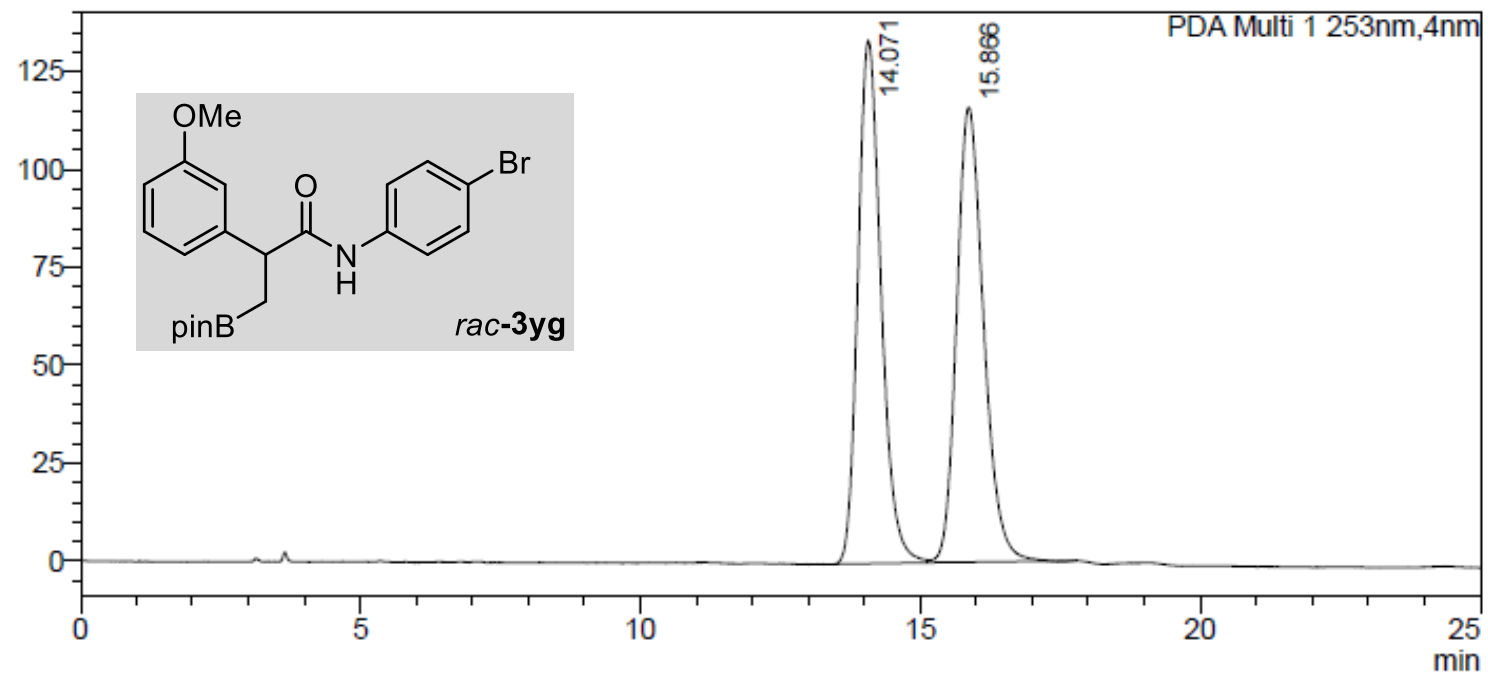

<Peak Table>

PDA Ch1 253nm

\begin{tabular}{|r|r|r|r|}
\hline Peak\# & Ret. Time & Area & \multicolumn{1}{|c|}{ Area\% } \\
\hline 1 & 14.071 & 3852148 & 49.729 \\
\hline 2 & 15.866 & 3894180 & 50.271 \\
\hline Total & & 7746328 & 100.000 \\
\hline
\end{tabular}

\section{<Chromatogram>}

$\mathrm{mAU}$

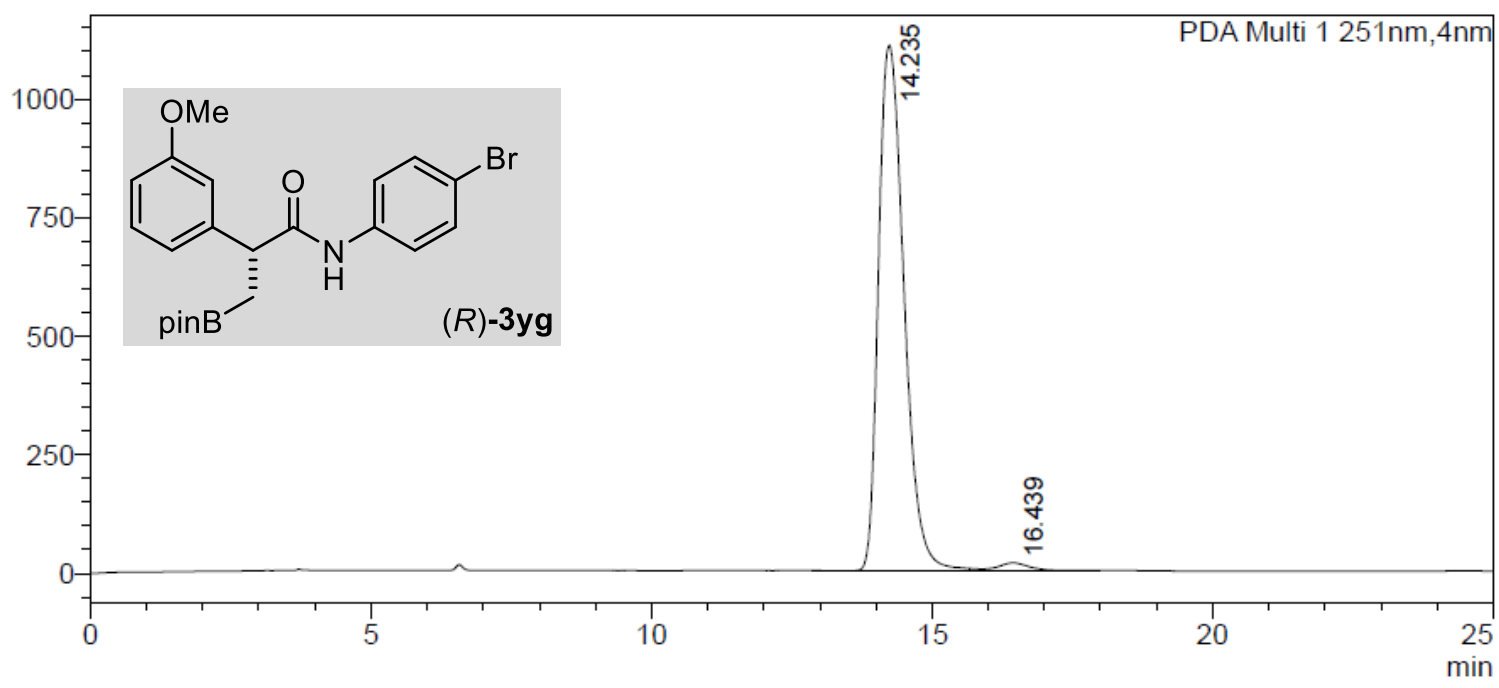

<Peak Table>

PDA Ch1 251nm

\begin{tabular}{|r|r|r|r|}
\hline Peak\# & Ret. Time & \multicolumn{1}{c|}{ Area } & \multicolumn{1}{c|}{ Area $\%$} \\
\hline 1 & 14.235 & 35022752 & 98.303 \\
\hline 2 & 16.439 & 604533 & 1.697 \\
\hline Total & & 35627285 & 100.000 \\
\hline
\end{tabular}


(R)-2-(3-methoxyphenyl)-3-(4,4,5,5-tetramethyl-1,3,2-dioxaborolan-2-yl)- N-(ptolyl)propanamide $3 y m$

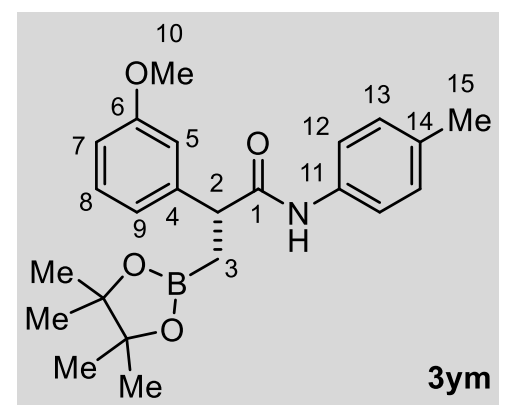

According to the general procedure for enantioselective coppercatalyzed borylative carboxamidation using 3-methoxystyrene 1y $(20 \mathrm{mg}, 0.15 \mathrm{mmol}, 1.0$ equiv.), $\mathrm{CuCl}(1.5 \mathrm{mg}, 0.015 \mathrm{mmol}$, $10 \mathrm{~mol} \%)$, ligand $(R, R)-6 \mathrm{k}(7.0 \mathrm{mg}, 0.017 \mathrm{mmol}, 11 \mathrm{~mol} \%)$, $\mathrm{LiOtBu}$ (24 mg, $0.3 \mathrm{mmol}, 2.0$ equiv.), $\mathrm{B}_{2} \mathrm{pin}_{2}(57 \mathrm{mg}, 0.23 \mathrm{mmol}$, 1.5 equiv.) and 1 -isocyanato-4-methylbenzene $2 \mathrm{~m}(0.076 \mathrm{~mL}$, 0.60 mmol, 4.0 equiv.) in 2-Me-THF and $\mathrm{Et}_{2} \mathrm{O}(1.5 \mathrm{~mL}, \mathrm{v}: \mathrm{v}=1: 1$,

$0.1 \mathrm{M}) .95 \%$ conversion into $3 \mathrm{ym}$ determined by ${ }^{1} \mathrm{H}$ NMR analysis of the crude reaction mixture in presence of an internal standard. Purification by flash chromatography (pentane/ethyl acetate $4: 1$ ) gave pure $3 y \mathrm{~m}$ as a white solid (45 $\mathrm{mg}, 0.11 \mathrm{mmol}, 75 \%$ yield).

TLC: pentane/ethyl acetate $4: 1, \mathrm{R}_{\mathrm{f}}=0.2$.

${ }^{1} \mathrm{H}$ NMR $\left(400 \mathrm{MHz}, \mathrm{CDCl}_{3}\right) \delta(\mathrm{ppm})=7.29(\mathrm{~m}, 3 \mathrm{H}, \mathrm{H}-A r), 7.25(\mathrm{~m}, 2 \mathrm{H}, \mathrm{H}-A r+\mathrm{N}-H), 7.05(\mathrm{~d}, J$ $=8.1 \mathrm{~Hz}, 3 \mathrm{H}), 6.93\left(\mathrm{~d},{ }^{3} J_{\mathrm{HH}}=7.7 \mathrm{~Hz}, 1 \mathrm{H}, H-9\right), 6.90(\mathrm{~m}, 1 \mathrm{H}, H-5), 6.81\left(\mathrm{dd},{ }^{3} J_{\mathrm{HH}}=8.2 \mathrm{~Hz},{ }^{4} J_{\mathrm{HH}}\right.$ $=2.4 \mathrm{~Hz}, 1 \mathrm{H}, H-7), 3.84\left(\mathrm{dd},{ }^{3} \mathrm{~J}_{\mathrm{HH}}=9.9,6.3 \mathrm{~Hz}, 1 \mathrm{H}, H-2\right), 3.79(\mathrm{~s}, 3 \mathrm{H}, H-10), 2.27(\mathrm{~s}, 3 \mathrm{H}, H-$ 15), $1.64\left(\mathrm{dd},{ }^{2} J_{\mathrm{HH}}=15.7,{ }^{3} \mathrm{JHH}_{\mathrm{H}}=9.9 \mathrm{~Hz}, 1 \mathrm{H}, H-3\right), 1.27$ (m, $1 \mathrm{H}, H-3$ '), 1.22 (s, 6H, H-MeBpin), 1.20 (s, 6H, H-MeBpin).

${ }^{13} \mathrm{C}\left\{{ }^{1} \mathrm{H}\right\}$ NMR $\left(101 \mathrm{MHz}, \mathrm{CDCl}_{3}\right) \delta(\mathrm{ppm})=172.3(C-1), 160.1(C-6), 143.3(C-4), 135.6(C-$ 11), 133.7 (C-14), 130.1 ( $\mathrm{CH}-8), 129.4$ ( $\mathrm{CH}-13), 120.4$ ( $\mathrm{CH}-9), 119.7(\mathrm{CH}-12), 113.5(\mathrm{CH}-5)$, 113.1 (CH-7), 83.4 (CO-Bpin), $55.3\left(\mathrm{CH}_{3}-10\right), 50.0$ ( $\left.\mathrm{CH}-2\right), 24.9\left(\mathrm{CH}_{3}-\mathrm{MeBpin}\right), 24.8\left(\mathrm{CH}_{3}-\right.$ MeBpin), $21.0\left(\mathrm{CH}_{3}-15\right), 16.78$ (bs, $\left.\mathrm{CH}_{2}-3\right)$.

${ }^{11}$ B NMR $\left(128 \mathrm{MHz}, \mathrm{CDCl}_{3}\right) \delta(\mathrm{ppm})=33.5$

HRMS $(E S I+)$ : calculated for $\mathrm{C}_{23} \mathrm{H}_{30} \mathrm{BNO}_{4}[\mathrm{M}+\mathrm{H}]^{+}:$: 396.2345; found: 396.2336.

IR (neat): $v\left(\mathrm{~cm}^{-1}\right)=3353,2978,1709,1680,1599,1512,1489,1434,1402,1370,1320$, 1257, 1140, 1044, 967.

m.p.: $128-129^{\circ} \mathrm{C}$

HPLC analysis of 3ym indicated $85: 15 \mathrm{er}$, chiral stationary column: IC, mobile phase: $n$ hexane $/ \mathrm{PrOH}=95: 5,1.0 \mathrm{~mL} / \mathrm{min}, 207 \mathrm{~nm}, 30^{\circ} \mathrm{C}, \mathrm{t}_{\mathrm{R}}=34.0 \mathrm{~min}$ (major), $\mathrm{t}_{\mathrm{R}}=50.7 \mathrm{~min}$ (minor). $[\alpha]^{20} \mathrm{D}=-40.9\left(c 0.25, \mathrm{CH}_{2} \mathrm{Cl}_{2}\right)$. 


\section{<Chromatogram>}

mAU

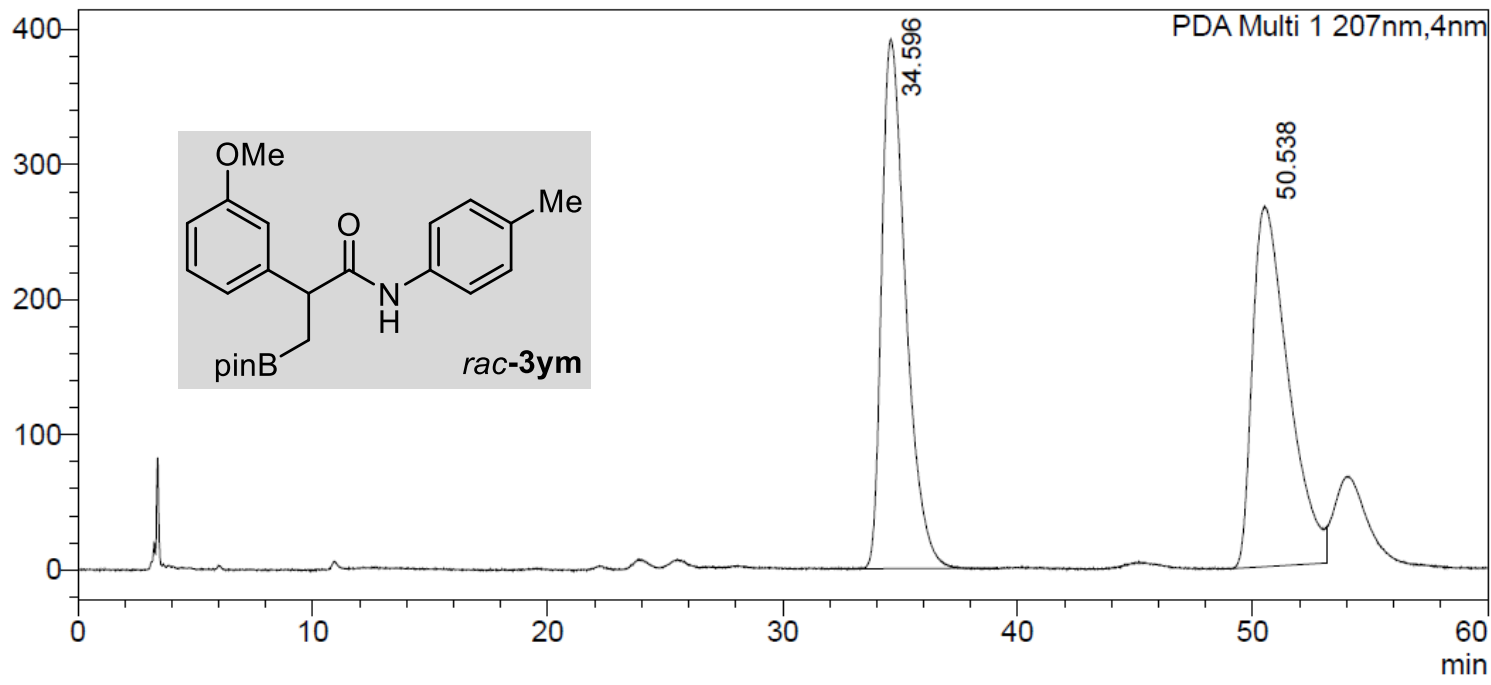

<Peak Table>

PDA Ch1 207nm

\begin{tabular}{|r|r|c|r|}
\hline Peak\# & Ret. Time & Area & \multicolumn{1}{|c|}{ Area $\%$} \\
\hline 1 & 34.596 & 27952834 & 49.600 \\
\hline 2 & 50.538 & 28404180 & 50.400 \\
\hline Total & & 56357014 & 100.000 \\
\hline
\end{tabular}

<Chromatogram>

$\mathrm{mAU}$

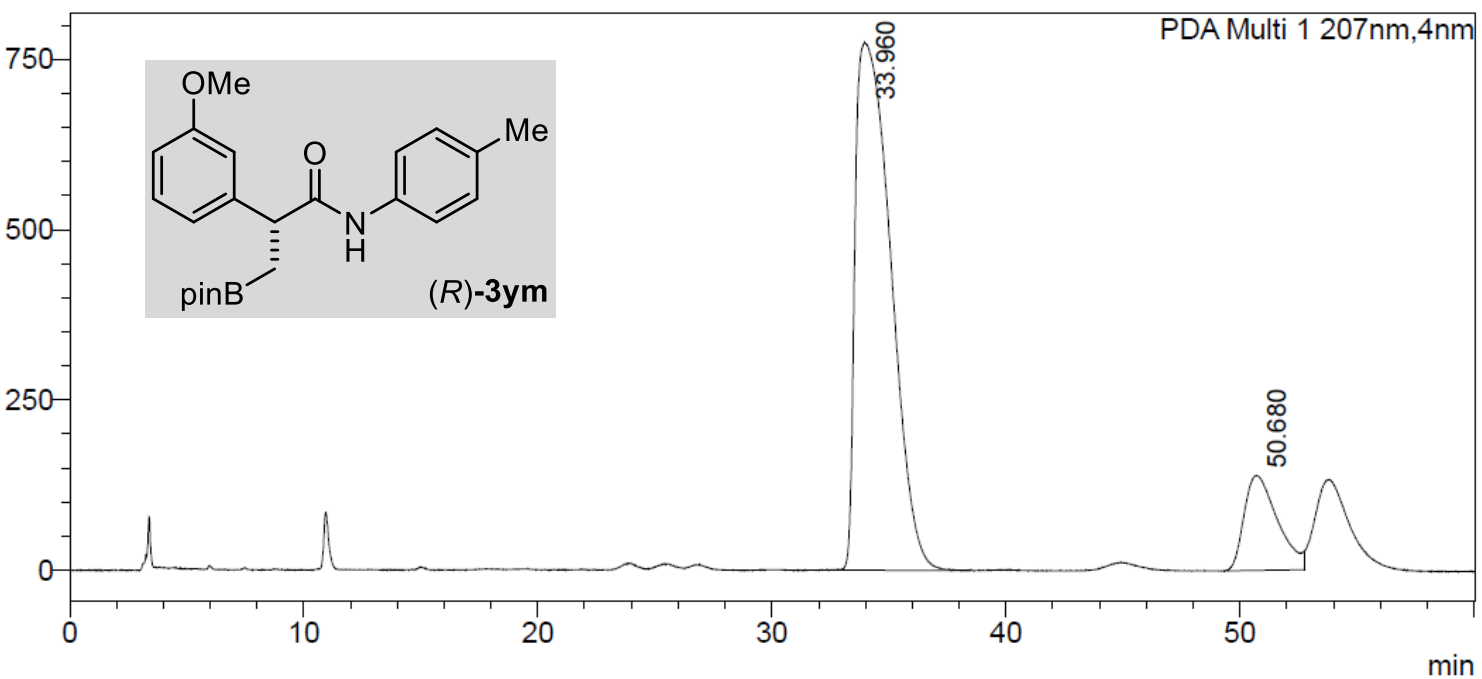

<Peak Table>

PDA Ch1 207nm

Peak\# Ret. Time

\begin{tabular}{|r|r|r|r|}
\hline 1 & 33.960 & 81846831 & 85.502 \\
\hline 2 & 50.680 & 13878267 & 14.498 \\
\hline Total & & 95725098 & 100.000 \\
\hline
\end{tabular}


(R)-N-(4-bromophenyl)-2-(6-morpholinopyridin-3-yl)-3-(4,4,5,5-tetramethyl-1,3,2dioxaborolan-2-yl)propanamide $\mathbf{3 z g}$

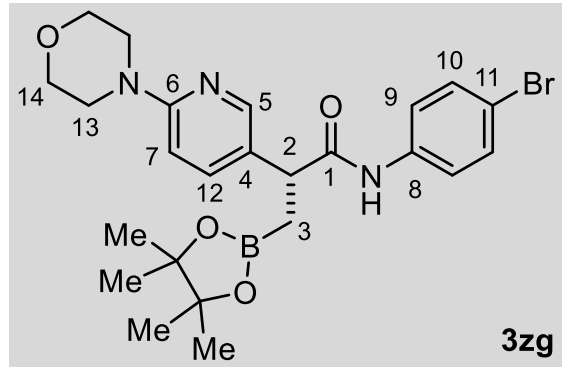

According to the general procedure for enantioselective copper-catalyzed borylative carboxamidation using 4-(5vinylpyridin-2-yl)morpholine $1 \mathrm{z}(29 \mathrm{mg}, 0.15 \mathrm{mmol}, 1.0$ equiv), ${ }^{11} \mathrm{CuCl}$ (1.5 mg, $\left.0.015 \mathrm{mmol}, 10 \mathrm{~mol} \%\right)$, ligand $(R, R)-6 \mathbf{k}(7.0 \mathrm{mg}, 0.017 \mathrm{mmol}, 11 \mathrm{~mol} \%)$, LiOtBu (24 mg, 0.3 mmol, 2.0 equiv.), $B_{2} p_{i n}(57 \mathrm{mg}, 0.23 \mathrm{mmol}, 1.5$ equiv.) and 4-isocyanato-1-bromobenzene $\mathbf{2 g}$ (119 $\mathrm{mg}, 0.60 \mathrm{mmol}, 4.0$ equiv.) in 2-Me-THF and $\mathrm{Et}_{2} \mathrm{O}(1.5 \mathrm{~mL}, \mathrm{v}: \mathrm{v}=1: 1,0.1 \mathrm{M}) .90 \%$ conversion into $3 \mathrm{zg}$ determined by ${ }^{1} \mathrm{H}$ NMR analysis of the crude reaction mixture in presence of an internal standard. Purification by flash chromatography (pentane/diethyl ether $4: 1$ ) gave pure $3 z g$ as a white solid (40 $\mathrm{mg}, 0.078$ $\mathrm{mmol}, 52 \%$ yield).

TLC: pentane/diethyl ether 7:3, $\mathrm{R}_{\mathfrak{f}}=0.3$.

${ }^{1} \mathrm{H}$ NMR $\left(400 \mathrm{MHz}, \mathrm{CDCl}_{3}\right) \delta(\mathrm{ppm})=8.15(\mathrm{~s}, 1 \mathrm{H}, \mathrm{H}-5), 7.53\left(\mathrm{dd},{ }^{3} J_{\mathrm{HH}}=8.8,2.3 \mathrm{~Hz}, 1 \mathrm{H}, \mathrm{H}-\right.$ 12), 7.39-7.32 (m, 5H), 7.23 (bs, $1 \mathrm{H}, \mathrm{N}-H), 6.65\left(\mathrm{~d},{ }^{3} \mathrm{~J}_{\mathrm{HH}}=9.9 \mathrm{~Hz}, 1 \mathrm{H}, H-7\right), 3.81(\mathrm{~m}, 4 \mathrm{H}, \mathrm{H}-$ 14), $3.74\left(\mathrm{dd},{ }^{3} J_{\mathrm{HH}}=9.9,6.1 \mathrm{~Hz}, 1 \mathrm{H}, H-2\right), 3.48(\mathrm{~m}, 4 \mathrm{H}, H-13), 1.60\left(\mathrm{dd},{ }^{2} J_{\mathrm{HH}}=15.9,{ }^{3} J_{\mathrm{HH}}=\right.$ $9.9 \mathrm{~Hz}, 1 \mathrm{H}, H-3), 1.22$ (s, 7H, H-MeBpin + H-3'), 1.19 (s, 6H, $H$-MeBpin).

${ }^{13} \mathrm{C}\left\{{ }^{1} \mathrm{H}\right\}$ NMR $\left(101 \mathrm{MHz}, \mathrm{CDCl}_{3}\right) \delta(\mathrm{ppm})=172.6(C-1), 159.1(C-6), 147.2(\mathrm{CH}-5), 137.2(C-$ 8), $137.1(\mathrm{CH}-12), 132.0(\mathrm{CH}-10), 126.6(\mathrm{C}-4), 121.2(\mathrm{CH}-9), 116.8(\mathrm{C}-11), 107.7(\mathrm{CH}-7), 83.6$ (CO-Bpin), 66.8 ( $\mathrm{CH}-14), 46.5$ ( $\mathrm{CH}-2), 45.8$ ( $\mathrm{CH}-13)$, 25.0 ( $\left.\mathrm{CH}_{3}-\mathrm{MeBpin}\right), 24.9\left(\mathrm{CH}_{3}-\mathrm{MeBpin}\right)$, 16.9 (bs, $\mathrm{CH}_{2}-3$ ).

${ }^{11} \mathbf{B}$ NMR $\left(128 \mathrm{MHz}, \mathrm{CDCl}_{3}\right) \delta(\mathrm{ppm})=32.6$.

HRMS (ESI +): calculated for $\mathrm{C}_{24} \mathrm{H}_{31} \mathrm{BBrN}_{3} \mathrm{O}_{4}[\mathrm{M}+\mathrm{H}]^{+}$: 516.1669 ; found: 516.1672 .

IR (neat): $v\left(\mathrm{~cm}^{-1}\right)=3336,2977,1681,1601,1527,1489,1394,1370,1325,1242,1139$, 1120, 1073, 942, 825.

m.p.: $199^{\circ} \mathrm{C}$

HPLC analysis of $\mathbf{3 z g}$ indicated 95:5 er, chiral stationary column: IC, mobile phase: $n$ hexane $/ \mathrm{PrOH}=90: 10,1.0 \mathrm{~mL} / \mathrm{min}, 255 \mathrm{~nm}, 30^{\circ} \mathrm{C}, \mathrm{t}_{\mathrm{R}}$ (major) $=20.3 \mathrm{~min}, \mathrm{t}_{\mathrm{R}}$ (minor) $=25.8$ $\min$.

$[\alpha]^{20}=-58.0\left(c 0.125, \mathrm{CH}_{2} \mathrm{Cl}_{2}\right)$. 


\section{<Chromatogram >}

mAU

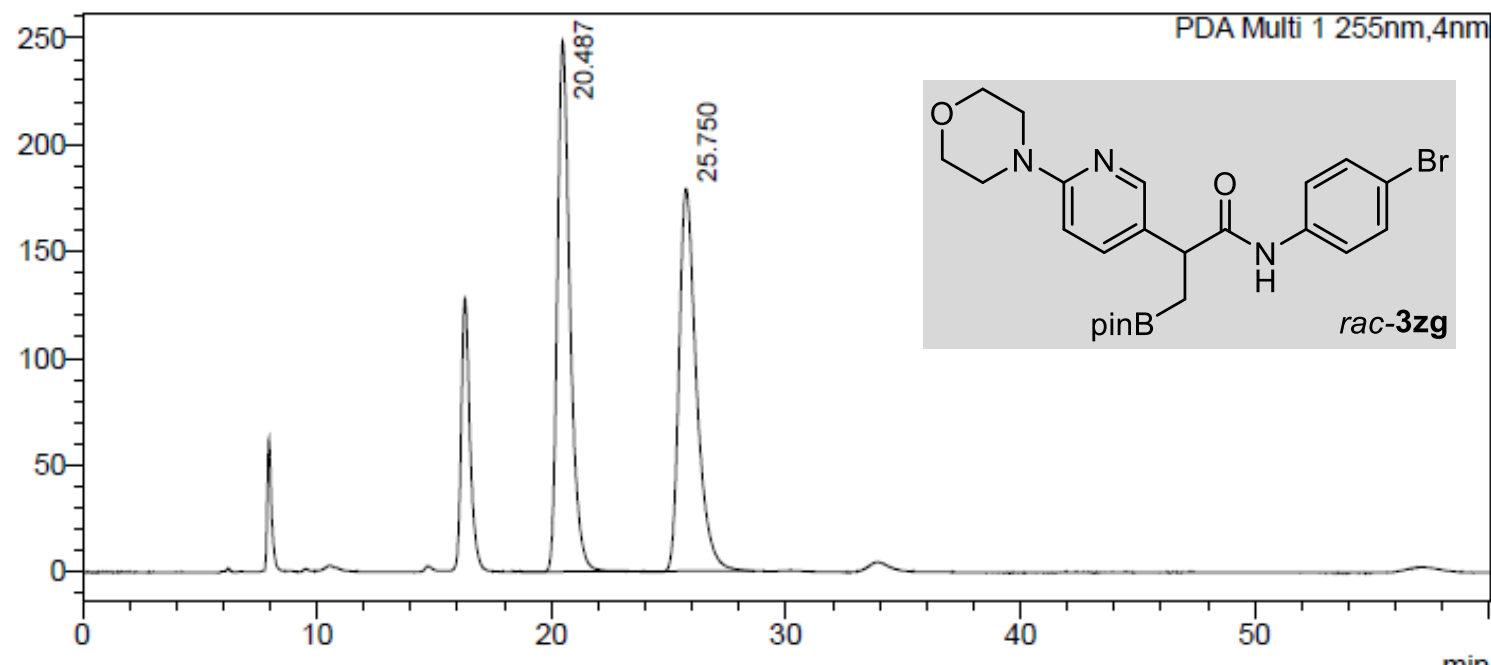

<Peak Table>

PDA Ch1 255nm

\begin{tabular}{|r|r|r|r|}
\hline Peak\# & Ret. Time & \multicolumn{1}{|c|}{ Area } & \multicolumn{1}{c|}{ Area $\%$} \\
\hline 1 & 20.487 & 9300396 & 50.274 \\
\hline 2 & 25.750 & 9198935 & 49.726 \\
\hline Total & & 18499331 & 100.000 \\
\hline
\end{tabular}

$<$ Chromatogram $>$

mAU

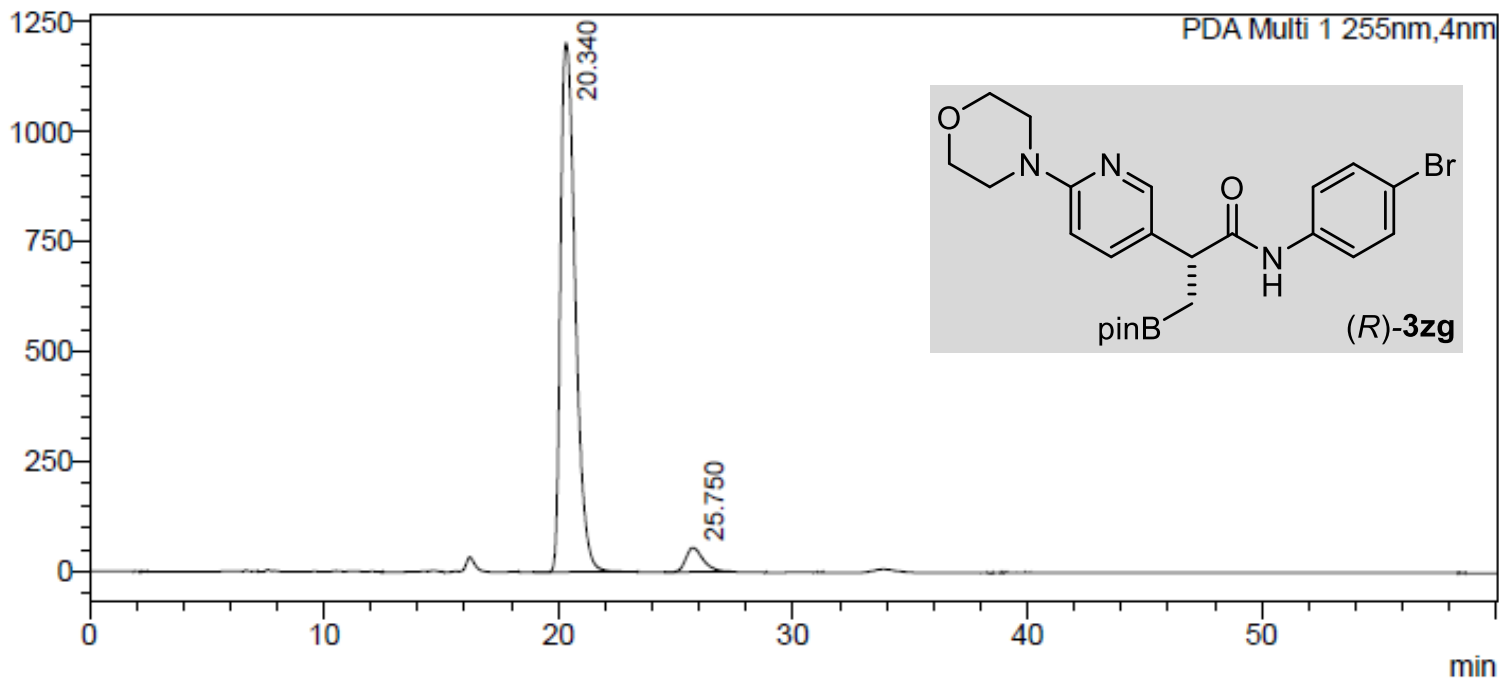

<Peak Table>

PDA Ch1 255nm

\begin{tabular}{|r|r|r|r|}
\hline Peak\# & Ret. Time & \multicolumn{1}{c|}{ Area } & \multicolumn{1}{c|}{ Area\% } \\
\hline 1 & 20.340 & 52639285 & 94.882 \\
\hline 2 & 25.750 & 2839512 & 5.118 \\
\hline Total & & 55478796 & 100.000 \\
\hline
\end{tabular}


(R)-2-(benzo[b]thiophen-5-yl)- $N$-(4-bromophenyl)-3-(4,4,5,5-tetramethyl-1,3,2-dioxaborolan2-yl)propanamide $\mathbf{3} \mathbf{a}_{2} \mathbf{g}$

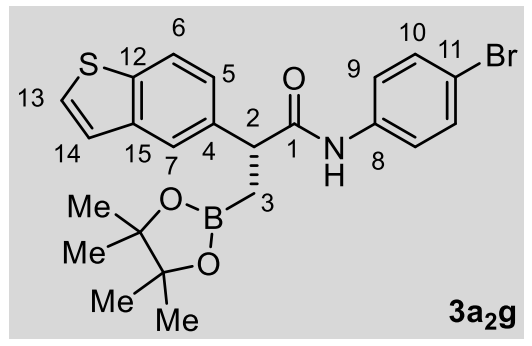

According to the general procedure for enantioselective copper-catalyzed borylative carboxamidation using 5vinylbenzo[b]thiophene $1 \mathbf{a}_{2}$ (24 mg, $0.15 \mathrm{mmol}, 1.0$ equiv.), ${ }^{12}$ $\mathrm{CuCl}(1.5 \mathrm{mg}, 0.015 \mathrm{mmol}, 10 \mathrm{~mol} \%)$, ligand $(R, R)-6 \mathbf{k}(7.0$ $\mathrm{mg}, 0.017 \mathrm{mmol}, 11 \mathrm{~mol} \%$ ), LiOtBu (24 mg, $0.3 \mathrm{mmol}, 2.0$ equiv.), $B_{2} \operatorname{pin}_{2}$ (57 mg, $0.23 \mathrm{mmol}, 1.5$ equiv.) and $4-$ isocyanato-1-bromobenzene $\mathbf{2 g}$ (119 $\mathrm{mg}, 0.60 \mathrm{mmol}, 4.0$ equiv.) in 2-Me-THF and $\mathrm{Et}_{2} \mathrm{O}$ (1.5 $\mathrm{mL}, \mathrm{v}: \mathrm{v}=1: 1,0.1 \mathrm{M}) .95 \%$ conversion into $3 \mathbf{a}_{2} \mathbf{g}$ determined by ${ }^{1} \mathrm{H}$ NMR analysis of the crude reaction mixture in presence of an internal standard. Purification by flash chromatography (pentane/diethyl ether 7:3) gave pure $3 \mathbf{a}_{2} \mathbf{g}$ as a white solid (65 $\mathrm{mg}, 0.13 \mathrm{mmol}, 89 \%$ yield).

TLC: pentane/diethyl ether 7:3, $\mathrm{R}_{\mathrm{f}}=0.3$.

${ }^{1} \mathbf{H}$ NMR $\left(400 \mathrm{MHz}, \mathrm{CDCl}_{3}\right) \delta(\mathrm{ppm})=7.86\left(\mathrm{~d},{ }^{3} \mathrm{~J}_{\mathrm{HH}}=8.3 \mathrm{~Hz}, 1 \mathrm{H}, \mathrm{H}-6\right), 7.80\left(\mathrm{~d},{ }^{4} \mathrm{~J}_{\mathrm{HH}}=1.3 \mathrm{~Hz}\right.$, $1 \mathrm{H}, H-7), 7.48\left(\mathrm{~d},{ }^{3} \mathrm{JHH}_{\mathrm{HH}}=5.4 \mathrm{~Hz}, 1 \mathrm{H}, H-13\right), 7.40-7.28(\mathrm{~m}, 5 \mathrm{H}, \mathrm{H}-\mathrm{Ar}), 7.11$ (bs, $\left.1 \mathrm{H}, \mathrm{N}-\mathrm{H}\right), 3.99$ $\left(\mathrm{dd},{ }^{3} \mathrm{~J}_{\mathrm{HH}}=9.8,6.2 \mathrm{~Hz}, 1 \mathrm{H}, \mathrm{H}-2\right), 1.71\left(\mathrm{dd},{ }^{2} \mathrm{~J}_{\mathrm{HH}}=15.8,{ }^{3} J_{\mathrm{HH}}=9.8 \mathrm{~Hz}, 1 \mathrm{H}, \mathrm{H}-3\right), 1.32\left(\mathrm{dd},{ }^{2} J_{\mathrm{HH}}\right.$ $=15.8,{ }^{3} J_{\mathrm{HH}}=6.2 \mathrm{~Hz}, 1 \mathrm{H}, H$-3'), 1.21 (s, 6H, H-MeBpin), 1.19 (s, 6H, H-MeBpin).

${ }^{13} \mathrm{C}\left\{{ }^{1} \mathrm{H}\right\}$ NMR $\left(101 \mathrm{MHz}, \mathrm{CDCl}_{3}\right) \delta(\mathrm{ppm})=172.8(C-1), 140.3(C-4), 139.0(C-\mathrm{Ar}), 137.6(C-$ $\mathrm{Ar}), 137.2$ (C-Ar), 131.9 ( $\mathrm{CH}-10), 127.5(\mathrm{CH}-13), 124.3$ (CH-Ar), $123.9(\mathrm{CH}-\mathrm{Ar}), 123.3(\mathrm{CH}-6)$, 123.0 ( $\mathrm{CH}-7), 121.2(\mathrm{CH}-9), 116.6$ (C-11), 83.5 (C-11), $50.0(\mathrm{CH}-2), 24.9$ ( $\left.\mathrm{CH}_{3}-\mathrm{MeBpin}\right), 24.8$ ( $\mathrm{CH}_{3}$-MeBpin), 17.3 (bs, $\mathrm{CH}_{2}-3$ ).

${ }^{11} \mathbf{B}$ NMR $\left(128 \mathrm{MHz}, \mathrm{CDCl}_{3}\right) \delta(\mathrm{ppm})=33.3$.

HRMS (ESI +): calculated for $\mathrm{C}_{23} \mathrm{H}_{25} \mathrm{BBrNO}_{3} \mathrm{~S}[\mathrm{M}+\mathrm{H}]^{+}: 504.1195$; found: 504.1091 .

IR (neat): $v\left(\mathrm{~cm}^{-1}\right)=3332,2980,1685,1591,1522,1488,1371,1322,1245,1140,842,700$.

m.p.: $194-196^{\circ} \mathrm{C}$

HPLC analysis of $\mathbf{3} \mathbf{a}_{2} \mathbf{g}$ indicated $87: 13 \mathrm{er}$, chiral stationary column: IC, mobile phase: $n$ hexane $/ \mathrm{PrOH}=90: 10,1.0 \mathrm{~mL} / \mathrm{min}, 232 \mathrm{~nm}, 30^{\circ} \mathrm{C}, \mathrm{t}_{\mathrm{R}}$ (major) $=10.6 \mathrm{~min}, \mathrm{t}_{\mathrm{R}}($ minor $)=13.0$ $\min$.

$[\alpha]^{20} \mathrm{D}=-65.1\left(c 0.20, \mathrm{CH}_{2} \mathrm{Cl}_{2}\right)$. 


\section{$<$ Chromatogram $>$}

mAU

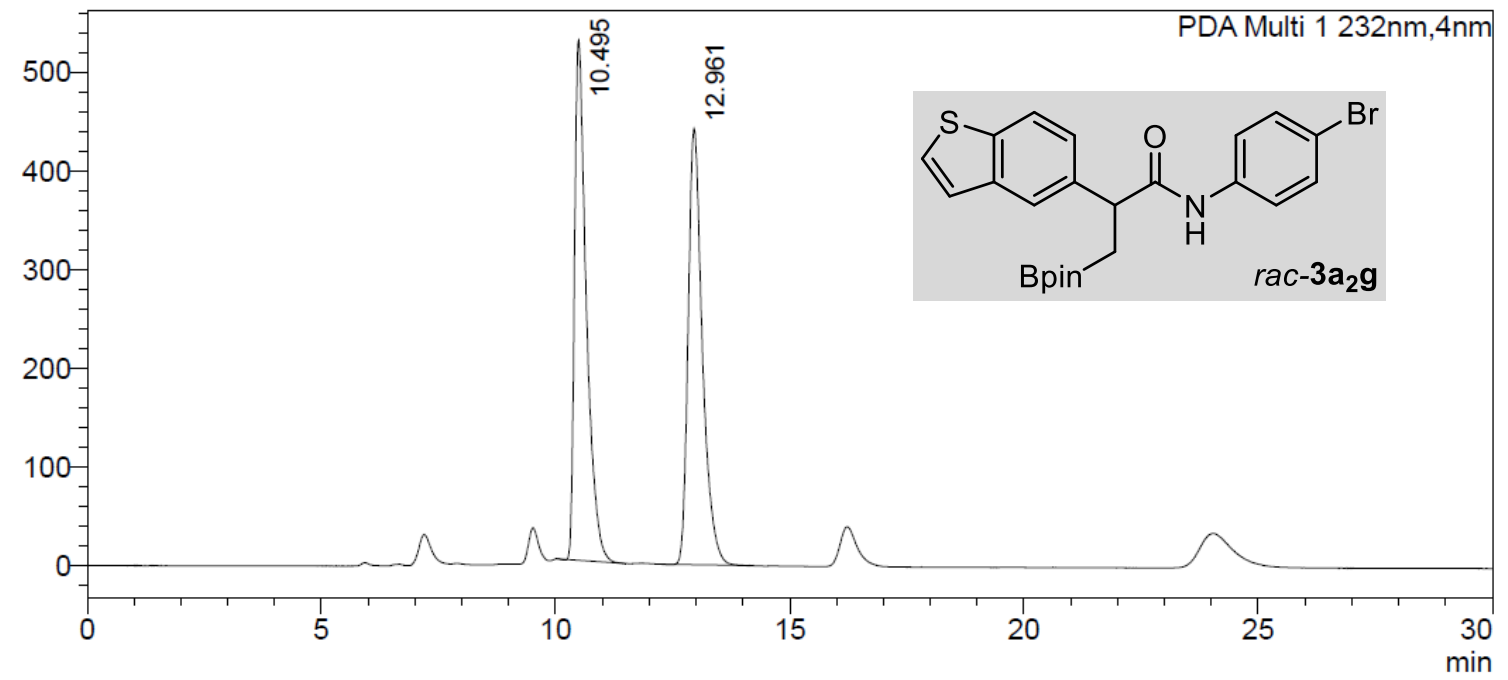

<Peak Table>

PDA Ch1 232nm

\begin{tabular}{|r|r|r|r|}
\hline Peak\# & Ret. Time & \multicolumn{1}{|c|}{ Area } & \multicolumn{1}{c|}{ Area\% } \\
\hline 1 & 10.495 & 9286428 & 49.858 \\
\hline 2 & 12.961 & 9339225 & 50.142 \\
\hline Total & & 18625653 & 100.000 \\
\hline
\end{tabular}

\section{$<$ Chromatogram $>$}

mAU

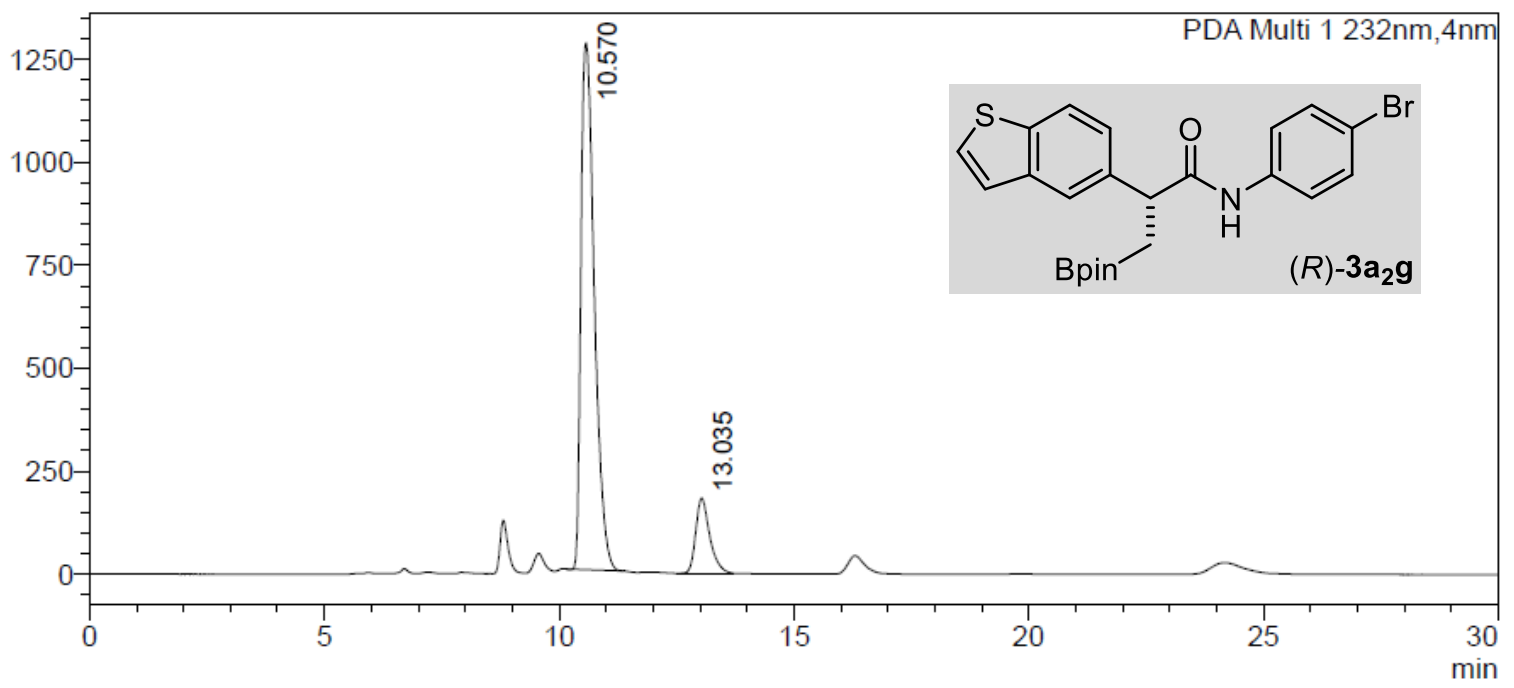

<Peak Table>

PDA Ch1 232nm

\begin{tabular}{|r|r|r|r|}
\hline Peak\# & Ret. Time & \multicolumn{1}{c|}{ Area } & \multicolumn{1}{c|}{ Area\% } \\
\hline 1 & 10.570 & 25378875 & 87.123 \\
\hline 2 & 13.035 & 3751039 & 12.877 \\
\hline Total & & 29129913 & 100.000 \\
\hline
\end{tabular}


(R)-2-(4-((((1R,2S,5R)-2-isopropyl-5-methylcyclohexyl)oxy)methyl)phenyl)- $N$-phenyl-3(4,4,5,5-tetramethyl-1,3,2-dioxaborolan-2-yl)propanamide $\mathbf{3} \mathbf{b}_{\mathbf{2}} \mathbf{a}$

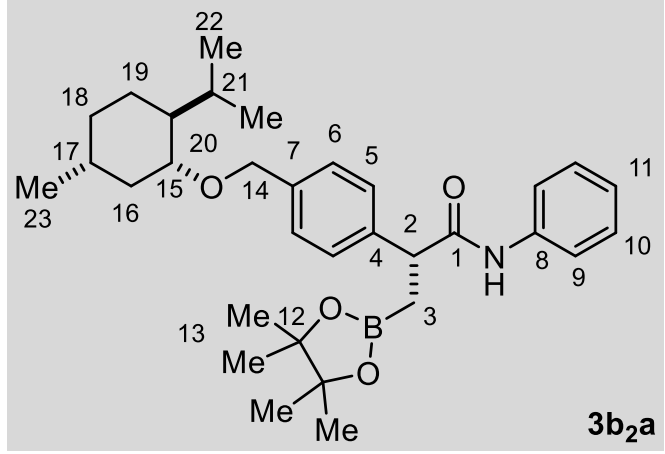

According to the general procedure for enantioselective copper-catalyzed borylative carboxamidation using styrene $\mathbf{1}_{\mathbf{2}}(82 \mathrm{mg}, 0.3$ mmol, 1.0 equiv.), $\mathrm{CuCl}(3.0 \mathrm{mg}, 0.03 \mathrm{mmol}, 10$ mol\%), ligand $(R, R)-6 \mathbf{k}(14 \mathrm{mg}, 0.033 \mathrm{mmol}, 11$ mol\%), LiOtBu (48 mg, 0.6 mmol, 2.0 equiv.), $B_{2}$ pin $_{2}$ (114 mg, $0.45 \mathrm{mmol}, \quad 1.5$ equiv.) and isocyanatobenzene $\mathbf{2 a}\left(0.132 \mathrm{~mL}, 1.2 \mathrm{mmol}, 4.0\right.$ equiv.) in 2-Me-THF and $\mathrm{Et}_{2} \mathrm{O}(3.0 \mathrm{~mL}$, v:v = $1: 1,0.1 \mathrm{M}) .83 \%$ conversion into $\mathbf{3} \mathbf{b}_{2} \mathbf{a}(d r>10: 1)$ determined by ${ }^{1} \mathrm{H}$ NMR analysis of the crude reaction mixture in presence of an internal standard. Purification by flash chromatography (pentane/diethyl ether $3: 1$ ) gave pure $3 \mathbf{b}_{2} \mathbf{a}$ as a white solid (114 $\mathrm{mg}, 0.22 \mathrm{mmol}, 73 \%$ yield, $d r$ 10:1).

TLC: pentane/ethyl acetate $4: 1, \mathrm{R}_{\mathrm{f}}=0.3$.

${ }^{1} \mathrm{H}$ NMR $\left(500 \mathrm{MHz}, \mathrm{CDCl}_{3}\right) \delta(\mathrm{ppm})=7.32(\mathrm{~m}, 2 \mathrm{H}), 7.26(\mathrm{~m}, 3 \mathrm{H}), 7.17(\mathrm{~m}, 3 \mathrm{H}), 6.96(\mathrm{~m}, 2 \mathrm{H})$, 4.57 (d, $J=11.6 \mathrm{~Hz}, 1 \mathrm{H}, H-14$ ), 4.33 (d, $J=11.6 \mathrm{~Hz}, 1 \mathrm{H}, H-14$ ), 3.80 (dd, $J=9.5,6.4 \mathrm{~Hz}, 1 \mathrm{H}$, $H-2), 3.11(\mathrm{td}, J=10.5,4.1 \mathrm{~Hz}, 1 \mathrm{H}, H-15), 2.26-2.18(\mathrm{~m}, 1 \mathrm{H}, H-17), 2.14-2.08(\mathrm{~m}, 1 \mathrm{H}, H-$ 20), $1.62-1.55(\mathrm{~m}, 3 \mathrm{H}, H-19, H-18, H-3), 1.33-1.22(\mathrm{~m}, 2 \mathrm{H}, H-18,16), 1.19(\mathrm{~m}, 1 \mathrm{H}, H-3)$, 1.15 (s, 6H, H-13), 1.13 (s, 6H, H-13), $0.93-0.79$ (m, 9H, H-22, H-21, H-19, H-16), 0.67 (s, $1.5 \mathrm{H}, \mathrm{H}-23), 0.66(\mathrm{~s}, 1.5 \mathrm{H}, \mathrm{H}-23)$.

${ }^{13} \mathrm{C}\left\{{ }^{1} \mathrm{H}\right\}$ NMR $\left(125 \mathrm{MHz}, \mathrm{CDCl}_{3}\right) \delta(\mathrm{ppm})=172.4(C-1), 140.8(C-4), 138.5(C-7), 138.2(C-8)$, 128.8 (C-10), 128.4 (C-6), 128.0 (C-5), 124.0 (C-11), 119.7 (C-9), 83.3 (C-12), 79.1 (C-15), 70.1 (C-14), 49.8 (C-2), 48.5 (C-20), 40.5 (C-19), 34.7 (C-18), $31.7(C-21), 25.7(C-17), 24.8$ (C-13), 24.7 (C-13), 23.5 (C-16), 22.3 (C-22), 21.0 (C-22), $16.8(C-3), 16.2(C-23)$.

${ }^{11} \mathbf{B}$ NMR $\left(128 \mathrm{MHz}, \mathrm{CDCl}_{3}\right) \delta(\mathrm{ppm})=33.8$.

$[\alpha]^{20}{ }_{D}=-86.9\left(c 0.47, \mathrm{CH}_{2} \mathrm{Cl}_{2}\right)$.

HRMS (ESI +): calculated for $\mathrm{C}_{32} \mathrm{H}_{46} \mathrm{BNO}_{4}[\mathrm{M}+\mathrm{H}]^{+}:$520.3599; found: 520.3581 .

IR (neat): $v\left(\mathrm{~cm}^{-1}\right)=3320,2955,2924,2869,1665,1600,1536,1499,1440,1370,1343$, $1320,1250,1216,1166,1143,1106,1083,1072$, 1055, 1021, 968, 890, 847, 790, 753, 692, 673.

m.p.: $132-136^{\circ} \mathrm{C}$

The innate bias imposed by styrene $\mathbf{1}_{\mathbf{2}}$ was assessed performing the copper-catalyzed borylative carboxamidation with $\mathrm{PCy}_{3}$ instead of $(R, R)$-6k under otherwise identical conditions (64\% yield, $d r 1.5: 1)$. 


\section{Copper-catalyzed borylative carboxamidation of alkynes}
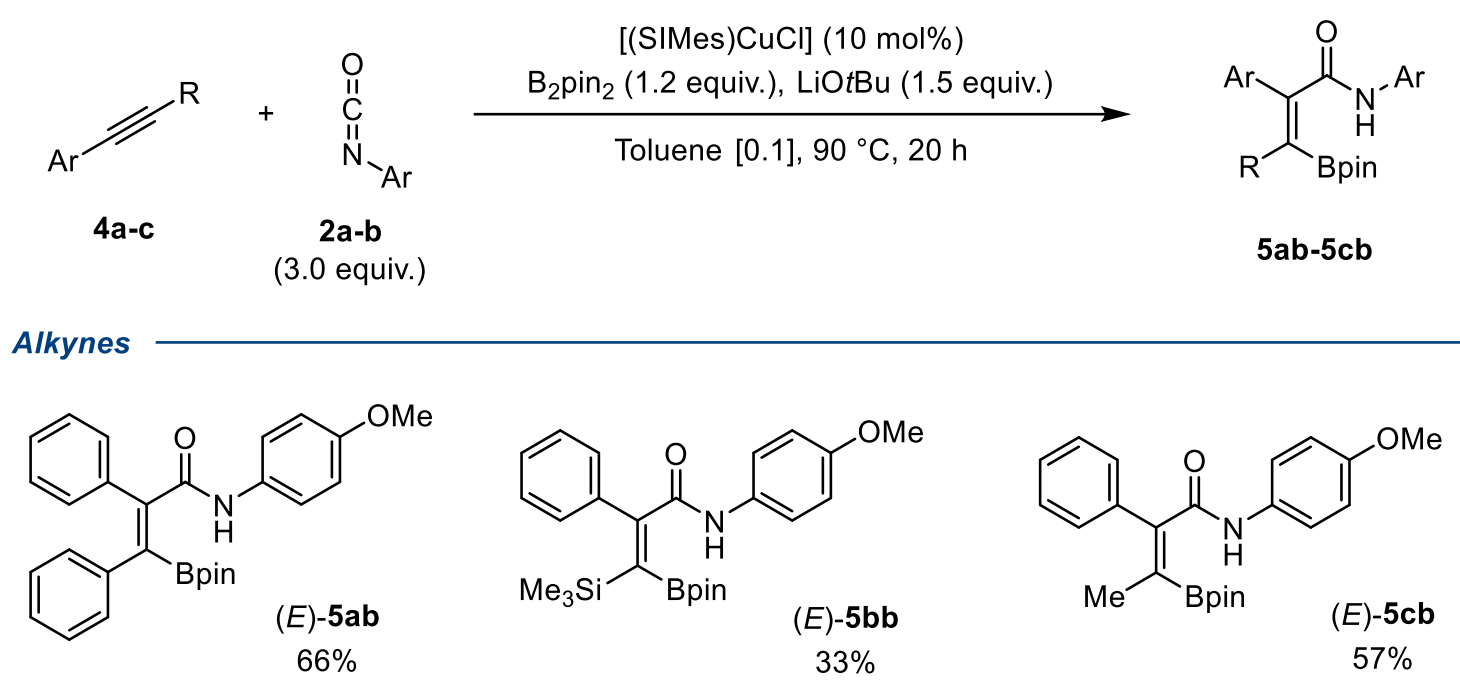

Limitations (<5\% conv.)<smiles>C#Cc1ccccc1</smiles><smiles>CCOC(=O)C#CC(=O)OCC</smiles><smiles>CCCC#CCCC</smiles>

Figure S8. Copper-catalyzed borylative carboxamidation of alkynes. Reactions run on 0.3 mmol scale. Isolated yield of $\mathbf{5}$ after purification by column chromatography.

\section{General procedure for the copper-catalyzed borylative carboxamidation of alkynes}

In a $10 \mathrm{~mL}$ Schlenk tube, [(SIMes)CuCl] (6.1 mg, $0.015 \mathrm{mmol}, 5.0 \mathrm{~mol} \%)$, LiOtBu (36 mg, 0.45 mmol, 1.5 equiv.) and $B_{2} p_{2}(91 \mathrm{mg}, 0.36 \mathrm{mmol}, 1.2$ equiv.) were dissolved in $3.0 \mathrm{~mL}$ of anhydrous and degassed toluene $(0.1 \mathrm{M})$. The resulting dark solution was stirred for $10 \mathrm{~min}$ at $23^{\circ} \mathrm{C}$, then alkyne $4(0.3 \mathrm{mmol}, 1.0$ equiv. $)$ was added neat with a micro-syringe, if liquid, or dissolved in $0.5 \mathrm{~mL}$ of toluene, if solid. The resulting mixture was stirred for $5 \mathrm{~min}$ at $23^{\circ} \mathrm{C}$, then the isocyanate $2(0.9 \mathrm{mmol}, 3.0$ equiv.) was added neat with a micro-syringe, if liquid, or dissolved in $0.5 \mathrm{~mL}$ of toluene, if solid. The sealed tube was stirred at $90^{\circ} \mathrm{C}$ for $20 \mathrm{~h}$. After 20 $\mathrm{h}$, the reaction mixture was cooled to $23^{\circ} \mathrm{C}$ and quenched by addition of a saturated aqueous $\mathrm{NH}_{4} \mathrm{Cl}$ solution $(1.0 \mathrm{~mL})$. The reaction mixture was diluted with ethyl acetate $(5 \mathrm{~mL})$ and water $(5 \mathrm{~mL})$ and extracted with ethyl acetate $(3 \times 10 \mathrm{~mL})$. The combined organic phases were dried over sodium sulfate and the solvent was removed under reduced pressure. Conversion into acrylamide 5 was assessed by ${ }^{1} \mathrm{H}$ NMR analysis of the crude residue in presence of an internal standard. Purification by flash chromatography on silica gel afforded pure acrylamide 5 . 
$(E)$-N-(4-methoxyphenyl)-2,3-diphenyl-3-(4,4,5,5-tetramethyl-1,3,2-dioxaborolan-2yl)acrylamide $(E)-5 a b$

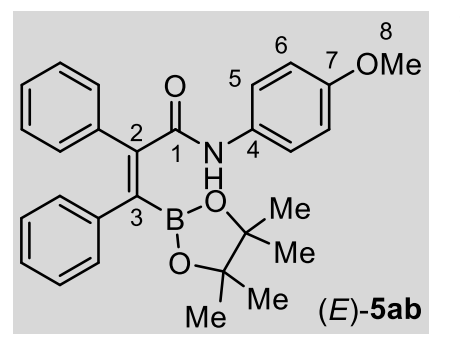

This product was prepared according to the general procedure for the copper-catalyzed borylative carboxamidation of alkynes using diphenylacetylene $4 \mathrm{a} \quad(162 \mathrm{mg}, 0.9 \mathrm{mmol}, 1.0$ equiv.), [(SIMes)CuCl] (36.6 mg, $0.09 \mathrm{mmol}, 10.0 \mathrm{~mol} \%)$, LiOtBu (108 mg, $1.35 \mathrm{mmol}, 1.5$ equiv.), $\mathrm{B}_{2}$ pin $_{2}$ (273 mg, $1.08 \mathrm{mmol}, 1.2$ equiv.) and 1-isocyanato-4-methoxybenzene $\mathbf{2 b}(0.360 \mathrm{~mL}, 2.7 \mathrm{mmol}, 3.0$ equiv. $)$ in toluene $(9.0 \mathrm{~mL}, 0.1$ M). Reaction time: 20 h. $80 \%$ conversion into $(E)-5 a b(E / Z>20: 1)$ determined by ${ }^{1} \mathrm{H}$ NMR analysis of the crude reaction mixture in presence of an internal standard. Purification by flash chromatography $\left(\mathrm{CH}_{2} \mathrm{Cl}_{2}\right.$ with $\left.\mathrm{MeOH} 1 \%\right)$ followed by second flash chromatography (pentane/acetone 85:15) gave pure $(E)-5 \mathrm{ab}(E / Z>20: 1)$ as a white solid (270 mg, $0.594 \mathrm{mmol}$, $66 \%$ yield). Stereochemistry of $(E)-5 a b(E / Z>20: 1)$ was determined by $X$-ray analysis of suitable crystals obtained layering with $n$-hexane a concentrated solution of $(E)-5 \mathbf{a b}$ in benzene.

TLC: $\mathrm{CH}_{2} \mathrm{Cl}_{2}$ with $\mathrm{MeOH} 1 \%, \mathrm{R}_{f}=0.2$. Pentane/acetone 85:15, $\mathrm{R}_{\mathrm{f}}=0.3$.

${ }^{1} \mathrm{H}$ NMR $\left(400 \mathrm{MHz}, \mathrm{CDCl}_{3}\right) \delta(\mathrm{ppm})=7.51$ (bs, $\left.1 \mathrm{H}, \mathrm{N}-H\right), 7.41\left(\mathrm{~d},{ }^{3} J_{\mathrm{HH}}=9.0 \mathrm{~Hz}, 2 \mathrm{H}, H-5\right), 7.38$ (m, 3H, H-Ar), $7.32(\mathrm{~m}, 2 \mathrm{H}, H-\mathrm{Ar}), 7.17(\mathrm{~m}, 5 \mathrm{H}, H-\mathrm{Ar}), 6.87\left(\mathrm{~d},{ }^{3} \mathrm{~J}_{\mathrm{HH}}=9.0 \mathrm{~Hz}, 2 \mathrm{H}, H-6\right), 3.80$ (s, 3H, H-8), 1.29 (s, 12H, H-MeBpin).

${ }^{13} \mathrm{C}\left\{{ }^{1} \mathrm{H}\right\}$ NMR $\left(101 \mathrm{MHz}, \mathrm{CDCl}_{3}\right) \delta(\mathrm{ppm})=171.8(C-1), 158.1(C-7), 137.9(C-\mathrm{Ar}), 133.1(C-$ Ar), 132.7(C-4), 130.0 (CH-Ar), 129.7 (CH-Ar), 128.8 ( $\mathrm{CH}-\mathrm{Ar}), 128.7$ (CH-Ar), $128.6(\mathrm{C}-2)$, 127.8 (CH-Ar), 127.7 ( $\mathrm{CH}-\mathrm{Ar}), 122.8$ ( $\mathrm{CH}-5), 114.5$ ( $\mathrm{CH}-6)$, 81.0 (CO-Bpin), $55.6\left(\mathrm{CH}_{3}-8\right), 25.9$ $\left(\mathrm{CH}_{3}-\mathrm{MeBpin}\right)$. The boron-bound carbons $\mathrm{C}-3$ was not detected due to quadrupolar relaxation. ${ }^{11} \mathbf{B}$ NMR $\left(128 \mathrm{MHz}, \mathrm{CDCl}_{3}\right) \delta(\mathrm{ppm})=15.9$

HRMS $(\mathrm{ESI}+)$ : calculated for $\mathrm{C}_{28} \mathrm{H}_{30} \mathrm{BNO}_{4}[\mathrm{M}+\mathrm{H}]^{+}:$: 456.2346; found: 456.2352 .

IR (neat): $v\left(\mathrm{~cm}^{-1}\right)=3378,2968,1623,1607,1607,1576,1213,1252,1179,1135$.

m.p.: $125-129^{\circ} \mathrm{C}$ 
(E)-N-(4-methoxyphenyl)-2-phenyl-3-(4,4,5,5-tetramethyl-1,3,2-dioxaborolan-2-yl)-3(trimethylsilyl)acrylamide $(E)-5 \mathbf{b} \mathbf{b}$

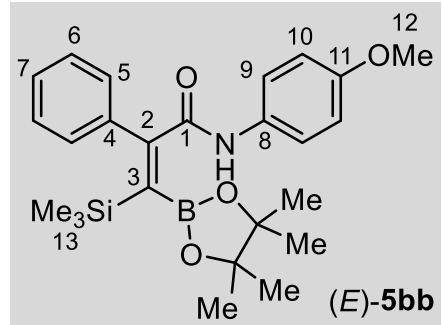

This product was prepared according to the general procedure for the copper-catalyzed borylative carboxamidation of alkynes using trimethyl(phenylethynyl)silane $\mathbf{4 b}$ (53 $\mathrm{mg}, 0.3 \mathrm{mmol}, 1.0$ equiv.), [(SIMes)CuCl] (12.2 mg, $0.03 \mathrm{mmol}, 10.0 \mathrm{~mol} \%$ ), LiOtBu (36 mg,

$0.45 \mathrm{mmol}, 1.5$ equiv.), $B_{2}$ pin $_{2}(91 \mathrm{mg}, 0.36 \mathrm{mmol}, 1.2$ equiv.) and 1-isocyanato-4-methoxybenzene $\mathbf{2 b}(0.117 \mathrm{~mL}, 0.9 \mathrm{mmol}, 3.0$ equiv.) in toluene (3.0 $\mathrm{mL}, 0.1$ M). Reaction time: 20 h. $50 \%$ conversion into $(E)-5 b b(E / Z>20: 1)$ determined by ${ }^{1} \mathrm{H}$ NMR analysis of the crude reaction mixture in presence of an internal standard. Purification by flash chromatography (pentane/ethyl acetate 7:3) gave pure $(E)-5 \mathbf{b b}(E / Z>20: 1)$ as a white solid (45 $\mathrm{mg}, 0.10 \mathrm{mmol}, 33 \%$ yield). Stereochemistry of $(E)-5 \mathbf{b b}(E / Z>20: 1)$ was assigned by analogy with $(E)-5 \mathbf{a b}$.

TLC: pentane/ethyl acetate 7:3, $\mathrm{R}_{\mathrm{f}}=0.3$.

${ }^{1} \mathrm{H}$ NMR $\left(400 \mathrm{MHz}, \mathrm{CDCl}_{3}\right) \delta(\mathrm{ppm})=7.76(\mathrm{bs}, 1 \mathrm{H}, \mathrm{N}-H), 7.47\left(\mathrm{~d},{ }^{3} J_{\mathrm{HH}}=9.0 \mathrm{~Hz}, 2 \mathrm{H}, H-9\right), 7.30$ (m, 2H, H-5), $7.21(\mathrm{~m}, 3 \mathrm{H}, H-6+H-7), 6.91\left(\mathrm{~d},{ }^{3} \mathrm{~J}_{\mathrm{HH}}=9.0 \mathrm{~Hz}, 2 \mathrm{H}, H-10\right), 3.82(\mathrm{~s}, 3 \mathrm{H}, H-12)$, 1.08 (s, 12H, H-MeBpin), 0.07 (s, 9H, $H$-13).

${ }^{13} \mathrm{C}\left\{{ }^{1} \mathrm{H}\right\}$ NMR $\left(101 \mathrm{MHz}, \mathrm{CDCl}_{3}\right) \delta(\mathrm{ppm})=175.2(C-1), 158.2(C-11), 141.8(C-4), 132.0(C-$ 8), 128.9 (C-2), $127.4(\mathrm{CH}-5), 126.8$ ( $\mathrm{CH}-7), 125.8(\mathrm{CH}-6), 123.0(\mathrm{CH}-9), 114.5(\mathrm{CH}-10), 80.5$ (CO-Bpin), $55.6\left(\mathrm{CH}_{3}-12\right), 25.4\left(\mathrm{CH}_{3}-\mathrm{MeBpin}\right), 1.0\left(\mathrm{CH}_{3}-13\right)$. The boron-bound carbons $\mathrm{C}-3$ was not detected due to quadrupolar relaxation.

${ }^{11} \mathbf{B}$ NMR $\left(128 \mathrm{MHz}, \mathrm{CDCl}_{3}\right) \delta(\mathrm{ppm})=14.3$

HRMS (ESI +): calculated for $\mathrm{C}_{25} \mathrm{H}_{34} \mathrm{BNO}_{4} \mathrm{Si}[\mathrm{M}+\mathrm{H}]^{+}:$: 452.2428 ; found: 452.2463 .

IR (neat): $v\left(\mathrm{~cm}^{-1}\right)=3424,2965,1609,1582,1512,1248,1179,1124,1033,837,769$.

m.p.: $140-145^{\circ} \mathrm{C}$ 
(E)-N-(4-methoxyphenyl)-2-phenyl-3-(4,4,5,5-tetramethyl-1,3,2-dioxaborolan-2-yl)but-2enamide $(E)-5 \mathbf{c b}$

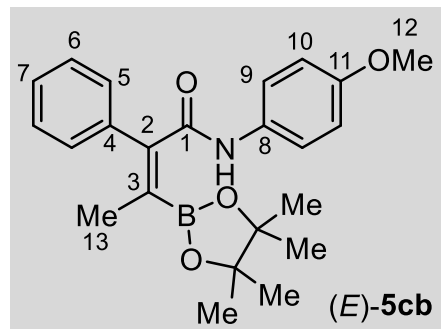

This product was prepared according to the general procedure for the copper-catalyzed borylative carboxamidation of alkynes using prop-1-yn-1-ylbenzene 4c (35 mg, $0.3 \mathrm{mmol}, 1.0$ equiv.), [(SIMes)CuCl] (12.2 mg, $0.03 \mathrm{mmol}, 10.0 \mathrm{~mol} \%$ ), LiOtBu (36 mg,

$0.45 \mathrm{mmol}, 1.5$ equiv.), $B_{2}$ pin $_{2}$ (91 mg, $0.36 \mathrm{mmol}, 1.2$ equiv.) and 1-isocyanato-4-methoxybenzene $2 \mathrm{~b}(0.117 \mathrm{~mL}, 0.9 \mathrm{mmol}, 3.0$ equiv.) in toluene $(3.0 \mathrm{~mL}, 0.1$ M). Reaction time: 20 h. $72 \%$ conversion into $(E)-5 \mathrm{cb}(E / Z>20: 1)$ determined by ${ }^{1} \mathrm{H}$ NMR analysis of the crude reaction mixture in presence of an internal standard. Purification by flash chromatography (pentane/diethyl ether 4:1) gave pure $(E)-5 \mathbf{c b}(E / Z>20: 1)$ as a white solid (65 mg, $0.17 \mathrm{mmol}, 57 \%$ yield). Stereochemistry of $(E)-5 \mathbf{c b}(E / Z>20: 1)$ was assigned by analogy with $(E)-5 \mathbf{a b}$.

TLC: pentane/diethyl ether $4: 1, R_{f}=0.3$.

${ }^{1} \mathrm{H}$ NMR $\left(400 \mathrm{MHz}, \mathrm{CDCl}_{3}\right) \delta(\mathrm{ppm})=7.47(\mathrm{~m}, 2 \mathrm{H}, \mathrm{H}-6), 7.42(\mathrm{~m}, 1 \mathrm{H}, \mathrm{H}-7), 7.37\left(\mathrm{~d},{ }^{3} \mathrm{JHH}_{\mathrm{HH}}=9.0\right.$ $\mathrm{Hz}, 2 \mathrm{H}, H-9), 7.36$ (bs, $1 \mathrm{H}, \mathrm{N}-H), 7.22\left(\mathrm{~d},{ }^{3} \mathrm{JHH}_{\mathrm{HH}}=6.8 \mathrm{~Hz}, 2 \mathrm{H}, \mathrm{H}-5\right), 6.84\left(\mathrm{~d},{ }^{3} \mathrm{~J}_{\mathrm{HH}}=9.0 \mathrm{~Hz}, 2 \mathrm{H}\right.$, $H-10$ ), 3.78 (s, 3H, $H-12$ ), 1.95 (s, 3H, $H-13$ ), 1.32 (s, 12H, H-MeBpin).

${ }^{13} \mathrm{C}\left\{{ }^{1} \mathrm{H}\right\}$ NMR $(101 \mathrm{MHz}, \mathrm{CDCl} 3) \delta(\mathrm{ppm})=171.8(C-1), 157.9(C-11), 133.2(C-8+C-4), 132.3$ (C-2), 129.8 (CH-5), $129.6(\mathrm{CH}-6), 128.7(\mathrm{CH}-7), 122.7(\mathrm{CH}-9), 114.4(\mathrm{CH}-10), 80.7$ (COBpin), $55.6\left(\mathrm{CH}_{3}-12\right), 25.5\left(\mathrm{CH}_{3}-\mathrm{MeBpin}\right), 16.1\left(\mathrm{CH}_{3}-13\right)$. The boron-bound carbons $C-3$ was not detected due to quadrupolar relaxation.

${ }^{11} \mathbf{B}$ NMR $\left(128 \mathrm{MHz}, \mathrm{CDCl}_{3}\right) \delta(\mathrm{ppm})=15.8$

HRMS (ESI +): calculated for $\mathrm{C}_{23} \mathrm{H}_{28} \mathrm{BNO}_{4}[\mathrm{M}+\mathrm{H}]^{+}:$: 394.2189; found: 394.2206.

IR (neat): $v\left(\mathrm{~cm}^{-1}\right)=3192,2969,1611,1581,1510,1246,1173,1158,1076,1034,1007,825$, 713.

m.p.: $179-182^{\circ} \mathrm{C}$ 


\section{Derivatization of amides}

A) Stereoretentive derivatizations<smiles>O=C(Nc1ccccc1)[C@@H](CBO)c1ccccc1</smiles>

$\mathrm{KHF}_{2}$

$\mathrm{CH}_{3} \mathrm{CN} / \mathrm{H}_{2} \mathrm{O}$,

$23{ }^{\circ} \mathrm{C}, 5 \mathrm{~h}$<smiles>O=C(Nc1ccccc1)[C@H](C[15F])c1ccccc1</smiles>

(quantitative)<smiles>O=C(Nc1ccccc1)[C@H](CO)c1ccccc1</smiles>

(quantitative)

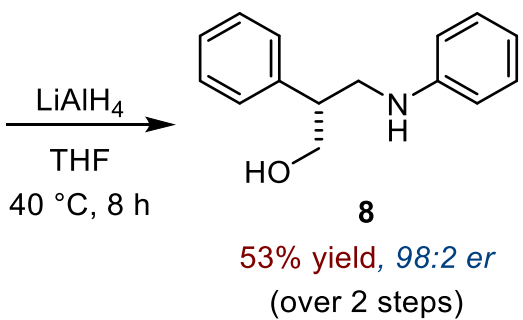

(over 2 steps)
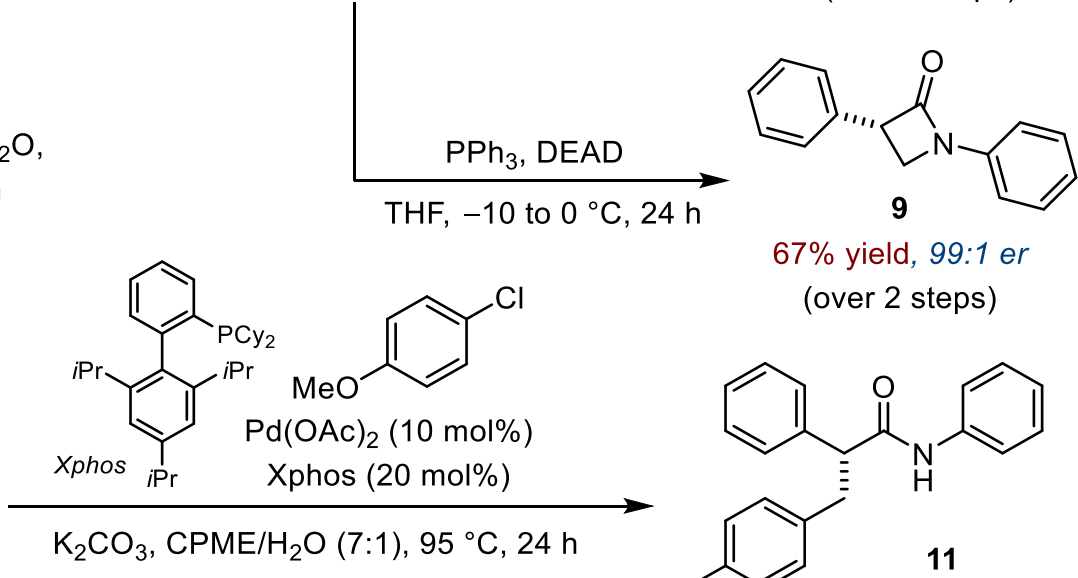

(over 2 steps)

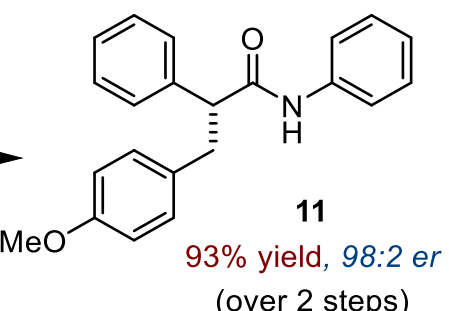

B) Polysubstituted acrylamide synthesis<smiles>O=C(Nc1ccccc1)/C(=C(\Cc1ccccc1)c1ccccc1)c1ccccc1</smiles>

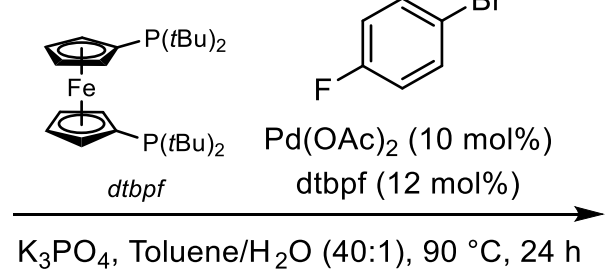<smiles>O=C(Nc1ccccc1)C(=C(c1ccccc1)c1ccccc1)c1ccc(F)cc1</smiles>

(Z)-12

C) N-deprotection<smiles>COc1ccc(NC(=O)C(C[Pb])c2ccc3ccccc3c2)cc1</smiles>

3ib
1) $\mathrm{H}_{2} \mathrm{O}_{2} / \mathrm{NaOH}$ (aq.), $\mathrm{THF}, 23^{\circ} \mathrm{C}, 30 \mathrm{~min}$

2) $\mathrm{TBSCl}$ Imidazole, $\mathrm{CH}_{2} \mathrm{Cl}_{2}, 23{ }^{\circ} \mathrm{C}, 12 \mathrm{~h}$

3) $\mathrm{CAN}, \mathrm{CH}_{3} \mathrm{CN} / \mathrm{H}_{2} \mathrm{O}, 0{ }^{\circ} \mathrm{C}, 30 \mathrm{~min}$<smiles>CS(=O)(=O)OCC(C(N)=O)c1ccc2ccccc2c1</smiles>

$32 \%$ over 3 steps

Figure S9. (A) Stereoretentive derivatizations. (B) Synthesis of a polysubstituted acrylamide.

(C) Deprotection of the $\mathrm{N}$-amide. Yields of isolated product after purification. Er determined by HPLC. 
(R)-2-phenyl-3-(phenylamino)propan-1-ol 8

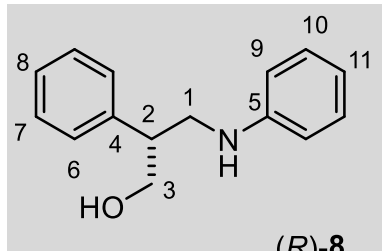

(R)-8

To a solution of 3aa $(35.1 \mathrm{mg}, 0.1 \mathrm{mmol})$ in THF $(1 \mathrm{~mL})$ and $\mathrm{H}_{2} \mathrm{O}(1$ $\mathrm{mL}$ ) was added $\mathrm{NaBO}_{3} \cdot 4 \mathrm{H}_{2} \mathrm{O}(56 \mathrm{mg}, 0.8 \mathrm{mmol})$ at $23^{\circ} \mathrm{C}$. The reaction mixture was stirred for $3 \mathrm{~h}$ and quenched by addition of saturated aqueous $\mathrm{Na}_{2} \mathrm{~S}_{2} \mathrm{O}_{3}(2 \mathrm{~mL})$. The mixture was extracted with ethyl acetate. The combined organic layers were washed with water and brine, dried over anhydrous sodium sulfate and concentrated under vacuum. The crude product 7 was used directly for the next step without further purification.

Amide 7 was dissolved in THF $(2 \mathrm{~mL})$ at $23^{\circ} \mathrm{C}$. $\mathrm{LiAlH}_{4}$ was added slowly to the reaction solution and warmed to $40{ }^{\circ} \mathrm{C}$ for $8 \mathrm{~h}$. The reaction was quenched with water and the mixture was adjusted to basic condition by addition $\mathrm{NaOH}(4 \mathrm{M})$, and extracted with ethyl acetate $(10 \mathrm{~mL} \times$ 3) and concentrated under reduced pressure. The crude product was purified by column chromatography (pentane/acetone 6:1) and $\beta$-aminol 8 was isolated as a colorless oil (12 mg, $0.053 \mathrm{mmol}, 53 \%$ yield, $98: 2 \mathrm{er})$.

TLC: pentane/acetone $2: 1, \mathrm{R}_{\mathrm{f}}=0.6$

${ }^{1} \mathrm{H}$ NMR $\left(400 \mathrm{MHz}, \mathrm{CDCl}_{3}\right) \delta(\mathrm{ppm})=7.36(\mathrm{~m}, 2 \mathrm{H}), 7.30(\mathrm{~m}, 1 \mathrm{H}), 7.27(\mathrm{~m}, 1 \mathrm{H}), 7.25(\mathrm{~m}, 1 \mathrm{H})$, $7.20(\mathrm{~m}, 2 \mathrm{H}), 6.77\left(\mathrm{t},{ }^{3} \mathrm{~J}_{\mathrm{HH}}=7.3 \mathrm{~Hz}, 1 \mathrm{H}\right), 6.69\left(\mathrm{~d},{ }^{3} \mathrm{~J}_{\mathrm{HH}}=7.6 \mathrm{~Hz}, 2 \mathrm{H}\right), 3.94\left(\mathrm{~d},{ }^{3} J_{\mathrm{HH}}=6.3 \mathrm{~Hz}\right.$, $2 \mathrm{H}, H-3), 3.63\left(\mathrm{dd},{ }^{2} J_{\mathrm{HH}}=12.5 \mathrm{~Hz},{ }^{3} J_{\mathrm{HH}}=7.1 \mathrm{~Hz}, 1 \mathrm{H}, H-1\right), 3.40\left(\mathrm{dd},{ }^{2} J_{\mathrm{HH}}=12.4 \mathrm{~Hz},{ }^{3} J_{\mathrm{HH}}=\right.$ $7.1 \mathrm{~Hz}, 1 \mathrm{H}, H-1), 3.22\left(\mathrm{t},{ }^{3} \mathrm{JHH}_{\mathrm{H}}=6.7 \mathrm{~Hz}, 1 \mathrm{H}, H-2\right)$.

${ }^{13} \mathrm{C}\left\{{ }^{1} \mathrm{H}\right\}$ NMR $\left(101 \mathrm{MHz}, \mathrm{CDCl}_{3}\right) \delta(\mathrm{ppm})=147.9(C-5), 140.0(C-4), 129.3(C-9), 128.9(C-6)$, 128.0 (C-10), 127.3 (C-11), 117.9 (C-8), 113.3 (C-7), 66.2 (C-3), $47.6(C-2), 46.8(C-1)$.

HRMS $(\mathrm{ESI}+)$ : calculated for $\mathrm{C}_{15} \mathrm{H}_{17} \mathrm{NO}[\mathrm{M}+\mathrm{H}]^{+}:$:228.1383; found: 228.1399.

IR (neat): $v\left(\mathrm{~cm}^{-1}\right)=3399,2923,2854,1601,1505,1495,1453,1432,1316,1258,1180$, 1154, 1121, 1053, 1027, 1003, 991, 911, 869, 748, 692, 611.

HPLC analysis of 8 indicated 98:2 er, chiral stationary column: AD-H, mobile phase: $n$ hexane $/ \mathrm{PrOH}=90: 10,1.0 \mathrm{~mL} / \mathrm{min}, 254 \mathrm{~nm}, 30^{\circ} \mathrm{C}, \mathrm{t}_{\mathrm{R}}$ (major) $=11.2 \mathrm{~min}, \mathrm{t}_{\mathrm{R}}$ (minor) $=9.1 \mathrm{~min}$. $[\alpha]^{20} \mathrm{D}=+26.7\left(\mathrm{c} 0.19, \mathrm{CH}_{2} \mathrm{Cl}_{2}\right)$. 


\section{<Chromatogram>}

mAU

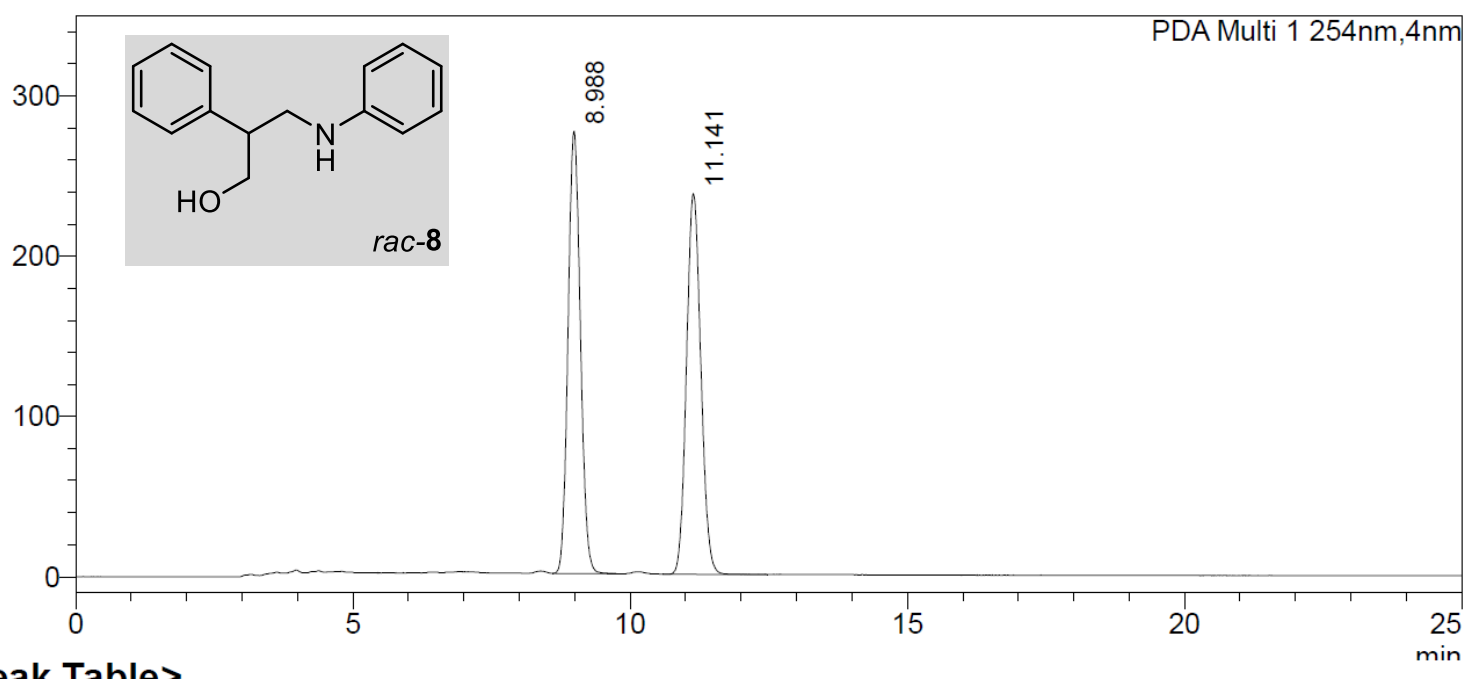

<Peak Table>

PDA Ch1 254nm

\begin{tabular}{|r|r|r|r|}
\hline Peak\# & Ret. Time & Area & \multicolumn{1}{c|}{ Area\% } \\
\hline 1 & 8.988 & 4273389 & 49.936 \\
\hline 2 & 11.141 & 4284379 & 50.064 \\
\hline Total & & 8557768 & 100.000 \\
\hline
\end{tabular}

\section{<Chromatogram>}

mAU

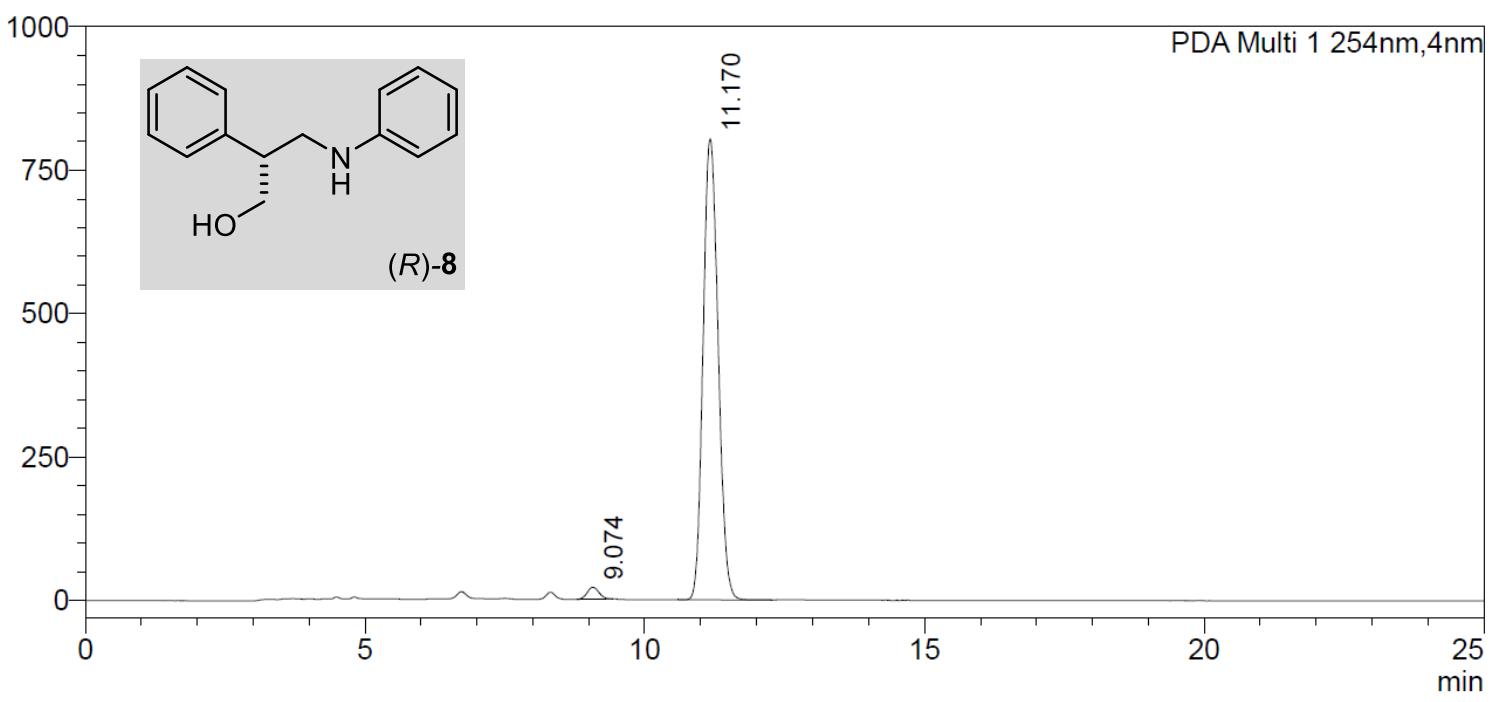

<Peak Table>

PDA Ch1 254nm

\begin{tabular}{|r|r|r|r|}
\hline Peak\# & Ret. Time & \multicolumn{1}{|c|}{ Area } & \multicolumn{1}{c|}{ Area\% } \\
\hline 1 & 9.074 & 302899 & 1.974 \\
\hline 2 & 11.170 & 15041337 & 98.026 \\
\hline Total & & 15344236 & 100.000 \\
\hline
\end{tabular}


(S)-1,3-diphenylazetidin-2-one $9^{10}$

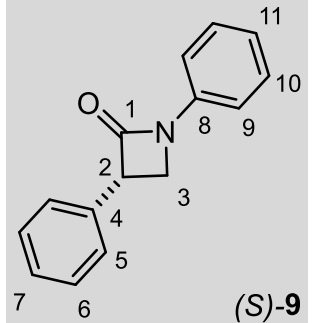

To a solution of 3aa $(35.1 \mathrm{mg}, 0.1 \mathrm{mmol})$ in THF $(1 \mathrm{~mL})$ and $\mathrm{H}_{2} \mathrm{O}(1 \mathrm{~mL})$ was added $\mathrm{NaBO}_{3} \cdot 4 \mathrm{H}_{2} \mathrm{O}(56 \mathrm{mg}, 0.8 \mathrm{mmol})$ at $23^{\circ} \mathrm{C}$. The reaction mixture was stirred for $3 \mathrm{~h}$ and quenched by addition of saturated aqueous $\mathrm{Na}_{2} \mathrm{~S}_{2} \mathrm{O}_{3}(2 \mathrm{~mL})$. The mixture was extracted with ethyl acetate. The combined organic layers were washed with water and brine, dried over anhydrous sodium sulfate and concentrated in vacuum. The crude product 7 was used directly for the next step without further purification.

Diethyl diazodicarboxylate $(17.4 \mathrm{mg}, 0.11 \mathrm{mmol}, 1.1$ equiv.) was added to a stirred solution of triphenylphosphine (26.2 mg, $0.1 \mathrm{mmol}, 1.0$ equiv.) and amide 7 in THF (2 mL), maintained under nitrogen at $-10^{\circ} \mathrm{C}$. The reaction mixture was slowly warmed to $0{ }^{\circ} \mathrm{C}$ and maintained at this temperature for $24 \mathrm{~h}$. The reaction was subsequently quenched by removing the solvent at reduced pressure and the residue subjected to flash chromatography (pentane/diethyl ether 6:1) to afford $\beta$-lactam 9 as a white solid (15 mg, $0.067 \mathrm{mmol}, 67 \%$ yield, $99: 1 \mathrm{er}$ ).

TLC: pentane/diethyl ether $2: 1, \mathrm{R}_{\mathrm{f}}=0.6$

${ }^{1} \mathrm{H}$ NMR $\left(400 \mathrm{MHz}, \mathrm{CDCl}_{3}\right) \delta(\mathrm{ppm})=7.48-7.27(\mathrm{~m}, 9 \mathrm{H}), 7.17-7.09(\mathrm{~m}, 1 \mathrm{H}), 4.53\left(\mathrm{dd},{ }^{3} \mathrm{JHH}_{\mathrm{H}}\right.$ $=5.9,2.9 \mathrm{~Hz}, 1 \mathrm{H}, \mathrm{H}-2), 4.09\left(\mathrm{dd},{ }^{2} J_{\mathrm{HH}}=5.8 \mathrm{~Hz},{ }^{3} \mathrm{~J}_{\mathrm{HH}}=5.9 \mathrm{~Hz}, 1 \mathrm{H}, \mathrm{H}-3\right), 3.69\left(\mathrm{dd},{ }^{2} \mathrm{~J}_{\mathrm{HH}}=5.8\right.$, $\left.{ }^{3} \mathrm{~J}_{\mathrm{HH}}=2.9 \mathrm{~Hz}, 1 \mathrm{H}, \mathrm{H}-3\right)$.

${ }^{13} \mathrm{C}\left\{{ }^{1} \mathrm{H}\right\}$ NMR $\left(75 \mathrm{MHz}, \mathrm{CDCl}_{3}\right) \delta(\mathrm{ppm})=165.3(C-1), 138.2(C-8), 135.3(C-4), 129.2(C-\mathrm{Ar})$, 128.9 (C-Ar), 127.7 (C-Ar), 127.4 (C-5), 124.1 (C-Ar), 116.4 (C-Ar), 53.6 (C-2), 46.7 (C-3).

HRMS $(E S I+)$ : calculated for $\mathrm{C}_{15} \mathrm{H}_{13} \mathrm{NO}[\mathrm{M}+\mathrm{H}]^{+}:$224.1070; found: 224.1105.

IR (neat): $v\left(\mathrm{~cm}^{-1}\right)=3457,3029,2971,2952,2926,2855,1738,1599,1497,1474,1455$, $1381,1229,1217,1206,1178,1144,1095,1034,898,780,753,734,692$

m.p.: $97-99^{\circ} \mathrm{C}$

HPLC analysis of 9 indicated 99:1 er, chiral stationary column: AD-H, mobile phase: $n$ hexane $/ \mathrm{PrOH}=95: 5,1.0 \mathrm{~mL} / \mathrm{min}, 254 \mathrm{~nm}, 30^{\circ} \mathrm{C}, \mathrm{t}_{\mathrm{R}}$ (major) $=20.8 \mathrm{~min}, \mathrm{t}_{\mathrm{R}}$ (minor) $=26.6 \mathrm{~min}$. $[\alpha]^{20} \mathrm{D}=-15.7\left(c 30, \mathrm{CH}_{2} \mathrm{Cl}_{2}\right)$. 


\section{<Chromatogram>}

mAU

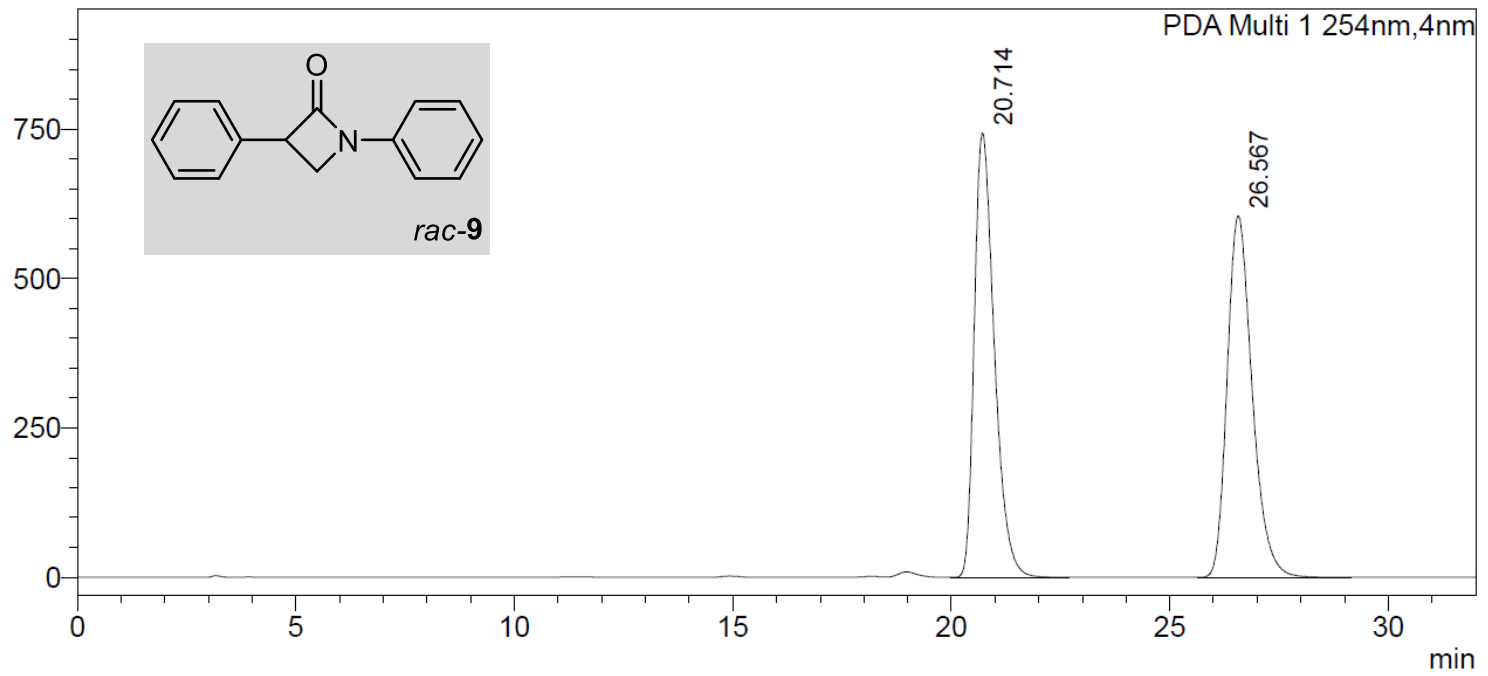

\section{<Peak Table>}

PDA Ch1 254nm

\begin{tabular}{|r|r|r|r|}
\hline Peak\# & Ret. Time & \multicolumn{1}{c|}{ Area } & \multicolumn{1}{c|}{ Area\% } \\
\hline 1 & 20.714 & 23714311 & 49.599 \\
\hline 2 & 26.567 & 24098231 & 50.401 \\
\hline Total & & 47812542 & 100.000 \\
\hline
\end{tabular}

\section{$<$ Chromatogram>} mAU

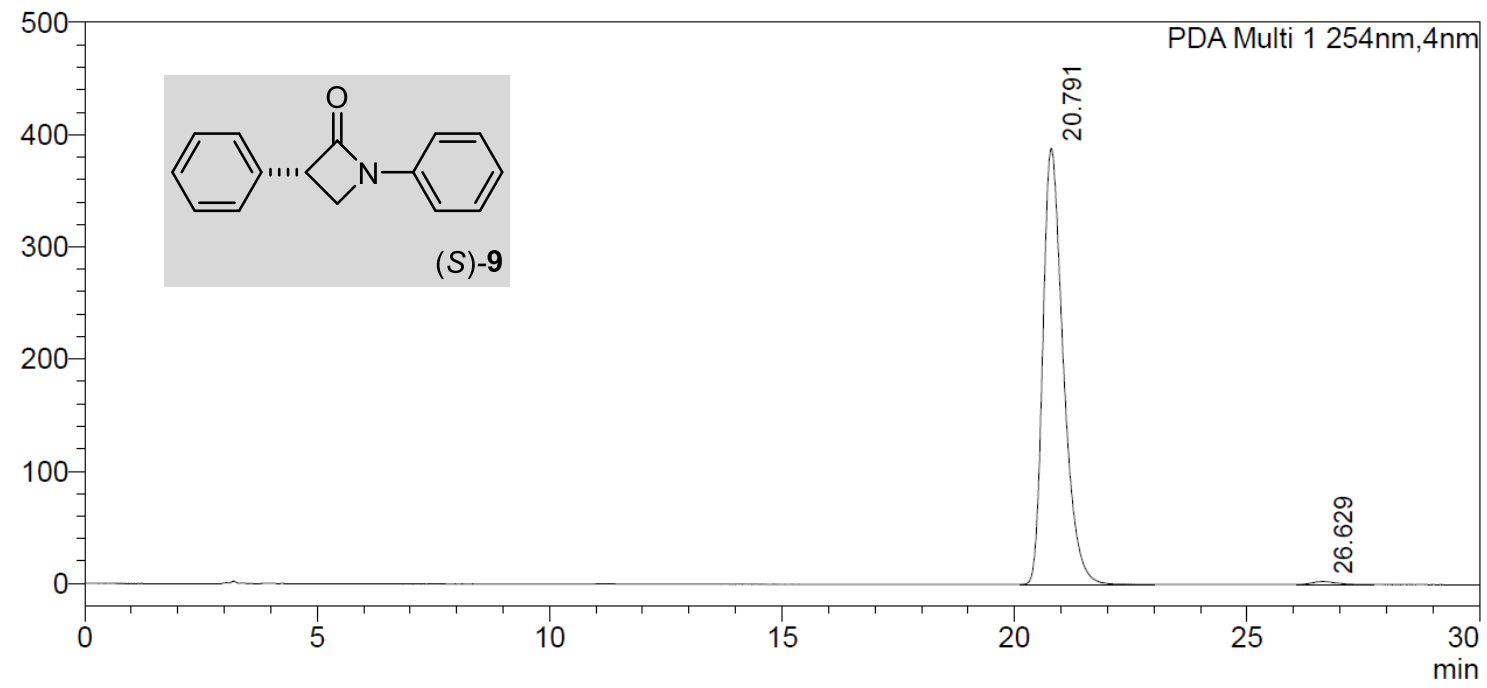

<Peak Table>

\section{PDA Ch1 254nm}

\begin{tabular}{|r|r|r|r|}
\hline Peak\# & Ret. Time & \multicolumn{1}{c|}{ Area } & \multicolumn{1}{c|}{ Area\% } \\
\hline 1 & 20.791 & 11867130 & 99.136 \\
\hline 2 & 26.629 & 103387 & 0.864 \\
\hline Total & & 11970517 & 100.000 \\
\hline
\end{tabular}


(R)- $N$,2-diphenyl-3-(trifluoro- $\lambda^{4}$-boraneyl)propanamide, potassium salt $10^{13}$

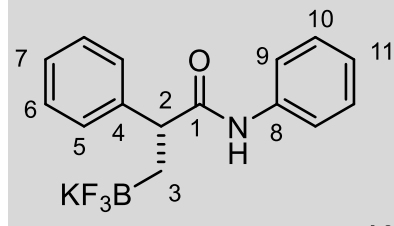

$(R)-10$

To a $10 \mathrm{~mL}$ reaction tube was added amide 3 aa $(70.2 \mathrm{mg}, 0.20$ mmol, 1.0 equiv.), $\mathrm{MeCN}(2.0 \mathrm{~mL})$, water (2.0 mL) and $\mathrm{KHF}_{2}(63$ $\mathrm{mg}, 0.80 \mathrm{mmol}, 4.0$ equiv.). The reaction was stirred at $23^{\circ} \mathrm{C}$ for $5 \mathrm{~h}$, then concentrated, and evaporated azeotropically with EtOH $(5 \mathrm{~mL} \times 3)$. The residue was then dried under vacuum overnight. The residue was dissolved with hot acetone, filtered and then concentrated under vacuum. The solid residue was suspended in $\mathrm{Et}_{2} \mathrm{O}(10 \mathrm{~mL})$ and the mixture was sonicated for 5 min. After filtration, the potassium $\beta$-trifluoroboratoamide 10 was obtained as a white solid in quantitative yield.

${ }^{1} \mathrm{H}$ NMR $\left(400 \mathrm{MHz}\right.$, Acetone- $\left.d_{6}\right) \delta(\mathrm{ppm})=9.10(\mathrm{~s}, 1 \mathrm{H}, \mathrm{N}-H), 7.75-7.57(\mathrm{~m}, 2 \mathrm{H}), 7.46-7.37$ $(\mathrm{m}, 2 \mathrm{H}), 7.32-7.16(\mathrm{~m}, 4 \mathrm{H}), 7.16-7.08(\mathrm{~m}, 1 \mathrm{H}), 7.02-6.89(\mathrm{~m}, 1 \mathrm{H}), 3.76\left(\mathrm{dd},{ }^{3} J_{H H}=11.2\right.$, $3.5 \mathrm{~Hz}, 1 \mathrm{H}, H-2), 1.27-1.17(\mathrm{~m}, 1 \mathrm{H}, H-3), 0.74-0.56(\mathrm{~m}, 1 \mathrm{H}, H-3)$.

${ }^{13} \mathrm{C}\left\{{ }^{1} \mathrm{H}\right\}$ NMR $\left(100 \mathrm{MHz}\right.$, Acetone- $\left.d_{6}\right) \delta(\mathrm{ppm})=175.1(C-1), 146.2(C-4), 140.3(C-8), 128.3$ (C-10), 127.9 (C-5), $127.7(C-6), 125.5$ (C-7), 122.5 (C-11), $119.2(C-9), 51.1(C-2)$.

${ }^{11} \mathrm{~B}$ NMR $\left(128 \mathrm{MHz}\right.$, Acetone- $\left.d_{6}\right) \delta(\mathrm{ppm})=4.6$

${ }^{19} \mathrm{~F}\left\{{ }^{1} \mathrm{H}\right\}$ NMR $\left(282 \mathrm{MHz}\right.$, Acetone- $\left.d_{6}\right) \delta(\mathrm{ppm})=-139.3$.

LRMS (ESI -): calculated for $\mathrm{C}_{15} \mathrm{H}_{14} \mathrm{BF}_{3} \mathrm{NO}$ [M]-: 292.1; found: 292.1 [M] ${ }^{-}$. 
(R)-3-(4-methoxyphenyl)-N,2-diphenylpropanamide $11^{13}$

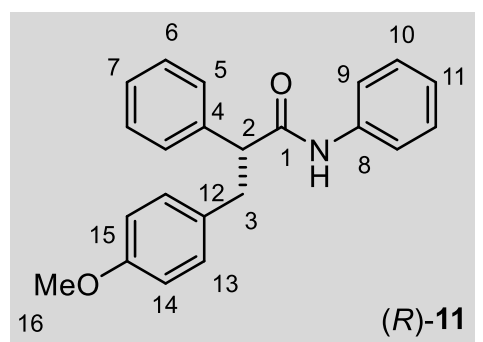

In a $10 \mathrm{~mL}$ Schlenck tube $\mathrm{Pd}(\mathrm{OAc})_{2}(2.2 \mathrm{mg}, 0.01 \mathrm{mmol}, 10$ mol\%), XPhos (9.5 mg, $0.02 \mathrm{mmol}, 20 \mathrm{~mol} \%), \mathrm{K}_{2} \mathrm{CO}_{3}$ (41.5 mg, $0.3 \mathrm{mmol}, 3.0$ equiv.), 1 -chloro-4-methoxybenzene (16 mg, 0.11 mmol, 1.1 equiv.), and potassium $\beta$-trifluoroboratoamide 10 (33 $\mathrm{mg}, 0.1 \mathrm{mmol}, 1.0$ equiv.). were dissolved in CPME $(0.4 \mathrm{~mL})$ and water $(0.06 \mathrm{~mL})$, and then the reaction was heated to $95^{\circ} \mathrm{C}$ for $24 \mathrm{~h}$. The reaction mixture was allowed to cool to $23{ }^{\circ} \mathrm{C}$ and quenched with $\mathrm{H}_{2} \mathrm{O}$. The organic layer was separated and the aqueous layer was extracted with Ethyl acetate. The combined organic phase was concentrated and purified by silica gel column chromatography (pentane/acetone 6:1) to afford the cross-coupling product 11 as a white solid (30.8 $\mathrm{mg}, 0.093$ mmol, 93\% yield, 98:2 er).

TLC: pentane/acetone $4: 1, \mathrm{R}_{\mathrm{f}}=0.5$

${ }^{1} \mathrm{H}$ NMR $\left(400 \mathrm{MHz}, \mathrm{CDCl}_{3}\right) \delta(\mathrm{ppm})=7.30\left(\mathrm{~d},{ }^{3} \mathrm{JHH}_{\mathrm{HH}}=7.4 \mathrm{~Hz}, 2 \mathrm{H}\right), 7.26(\mathrm{~s}, 2 \mathrm{H}), 7.24-7.14(\mathrm{~m}$, $4 \mathrm{H}), 7.01-6.92(\mathrm{~m}, 4 \mathrm{H}), 6.70-6.65(\mathrm{~m}, 2 \mathrm{H}), 3.67(\mathrm{~s}, 3 \mathrm{H}, \mathrm{H}-16), 3.61(\mathrm{~m}, 1 \mathrm{H}, \mathrm{H}-2), 3.48$ (dd, $\left.{ }^{2} J_{\mathrm{HH}}=13.7,{ }^{3} \mathrm{JHH}_{\mathrm{HH}}=7.8 \mathrm{~Hz}, 1 \mathrm{H}, \mathrm{H}-3\right), 2.92\left(\mathrm{dd},{ }^{2} J_{\mathrm{HH}}=13.7,{ }^{3} \mathrm{JHH}_{\mathrm{HH}}=6.9 \mathrm{~Hz}, 1 \mathrm{H}, \mathrm{H}-3\right)$.

${ }^{13} \mathrm{C}\left\{{ }^{1} \mathrm{H}\right\}$ NMR $\left(100 \mathrm{MHz}, \mathrm{CDCl}_{3}\right) \delta(\mathrm{ppm})=171.0(C-1), 158.1(C-15), 139.3(C-4), 137.7(C-$ 8), $131.6(C-12), 130.0$ (C-13), 128.9 (C-10), $128.8(C-6), 128.1(C-5), 127.6(C-7), 124.3(C$ 11), 119.9 (C-9), 113.8 (C-14), 56.9 (C-16), 55.2 (C-2), $38.9(C-3)$.

HRMS (ESI +): calculated for $\mathrm{C}_{22} \mathrm{H}_{21} \mathrm{NO}_{2}[\mathrm{M}+\mathrm{H}]^{+}:$:332.1645; found: 332.1666.

IR (neat): $v\left(\mathrm{~cm}^{-1}\right)=3457,3303,3027,3017,2971,2947,2856,1741,1659,1598,1536$, 1511, 1496, 1440, 1366, 1301, 1230, 1217, 1177, 1107, 1033, 897, 821, 754, 733, 694.

m.p.: $153-155^{\circ} \mathrm{C}$

HPLC analysis of $\mathbf{1 1}$ indicated 98:2 er, chiral stationary column: OD-H, mobile phase: $n$ hexane $/ \mathrm{PrOH}=90: 10,1.0 \mathrm{~mL} / \mathrm{min}, 254 \mathrm{~nm}, 30^{\circ} \mathrm{C}, \mathrm{t}_{\mathrm{R}}$ (major) $=9.7 \mathrm{~min}, \mathrm{t}_{\mathrm{R}}$ (minor) $=7.7 \mathrm{~min}$. $[\alpha]^{20} \mathrm{D}=-58.2\left(c 0.74, \mathrm{CH}_{2} \mathrm{Cl}_{2}\right)$. 


\section{<Chromatogram>}

mAU

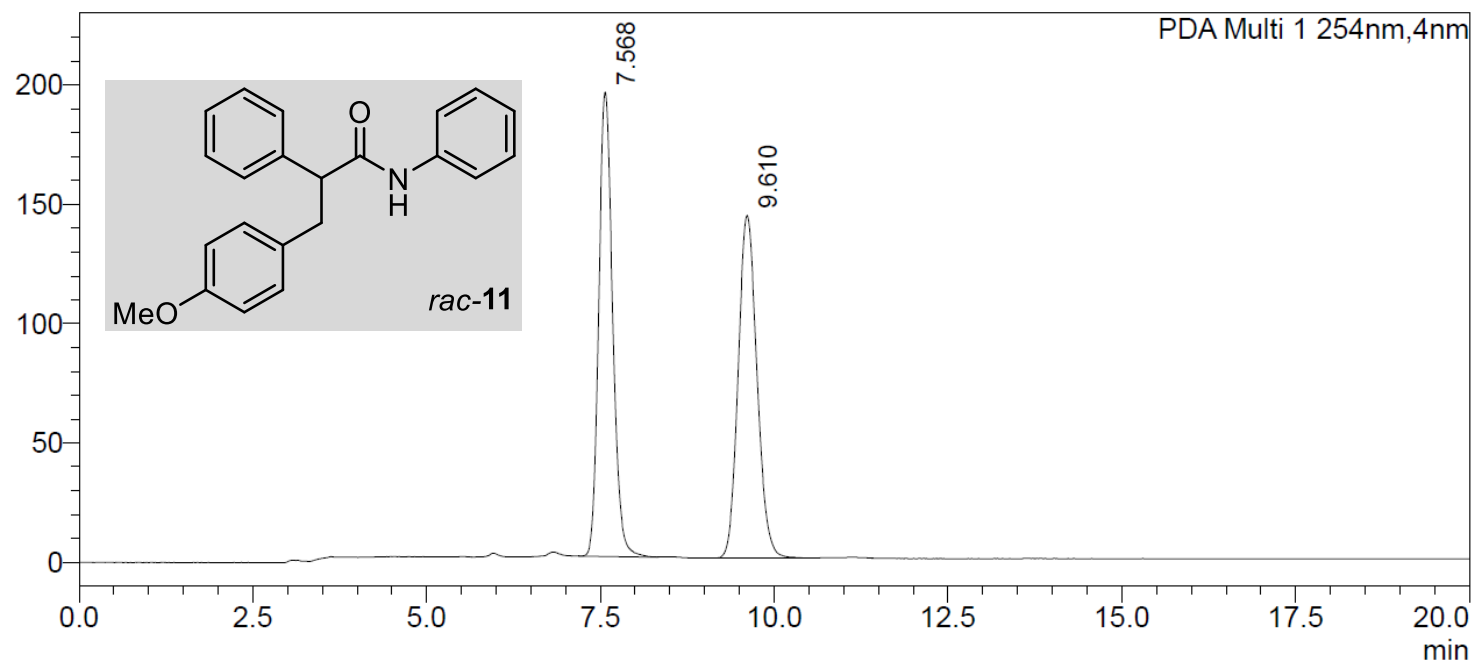

<Peak Table>

PDA Ch1 254nm

\begin{tabular}{|r|r|l|r|}
\hline Peak\# & Ret. Time & \multicolumn{1}{|c|}{ Area } & \multicolumn{1}{c|}{ Area\% } \\
\hline 1 & 7.568 & 2703524 & 50.037 \\
\hline 2 & 9.610 & 2699515 & 49.963 \\
\hline Total & & 5403039 & 100.000 \\
\hline
\end{tabular}

\section{<Chromatogram>}

mAU

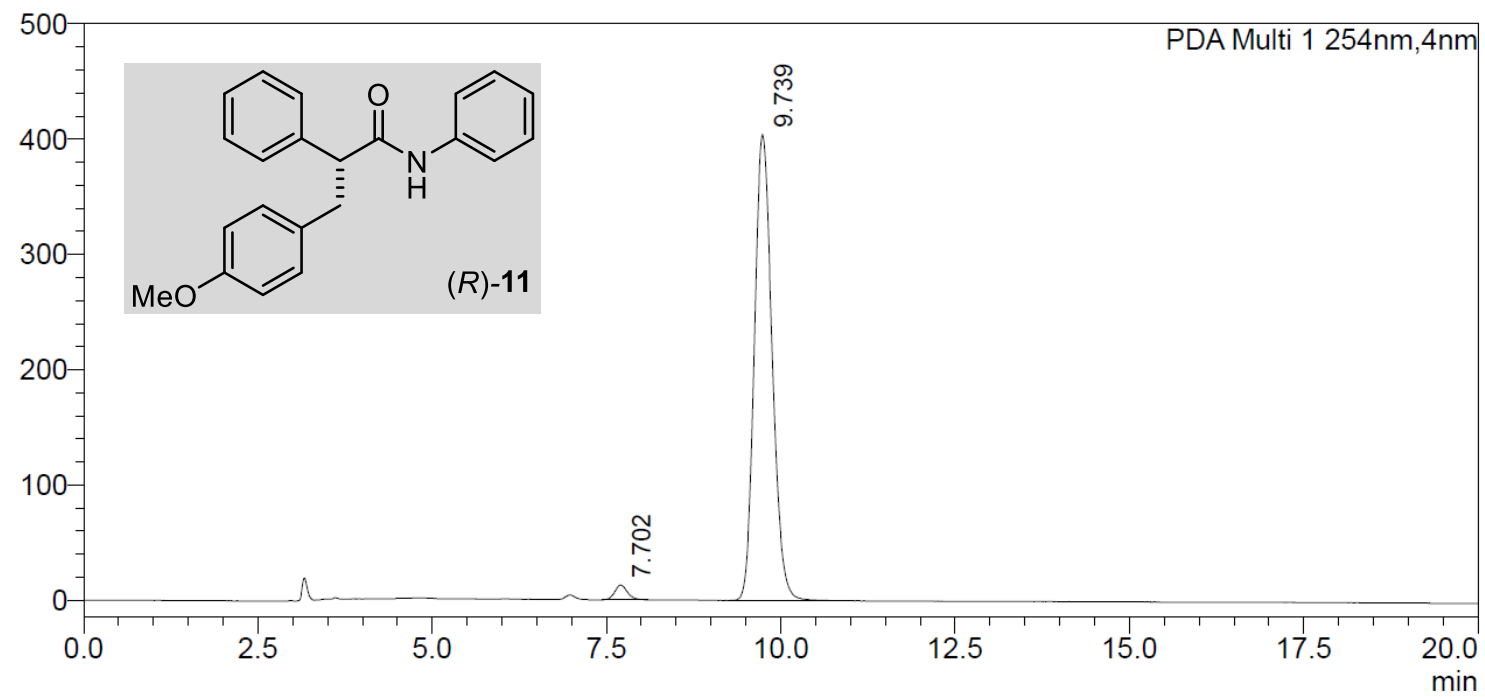

<Peak Table>

PDA Ch1 254nm

\begin{tabular}{|r|r|r|r|}
\hline Peak\# & Ret. Time & \multicolumn{1}{|c|}{ Area } & \multicolumn{1}{|c|}{ Area\% } \\
\hline 1 & 7.702 & 160829 & 2.196 \\
\hline 2 & 9.739 & 7164255 & 97.804 \\
\hline Total & & 7325084 & 100.000 \\
\hline
\end{tabular}


(Z)-3-(4-fluorophenyl)-N-(4-methoxyphenyl)-2,3-diphenylacrylamide (Z)-12 ${ }^{14}$

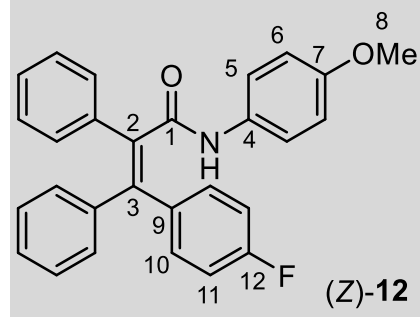

In a $10 \mathrm{~mL}$ Schlenk tube $\mathrm{Pd}(\mathrm{OAc})_{2}(2.2 \mathrm{mg}, 0.01 \mathrm{mmol}, 10.0$ mol\%), 1,1'-bis(di-tert-butylphosphino)ferrocene dtbpf (5.6 mg, $0.012 \mathrm{mmol}, 10.0 \mathrm{~mol} \%$ ) and $\mathrm{K}_{3} \mathrm{PO}_{4}(42 \mathrm{mg}, 0.20 \mathrm{mmol}, 2.0$ equiv.) were dissolved in $1.0 \mathrm{~mL}$ of toluene $(0.5 \mathrm{~mL}, 0.2 \mathrm{M})$, then alkenyl boronate (E)-5ab (46 mg, $0.1 \mathrm{mmol}, 1.0$ equiv.), 1-bromo-4fluorobenzene (26 mg, $0.15 \mathrm{mmol}, 1.5$ equiv.) and water (13 mg,

$0.7 \mathrm{mmol}, 7$ equiv.) were added. The resulting reaction mixture was stirred at $90{ }^{\circ} \mathrm{C}$ for $24 \mathrm{~h}$. After $24 \mathrm{~h}$, the reaction mixture was cooled to $23^{\circ} \mathrm{C}$ and quenched by dropwise addition of a saturated aqueous $\mathrm{NH}_{4} \mathrm{Cl}$ solution $(1.0 \mathrm{~mL})$. The reaction mixture was diluted with ethyl acetate $(5 \mathrm{~mL})$ and water $(5 \mathrm{~mL})$ and extracted with ethyl acetate $(3 \times 5 \mathrm{~mL})$. The combined organic phases were dried over sodium sulfate and the solvent was removed under reduced pressure. Purification by flash chromatography (pentane/ethyl acetate 4:1) gave pure acrylamide (Z)-12 as a white solid (35 $\mathrm{mg}, 0.083 \mathrm{mmol}, 83 \%$ yield, Z/E >20:1).

TLC: pentane/ethyl acetate $4: 1, R_{\mathrm{f}}=0.2$

${ }^{1} \mathbf{H}$ NMR $\left(400 \mathrm{MHz}, \mathrm{CDCl}_{3}\right) \delta(\mathrm{ppm})=7.34(\mathrm{~m}, 2 \mathrm{H}, \mathrm{H}-\mathrm{Ar}), 7.18(\mathrm{~m}, 8 \mathrm{H}, \mathrm{H}-\mathrm{Ar}), 7.12\left(\mathrm{~d},{ }^{3} \mathrm{~J}_{\mathrm{HH}}=\right.$ $9.0 \mathrm{~Hz}, 2 \mathrm{H}, H-5), 7.05$ (bs, $1 \mathrm{H}, \mathrm{N}-H), 7.00(\mathrm{~m}, 4 \mathrm{H}, H-\mathrm{Ar}), 6.78\left(\mathrm{~d},{ }^{3} \mathrm{~J}_{\mathrm{HH}}=9.0 \mathrm{~Hz}, 2 \mathrm{H}, H-6\right), 3.76$ (s, $3 \mathrm{H}, \mathrm{H}-8)$.

${ }^{13} \mathrm{C}\left\{{ }^{1} \mathrm{H}\right\}$ NMR $\left(101 \mathrm{MHz}, \mathrm{CDCl}_{3}\right) \delta(\mathrm{ppm})=168.5(C-1), 162.73\left(\mathrm{~d},{ }^{1} \mathrm{~J}_{\mathrm{CF}}=248.4 \mathrm{~Hz}, C-12\right)$, 156.8 (C-7), 143.5 (C-3), 141.0 (C-Ar), 138.07 (d, $\left.{ }^{4} J_{\mathrm{CF}}=3.3 \mathrm{~Hz}, C-9\right), 138.0$ (C-Ar), 137.6 (C2), $131.3\left(\mathrm{~d},{ }^{2} J_{\mathrm{CF}}=8.3 \mathrm{~Hz}, \mathrm{CH}-11\right), 131.1(\mathrm{CH}-\mathrm{Ar}), 130.7$ (CH-Ar), $130.3(\mathrm{CH}-\mathrm{Ar}), 128.5(\mathrm{CH}-$ $\mathrm{Ar}), 128.1$ (CH-Ar), 127.9 (CH-Ar), $127.8(\mathrm{C}-2), 122.0(\mathrm{CH}-5), 115.7$ (d, ${ }^{3} \mathrm{~J}_{\mathrm{CF}}=21.3 \mathrm{~Hz}, \mathrm{CH}-$ 10), $114.3(\mathrm{CH}-6), 55.6\left(\mathrm{CH}_{3}-8\right)$.

${ }^{19} \mathrm{~F}\left\{{ }^{1} \mathrm{H}\right\}$ NMR $\left(282 \mathrm{MHz}, \mathrm{CDCl}_{3}\right) \delta(\mathrm{ppm})=-112.9$

HRMS $(\mathrm{ESI}+)$ : calculated for $\mathrm{C}_{28} \mathrm{H}_{22} \mathrm{FNO}_{2}[\mathrm{M}+\mathrm{H}]^{+}:$423.1708; found: 423.1689 .

IR (neat): $v\left(\mathrm{~cm}^{-1}\right)=3350,2985,1681,1590,1523,1490,1391,1370,1332,1141,827,699$.

m.p.: $166-169^{\circ} \mathrm{C}$ 
3-((tert-butyldimethylsilyl)oxy)-N-(4-methoxyphenyl)-2-(naphthalen-2-yl)propanamide 14

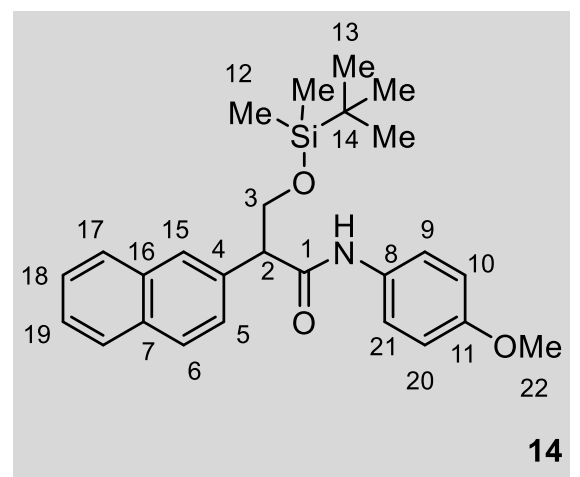

14

The amide 3ib (258 mg, $0.6 \mathrm{mmol}, 1.0$ equiv.) was oxidized by $\mathrm{NaOH} 4 \mathrm{M}(4 \mathrm{~mL})$ and $\mathrm{H}_{2} \mathrm{O}_{2} 30 \% \mathrm{v} / \mathrm{v}(2 \mathrm{~mL})$ in THF $(20 \mathrm{~mL})$ at $0{ }^{\circ} \mathrm{C}$ for $30 \mathrm{~min}$. The alcohol intermediate was used directly to the next step without further purification. Imidazole (68 mg, $2.4 \mathrm{mmol}, 4.0$ equiv.) and TBSCl (181 mg, $1.2 \mathrm{mmol}, 2.0$ equiv.) were added sequentially to the solution of alcohol in $\mathrm{CH}_{2} \mathrm{Cl}_{2}(10 \mathrm{~mL})$ at $23^{\circ} \mathrm{C}$. After stirring overnight, the reaction mixture was poured into saturated $\mathrm{NaHCO}_{3}$ (aq.) and extracted with $\mathrm{CH}_{2} \mathrm{Cl}_{2}$ for three times. The combined organic layers were then washed with brine and dried over sodium sulfate. The solvent was removed under reduced pressure. Purification by flash chromatography (pentane/diethyl ether $3: 1)$ gave protected alcohol 14 as a light yellow solid (220 mg, $0.51 \mathrm{mmol}, 84 \%$ yield).

TLC: pentane/diethyl ether $1: 1, R_{f}=0.4$

${ }^{1} \mathrm{H}$ NMR $\left(300 \mathrm{MHz}, \mathrm{CDCl}_{3}\right) \delta(\mathrm{ppm})=8.10(\mathrm{~s}, 1 \mathrm{H}, \mathrm{N}-H), 7.87-7.78(\mathrm{~m}, 4 \mathrm{H}), 7.53-7.40(\mathrm{~m}$, $5 \mathrm{H}), 6.86-6.78(\mathrm{~m}, 2 \mathrm{H}), 4.30\left(\mathrm{dd},{ }^{2} J_{\mathrm{HH}}=10.2,{ }^{3} J_{\mathrm{HH}}=6.9 \mathrm{~Hz}, 1 \mathrm{H}, H-3\right), 4.11\left(\mathrm{dd},{ }^{2} J_{H H}=10.2\right.$, $\left.{ }^{3} J_{\mathrm{HH}}=4.6 \mathrm{~Hz}, 1 \mathrm{H}, H-3\right), 3.88\left(\mathrm{dd},{ }^{3} \mathrm{~J}_{\mathrm{HH}}=6.9,4.6 \mathrm{~Hz}, 1 \mathrm{H}, H-2\right), 3.77(\mathrm{~s}, 3 \mathrm{H}, H-22), 0.89(\mathrm{~s}, 9 \mathrm{H}$, $H-13), 0.03$ (s, 3H, H-12), 0.01 (s, 3H, H-12).

${ }^{13} \mathrm{C}\left\{{ }^{1} \mathrm{H}\right\}$ NMR $(75 \mathrm{MHz}, \mathrm{CDCl}) \delta(\mathrm{ppm})=170.2(C-1), 156.3(C-11), 134.7(C-4), 133.4,132.7$, $131.2(C-8), 128.3,127.8,127.6,127.5,126.6,126.2,125.9,121.5$ (C-10), 114.1 (C-9), 65.1 (C-3), 56.0 (C-2), 55.4 (C-22), 25.8 (C-13), 18.2 (C-14), -5.6 (C-12).

HRMS (ESI +): calculated for $\mathrm{C}_{26} \mathrm{H}_{33} \mathrm{NO}_{3} \mathrm{Si}[\mathrm{M}+\mathrm{H}]^{+}: 436.2303$; found: 436.2347 .

IR (neat): $v\left(\mathrm{~cm}^{-1}\right)=3295,2953,2930,2856,1655,1603,1538,1510,1463,1442,1412$, 1299, 1243, 1172, 1102, 1036, 1007, 830, 776. 738, 704, 665, 643.

m.p.: $112-114^{\circ} \mathrm{C}$ 
3-((tert-butyldimethylsilyl)oxy)-2-(naphthalen-2-yl)propanamide $\mathbf{1 3}^{15}$

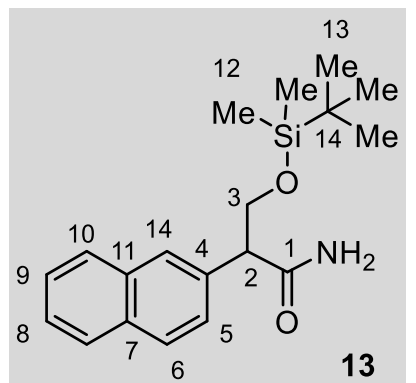

To a solution of protected alcohol 14 (87 $\mathrm{mg}, 0.2 \mathrm{mmol}, 1.0$ equiv.) in $\mathrm{CH}_{3} \mathrm{CN}(4 \mathrm{ml})$ and $\mathrm{H}_{2} \mathrm{O}(2 \mathrm{~mL})$ was added a solution of ceric ammonium nitrate CAN (230 mg, 0.42 mmol, 2.1 equiv.) in $\mathrm{H}_{2} \mathrm{O}(2$ $\mathrm{ml}$ ) at $0 \stackrel{\circ}{\circ}$. After stirring 30 minutes at $0 \stackrel{\circ}{\circ}$, sat. $\mathrm{NaHCO}_{3}$ aq. $(4 \mathrm{~mL})$ was added at same temperature. The mixture was extracted with ehyl acetate and organic layer was dried over sodium sulfate. After evaporation, the crude material was purified by preparative TLC (pentane/ethyl acetate 4:1) to afford primary amide 13 (25 $\mathrm{mg}, 0.075 \mathrm{mmol}, 38 \%$ yield) as a white solid.

TLC: pentane/ethyl acetate $1: 4, \mathrm{R}_{\mathrm{f}}=0.5$

${ }^{1} \mathrm{H}$ NMR $\left(400 \mathrm{MHz}, \mathrm{CDCl}_{3}\right) \delta(\mathrm{ppm})=7.85-7.78(\mathrm{~m}, 4 \mathrm{H}), 7.51-7.42(\mathrm{~m}, 3 \mathrm{H}), 6.17(\mathrm{~s}, 1 \mathrm{H}, \mathrm{N}-$ H), $5.76(\mathrm{~s}, 1 \mathrm{H}, \mathrm{N}-H), 4.23\left(\mathrm{dd},{ }^{2} J_{\mathrm{HH}}=10.1 \mathrm{~Hz},{ }^{3} J_{\mathrm{HH}}=7.1 \mathrm{~Hz}, 1 \mathrm{H}, H-3\right), 4.02\left(\mathrm{dd},{ }^{3} J_{\mathrm{HH}}=10.1\right.$ $\left.\mathrm{Hz},{ }^{3} \mathrm{~J}_{\mathrm{HH}}=5.1 \mathrm{~Hz}, 1 \mathrm{H}, H-3\right), 3.79\left(\mathrm{dd},{ }^{3} \mathrm{~J}_{\mathrm{HH}}=7.1,5.1 \mathrm{~Hz}, 1 \mathrm{H}, H-2\right), 0.87(\mathrm{~s}, 9 \mathrm{H}, H-13), 0.00$ (s, $6 \mathrm{H}, \mathrm{H}-12)$.

${ }^{13} \mathrm{C}\left\{{ }^{1} \mathrm{H}\right\}$ NMR $(75 \mathrm{MHz}, \mathrm{CDCl}) \delta(\mathrm{ppm})=174.6(C-1), 134.7(C-4), 133.4(C-\mathrm{Ar}), 132.7(C-\mathrm{Ar})$, 128.3 (C-Ar), 127.8 (C-Ar), 127.6 (C-Ar), 127.5 (C-Ar), 126.5 (C-Ar), 126.2 (C-Ar), 125.9 (CAr), 64.9 (C-3), 55.1 (C-2), 25.8 (C-13), 18.2 (C-14), -5.6 (C-12).

HRMS $(E S I+)$ : calculated for $\mathrm{C}_{19} \mathrm{H}_{27} \mathrm{NO}_{2} \mathrm{Si}[\mathrm{M}+\mathrm{H}]^{+}: 330.1884$; found: 330.1899 .

IR (neat): $v\left(\mathrm{~cm}^{-1}\right)=3458,3313,3182,3025,2952,2929,2857,1740,1672,1602,1462$, $1367,1301,1254,1229,1217,1103,1007,897,837,779,745$.

m.p.: $120-122^{\circ} \mathrm{C}$ 


\section{Determination of diastereoisomeric ratio for steroid 3pb via Mosher acid method}

To a solution of $3 \mathrm{pb}(22 \mathrm{mg}, 0.033 \mathrm{mmol})$ in THF $(0.7 \mathrm{~mL})$ and $\mathrm{H}_{2} \mathrm{O}(0.7 \mathrm{~mL})$ was added $\mathrm{NaBO}_{3} \cdot 4 \mathrm{H}_{2} \mathrm{O}(41 \mathrm{mg}, 0.26 \mathrm{mmol}, 8$ equiv. $)$ at $23^{\circ} \mathrm{C}$. The reaction mixture was stirred for $2 \mathrm{~h}$ and quenched by addition of saturated aqueous $\mathrm{Na}_{2} \mathrm{~S}_{2} \mathrm{O}_{3}(2 \mathrm{~mL})$. The mixture was extracted with ethyl acetate $(2 \times 5 \mathrm{~mL})$. The combined organic layers were washed with water and brine, dried by anhydrous sodium sulfate and concentrated in vacuum. Purification by flash chromatography (pentane/ethyl acetate 3:2 with $\mathrm{Et}_{3} \mathrm{~N} 0.1 \%$ ) gave alcohol 15 as a white foam (17 mg, $0.030 \mathrm{mmol}, 91 \%$ yield). Alcohol 15 (12 mg, $0.02 \mathrm{mmol}$ ) was esterified with $(R)$-MTPA$\mathrm{Cl}$ (15 mg, $0.06 \mathrm{mmol}, 3$ equiv.) and pyridine $\left(0.0065 \mathrm{~mL}, 0.08 \mathrm{mmol}, 4\right.$ equiv.) in $\mathrm{CDCl}_{3}(0.5$ $\mathrm{mL})$ at $23^{\circ} \mathrm{C}$ for $14 \mathrm{~h}$. The reaction mixture was quenched with water $(0.5 \mathrm{~mL})$, extracted with ethyl acetate $(2 \times 2 \mathrm{~mL})$ and dried over sodium sulfate. After evaporation, the crude residue was purified by flash chromatography (pentane/ethyl acetate 10:1) affording pure (S)-MTPA15 ester (12 mg, 80\% yield), that allowed to determine the diastereoisomeric ratio ( $d r$ 1.2:1) both by ${ }^{1} \mathrm{H}$ and ${ }^{19} \mathrm{~F}$ NMR analysis (spectra enclosed).

Alcohol 15

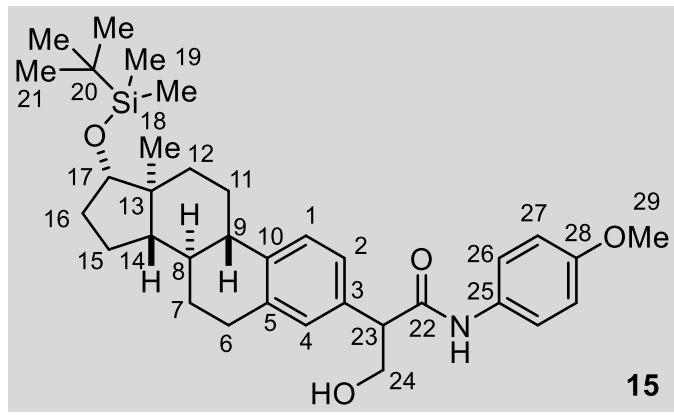

$3.77(\mathrm{~s}, 3 \mathrm{H}, \mathrm{H}-29), 3.75\left(\mathrm{dd},{ }^{3} \mathrm{~J}_{\mathrm{HH}}=8.8,4.5 \mathrm{~Hz}, 1 \mathrm{H}, \mathrm{H}-23\right), 3.65$ (t, $\left.{ }^{3} J_{\mathrm{HH}}=8.3 \mathrm{~Hz}, 1 \mathrm{H}, H-17\right)$, 3.34 (bs, 1H, OH), $2.85(\mathrm{~m}, 2 \mathrm{H}, H-6), 2.30(\mathrm{~m}, 1 \mathrm{H}, H-11), 2.21(\mathrm{~m}, 1 \mathrm{H}, H-9), 1.91(\mathrm{~m}, 3 \mathrm{H}, H-7$ $+H-16+H-12), 1.66(\mathrm{~m}, 1 \mathrm{H}, H-15), 1.49\left(\mathrm{~m}, 3 \mathrm{H}, \mathrm{H}-8+H-11^{\prime}+H-16^{\prime}\right), 1.36\left(\mathrm{~m}, 3 \mathrm{H}, \mathrm{H}-7^{\prime}+H^{-}\right.$ $12^{\prime}+H-15$ '), $1.16(\mathrm{~m}, 1 \mathrm{H}, H-14), 0.90$ (s, 9H, $\left.H-21\right), 0.75$ (s, 3H, $\left.H-18\right), 0.04$ (s, 3H, $\left.H-19\right)$, $0.03(\mathrm{~s}, 3 \mathrm{H}, \mathrm{H}-19)$.

${ }^{13} \mathrm{C}\left\{{ }^{1} \mathrm{H}\right\}$ NMR $(126 \mathrm{MHz}, \mathrm{CDCl}) \delta(\mathrm{ppm})=172.1(C-22), 156.8(C-28), 140.8(C-10), 138.1(C-$ 3), $133.5(\mathrm{C}-5), 130.5$ (C-25), $129.1(\mathrm{CH}-4), 126.5(\mathrm{CH}-1), 125.8(\mathrm{CH}-2), 122.2(\mathrm{CH}-26), 114.3$ ( $\mathrm{CH}-27), 81.9(\mathrm{CH}-17), 65.2\left(\mathrm{CH}_{2}-24\right), 55.6\left(\mathrm{CH}_{3}-29\right), 54.9(\mathrm{CH}-23), 49.9(\mathrm{CH}-4), 44.6(\mathrm{C}-13)$, 43.7 (CH-9), 38.7 (CH-8), 37.3 $\left(\mathrm{CH}_{2}-12\right)$, $31.1\left(\mathrm{CH}_{2}-16\right), 29.7\left(\mathrm{CH}_{2}-6\right), 27.3\left(\mathrm{CH}_{2}-7\right), 26.3\left(\mathrm{CH}_{2}-\right.$ 11), 26.0 ( $\left.\mathrm{CH}_{3}-21\right), 23.4\left(\mathrm{CH}_{2}-15\right), 18.3$ (C-20), $11.5\left(\mathrm{CH}_{3}-18\right),-4.3\left(\mathrm{CH}_{3}-19\right),-4.7\left(\mathrm{CH}_{3}-19\right)$. HRMS (ESI +): calculated for $\mathrm{C}_{34} \mathrm{H}_{49} \mathrm{NO}_{4} \mathrm{Si}[\mathrm{M}+\mathrm{H}]^{+}: 564.3504$; found: 564.3512 .

IR (neat): $v\left(\mathrm{~cm}^{-1}\right)=3285,2929,2856,1654,1606,1551,1512,1463,1248,1141,10196$, $884,834,775$.

TLC: pentane/ethyl acetate $3: 2, R_{f}=0.3$

${ }^{1} \mathbf{H}$ NMR $\left(500 \mathrm{MHz}, \mathrm{CDCl}_{3}\right) \delta(\mathrm{ppm})=7.33\left(\mathrm{~d},{ }^{3} \mathrm{JHH}_{\mathrm{HH}}=\right.$ $9.0 \mathrm{~Hz}, 2 \mathrm{H}, \mathrm{H}-26), 7.30\left(\mathrm{~d},{ }^{3} \mathrm{~J}_{\mathrm{HH}}=8.0 \mathrm{~Hz}, 1 \mathrm{H}, H-1\right)$, $7.14(\mathrm{~s}, 1 \mathrm{H}, \mathrm{N}-H), 7.08\left(\mathrm{~d},{ }^{3} J_{H H}=8.0 \mathrm{~Hz}, 1 \mathrm{H}, H-2\right)$, $7.02(\mathrm{~s}, 1 \mathrm{H}, H-4), 6.82\left(\mathrm{~d},{ }^{3} J_{\mathrm{HH}}=9.0 \mathrm{~Hz}, 2 \mathrm{H}, \mathrm{H}-27\right)$, $4.21\left(\mathrm{dd},{ }^{2} J_{\mathrm{HH}}=11.0 \mathrm{~Hz},{ }^{3} \mathrm{JHH}_{\mathrm{HH}}=8.8 \mathrm{~Hz}, 1 \mathrm{H}, \mathrm{H}-24\right)$, $3.81\left(\mathrm{dd},{ }^{2} \mathrm{~J}_{\mathrm{HH}}=11.0 \mathrm{~Hz},{ }^{3} \mathrm{~J}_{\mathrm{HH}}=4.5 \mathrm{~Hz}, 1 \mathrm{H}, \mathrm{H}-24\right.$ '), 


\section{X-ray analyses}

All data were collected on an Agilent Supernova diffractometer equipped with an ATLAS CCD detector using Cu radiation. The crystal was kept at 180.01(10) K during data collection. Using Olex2, the structure was solved with the ShelXT structure solution program using Intrinsic Phasing and refined with the ShelXL refinement package using Least Squares minimization. 16, 17, 18 Crystallographic data are found in Table S8-10. 
Table S13. Crystal structure and data refinement for 3wa (CCDC 1914321)

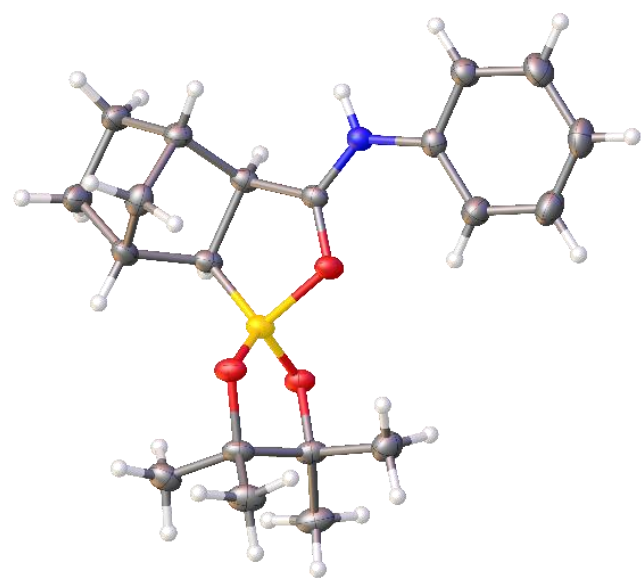

Identification code

CCDC

Empirical formula

Formula weight

Temperature/K

Crystal system

Space group

$a / \AA$

$\mathrm{b} / \AA ̊ \AA$

$\mathrm{c} / \AA ̊ A$

$\alpha /^{\circ}$

$\beta /^{\circ}$

$\mathrm{Y} /^{\circ}$

Volume/ $\AA^{3}$

Z

$\rho_{\text {calc }} \mathrm{g} / \mathrm{cm}^{3}$

$\mu / \mathrm{mm}^{-1}$

$\mathrm{F}(000)$

Crystal size/ $/ \mathrm{mm}^{3}$

Radiation

$2 \Theta$ range for data collection $/^{\circ}$

Index ranges

Reflections collected

Independent reflections

Data/restraints/parameters

Goodness-of-fit on $\mathrm{F}^{2}$

Final $R$ indexes [l>=2 $\sigma(\mathrm{I})]$

Final $R$ indexes [all data]

Largest diff. peak/hole / e $\AA^{-3}$ 3wa

1914321

$\mathrm{C}_{20} \mathrm{H}_{28} \mathrm{BNO}_{3}$

341.24

140.00(10)

monoclinic

$\mathrm{P} 2{ }_{1} / \mathrm{c}$

17.9466(3)

8.57729(14)

11.94065(18)

90

95.3190(15)

90

$1830.15(5)$

4

1.238

0.643

736.0

$0.488 \times 0.024 \times 0.017$

CuKa $(\lambda=1.54184)$

9.9 to 147.148

$-17 \leq \mathrm{h} \leq 22,-10 \leq \mathrm{k} \leq 6,-14 \leq \mathrm{I} \leq 14$

7081

$3604\left[R_{\text {int }}=0.0250, R_{\text {sigma }}=0.0341\right]$

$3604 / 0 / 234$

1.015

$\mathrm{R}_{1}=0.0411, \mathrm{wR}_{2}=0.1014$

$\mathrm{R}_{1}=0.0512, \mathrm{wR}_{2}=0.1083$

$0.26 /-0.23$ 
Table S14. Crystal structure and data refinement for (E)-5ab (CCDC 1914322)

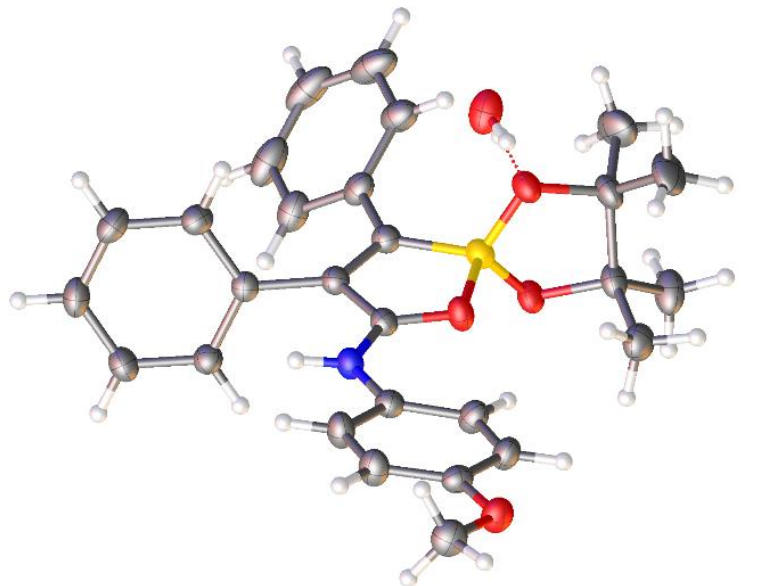

Identification code

$(E)-5 a b$

CCDC

1914322

Empirical formula

$\mathrm{C}_{28} \mathrm{H}_{30} \mathrm{BNO}_{4}, \mathrm{H}_{2} \mathrm{O}$

Formula weight

473.35

Temperature/K

180.01(10)

Crystal system

monoclinic

Space group

$\mathrm{P} 2{ }_{1} / \mathrm{n}$

$\mathrm{a} / \AA ̊ \AA$

14.3572(2)

$\mathrm{b} / \mathrm{A}$

$10.32740(10)$

$c / \AA$

17.3961(2)

$\alpha /^{\circ}$

90

$\beta /^{\circ}$

$91.6200(10)$

$\mathrm{Y} /{ }^{\circ}$

90

Volume/ $\AA^{3}$

2578.33(5)

Z

$\rho_{\text {calc }} \mathrm{g} / \mathrm{cm}^{3}$

4

1.219

$\mu / \mathrm{mm}^{-1}$

0.663

$\mathrm{F}(000)$

1008.0

Crystal size $/ \mathrm{mm}^{3}$

$0.637 \times 0.31 \times 0.241$

Radiation

$2 \Theta$ range for data collection $/^{\circ}$

CuKa $(\lambda=1.54184)$

Index ranges

8.098 to 141.78

$-15 \leq h \leq 17,-8 \leq k \leq 12,-20 \leq \mathrm{I} \leq 20$

10786

Reflections collected

$4847\left[R_{\text {int }}=0.0116, R_{\text {sigma }}=0.0128\right]$

Independent reflections

Data/restraints/parameters

Goodness-of-fit on $\mathrm{F}^{2}$

$4847 / 0 / 330$

1.041

Final $R$ indexes $[I>=2 \sigma(\mathrm{I})]$

$\mathrm{R}_{1}=0.0345, w R_{2}=0.0888$

Final $R$ indexes [all data]

$\mathrm{R}_{1}=0.0368, w R_{2}=0.0906$

Largest diff. peak/hole / e $\AA^{-3}$

$0.24 /-0.21$ 
Table S15. Crystal structure and data refinement for $(R)$-3ja (CCDC 1914320)

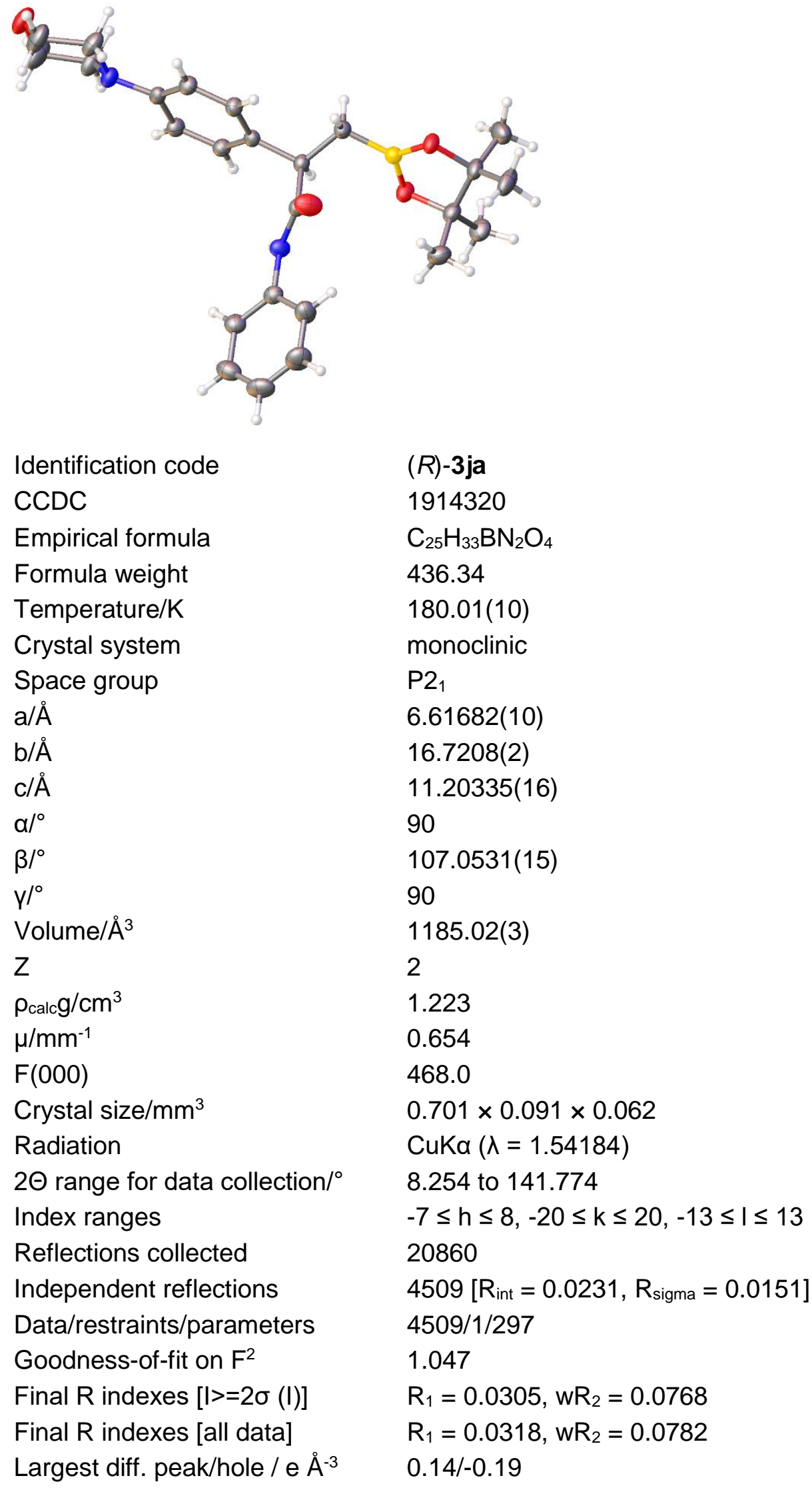




\section{References}

${ }^{1}$ [(SIMes)CuCl], [(IMes)CuCl], [(SIPr)CuCl], [(IPr)CuCl], [(ICy)CuCl] and [(ItBu)CuBr] were prepared following the literature. For details, see: (a) McIntosh, M. L.; Moore, C. M.; Clark, T. B. Copper-Catalyzed Diboration of Ketones: Facile Synthesis of Tertiary a-Hydroxyboronate Esters. Org. Lett. 2010,12, 1996. (b) Landers, B.; Navarro, O. Microwave-Assisted Synthesis of (N-Heterocyclic carbene) MCl Complexes of Group 11 Metals. Eur. J. Inorg. Chem. 2012, 2980.

2 Styrene $1 \mathrm{j}$ was prepared from 4-chloro styrene by Buchwald-Hartwig amination with morpholine (1.2 equiv.) using $\mathrm{Pd}(\mathrm{OAc})_{2}(2.5 \mathrm{~mol} \%)$, XPhos (5.0 mol\%) and $\mathrm{NaO}$ tBu (1.5 equiv.) in toluene (1 M). Styrene 1j is a known compound. For details, see: Zhao, X.-Y.; Zhou, Q., Lu, J.-M. Synthesis and Characterization of $\mathrm{N}$-heterocyclic Carbene-Palladium(II) Chlorides-1-Methylindazole and -1Methylpyrazole Complexes and their Catalytic Activity Toward C-N Coupling of Aryl Chlorides. RSC Adv. 2016, 6, 24484.

${ }^{3}$ Vinylarene 1k was prepared following the literature. For details, see: Puleo, T. R.; Strong, A. J.; Bandar, J. S. Catalytic $\alpha$-Selective Deuteration of Styrene Derivatives. J. Am. Chem. Soc. 2019, 141, 1467.

${ }^{4}$ Styrene 11 was prepared following the literature. For details, see: Seo, H.; Liu, A.; Jamison, T. F. Direct $\beta$-Selective Hydrocarboxylation of Styrenes with $\mathrm{CO}_{2}$ Enabled by Continuous Flow Photoredox Catalysis. J. Am. Chem. Soc. 2017,139, 13969.

${ }^{5}$ Styrene $1 \mathrm{n}$ was prepared following the literature. For details, see: Cao, H.; Jiang, H.; Feng, H.; Kwan, J. M. C.; Liu, X.; Wu, J. Photo-Induced Decarboxylative Heck-Type Coupling of Unactivated Aliphatic Acids and Terminal Alkenes in the Absence of Sacrificial Hydrogen Acceptors. J. Am. Chem. Soc. 2018, 140, 16360.

${ }^{6}$ Styrene $1 \mathrm{p}$ was prepared from $\beta$-estradiol following the literature. For details, see: Martin, S. E. S.; Watson, D. A. Preparation of Vinyl Silyl Ethers and Disiloxanes via the Silyl-Heck Reaction of Silyl Ditriflates. J. Am. Chem. Soc. 2013, 135, 13330.

7 Kitagawa, O.; Momose, S.; Yamada, Y.; Shiro, M.; Taguchi, T. Synthesis of Optically Active Aldol Derivatives Through Chirality Transfer Type 1,2-Wittig Rearrangement of $\alpha$-Alkoxycarboxamides. Tetrahedron Lett. 2001, 42, 4865.

8 2-cyclohexyl 1,3-butadiene $\mathbf{1} \mathbf{u}$ and diene $(R)$-1 $\mathbf{v}$ were prepared following the literature. For details, see: Fiorito, D.; Folliet, S.; Liu, Y.; Mazet, C. A General Nickel-Catalyzed Kumada Vinylation for the Preparation of 2-Substituted 1,3-Dienes. ACS Catal. 2018, 8, 1392.

${ }^{9}$ Preparation according to the procedure reported in the literature: Müller, D.; Guénée, L.; Alexakis, A. Practical Synthesis of SimplePhos Ligands: Further Development of Alkyl-Substituted Phosphanamines. Eur. J. Org. Chem. 2013, 6335.

${ }^{10}$ Lange, J.; Bissember, A. C.; Banwell, M. G.; Cade, I. A. Synthesis of 2,3-Dihydro-4(1H)-Quinolones and the Corresponding $4(1 \mathrm{H})$-Quinolones via Low-Temperature Fries Rearrangement of $\mathrm{N}$-Arylazetidin2-ones. Aust. J. Chem. 2011, 64, 454.

11 Vinylarenes $1 z$ was prepared following the literature. For details, see: Gribble, M. W.; Pirnot, M. T.; Bandar, J. S.; Liu, R. Y.; Buchwald, S. L. Asymmetric Copper Hydride-Catalyzed Markovnikov Hydrosilylation of Vinylarenes and Vinyl Heterocycles. J. Am. Chem. Soc. 2017, 139, 2192.

12 Vinylarene $1 \mathbf{a}_{2}$ was prepared following the literature. For details, see: Cooper, P.; Crisenza, G. E. M.;

Feron, L. J.; Bower, J. F. Iridium-Catalyzed $\alpha$-Selective Arylation of Styrenes by Dual C-H Functionalization. Angew. Chem., Int. Ed. 2018, 57, 14198.

${ }^{13}$ (a) Gao, T. T.; Zhang, W. W.; Sun, X.; Lu, H. X.; Li. B. J. Stereodivergent Synthesis through Catalytic Asymmetric Reversed Hydroboration. J. Am. Chem. Soc. 2019, 141, 4670. (b) Sandrock, D. L.; JeanGérard, L.; Chen, C.; Dreher, S. D.; Molander, G. A. Stereospecific Cross-Coupling of Secondary Alkyl $\beta$-Trifluoroboratoamides. J. Am. Chem. Soc. 2010, 132, 17108.

14 Nagao, K.; Ohmiya, H.; Sawamura, M. Anti-Selective Vicinal Silaboration and Diboration of Alkynoates through Phosphine Organocatalysis. Org. Lett. 2015, 17, 1304.

15 Tanaka, Y.; Hasui, T.; Suginome, M. Acid-Free, Aminoborane-Mediated Ugi-Type Reaction Leading to General Utilization of Secondary Amines. Org. Lett. 2007, 9, 4407.

${ }^{16}$ Dolomanov, O.V.; Bourhis, L.J.; Gildea, R.J; Howard, J.A.K.; Puschmann, H. OLEX2: A Complete Structure Solution, Refinement and Analysis Program. J. Appl. Cryst. 2009, 42, 339.

17 Sheldrick, G.M. SHELXT - Integrated Space-Group and Crystal-Structure Determination. Acta Cryst. 2015, A71, 3.

${ }^{18}$ Sheldrick, G.M. Crystal Structure Refinement with SHELXL. Acta Cryst. 2015, C71, 3. 
8. NMR spectra of new compounds 


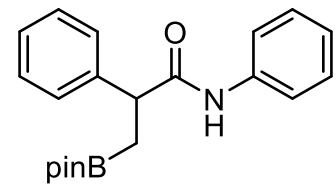

$3 a a$

${ }^{1} \mathrm{H}$ NMR (400 MHz, $\mathrm{CDCl}_{3}$ )

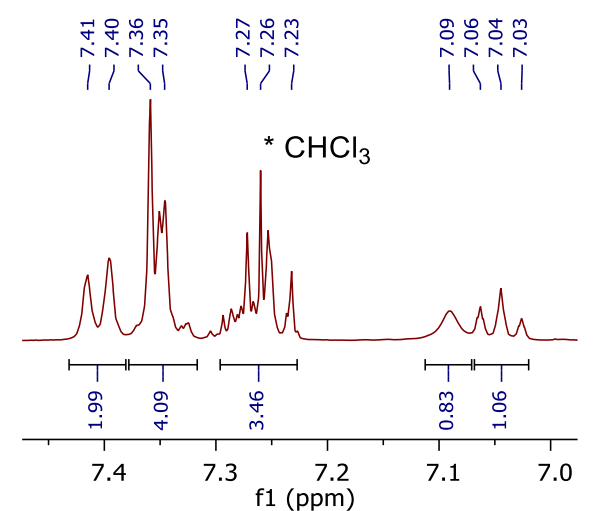

喅

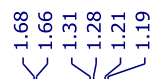

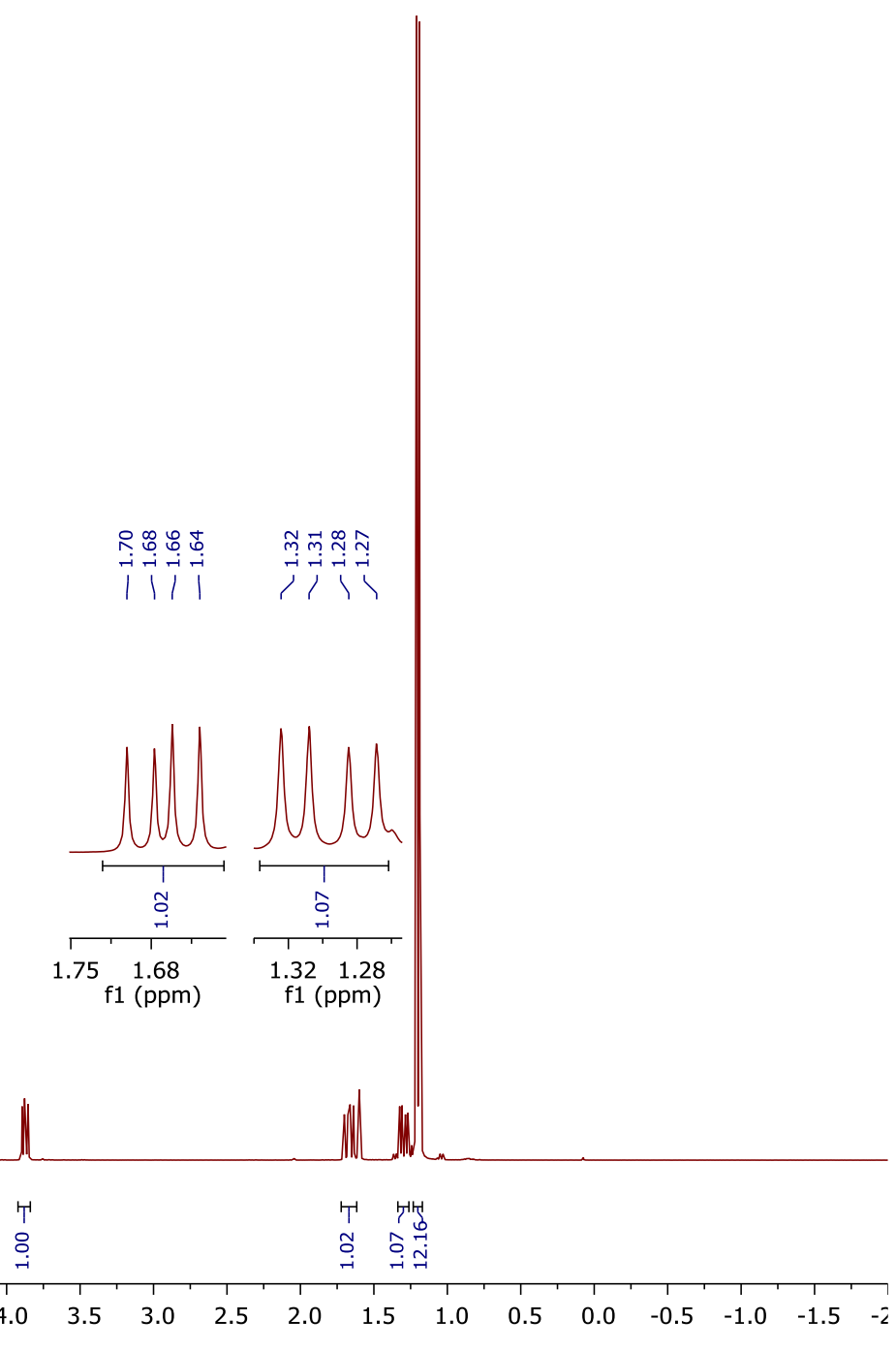

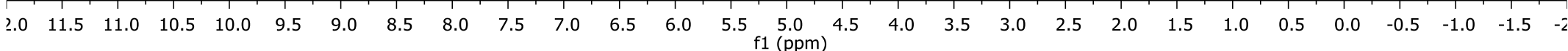



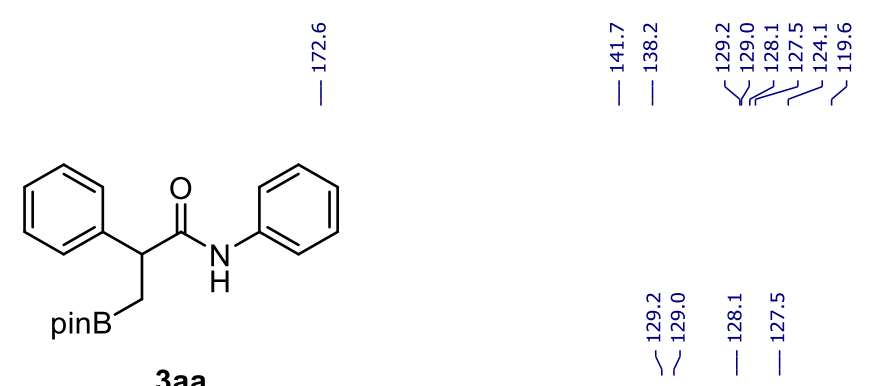

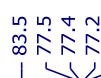

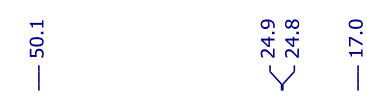

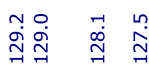

3 aa

$1 / 11$

${ }^{13} \mathrm{C}\left\{{ }^{1} \mathrm{H}\right\}$ NMR $\left(101 \mathrm{MHz}, \mathrm{CDCl}_{3}\right)$

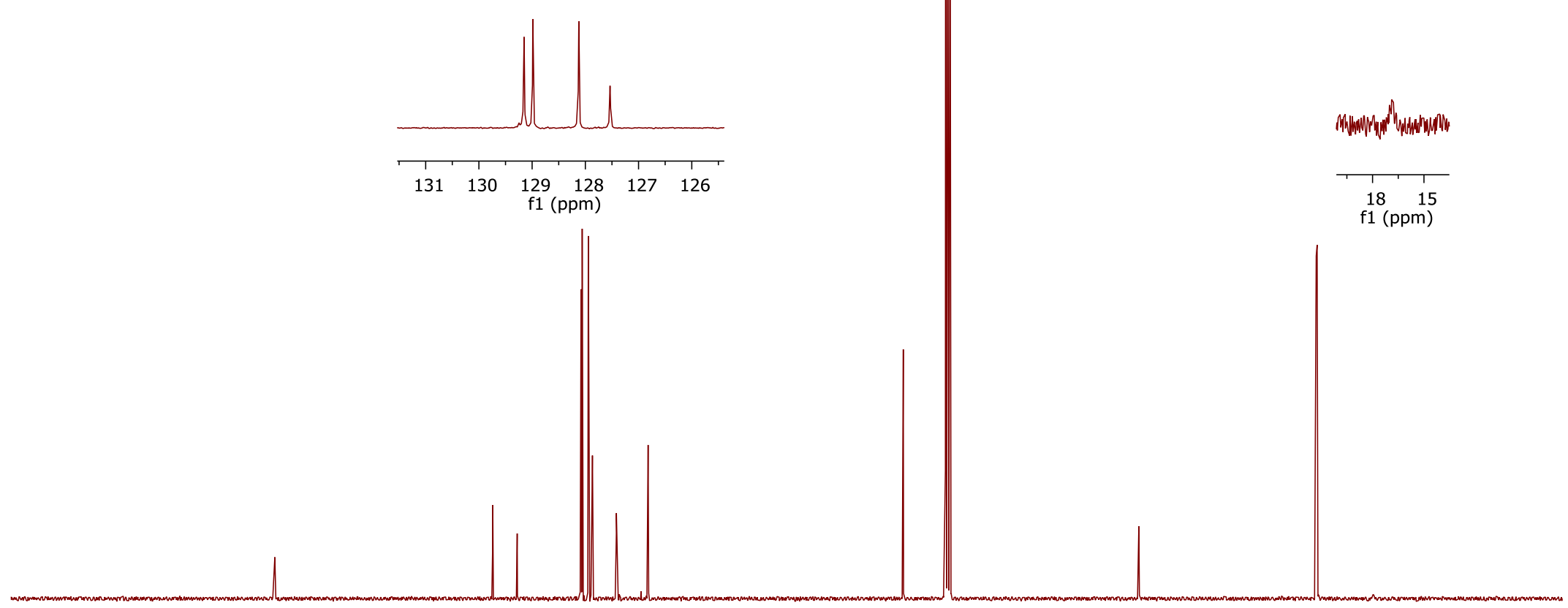

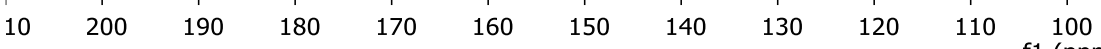
$\stackrel{100}{\mathrm{f} 1(\mathrm{ppm})}$

80

60

50 


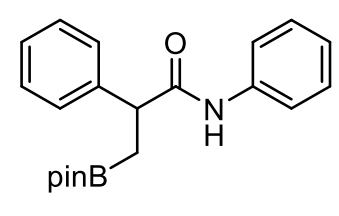

$3 a a$

${ }^{11} \mathrm{~B}$ NMR $\left(128 \mathrm{MHz}, \mathrm{CDCl}_{3}\right)$

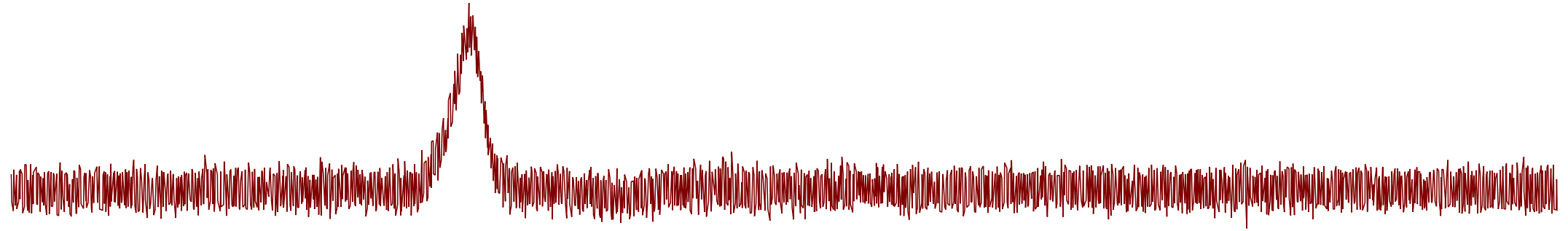




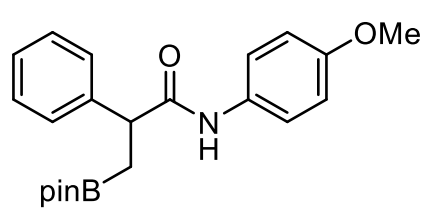

$3 a b$

${ }^{1} \mathrm{H}$ NMR $\left(500 \mathrm{MHz}, \mathrm{CDCl}_{3}\right)$
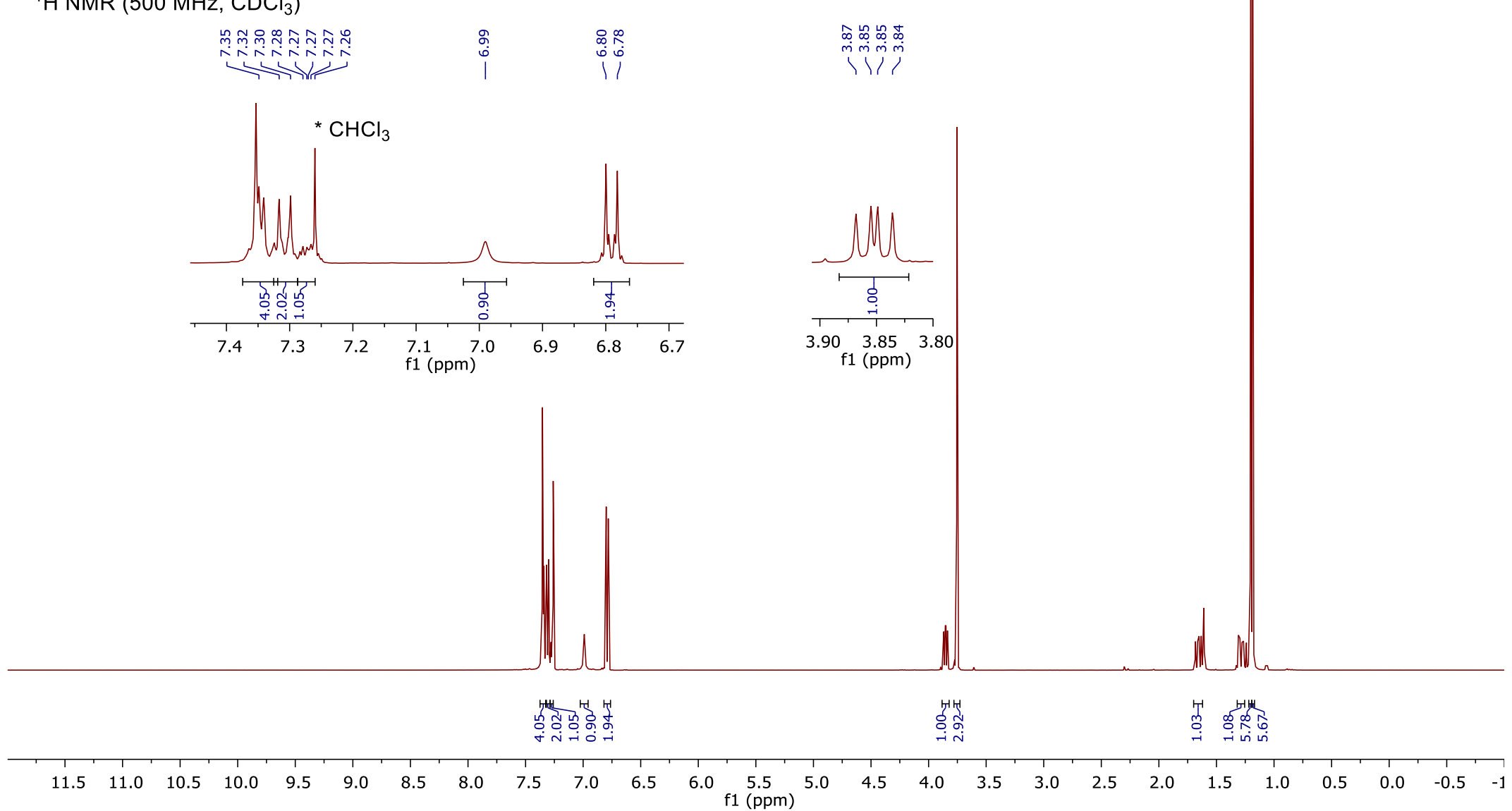

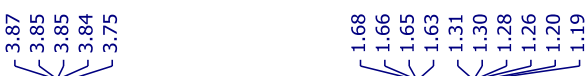

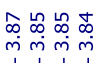

$90 \quad 3.85 \quad 3.80$
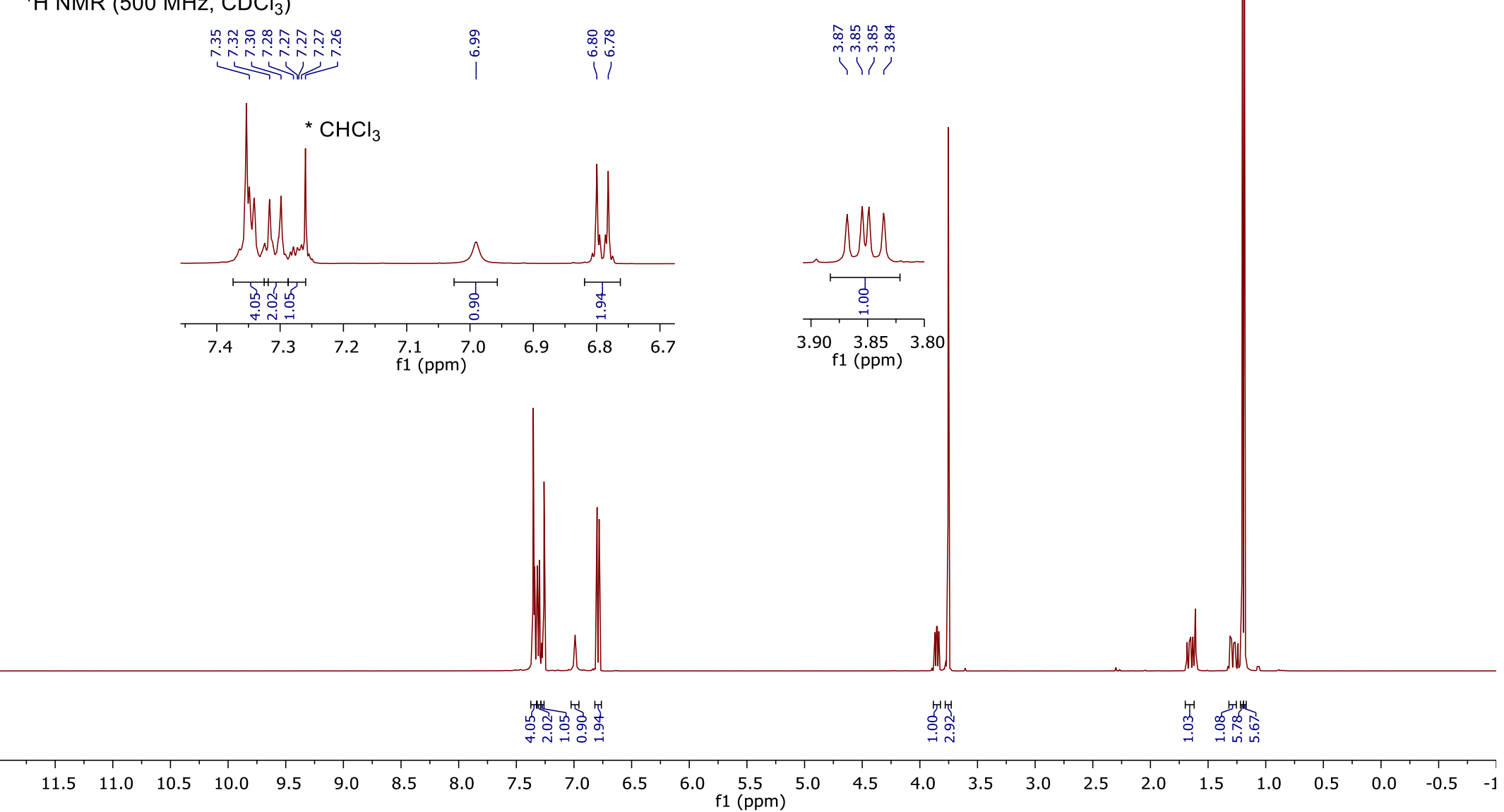


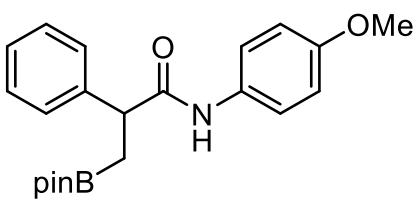

$3 a b$

${ }^{13} \mathrm{C}\left\{{ }^{1} \mathrm{H}\right\}$ NMR $\left(126 \mathrm{MHz}, \mathrm{CDCl}_{3}\right)$

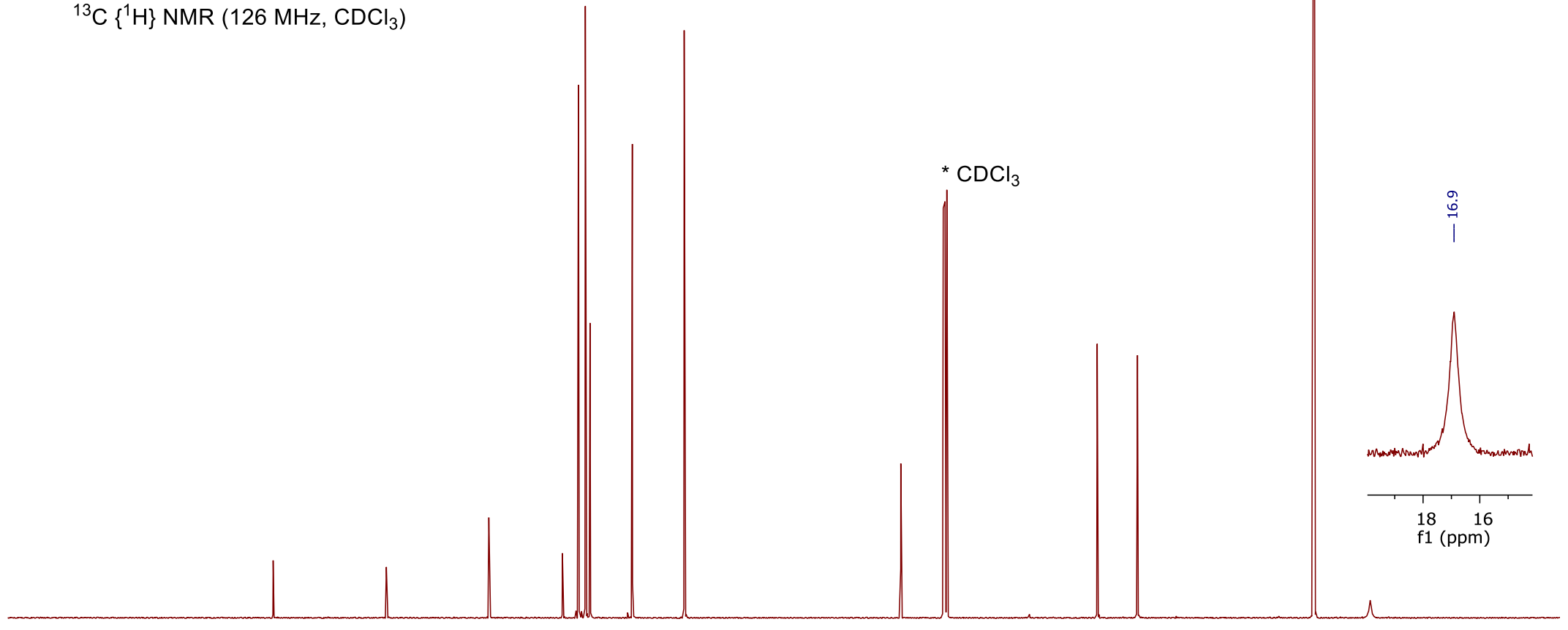

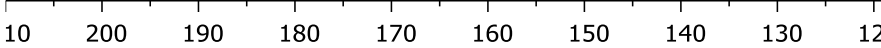

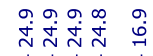

I 


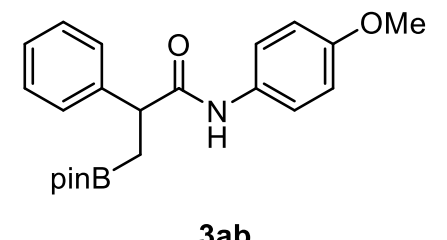

${ }^{11} \mathrm{~B}$ NMR $\left(96 \mathrm{MHz}, \mathrm{CDCl}_{3}\right)$

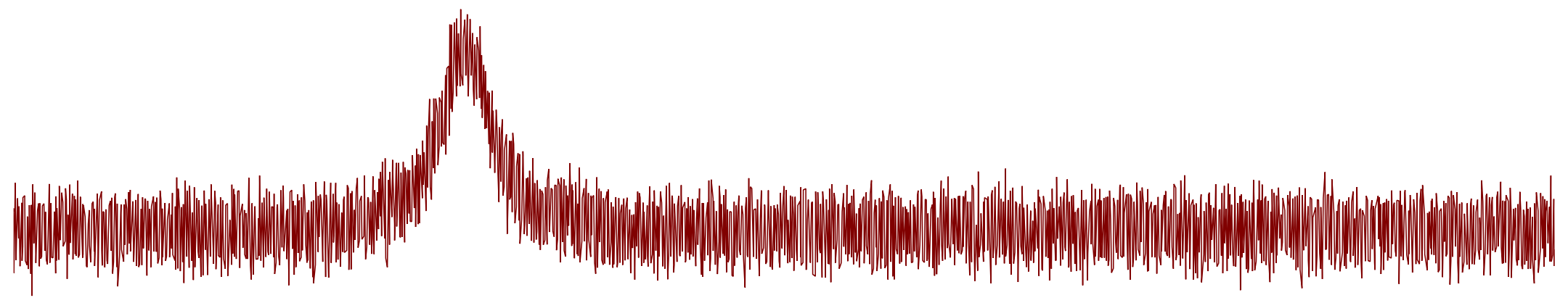

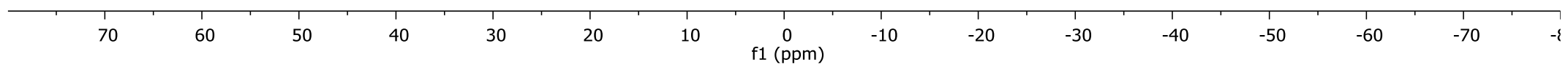




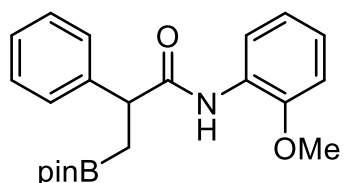

$3 a c$

${ }^{1} \mathrm{H}$ NMR (400 MHz, $\mathrm{CDCl}_{3}$ )

$m_{\infty}^{\infty}$

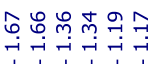

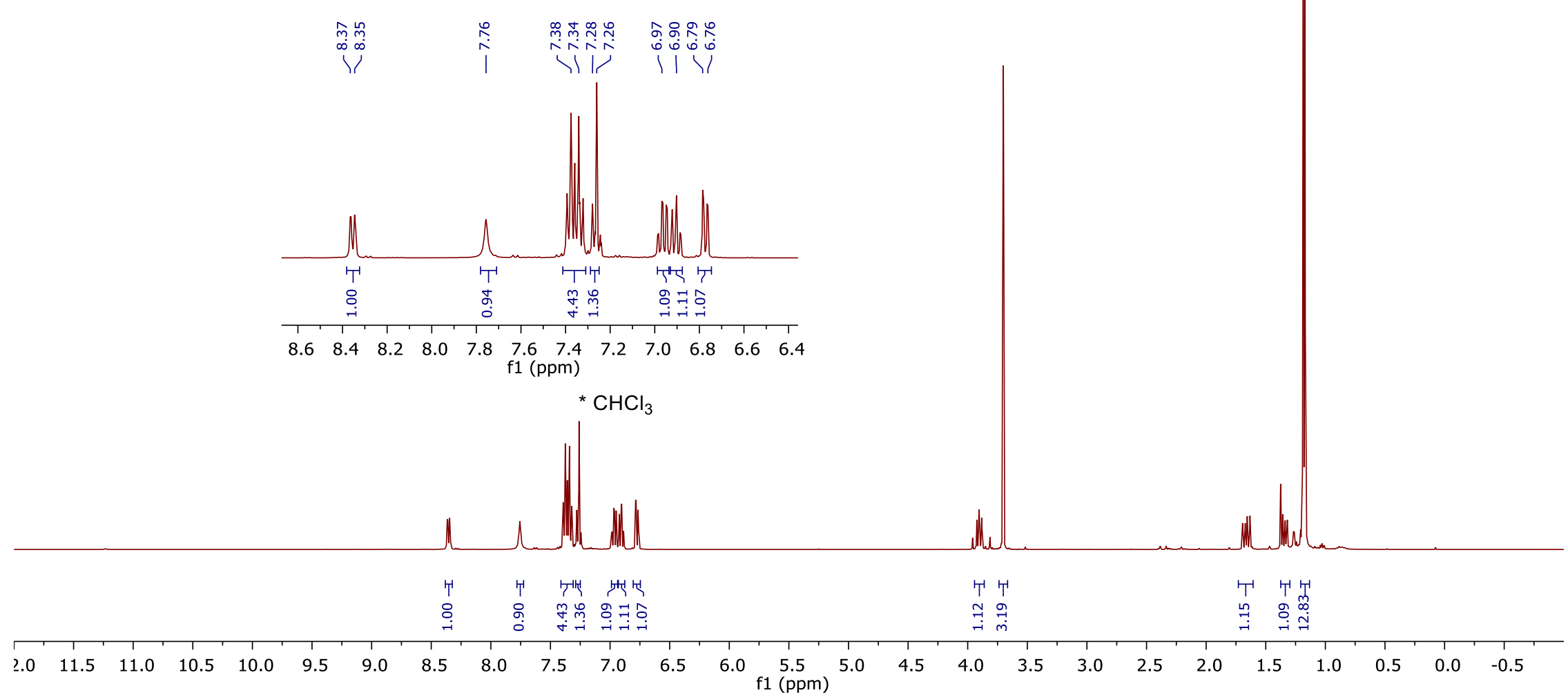




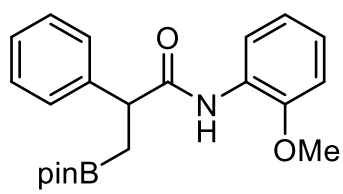

* $\mathrm{CDCl}_{3}$

${ }^{13} \mathrm{C}\left\{{ }^{1} \mathrm{H}\right\}$ NMR $\left(101 \mathrm{MHz}, \mathrm{CDCl}_{3}\right)$

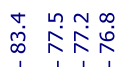

I V।

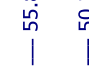

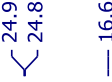

$3 a c$

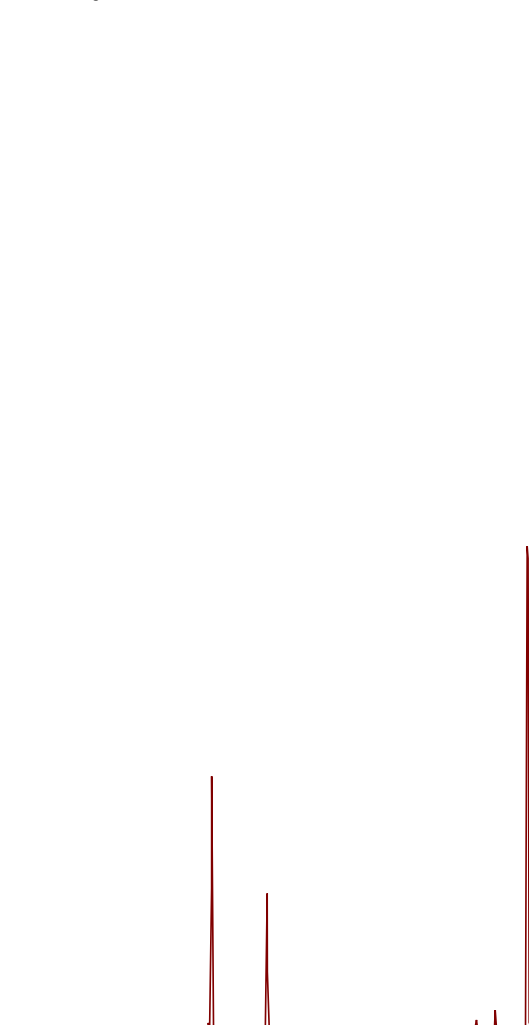




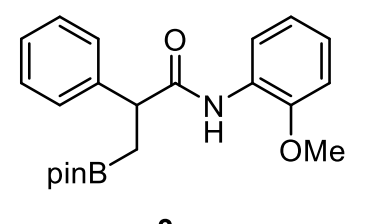

${ }^{11} \mathrm{~B}$ NMR $\left(96 \mathrm{MHz}, \mathrm{CDCl}_{3}\right)$

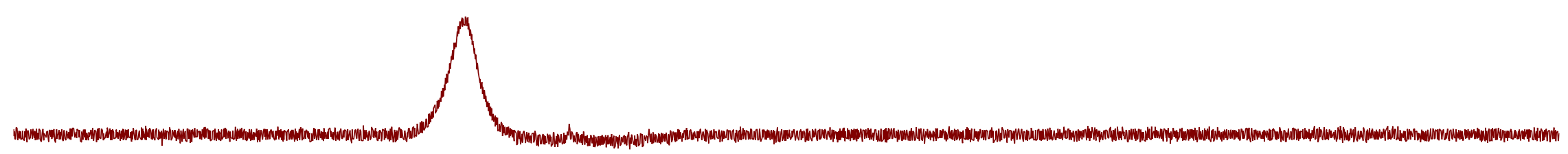

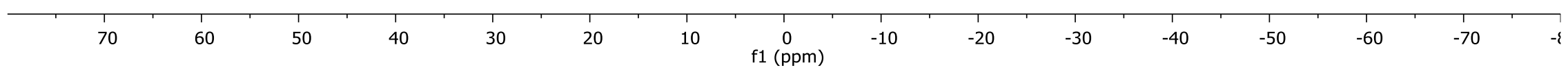




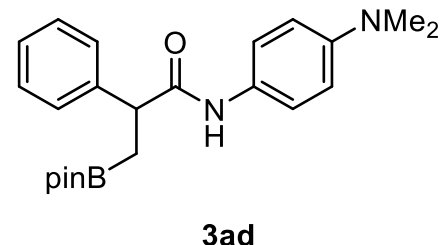

${ }^{1} \mathrm{H}$ NMR $\left(400 \mathrm{MHz}, \mathrm{CDCl}_{3}\right)$

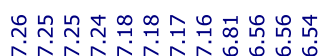

筮

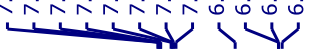

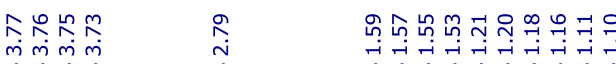 \\ in}

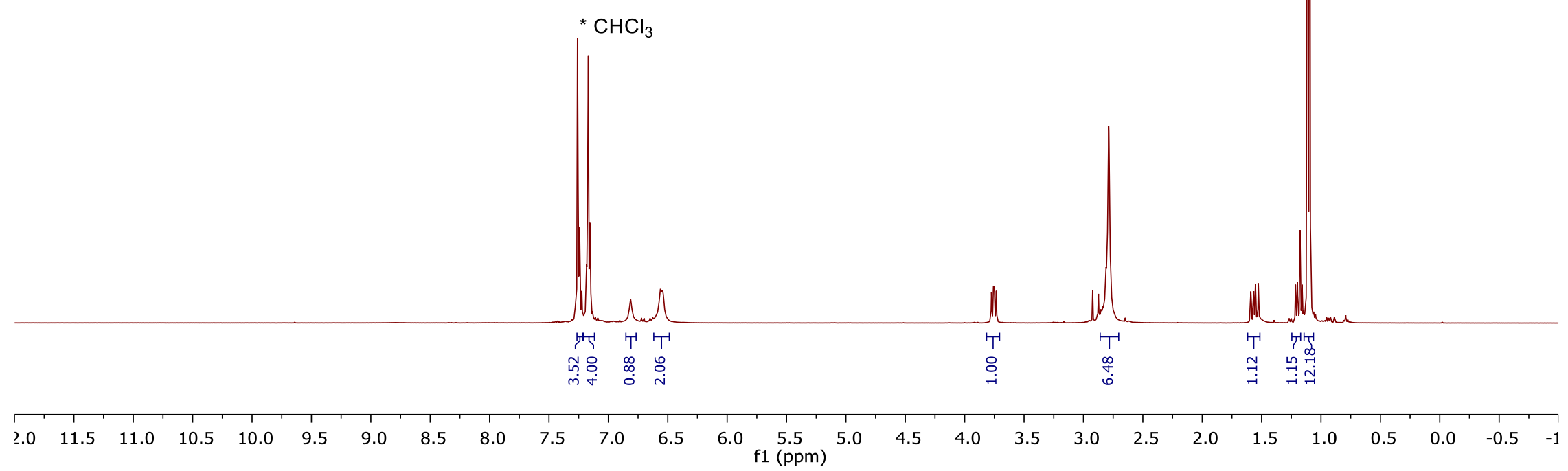



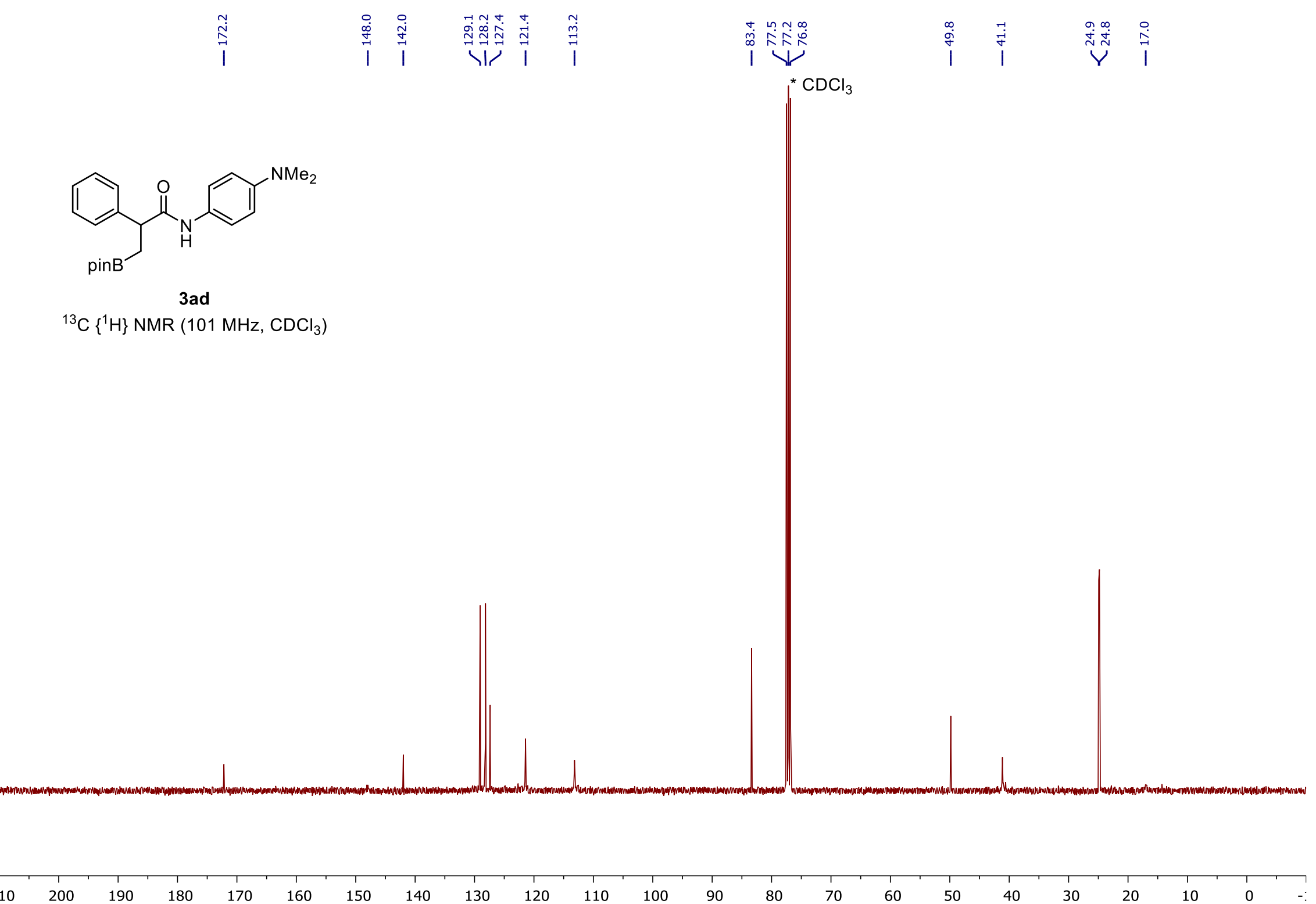
Supporting Information

S154

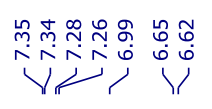

오ำ

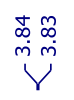

计
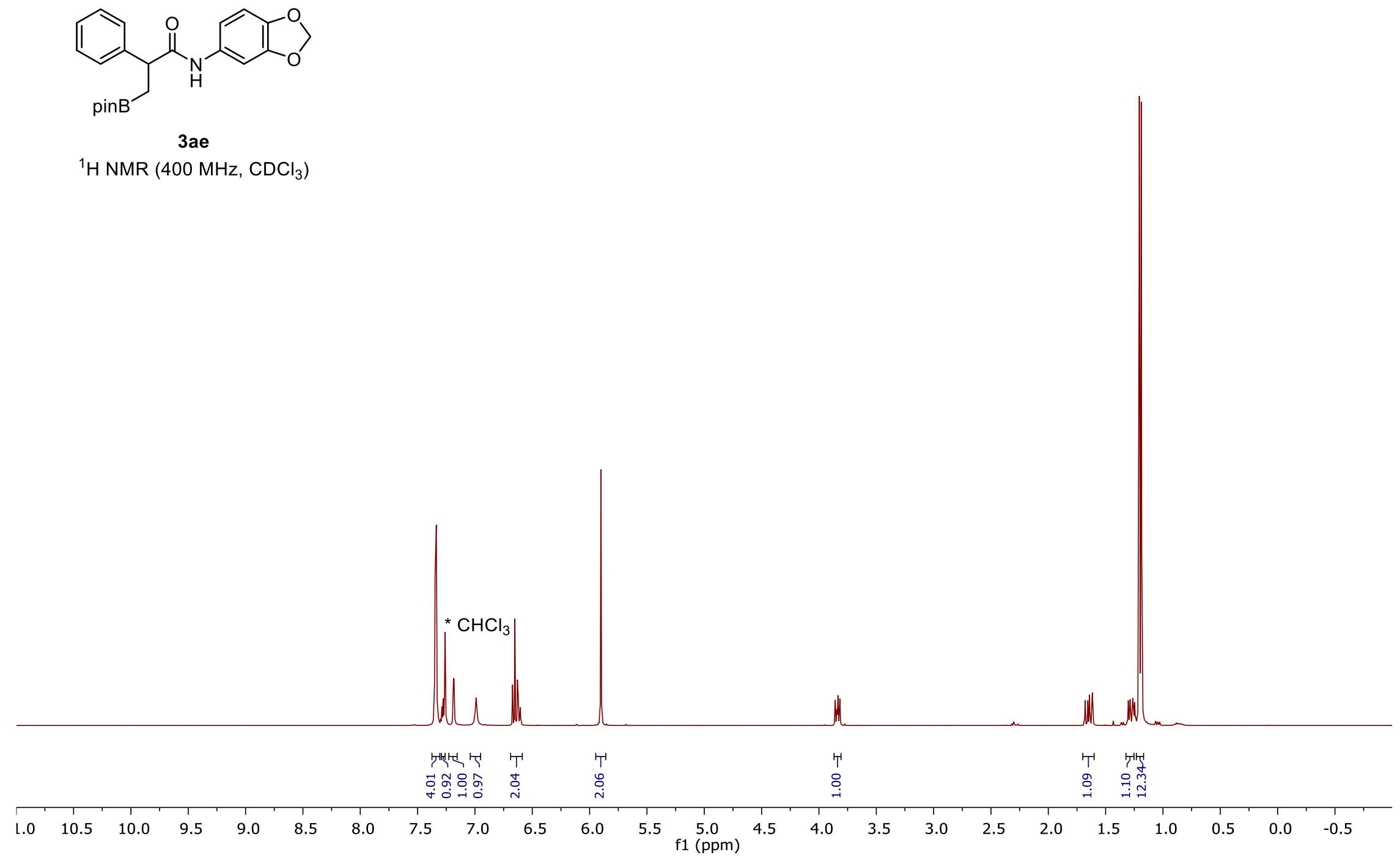


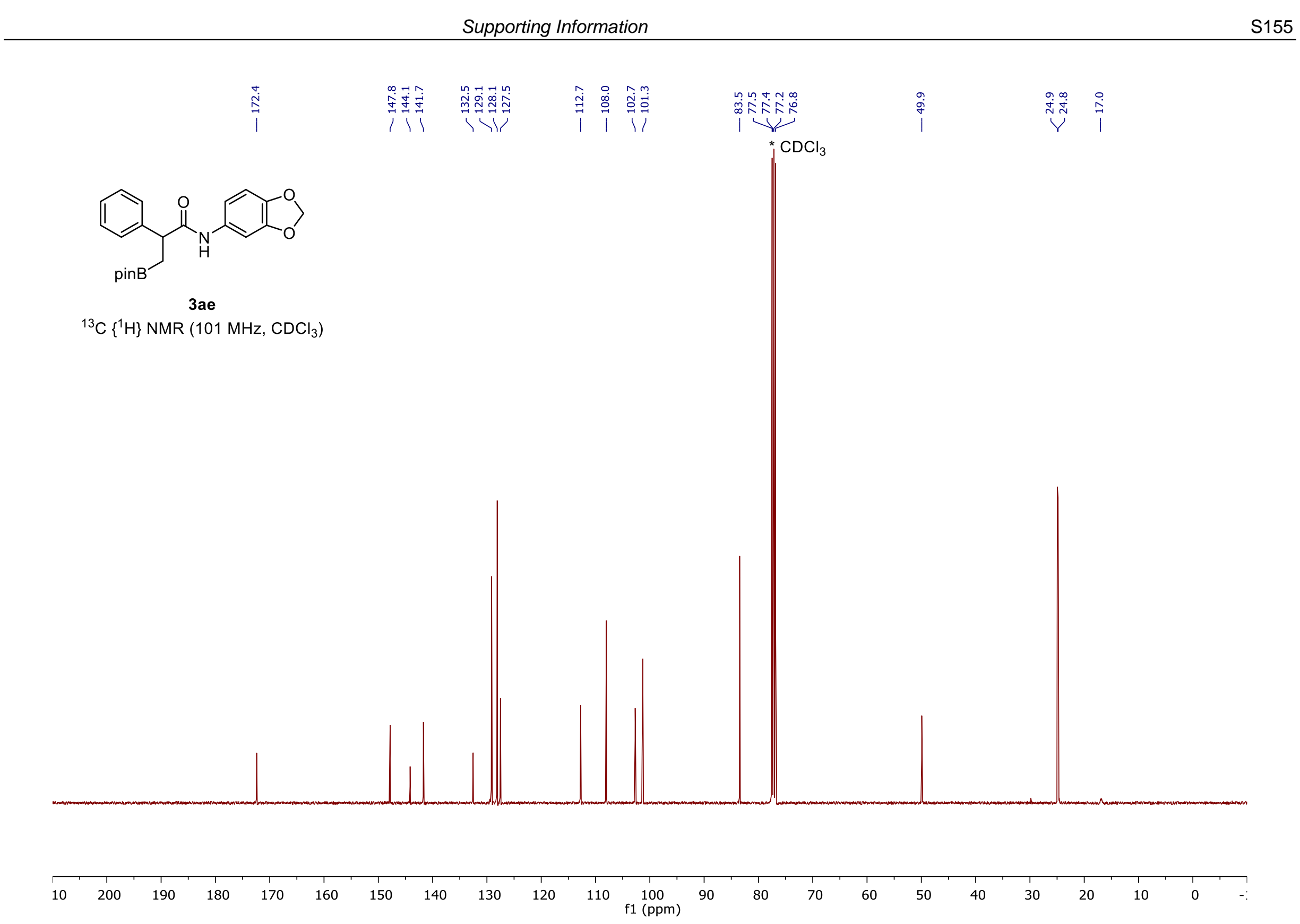




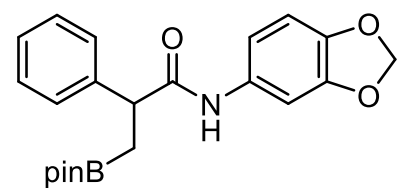

3 ae

${ }^{11} \mathrm{~B}$ NMR $\left(96 \mathrm{MHz}, \mathrm{CDCl}_{3}\right)$

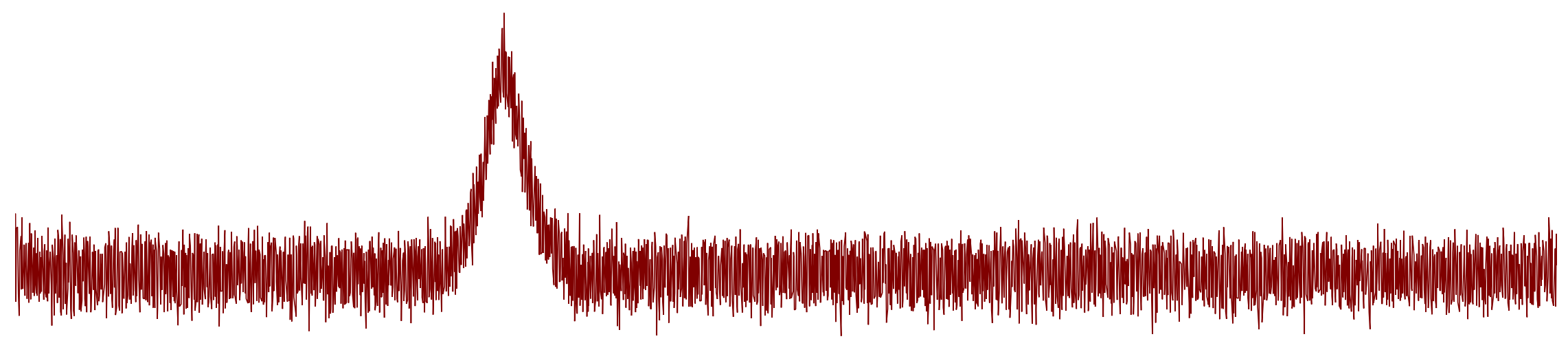




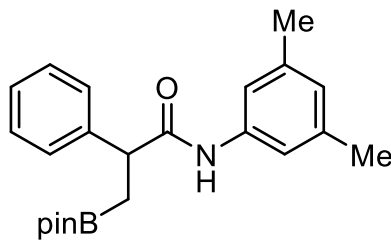

3 af

${ }^{1} \mathrm{H}$ NMR (400 MHz, $\mathrm{CDCl}_{3}$ )

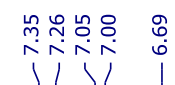

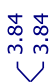

I

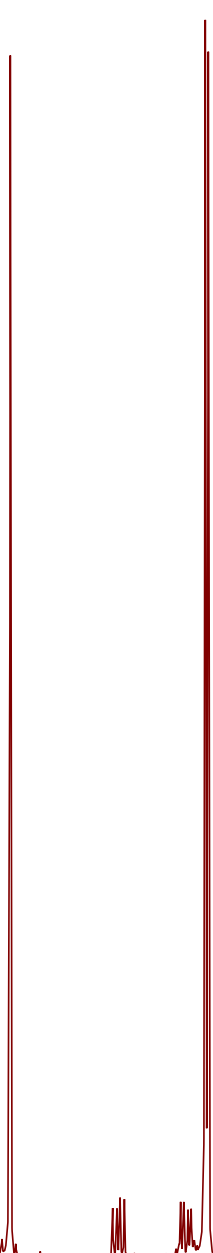

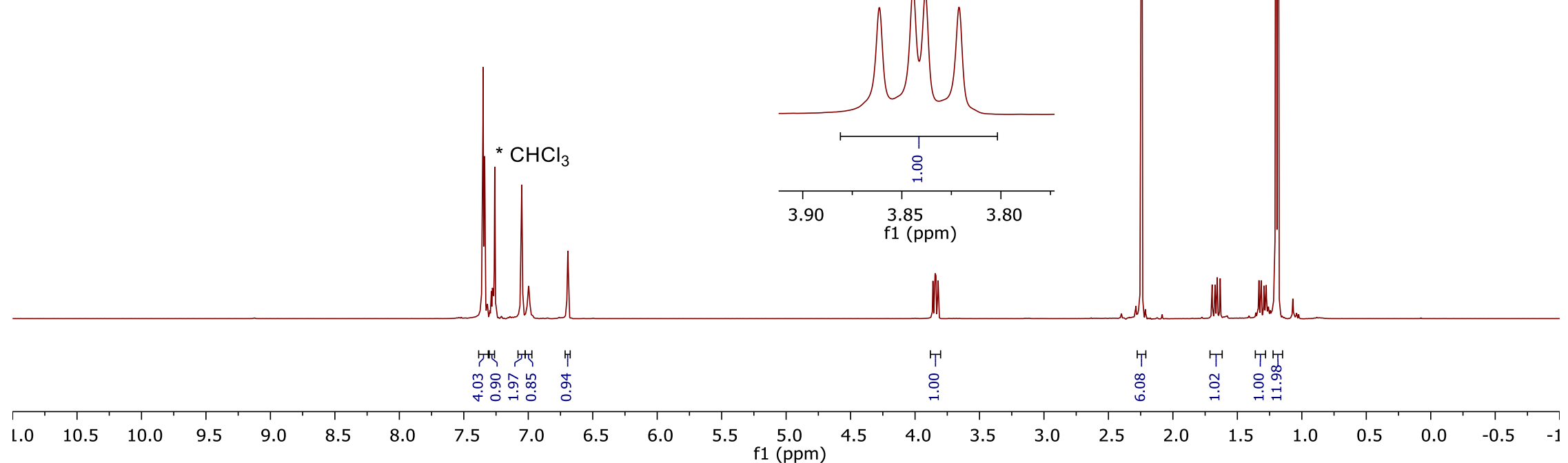




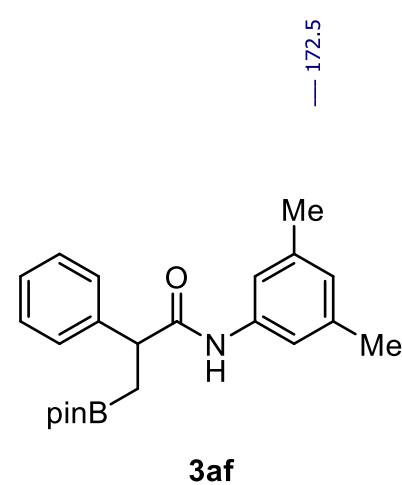

|

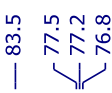

宫

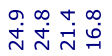

$\mathrm{CDCl}_{3}$

r'

${ }^{13} \mathrm{C}\left\{{ }^{1} \mathrm{H}\right\}$ NMR $\left(101 \mathrm{MHz}, \mathrm{CDCl}_{3}\right)$

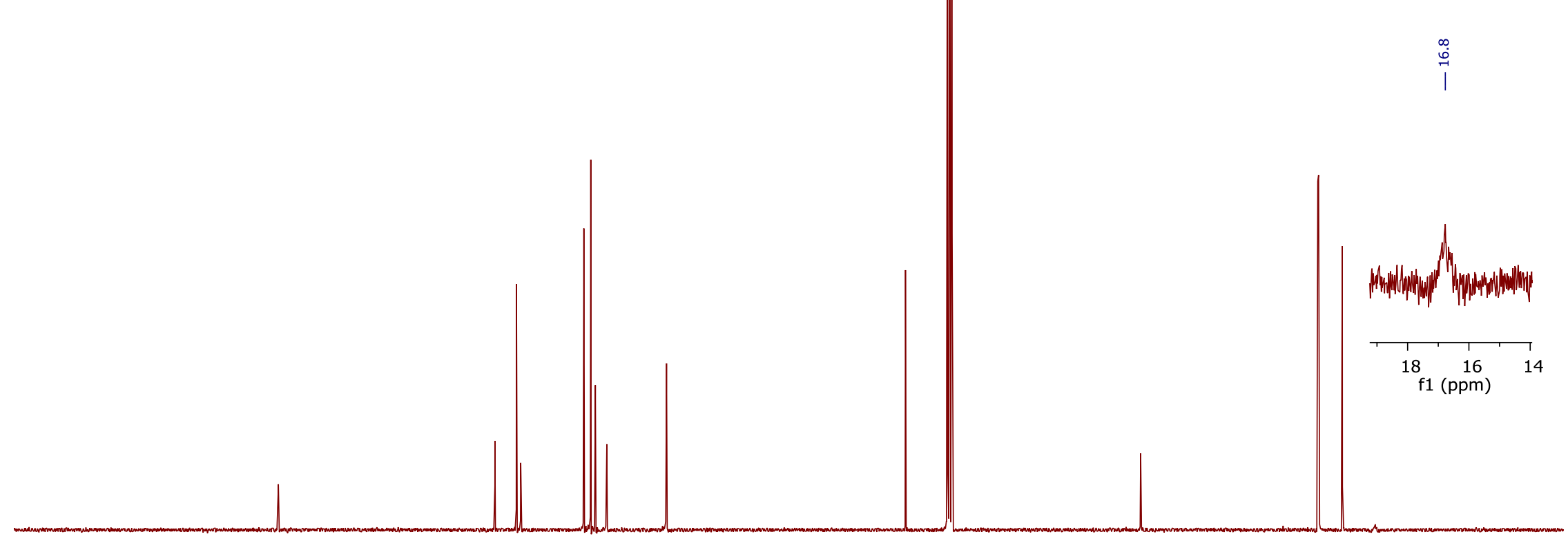




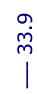

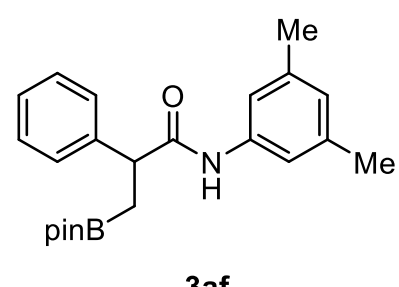

${ }^{11} \mathrm{~B}$ NMR (128 MHz, $\mathrm{CDCl}_{3}$ )

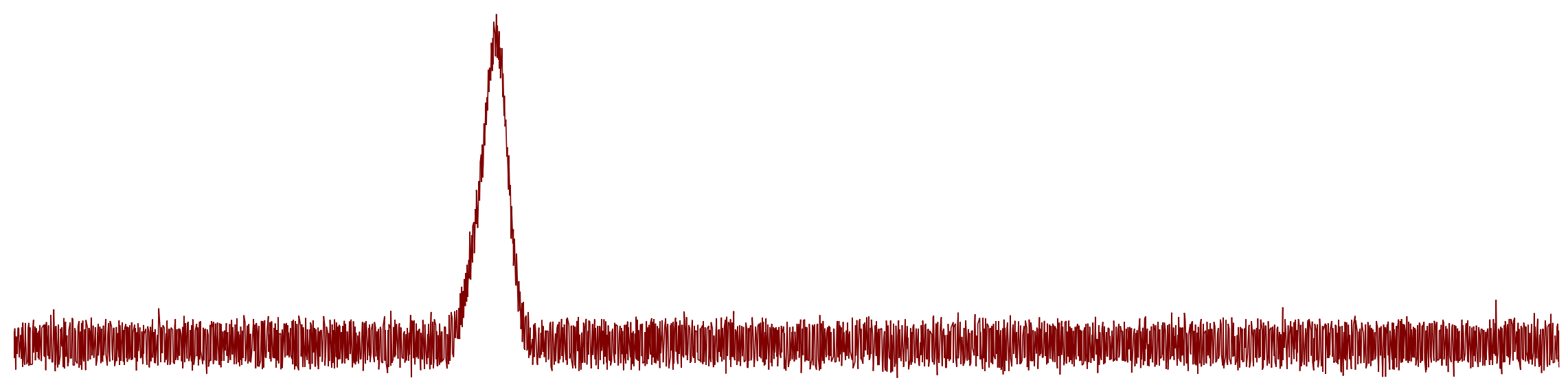




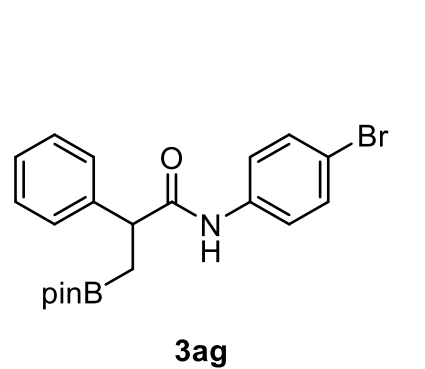

ำ

पiji

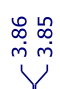

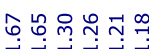

$\checkmark$ 人)

${ }^{1} \mathrm{H}$ NMR $\left(300 \mathrm{MHz}, \mathrm{CDCl}_{3}\right)$

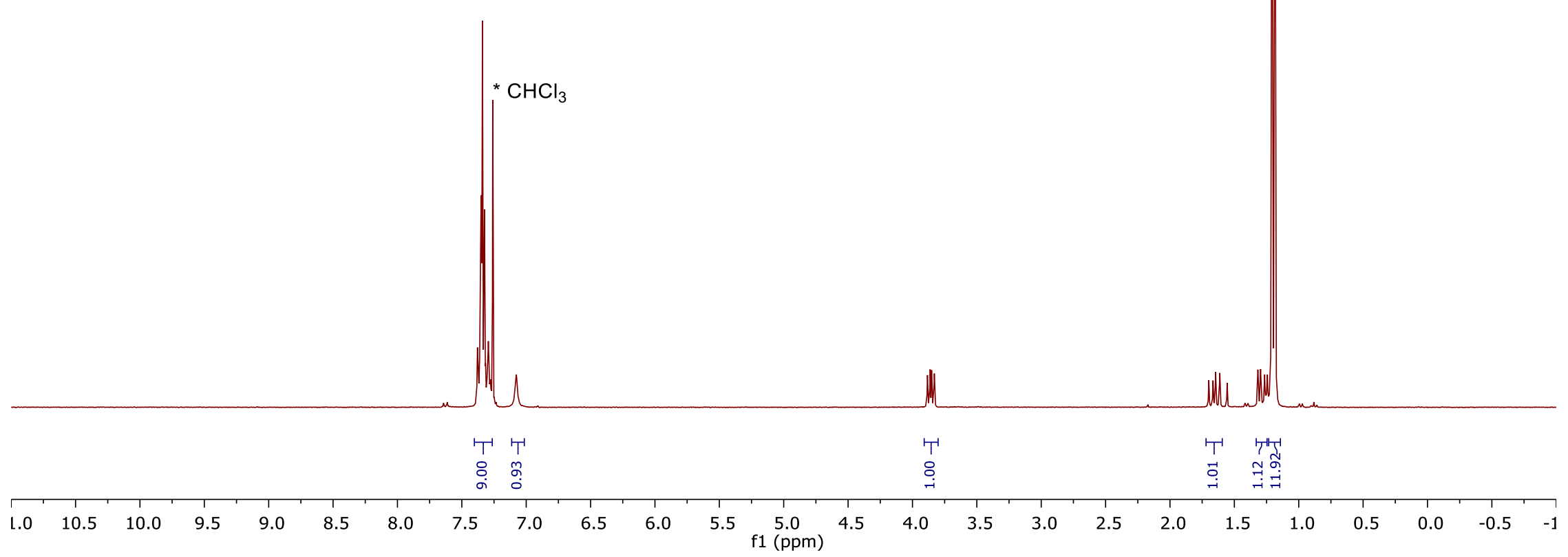




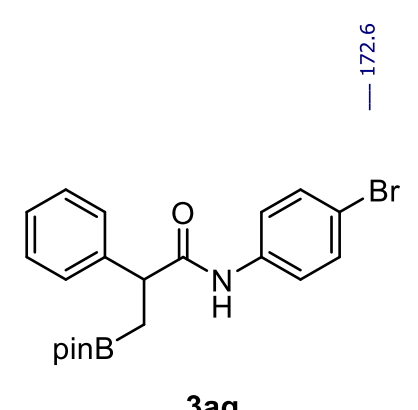

|

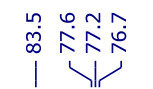

$* \mathrm{CDCl}_{3}$

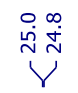

${ }^{13} \mathrm{C}\left\{{ }^{1} \mathrm{H}\right\} \operatorname{NMR}\left(75 \mathrm{MHz}, \mathrm{CDCl}_{3}\right)$
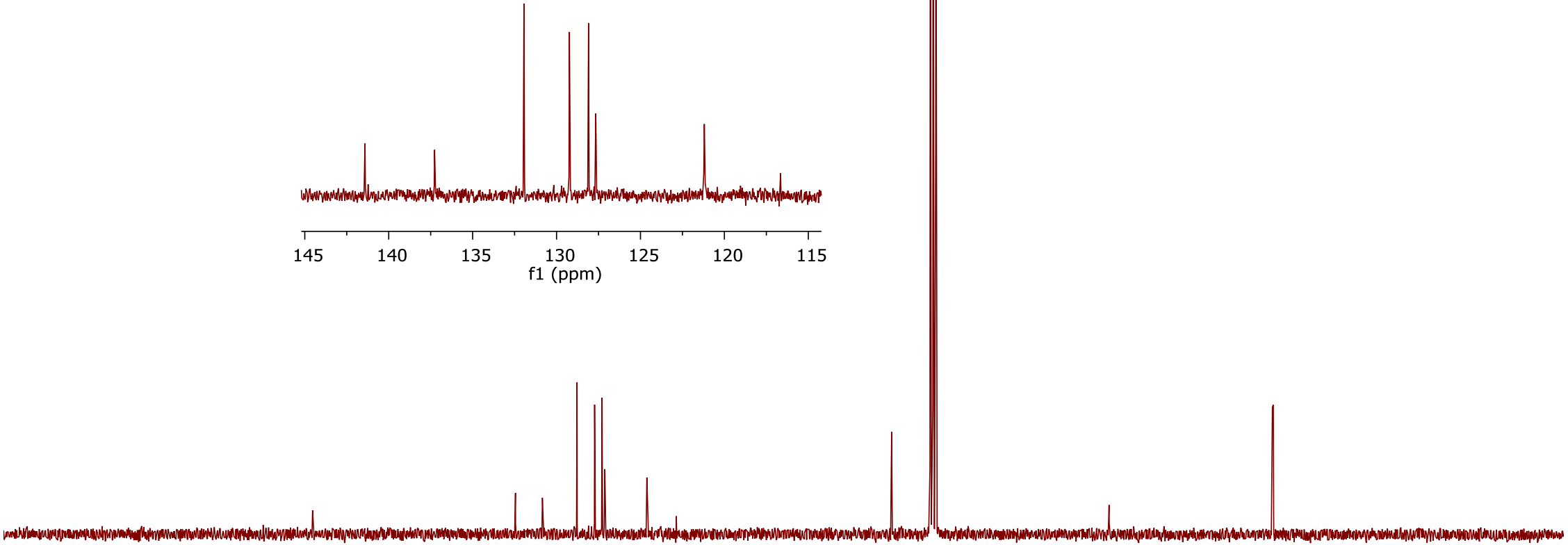


\section{$\stackrel{\sim}{m}$}

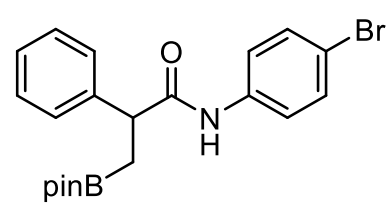

$3 a g$

${ }^{11} \mathrm{~B}$ NMR (96 MHz, $\mathrm{CDCl}_{3}$ )

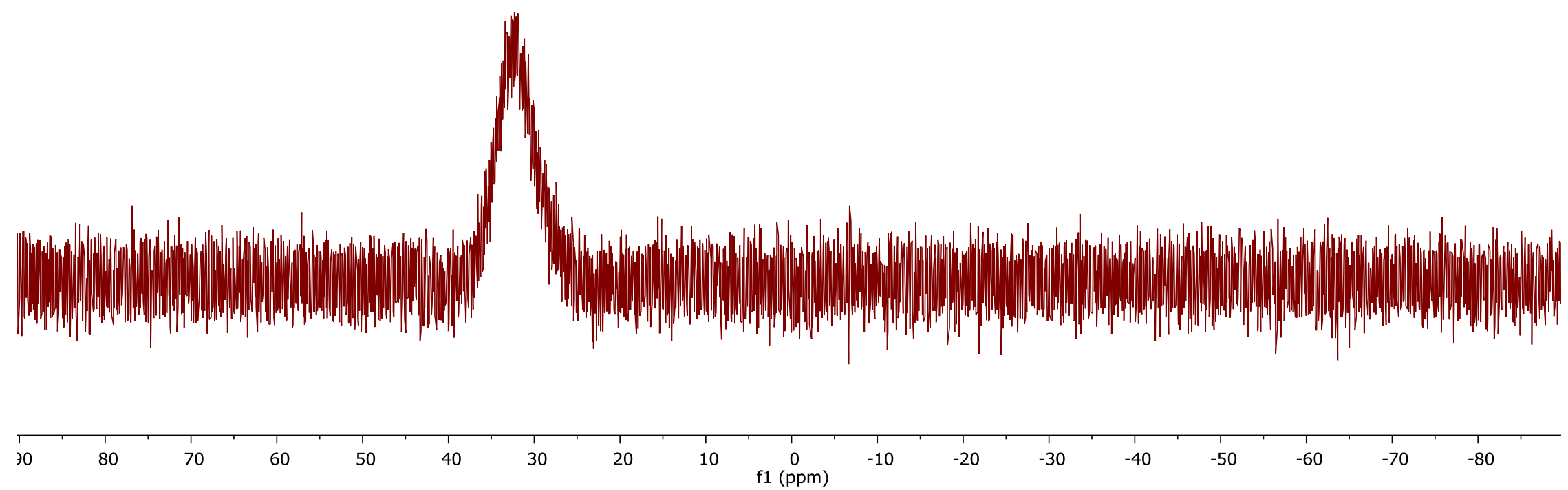




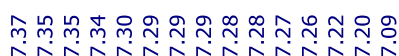

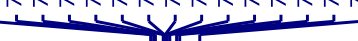

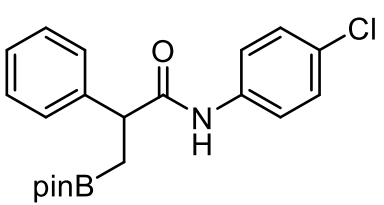

3ah

${ }^{1} \mathrm{H}$ NMR $\left(500 \mathrm{MHz}, \mathrm{CDCl}_{3}\right.$ )

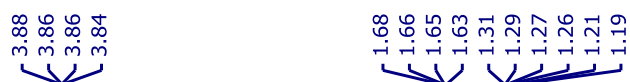

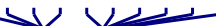

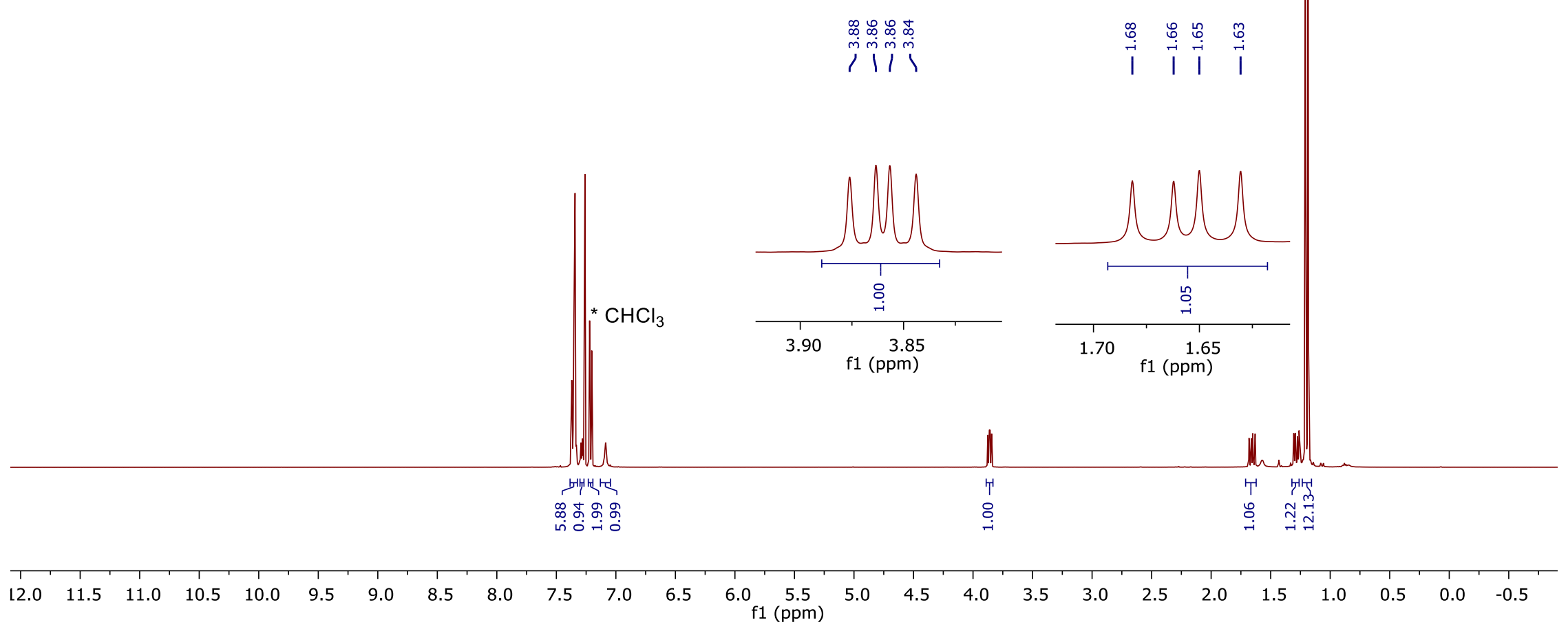




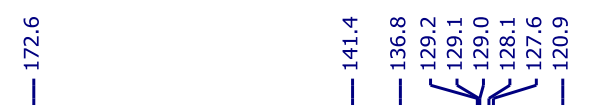

is

11 牙।

14

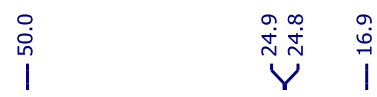

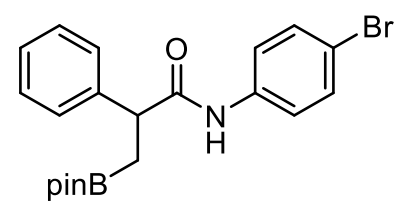

3ah

${ }^{13} \mathrm{C}\left\{{ }^{1} \mathrm{H}\right\} \operatorname{NMR}\left(126 \mathrm{MHz}, \mathrm{CDCl}_{3}\right)$
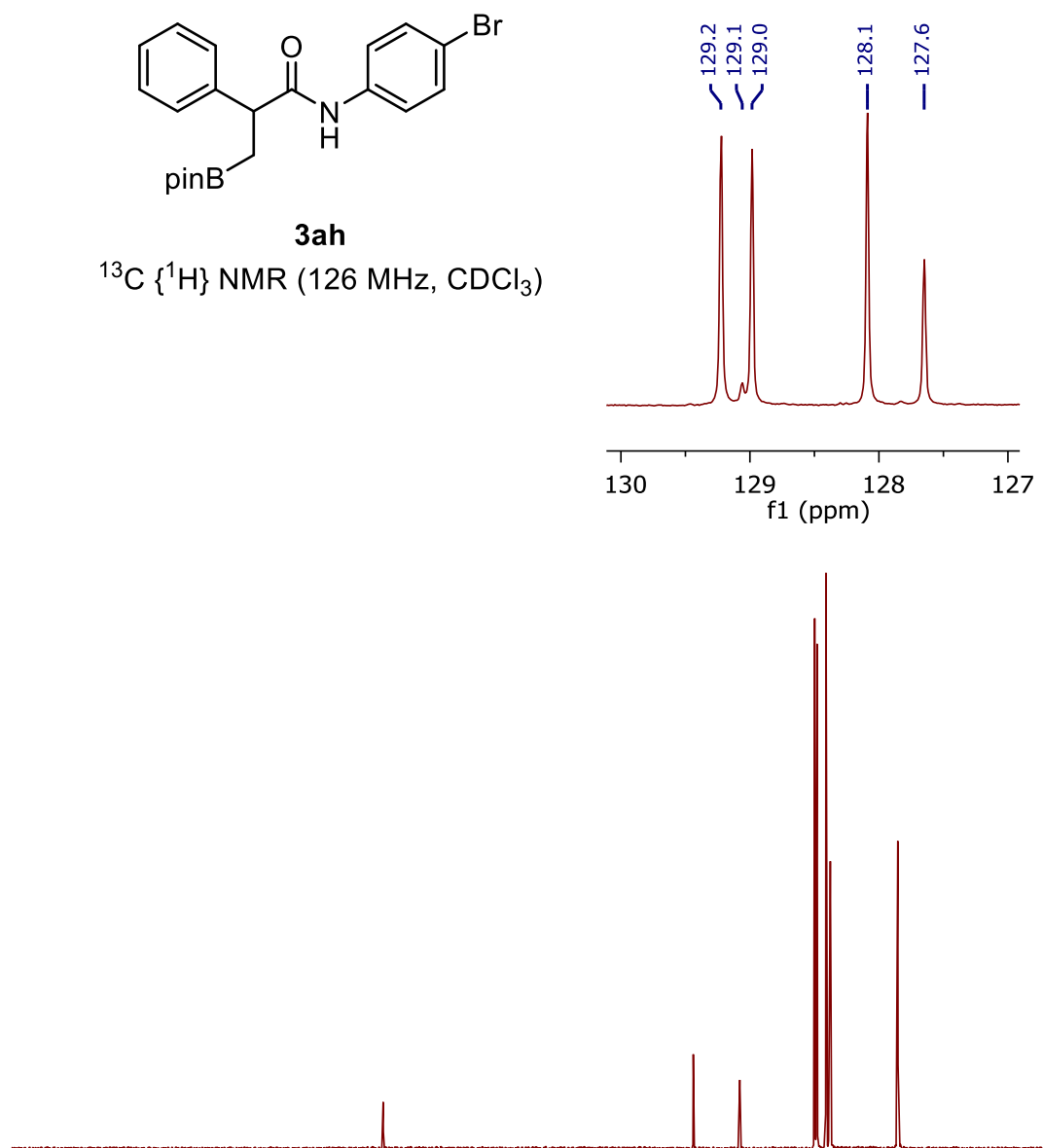

${ }^{*} \mathrm{CDCl}_{3}$

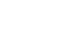

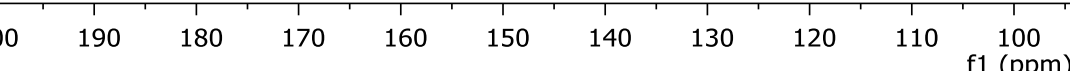

\section{$90 \quad 80$}




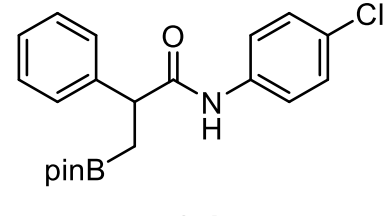

${ }^{11} \mathrm{~B}$ NMR (96 MHz, $\mathrm{CDCl}_{3}$ )

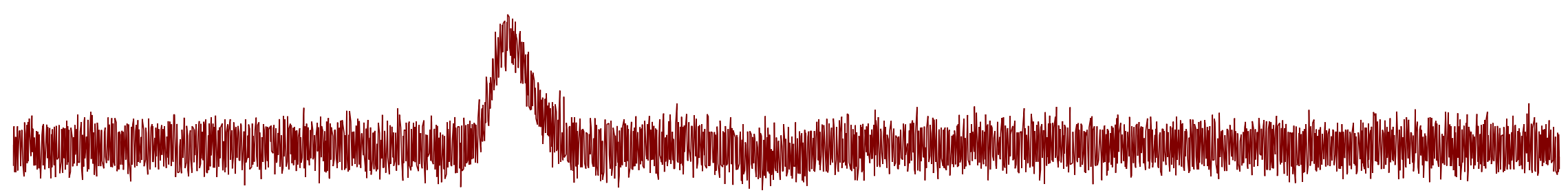




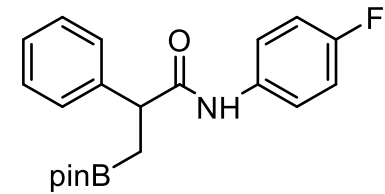

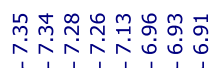

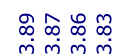

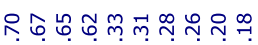

पर।

Ver

$\longrightarrow$

${ }^{1} \mathrm{H}$ NMR $\left(300 \mathrm{MHz}, \mathrm{CDCl}_{3}\right)$

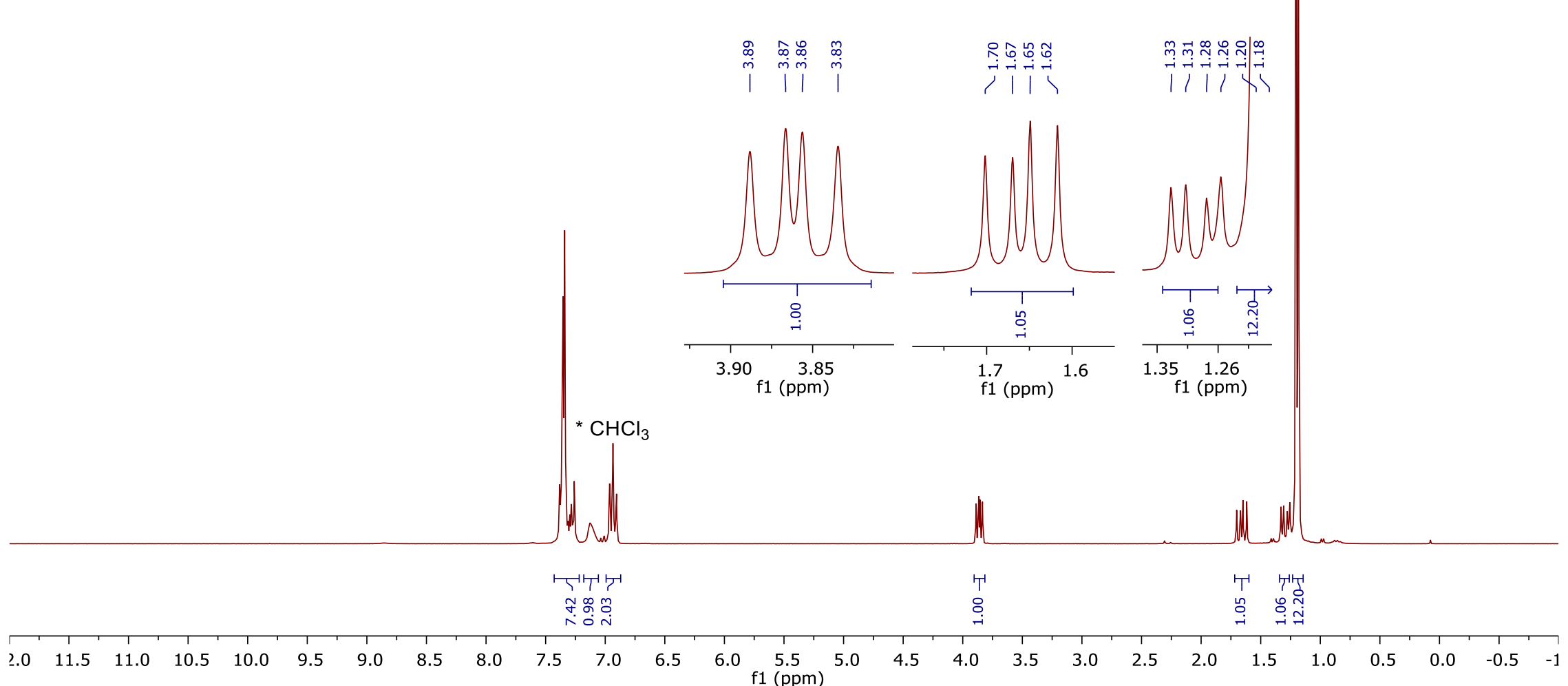




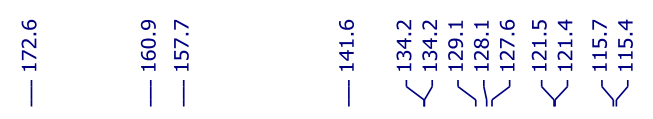

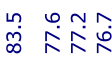

和

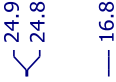

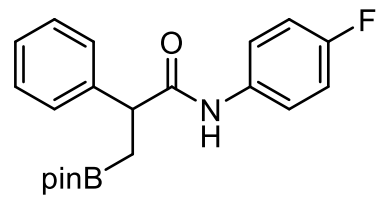

$3 a i$

${ }^{13} \mathrm{C}\left\{{ }^{1} \mathrm{H}\right\}$ NMR $\left(75 \mathrm{MHz}, \mathrm{CDCl}_{3}\right)$

${ }^{*} \mathrm{CDCl}_{3}$

4

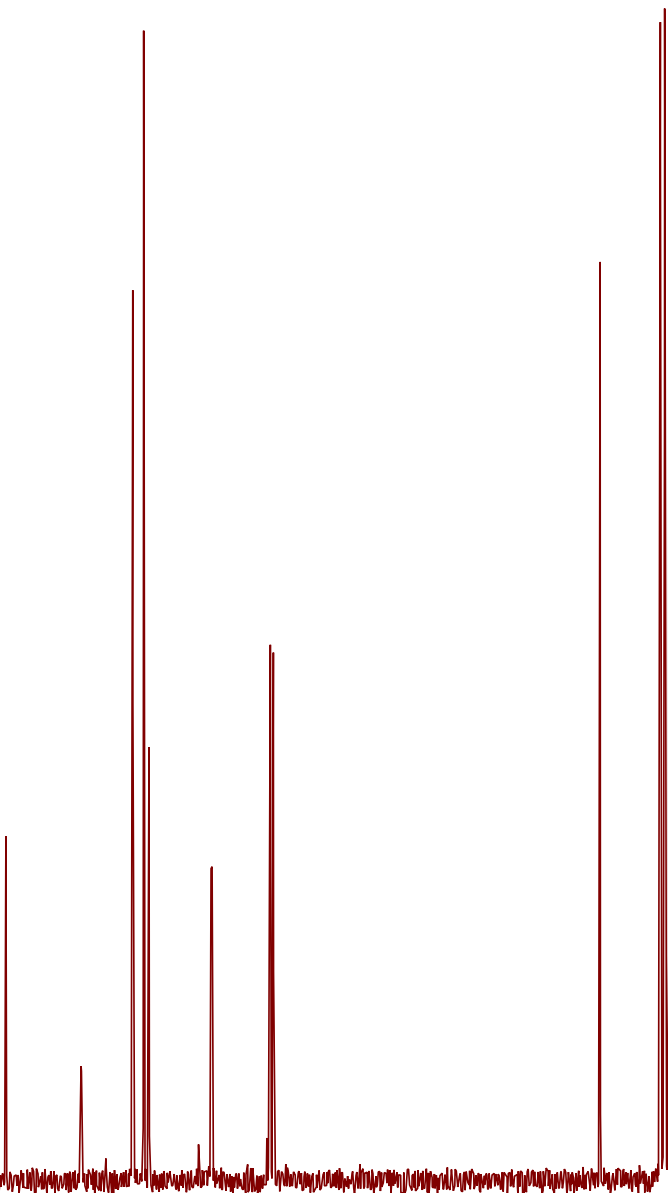

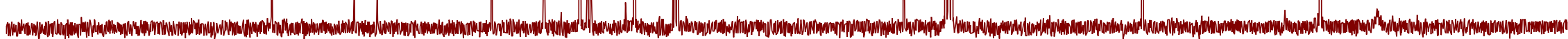

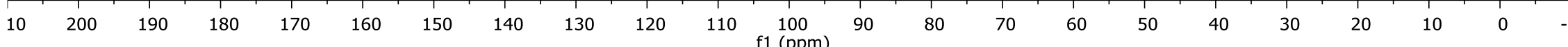




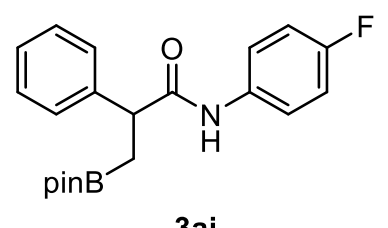

${ }^{11} \mathrm{~B}$ NMR $\left(96 \mathrm{MHz}, \mathrm{CDCl}_{3}\right.$ )

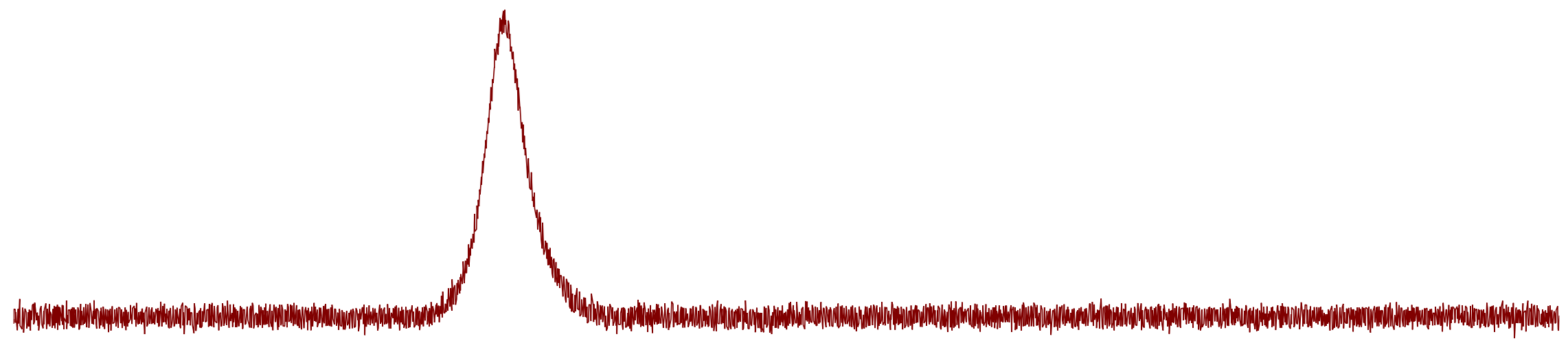




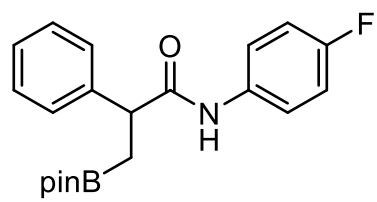

$3 \mathrm{ai}$

${ }^{19} \mathrm{~F}\left\{{ }^{1} \mathrm{H}\right\} \mathrm{NMR}\left(282 \mathrm{MHz}, \mathrm{CDCl}_{3}\right)$

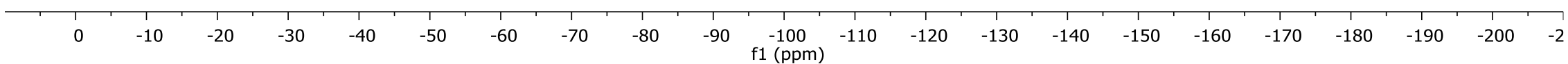




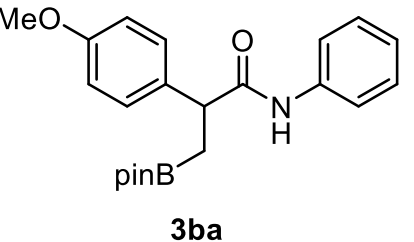

${ }^{1} \mathrm{H}$ NMR $\left(400 \mathrm{MHz}, \mathrm{CDCl}_{3}\right)$

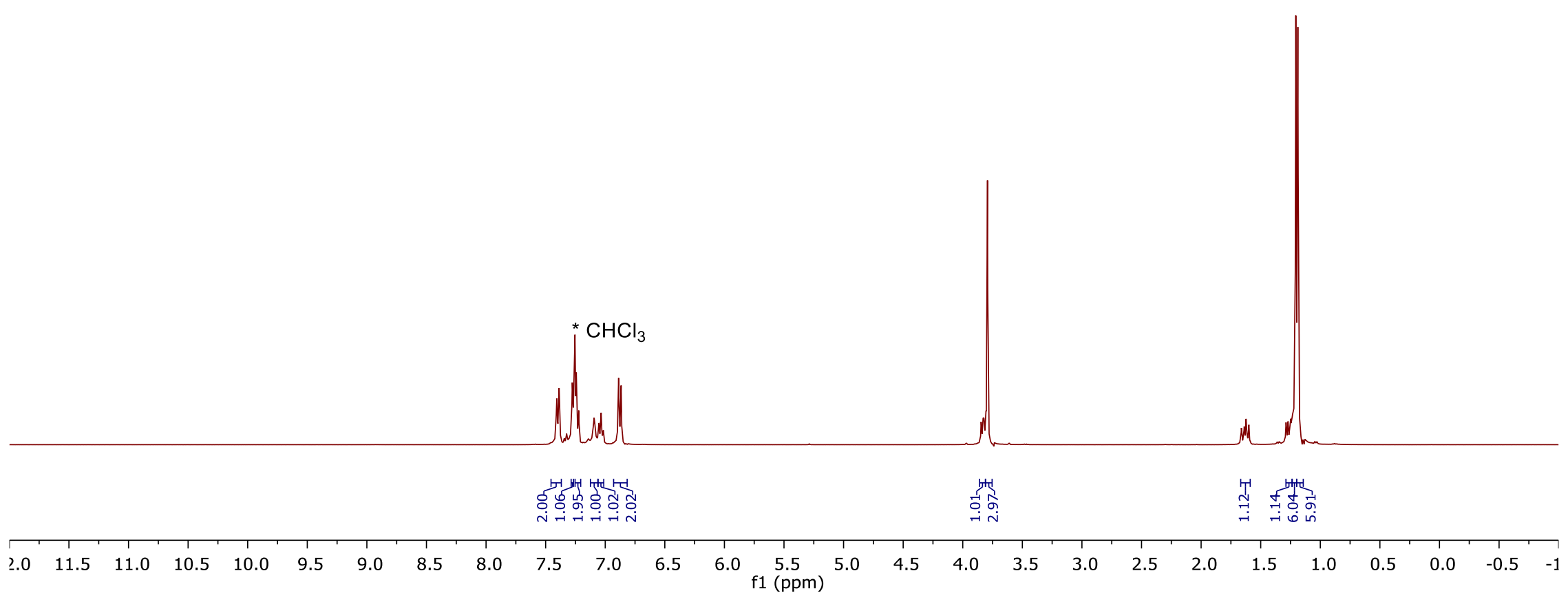



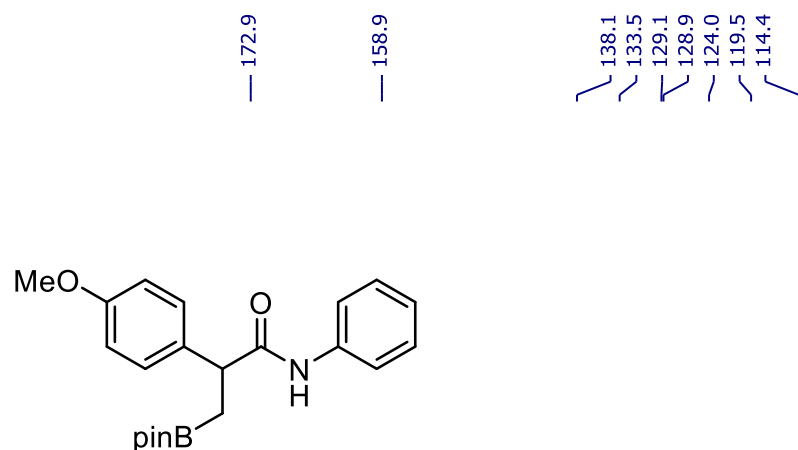

$$
3 \text { ba }
$$

${ }^{13} \mathrm{C}\left\{{ }^{1} \mathrm{H}\right\}$ NMR $\left(75 \mathrm{MHz}, \mathrm{CDCl}_{3}\right)$

${ }^{*} \mathrm{CDCl}_{3}$

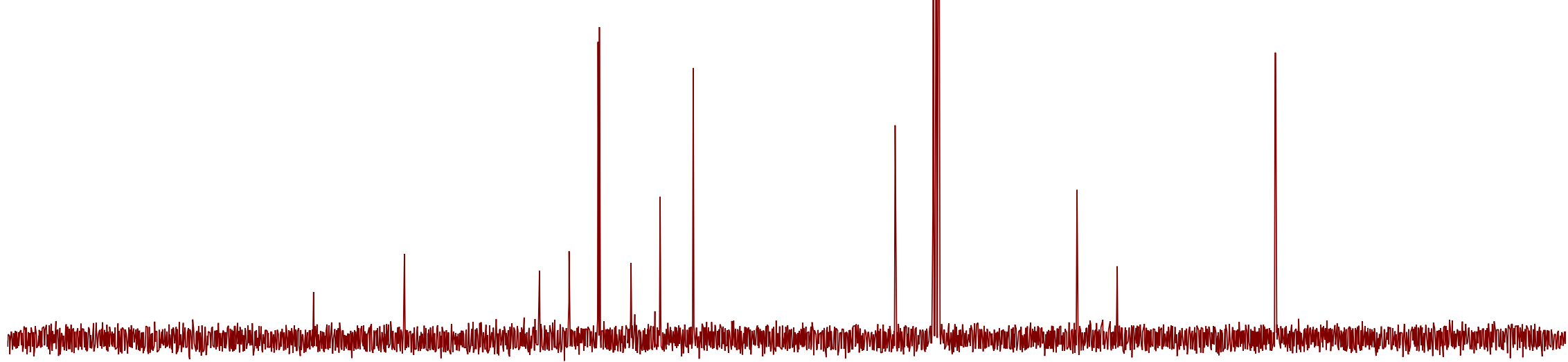




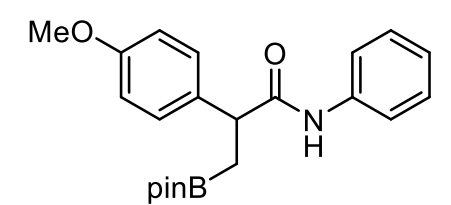

3 ba

${ }^{11} \mathrm{~B}$ NMR $\left(128 \mathrm{MHz}, \mathrm{CDCl}_{3}\right)$

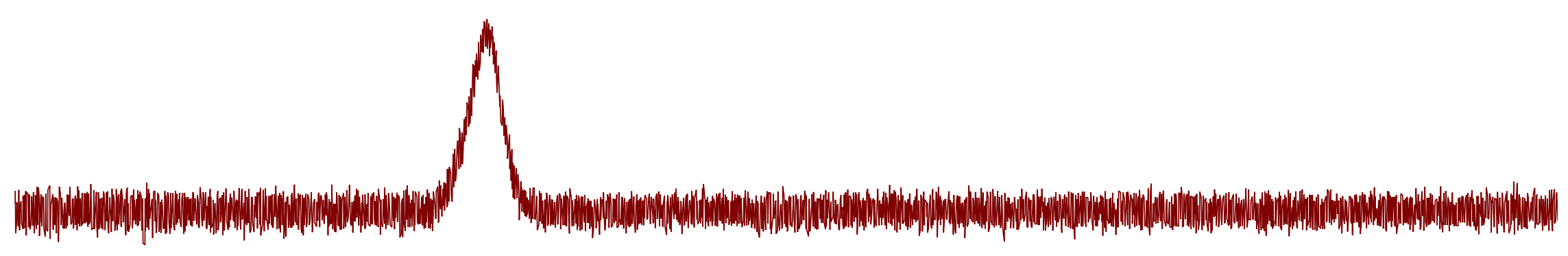

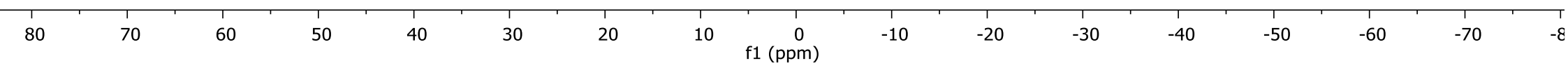




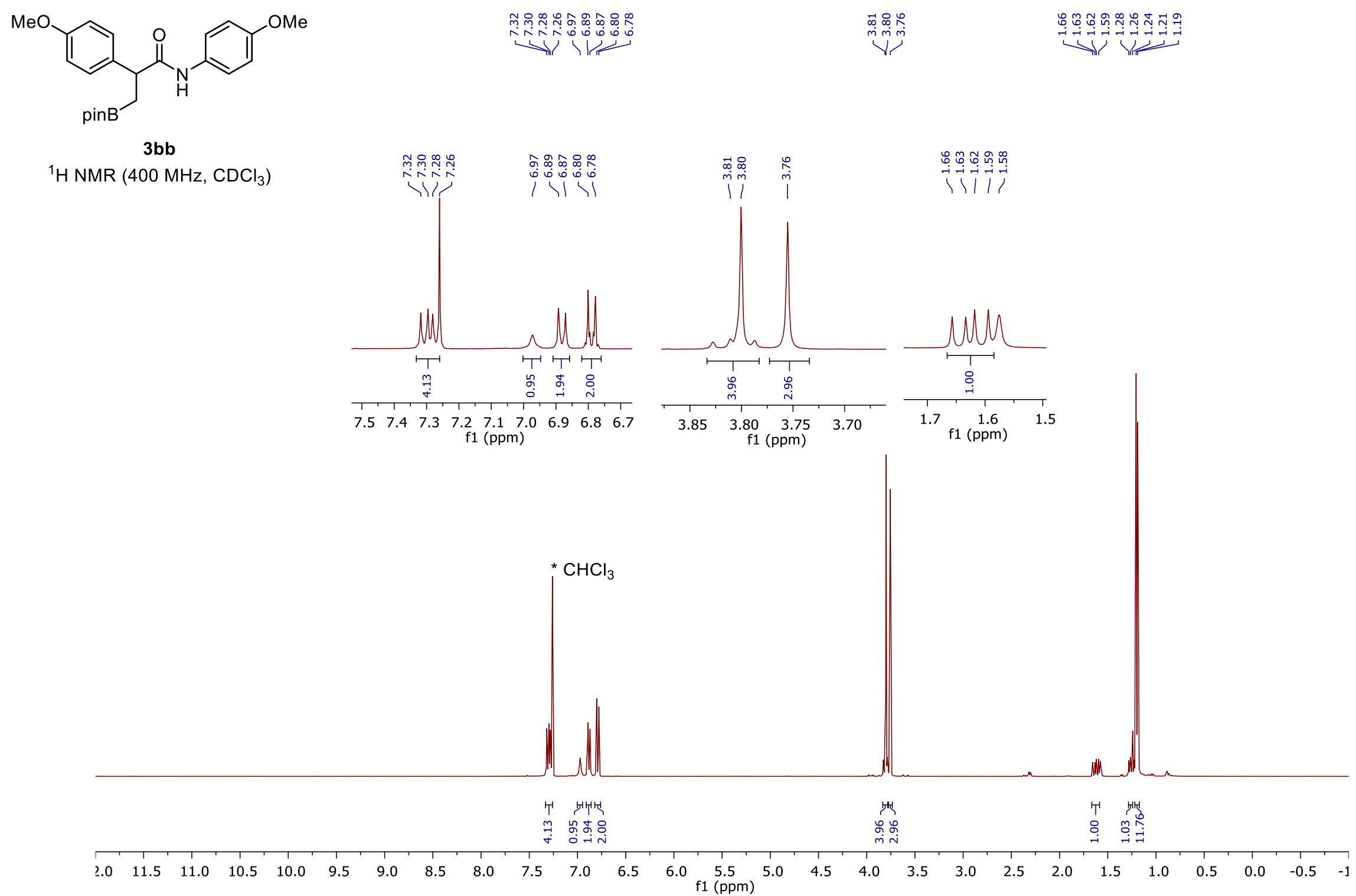




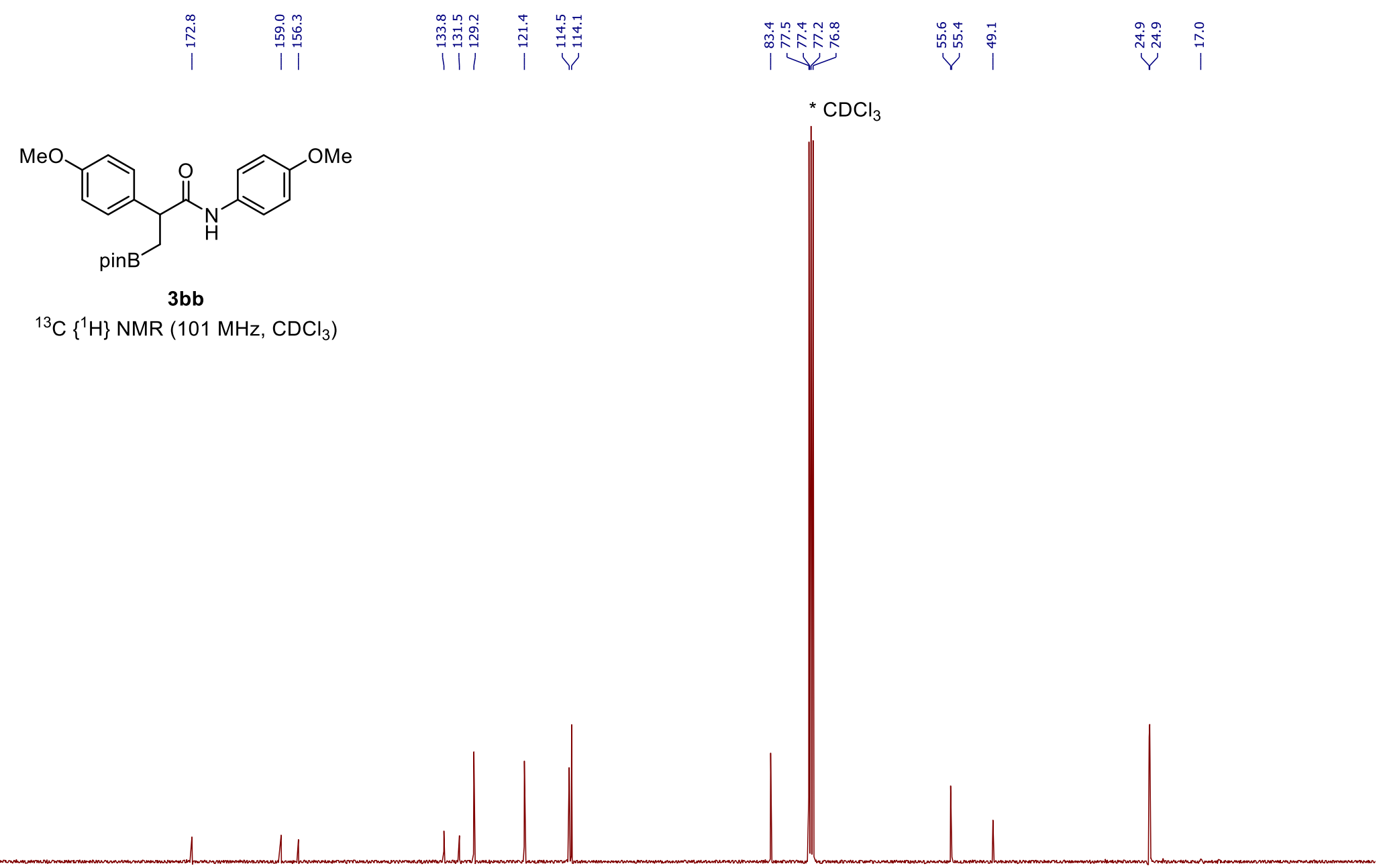

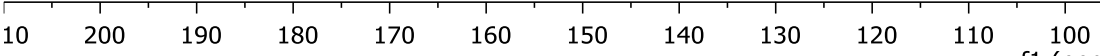
100
$\mathrm{f} 1(\mathrm{ppm})$

80 $\begin{array}{lll}70 & 60 & 50\end{array}$ 


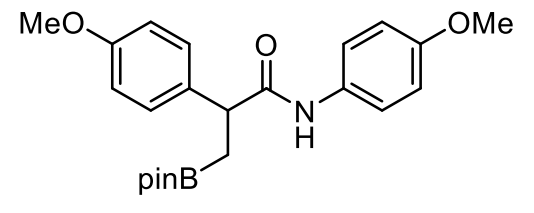

$3 \mathrm{bb}$

${ }^{11} \mathrm{~B}$ NMR (96 MHz, $\mathrm{CDCl}_{3}$ )

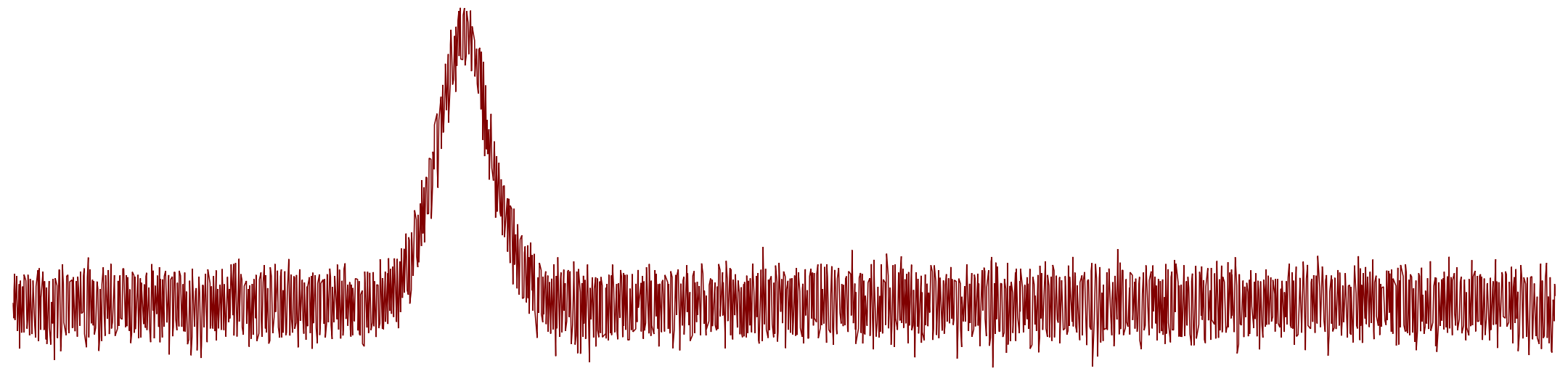

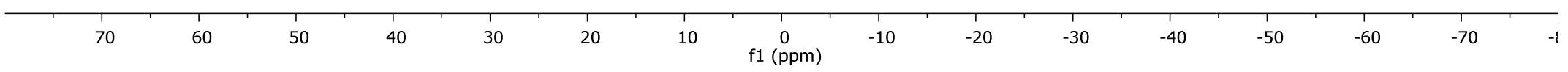




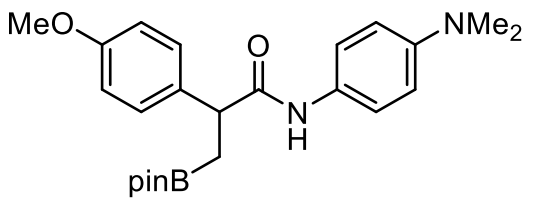

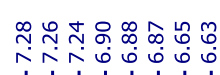

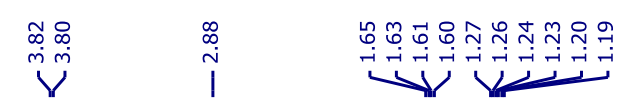

\section{3bd}

${ }^{1} \mathrm{H}$ NMR (500 MHz, $\mathrm{CDCl}_{3}$ )

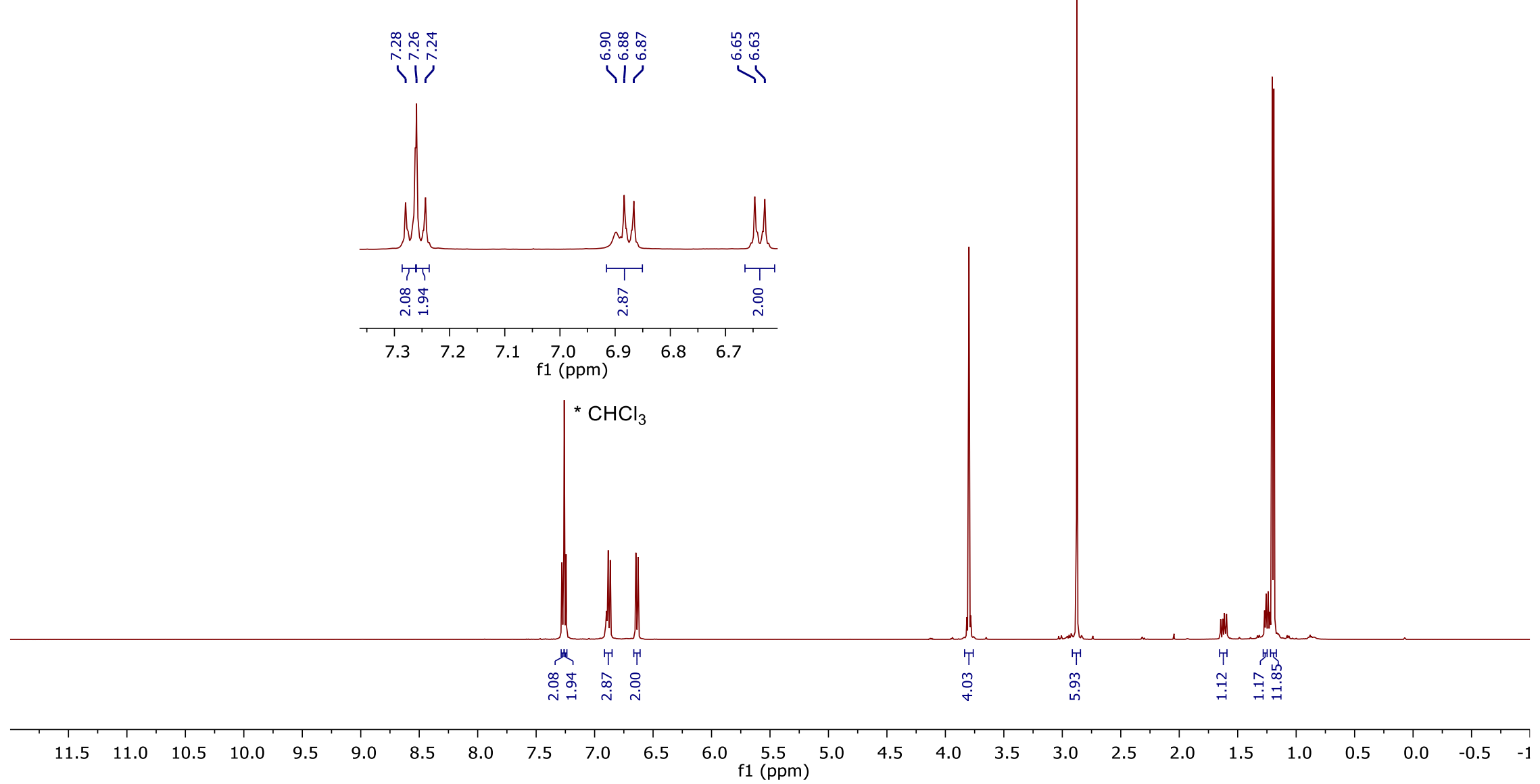




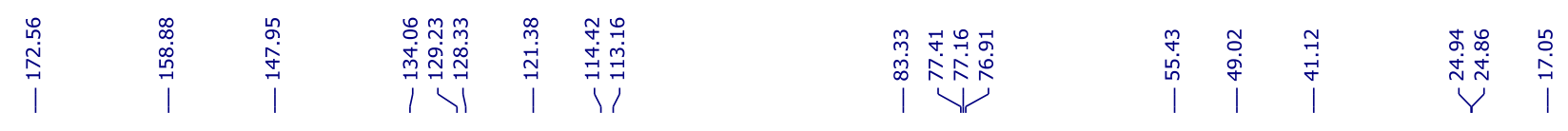

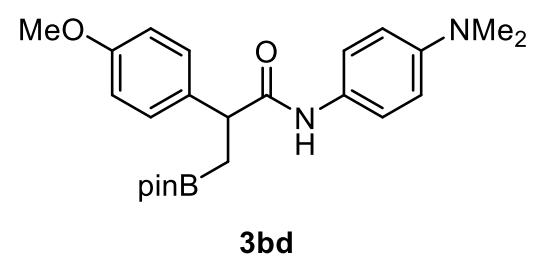

${ }^{13} \mathrm{C}\left\{{ }^{1} \mathrm{H}\right\}$ NMR $\left(126 \mathrm{MHz}, \mathrm{CDCl}_{3}\right)$

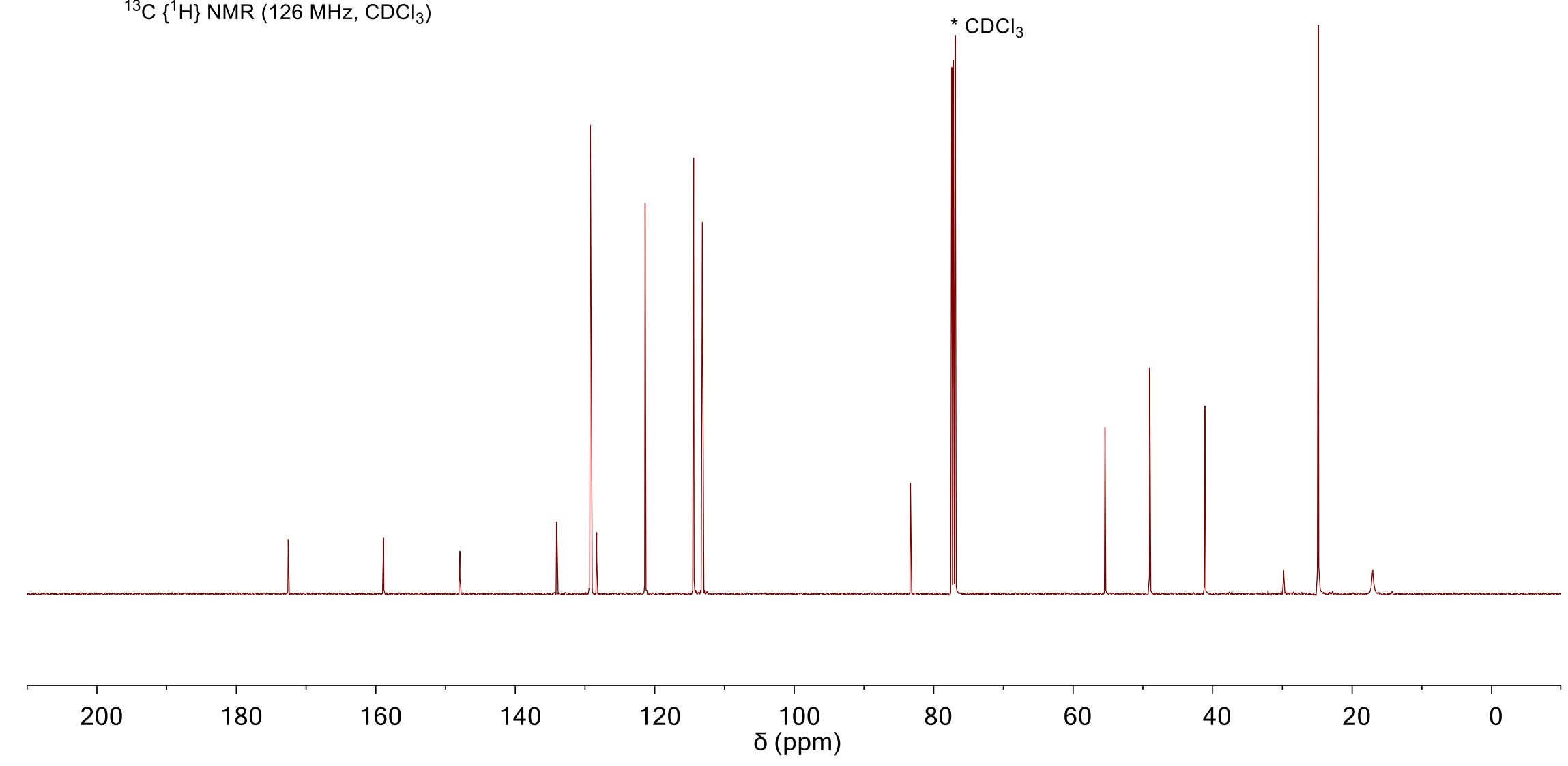




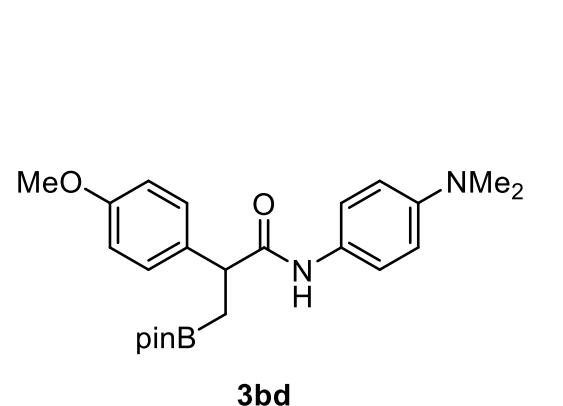

$\stackrel{n}{\mathfrak{m}}$

${ }^{11}$ B NMR (128 MHz, $\mathrm{CDCl}_{3}$ )

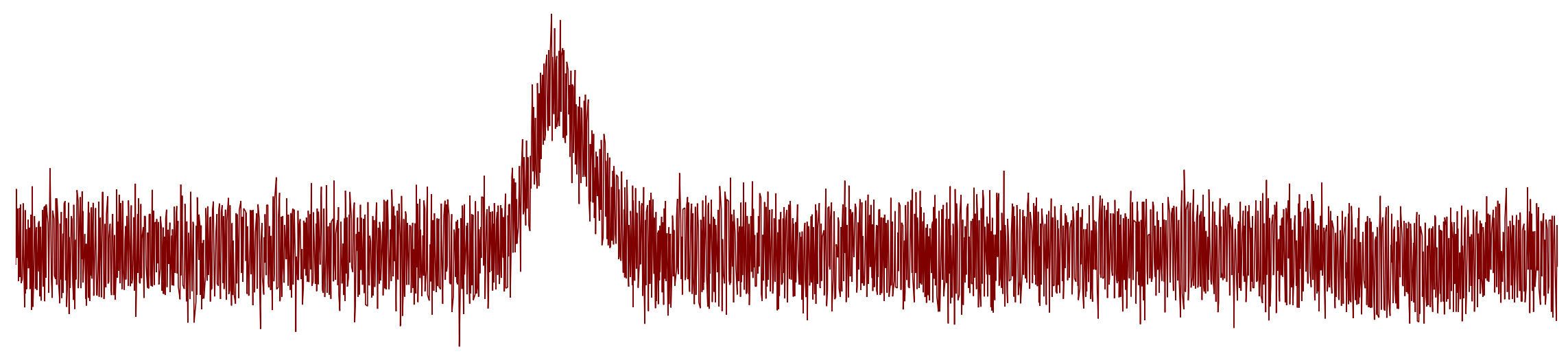


Supporting Information

S179

$\underbrace{\underbrace{2}}$

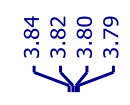

|
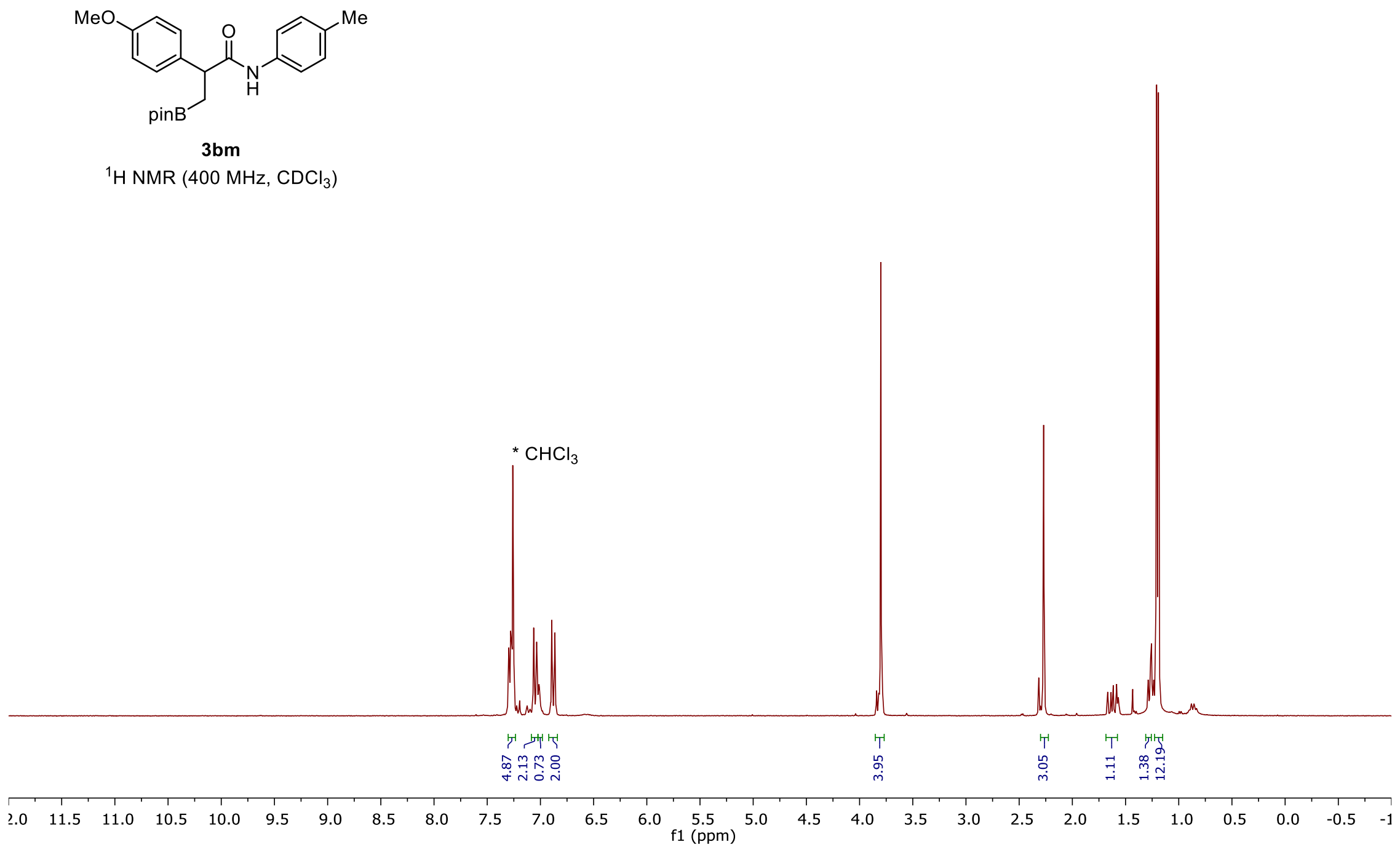
Supporting Information

S180

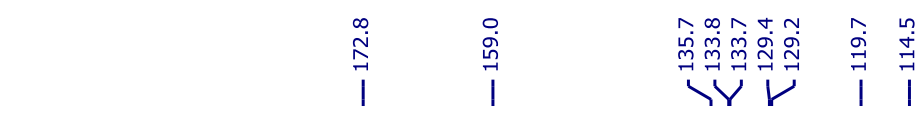<smiles>COc1ccc(C(C[PbH])C(=O)Nc2ccc(C)cc2)cc1</smiles>

3bm

${ }^{13} \mathrm{C}\left\{{ }^{1} \mathrm{H}\right\}$ NMR (101 MHz, $\mathrm{CDCl}_{3}$ )

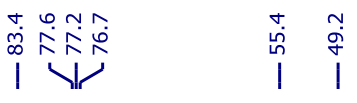

ìn

ฟับ
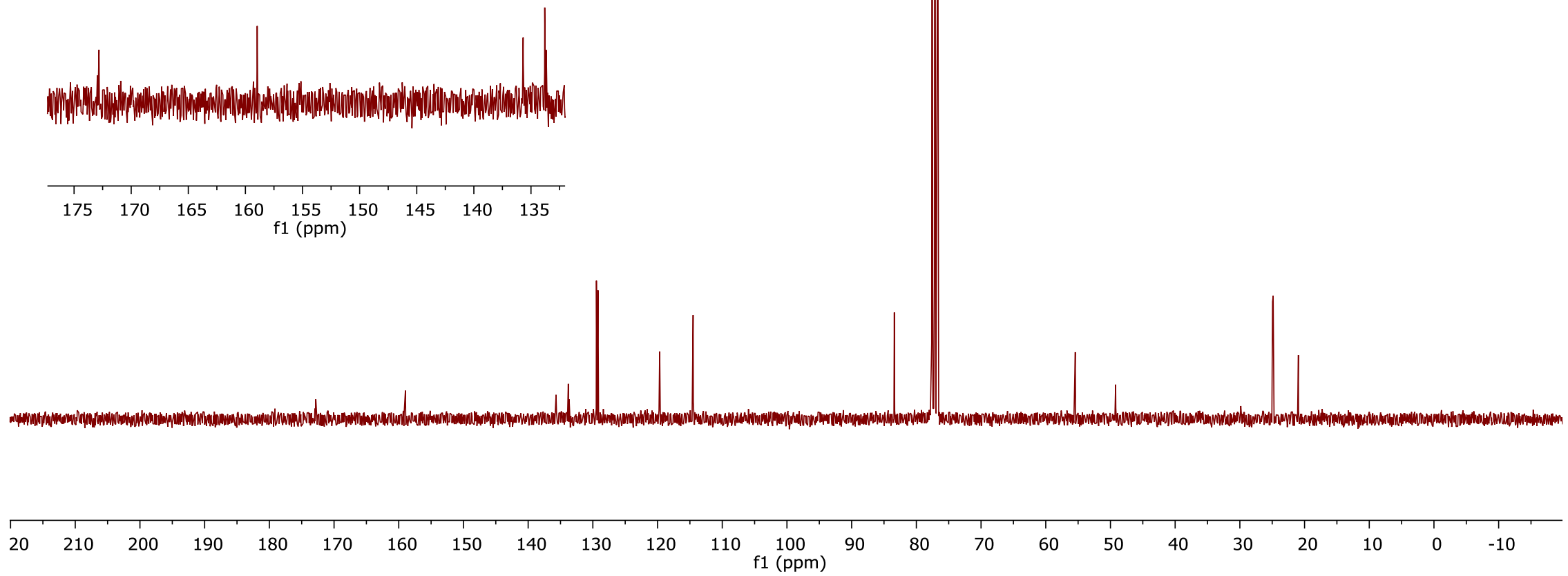


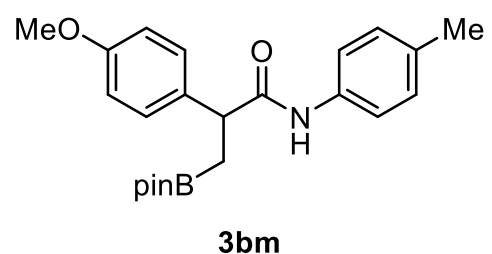

${ }^{11} \mathrm{~B}$ NMR $\left(128 \mathrm{MHz}, \mathrm{CDCl}_{3}\right)$

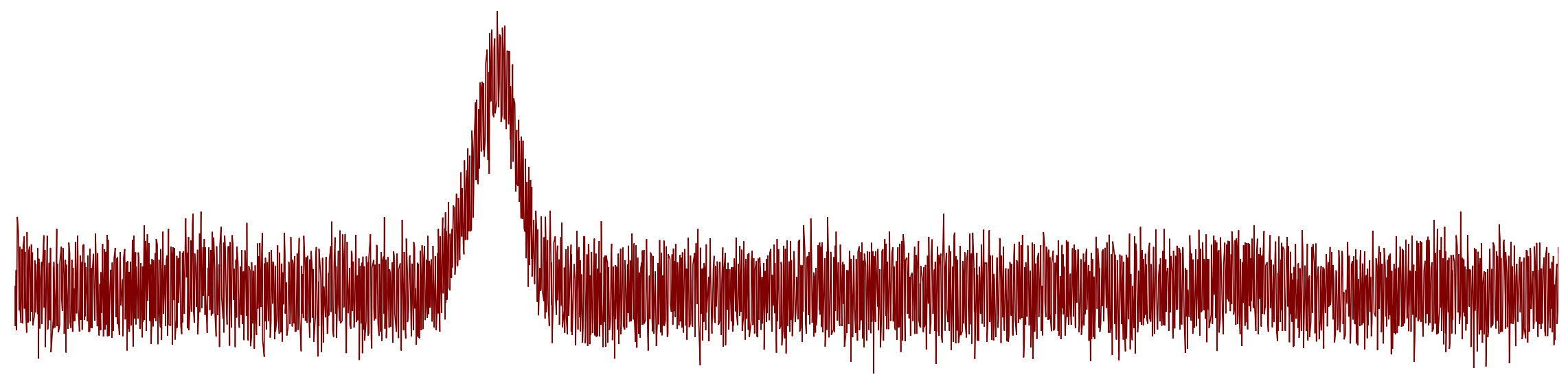

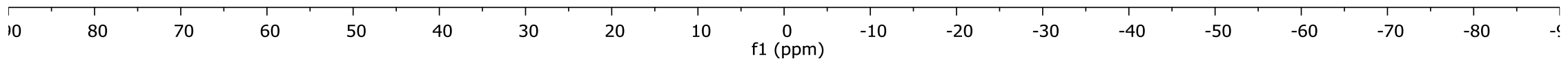




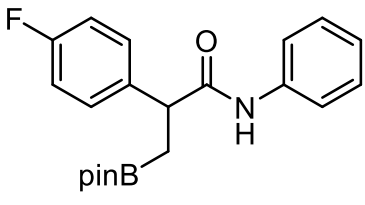

$3 \mathrm{ca}$

${ }^{1} \mathrm{H}$ NMR $\left(400 \mathrm{MHz}, \mathrm{CDCl}_{3}\right)$

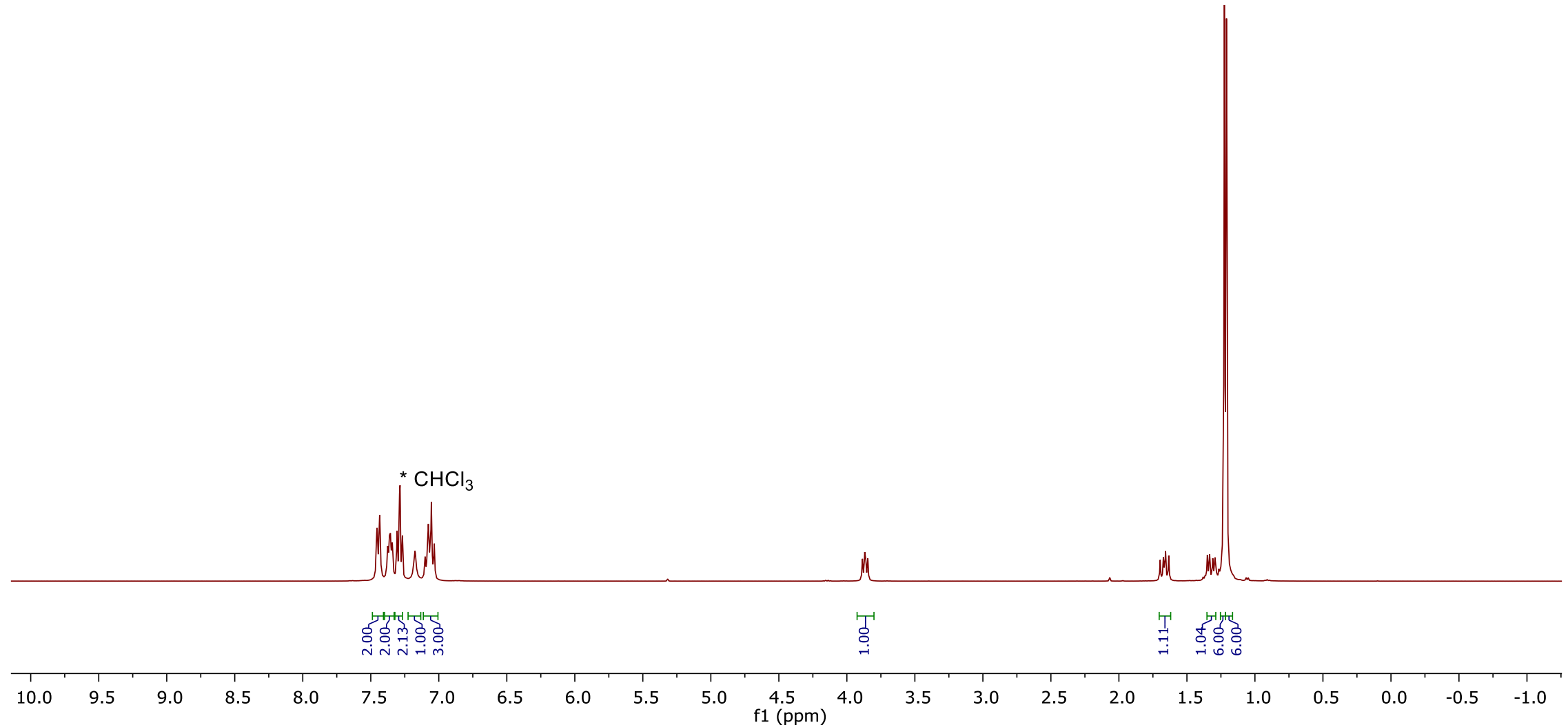




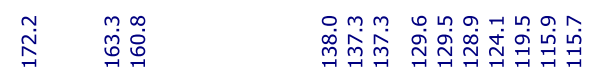

1 । रा अं।

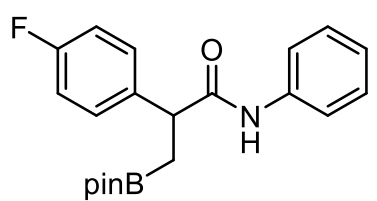

3 ca

${ }^{13} \mathrm{C}\left\{{ }^{1} \mathrm{H}\right\}$ NMR $\left(101 \mathrm{MHz}, \mathrm{CDCl}_{3}\right)$
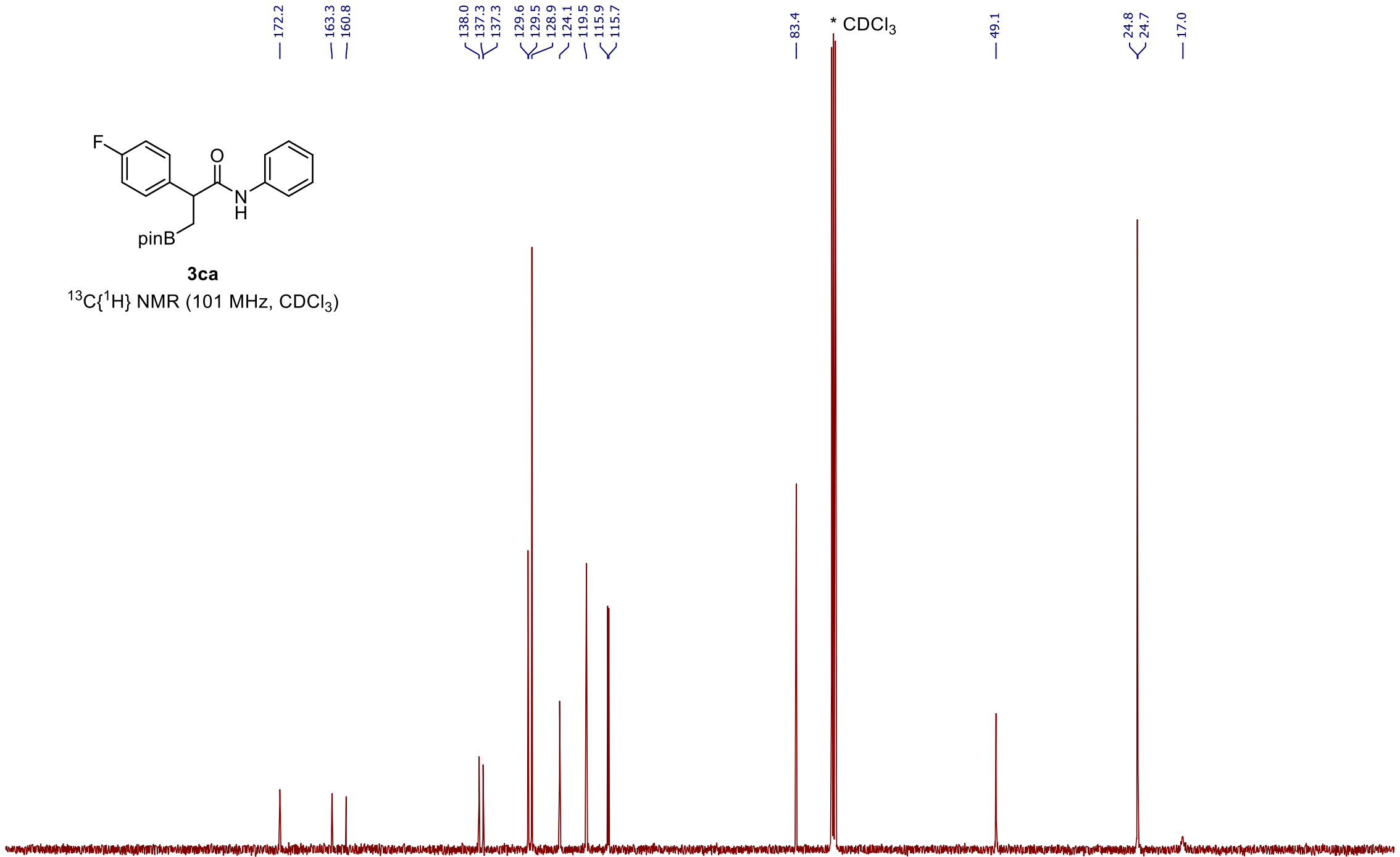

$\begin{array}{llllllllllllllllllllllll}210 & 200 & 190 & 180 & 170 & 160 & 150 & 140 & 130 & 120 & 110 & 100 & 90 & 80 & 70 & 60 & 50 & 40 & 30 & 20 & 10 & 0 & -10 & \end{array}$ f1 (ppm) 


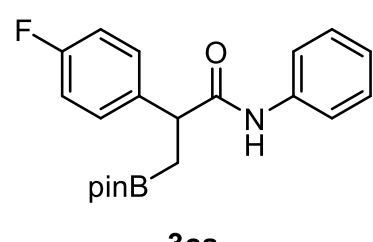

${ }^{11} \mathrm{~B}$ NMR (128 MHz, $\mathrm{CDCl}_{3}$ )

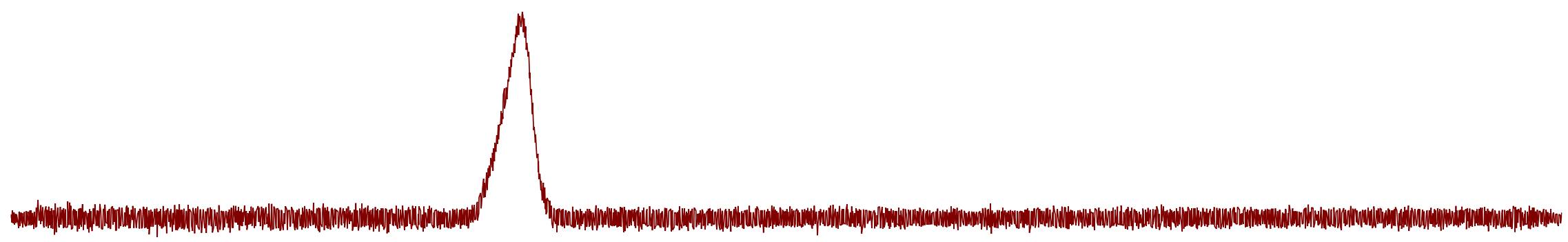




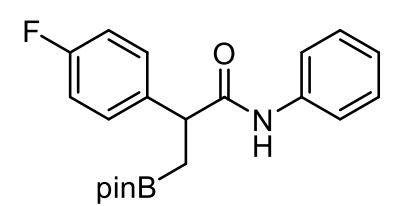

3ca

${ }^{19} \mathrm{~F}\left\{{ }^{1} \mathrm{H}\right\}$ NMR $\left(282 \mathrm{MHz}, \mathrm{CDCl}_{3}\right)$

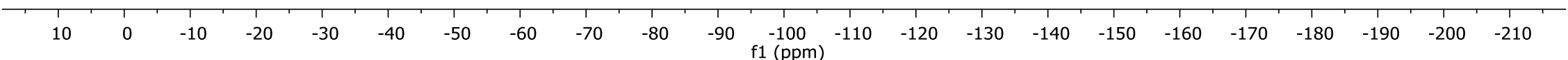




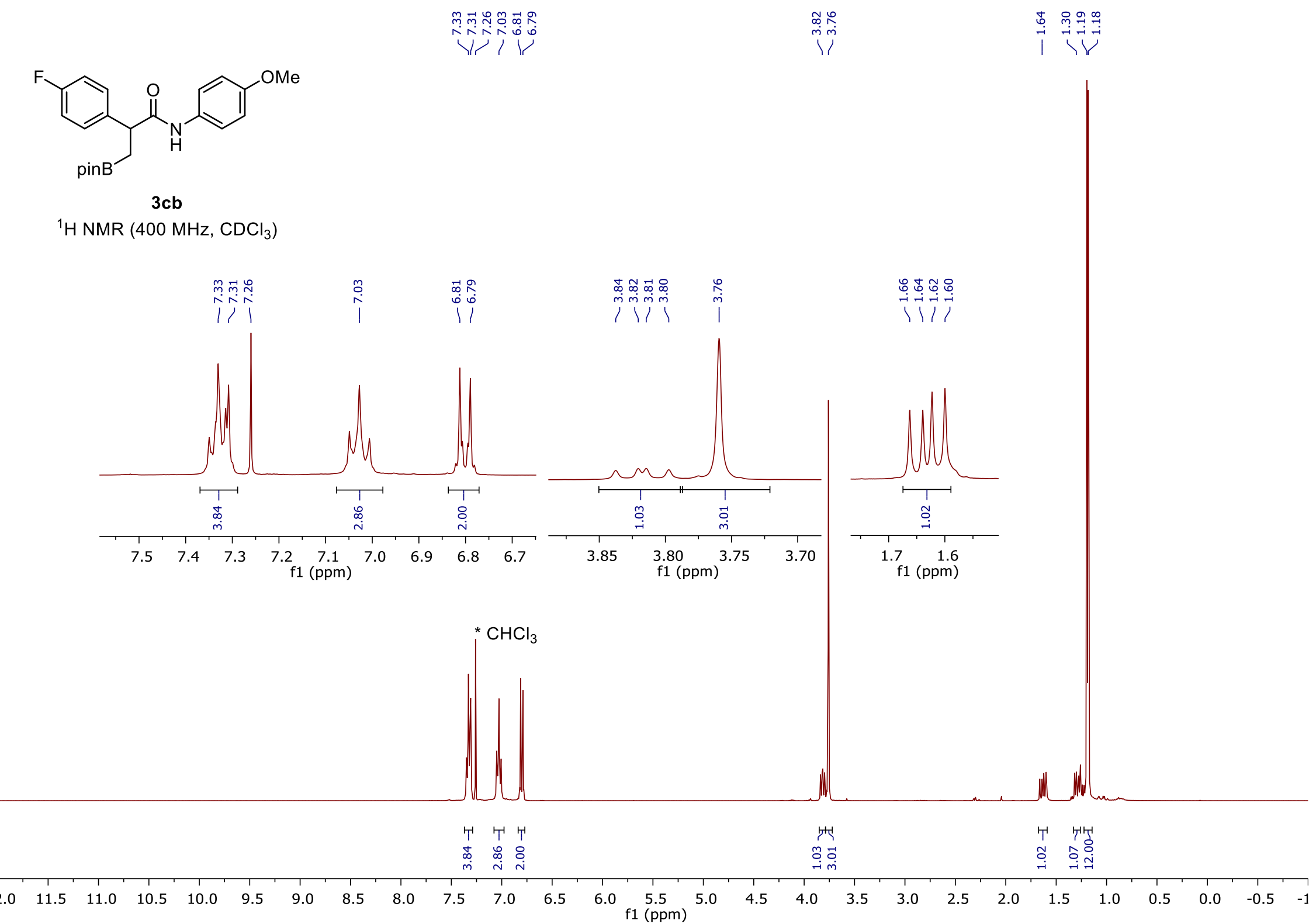



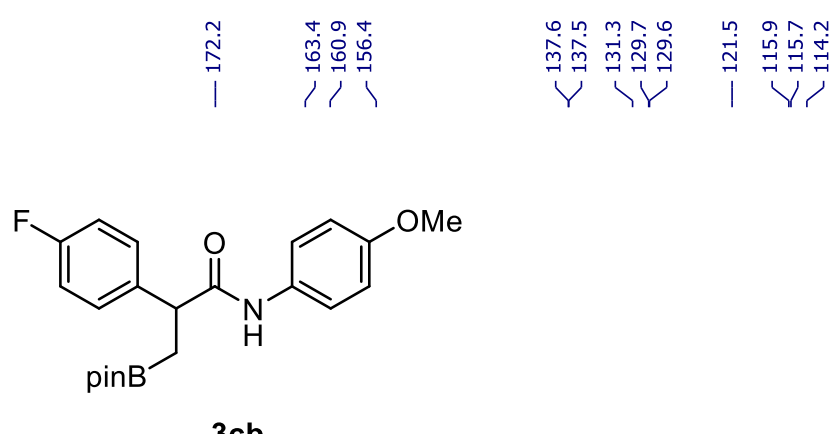

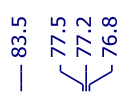

耑

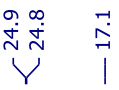

${ }^{13} \mathrm{C}\left\{{ }^{1} \mathrm{H}\right\}$ NMR $\left(101 \mathrm{MHz}, \mathrm{CDCl}_{3}\right)$

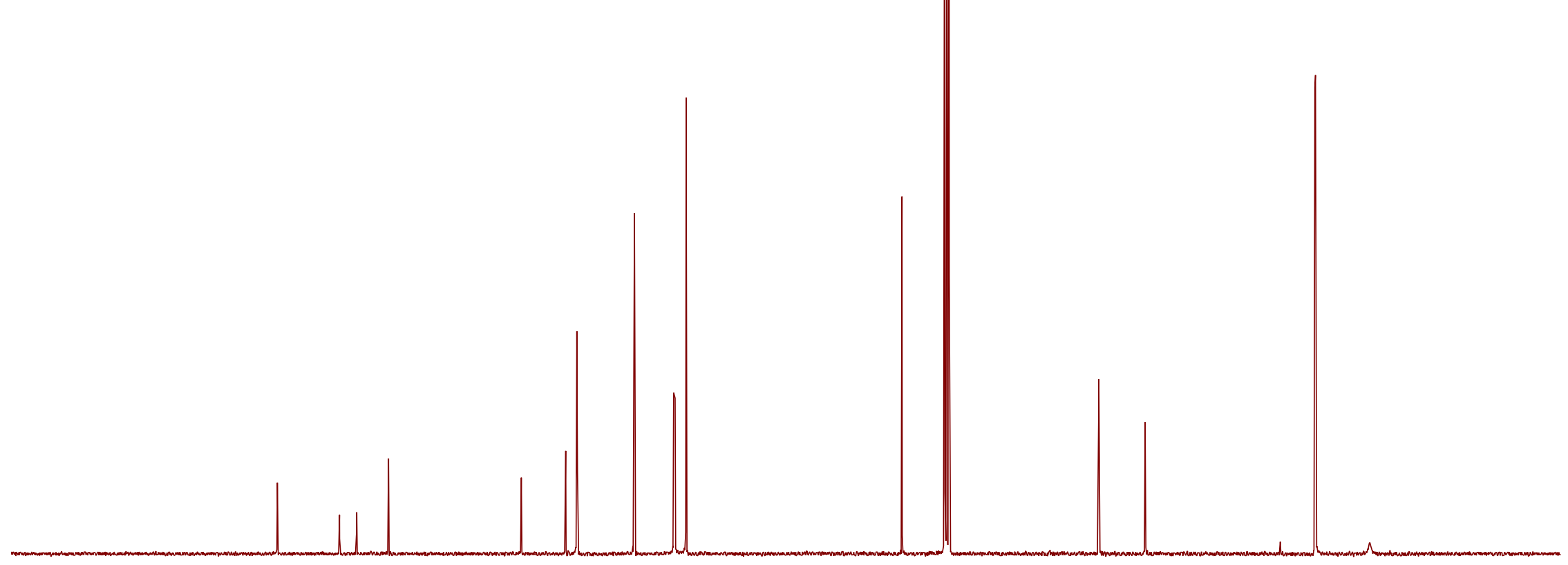
$10 \quad 200$ $200 \quad 190 \quad 180 \quad 180$ 160 $150 \quad 140$ $130 \quad 120$ $110 \quad 100$ f1 $(\mathrm{ppm})$ 


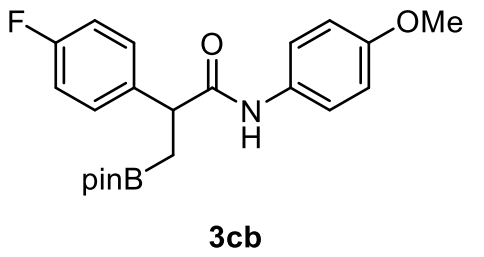

${ }^{11} \mathrm{~B}$ NMR $\left(128 \mathrm{MHz}, \mathrm{CDCl}_{3}\right.$ )
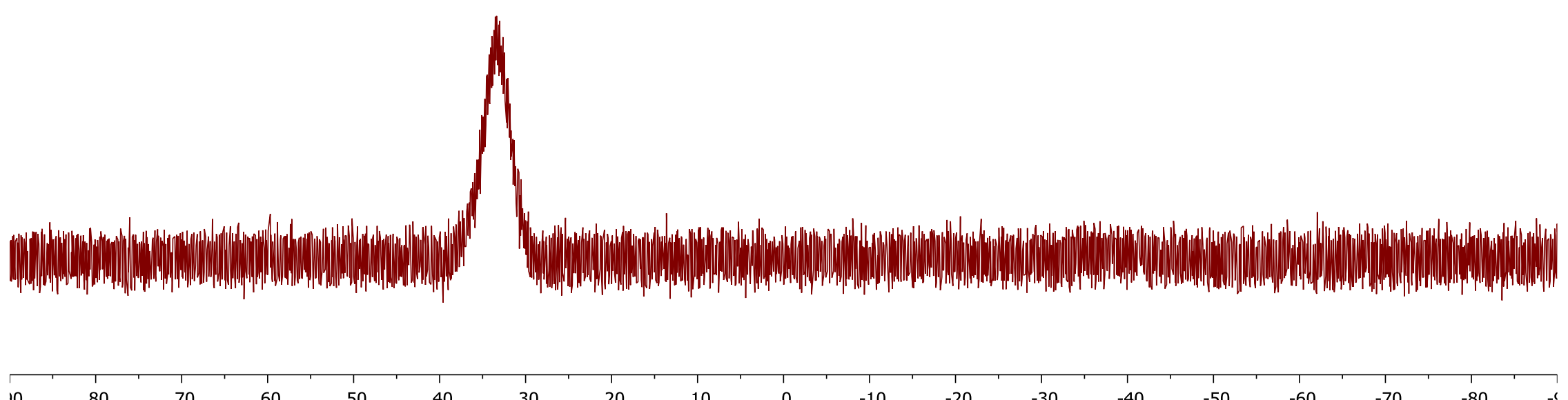

30

20 


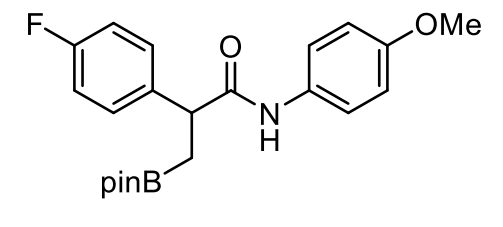

$$
3 \mathrm{cb}
$$

${ }^{19} \mathrm{~F}\left[{ }^{1} \mathrm{H}\right] \mathrm{NMR}\left(282 \mathrm{MHz}, \mathrm{CDCl}_{3}\right)$

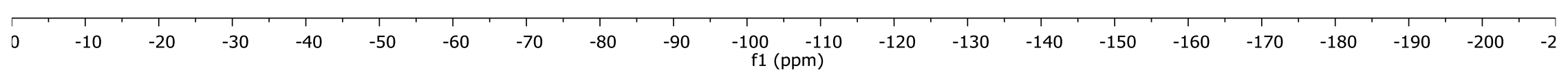


Supporting Information

S190<smiles>COc1ccc(NC(=O)C(C[Pb])c2cccc(Br)c2)cc1</smiles>

ن.

yo
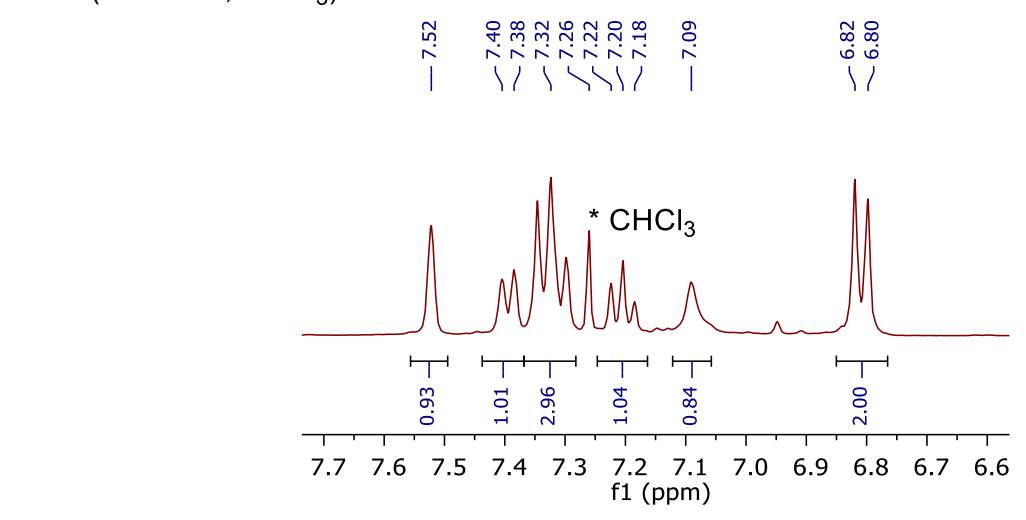

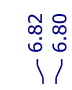

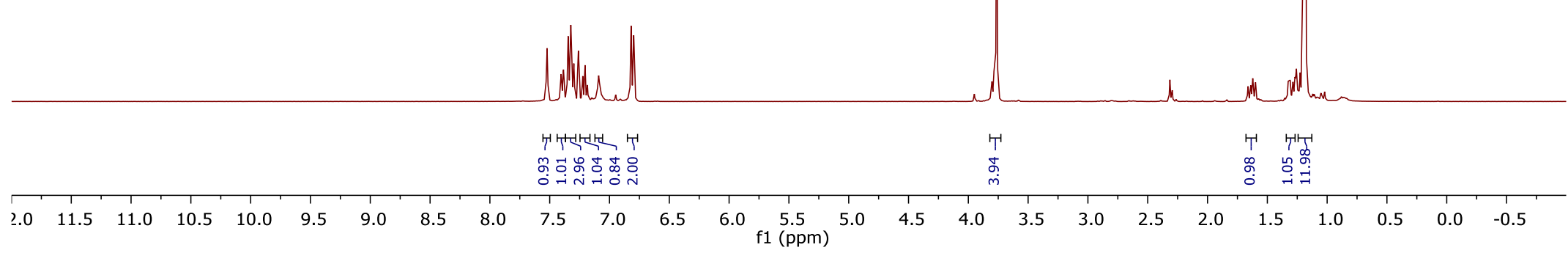




\section{|}

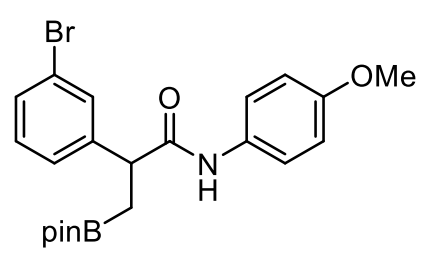

$3 \mathrm{db}$

${ }^{13} \mathrm{C}\left\{{ }^{1} \mathrm{H}\right\}$ NMR $\left(101 \mathrm{MHz}, \mathrm{CDCl}_{3}\right)$

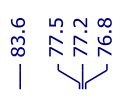

耑

${ }^{*} \mathrm{CDCl}_{3}$
\ั่
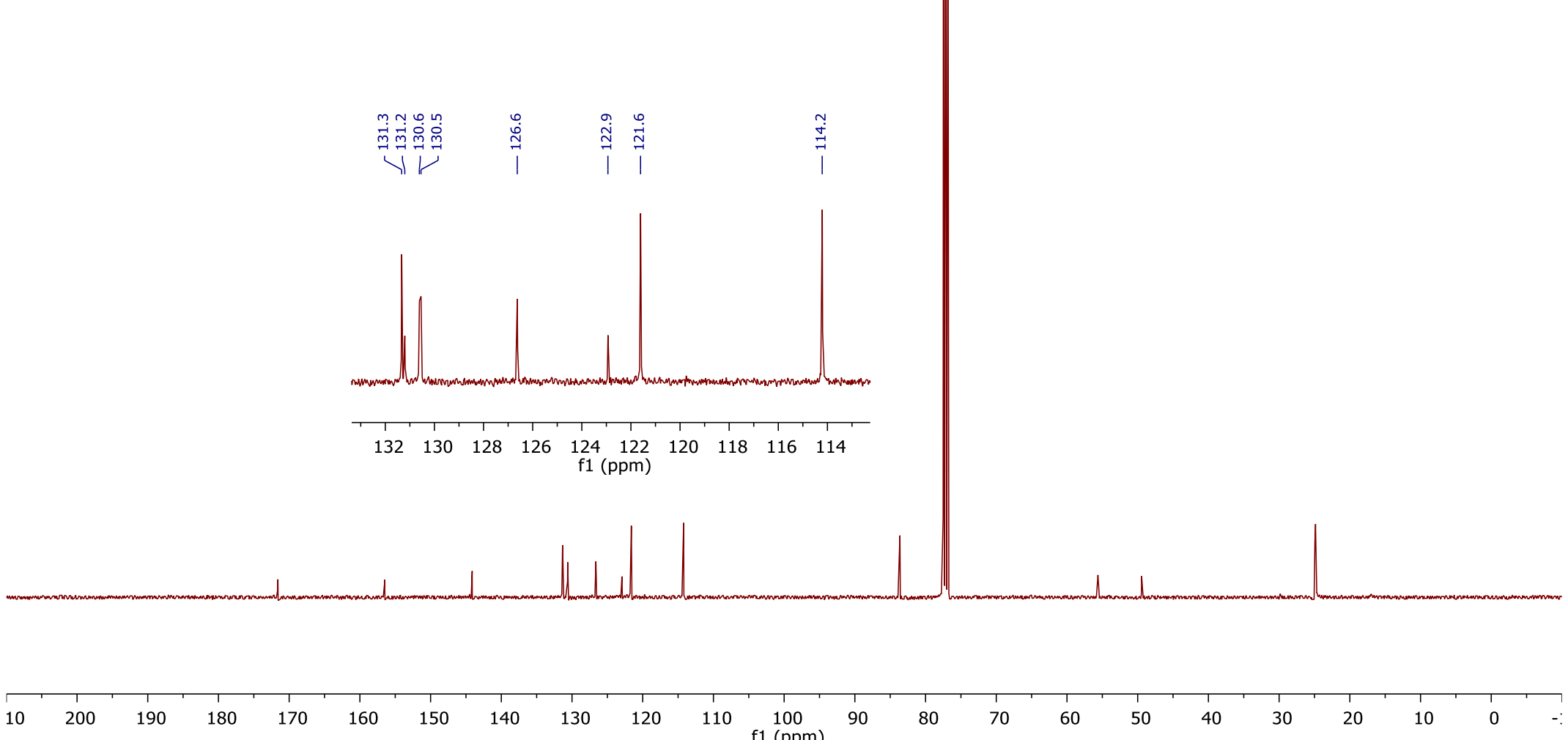

100
$\mathrm{f} 1(\mathrm{ppm})$

80

70

60

50 


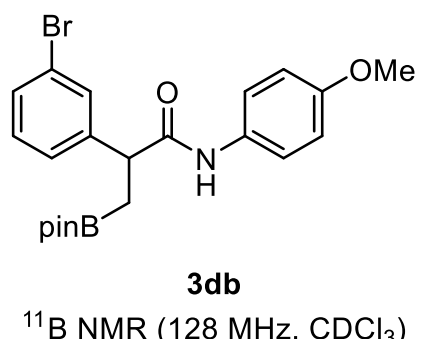

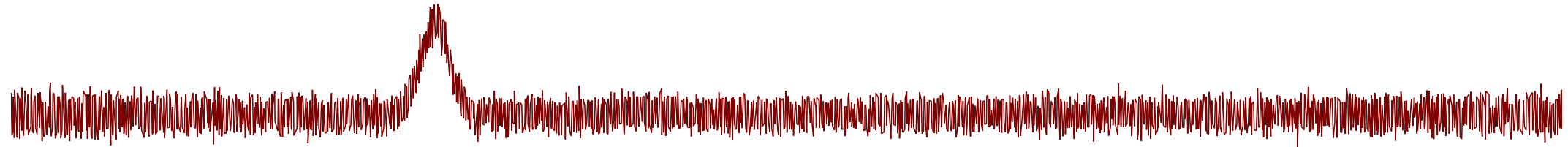

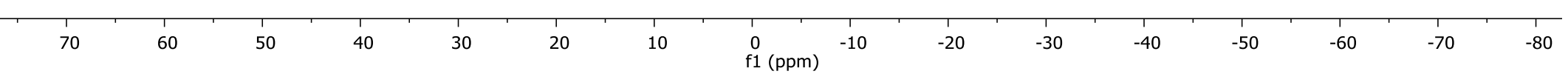




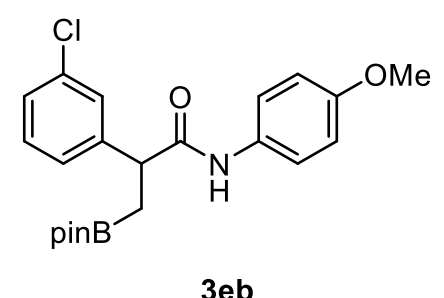

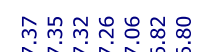

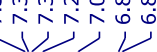

$\ln _{\substack{\infty \\ m}}^{\stackrel{p}{m}}$

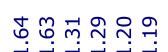

\।

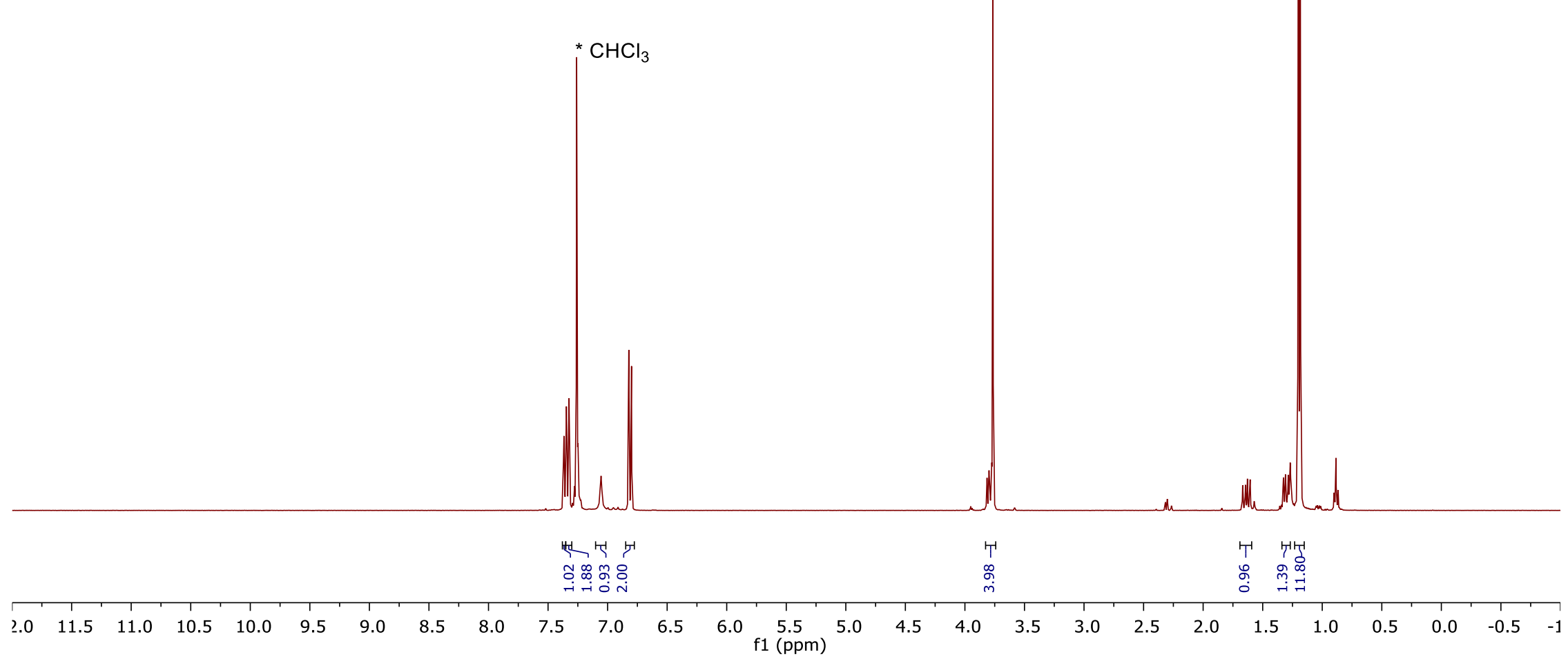




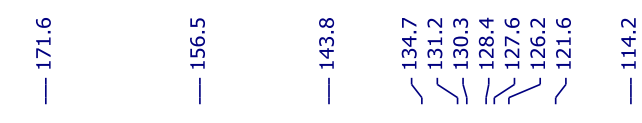

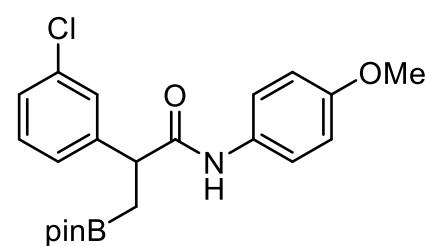

3eb

${ }^{13} \mathrm{C}\left\{{ }^{1} \mathrm{H}\right\}$ NMR $\left(101 \mathrm{MHz}, \mathrm{CDCl}_{3}\right)$

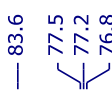

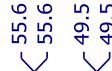

$\mathrm{CDCl}_{3}$

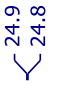

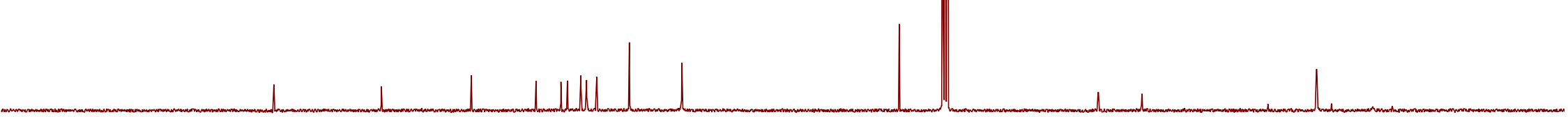

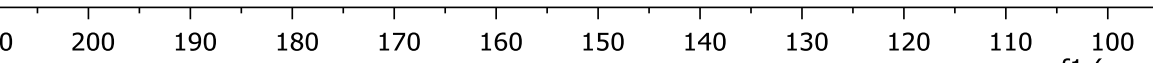
f1 $\stackrel{100}{(\mathrm{ppm})}$

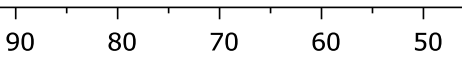




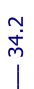

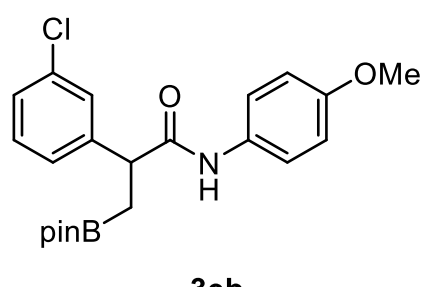

${ }^{11} \mathrm{~B}$ NMR (128 MHz, $\mathrm{CDCl}_{3}$ )

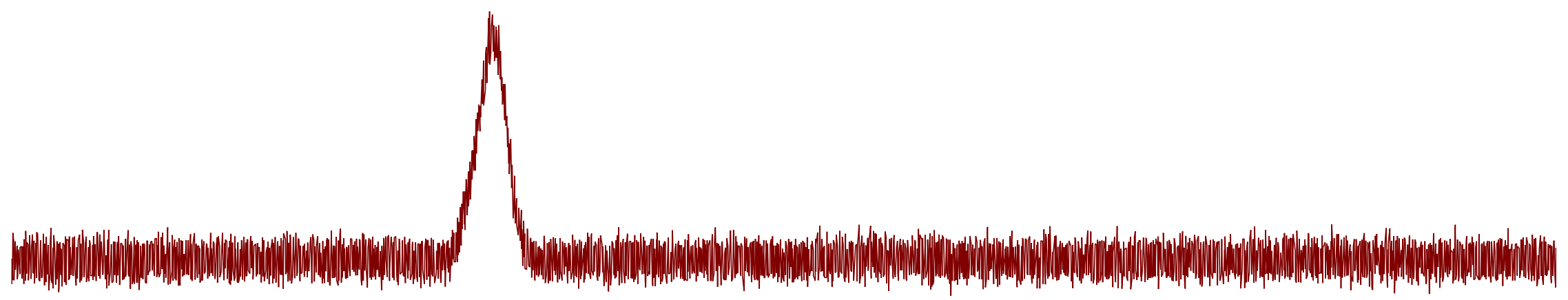




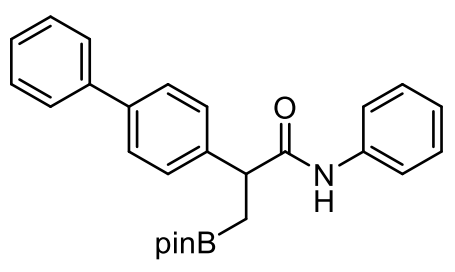

슬ำ

पili

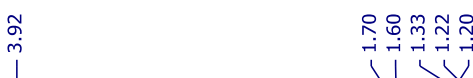

$3 \mathrm{fa}$

${ }^{1} \mathrm{H}$ NMR $\left(500 \mathrm{MHz}, \mathrm{CDCl}_{3}\right)$

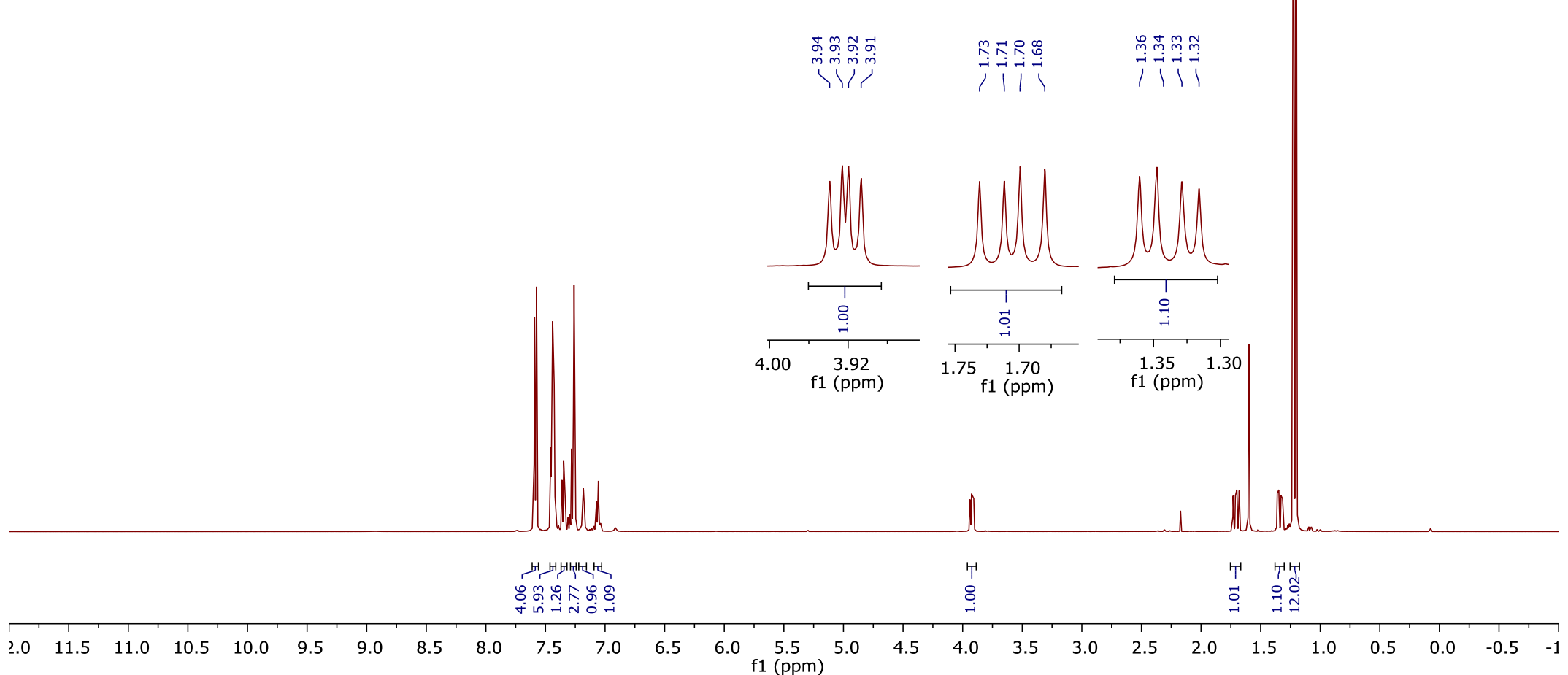




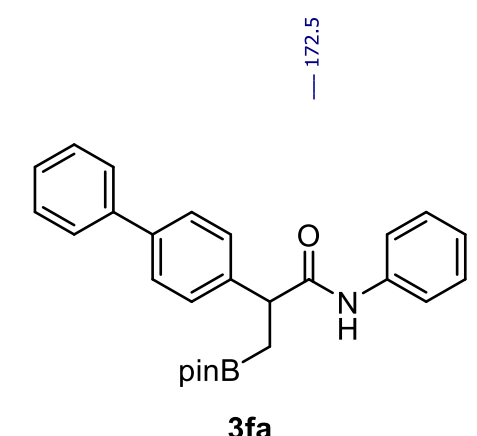

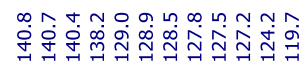

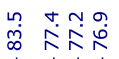

क्ष

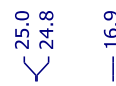

${ }^{13} \mathrm{C}\left\{{ }^{1} \mathrm{H}\right\}$ NMR $\left(126 \mathrm{MHz}, \mathrm{CDCl}_{3}\right)$

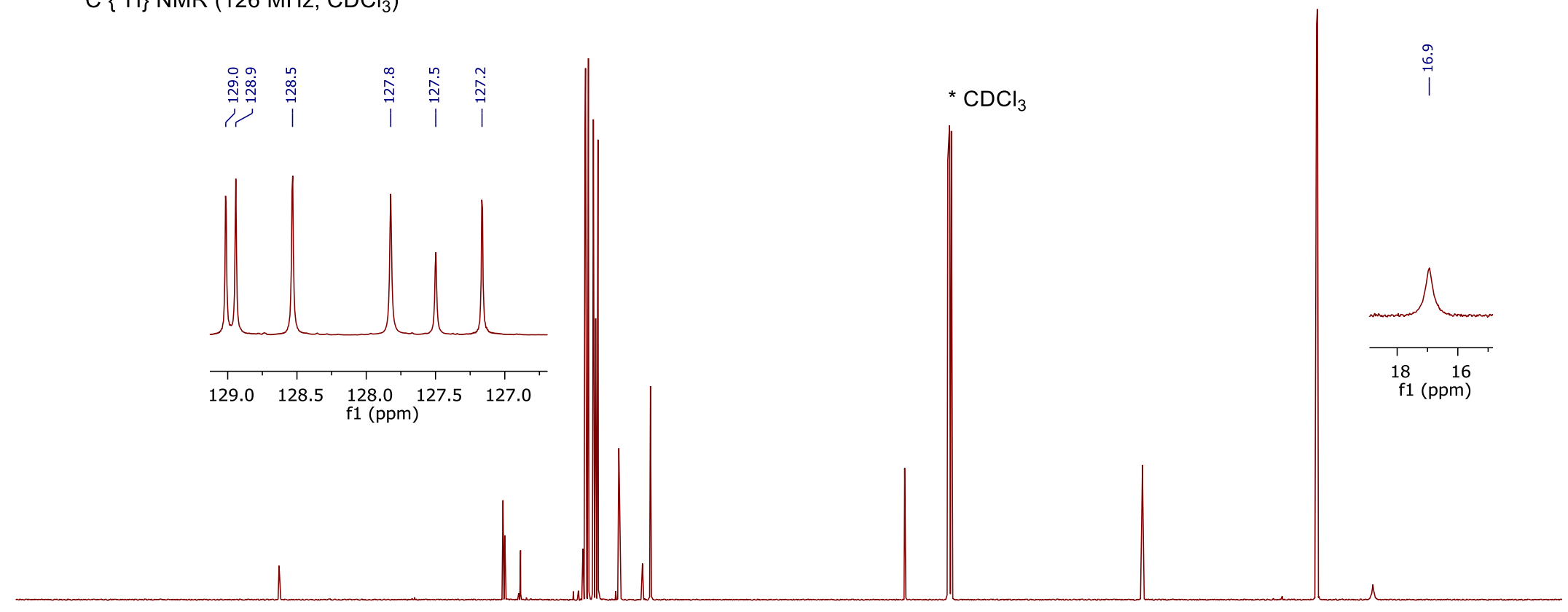

$10 \quad 200 \quad 190$

$\begin{array}{lll}170 & 160\end{array}$

$150 \quad 140$

$130 \quad 120$

$110 \quad 100$

80

$\begin{array}{lll}70 & 60 & 50\end{array}$

$40 \quad 30$

20 


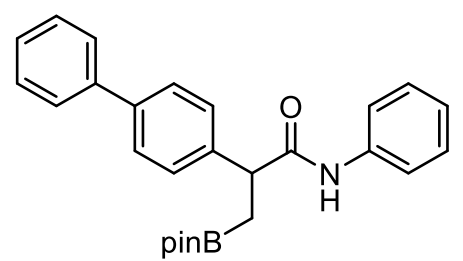

$3 \mathrm{fa}$

${ }^{11} \mathrm{~B}$ NMR $\left(128 \mathrm{MHz}, \mathrm{CDCl}_{3}\right)$

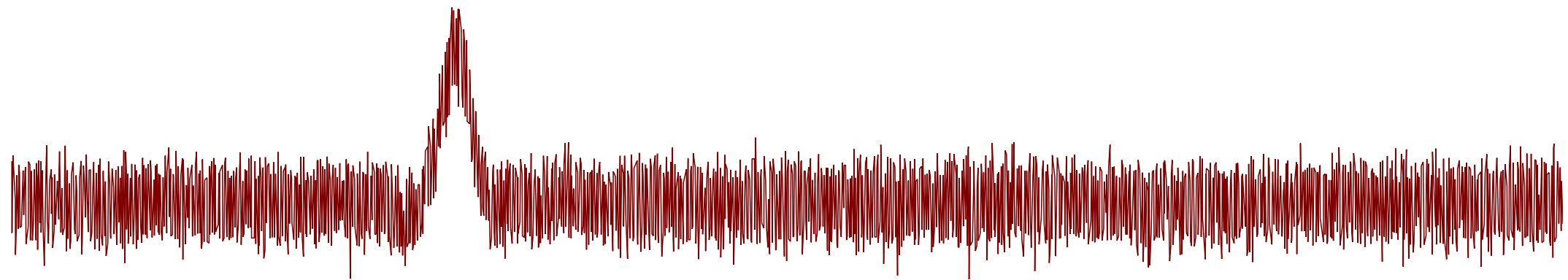

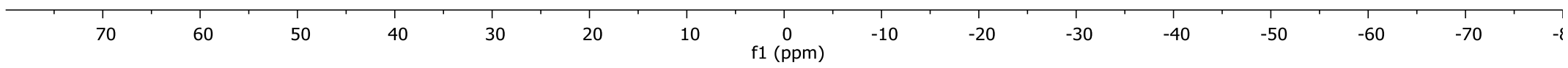




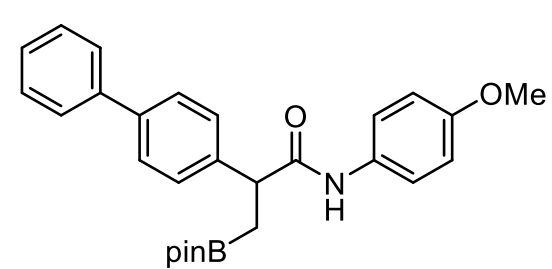

3fb

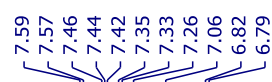

(

${ }^{1} \mathrm{H}$ NMR $\left(400 \mathrm{MHz}, \mathrm{CDCl}_{3}\right)$

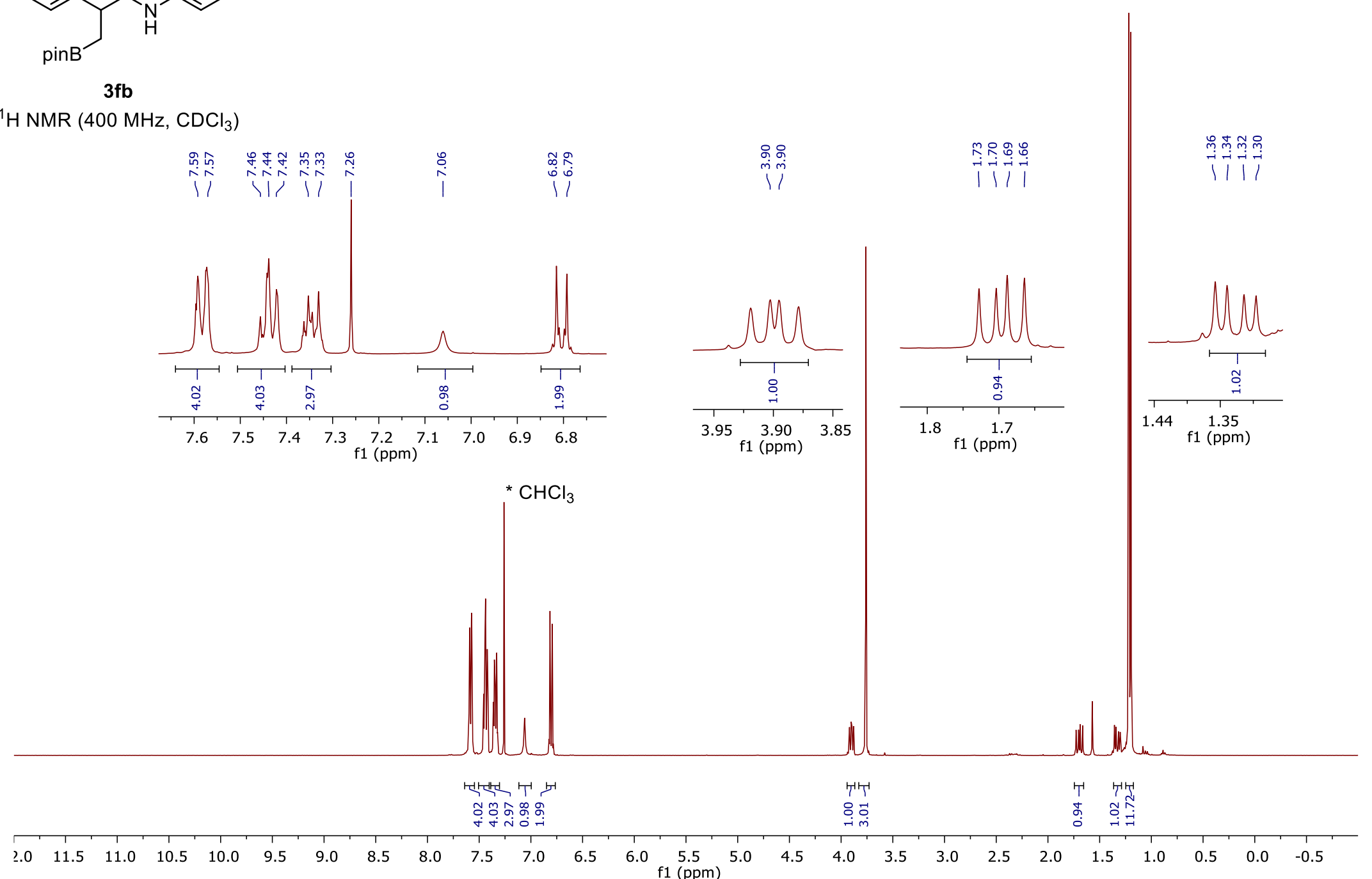

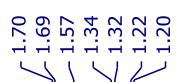

लंग 


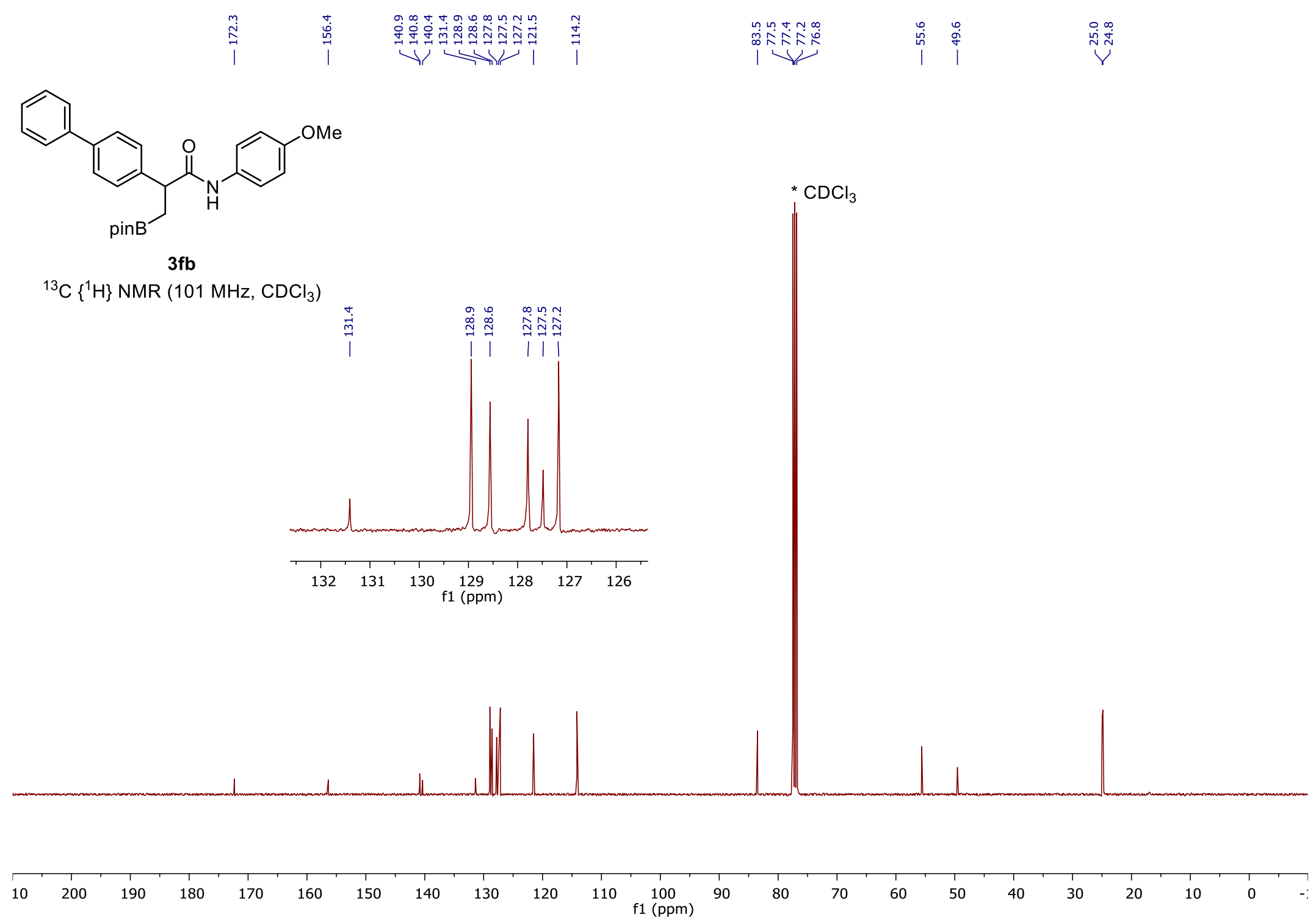




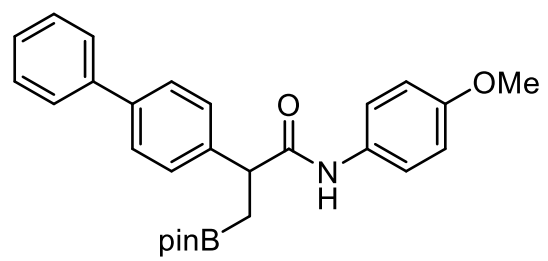

$3 \mathrm{fb}$

${ }^{11} \mathrm{~B}$ NMR (96 MHz, $\mathrm{CDCl}_{3}$ )

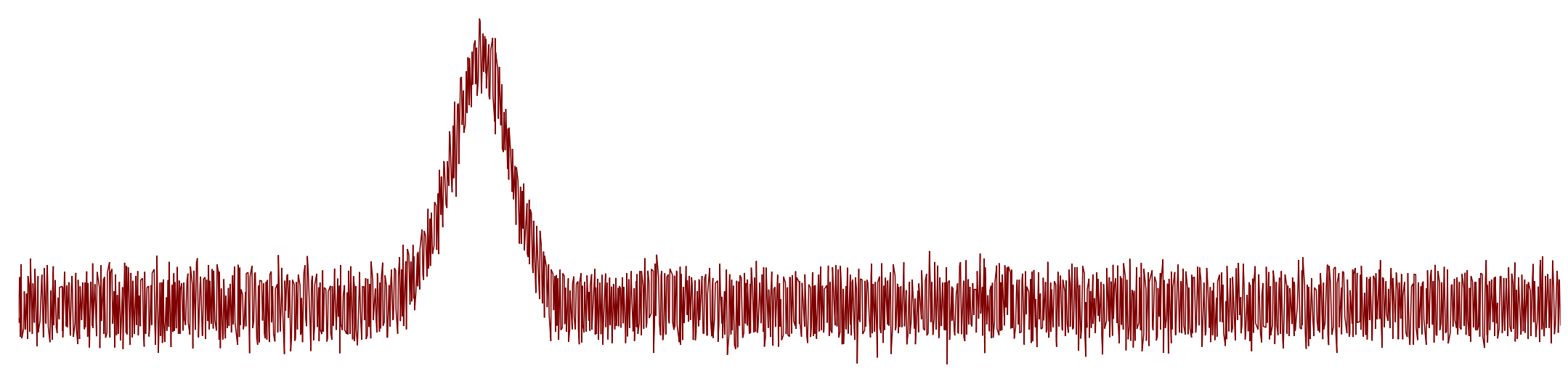

70 60 


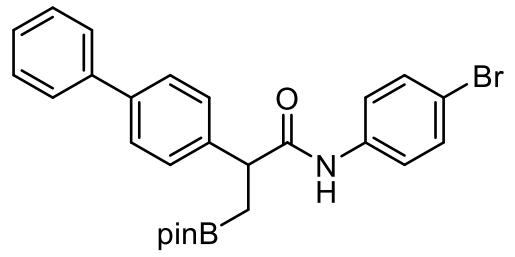

$3 f g$

${ }^{1} \mathrm{H}$ NMR $\left(400 \mathrm{MHz}, \mathrm{CDCl}_{3}\right)$

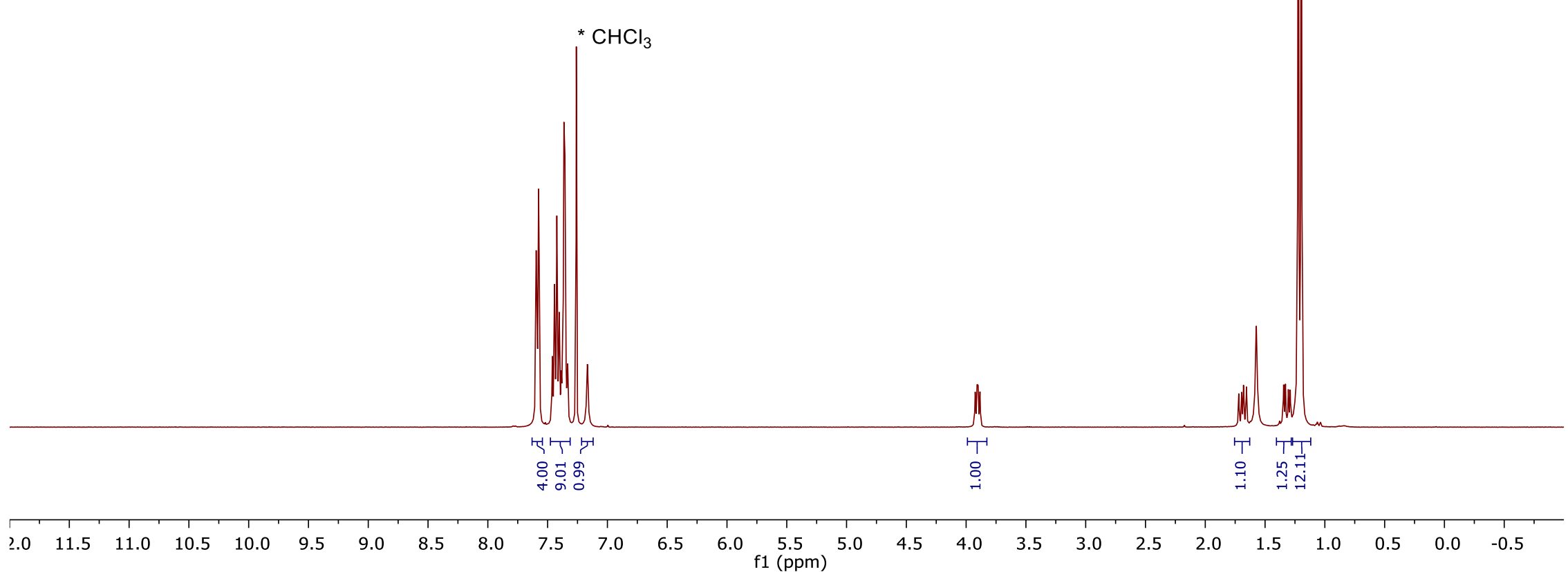




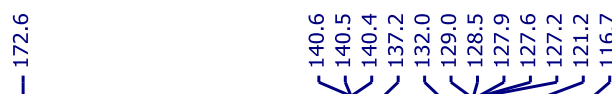

辛

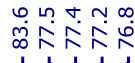

ind

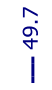

$\stackrel{\substack{\infty \\ \sim}}{\stackrel{\infty}{i}}$

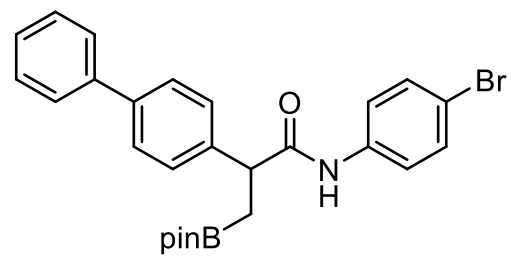

$3 \mathrm{fg}$

${ }^{13} \mathrm{C}\left\{{ }^{1} \mathrm{H}\right\}$ NMR $\left(101 \mathrm{MHz}, \mathrm{CDCl}_{3}\right)$
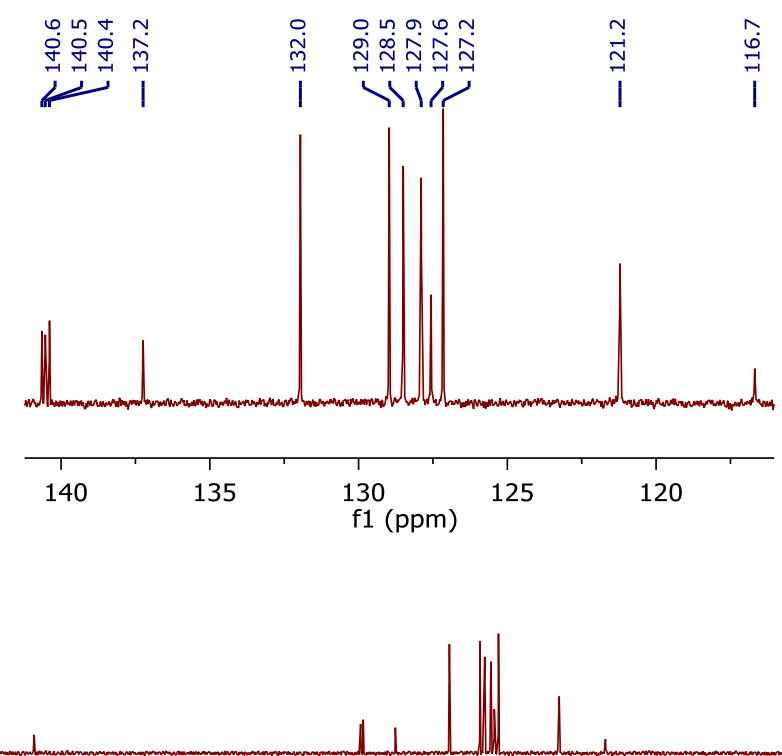

${ }^{*} \mathrm{CDCl}_{3}$ 


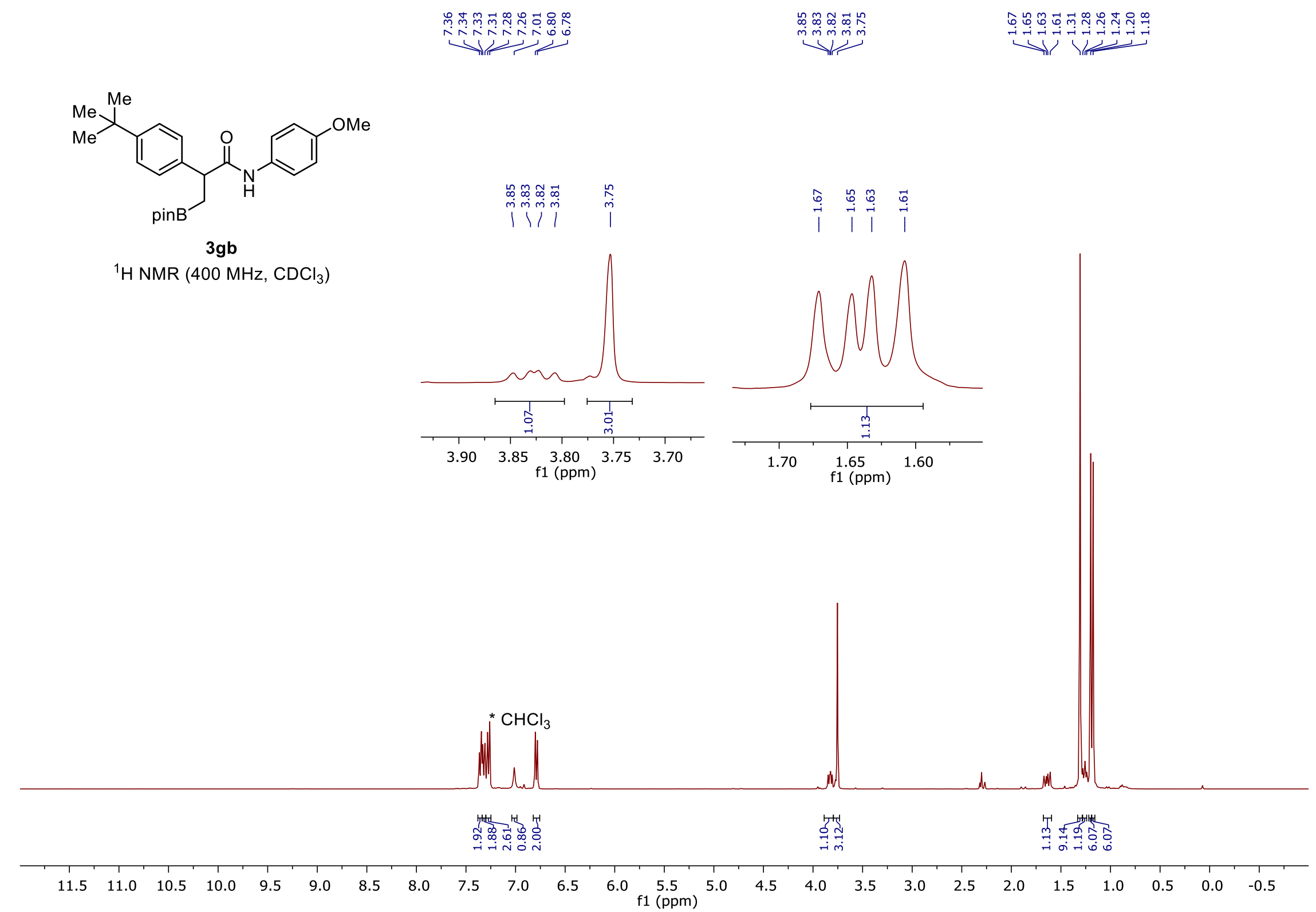


Supporting Information

S205

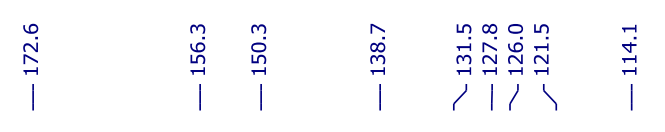
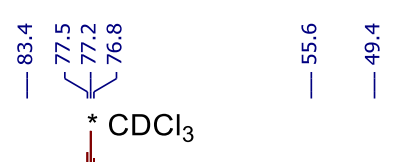

|

$\stackrel{9}{\stackrel{9}{0}}$<smiles>COc1ccc(NC(=O)C(CPc2ccccc2)c2ccc(C(C)(C)C)cc2)cc1</smiles>

3 gb
${ }^{13} \mathrm{C}\left\{{ }^{1} \mathrm{H}\right\}$ NMR $\left(101 \mathrm{MHz}, \mathrm{CDCl}_{3}\right)$

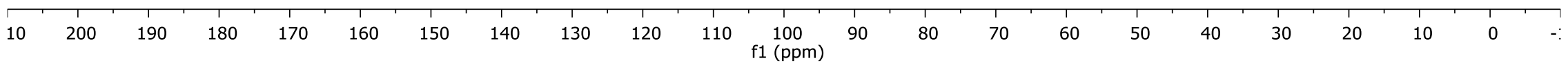




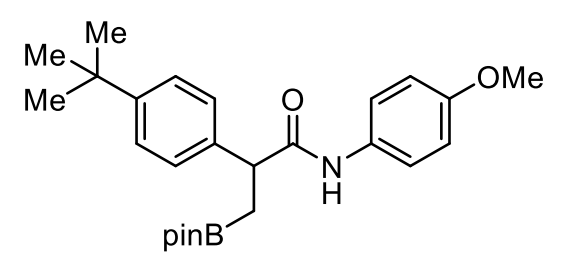

$3 g b$

${ }^{11} \mathrm{~B}$ NMR (96 MHz, $\mathrm{CDCl}_{3}$ )

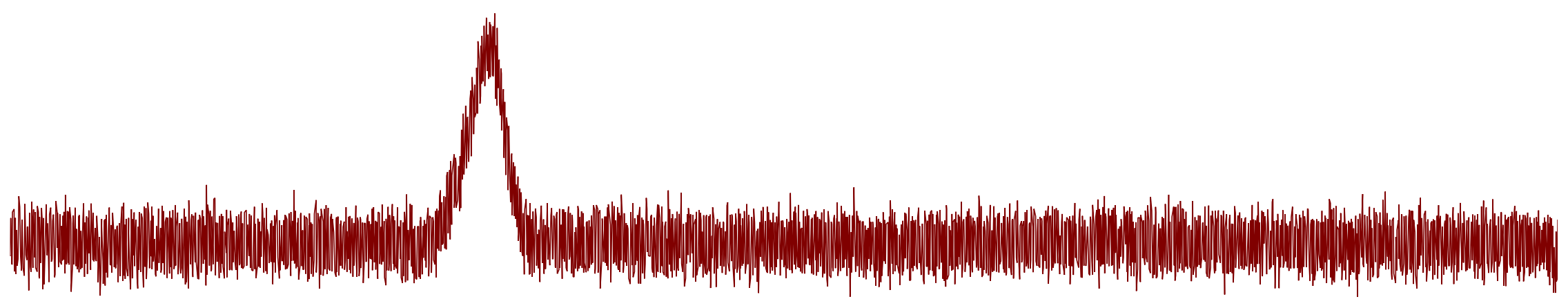




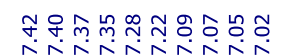

inivinin

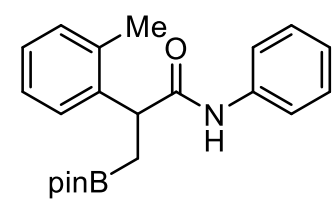

3ha

${ }^{1} \mathrm{H} \mathrm{NMR}\left(400 \mathrm{MHz}, \mathrm{CDCl}_{3}\right)$
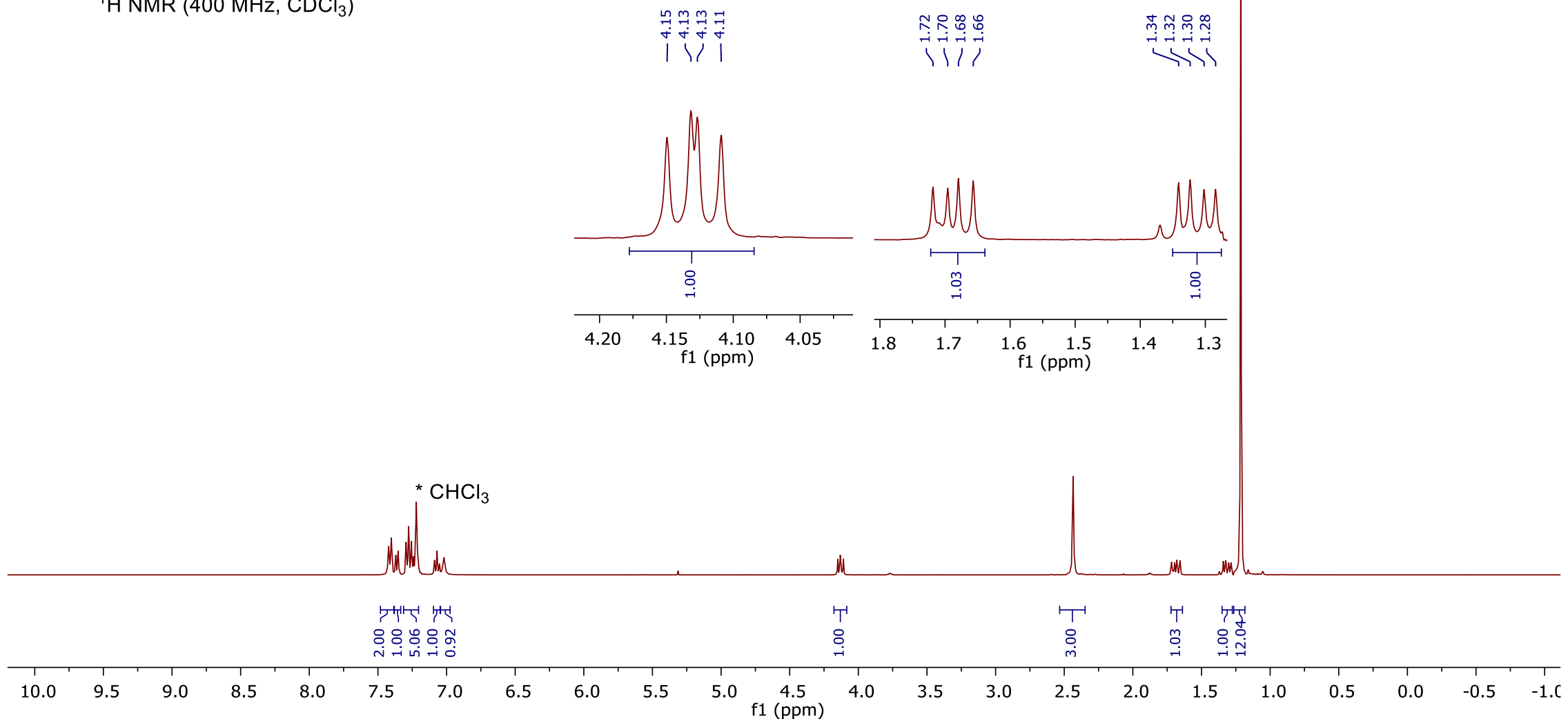


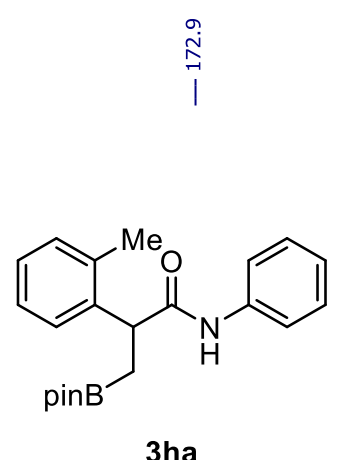

${ }^{13} \mathrm{C}\left\{{ }^{1} \mathrm{H}\right\}$ NMR $\left(101 \mathrm{MHz}, \mathrm{CDCl}_{3}\right)$

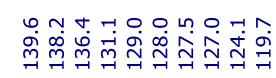

111 रो।

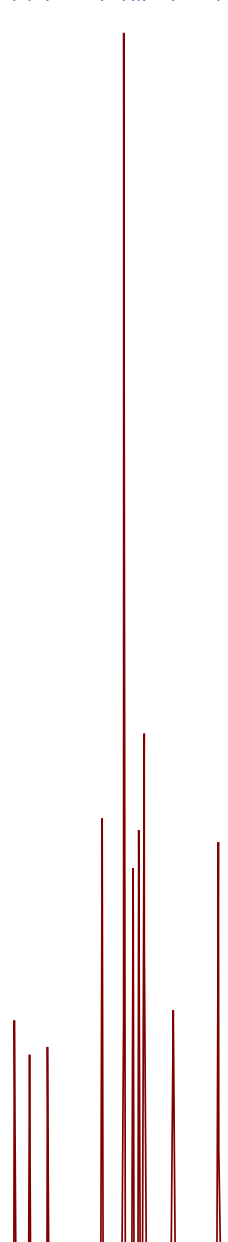

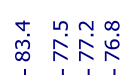

1

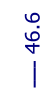

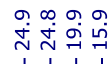

${ }^{*} \mathrm{CDCl}_{3}$

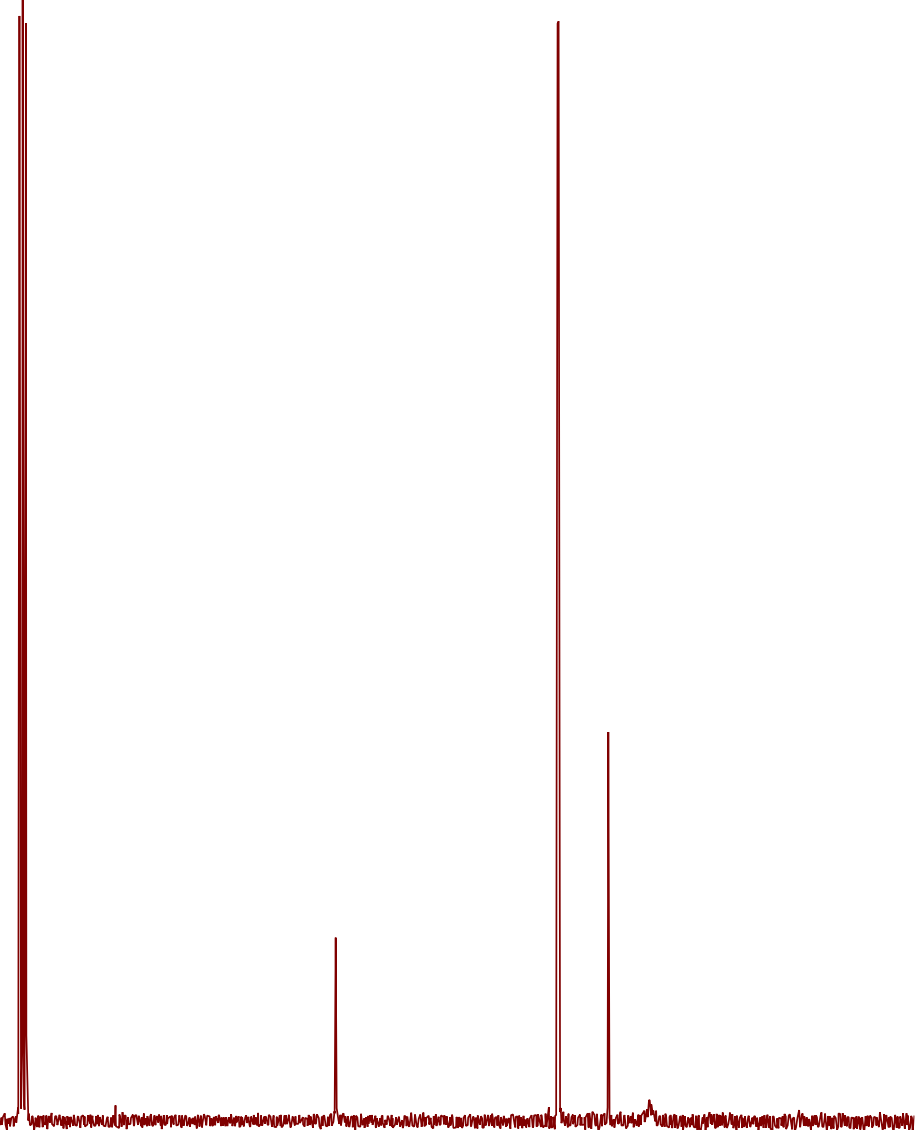


$\stackrel{\circ}{\dot{m}}$

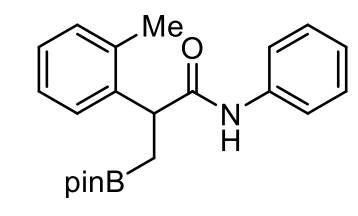

3ha

${ }^{11} \mathrm{~B}$ NMR (128 MHz, $\mathrm{CDCl}_{3}$ )

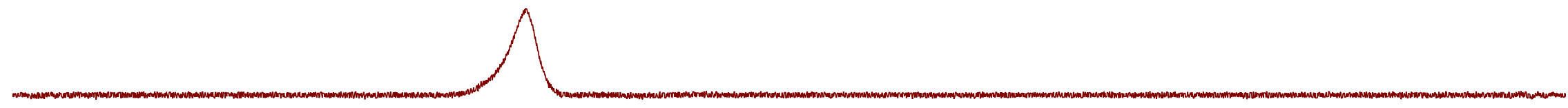

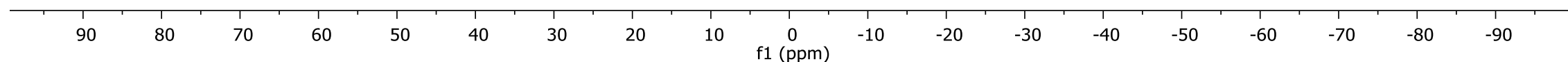




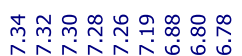

近,

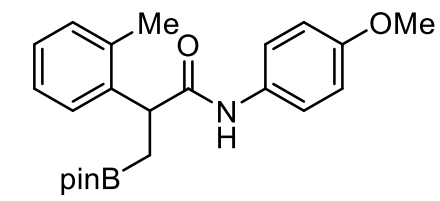

$3 \mathrm{hb}$

${ }^{1} \mathrm{H}$ NMR (400 MHz, $\mathrm{CDCl}_{3}$ )

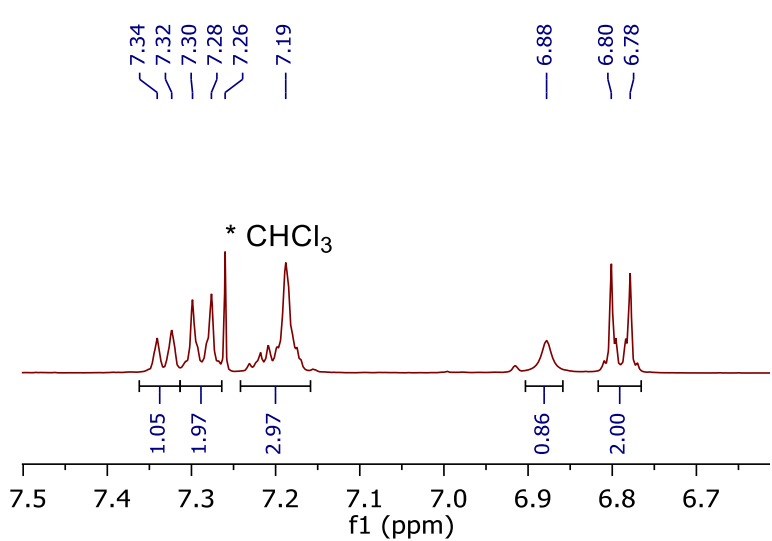

(ppm)

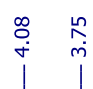

1.

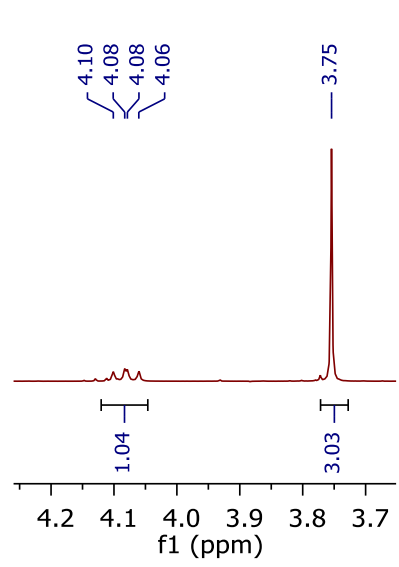

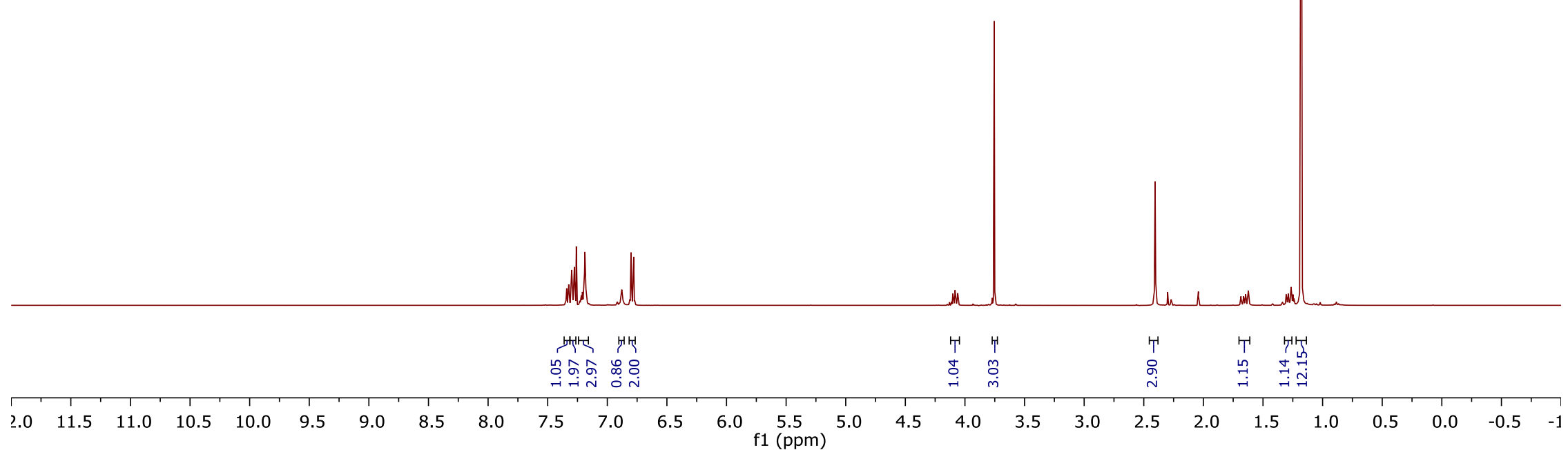




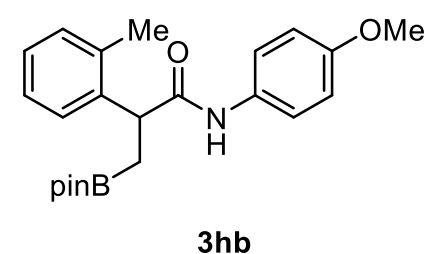

${ }^{13} \mathrm{C}\left\{{ }^{1} \mathrm{H}\right\}$ NMR $\left(101 \mathrm{MHz}, \mathrm{CDCl}_{3}\right)$
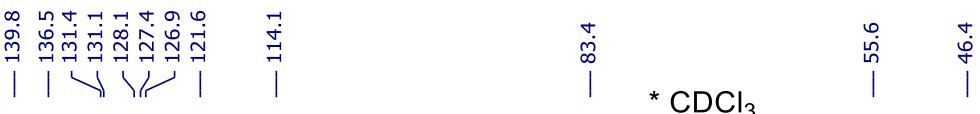

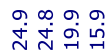

商

सi
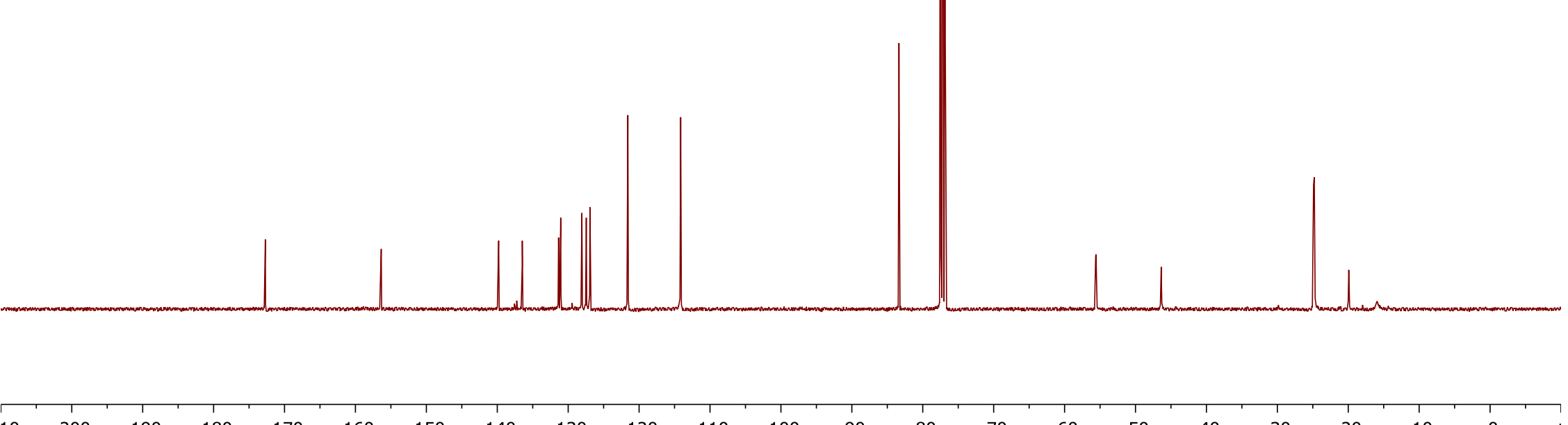

180

160

$150 \quad 140$

$130 \quad 120$

$110 \quad 100$

f1 $\stackrel{100}{(\mathrm{ppm})}$
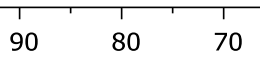

$60 \quad 50$

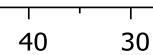


$\stackrel{\text { m }}{\text { I }}$

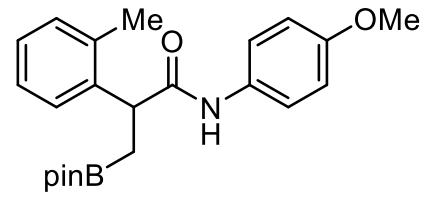

3hb

${ }^{11} \mathrm{~B}$ NMR (96 MHz, CDCl${ }_{3}$ )

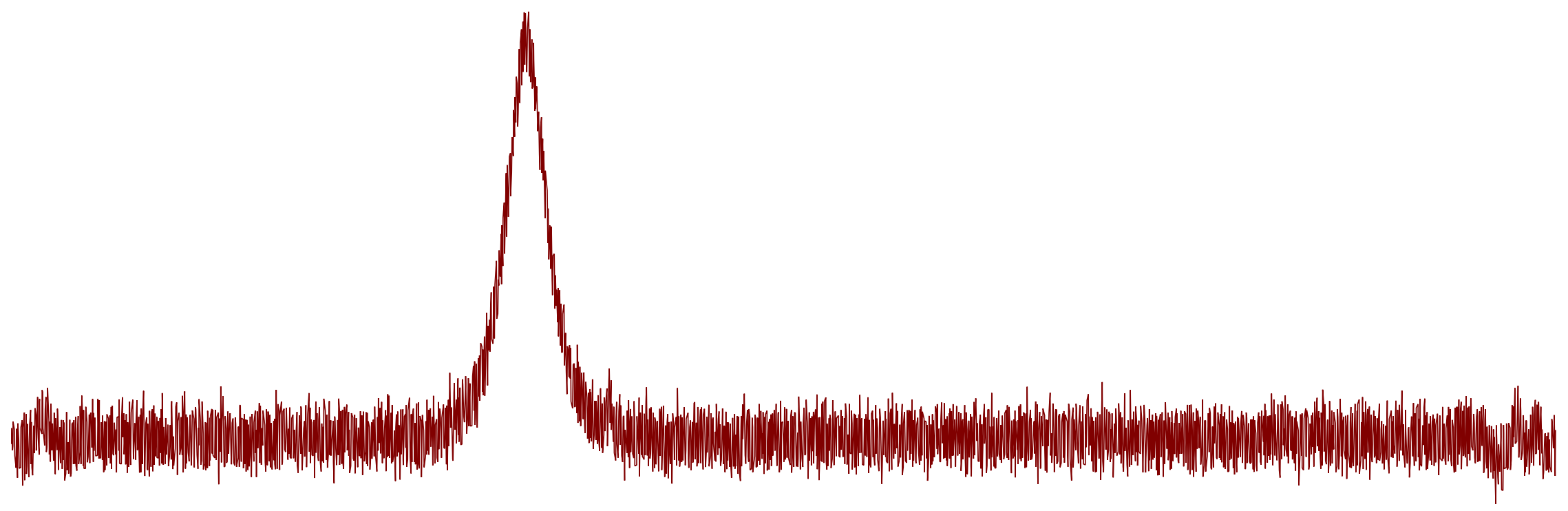




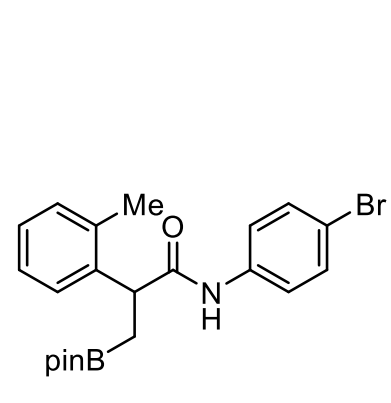

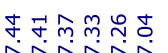

पilf

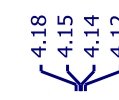

空

$3 \mathrm{hg}$

${ }^{1} \mathrm{H}$ NMR $\left(300 \mathrm{MHz}, \mathrm{CDCl}_{3}\right)$

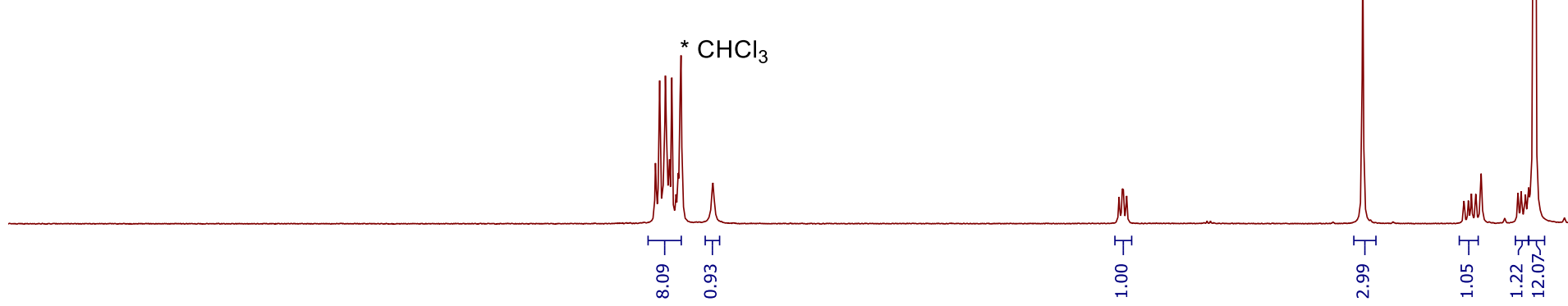

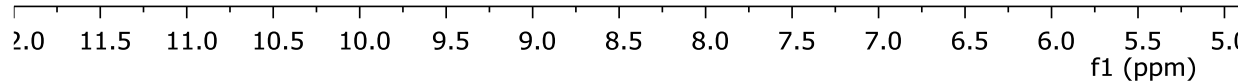

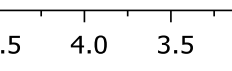

$2.5 \quad 2.0$

$\begin{array}{llllll}1.0 & 0.5 & 0.0 & -0.5 & -1\end{array}$ 

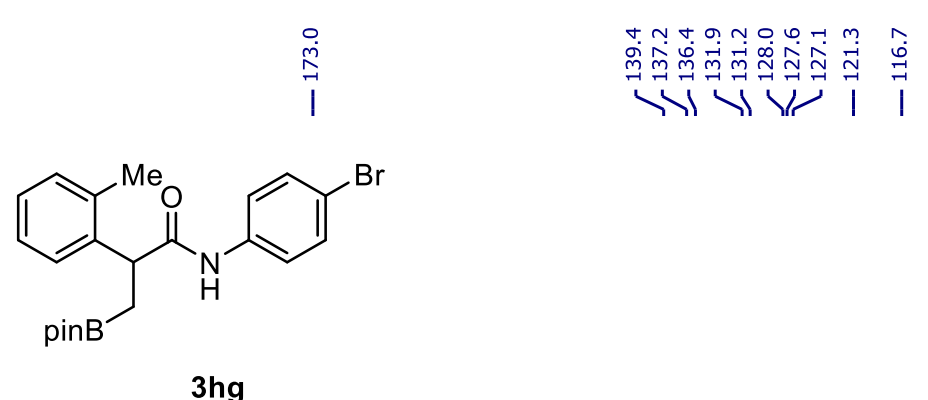

रो परें।

i

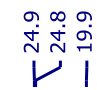

${ }^{13} \mathrm{C}\left\{{ }^{1} \mathrm{H}\right\}$ NMR $\left(75 \mathrm{MHz}, \mathrm{CDCl}_{3}\right)$

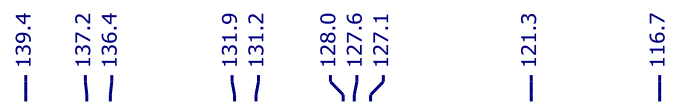
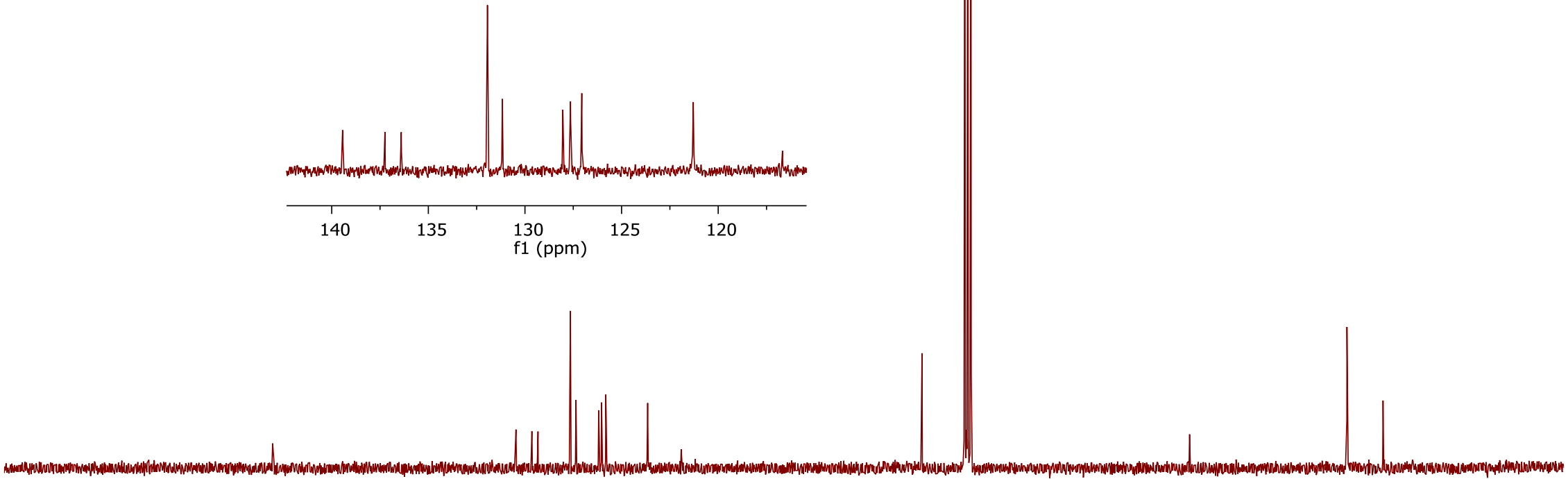

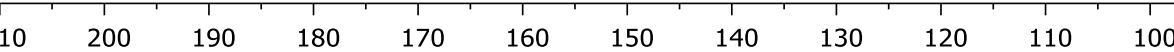
$1(\mathrm{ppm})$

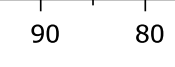

70 


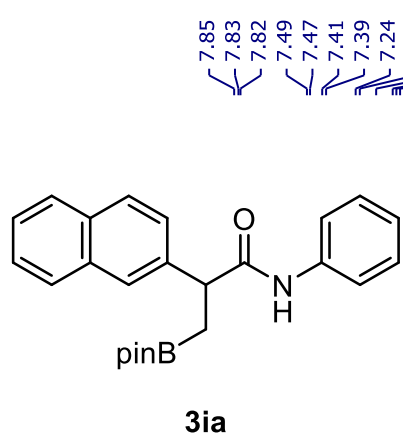

${ }^{1} \mathrm{H} \mathrm{NMR}\left(400 \mathrm{MHz}, \mathrm{CDCl}_{3}\right)$

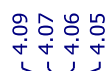 \\ 4}

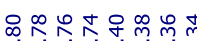

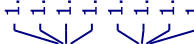

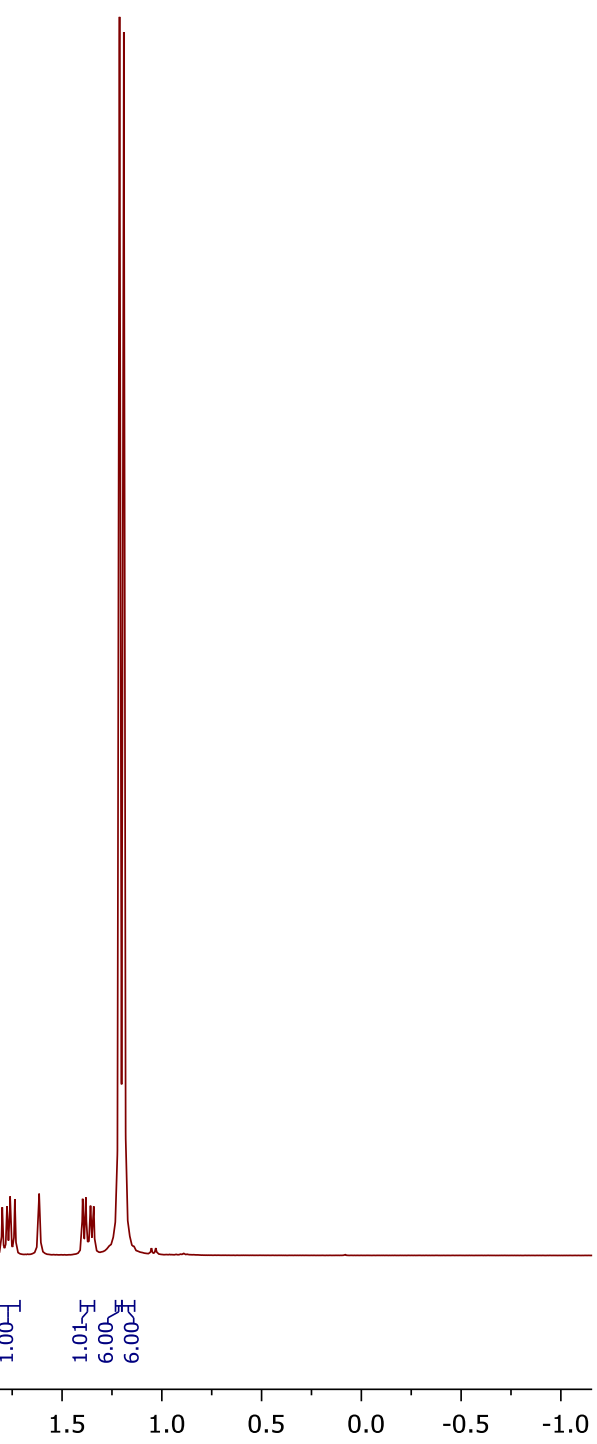




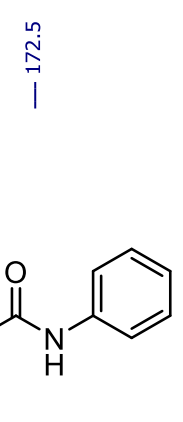

3 ia

${ }^{13} \mathrm{C}\left\{{ }^{1} \mathrm{H}\right\}$ NMR $\left(101 \mathrm{MHz}, \mathrm{CDCl}_{3}\right)$

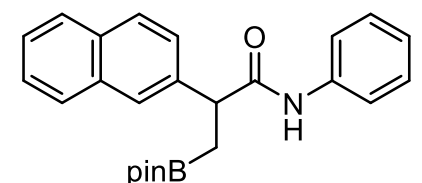

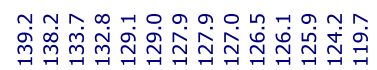

$+1$

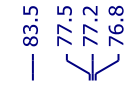

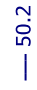

${ }^{*} \mathrm{CDCl}_{3}$

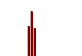

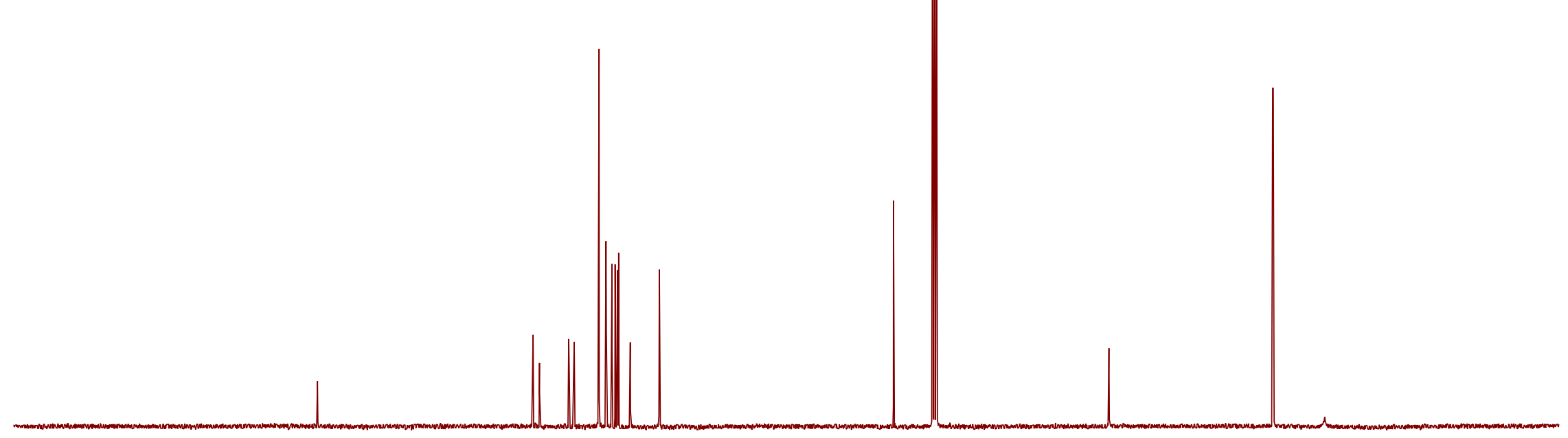

$210 \quad 200 \quad 190 \quad 180$ 


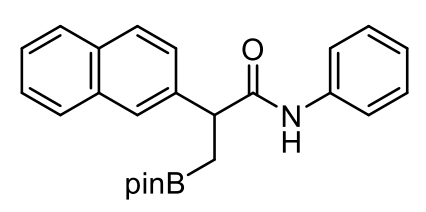

3ia

${ }^{11} \mathrm{~B}$ NMR (128 MHz, $\mathrm{CDCl}_{3}$ )

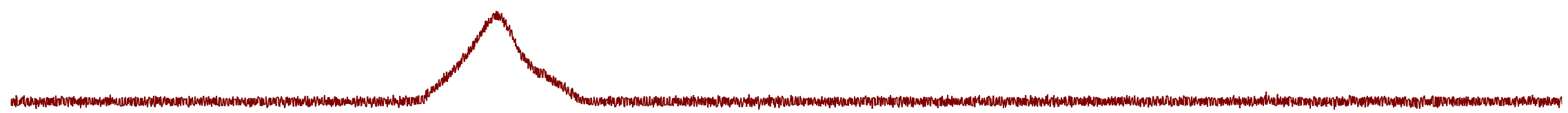




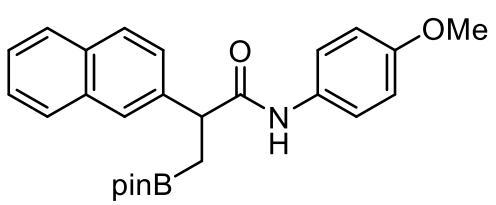

3ib

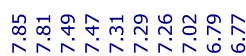

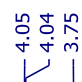

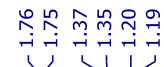

$\mathrm{H}$ NMR (400 MHz, $\mathrm{CDCl}_{3}$ )

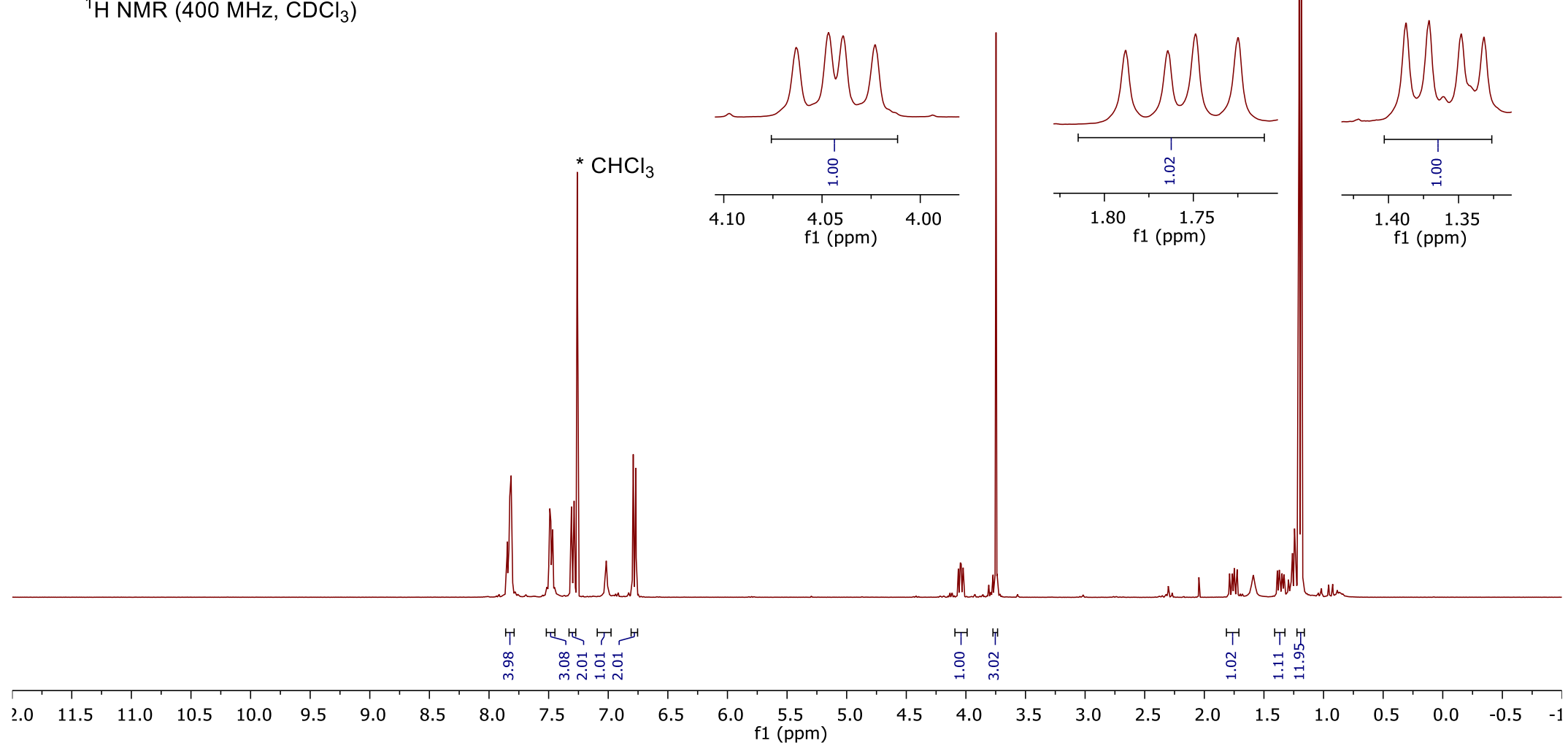



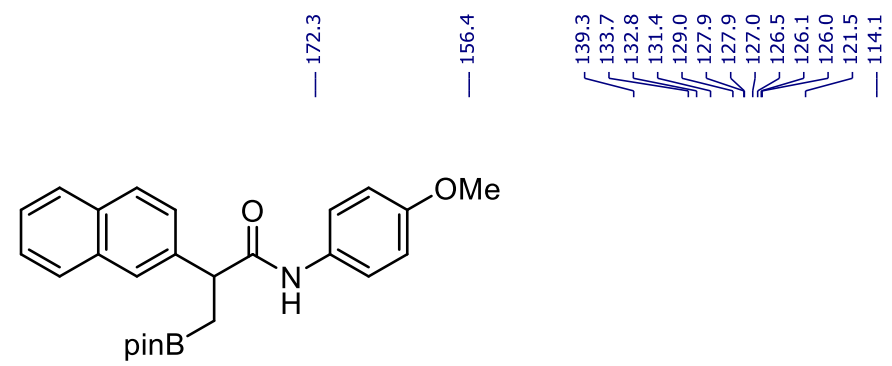

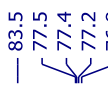

品 品

ஸํํำ

$3 i b$

${ }^{13} \mathrm{C}\left\{{ }^{1} \mathrm{H}\right\}$ NMR $\left(101 \mathrm{MHz}, \mathrm{CDCl}_{3}\right)$

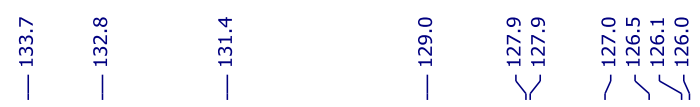

${ }^{*} \mathrm{CDCl}_{3}$
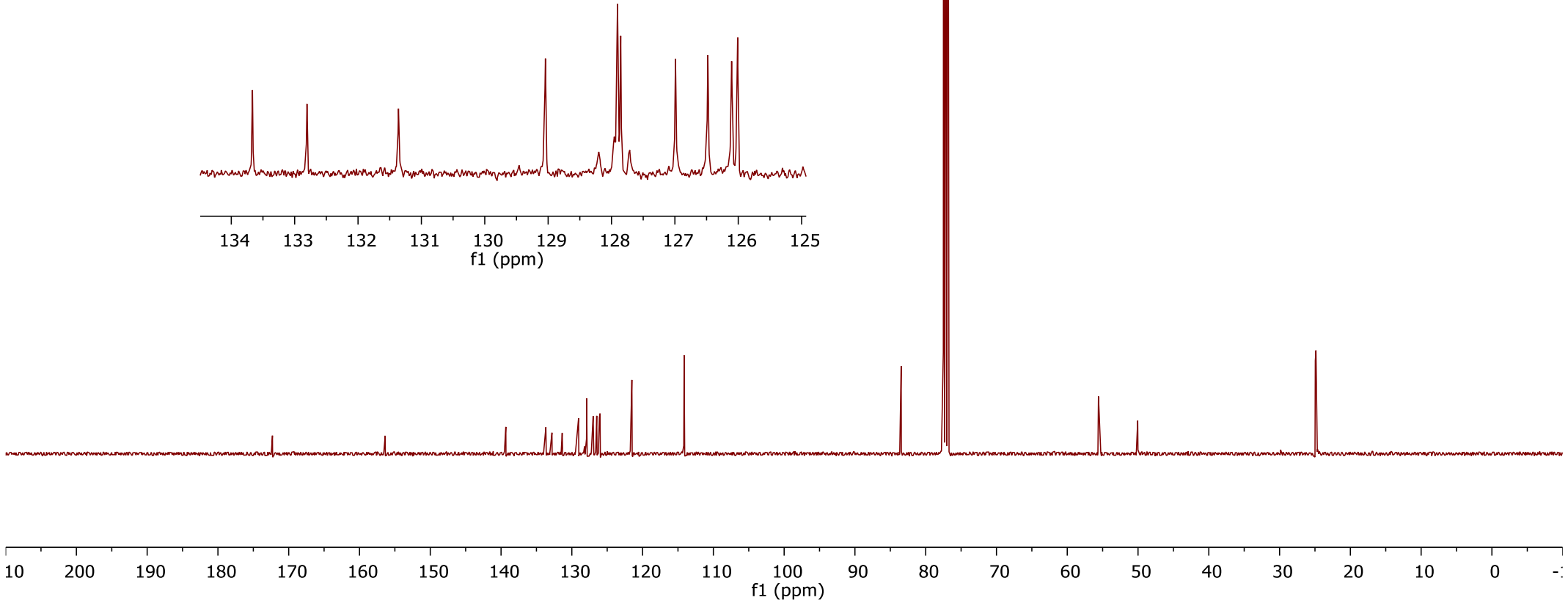

80 


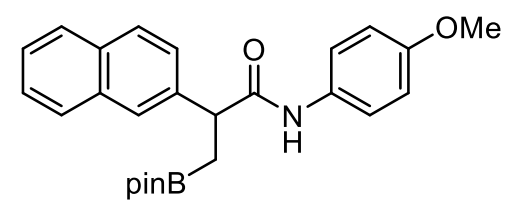

$3 i b$

${ }^{11} \mathrm{~B}$ NMR $\left(96 \mathrm{MHz}, \mathrm{CDCl}_{3}\right.$ )

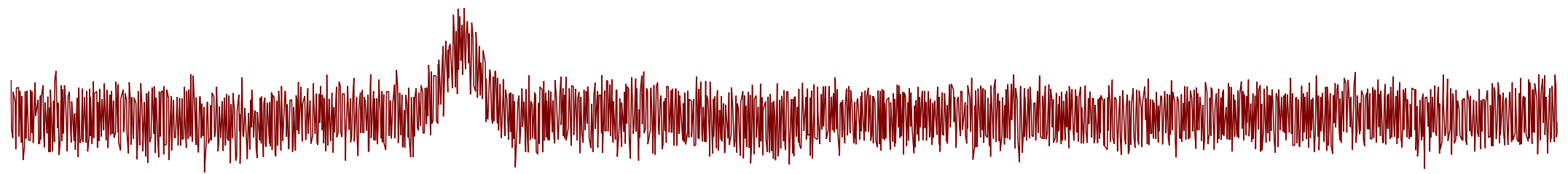

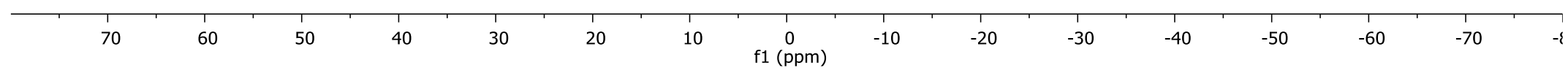




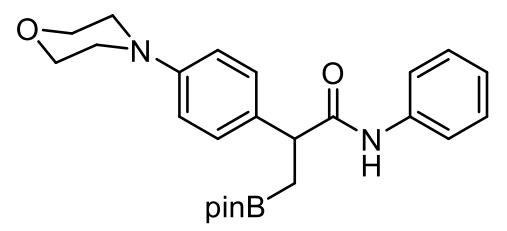

3ja

${ }^{1} \mathrm{H}$ NMR $\left(400 \mathrm{MHz}, \mathrm{CDCl}_{3}\right)$

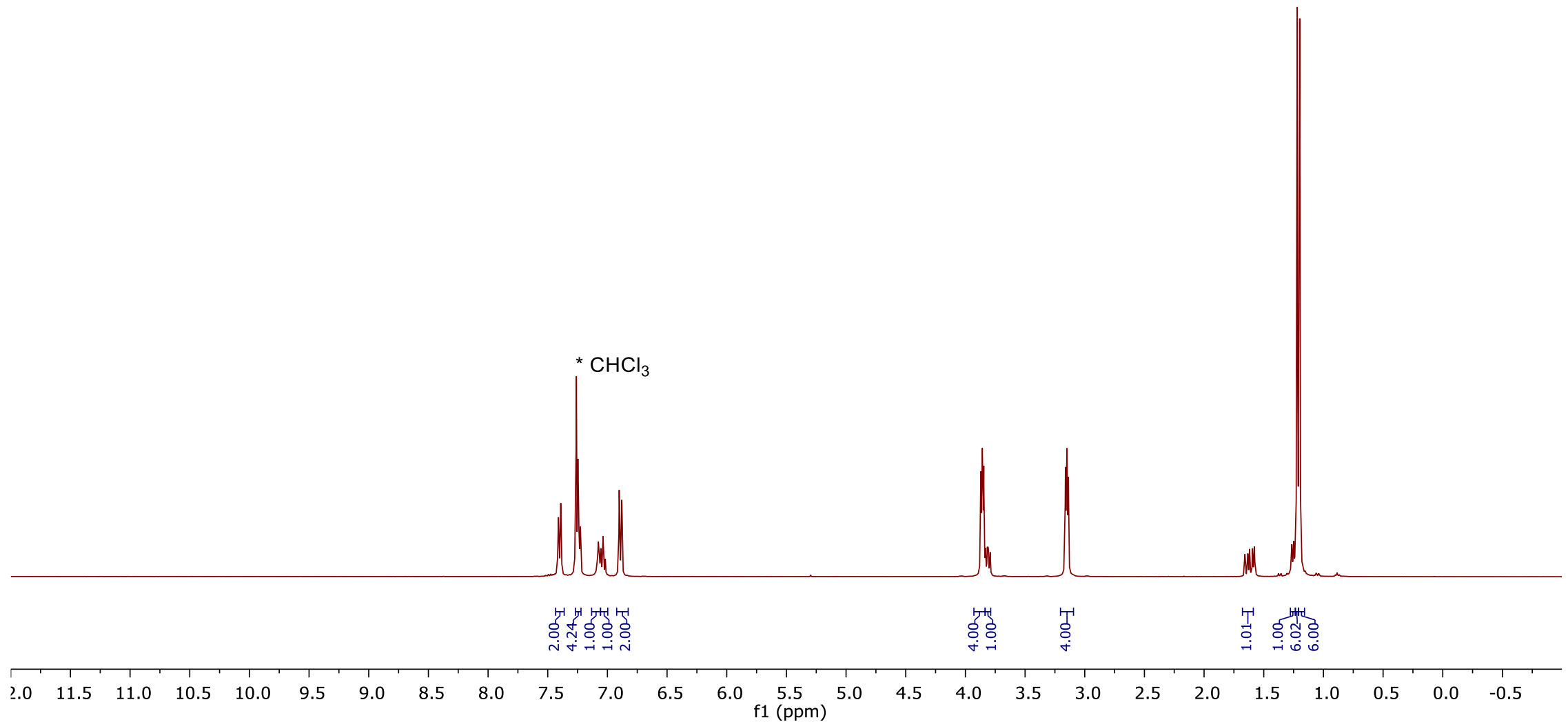




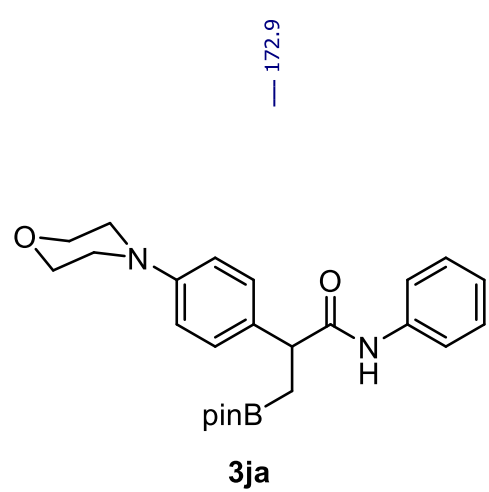

${ }^{13} \mathrm{C}\left\{{ }^{1} \mathrm{H}\right\}$ NMR (101 MHz, $\mathrm{CDCl}_{3}$ )

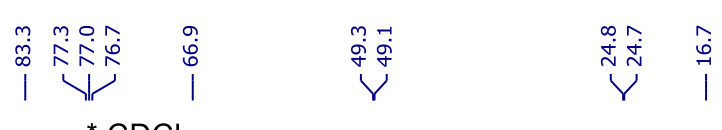

$\mathrm{CDCl}_{3}$

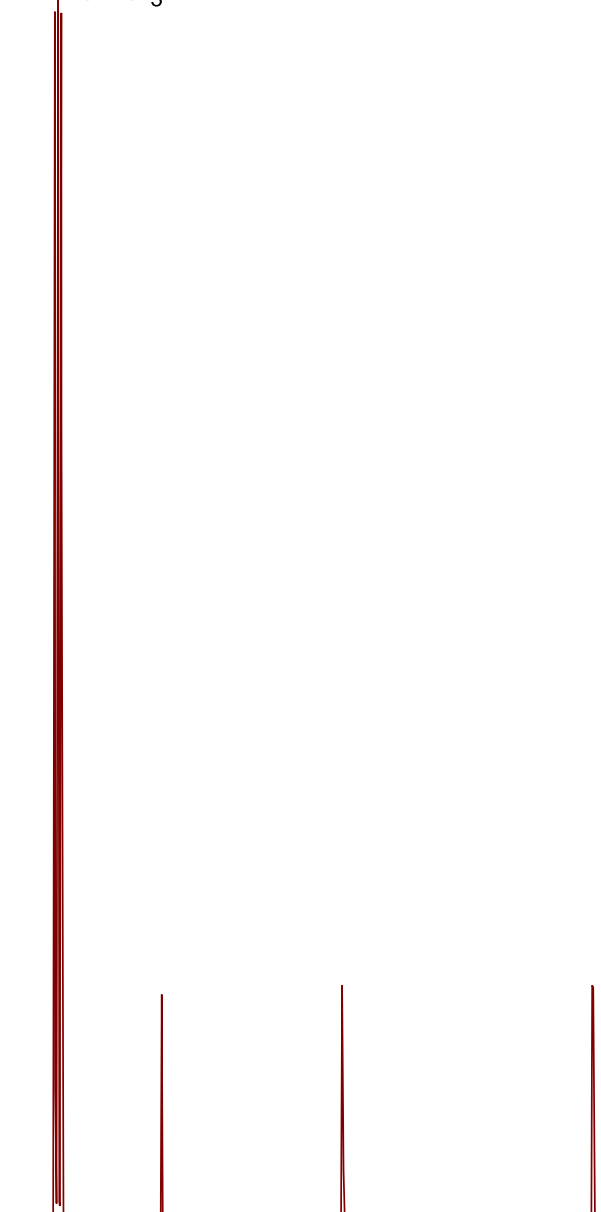

$10 \quad 200-190$

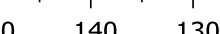


$\stackrel{\substack{+\infty \\ m}}{1}$

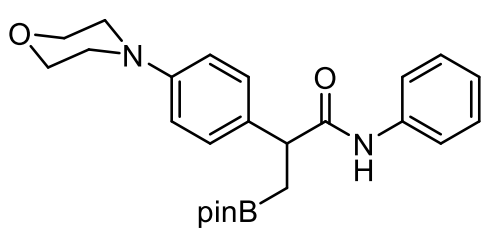

$3 j a$

${ }^{11} \mathrm{~B}$ NMR $\left(128 \mathrm{MHz}, \mathrm{CDCl}_{3}\right)$

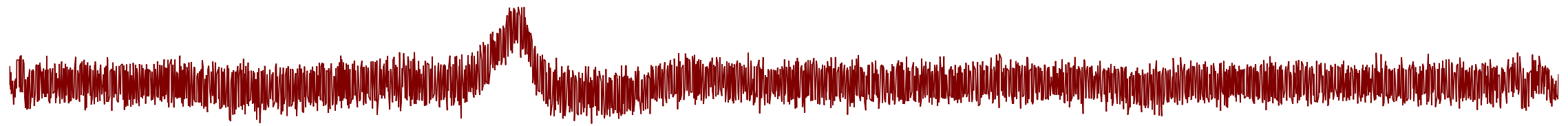

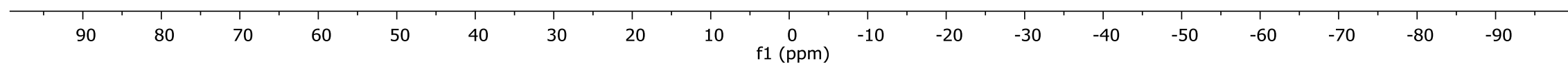




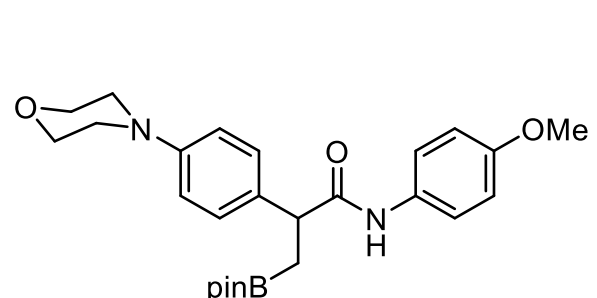

3jb

${ }^{1} \mathrm{H}$ NMR (400 MHz, $\mathrm{CDCl}_{3}$ )
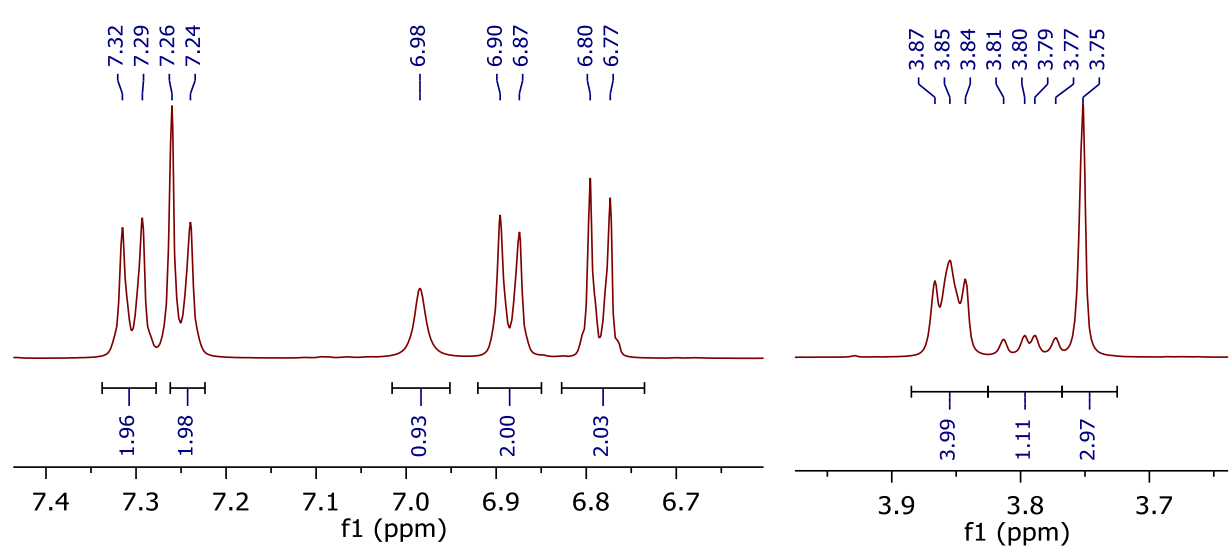

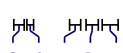

$\begin{array}{lllllll}2.0 & 11.5 & 11.0 & 10.5 & 10.0 & 9.5 & 9.0\end{array}$

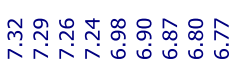

垈।

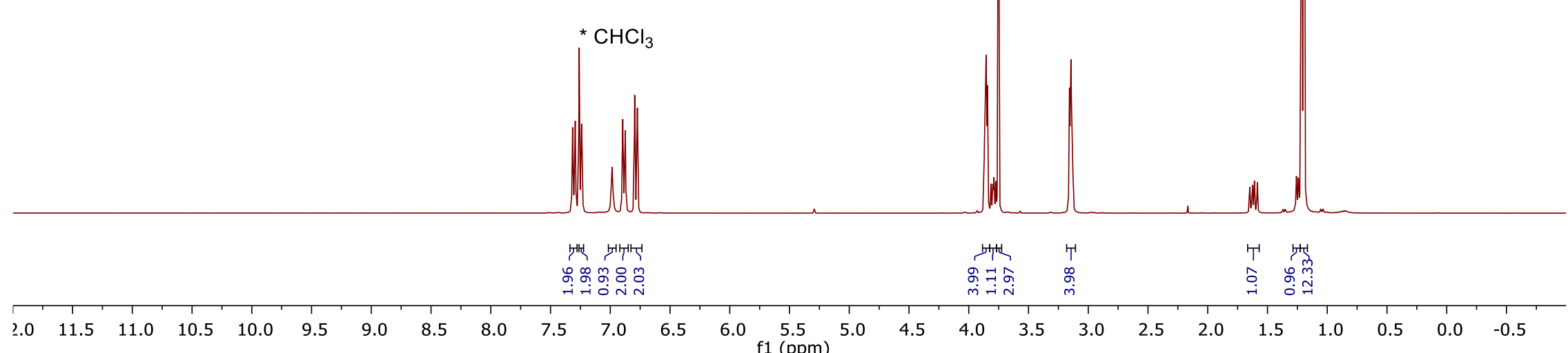

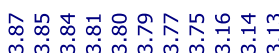

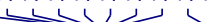

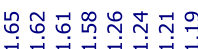

औ 


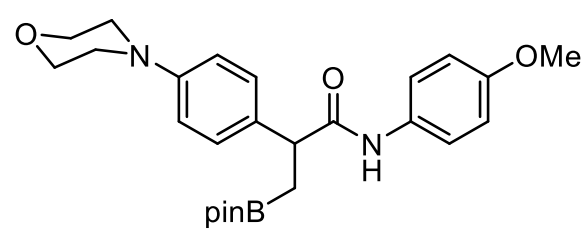

$3 \mathrm{jb}$

${ }^{13} \mathrm{C}\left\{{ }^{1} \mathrm{H}\right\}$ NMR $\left(101 \mathrm{MHz}, \mathrm{CDCl}_{3}\right)$
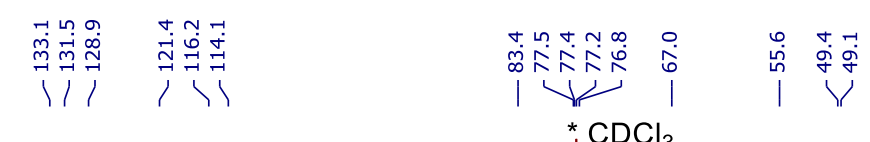

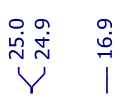

I

${ }^{*} \mathrm{CDCl}_{3}$

|

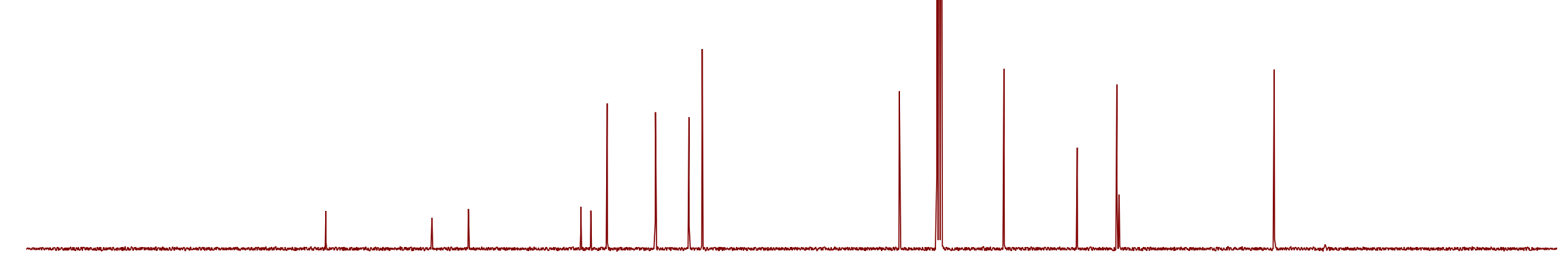

$210 \quad 200 \quad 190$

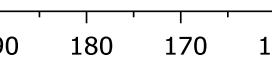

150

$140 \quad 130$

$120 \quad 110$ $10 \quad 100$ f1 100 80 60 40

30 $10 \quad 0 \quad-10$ 


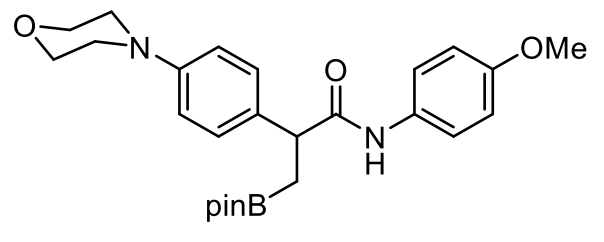

3jb

${ }^{11}$ B NMR (96 MHz, $\mathrm{CDCl}_{3}$ )

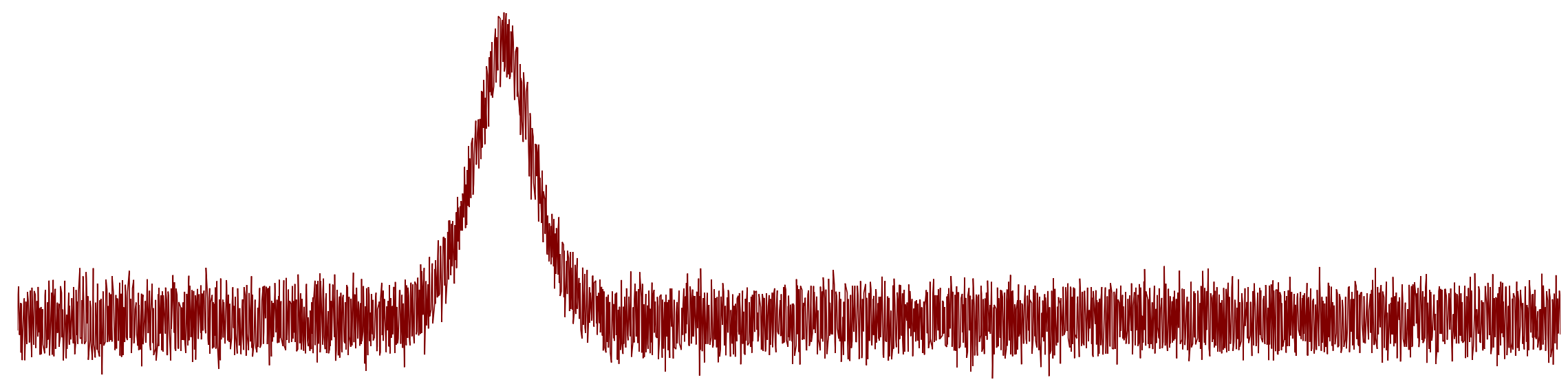




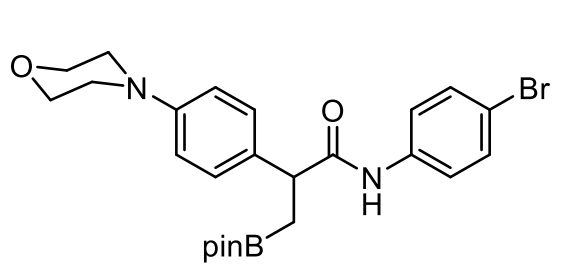

$3 j g$

${ }^{1} \mathrm{H}$ NMR $\left(500 \mathrm{MHz}, \mathrm{CDCl}_{3}\right)$
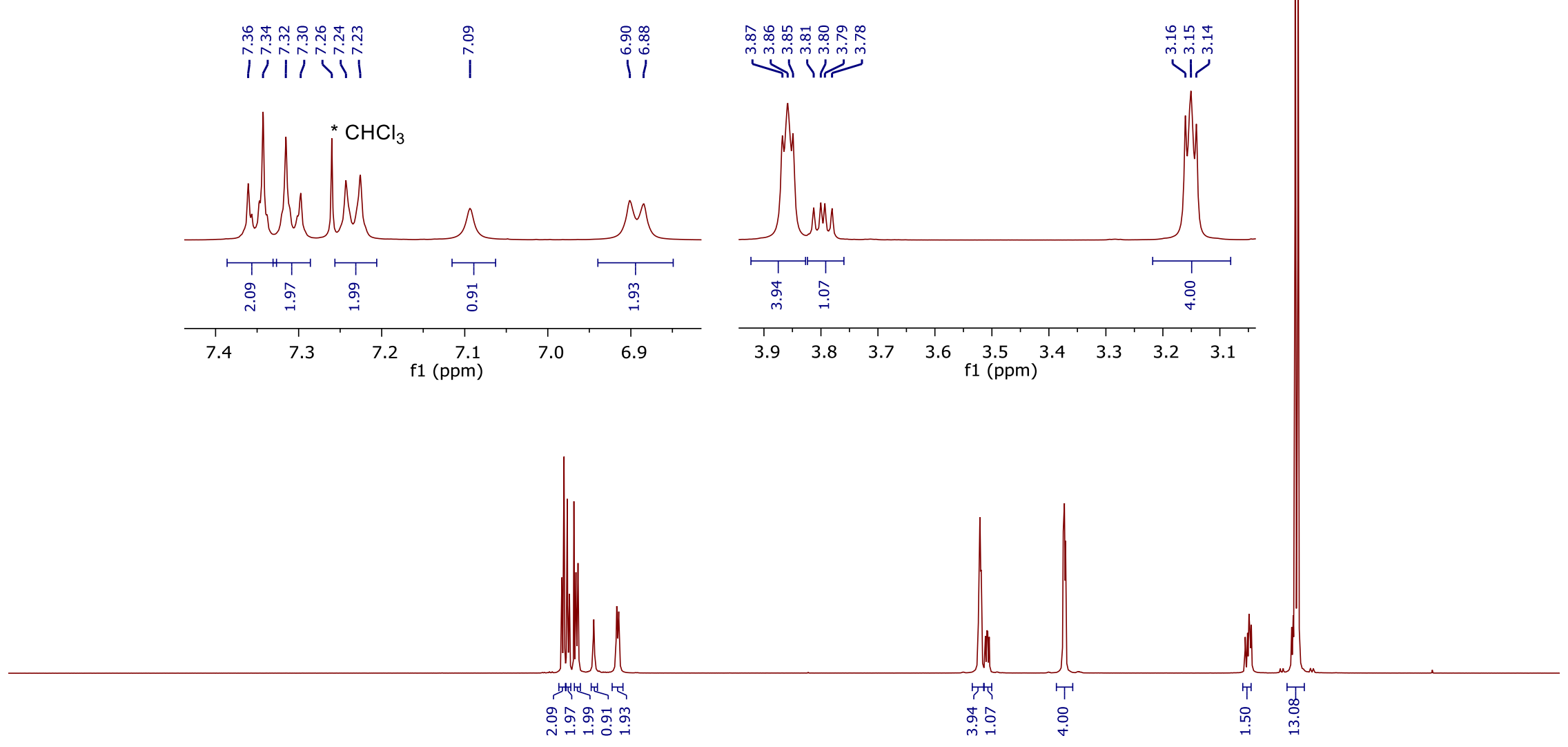

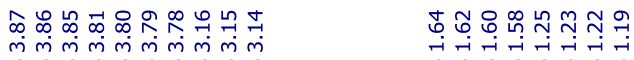

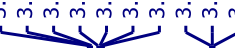

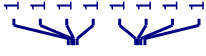

$\begin{array}{lllllllllllllllllllllllllllll}2.0 & 11.5 & 11.0 & 10.5 & 10.0 & 9.5 & 9.0 & 8.5 & 8.0 & 7.5 & 7.0 & 6.5 & 6.0 & 5.5 & 5.0 & 4.5 & 4.0 & 3.5 & 3.0 & 2.5 & 2.0 & 1.5 & 1.0 & 0.5 & 0.0 & -0.5 & -1\end{array}$ f1 $(\mathrm{ppm})$ 


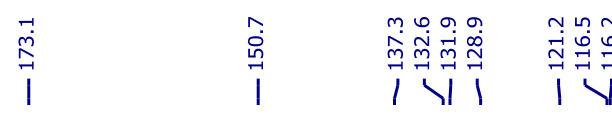

i لv

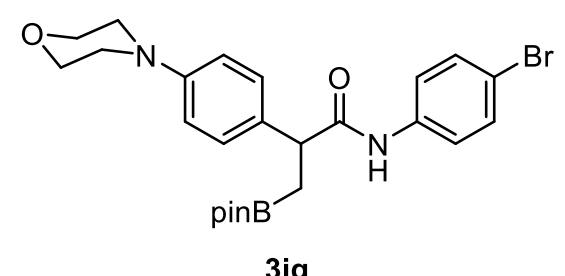

3 jg

${ }^{13} \mathrm{C}\left\{{ }^{1} \mathrm{H}\right\}$ NMR $\left(126 \mathrm{MHz}, \mathrm{CDCl}_{3}\right)$

$\mathrm{CDCl}_{3}$

i 


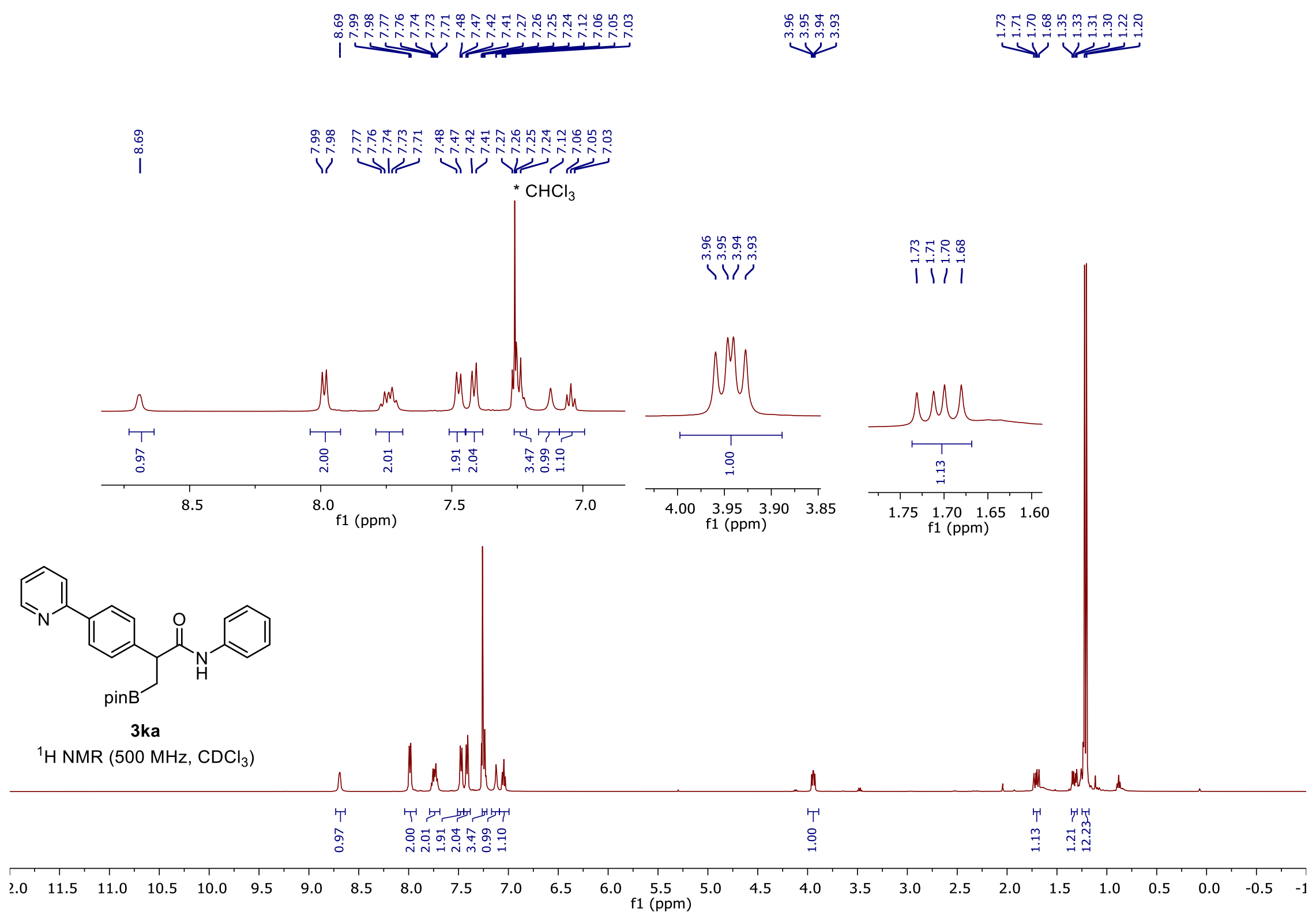



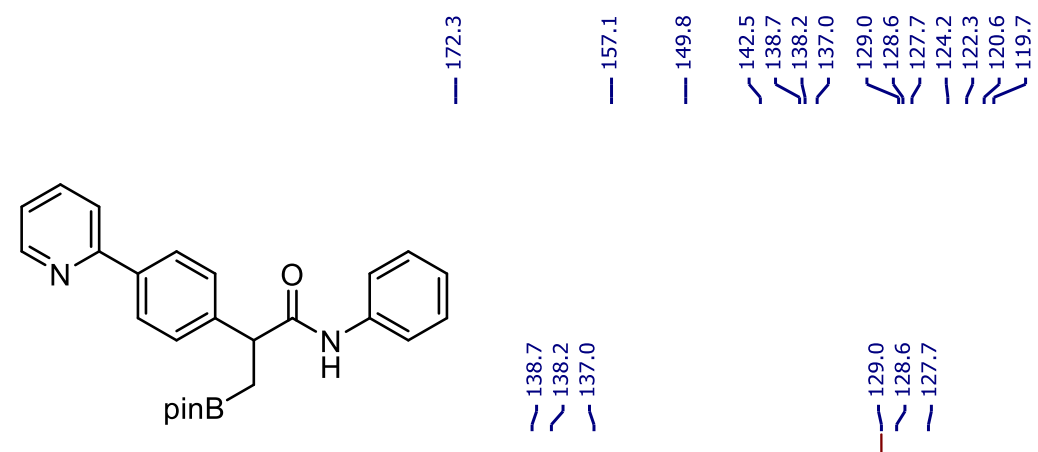

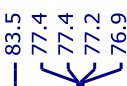

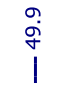

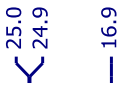

3 ka

${ }^{13} \mathrm{C}\left\{{ }^{1} \mathrm{H}\right\}$ NMR $\left(126 \mathrm{MHz}, \mathrm{CDCl}_{3}\right)$

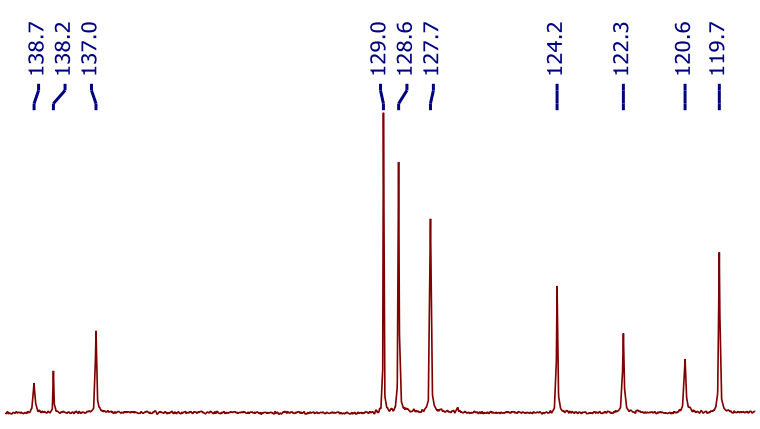

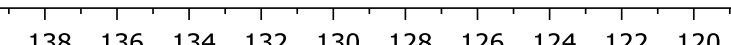
f1 (ppm)
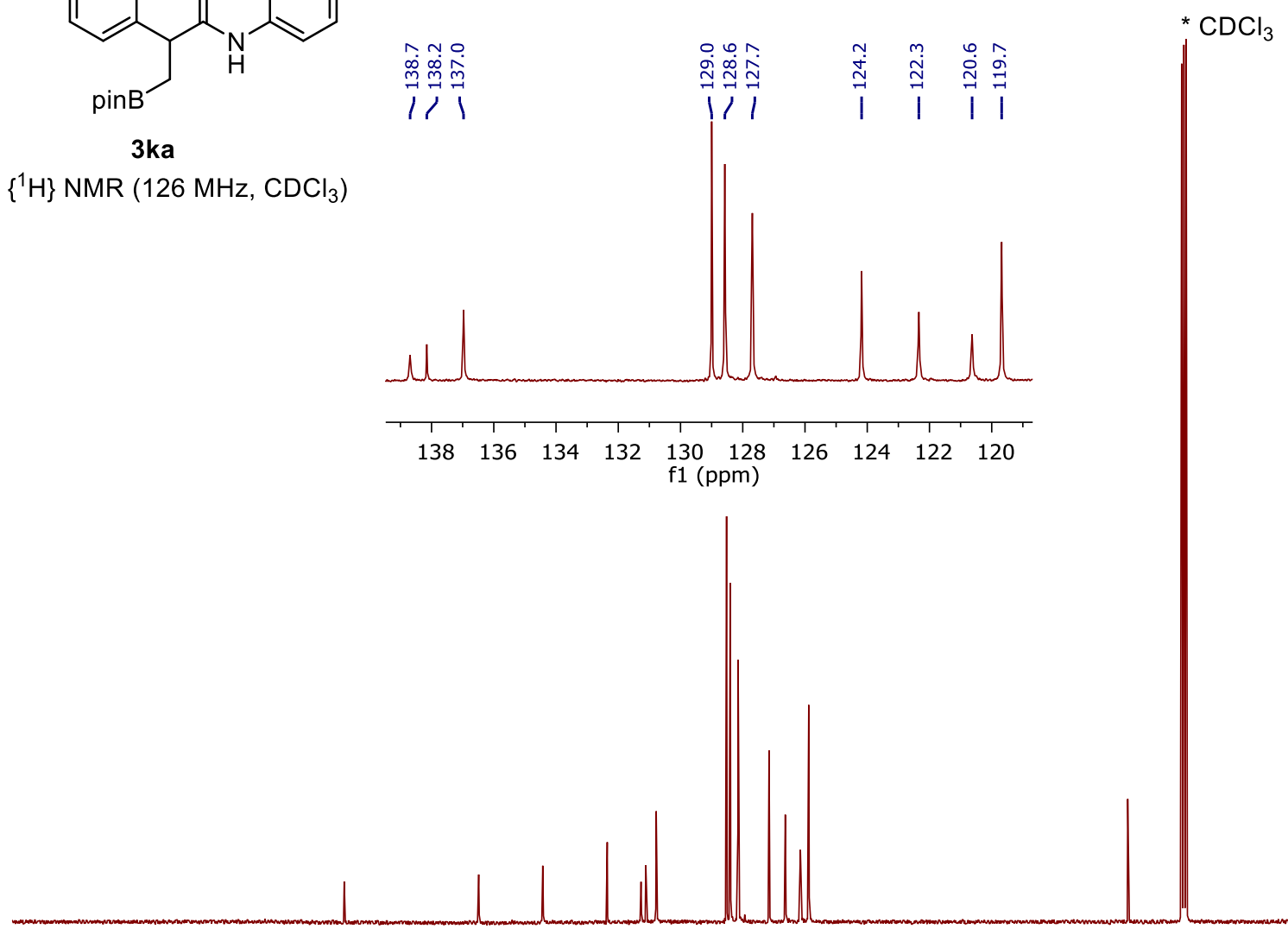

$200 \quad 190$

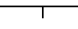

150

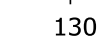

120

$110 \quad 100$

$90 \quad 80$

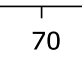

60

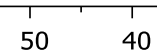

30

20

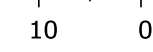




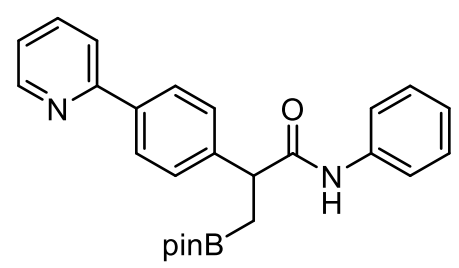

3ka

${ }^{11} \mathrm{~B}$ NMR $\left(96 \mathrm{MHz}, \mathrm{CDCl}_{3}\right)$

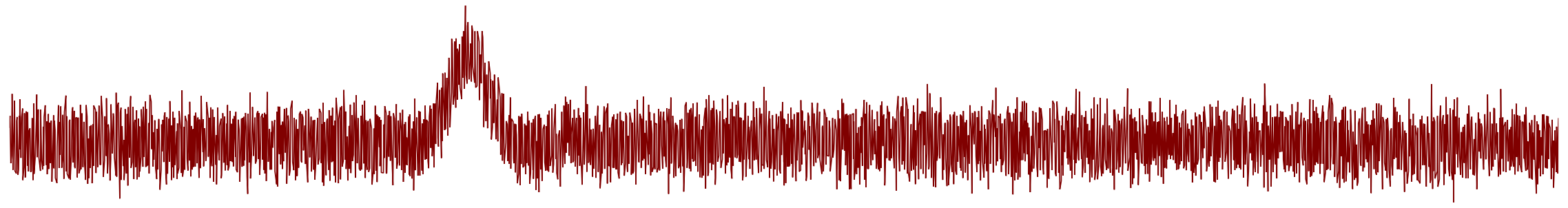

\begin{tabular}{|c|c|c|c|c|c|c|c|c|c|c|c|c|c|c|}
\hline 70 & 60 & 50 & 40 & 30 & 20 & 10 & $\begin{array}{c}0 \\
\mathrm{f} 1(\mathrm{ppm})\end{array}$ & -10 & -20 & -30 & -40 & -50 & -60 & -70 \\
\hline
\end{tabular}




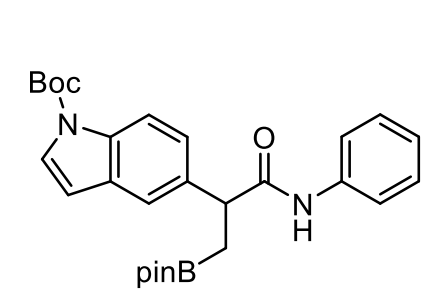

3 la

${ }^{1} \mathrm{H}$ NMR (400 MHz, $\mathrm{CDCl}_{3}$ )

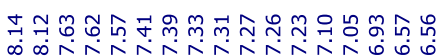

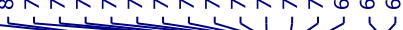

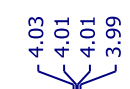

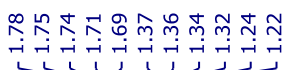
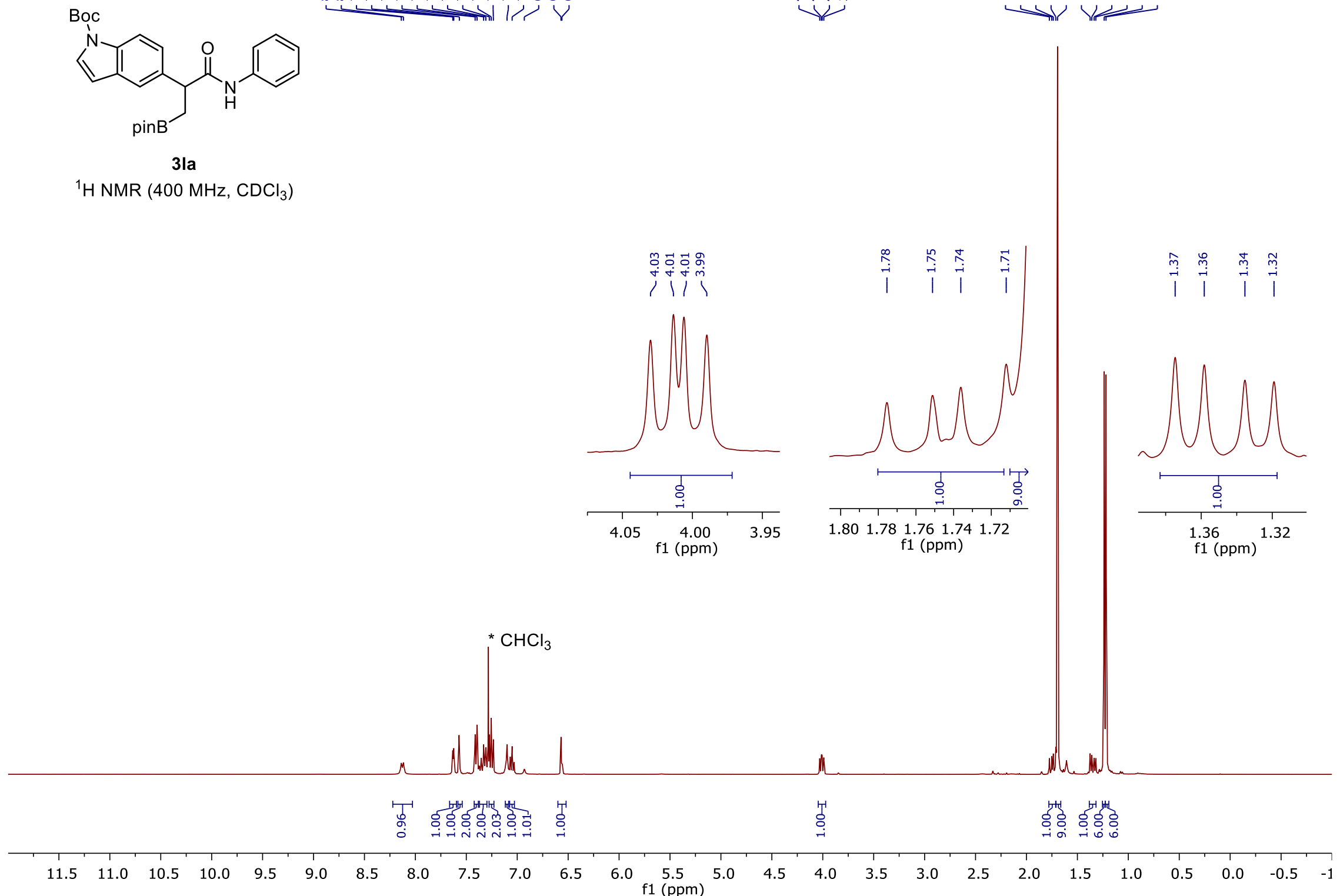
Boc

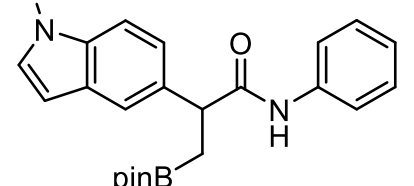

319

${ }^{13} \mathrm{C}\left\{{ }^{1} \mathrm{H}\right\}$ NMR $\left(100 \mathrm{MHz}, \mathrm{CDCl}_{3}\right)$

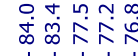

YV

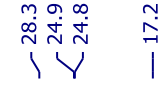

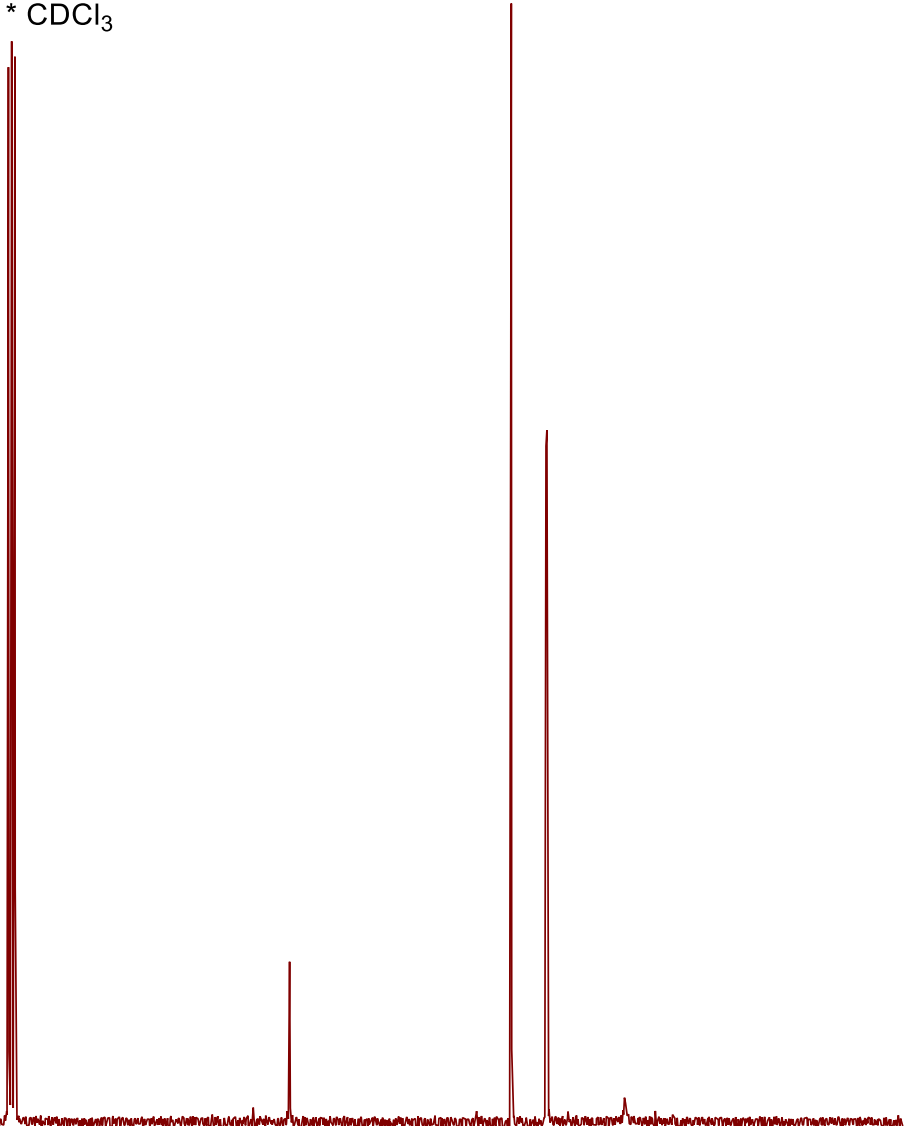

$10200 \quad 190 \quad 180$

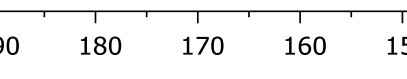

$\begin{array}{rrr}150 & 140 \quad 130\end{array}$
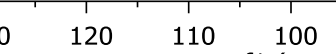

$\stackrel{100}{\mathrm{f} 1}(\mathrm{ppm})$

$90 \quad 80$ 
$\stackrel{\substack{m \\ m}}{1}$

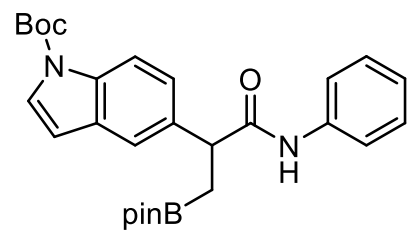

$31 \mathrm{a}$

${ }^{11} \mathrm{~B}$ NMR $\left(128 \mathrm{MHz}, \mathrm{CDCl}_{3}\right)$

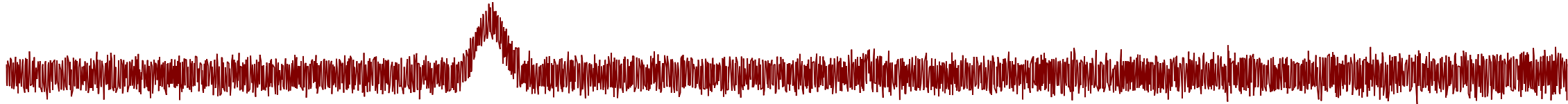

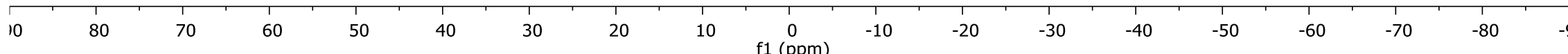




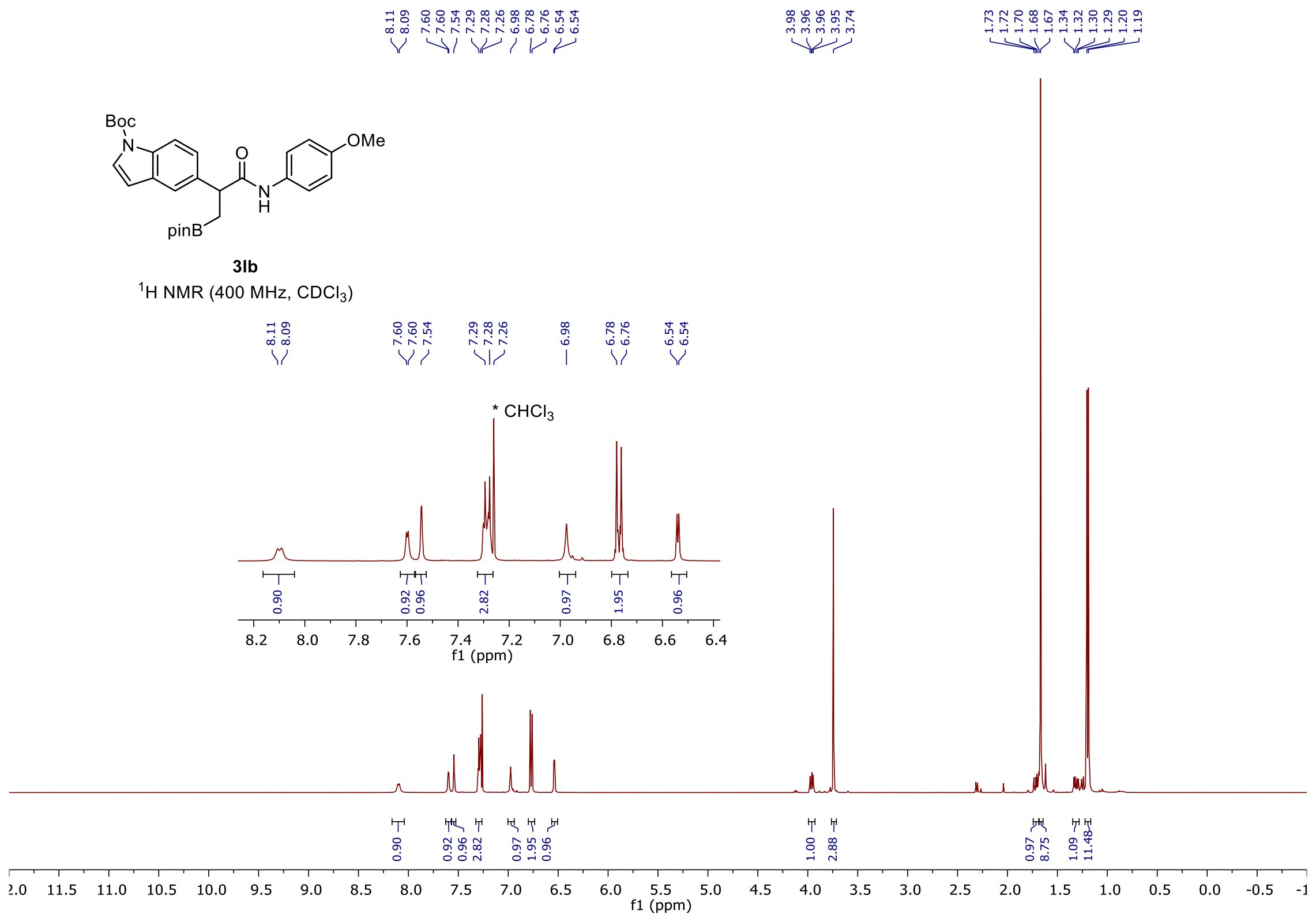




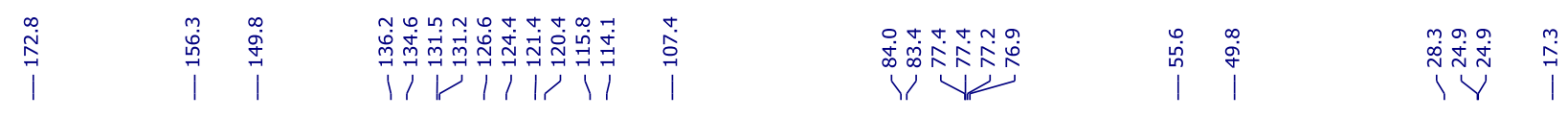

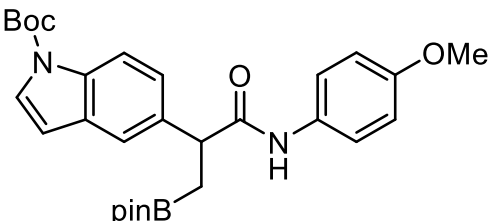

3lb

${ }^{13} \mathrm{C}\left\{{ }^{1} \mathrm{H}\right\}$ NMR $\left(101 \mathrm{MHz}, \mathrm{CDCl}_{3}\right)$

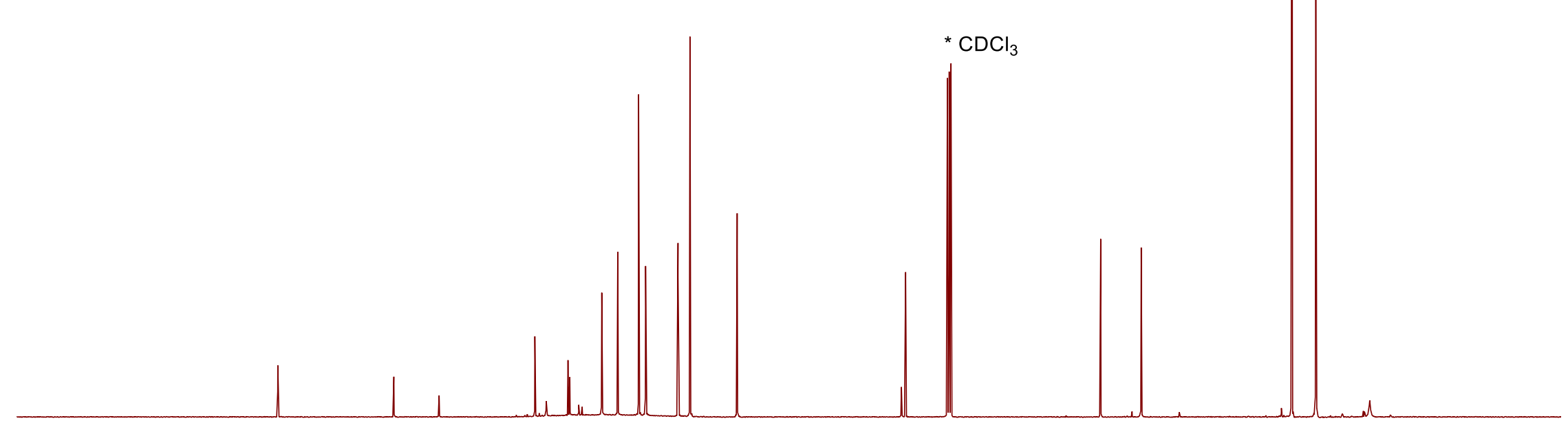




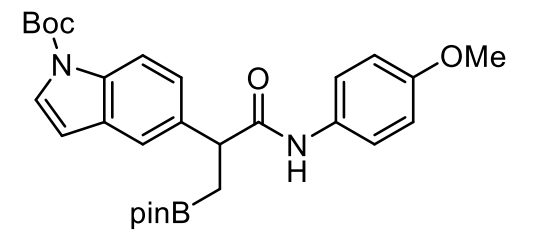

3 lb

${ }^{11} \mathrm{~B}$ NMR (96 MHz, $\mathrm{CDCl}_{3}$ )
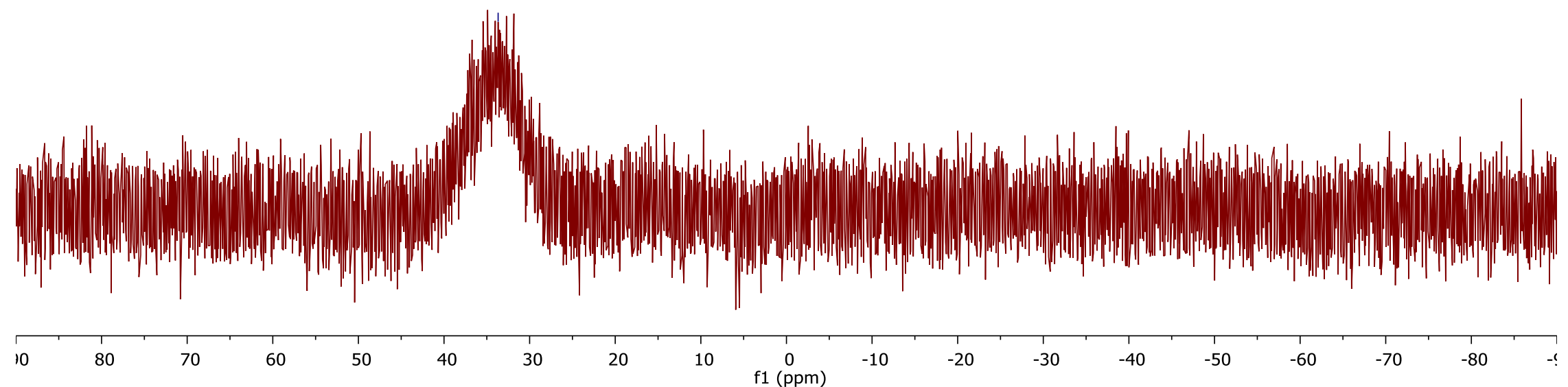

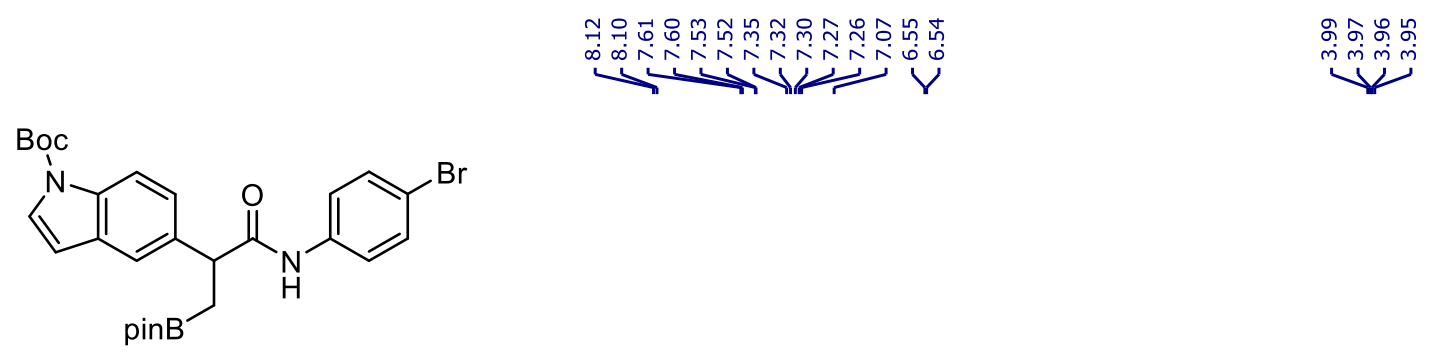

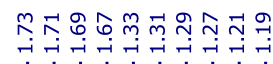

$31 \mathrm{~g}$

${ }^{1} \mathrm{H}$ NMR $\left(400 \mathrm{MHz}, \mathrm{CDCl}_{3}\right)$
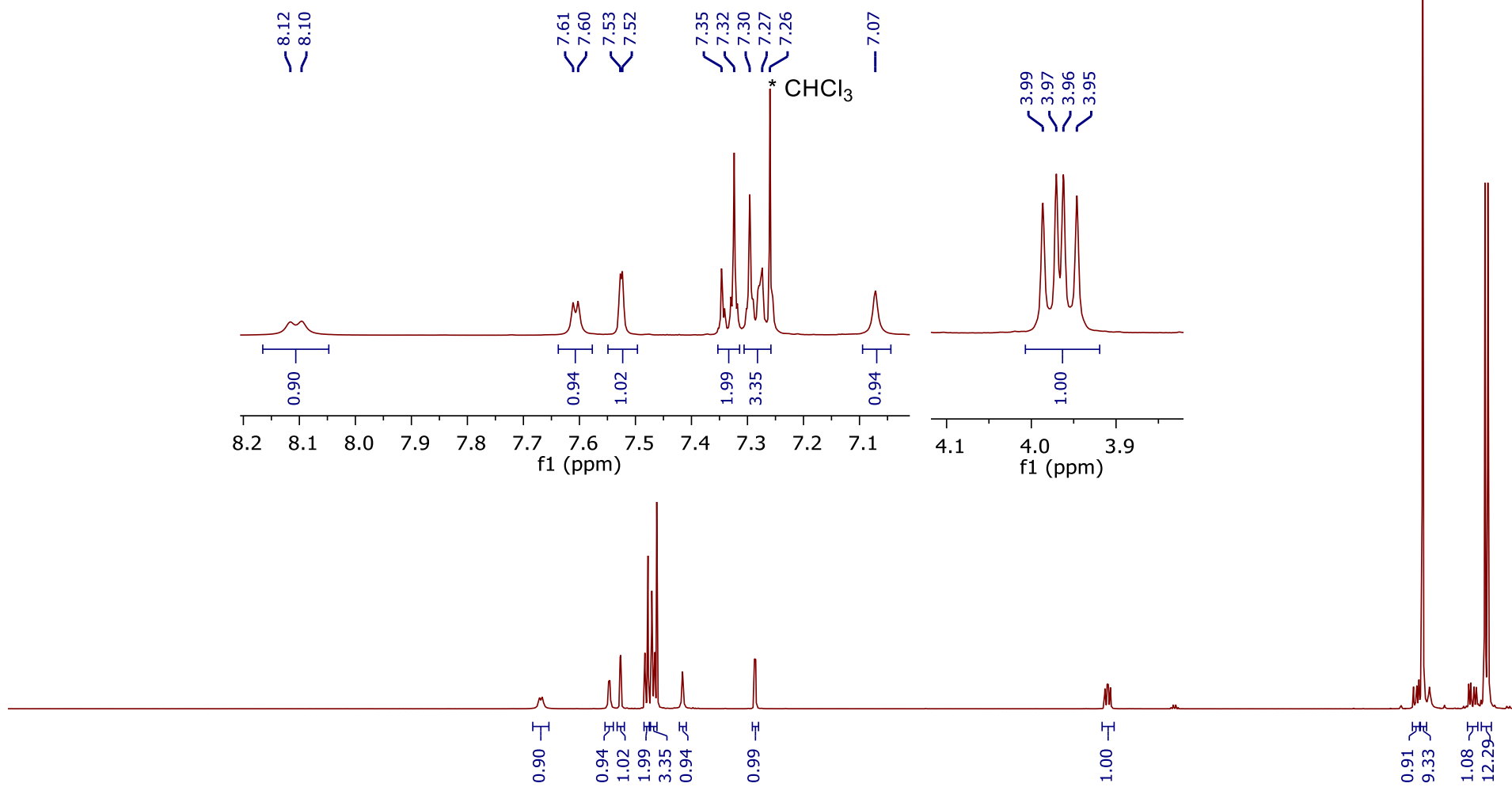

\begin{tabular}{lllllllllllllllllllllllllllllllllllllllllll}
\hline 2.0 & 11.5 & 11.0 & 10.5 & 10.0 & 9.5 & 9.0 & 8.5 & 8.0 & 7.5 & 7.0 & 6.5 & 6.0 & 5.5 & 5.0 & 4.5 & 4.0 & 3.5 & 3.0 & 2.5 & 2.0 & 1.5 & 1.0 & 0.5 & 0.0 & -0.5 &
\end{tabular} 


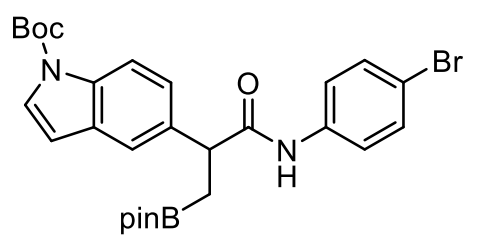

319

${ }^{13} \mathrm{C}\left\{{ }^{1} \mathrm{H}\right\}$ NMR $\left(101 \mathrm{MHz}, \mathrm{CDCl}_{3}\right)$

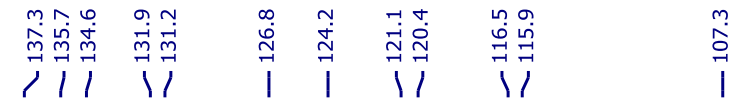
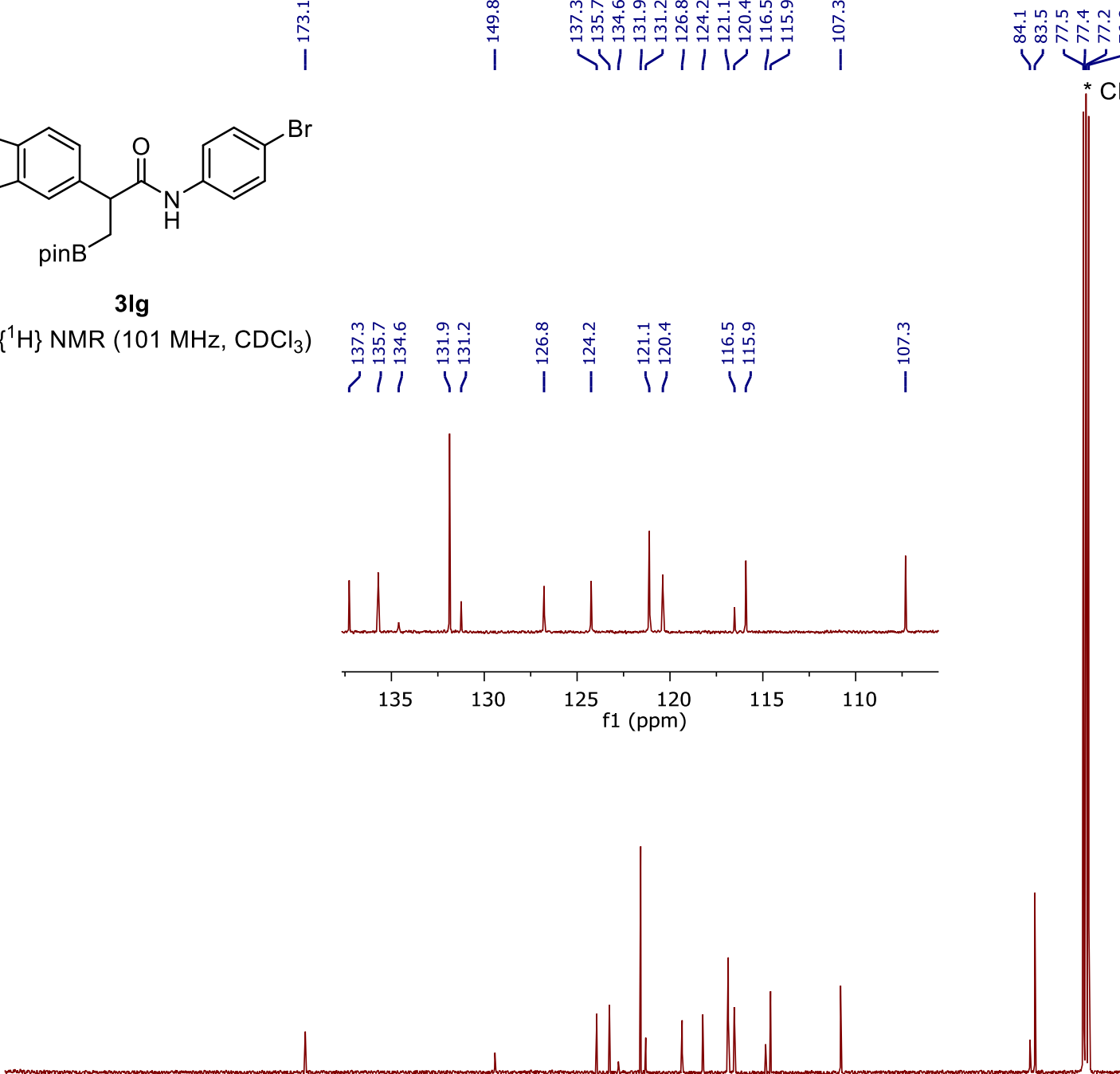

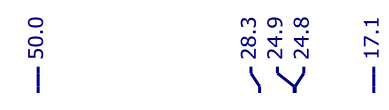

$\mathrm{CDCl}_{3}$

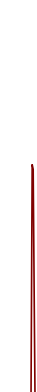

, 1

180

160

$150 \quad 140$

$130 \quad 120$

$110 \quad 100$

f1 $(\mathrm{ppm})$

$90 \quad 80$

70

60

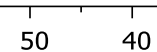

30

20 


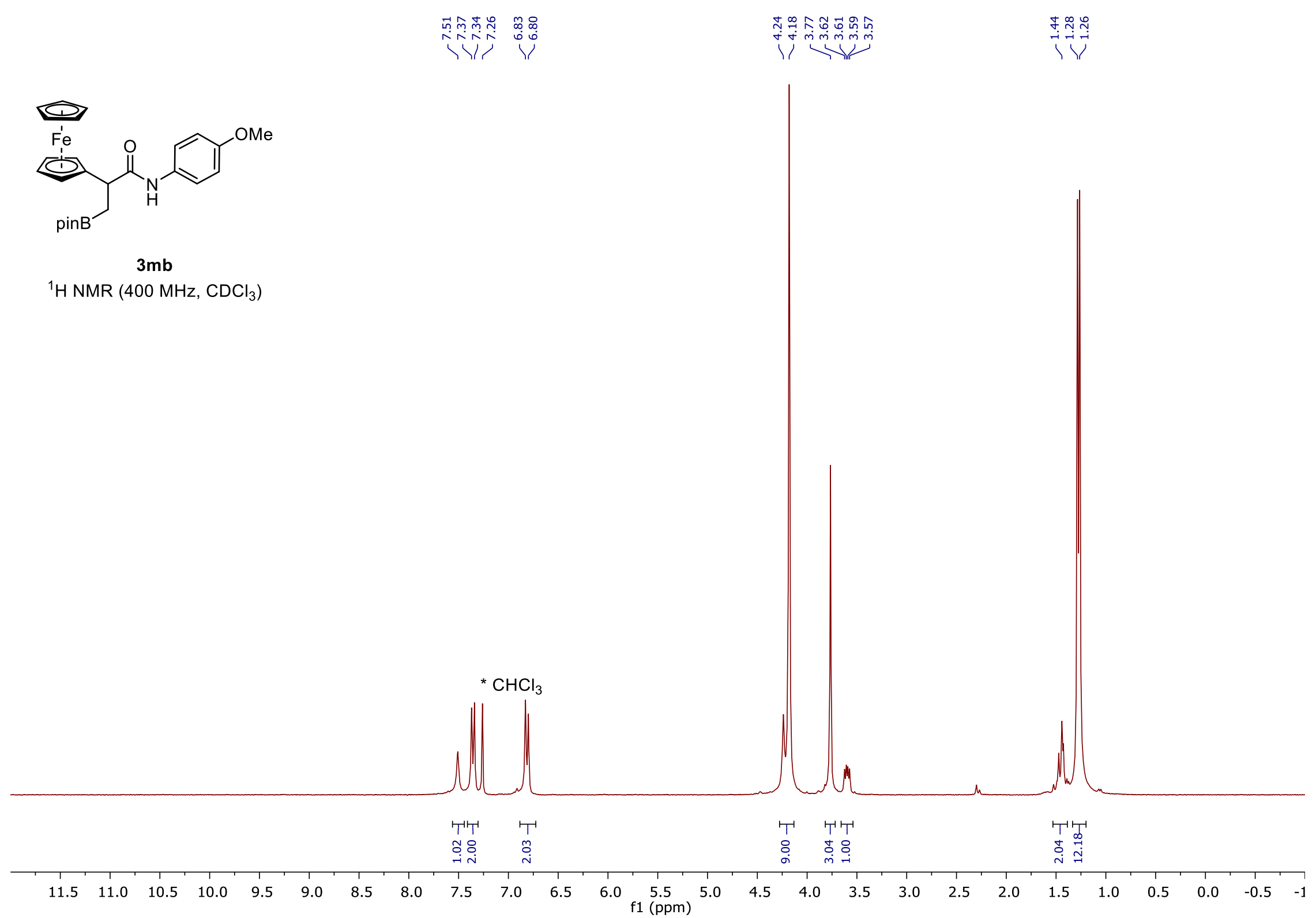




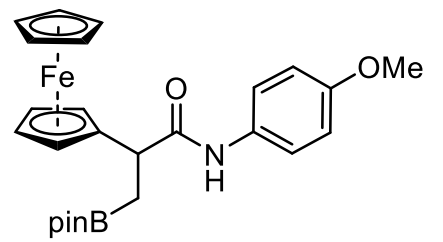

$3 \mathrm{mb}$

${ }^{13} \mathrm{C}\left\{{ }^{1} \mathrm{H}\right\}$ NMR $\left(101 \mathrm{MHz}, \mathrm{CDCl}_{3}\right)$

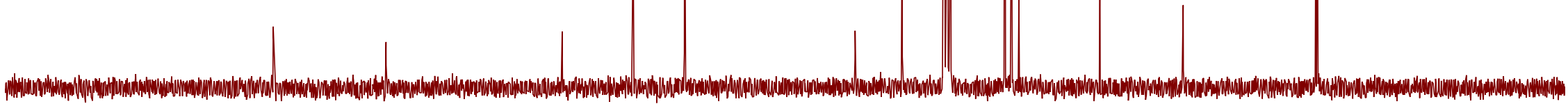




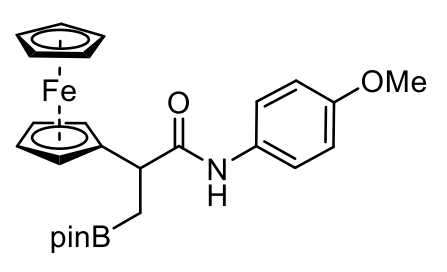

$3 \mathrm{mb}$

${ }^{11}$ B NMR (96 MHz, $\mathrm{CDCl}_{3}$ )

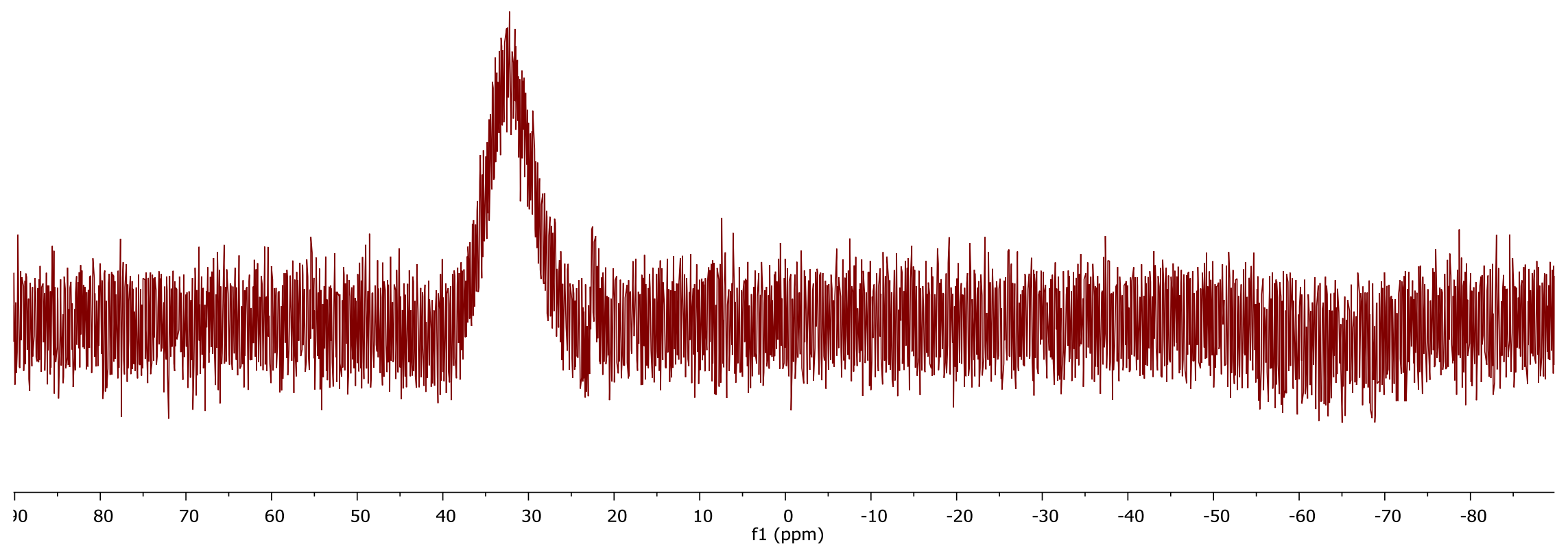




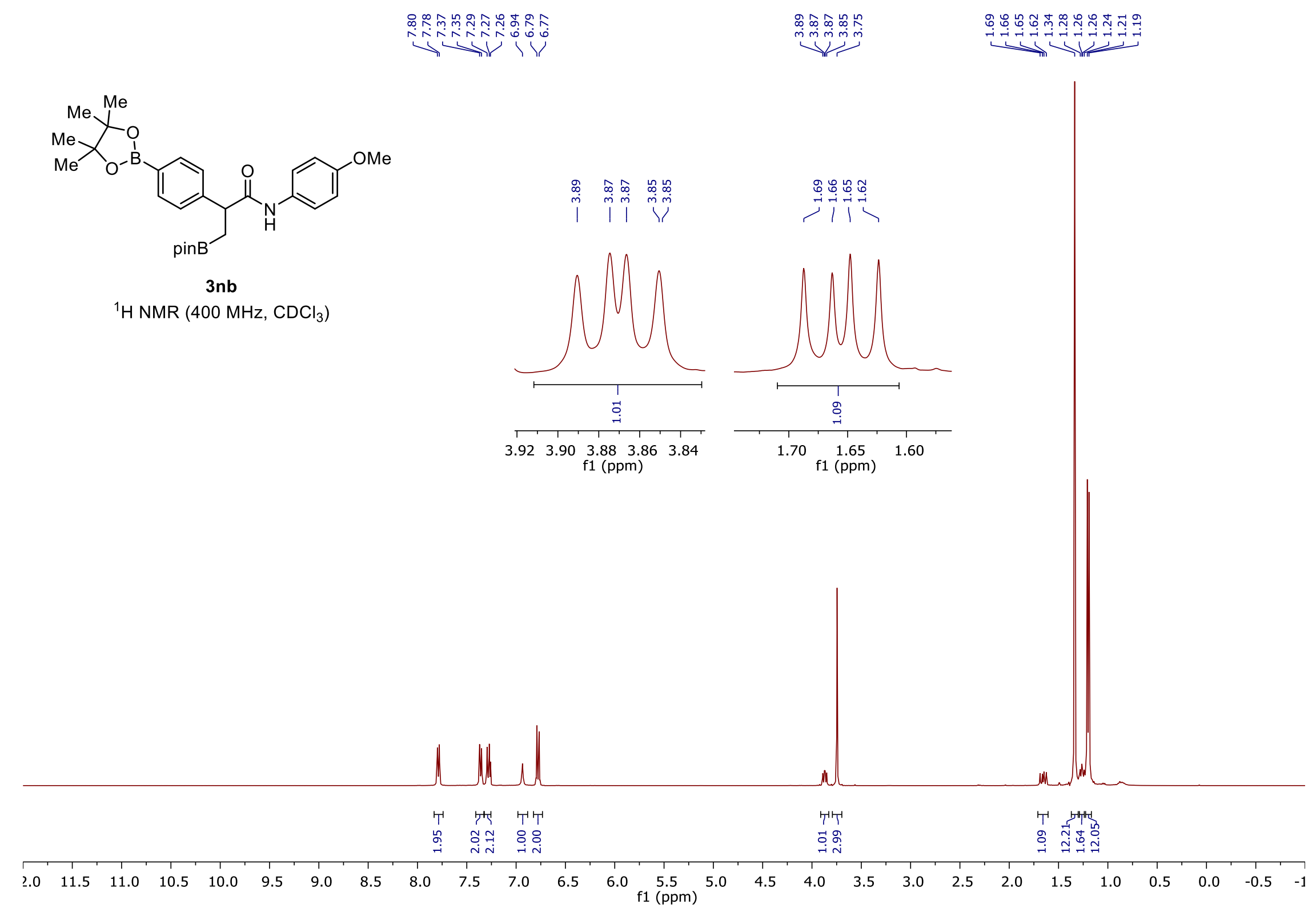




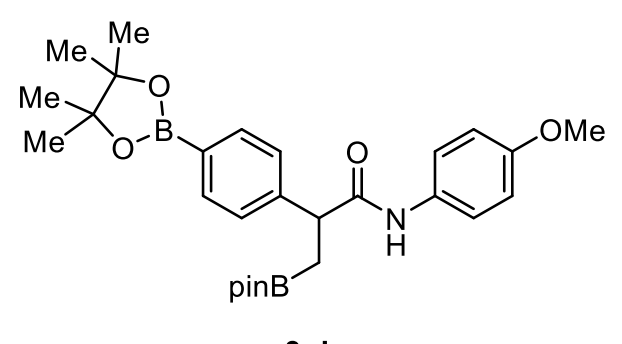

${ }^{13} \mathrm{C}\left\{{ }^{1} \mathrm{H}\right\}$ NMR $\left(101 \mathrm{MHz}, \mathrm{CDCl}_{3}\right)$
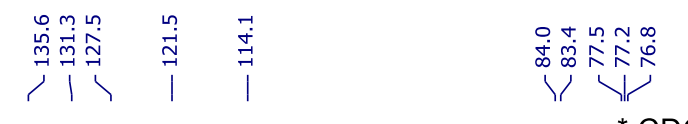

䓦

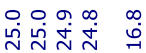

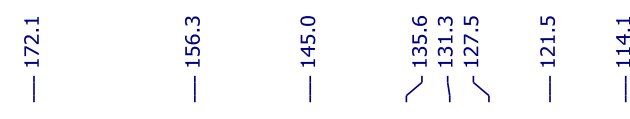

$\mathrm{CDCl}_{3}$

$\sqrt{2}$

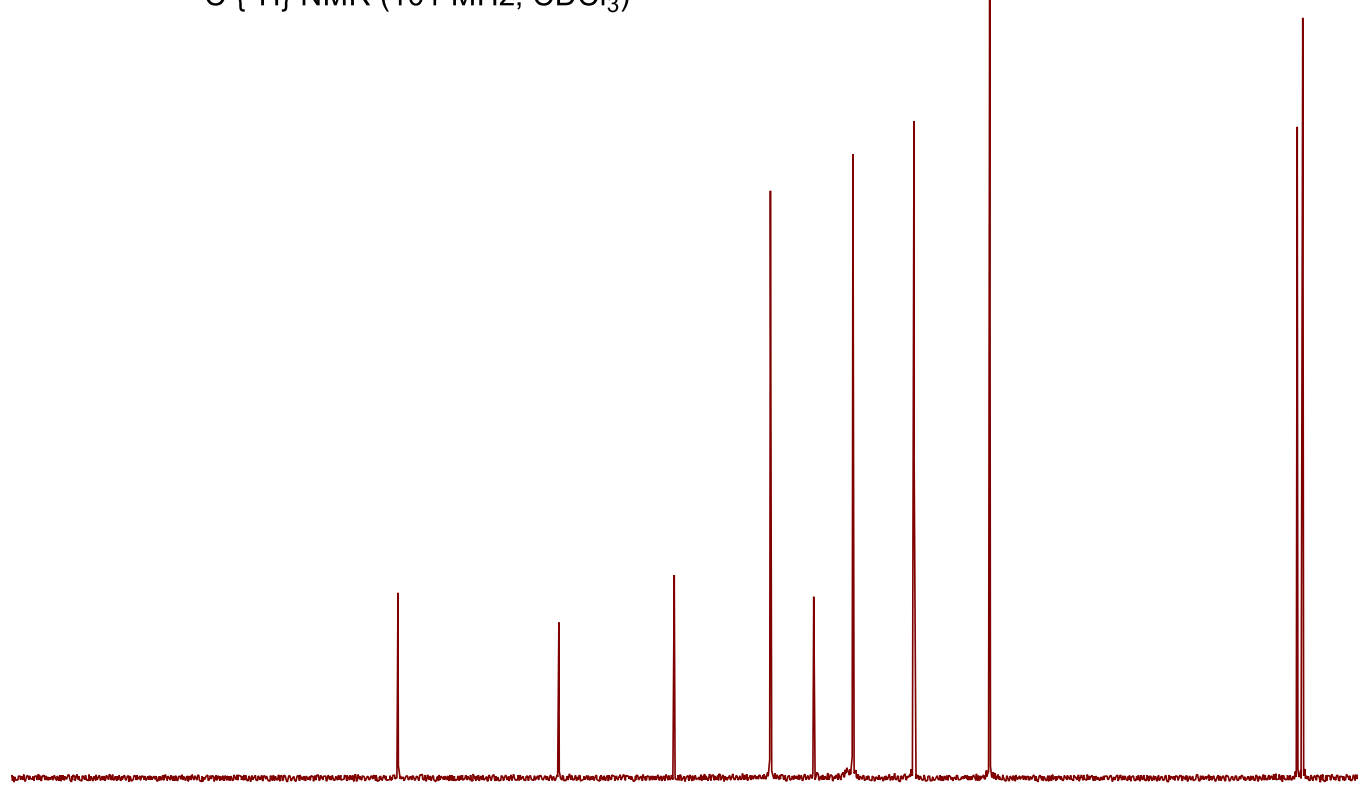




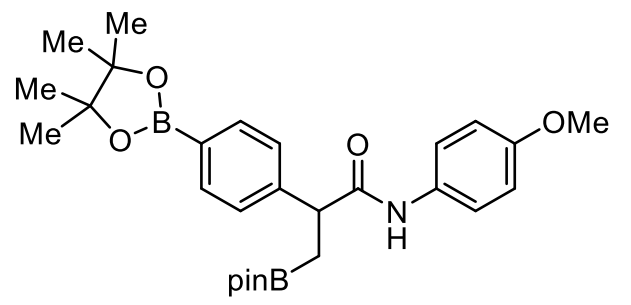

3nb

${ }^{11} \mathrm{~B}$ NMR (128 MHz, $\mathrm{CDCl}_{3}$ )

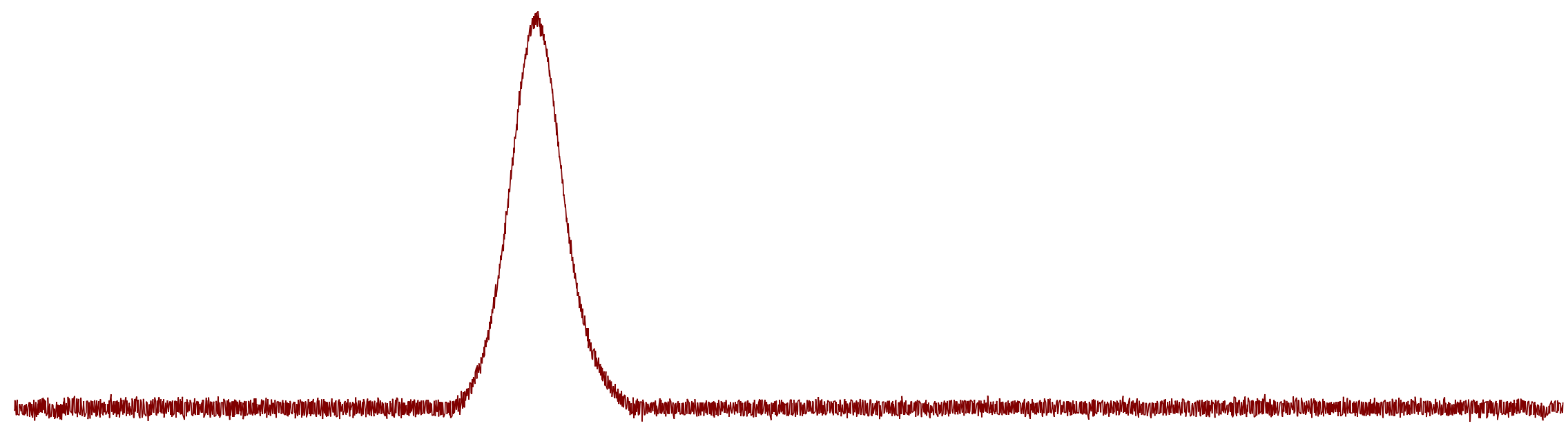


Supporting Information

S246

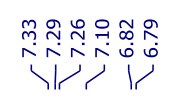<smiles>COc1ccc(NC(=O)C(C[Pb])c2ccc(P(c3ccccc3)c3ccccc3)cc2)cc1</smiles>
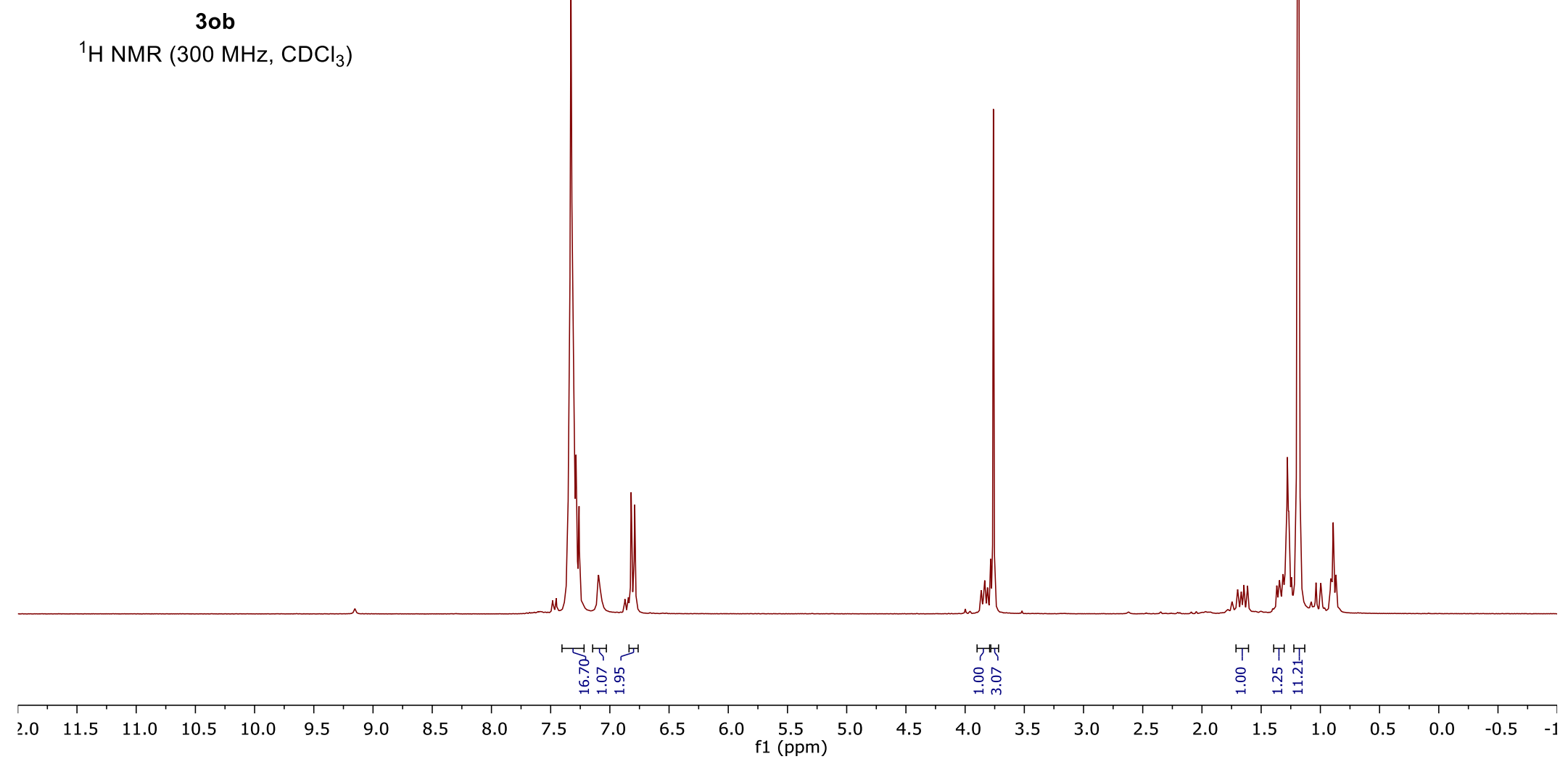

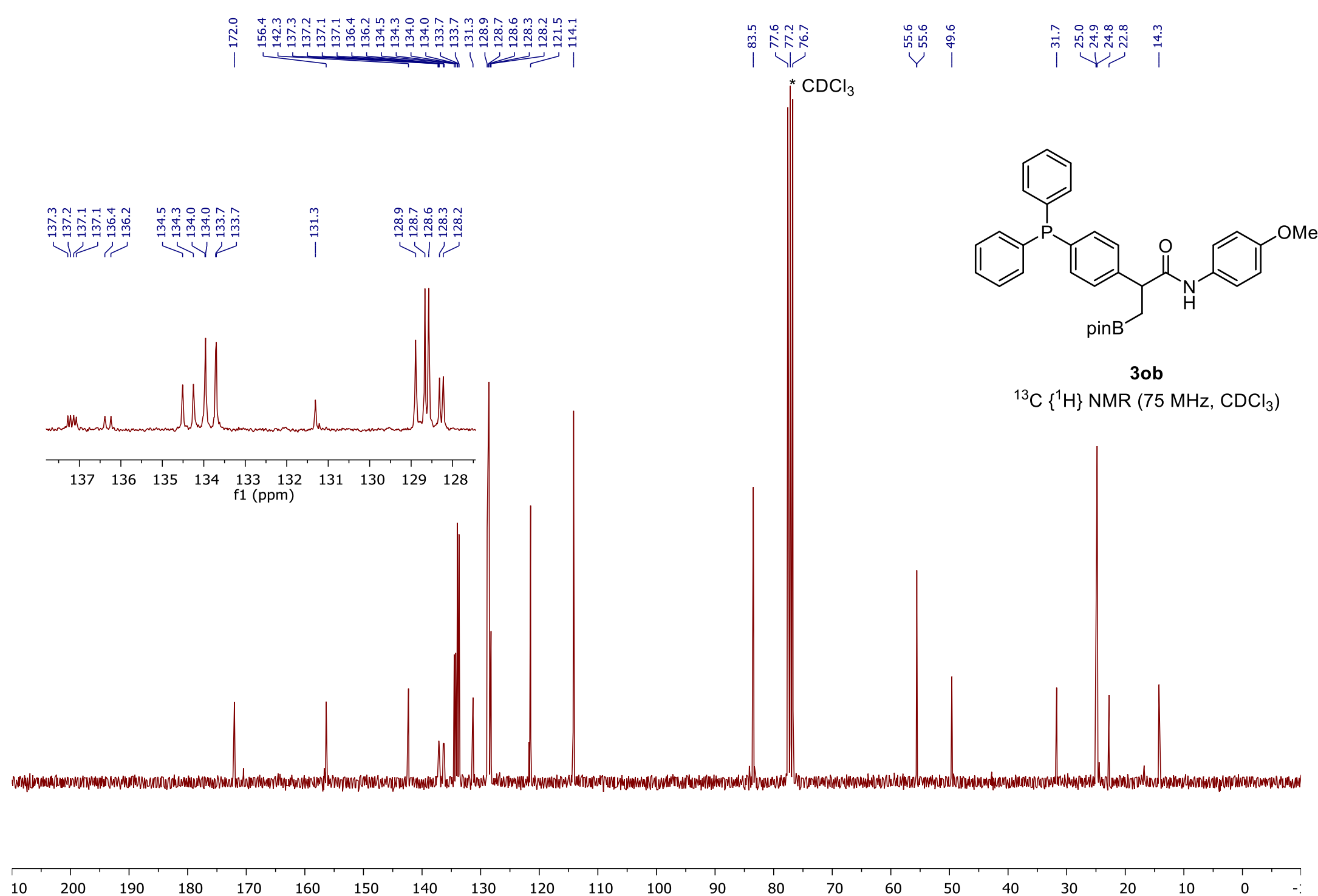

$10 \quad 100$ 100
$\mathrm{f} 1(\mathrm{ppm})$ 


$$
\text { 工 }
$$




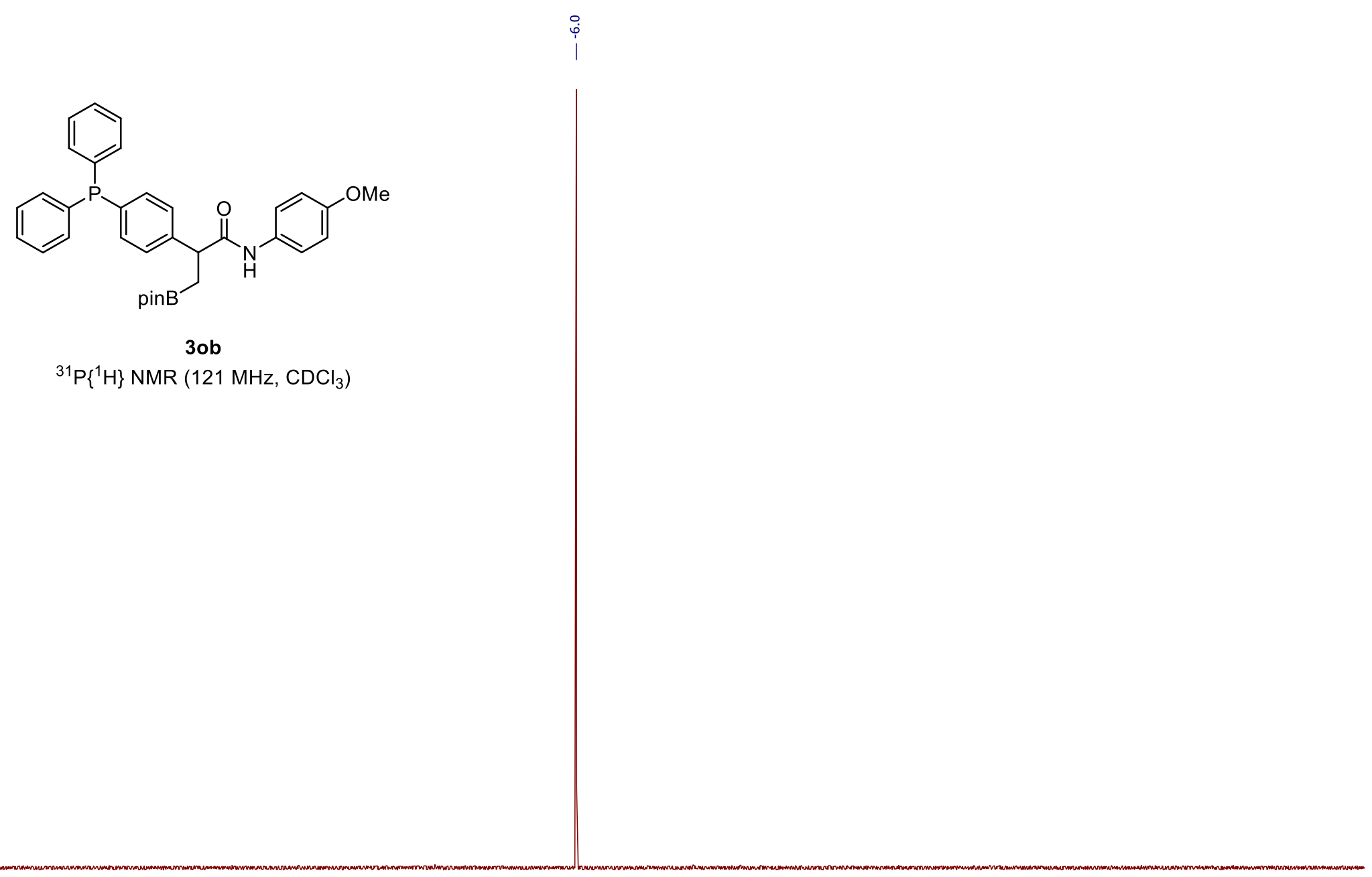

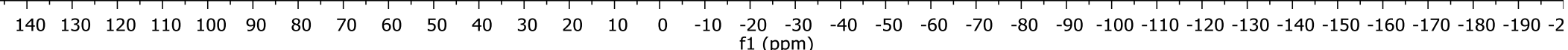




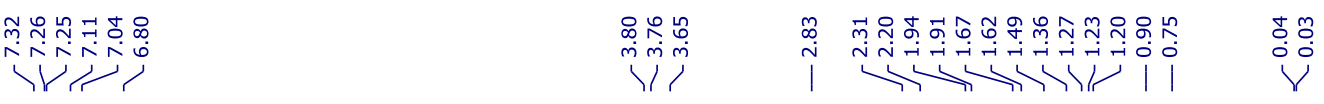

TBSO $\mathrm{Me}$

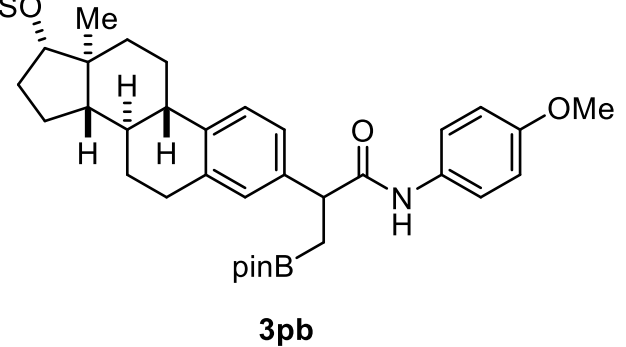

${ }^{1} \mathrm{H}$ NMR $\left(500 \mathrm{MHz}, \mathrm{CDCl}_{3}\right.$ )

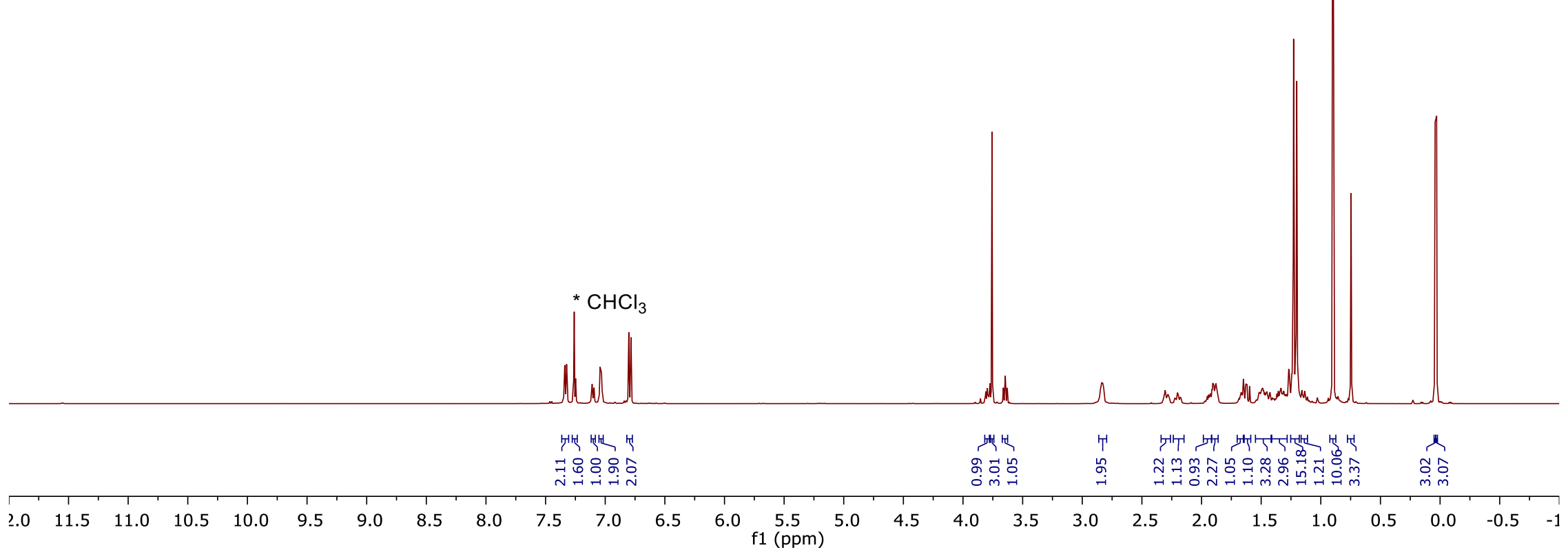




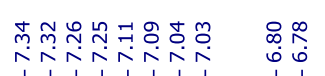

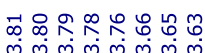

रो人̃⿰亻弋

ज1)

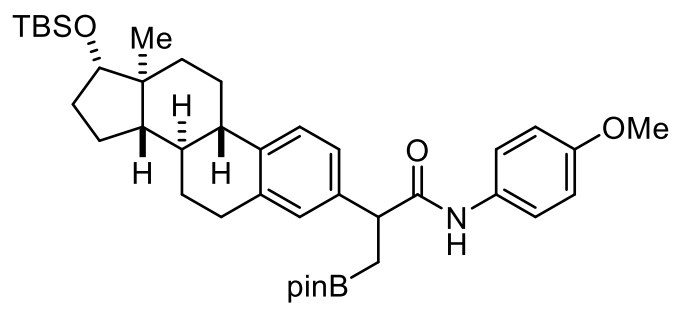

$3 \mathrm{pb}$

${ }^{1} \mathrm{H} \mathrm{NMR}\left(500 \mathrm{MHz}, \mathrm{CDCl}_{3}\right)$

aromatic region expansion

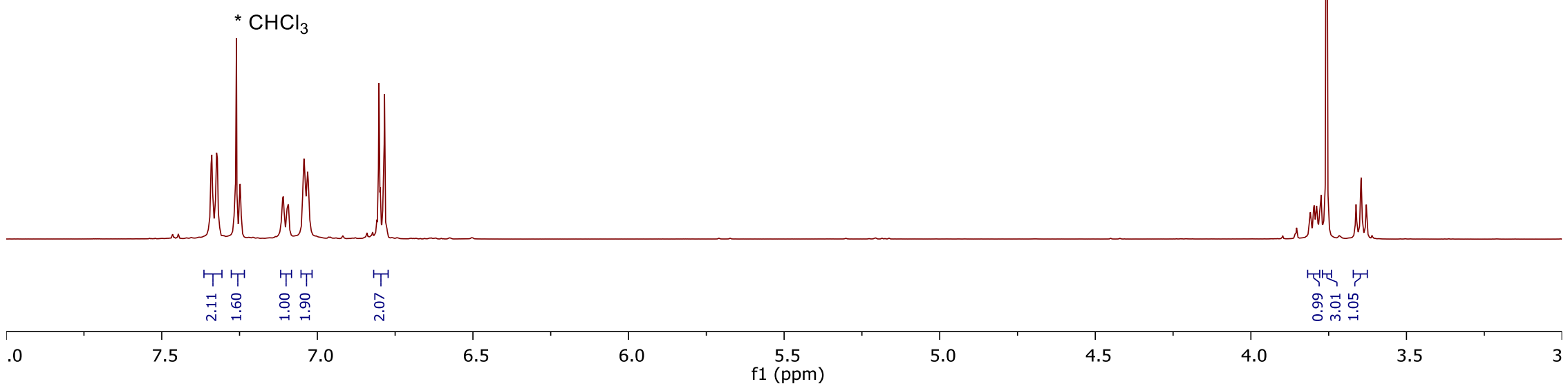




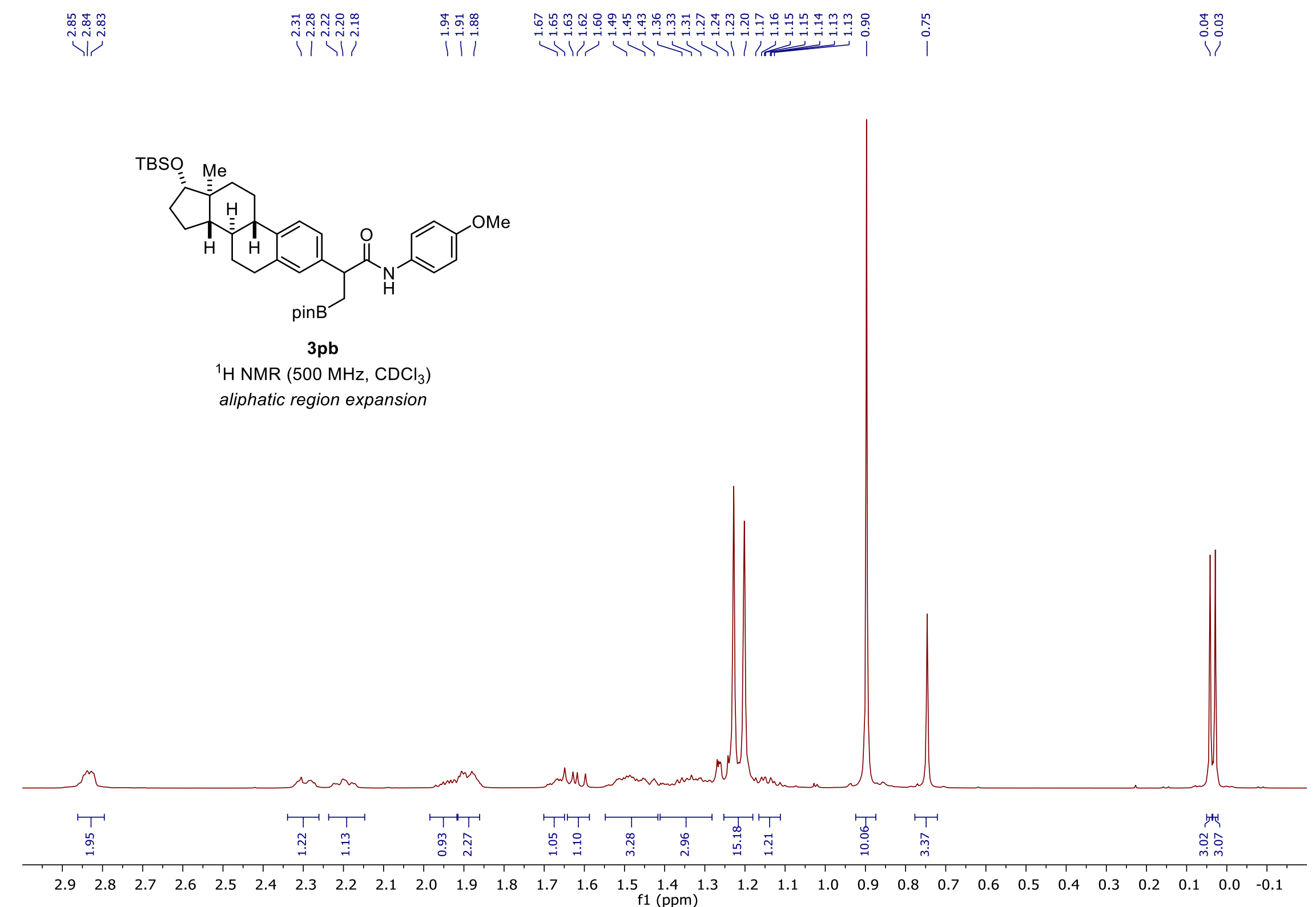




إن

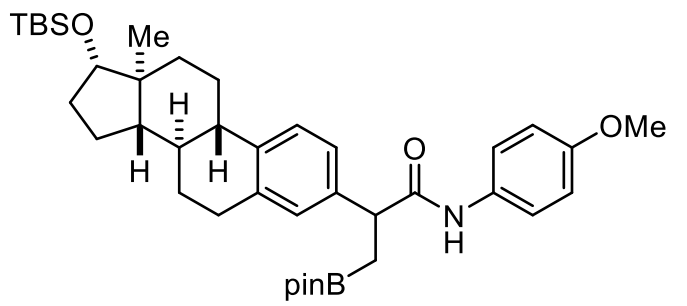

$3 p b$

${ }^{13} \mathrm{C}\left\{{ }^{1} \mathrm{H}\right\}$ NMR $\left(126 \mathrm{MHz}, \mathrm{CDCl}_{3}\right)$

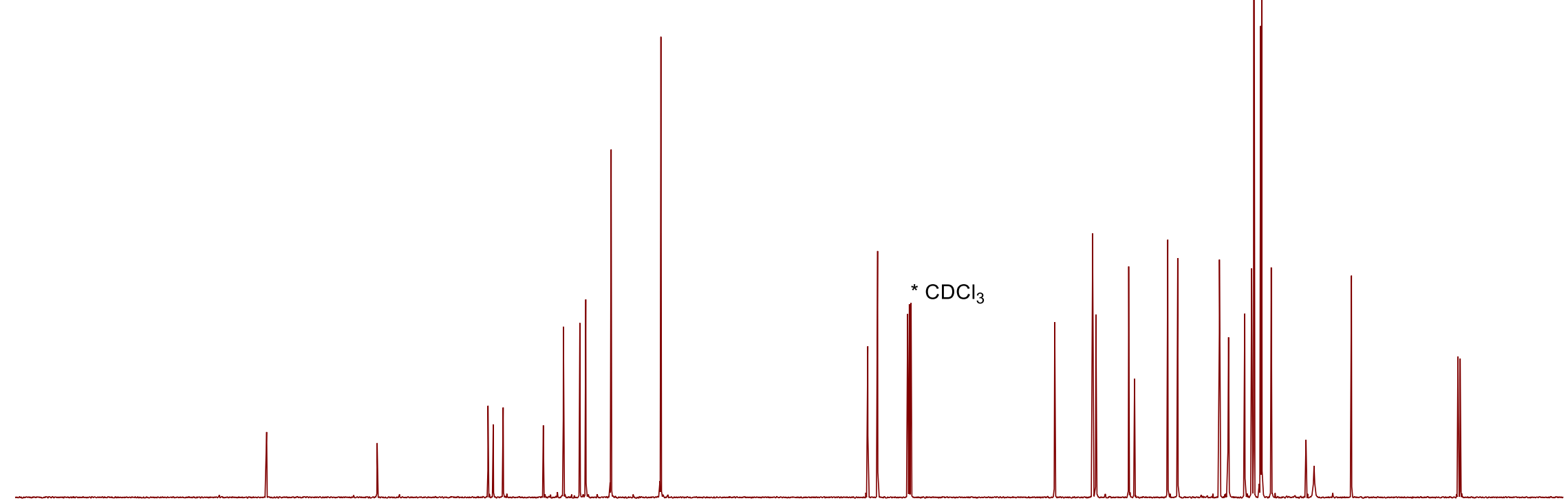

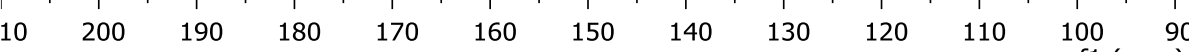

f1 (ppm) 


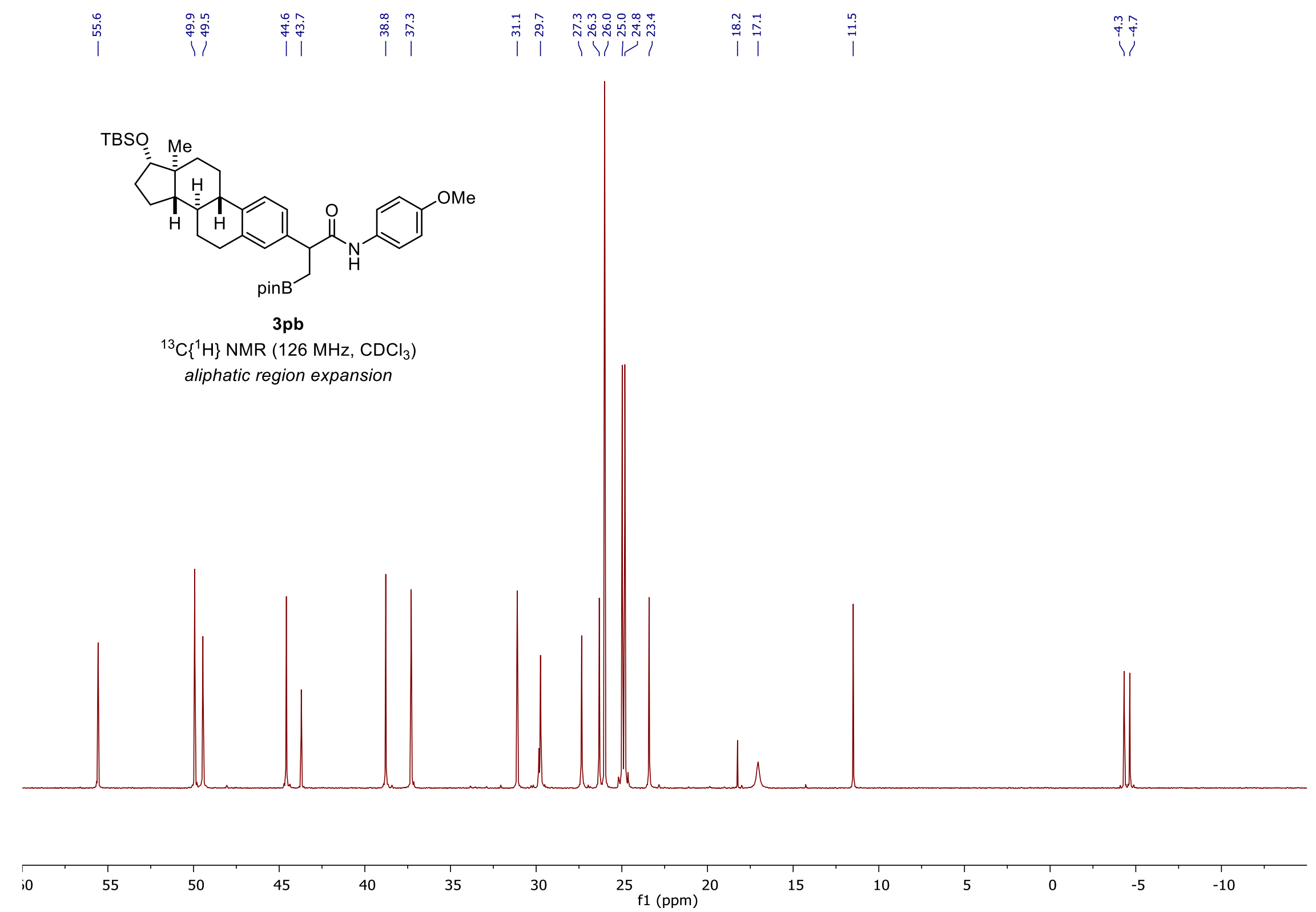




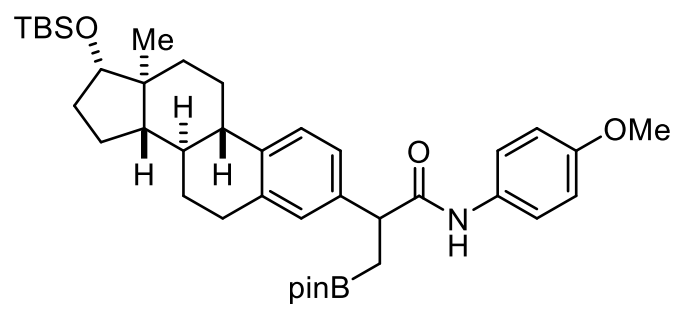

$3 p b$

${ }^{11} \mathrm{~B}$ NMR $\left(96 \mathrm{MHz}, \mathrm{CDCl}_{3}\right)$

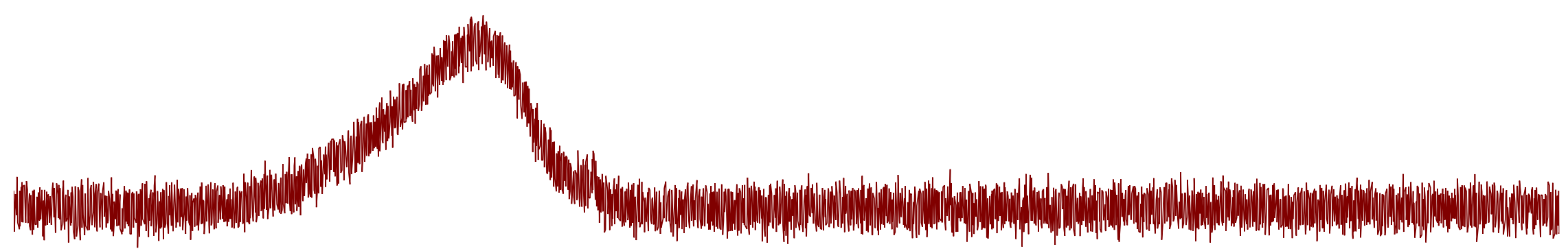

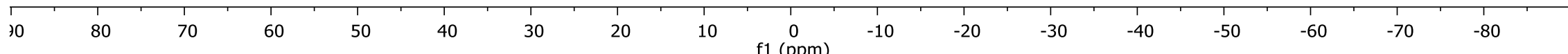




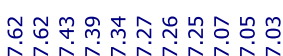

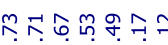

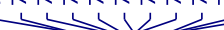

पil

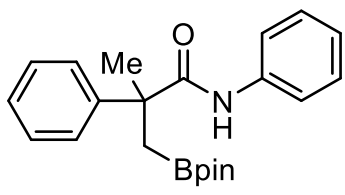

3qa

${ }^{1} \mathrm{H} \mathrm{NMR}\left(400 \mathrm{MHz}, \mathrm{CDCl}_{3}\right)$
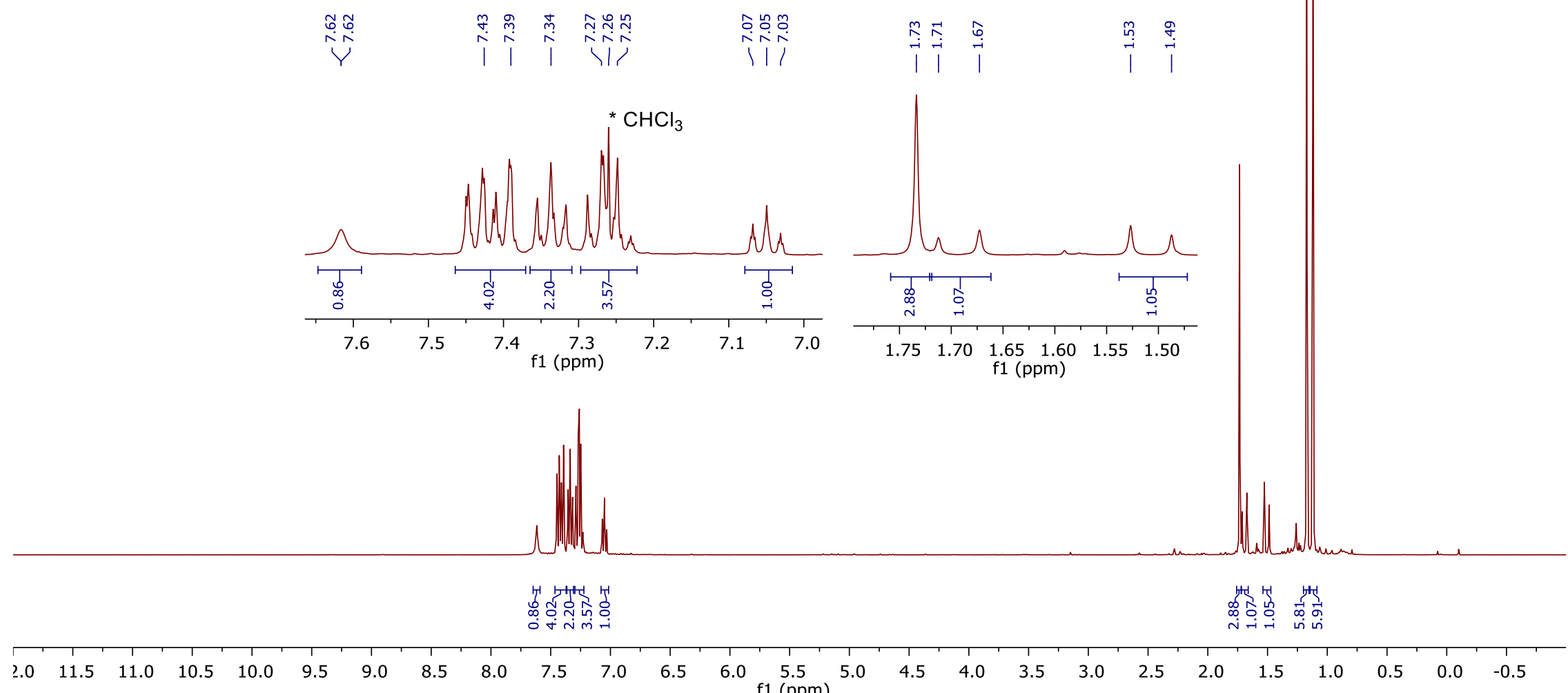

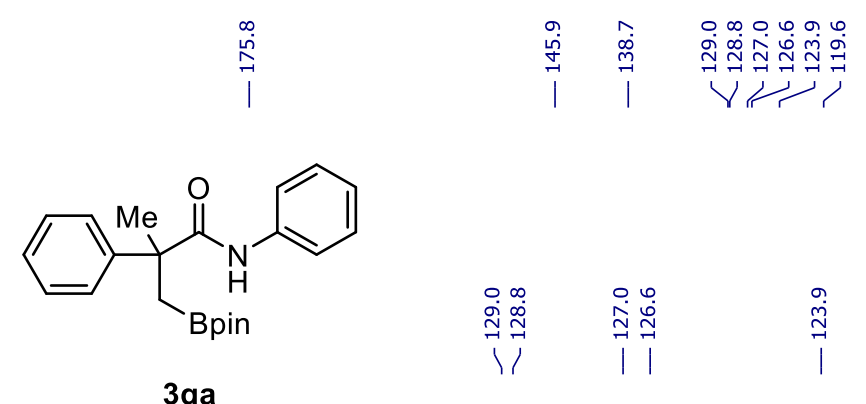

侢

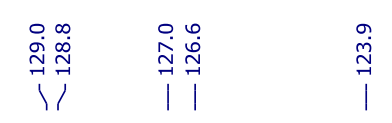

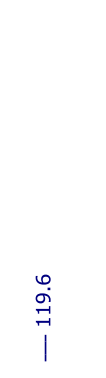

${ }^{13} \mathrm{C}\left\{{ }^{1} \mathrm{H}\right\}$ NMR $\left(75 \mathrm{MHz}, \mathrm{CDCl}_{3}\right)$

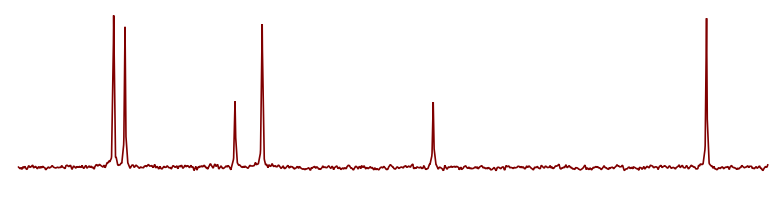

$\begin{array}{lllllllllllll}130 & 129 & 128 & 127 & 126 & 125 & 124 & 123 & 122 & 121 & 120 & 119\end{array}$ f1 (ppm)

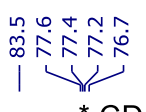

* $\mathrm{CDCl}_{3}$

I

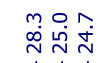

|

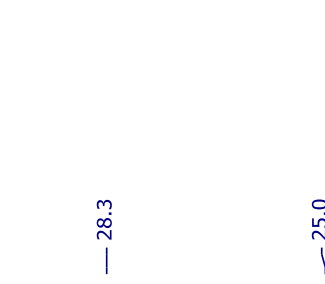

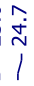
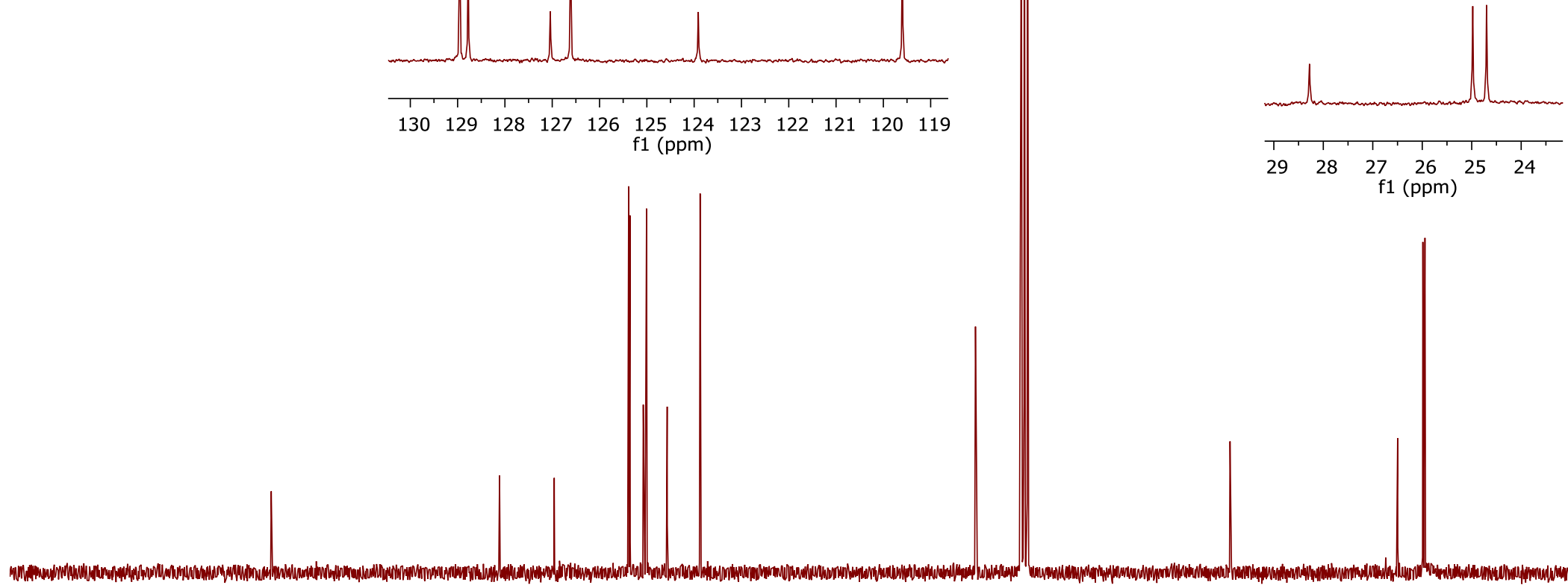

(ppm)

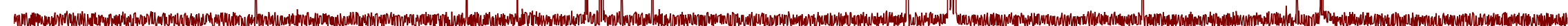

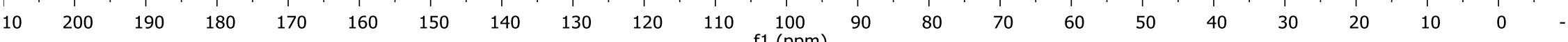




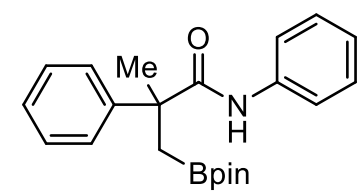

$3 q a$

${ }^{11} \mathrm{~B}$ NMR $\left(96 \mathrm{MHz}, \mathrm{CDCl}_{3}\right)$

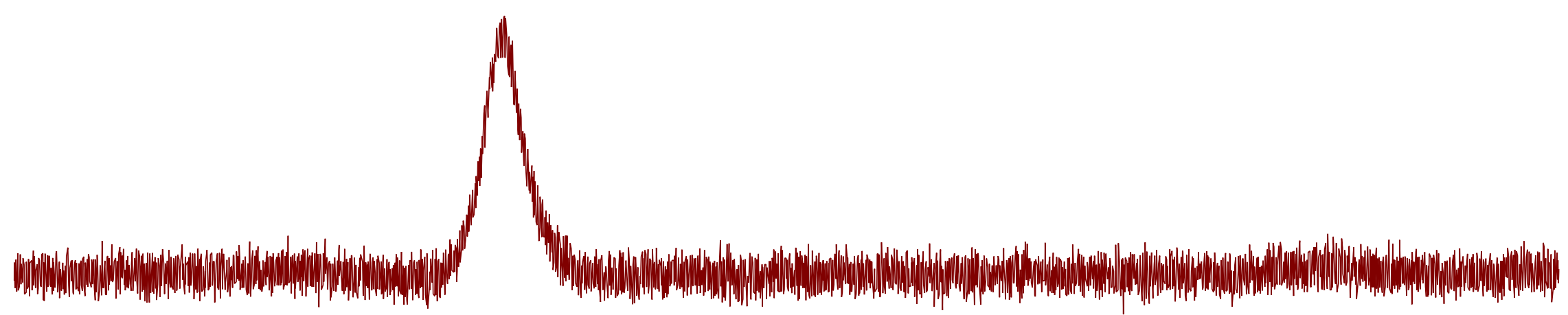

$\begin{array}{llllllllllllllllllllllllllllllllllllllllllllllllll}75 & 70 & 65 & 60 & 55 & 50 & 45 & 40 & 35 & 30 & 25 & 20 & 15 & 10 & 5 & 0 & -5 & -10 & -15 & -20 & -25 & -30 & -35 & -40 & -45 & -50 & -55 & -60 & -65 & \end{array}$




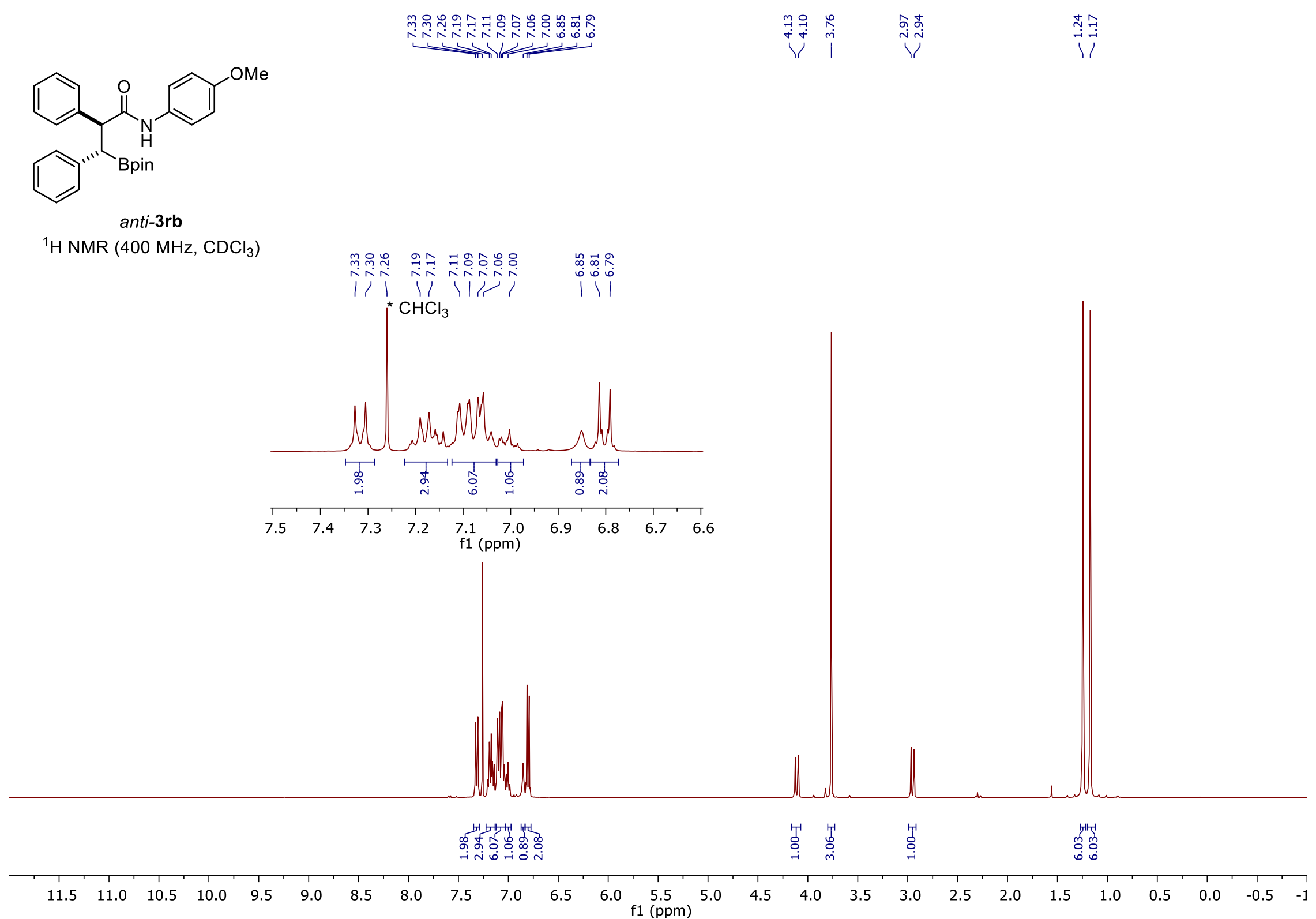




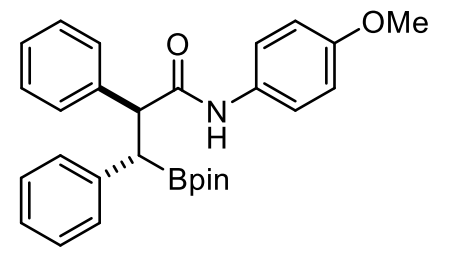

anti-3rb

${ }^{13} \mathrm{C}\left\{{ }^{1} \mathrm{H}\right\}$ NMR $\left(101 \mathrm{MHz}, \mathrm{CDCl}_{3}\right)$

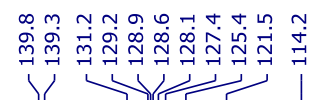

mis

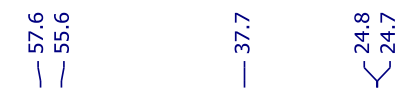

$\mathrm{CDCl}_{3}$

|

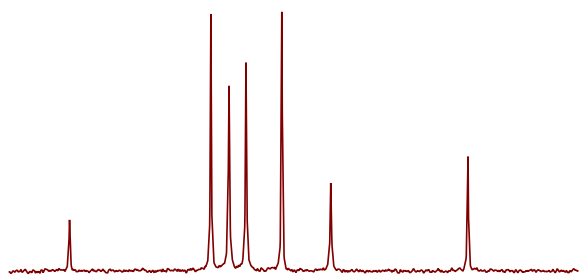

$\begin{array}{lllllllll}132 & 131 & 130 & 129 & 128 & 127 & 126 & 125 & 124\end{array}$ f1 (ppm)
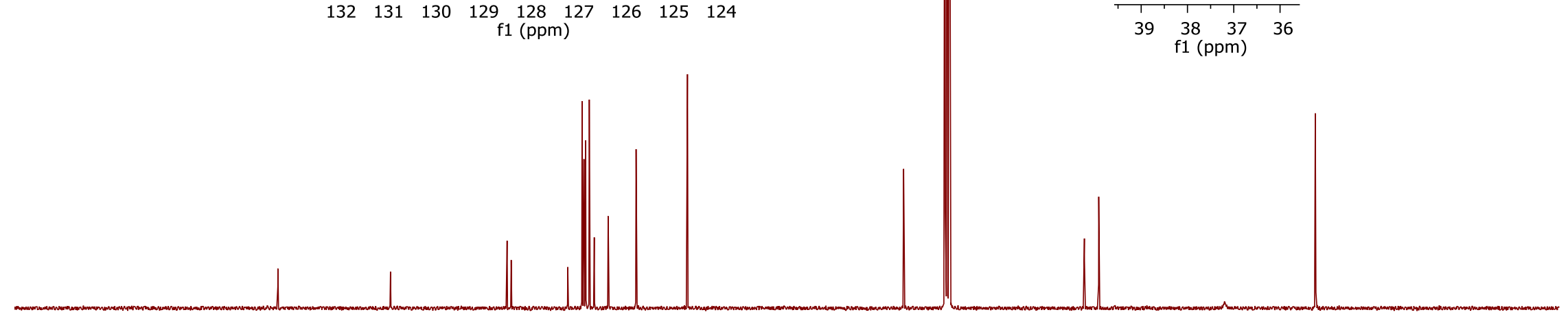

$10 \quad 200$

170

160

$150 \quad 140$

$130 \quad 120$

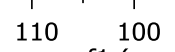

f1 $\stackrel{100}{(\mathrm{ppm})}$

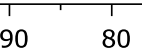

70

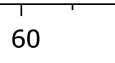




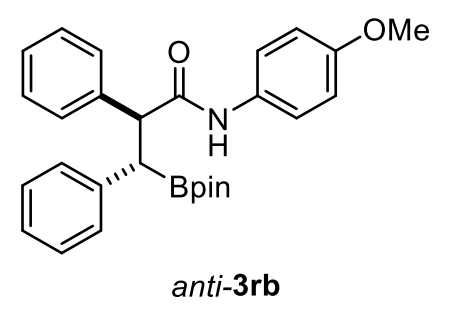

${ }^{11} \mathrm{~B}$ NMR $\left(96 \mathrm{MHz}, \mathrm{CDCl}_{3}\right)$

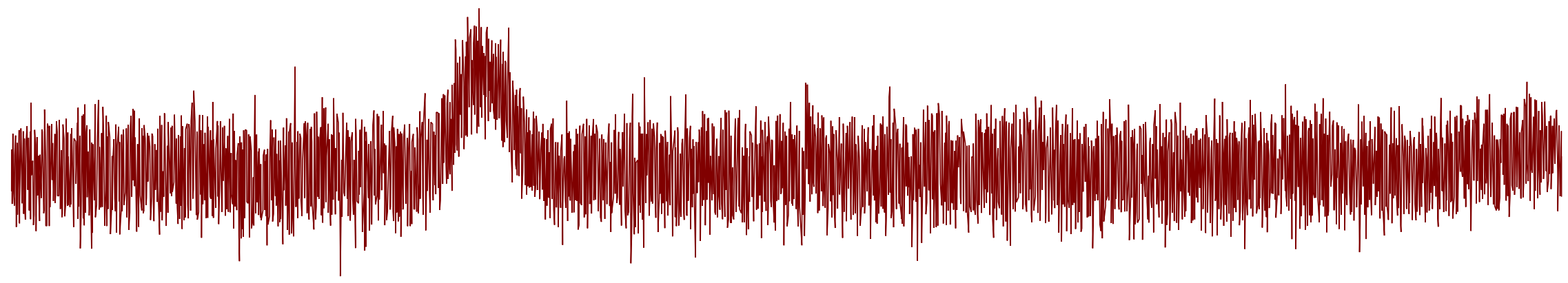

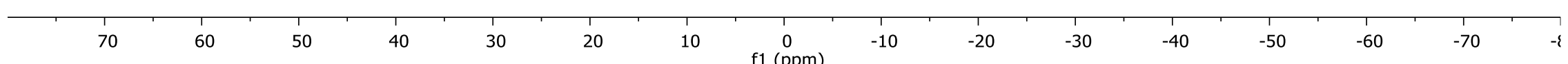




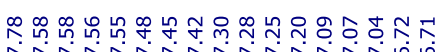
L $Y$

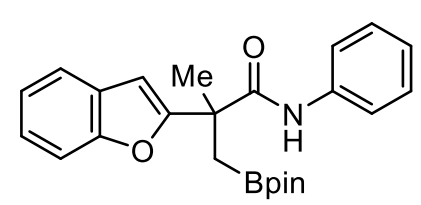

3 sa

${ }^{1} \mathrm{H}$ NMR $\left(300 \mathrm{MHz}, \mathrm{CD}_{2} \mathrm{Cl}_{2}\right)$
ำ

-1/1/

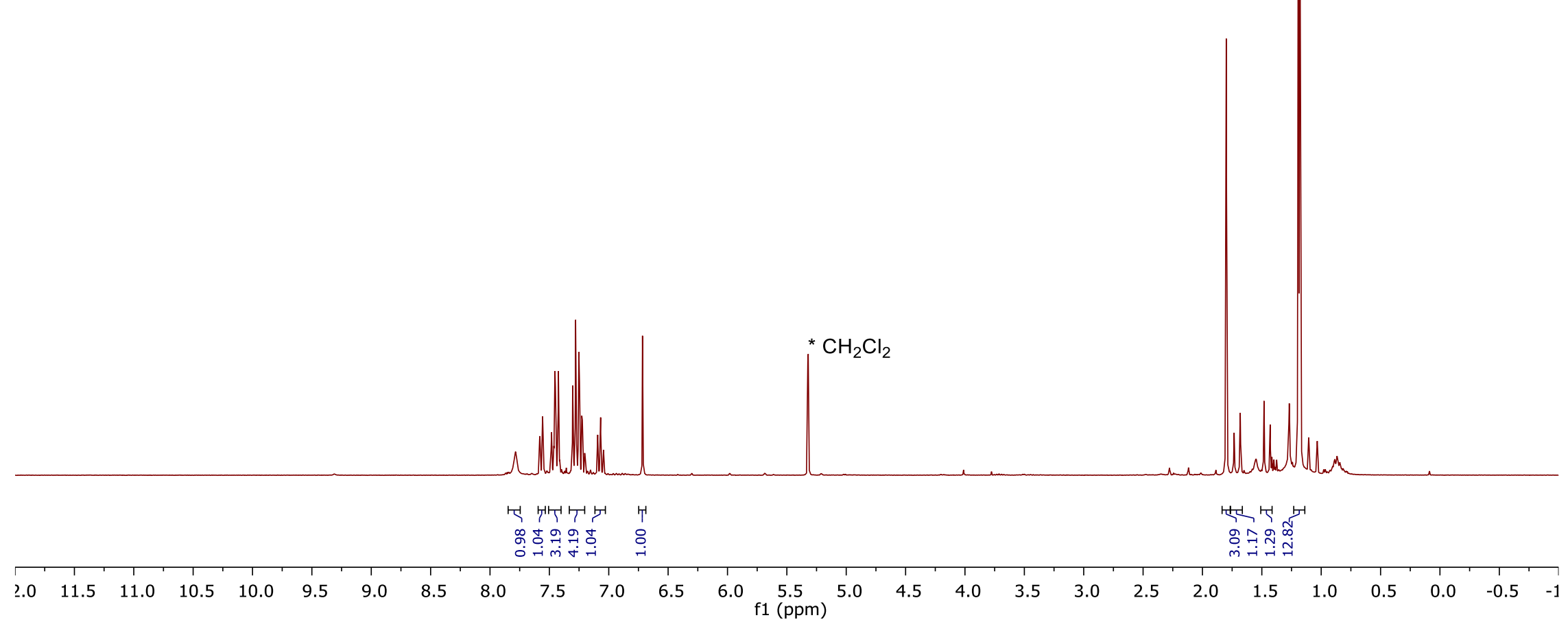




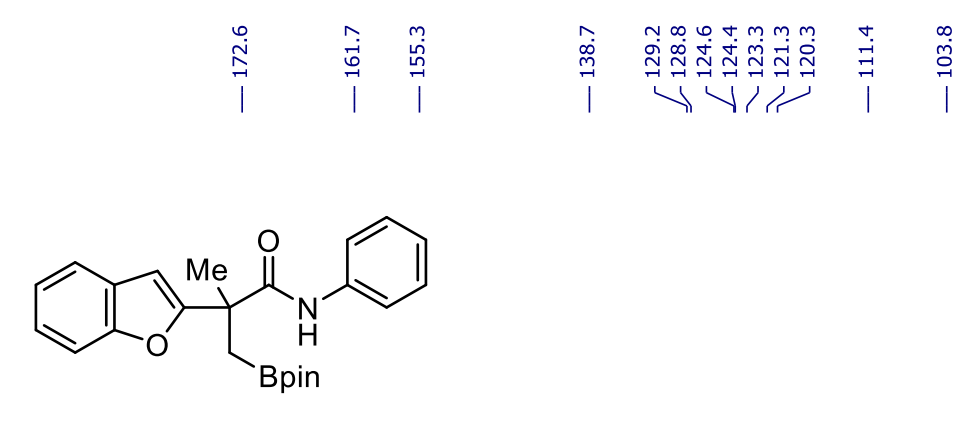

3sa

${ }^{13} \mathrm{C}\left\{{ }^{1} \mathrm{H}\right\}$ NMR $\left(75 \mathrm{MHz}, \mathrm{CD}_{2} \mathrm{Cl}_{2}\right)$
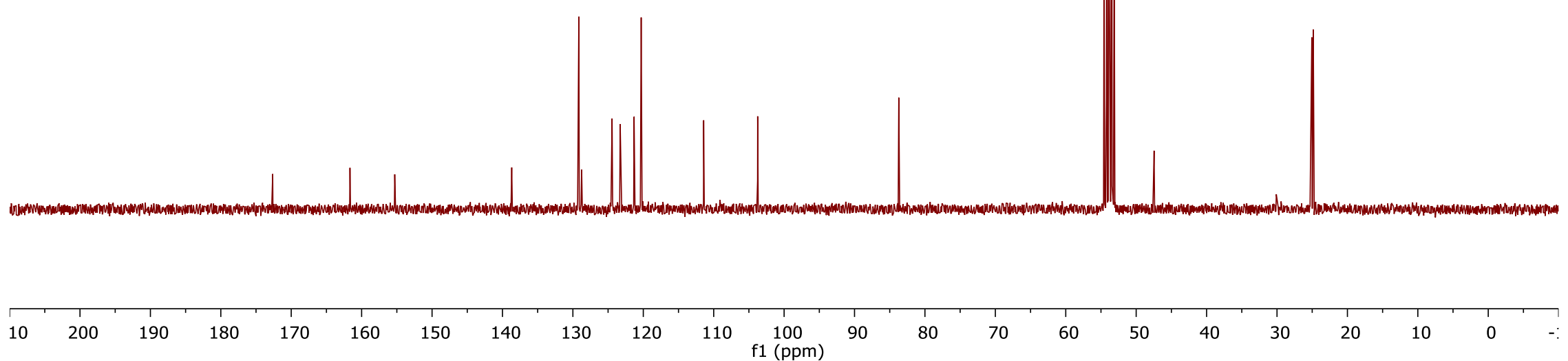
$\stackrel{0}{\dot{m}}$

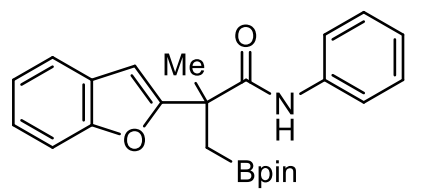

3sa

${ }^{11} \mathrm{~B}$ NMR $\left(96 \mathrm{MHz}, \mathrm{CD}_{2} \mathrm{Cl}_{2}\right)$

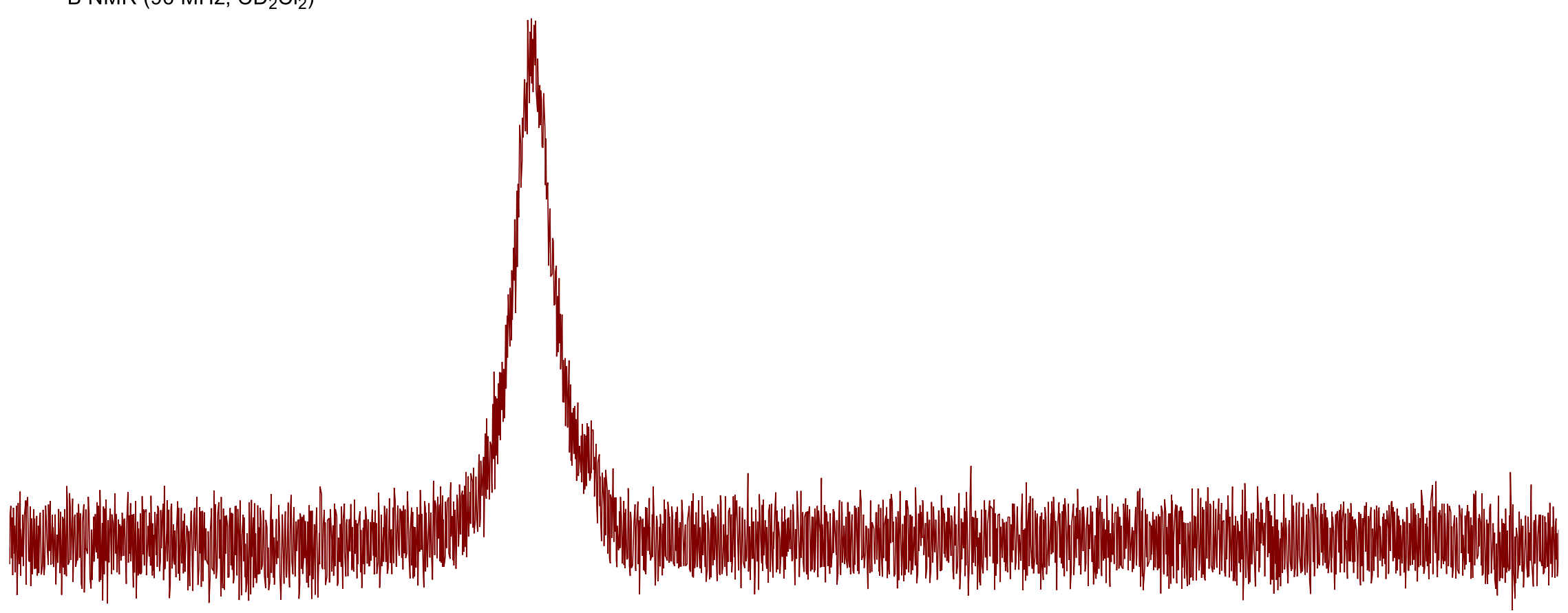

$\begin{array}{lllllllllllllllllllllllllllllllllllllllllllllll}75 & 70 & 65 & 60 & 55 & 50 & 45 & 40 & 35 & 30 & 25 & 20 & 15 & 10 & 5 & 0 & -5 & -10 & -15 & -20 & -25 & -30 & -35 & -40 & -45 & -50 & -55 & -1\end{array}$




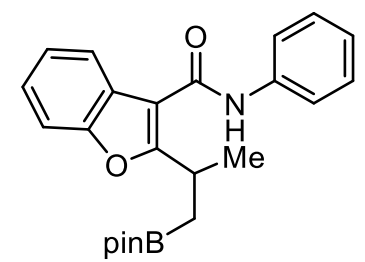

3sa'

${ }^{1} \mathrm{H}$ NMR (400 MHz, $\mathrm{CDCl}_{3}$ )

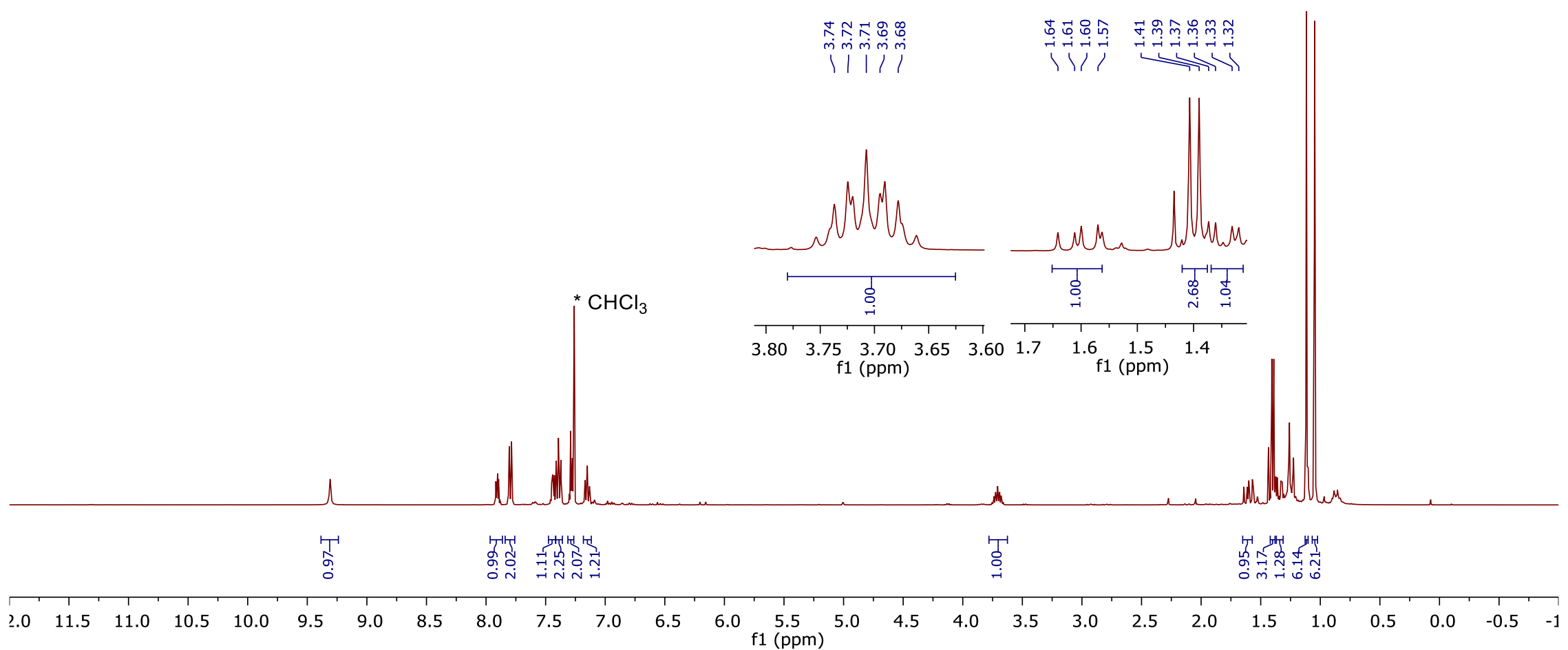




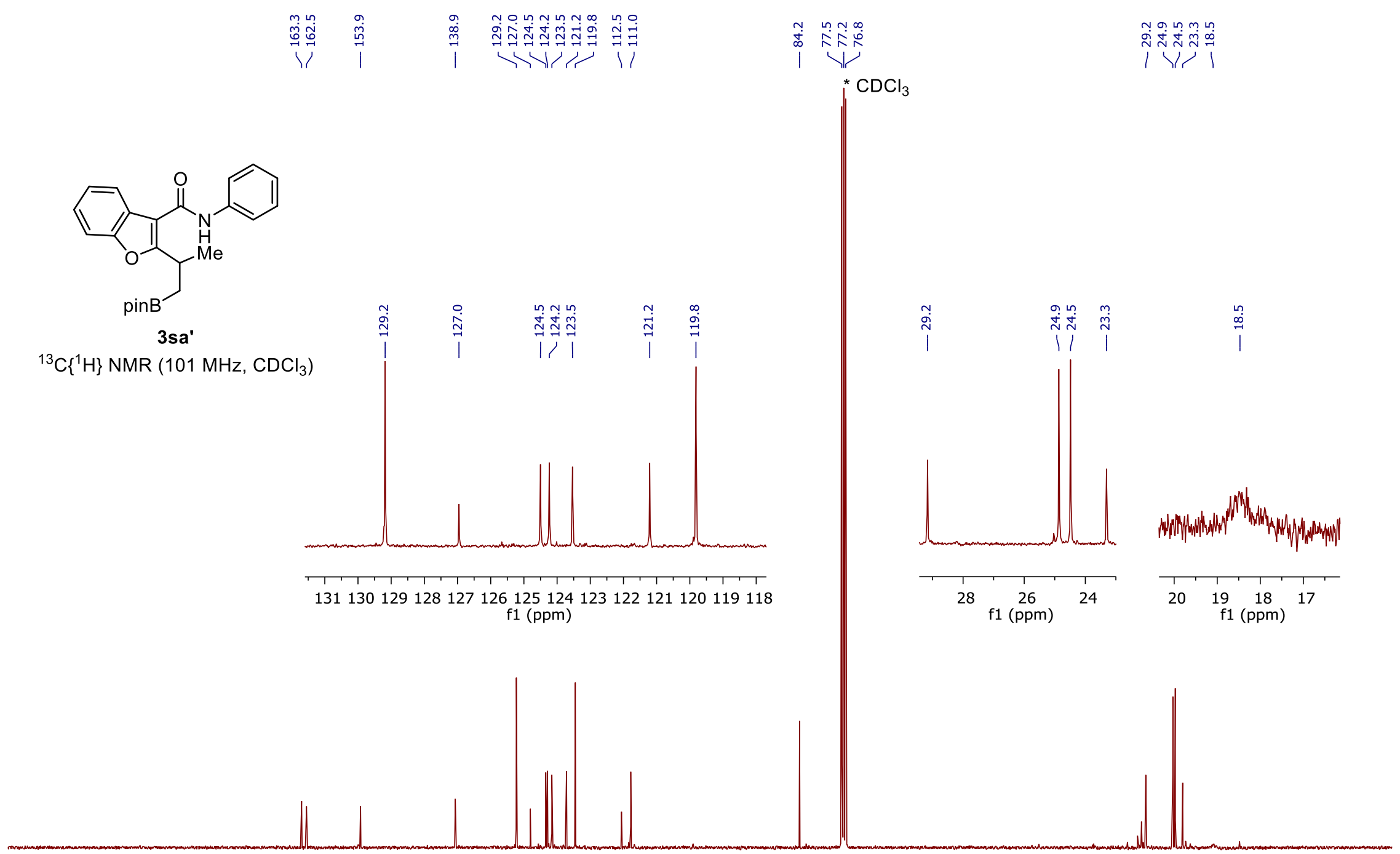

$\begin{array}{llllll}150 & 140 & 130 & 120 & 110 & 100 \\ \mathrm{f} 1(\mathrm{ppm})\end{array}$

$90 \quad 80$

$70 \quad 60$

50

$40 \quad 30$

20

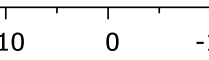




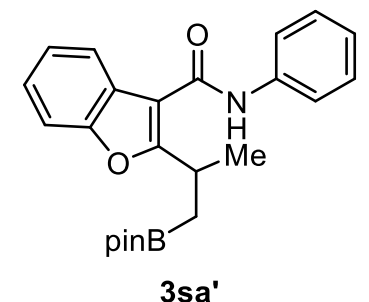

${ }^{11} \mathrm{~B}$ NMR $\left(96 \mathrm{MHz}, \mathrm{CDCl}_{3}\right)$

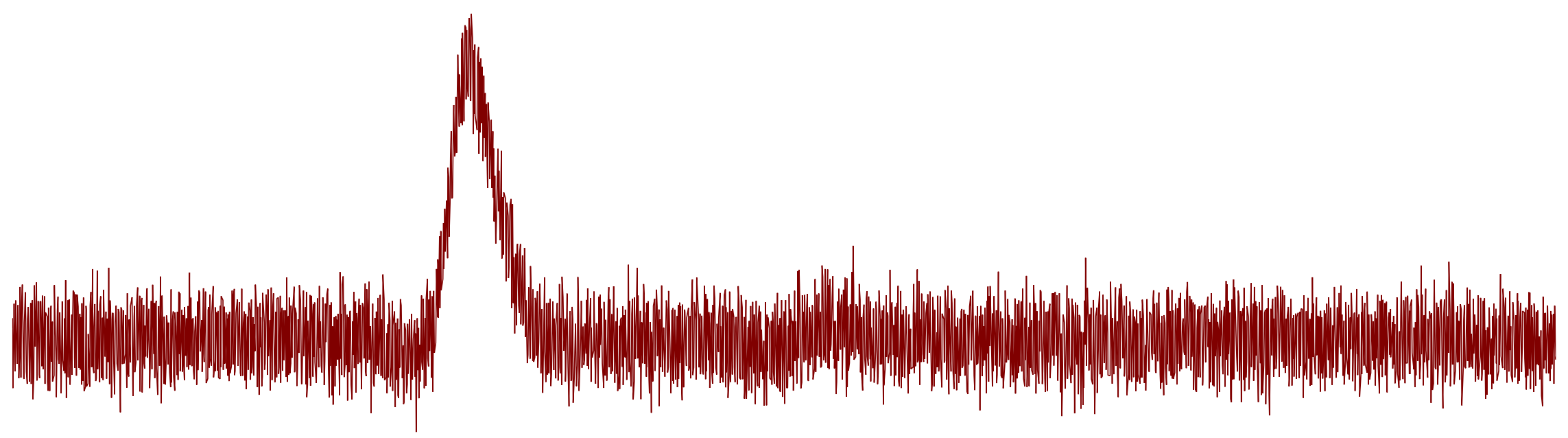

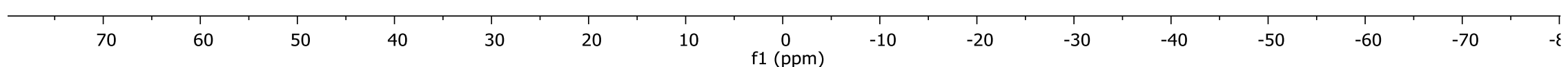




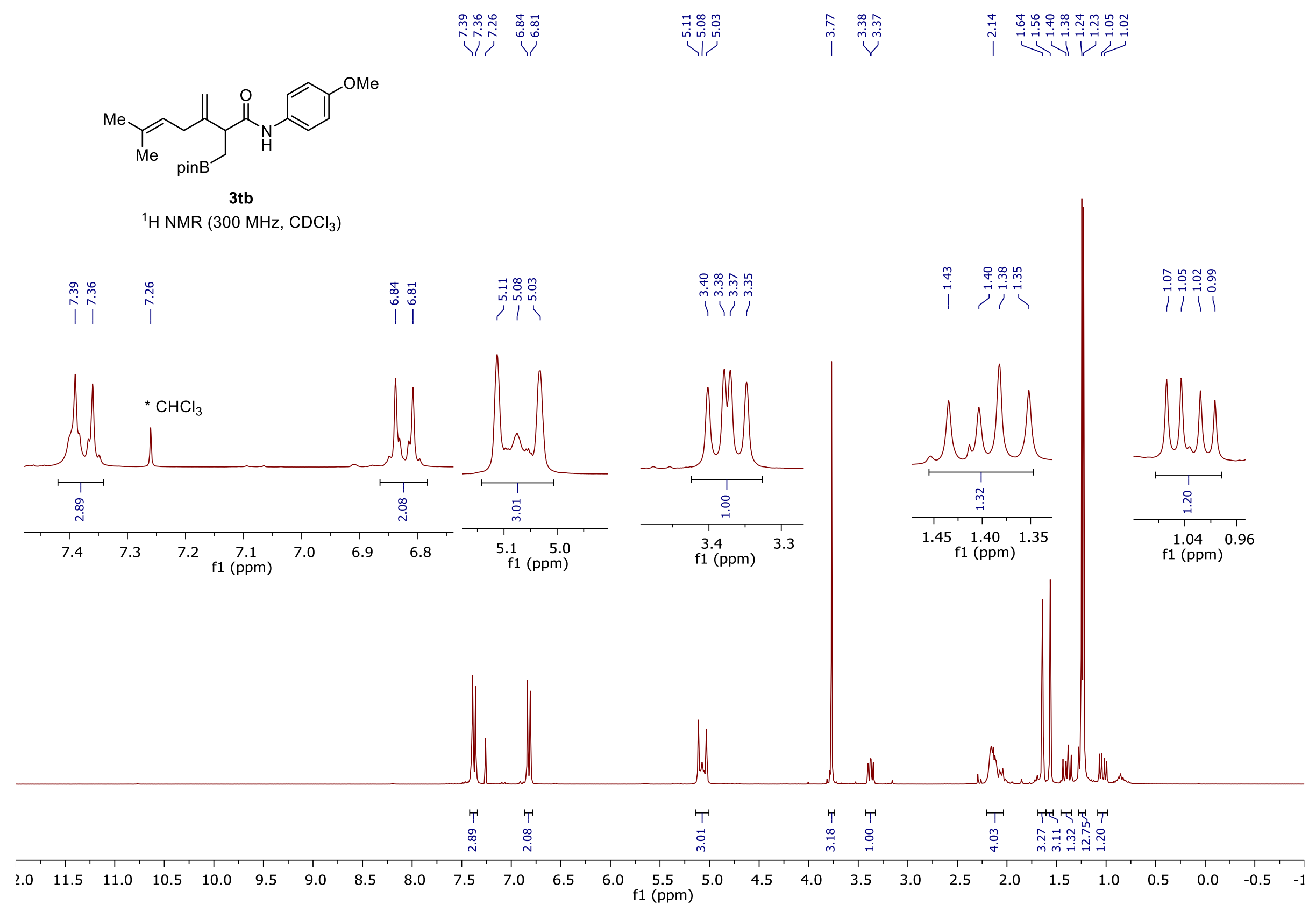



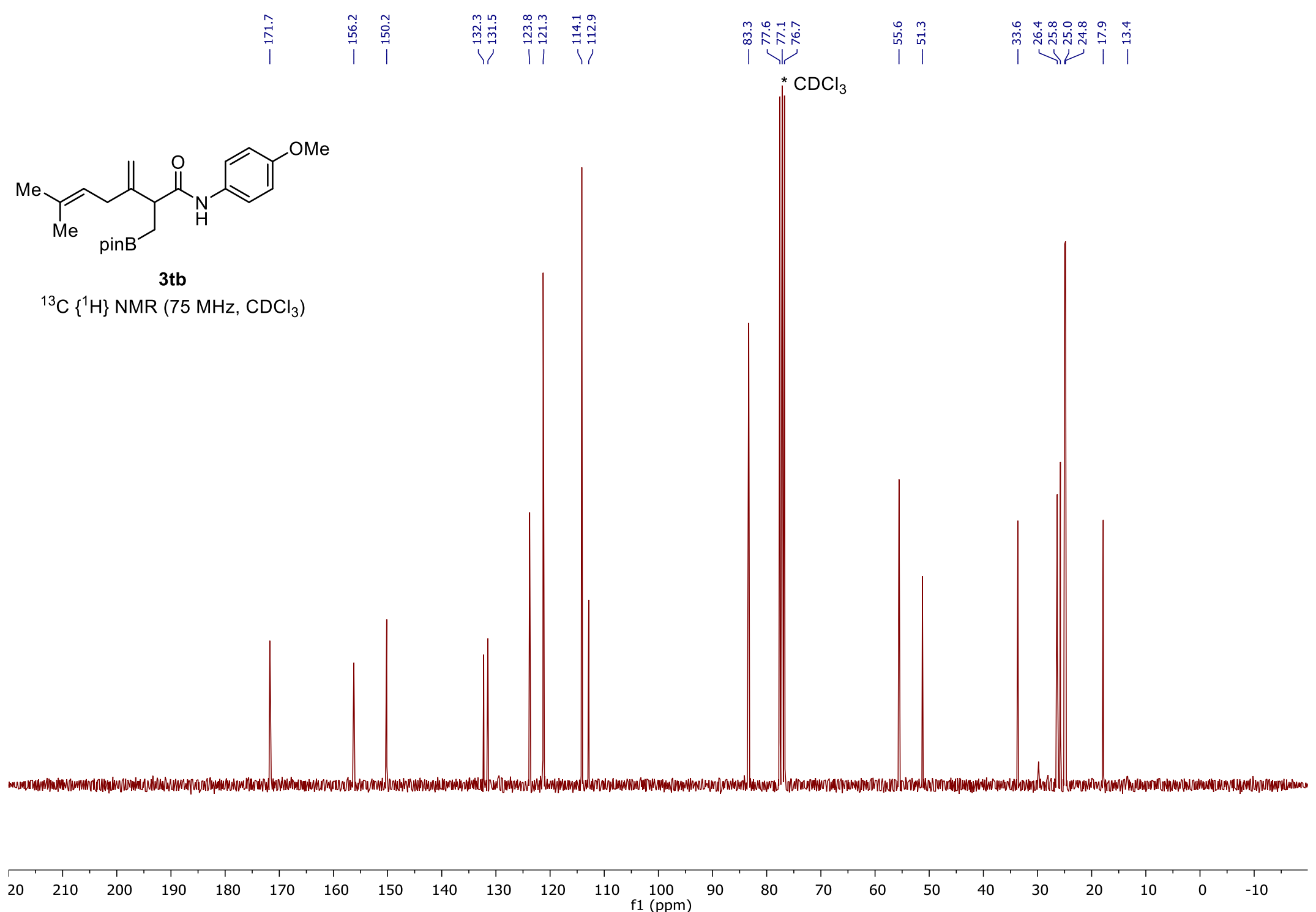


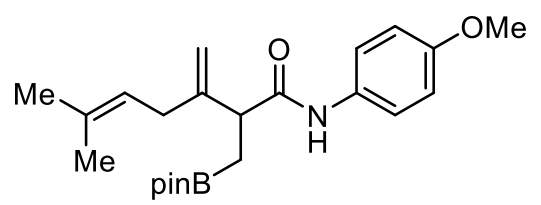

3tb

${ }^{11} \mathrm{~B}$ NMR (96 MHz, $\mathrm{CDCl}_{3}$ )

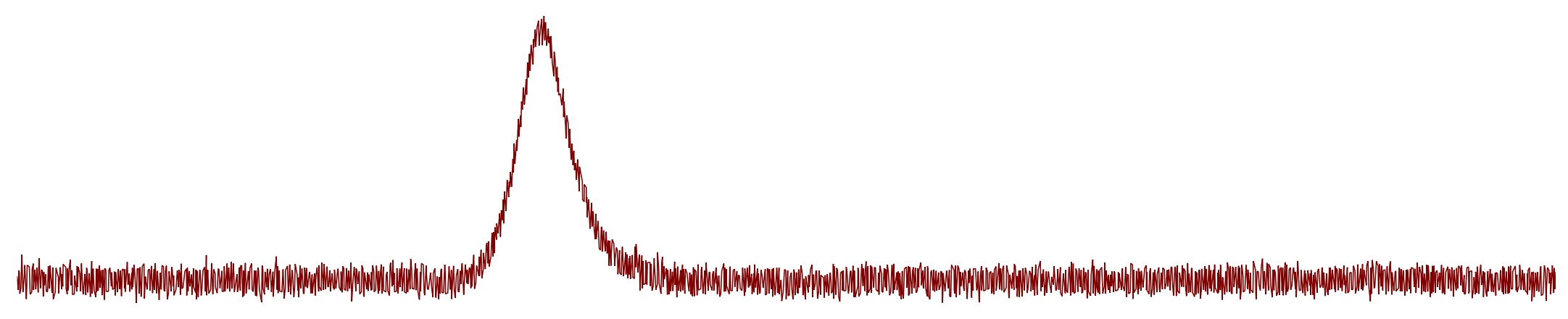




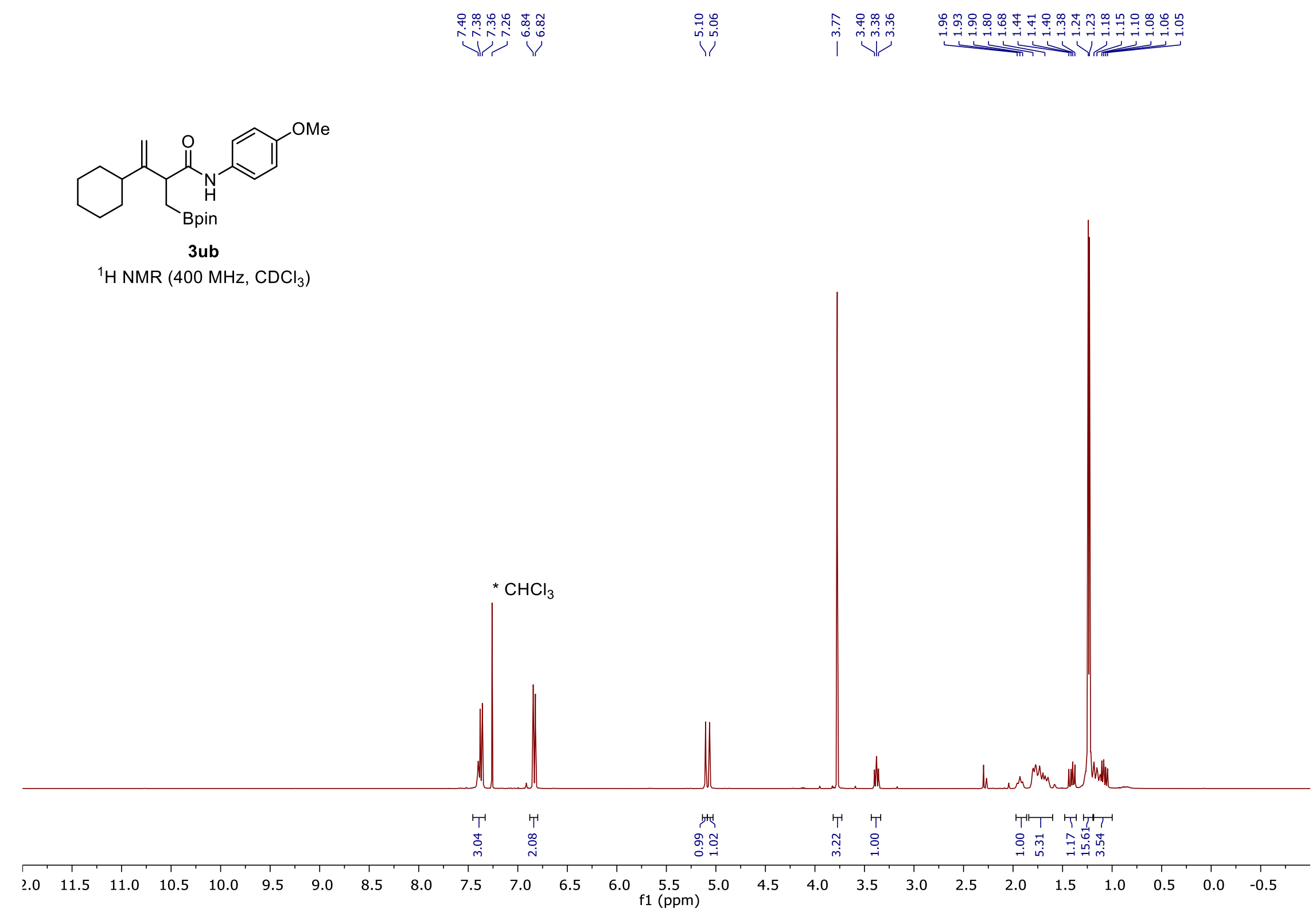




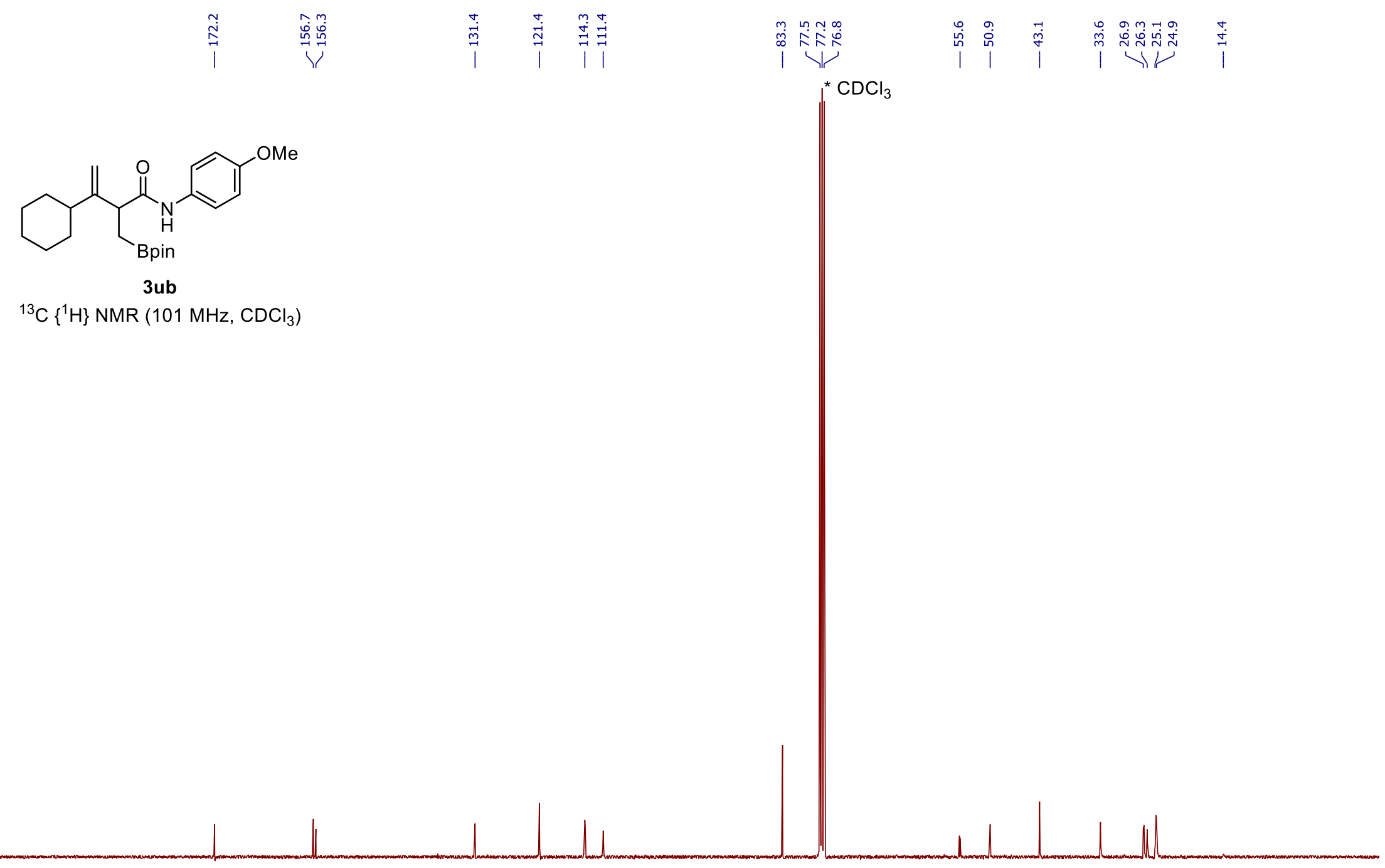




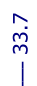

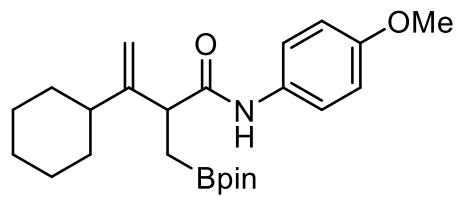

$3 u b$

${ }^{11} \mathrm{~B}$ NMR $\left(96 \mathrm{MHz}, \mathrm{CDCl}_{3}\right.$ )

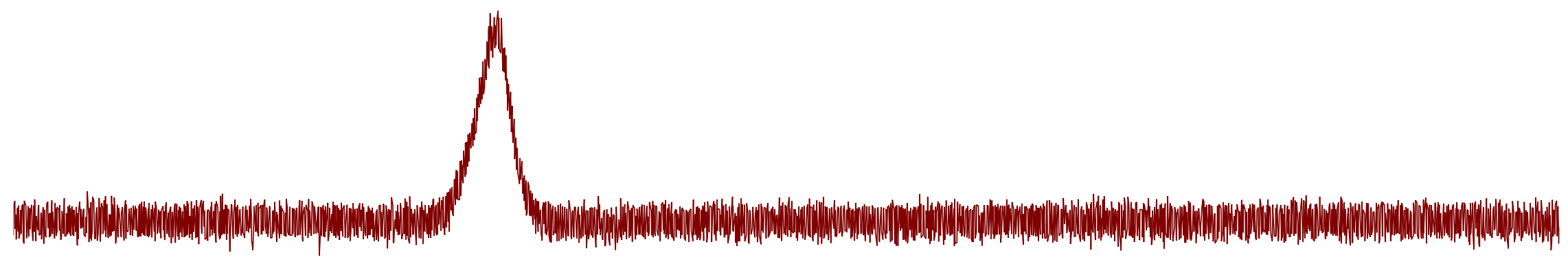



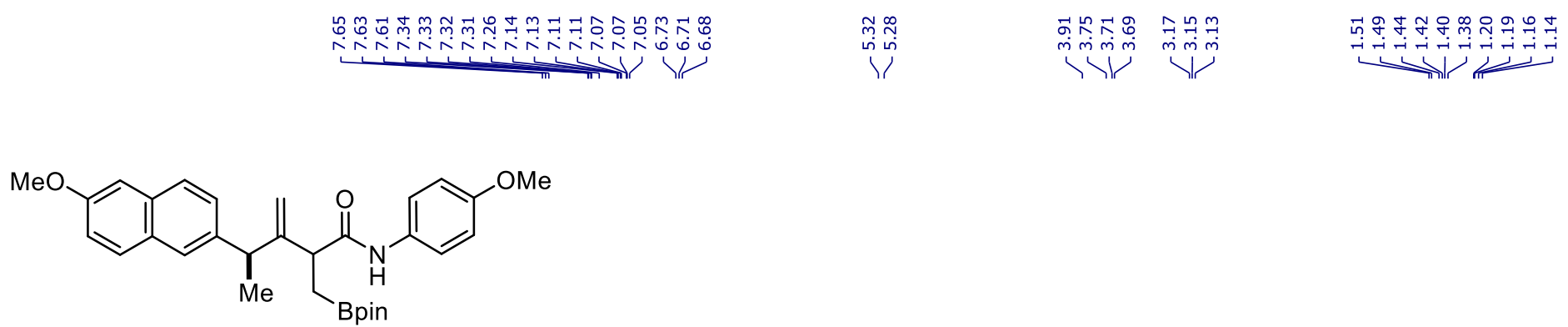

$3 \mathbf{v b}(d r 5: 1)$

${ }^{1} \mathrm{H}$ NMR (400 MHz, $\mathrm{CDCl}_{3}$ )

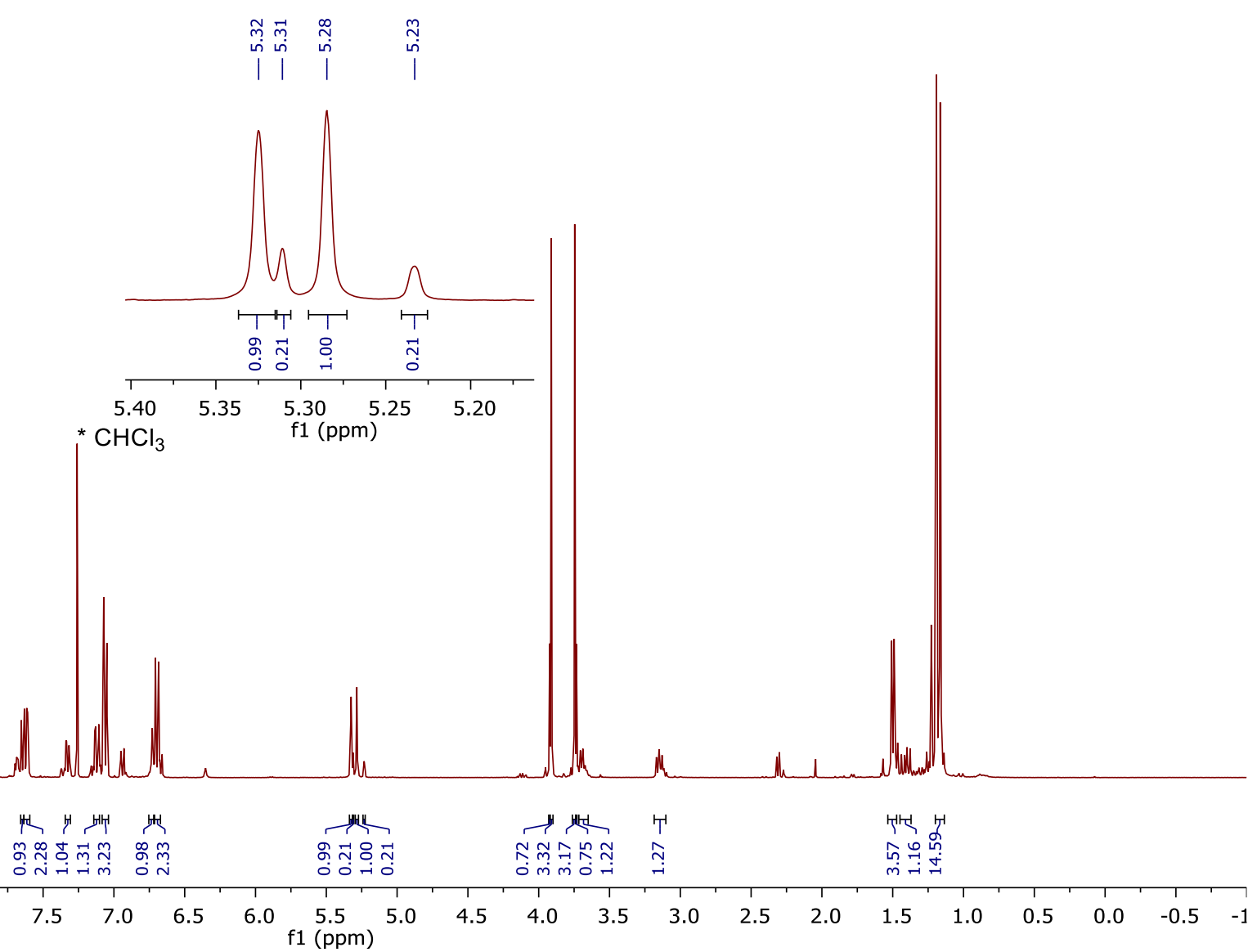



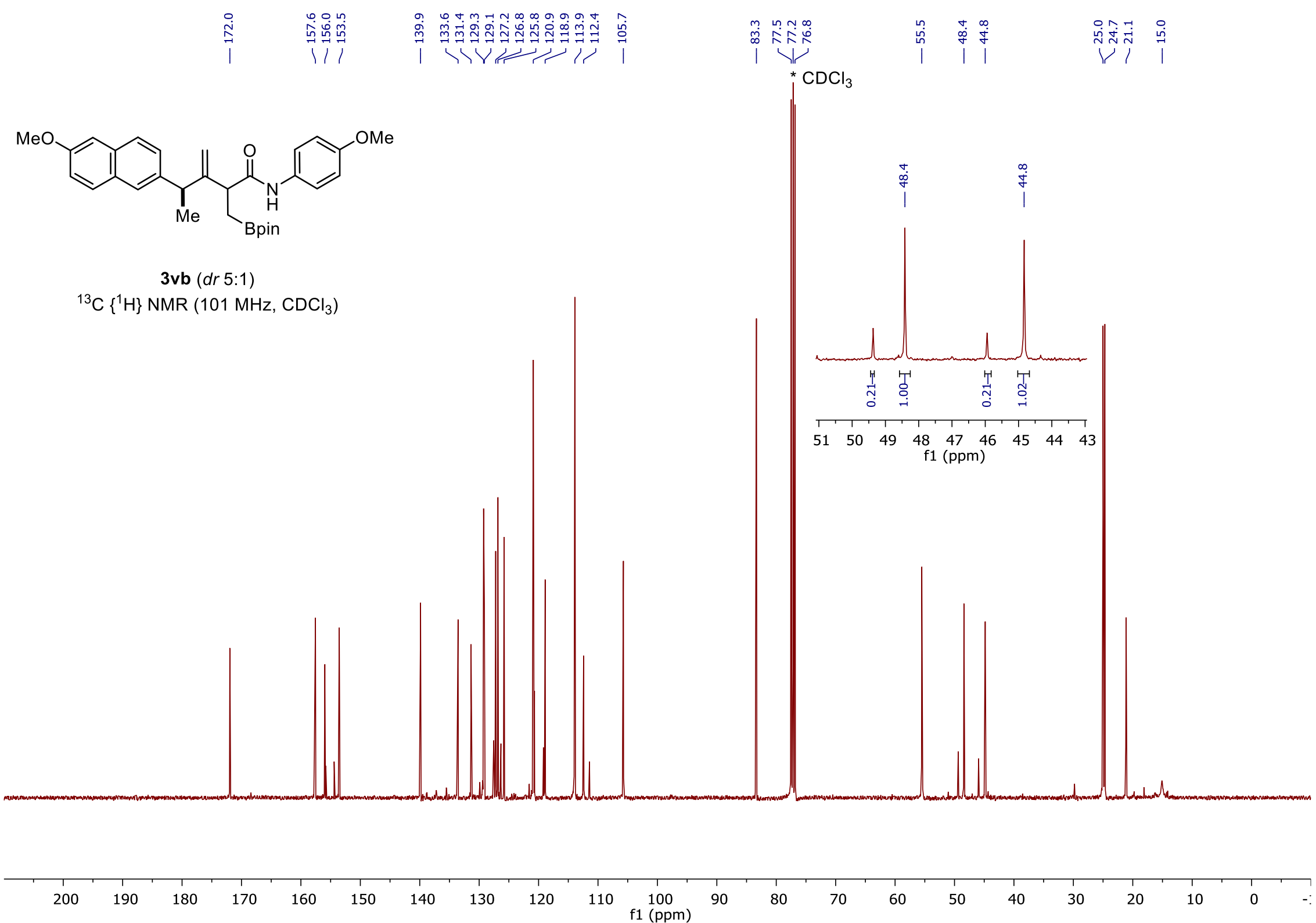


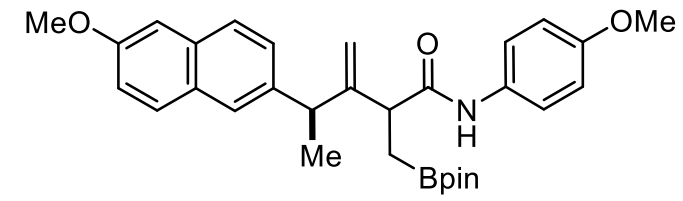

3vb (dr 5:1)

${ }^{11} \mathrm{~B}$ NMR $\left(128 \mathrm{MHz}, \mathrm{CDCl}_{3}\right)$
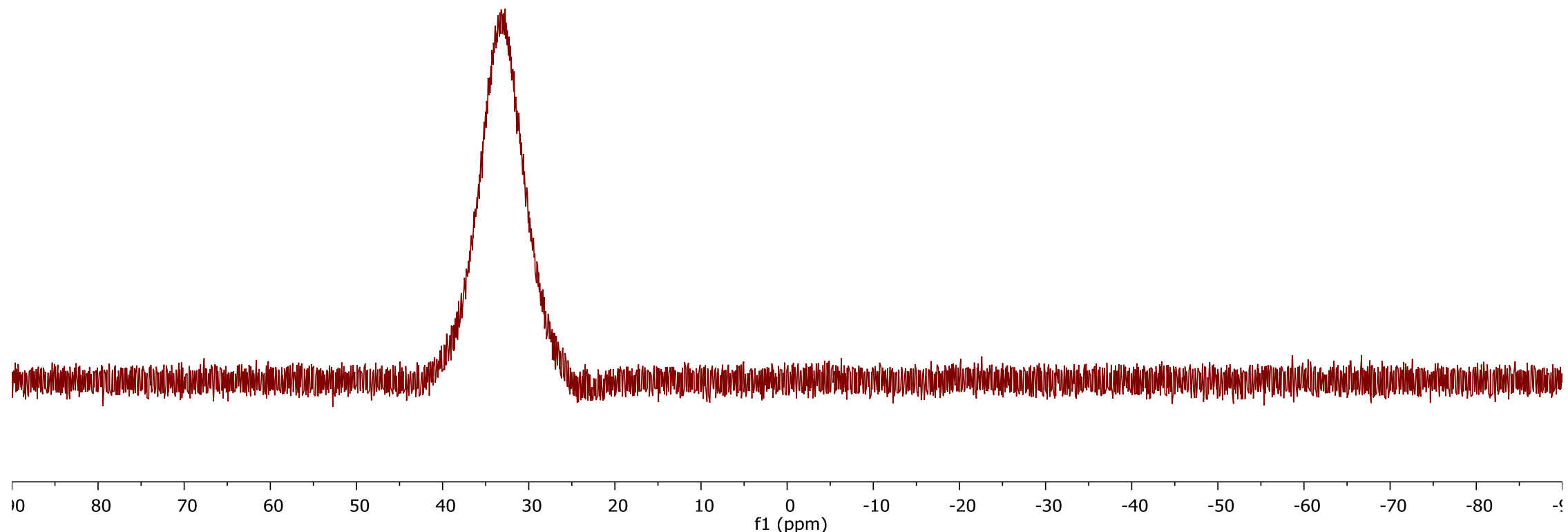


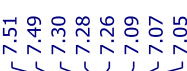

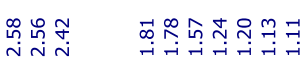

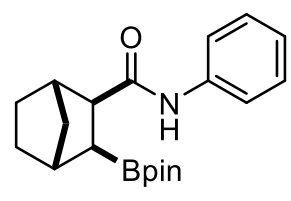

3wa

${ }^{1} \mathrm{H}$ NMR $\left(300 \mathrm{MHz}, \mathrm{CDCl}_{3}\right)$

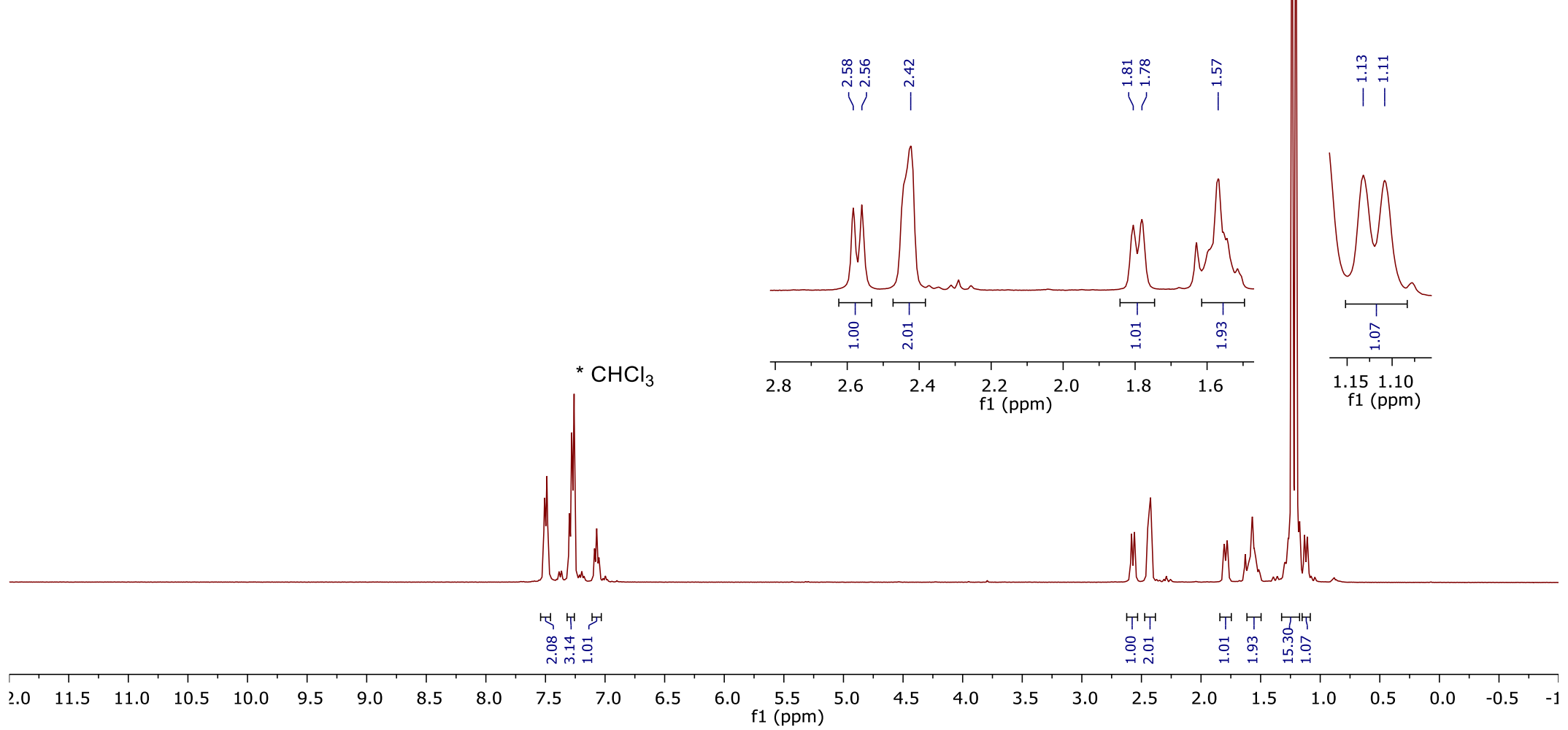


Supporting Information

S278
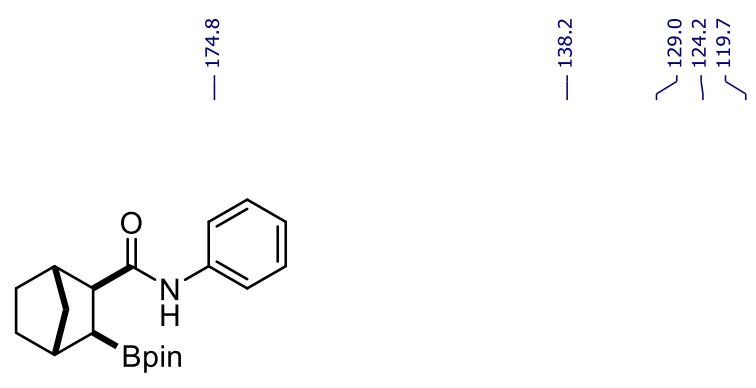

3wa

${ }^{13} \mathrm{C}\left\{{ }^{1} \mathrm{H}\right\}$ NMR $\left(75 \mathrm{MHz}, \mathrm{CDCl}_{3}\right)$

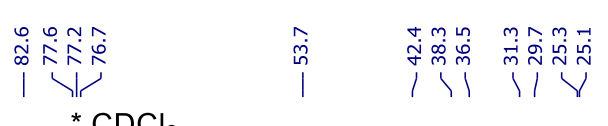

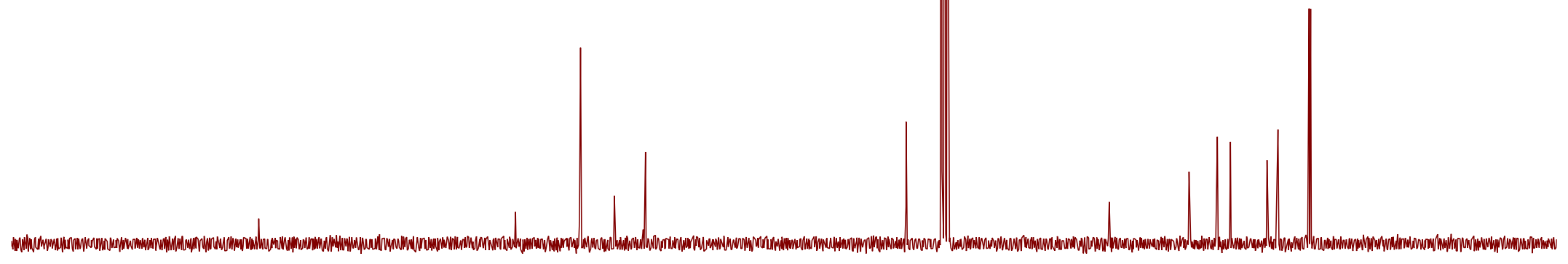

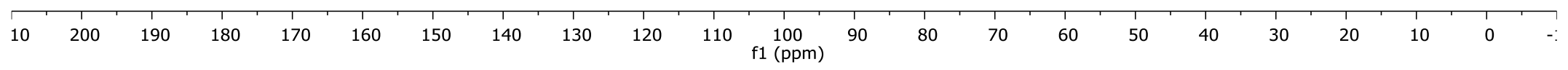




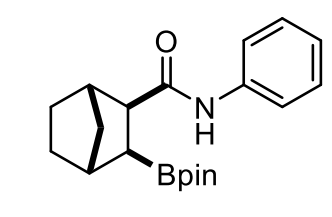

ì

${ }^{11} \mathrm{~B}$ NMR $\left(96 \mathrm{MHz}, \mathrm{CDCl}_{3}\right)$

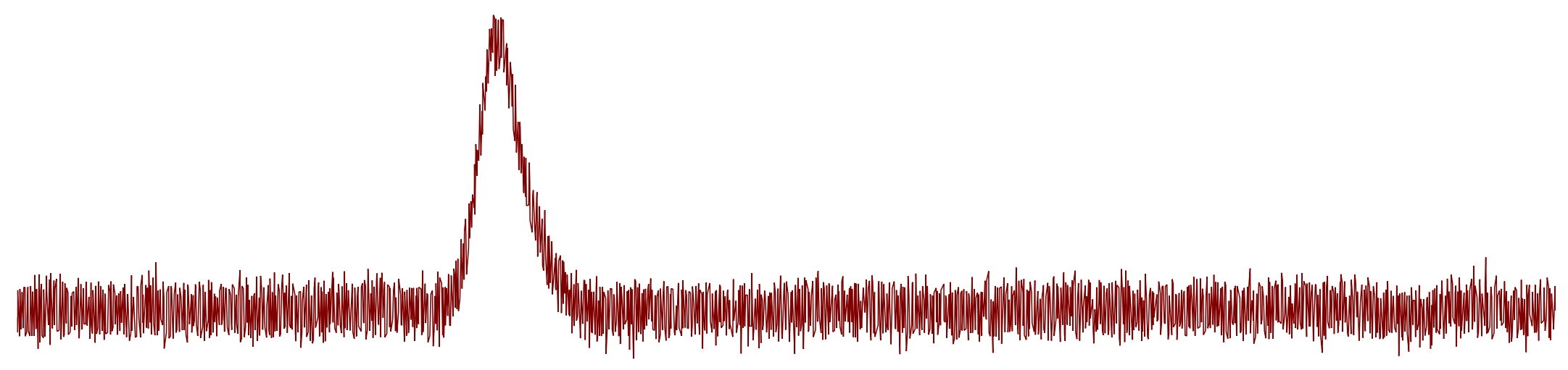

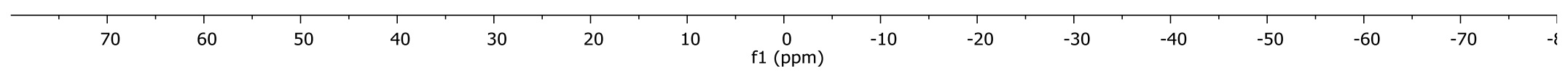




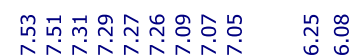

i্লj

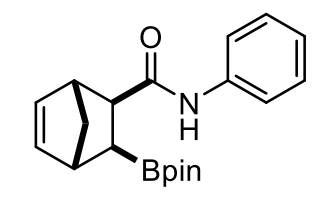

$3 \times a$

${ }^{1} \mathrm{H}$ NMR $\left(400 \mathrm{MHz}, \mathrm{CDCl}_{3}\right)$
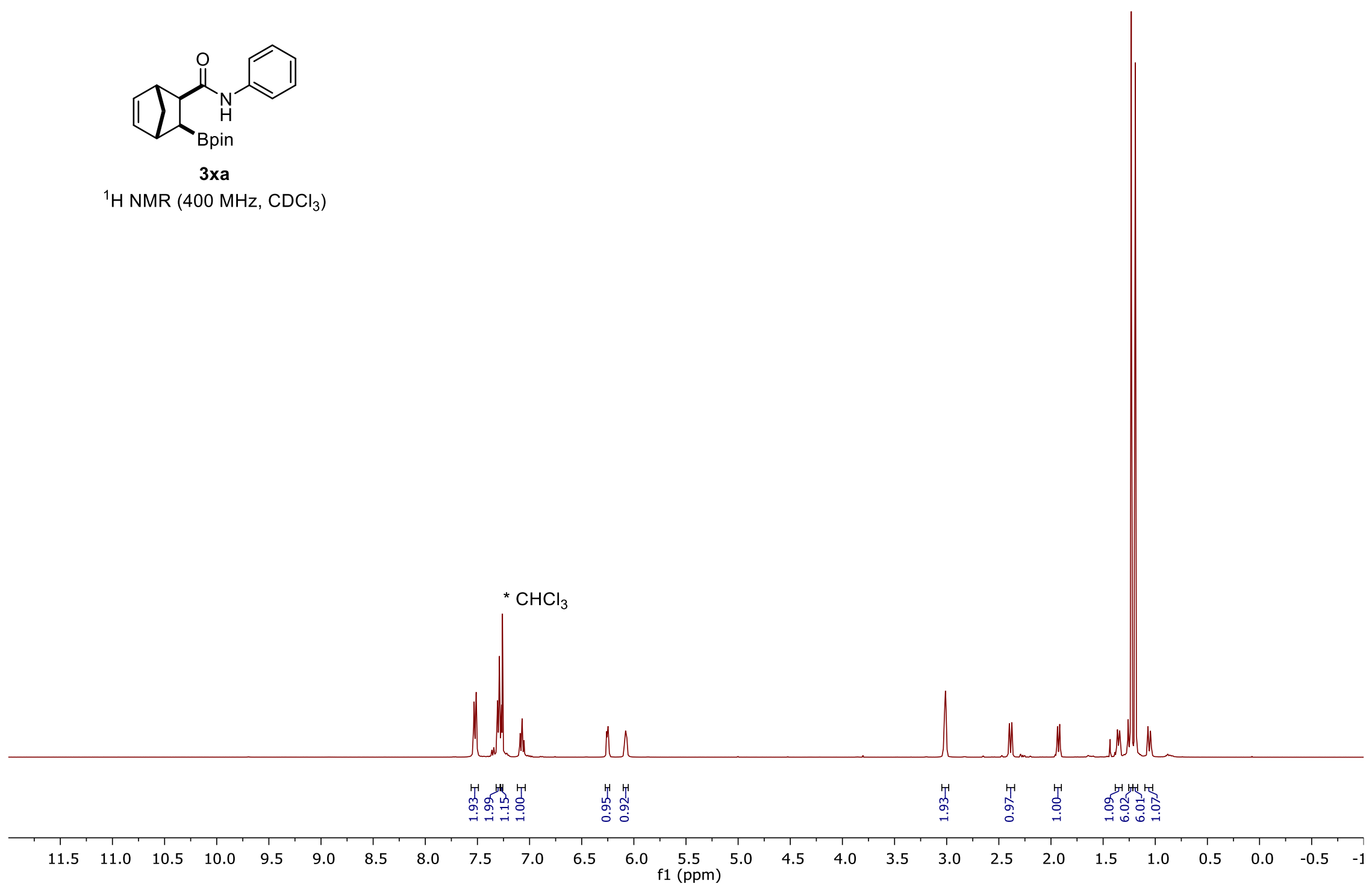

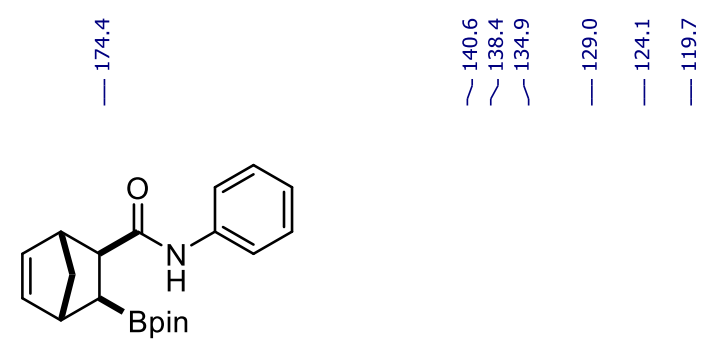

3xa

${ }^{13} \mathrm{C}\left\{{ }^{1} \mathrm{H}\right\}$ NMR $\left(101 \mathrm{MHz}, \mathrm{CDCl}_{3}\right.$ )

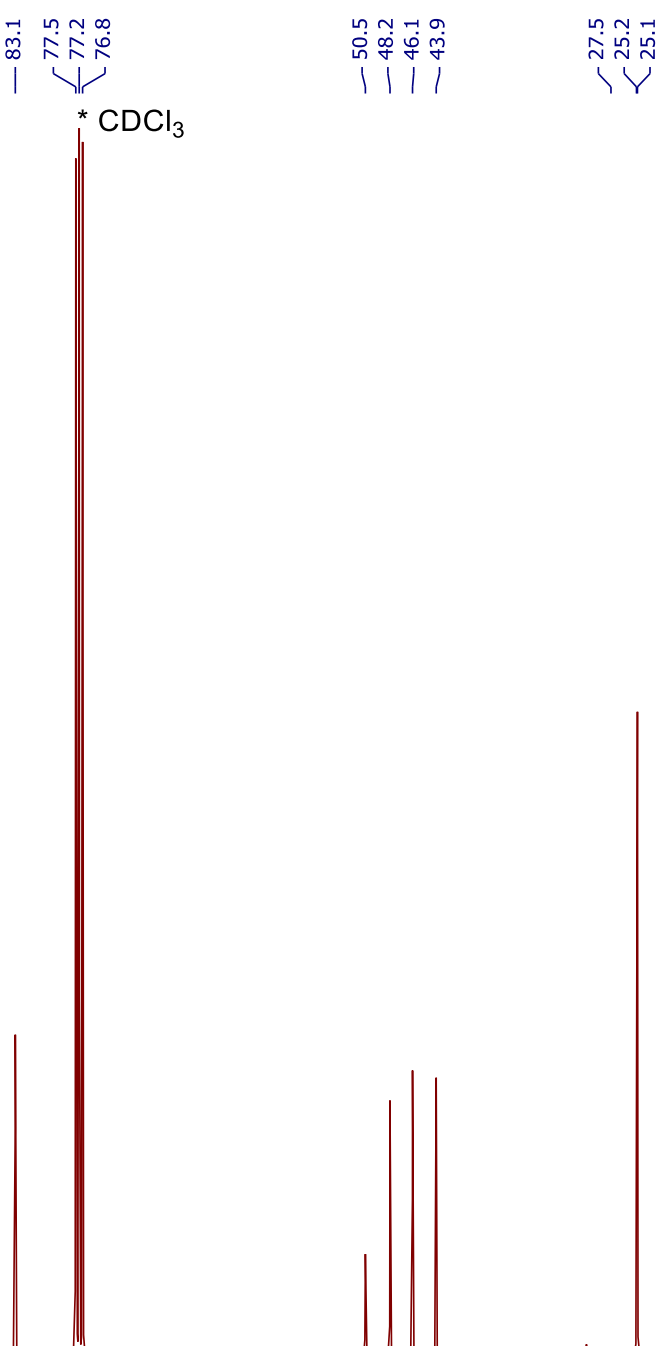

$00 \quad 190$

$180 \quad 170$

160

$150 \quad 140$

130

120

$110 \quad 100 \quad 90$ 


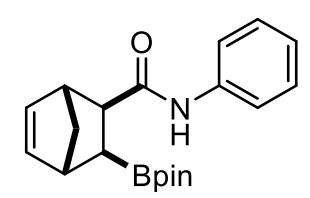

$3 \times a$

${ }^{11} \mathrm{~B}$ NMR $\left(128 \mathrm{MHz}, \mathrm{CDCl}_{3}\right)$

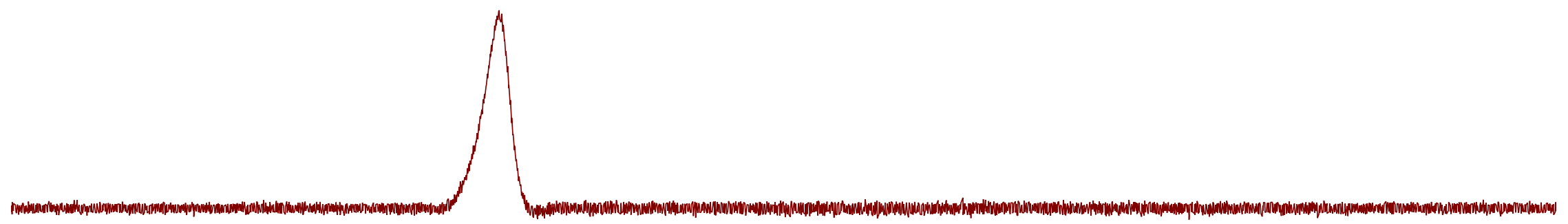

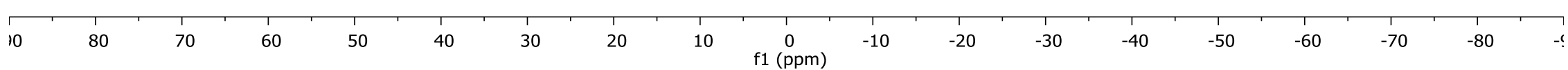




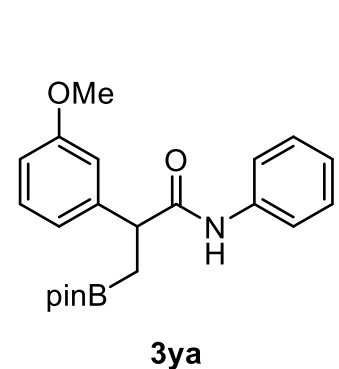

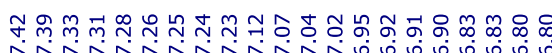

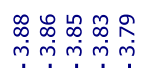

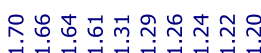

NMR $\left(300 \mathrm{MHz}, \mathrm{CDCl}_{3}\right)$
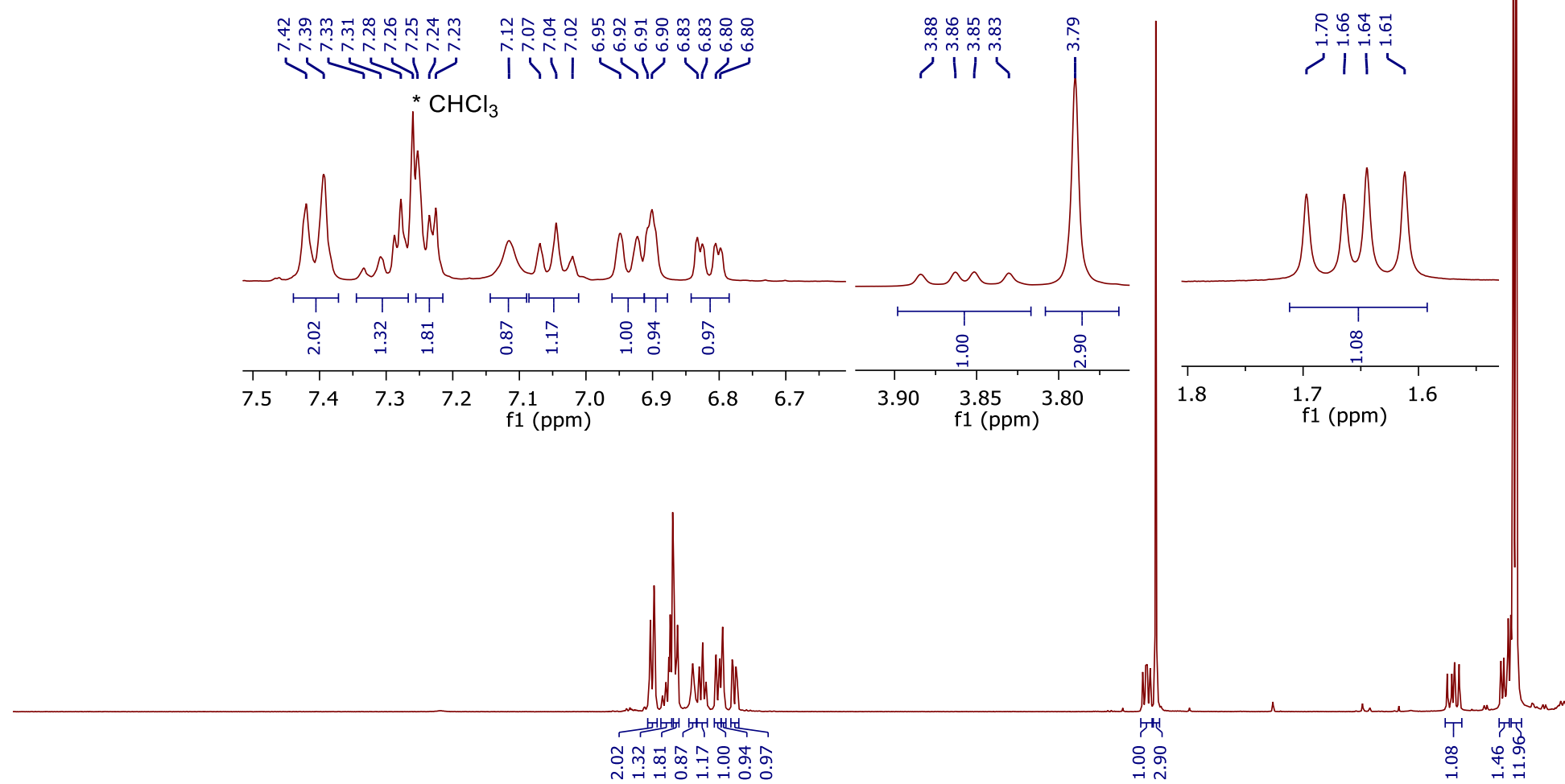

$\begin{array}{lllllll}2.0 & 11.5 & 11.0 & 10.5 & 10.0 & 9.5 & 9.0\end{array}$ 
Supporting Information

S284

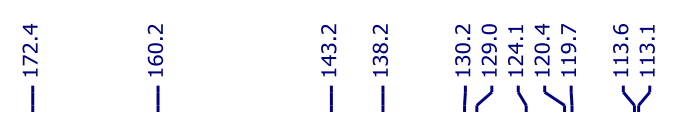<smiles>COc1cccc(C(CP)C(=O)Nc2ccccc2)c1</smiles>
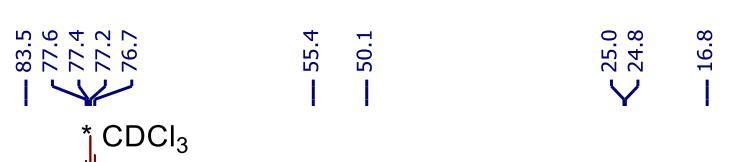
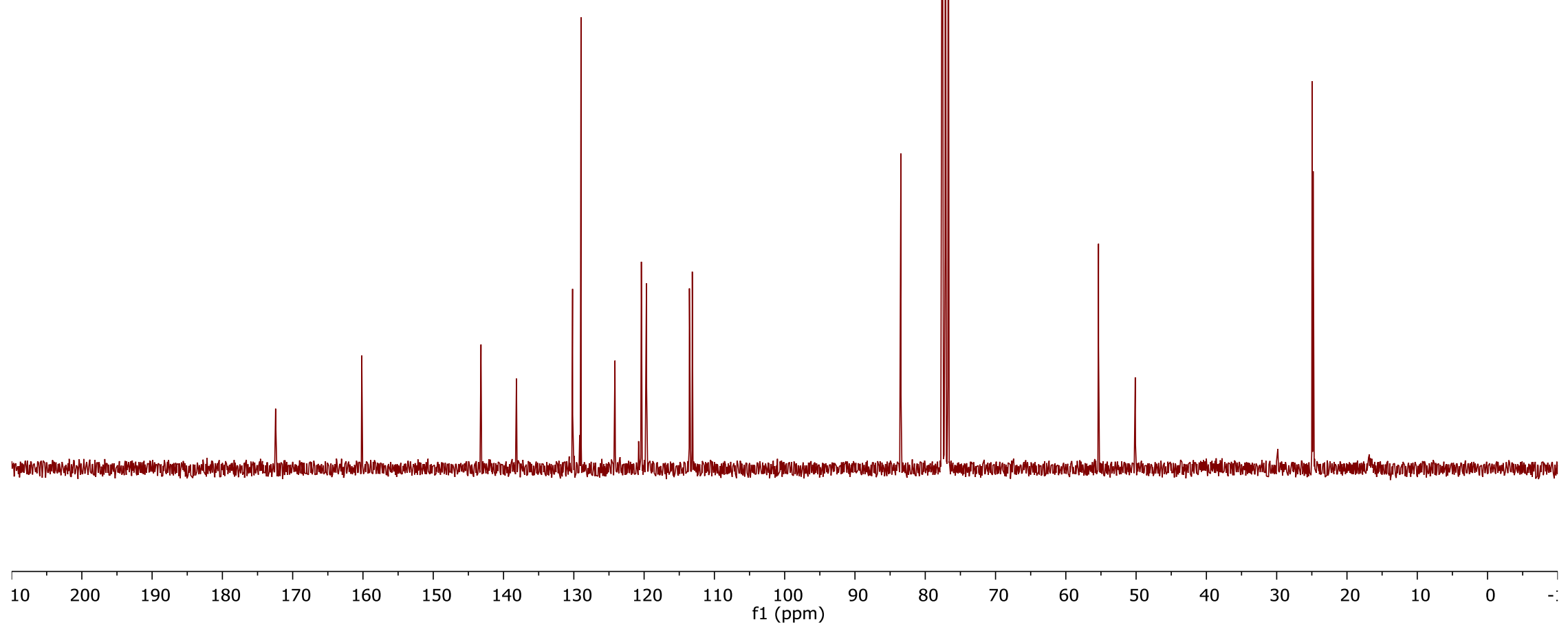


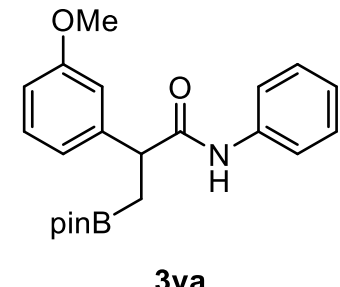

${ }^{11} \mathrm{~B}$ NMR (96 MHz, CDCl 3 )

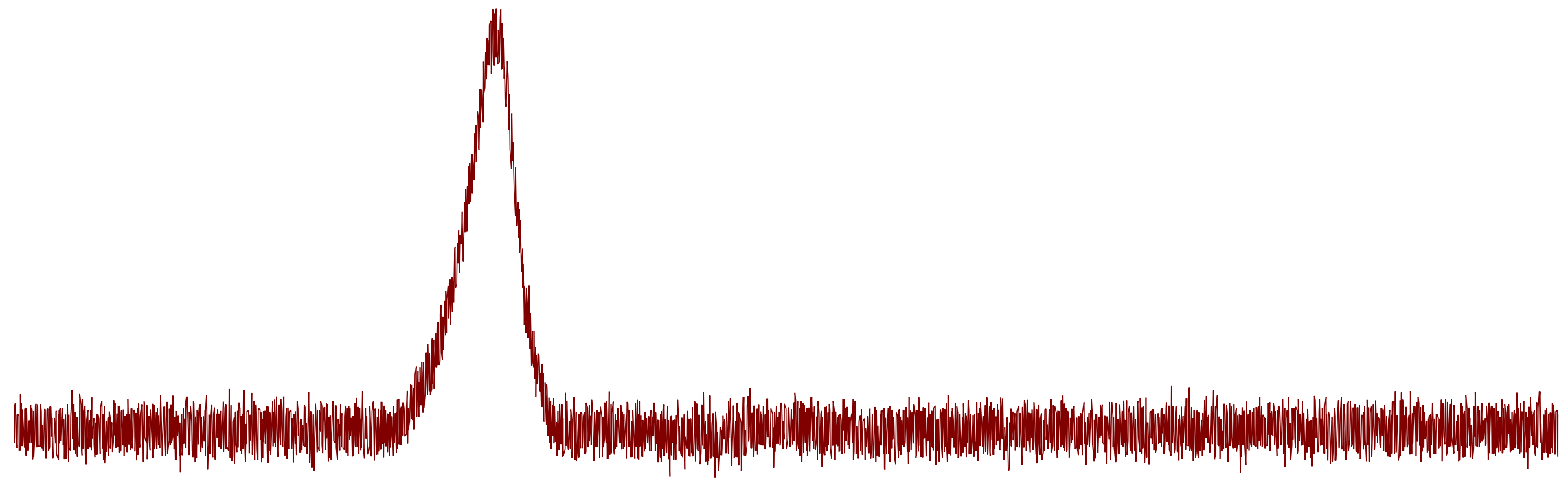




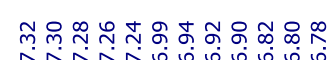

管

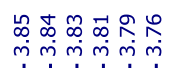

คํำ

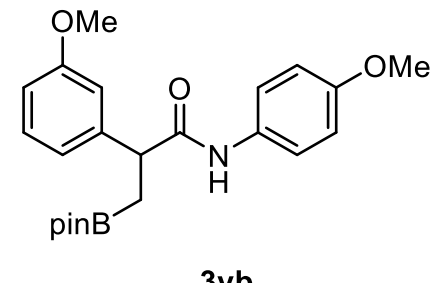

${ }^{1} \mathrm{H}$ NMR $\left(400 \mathrm{MHz}, \mathrm{CDCl}_{3}\right)$
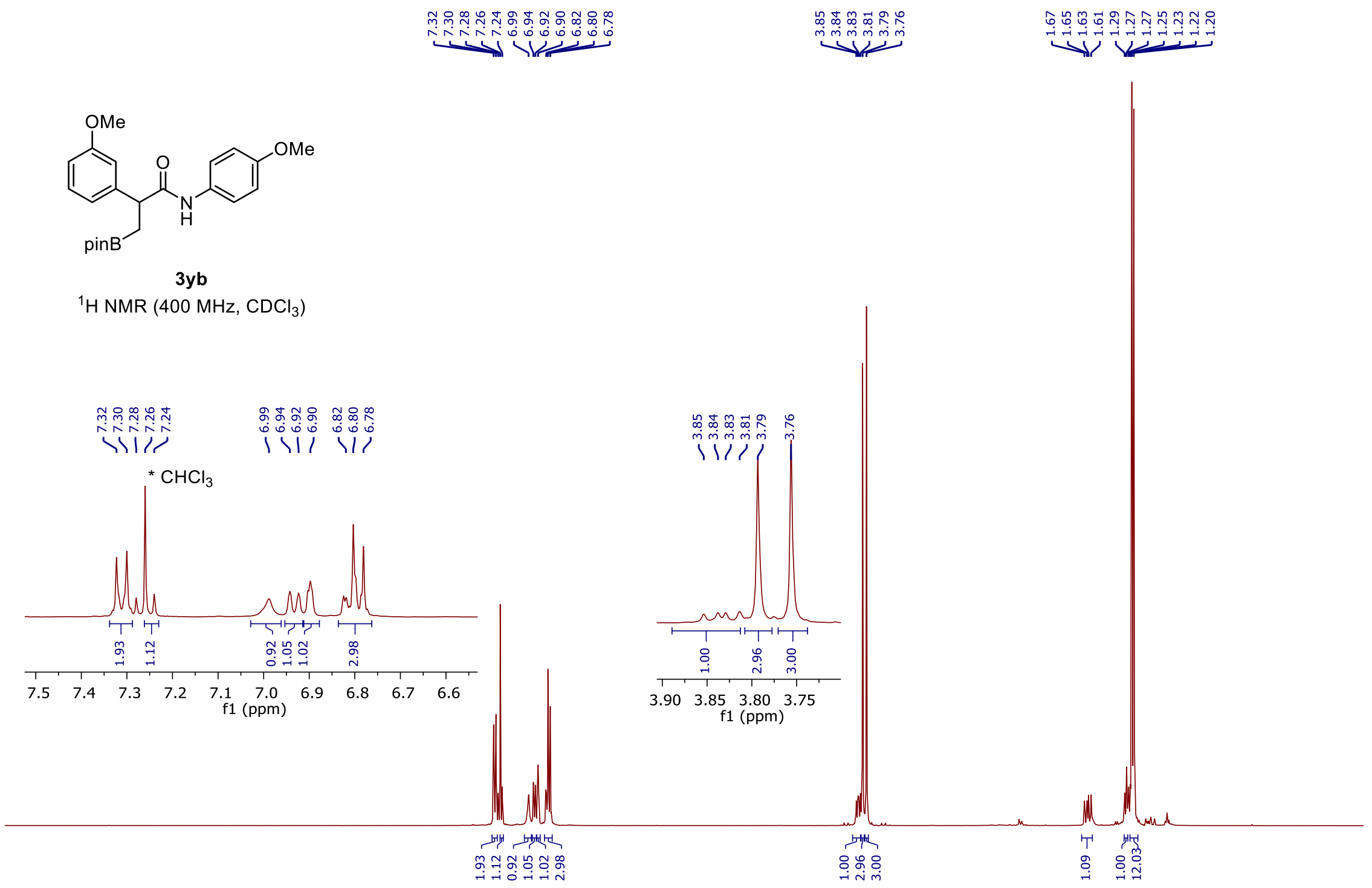

$\begin{array}{lllllllllll}2.0 & 11.5 & 11.0 & 10.5 & 10.0 & 9.5 & 9.0 & 8.5 & 8.0 & 7.5 & 7.0\end{array}$

$6.0 \begin{gathered}5.5 \\ f 1(\mathrm{ppm})\end{gathered}$

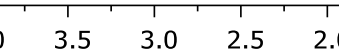

$\begin{array}{lllll}1.5 & 1.0 & 0.5 & 0.0 & -0.5\end{array}$ 


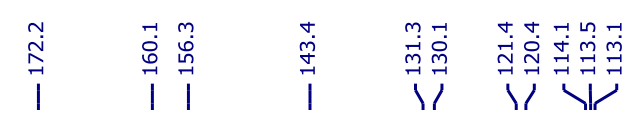

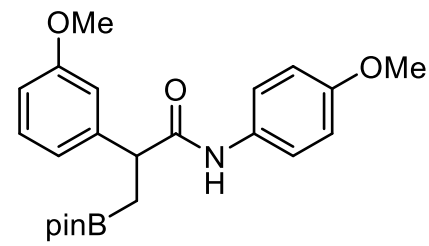

$3 y b$

${ }^{13} \mathrm{C}\left\{{ }^{1} \mathrm{H}\right\}$ NMR $\left(101 \mathrm{MHz}, \mathrm{CDCl}_{3}\right)$
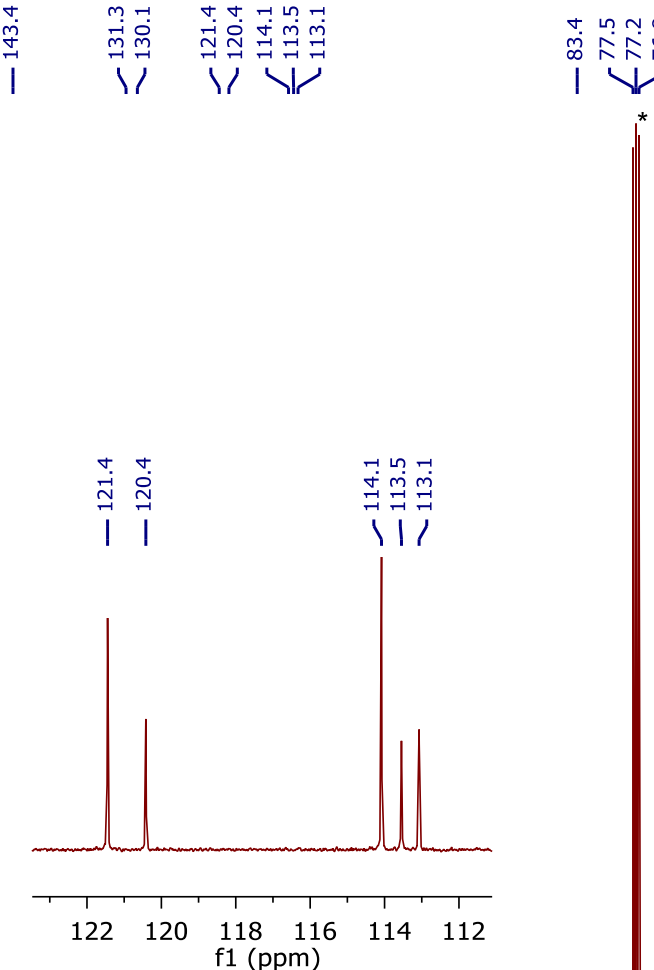

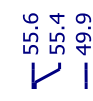

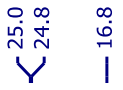

${ }^{*} \mathrm{CDCl}_{3}$
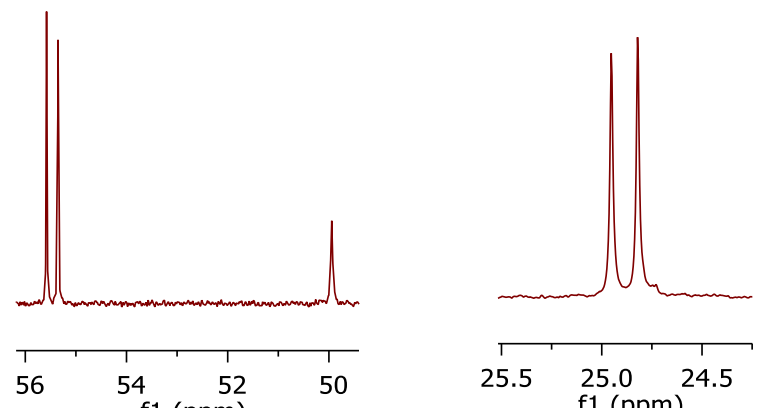

$25.5 \quad 25.0 \quad 24.5$

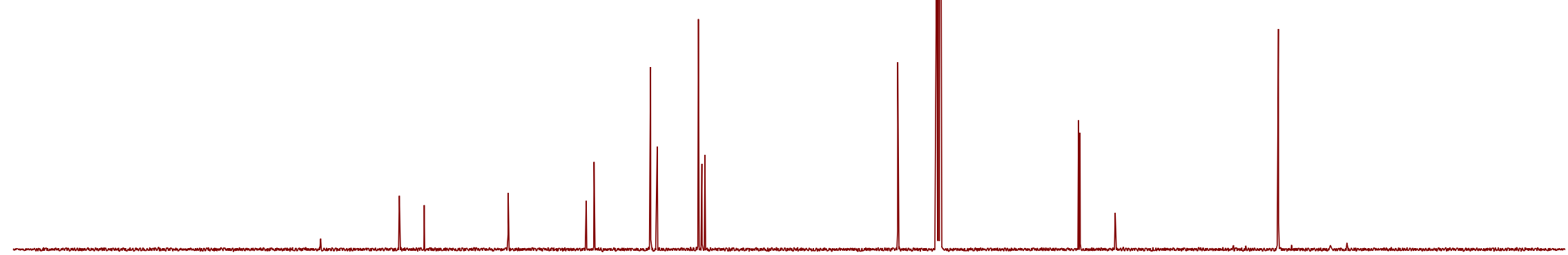




\section{i}

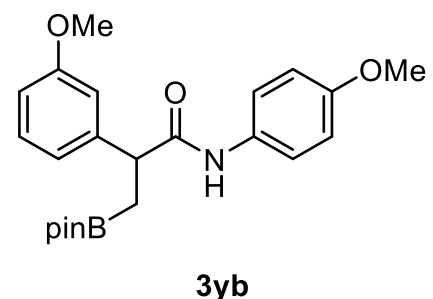

${ }^{11}$ B NMR (96 MHz, $\mathrm{CDCl}_{3}$ )

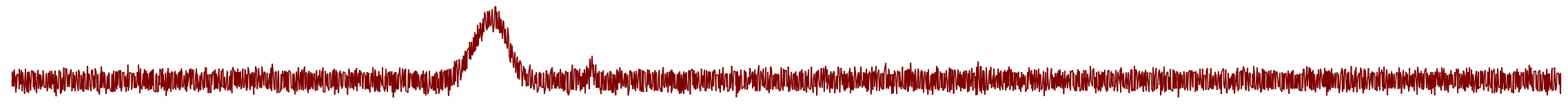




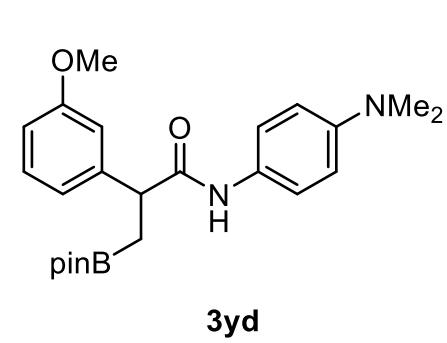

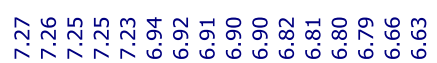

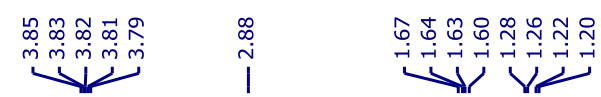

${ }^{1} \mathrm{H}$ NMR $\left(400 \mathrm{MHz}, \mathrm{CDCl}_{3}\right)$
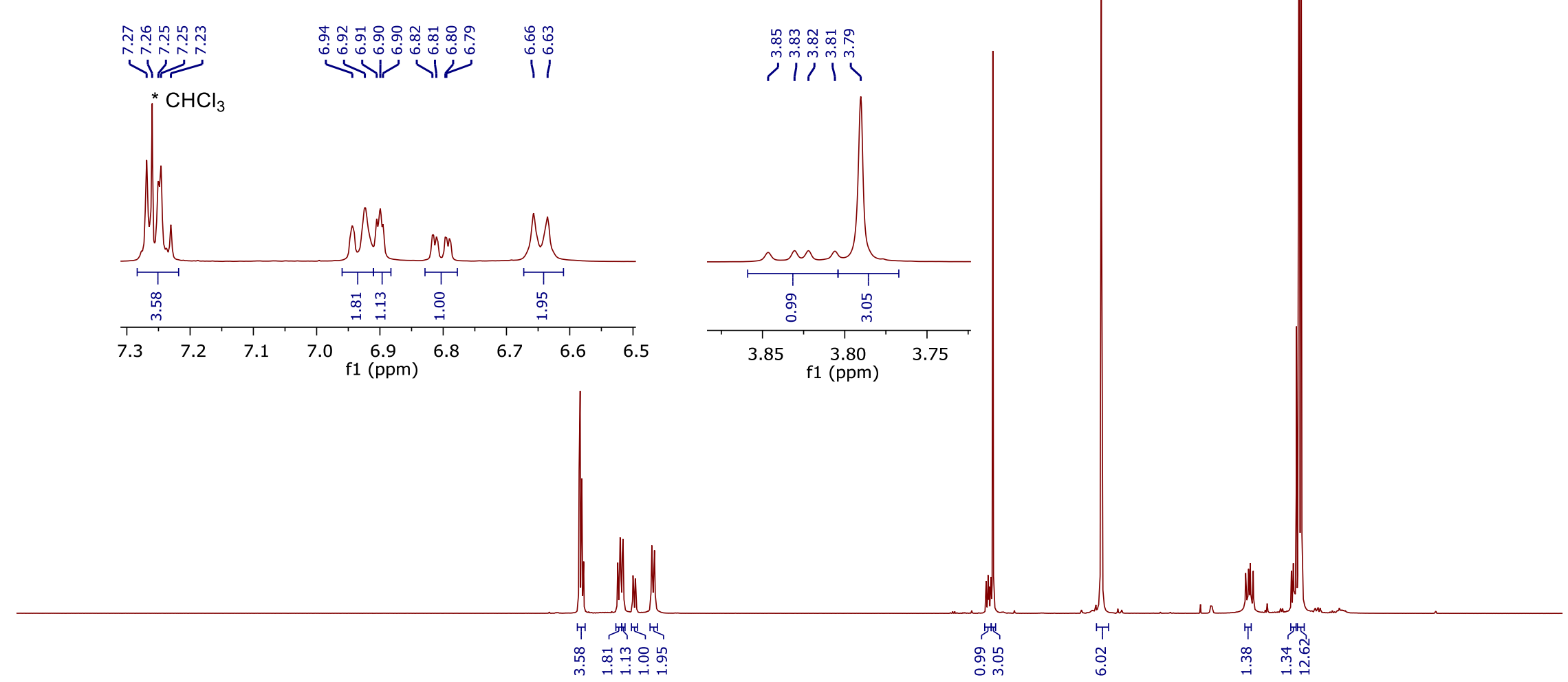

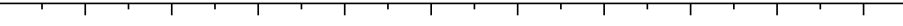

$\begin{array}{llllllllllllll}2.0 & 11.5 & 11.0 & 10.5 & 10.0 & 9.5 & 9.0 & 8.5 & 8.0 & 7.5 & 7.0 & 6.5 & 6.0 & \begin{array}{c}5.5 \\ \mathrm{f} 1\end{array}(\mathrm{ppm}) \\ 5.0 & \end{array}$ 

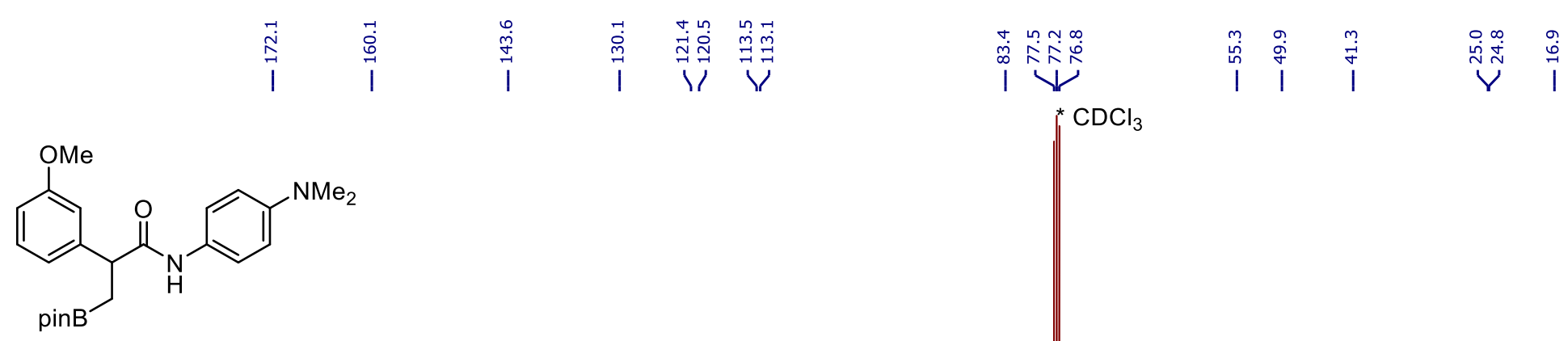

\section{3yd}

${ }^{13} \mathrm{C}\left\{{ }^{1} \mathrm{H}\right\}$ NMR $\left(101 \mathrm{MHz}, \mathrm{CDCl}_{3}\right)$

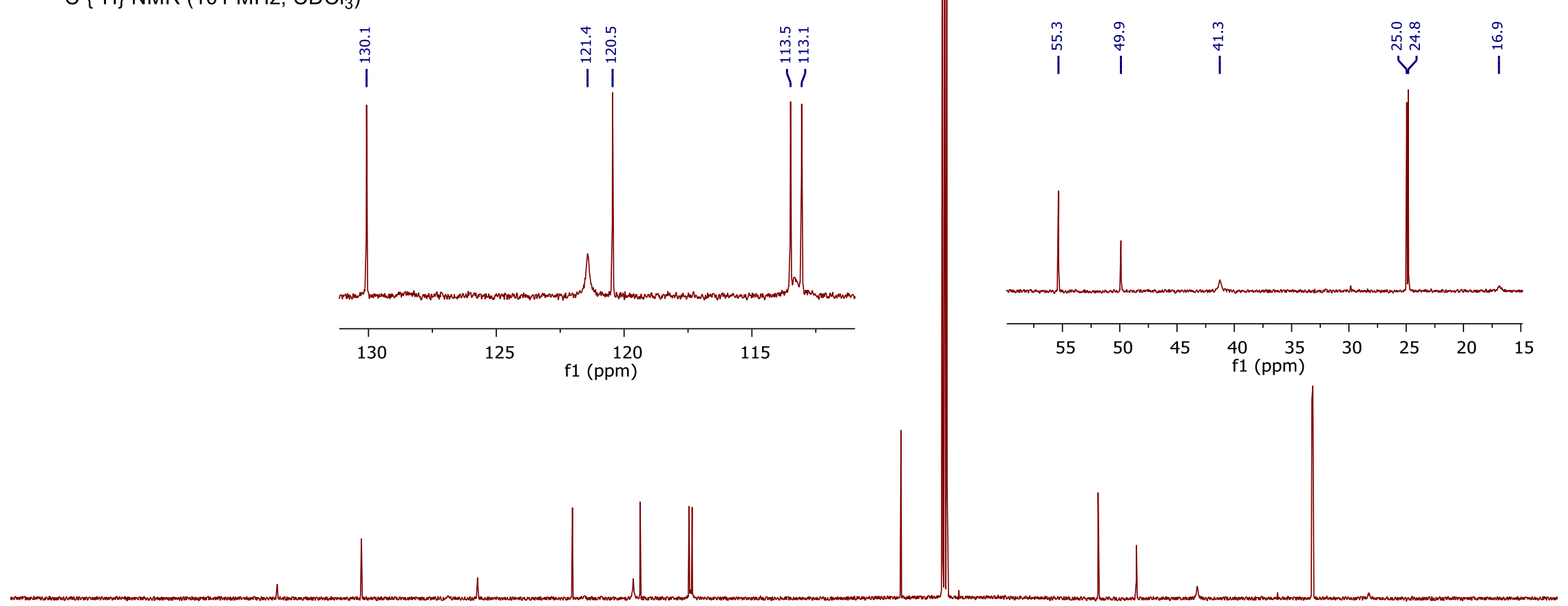

(1) 


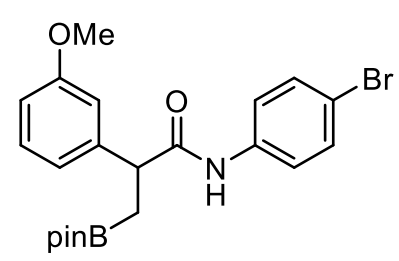

$3 y g$

${ }^{1} \mathrm{H}$ NMR $\left(400 \mathrm{MHz}, \mathrm{CDCl}_{3}\right)$
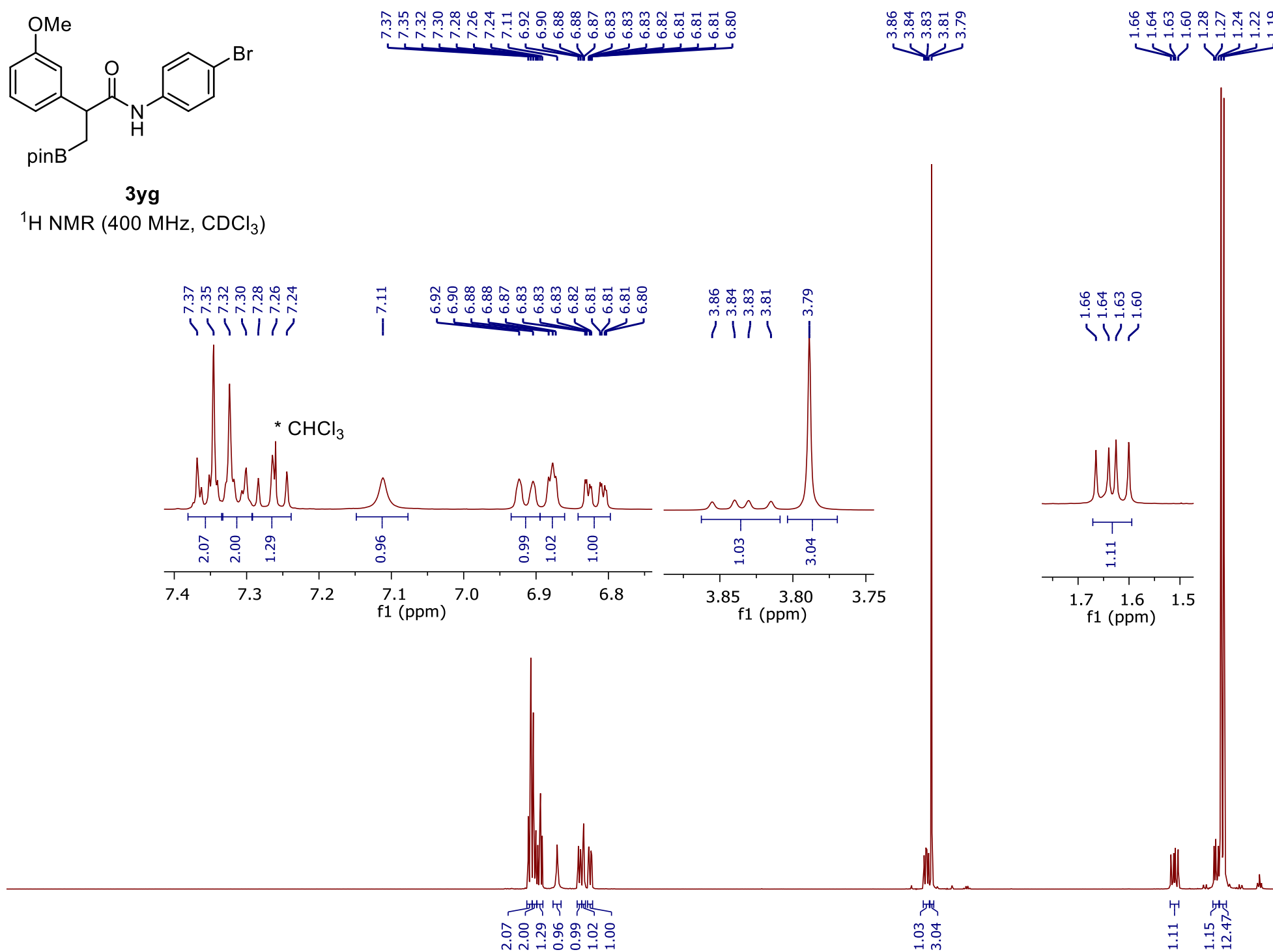

$\begin{array}{lllllllllllllll}2.0 & 11.5 & 11.0 & 10.5 & 10.0 & 9.5 & 9.0 & 8.5 & 8.0 & 7.5 & 7.0 & 6.5 & 6.0 & 5.5 & 5.0\end{array}$ 
Supporting Information

S292

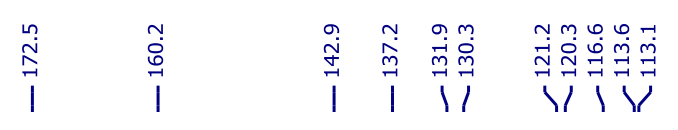<smiles>COc1cccc(C(C[Pb])C(=O)Nc2ccc(Br)cc2)c1</smiles>
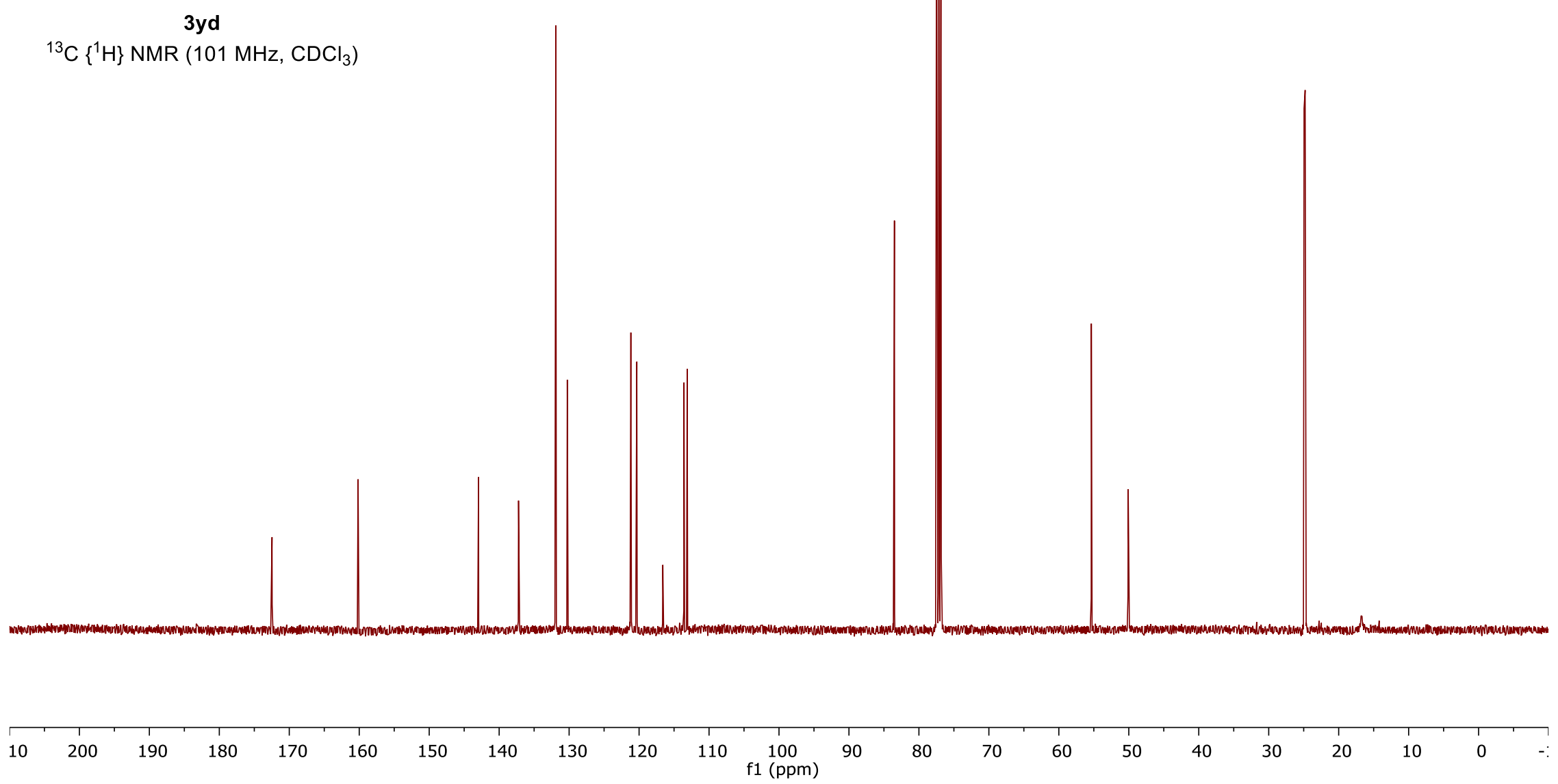
$\stackrel{\sim}{\stackrel{n}{m}}$

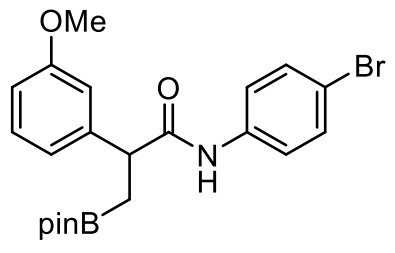

3yd

${ }^{11} \mathrm{~B}$ NMR $\left(128 \mathrm{MHz}, \mathrm{CDCl}_{3}\right)$

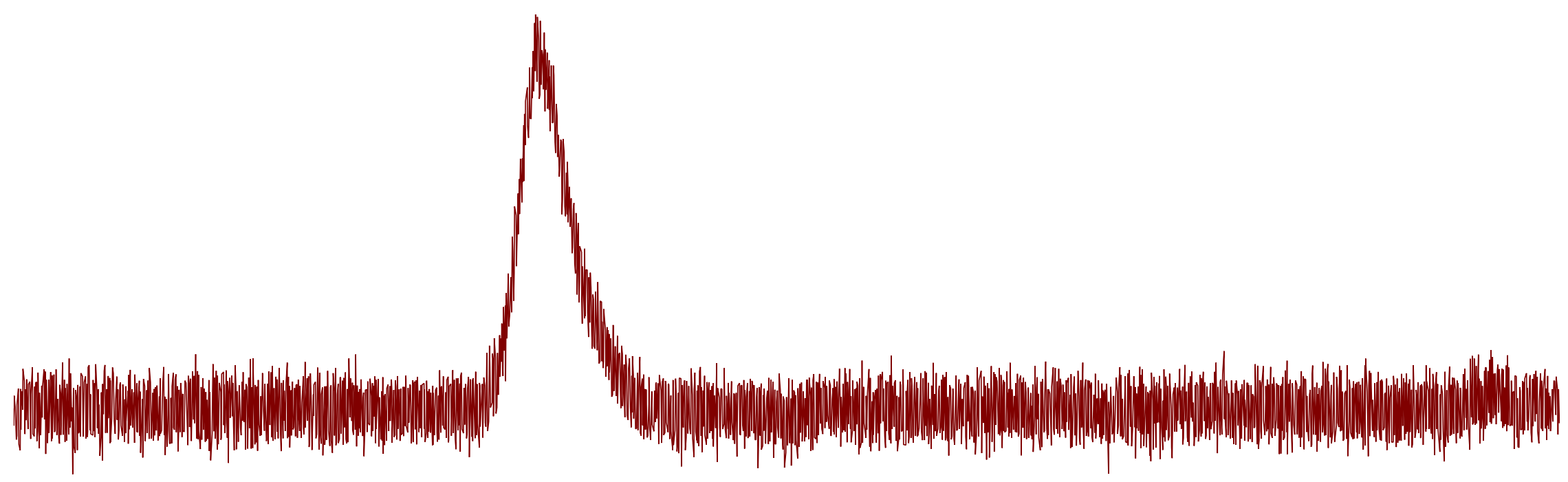



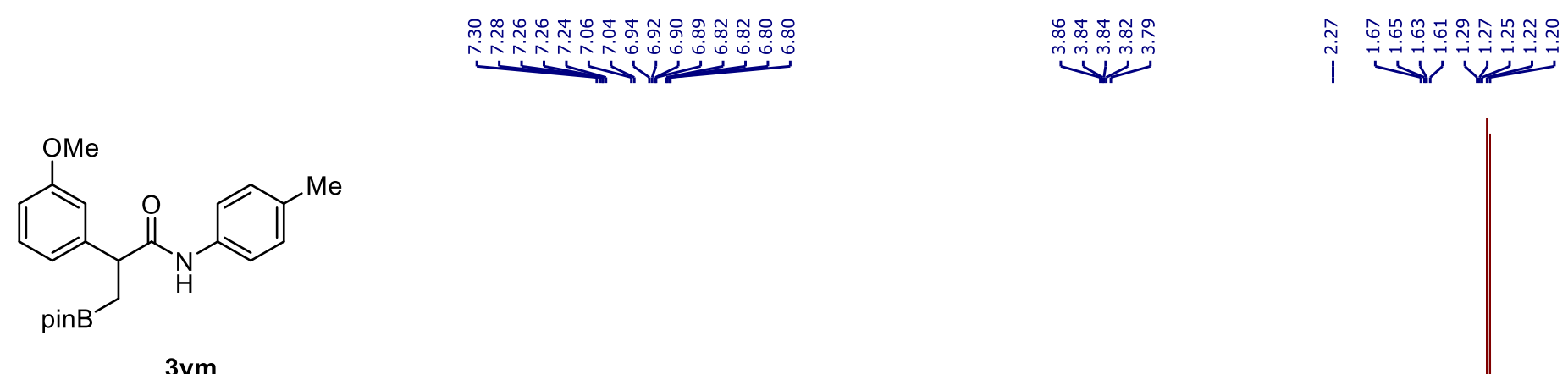

${ }^{1} \mathrm{H}$ NMR (400 MHz, $\mathrm{CDCl}_{3}$ )

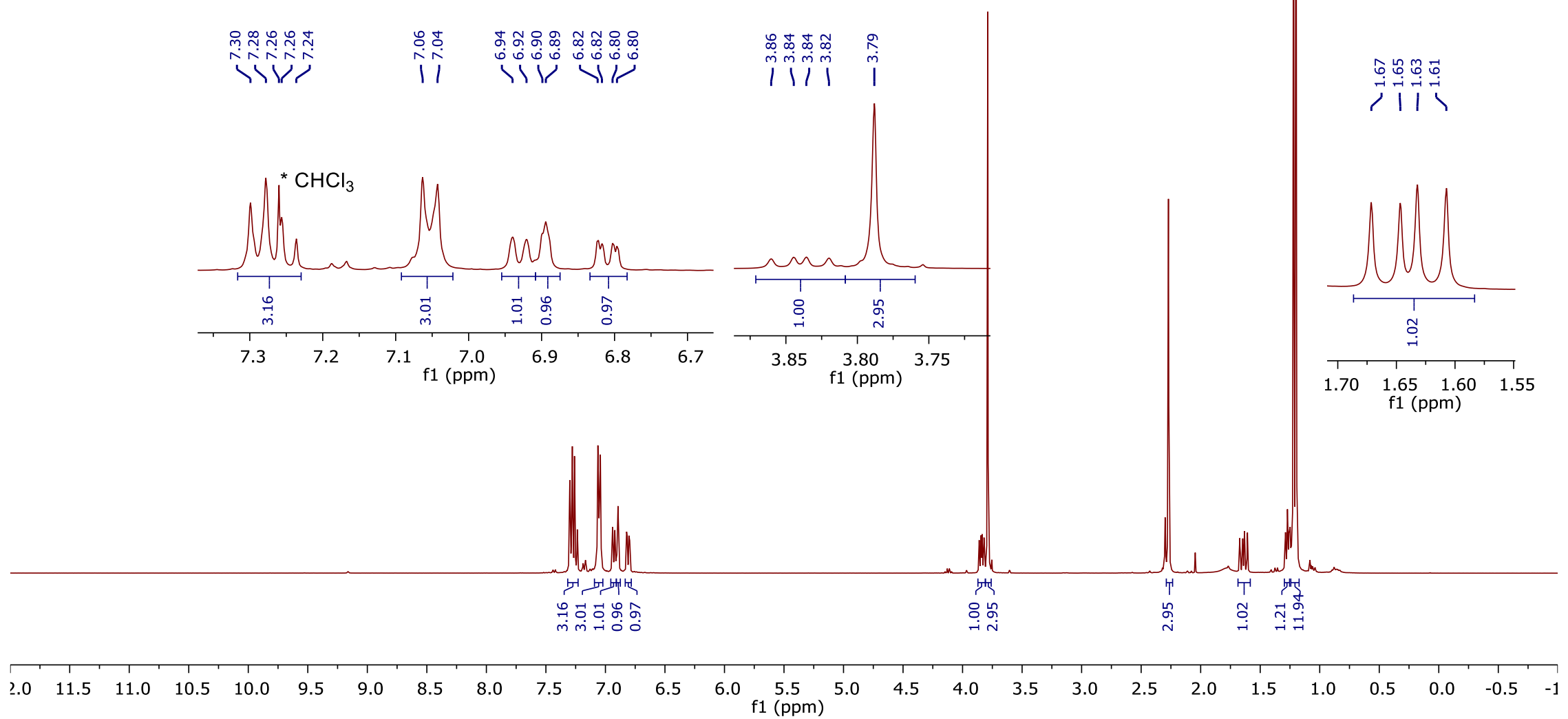




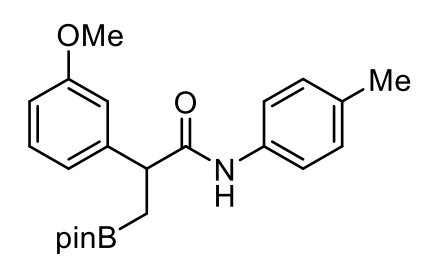

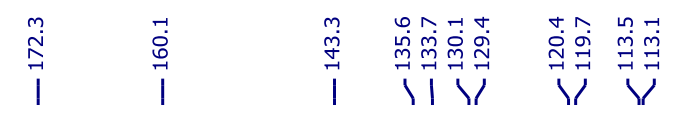

$3 y m$

${ }^{13} \mathrm{C}\left\{{ }^{1} \mathrm{H}\right\}$ NMR $\left(101 \mathrm{MHz}, \mathrm{CDCl}_{3}\right)$

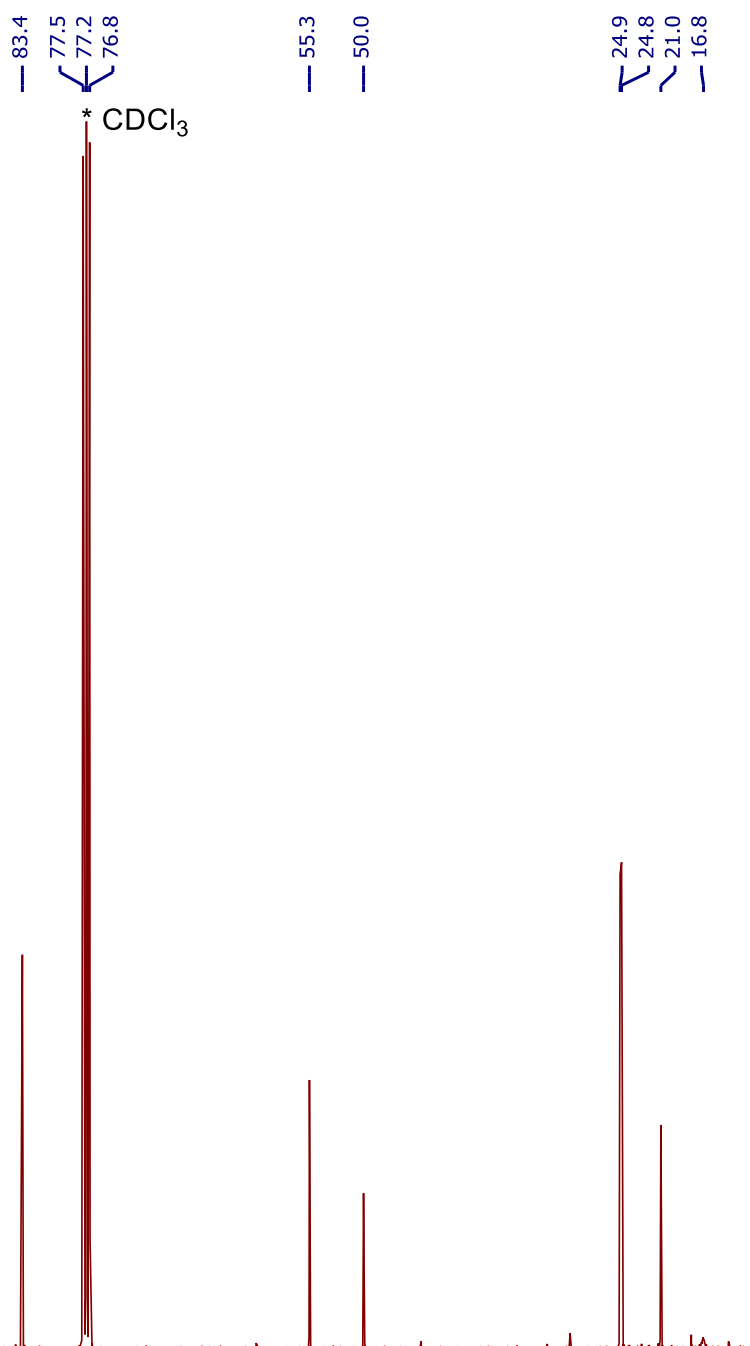

$180 \quad 1$

160

$150 \quad 140$

$130 \quad 120$

$110 \quad 100$

f1 (ppm)

$90 \quad 80$

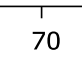

60

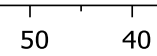

30

20

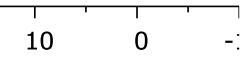



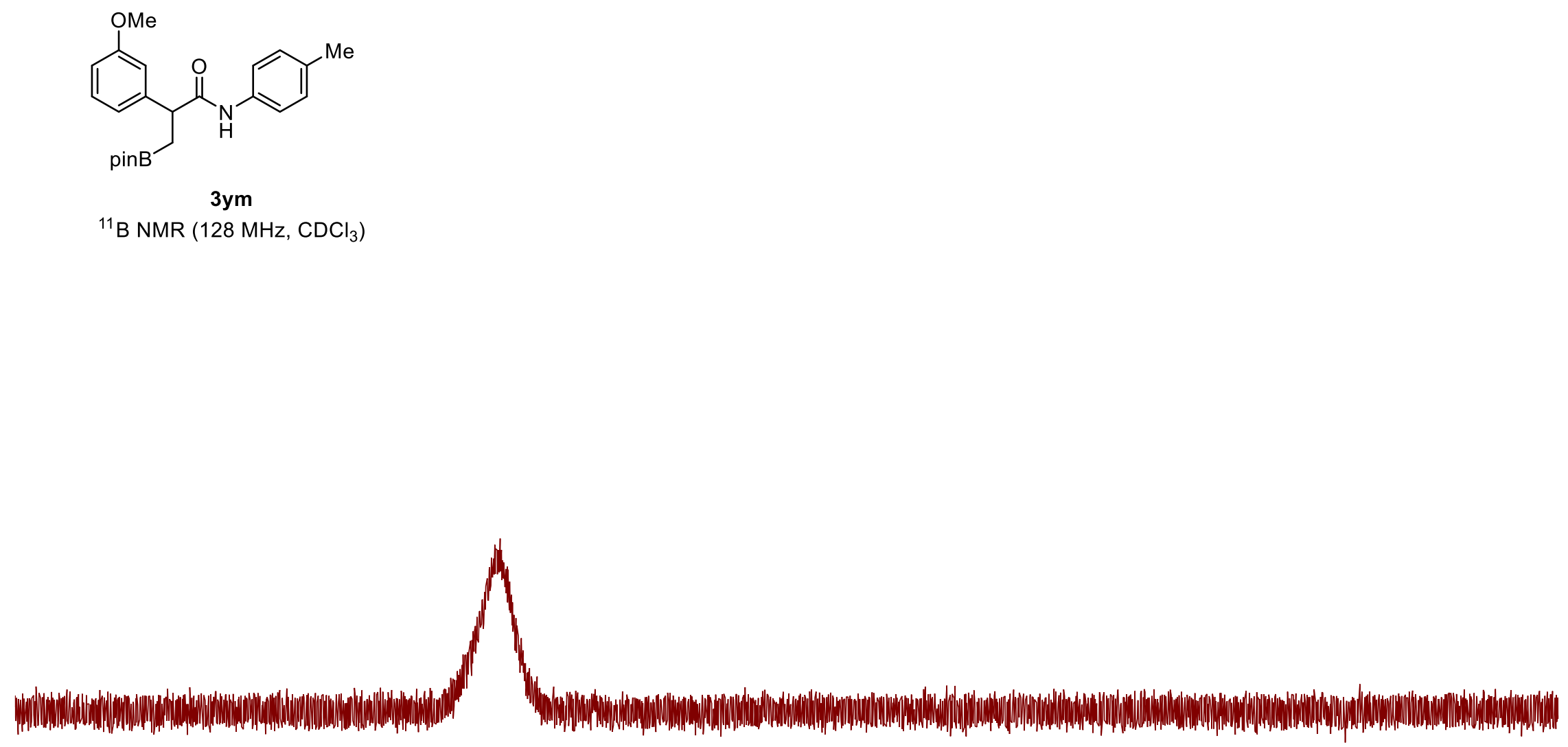

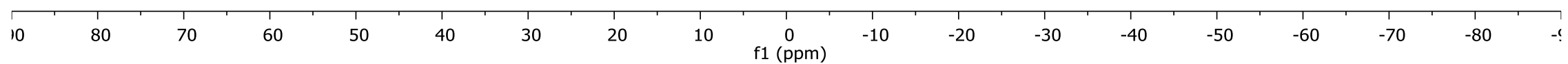



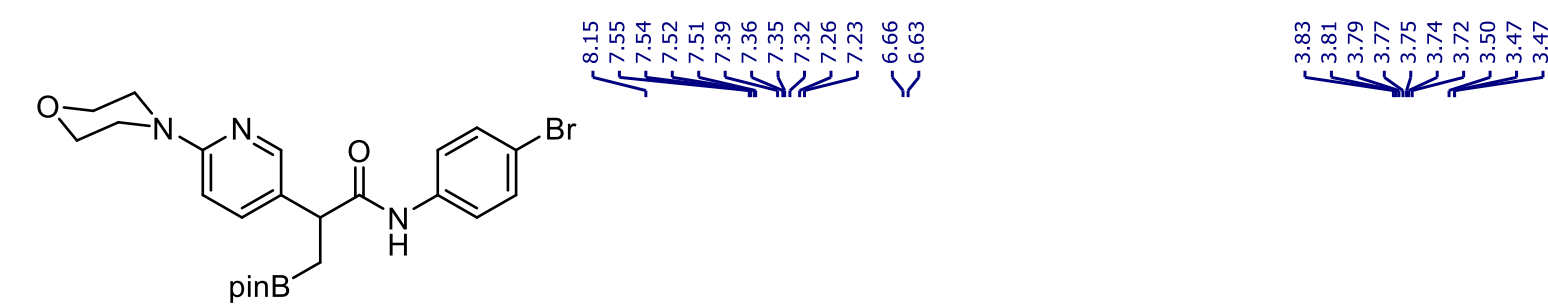

\section{苞}

$3 z g$

${ }^{1} \mathrm{H}$ NMR $\left(400 \mathrm{MHz}, \mathrm{CDCl}_{3}\right)$
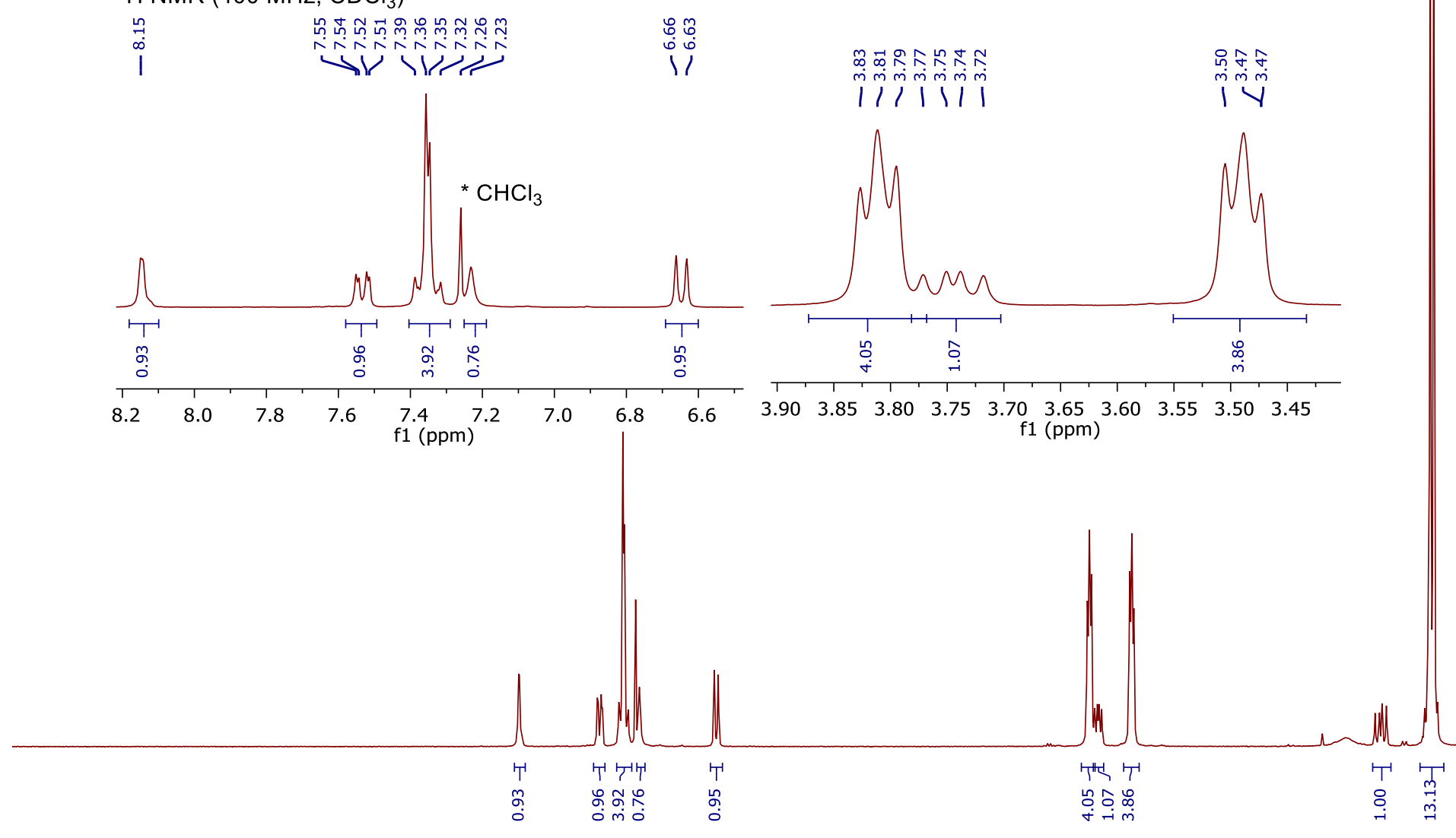

$\begin{array}{lllllllllllllllllllllllllllllllllllll}2.0 & 11.5 & 11.0 & 10.5 & 10.0 & 9.5 & 9.0 & 8.5 & 8.0 & 7.5 & 7.0 & 6.5 & 6.0 & 5.5 & 5.0 & 4.5 & 4.0 & 3.5 & 3.0 & 2.5 & 2.0 & 1.5 & 1.0 & 0.5 & 0.0 & -0.5 & -1\end{array}$ 

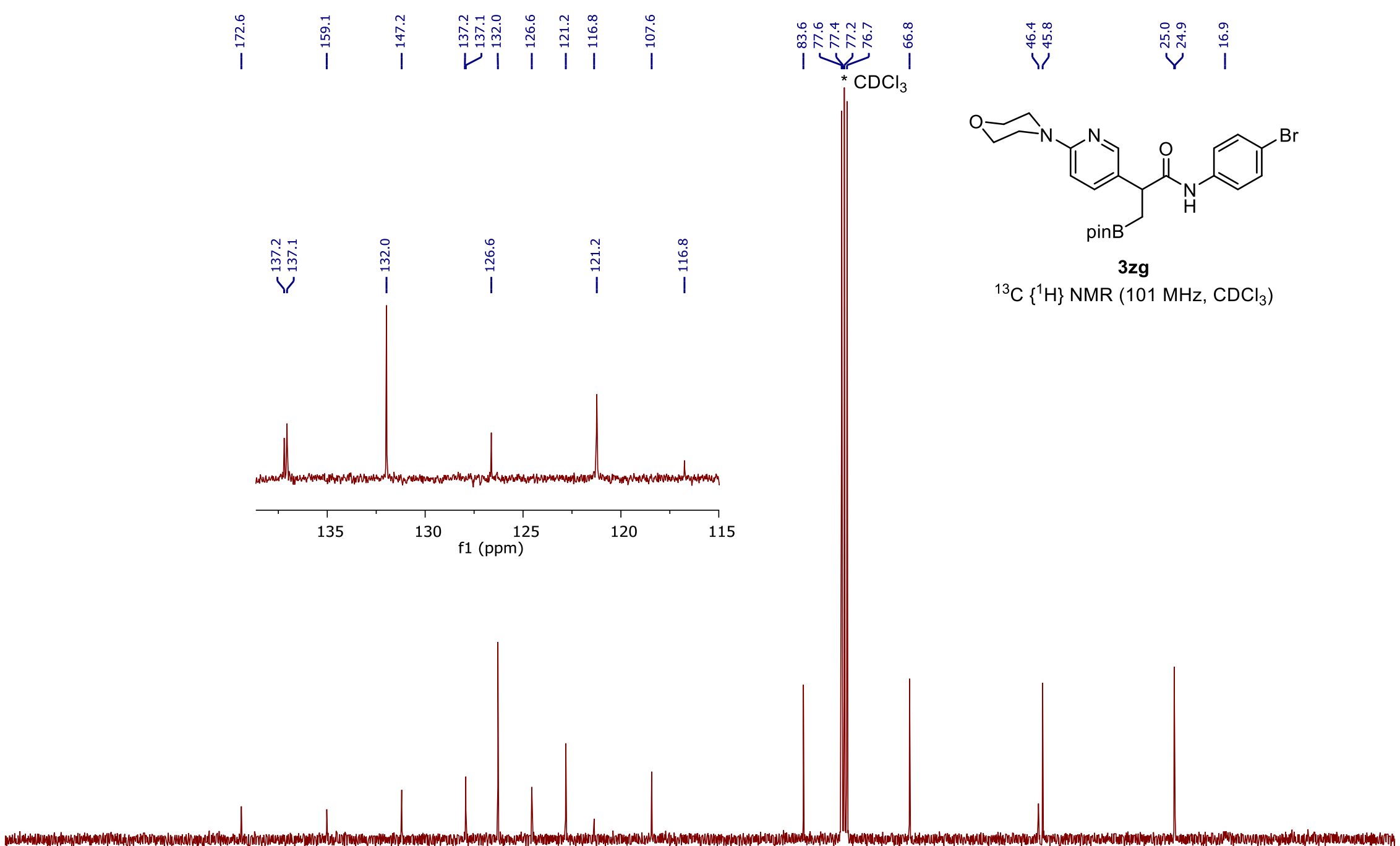


\section{$\stackrel{0}{\stackrel{m}{m}}$}

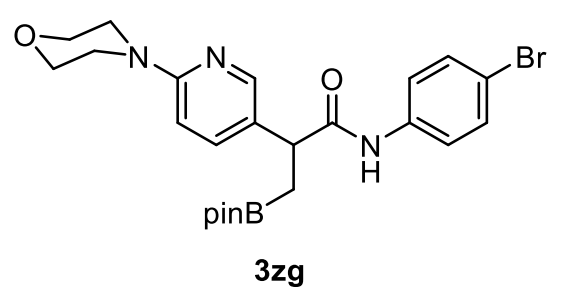

${ }^{11} \mathrm{~B}$ NMR $\left(128 \mathrm{MHz}, \mathrm{CDCl}_{3}\right)$

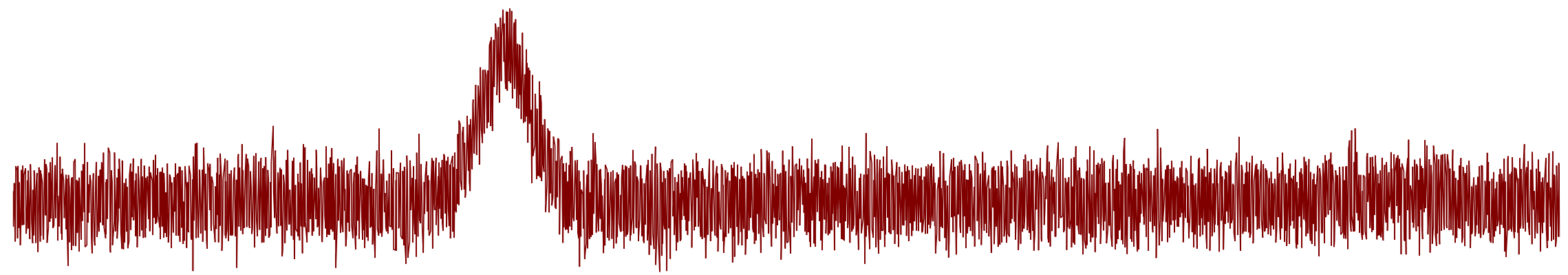




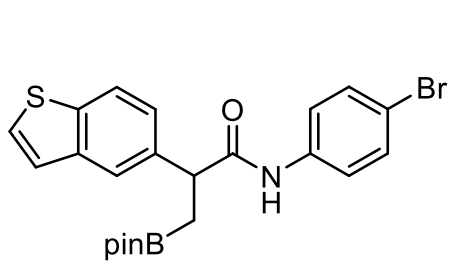

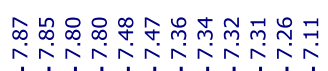

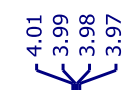

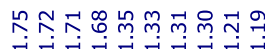

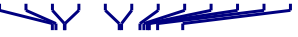

$3 \mathrm{a}_{2} \mathrm{~g}$

${ }^{1} \mathrm{H}$ NMR $\left(400 \mathrm{MHz}, \mathrm{CDCl}_{3}\right)$

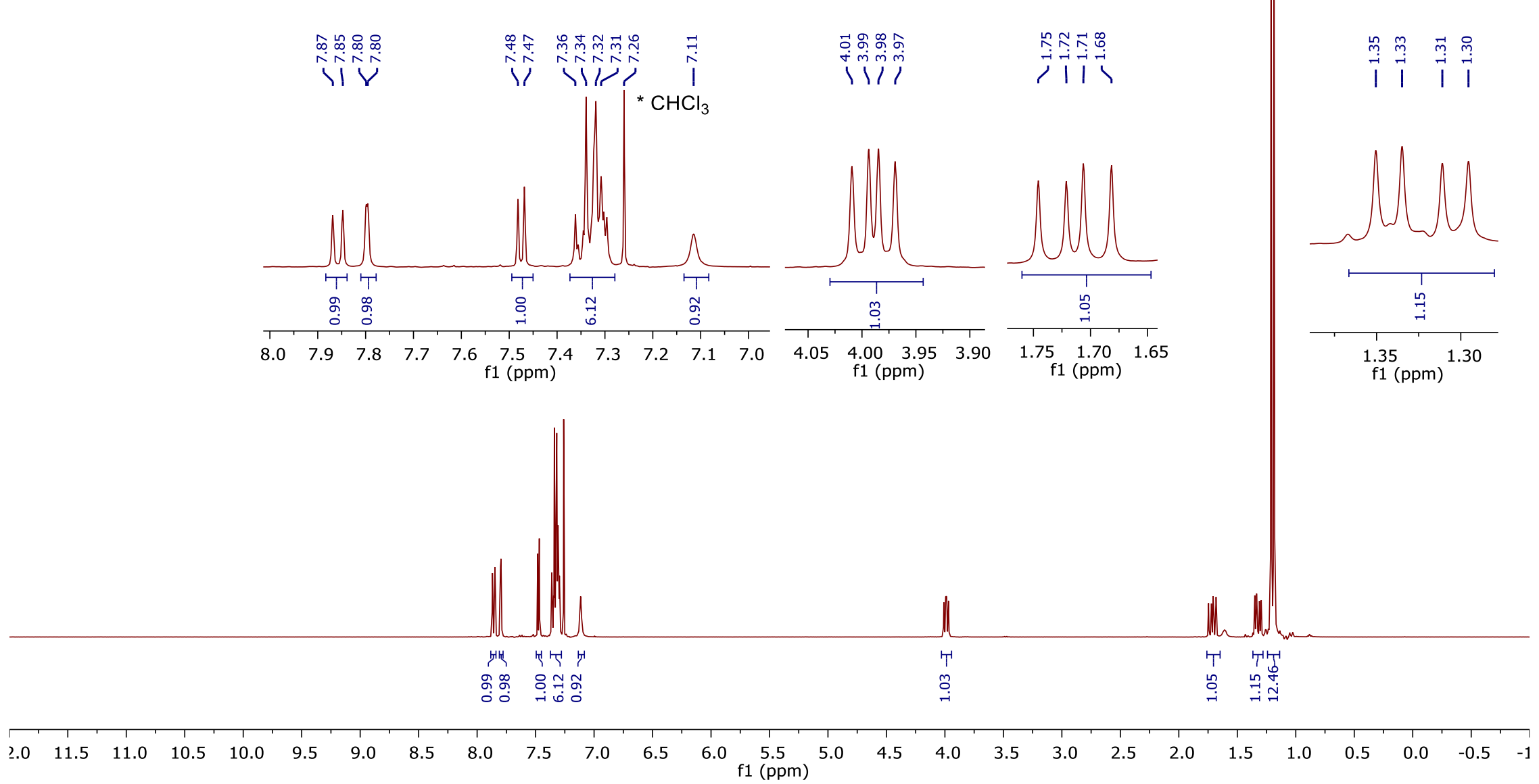




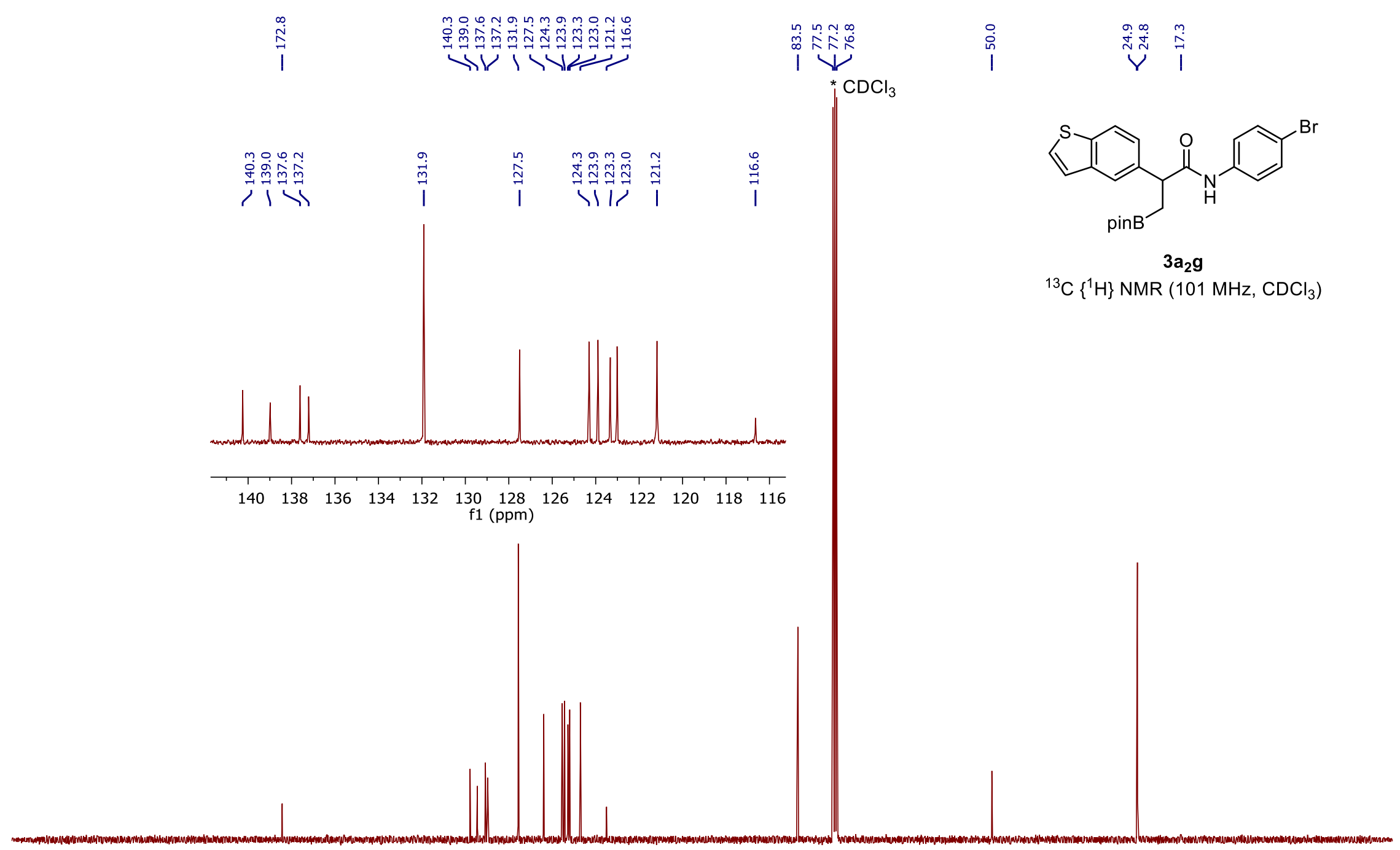

$\begin{array}{llllllllllllllllllllll}210 & 200 & 190 & 180 & 170 & 160 & 150 & 140 & 130 & 120 & 110 & \underset{f 1}{100}(\mathrm{ppm}) & 90 & 80 & 70 & 60 & 50 & 40 & 30 & 20 & 10 & 0\end{array}$ 


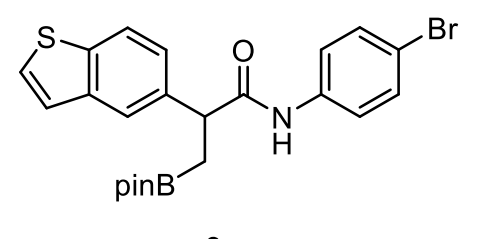

$\stackrel{3 \mathbf{a}_{2} \mathbf{g}}{{ }^{11} \mathrm{~B} \text { NMR }\left(128 \mathrm{MHz}, \mathrm{CDCl}_{3}\right)}$

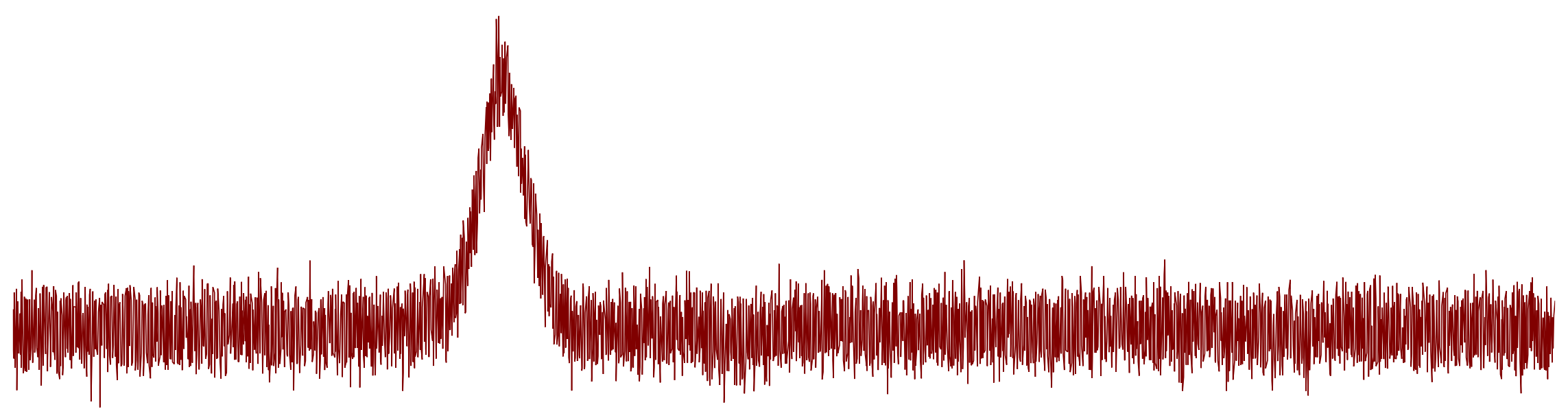

\begin{tabular}{|c|c|c|c|c|c|c|c|c|c|c|c|c|c|c|c|c|c|}
\hline 10 & 80 & 70 & 60 & 50 & 40 & 30 & 20 & 10 & $\begin{array}{l}0 \\
f 1\end{array}$ & -10 & -20 & -30 & -40 & -50 & -60 & -70 & -80 \\
\hline
\end{tabular}




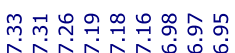

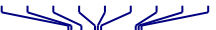

$\underbrace{M e}_{C_{M e}}$

$3 \mathrm{~b}_{2} \mathrm{a}$

${ }^{1} \mathrm{H}$ NMR $\left(500 \mathrm{MHz}, \mathrm{CDCl}_{3}\right.$ )

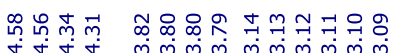

पर

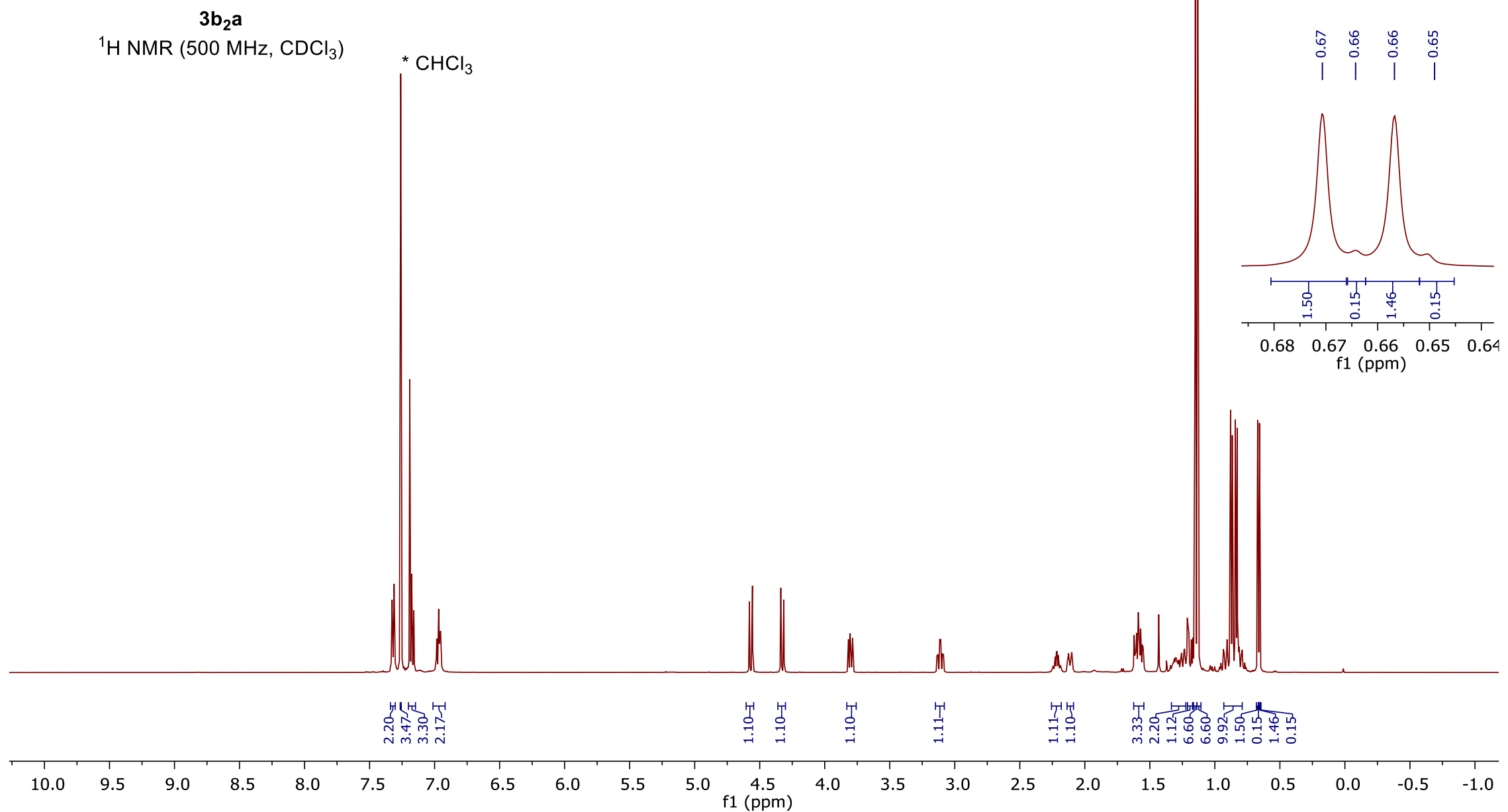




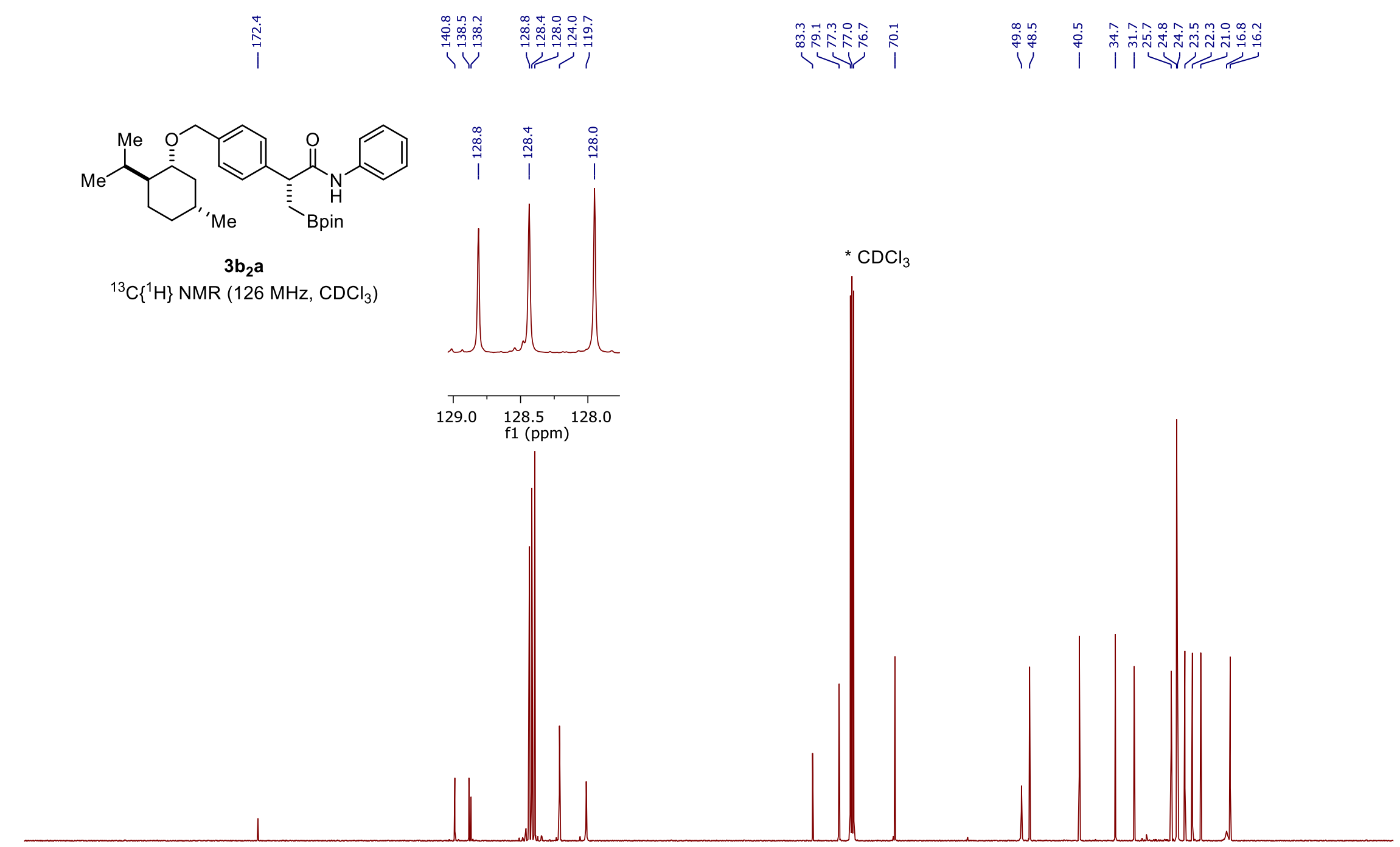

$10 \quad 200+190+180$

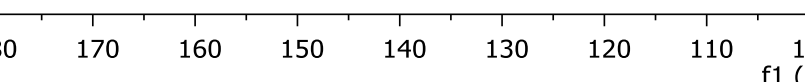

100
$\mathrm{f} 1(\mathrm{ppm})$

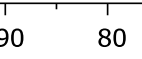

70

$60 \quad 50$

40

20 


\section{$\stackrel{\infty}{\stackrel{\infty}{i}}$}

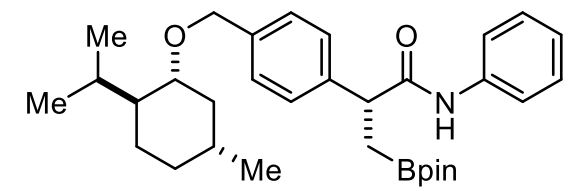

$3 \mathbf{b}_{2} \mathbf{a}$

${ }^{11} \mathrm{~B}$ NMR $\left(128 \mathrm{MHz}, \mathrm{CDCl}_{3}\right)$

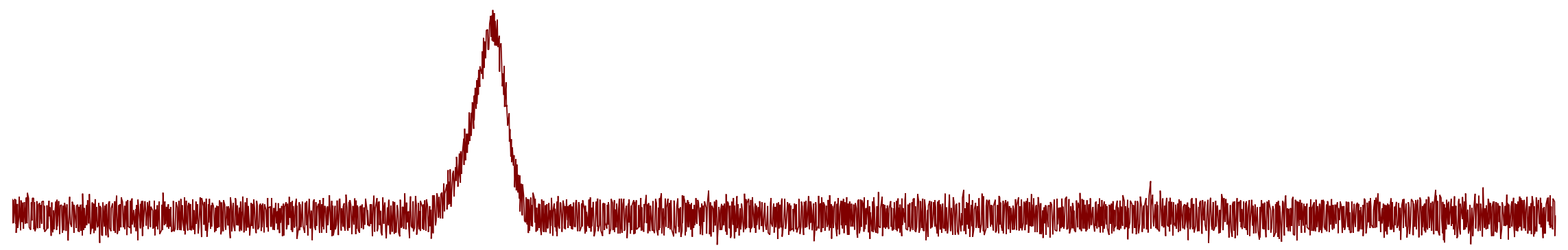




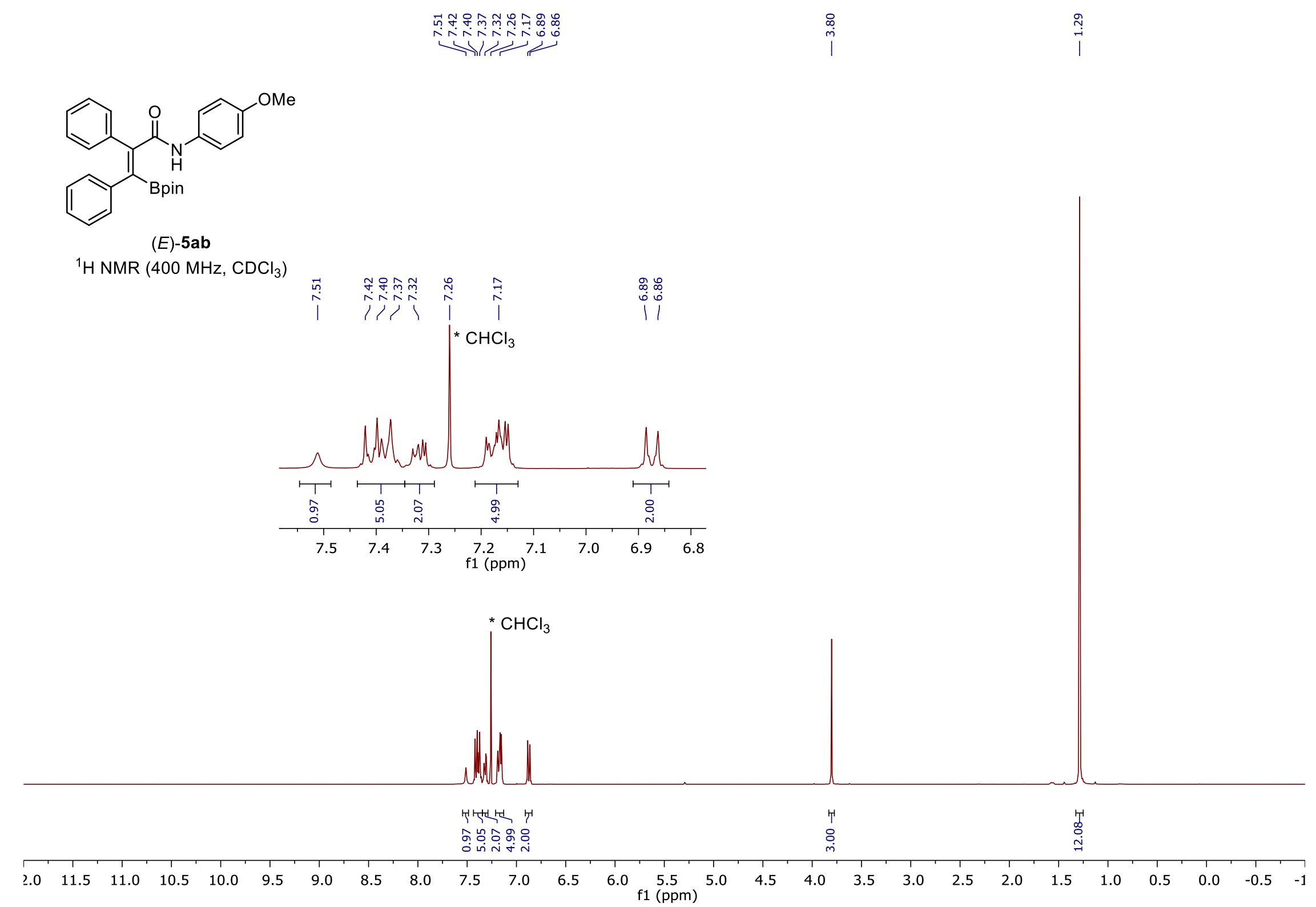




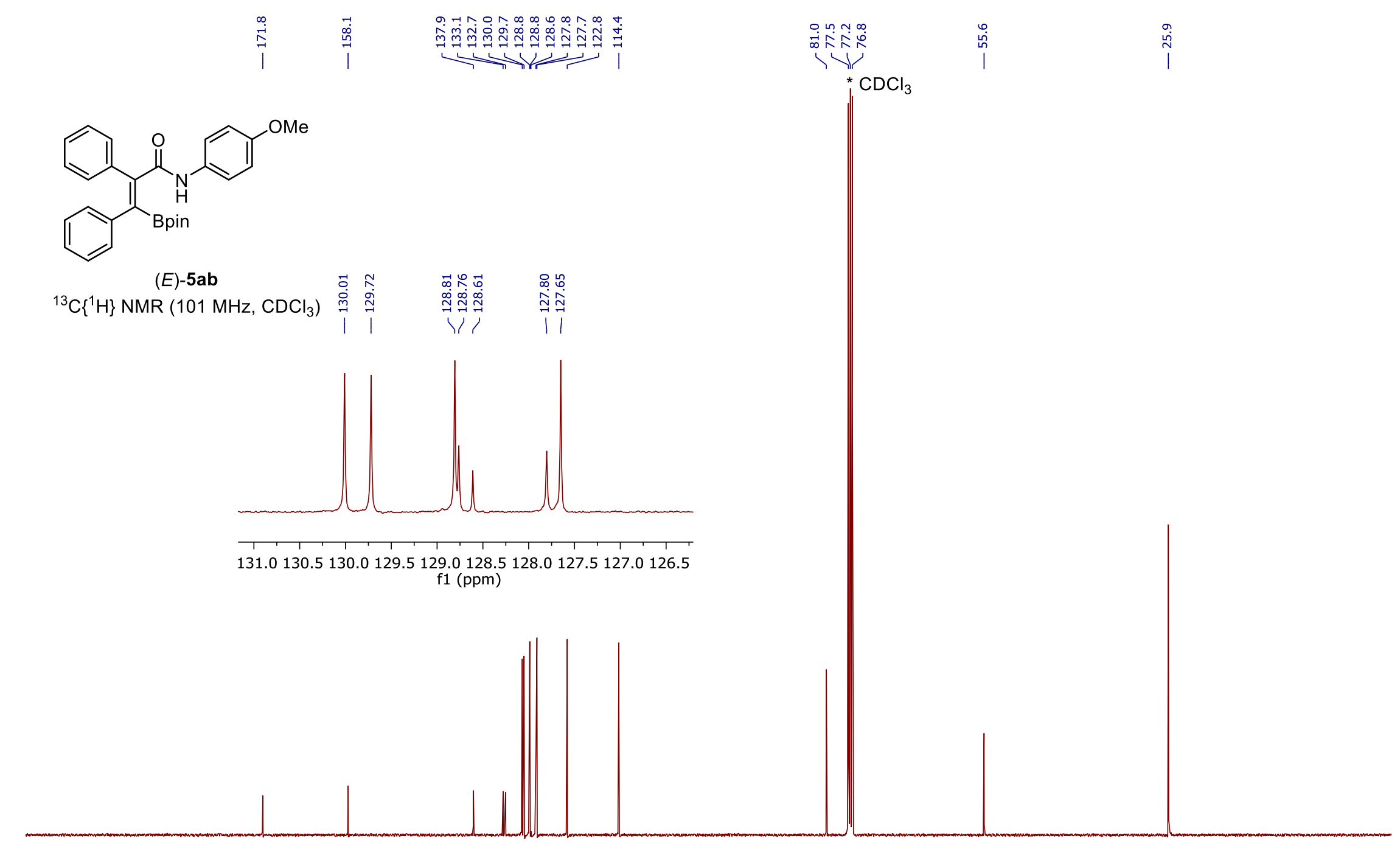

$200 \quad 190 \quad 180 \quad 170 \quad 160 \quad 150 \quad 140 \quad 130$ $110 \quad 100$ f1 $(\mathrm{ppm})$ 


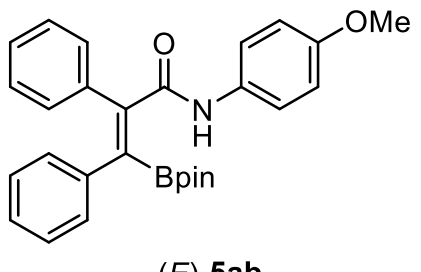

(E)-5ab

${ }^{11} \mathrm{~B}$ NMR $\left(128 \mathrm{MHz}, \mathrm{CDCl}_{3}\right)$

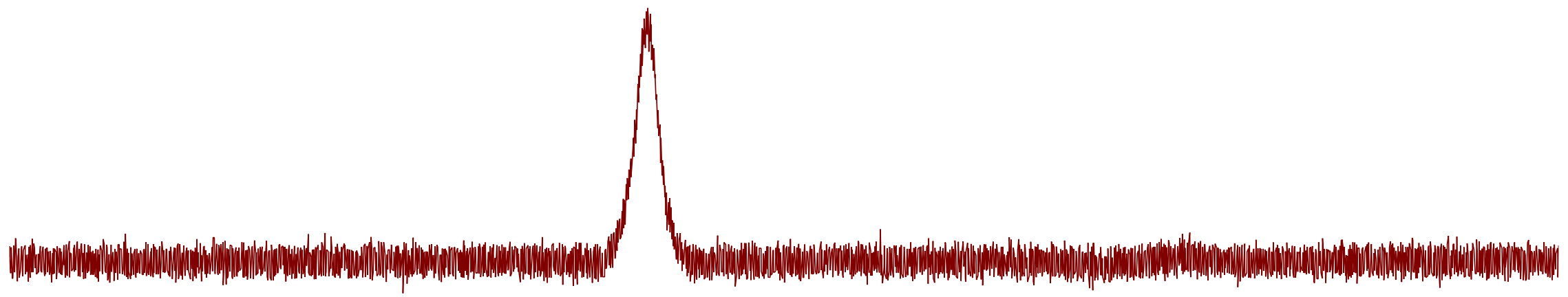




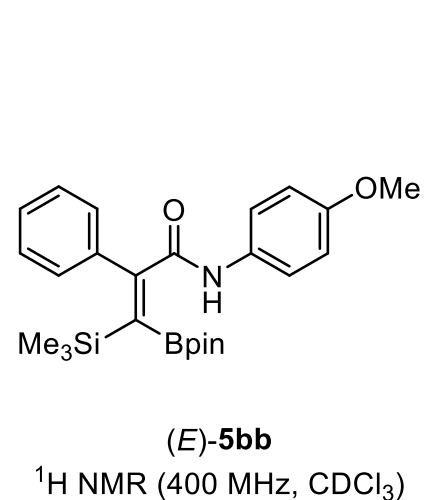

ํㅏㅅำ

jonitiong

$\stackrel{\sim}{\infty}$

\section{òj}

${ }^{1} \mathrm{H}$ NMR $\left(400 \mathrm{MHz}, \mathrm{CDCl}_{3}\right)$
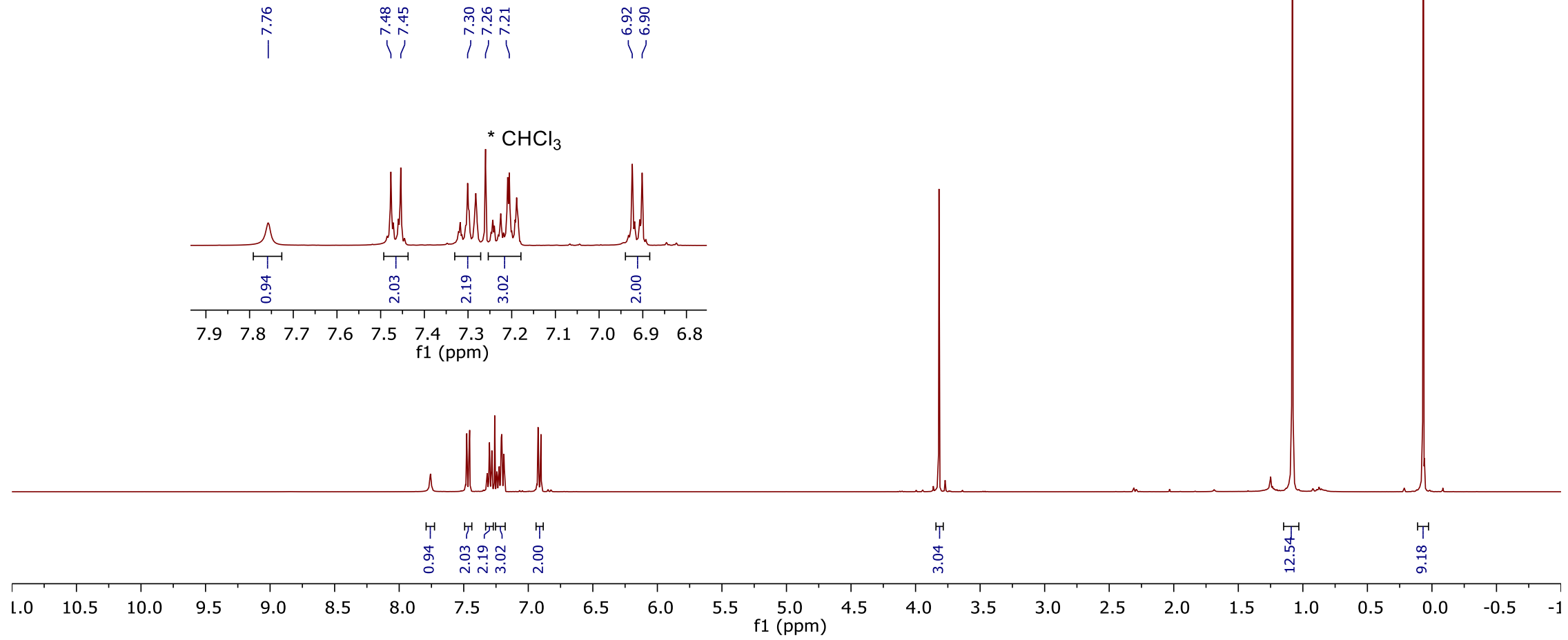


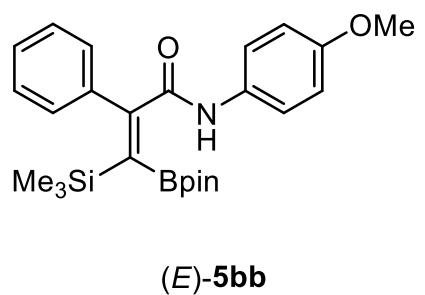

${ }^{13} \mathrm{C}\left\{{ }^{1} \mathrm{H}\right\}$ NMR $\left(101 \mathrm{MHz}, \mathrm{CDCl}_{3}\right)$

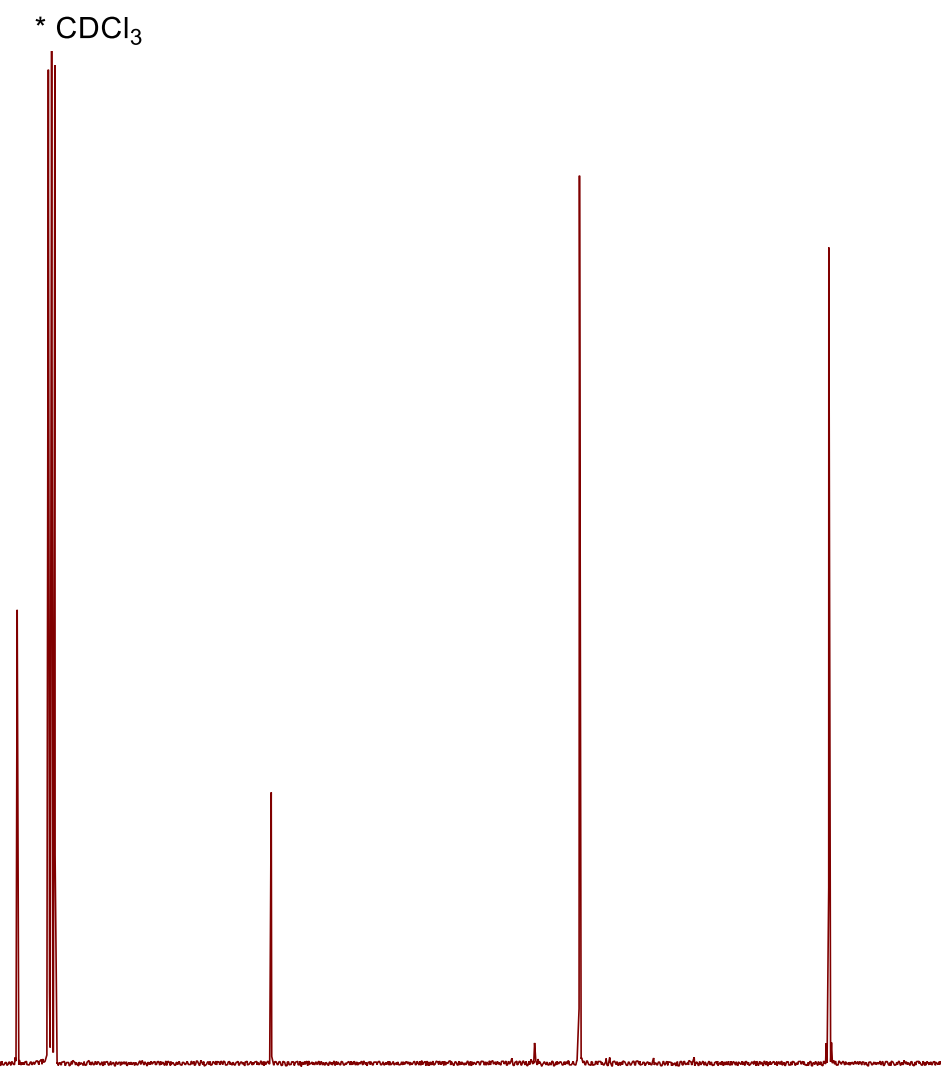

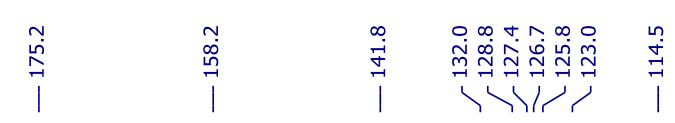

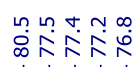

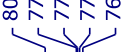

|

$\stackrel{\stackrel{+}{\dot{n}}}{1}$

$\stackrel{\circ}{i}$

(1)

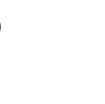




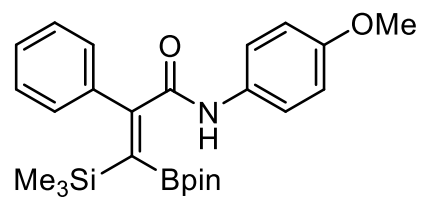

(E)-5bb

${ }^{11} \mathrm{~B}$ NMR $\left(128 \mathrm{MHz}, \mathrm{CDCl}_{3}\right)$

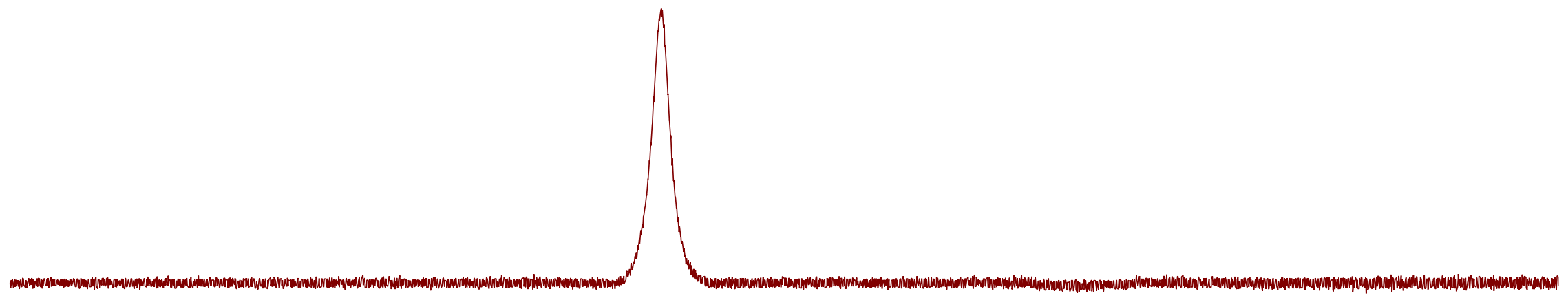

$\begin{array}{lllllll}-30 & -40 & -50 & -60 & -70 & -80 & -!\end{array}$




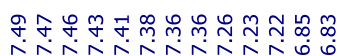

L
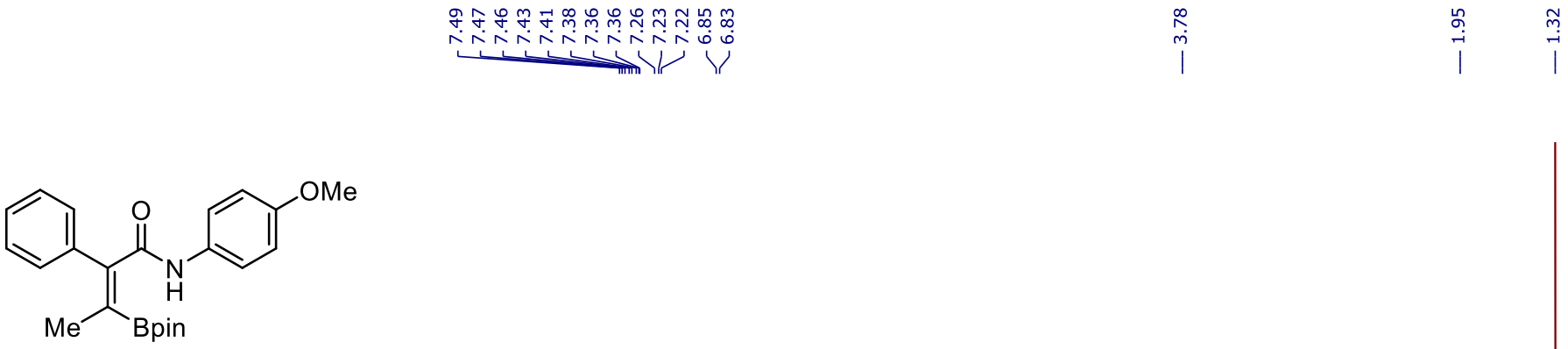

$(E)-5 \mathbf{c b}$

${ }^{1} \mathrm{H}$ NMR $\left(400 \mathrm{MHz}, \mathrm{CDCl}_{3}\right)$

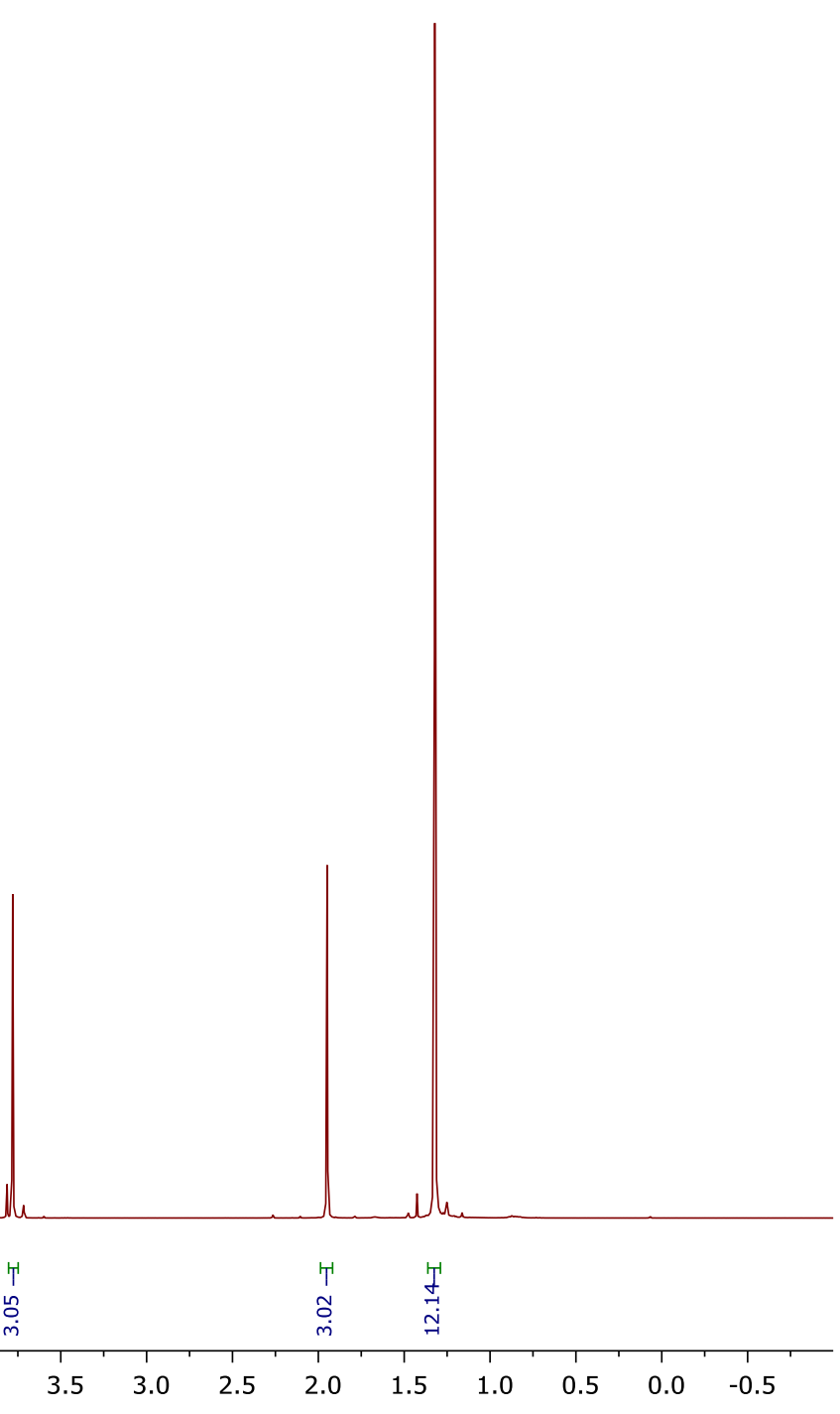

$\begin{array}{llllllllllllllllllllllllll}2.0 & 11.5 & 11.0 & 10.5 & 10.0 & 9.5 & 9.0 & 8.5 & 8.0 & 7.5 & 7.0 & 6.5 & 6.0 & 5.5 & 5.0 & 4.5 & 4.0 & 3.5 & 3.0 & 2.5 & 2.0 & 1.5 & 1.0 & 0.5 & 0.0 & -0.5\end{array}$ 

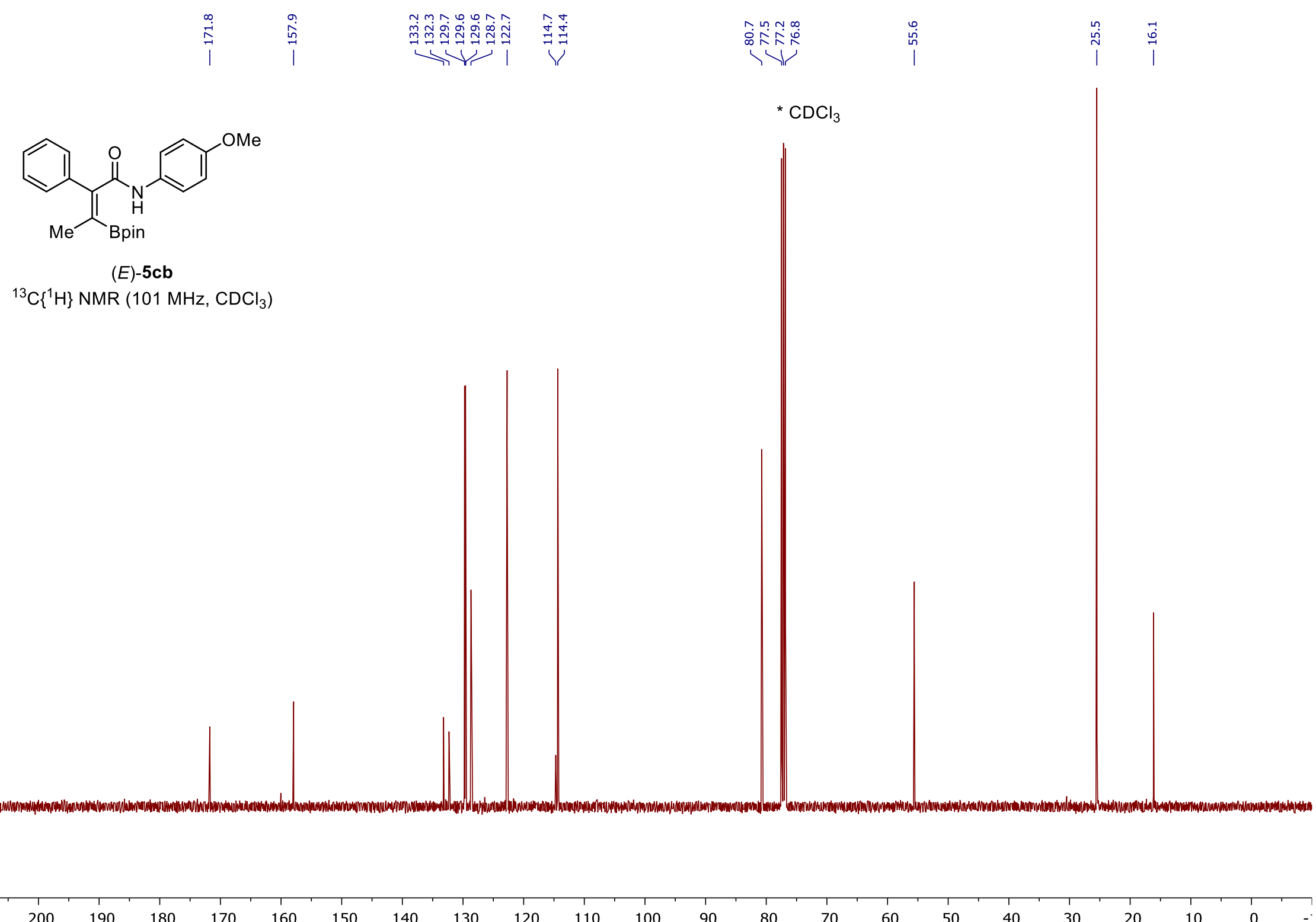

$150 \quad 140$

130

120

$110 \quad 100$

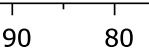

70

$60 \quad 50$

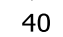

30 


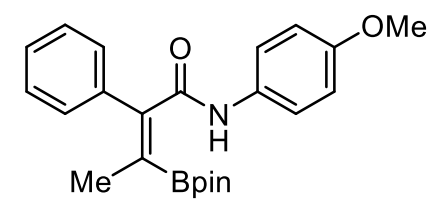

(E)-5cb

${ }^{11} \mathrm{~B}$ NMR $\left(128 \mathrm{MHz}, \mathrm{CDCl}_{3}\right)$

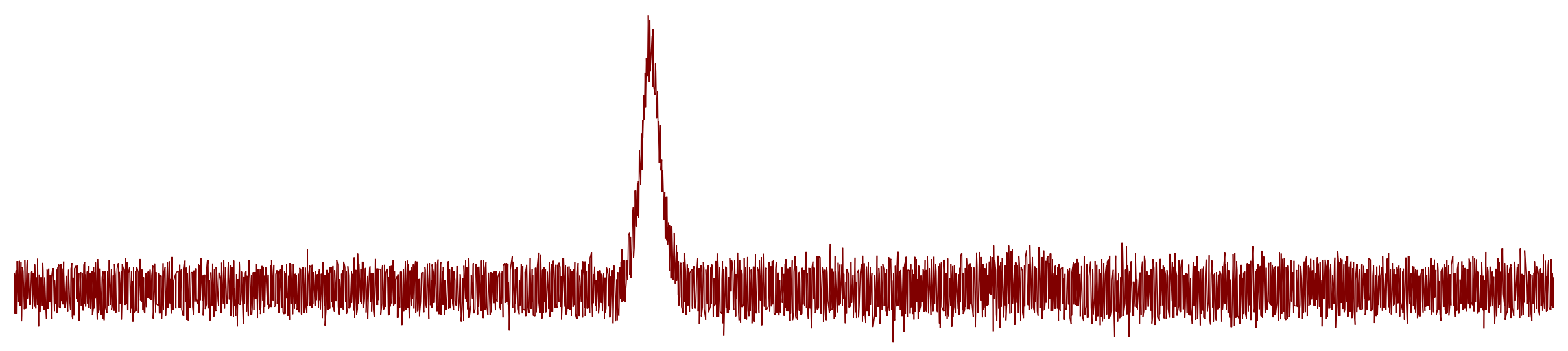



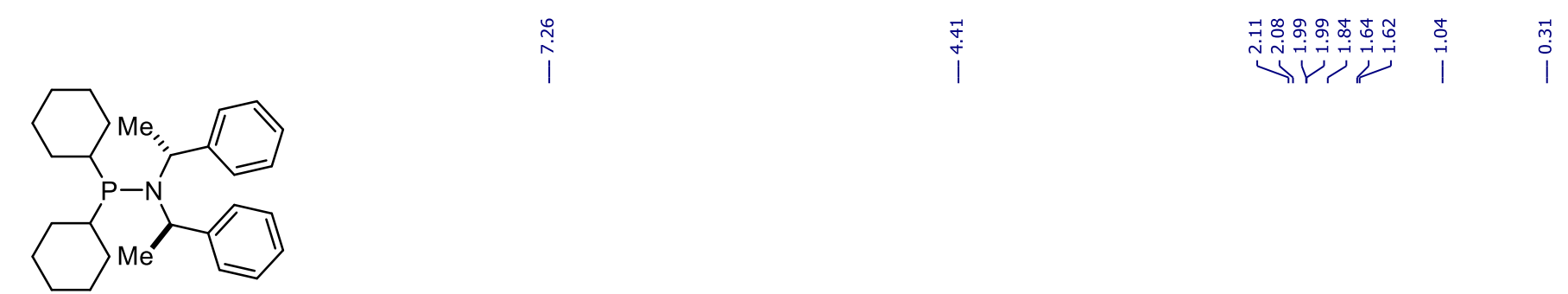

$(R, R)-6 \mathrm{k}$

${ }^{1} \mathrm{H}$ NMR $\left(400 \mathrm{MHz}, \mathrm{CDCl}_{3}\right)$

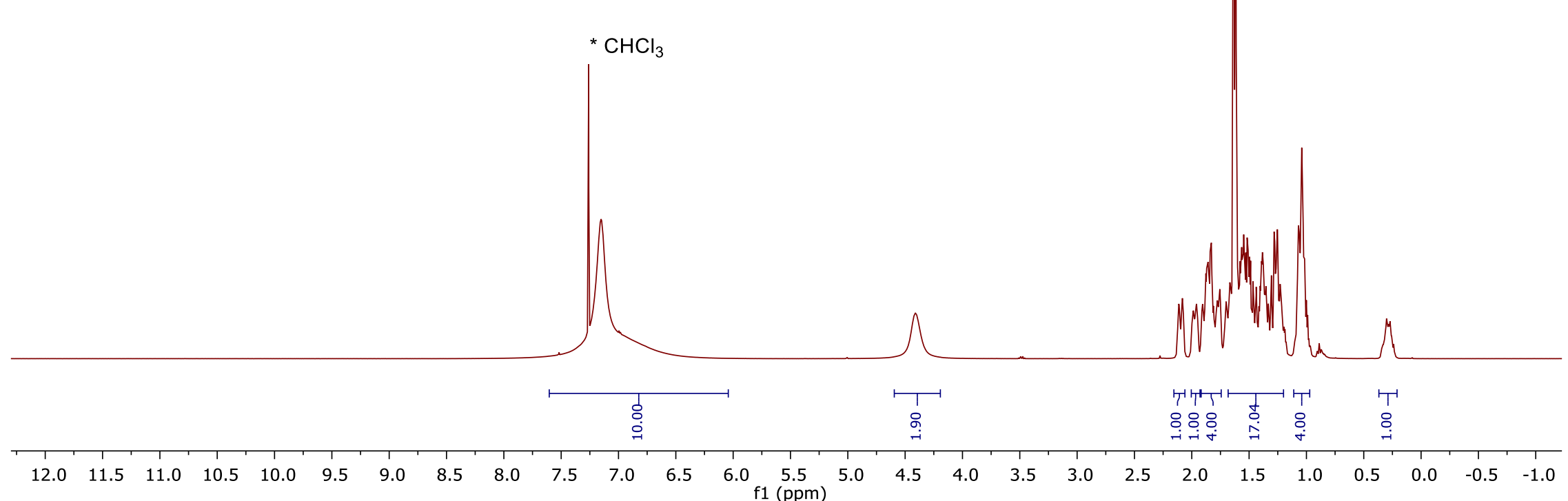




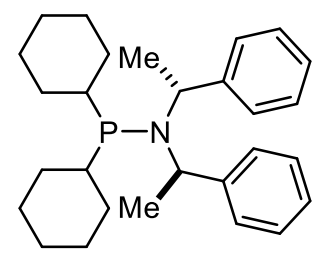

${ }^{*} \mathrm{CDCl}_{3}$

$(R, R)-6 \mathbf{k}$

${ }^{13} \mathrm{C}\left\{{ }^{1} \mathrm{H}\right\}$ NMR $\left(101 \mathrm{MHz}, \mathrm{CDCl}_{3}\right)$

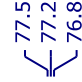

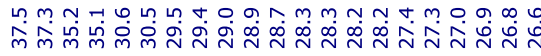

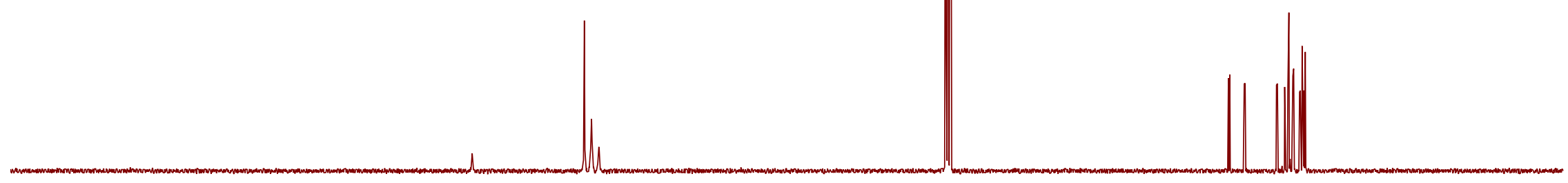

$10 \quad 200$

$180 \quad 170$ 
管

$\stackrel{m}{i m}$

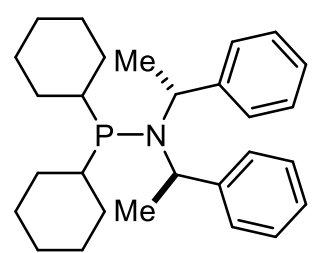

$(R, R)-\mathbf{6 k}$

${ }^{13} \mathrm{C}\left\{{ }^{1} \mathrm{H}\right\}$ NMR $\left(101 \mathrm{MHz}, \mathrm{CDCl}_{3}\right)$

aliphatic region expansion
11

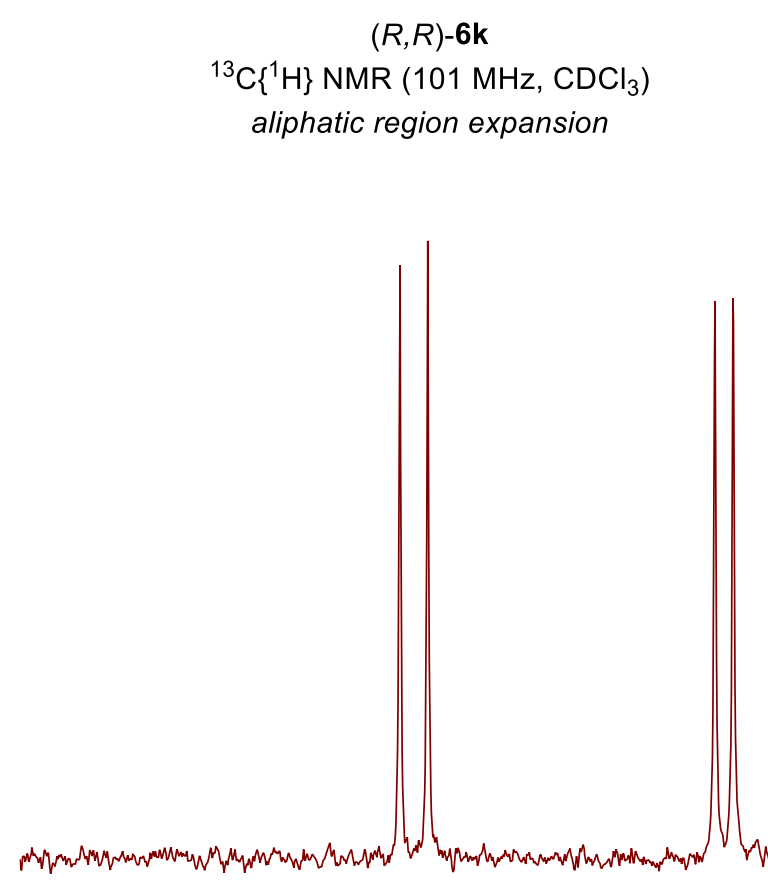

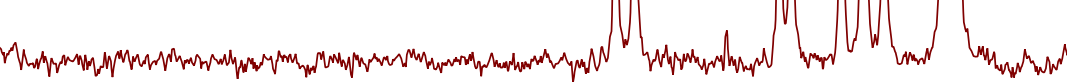

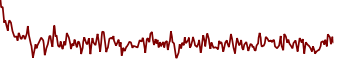




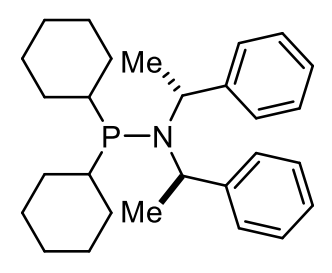

$(R, R)-6 \mathbf{k}$

${ }^{31} \mathrm{P}\left\{{ }^{1} \mathrm{H}\right\} \mathrm{NMR}\left(162 \mathrm{MHz}, \mathrm{CDCl}_{3}\right)$

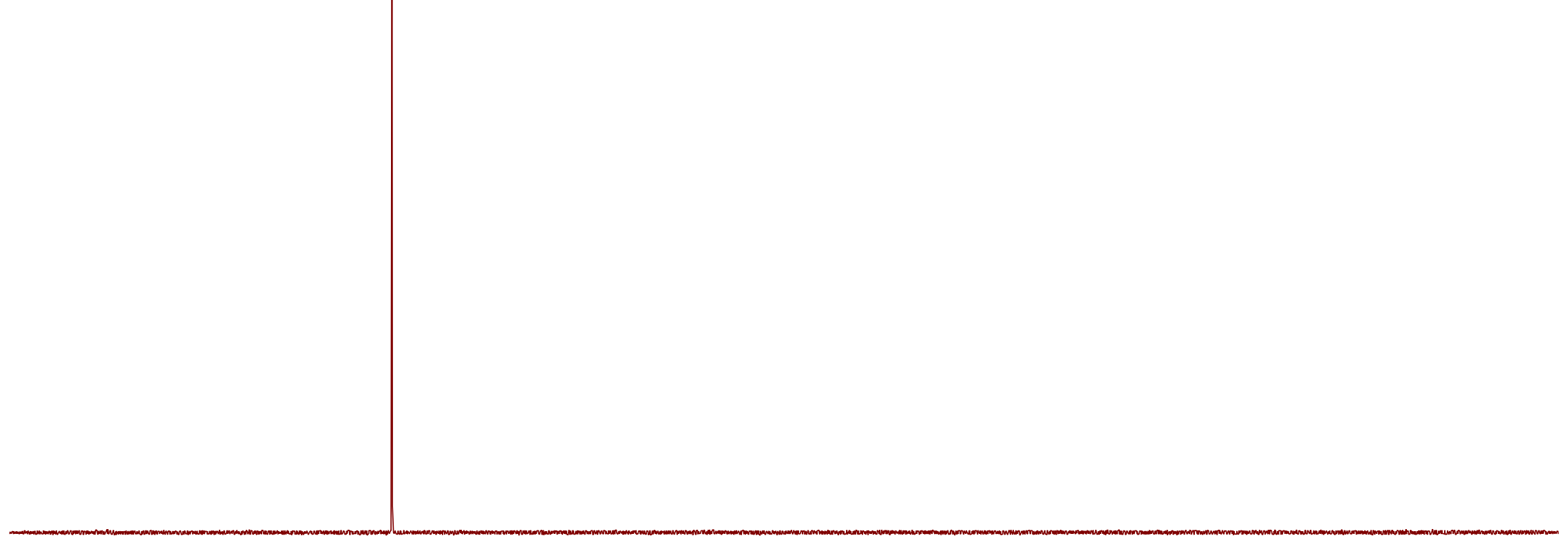




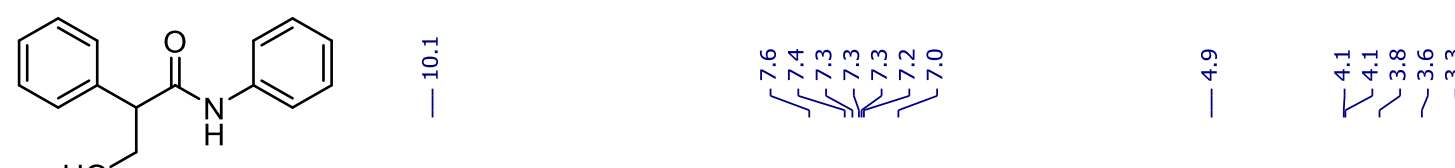

${ }^{1} \mathrm{H}$ NMR $\left(500 \mathrm{MHz}\right.$, DMSO-d $\left.\mathrm{d}_{6}\right)$

il

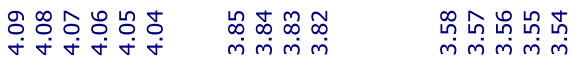

$\underbrace{2}$
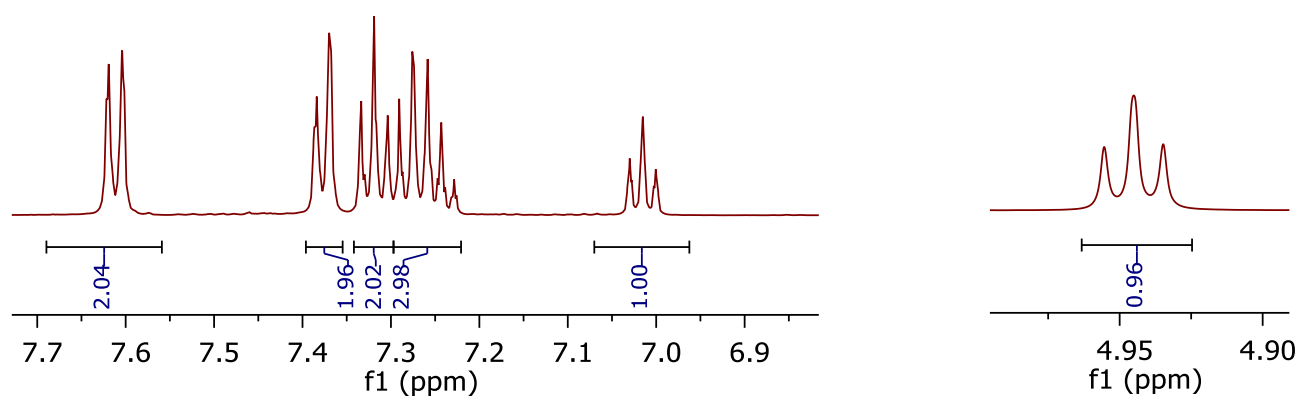

${ }^{*} \mathrm{H}_{2} \mathrm{O}$

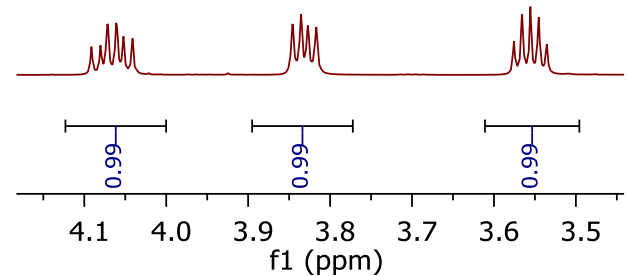

* $\left(\mathrm{CH}_{3}\right)_{2} \mathrm{SO}$

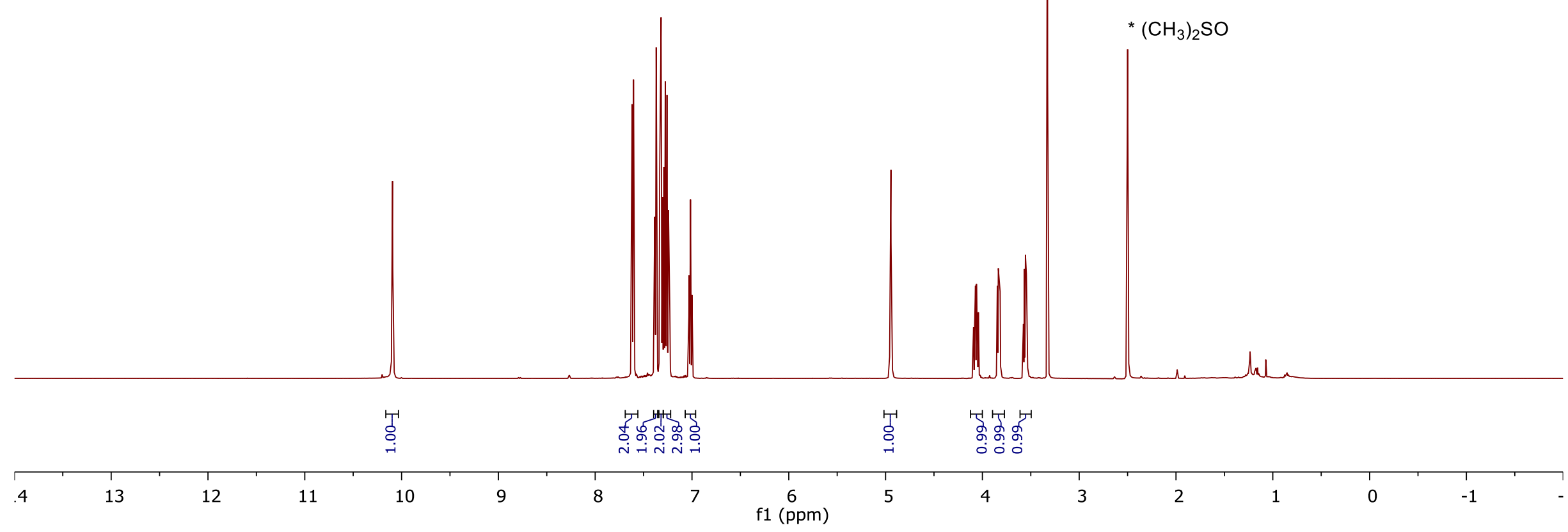




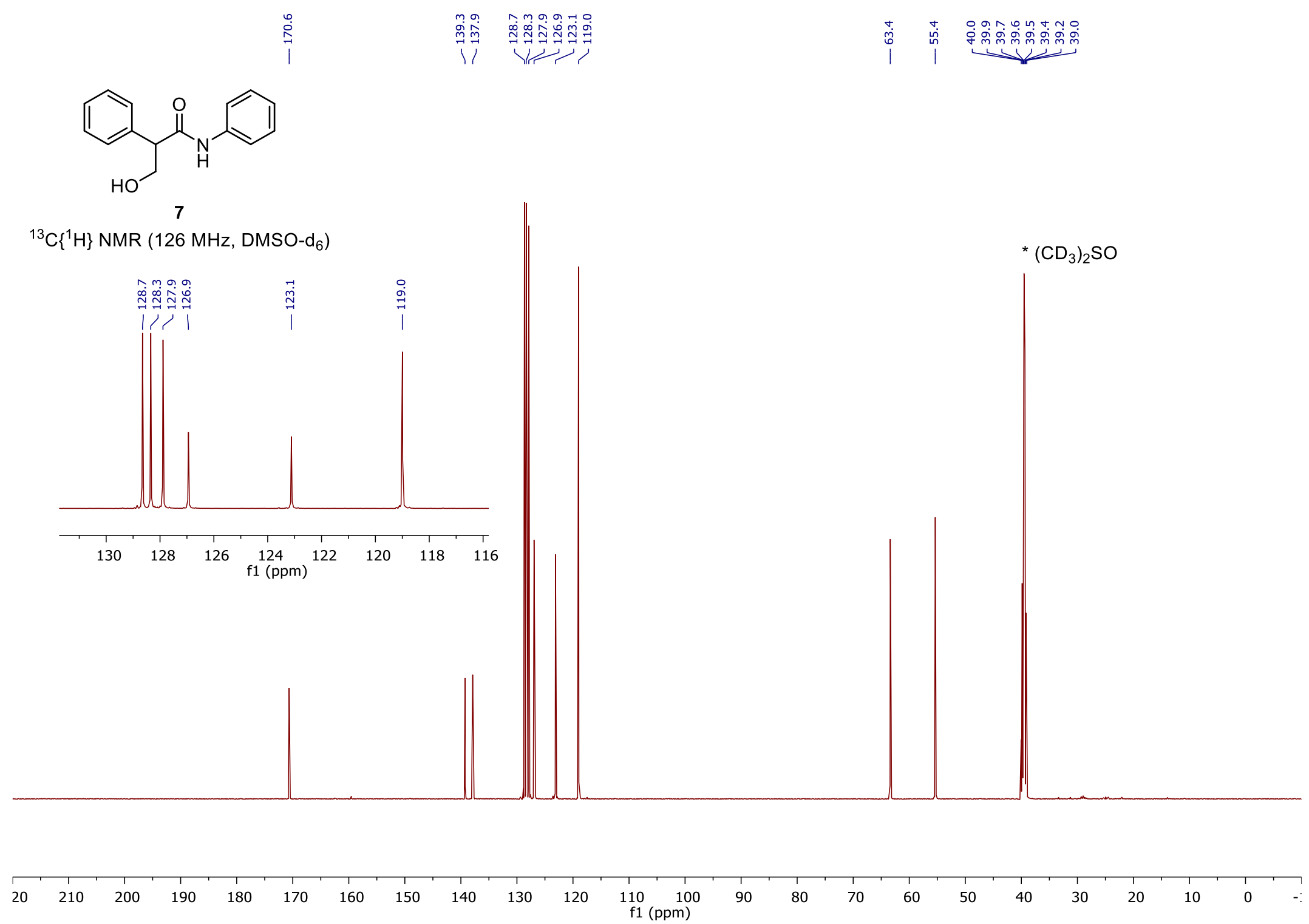




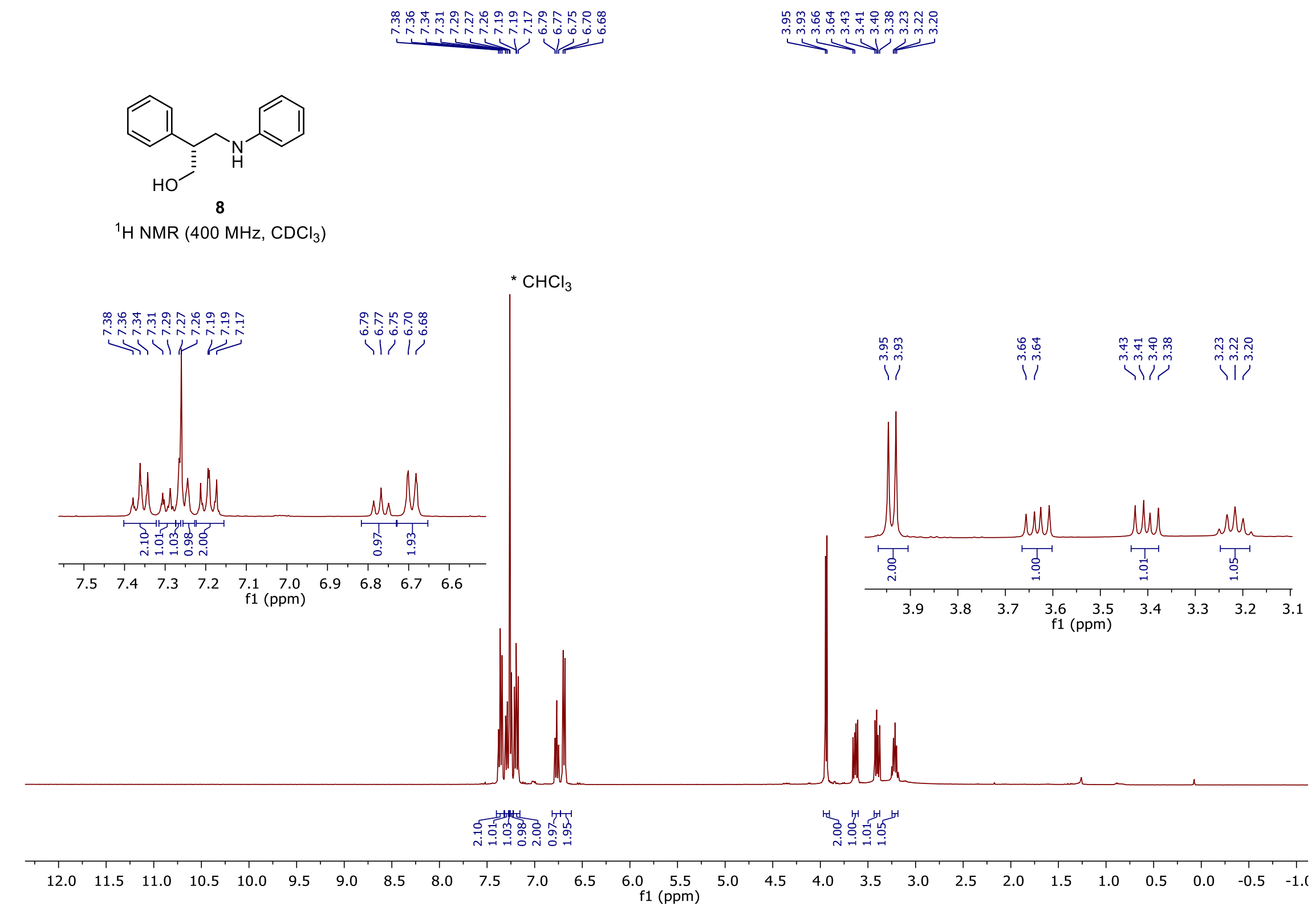




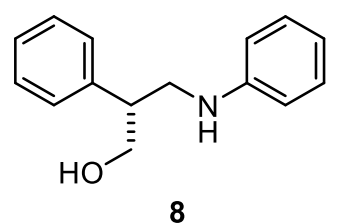

|
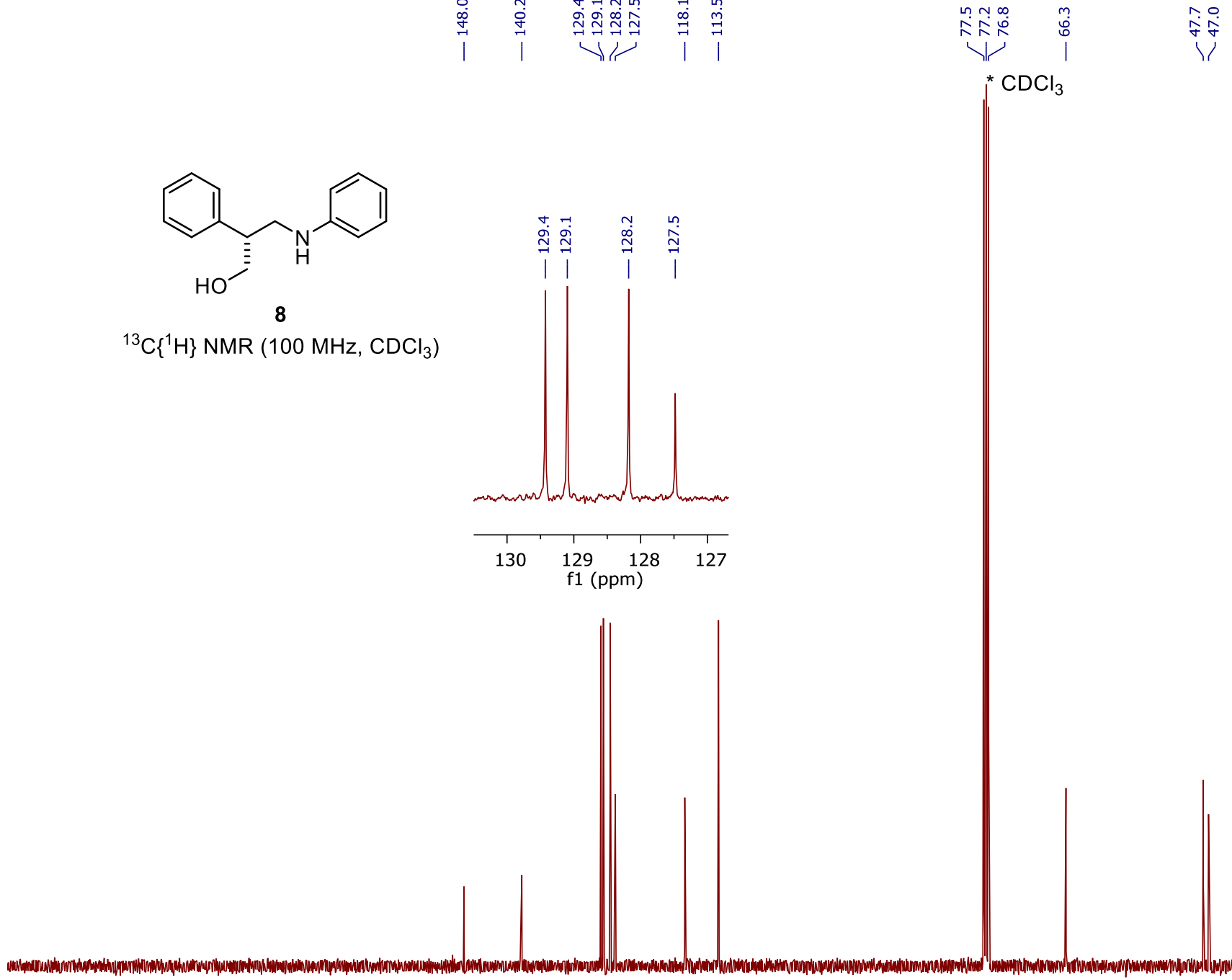

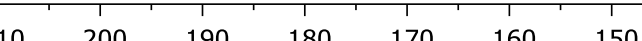




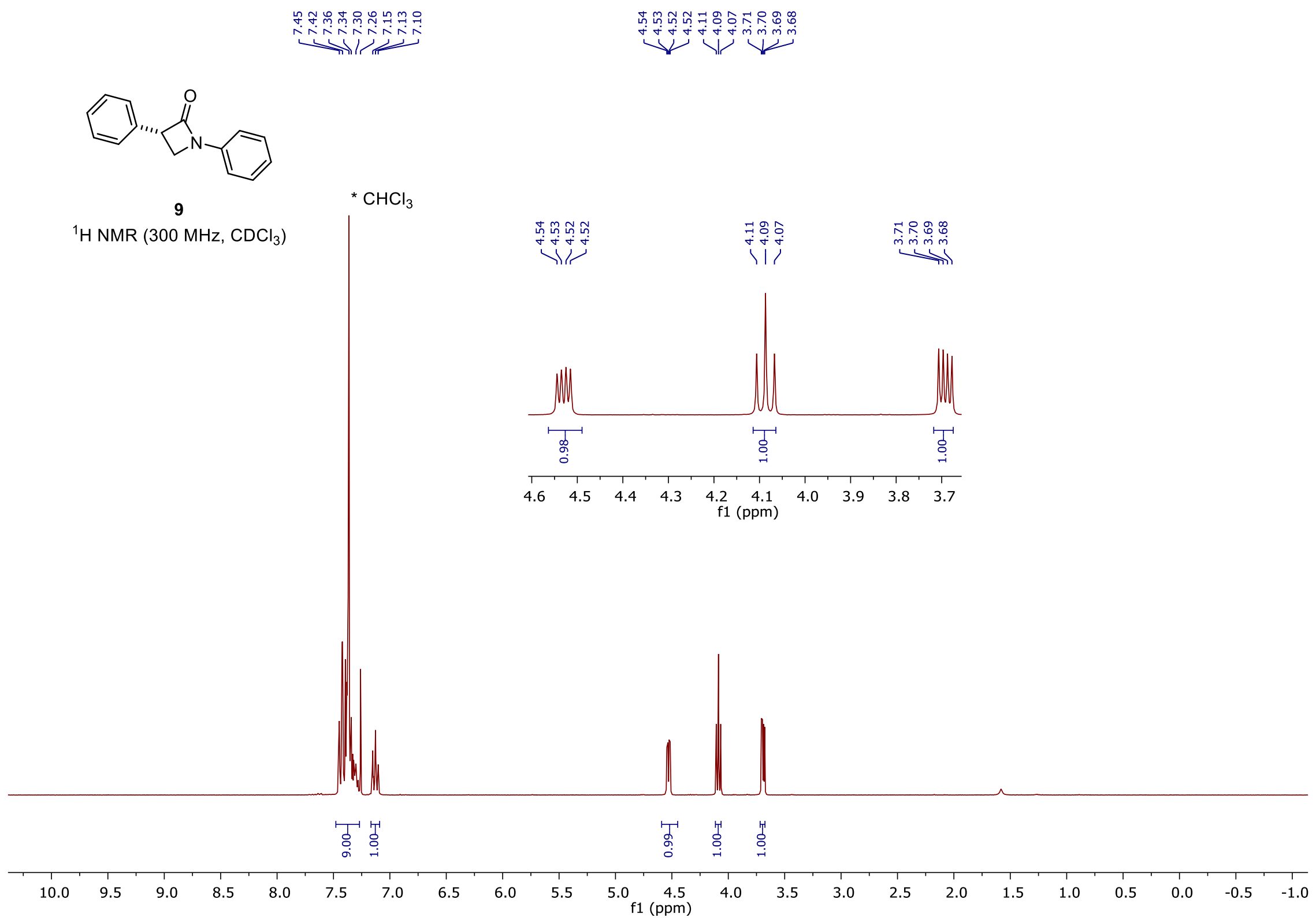


Supporting Information

S324

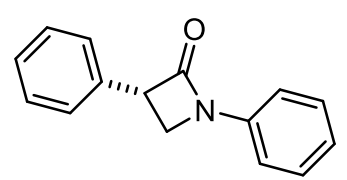

9

${ }^{13} \mathrm{C}\left\{{ }^{1} \mathrm{H}\right\}$ NMR $\left(75 \mathrm{MHz}, \mathrm{CDCl}_{3}\right)$

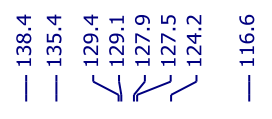
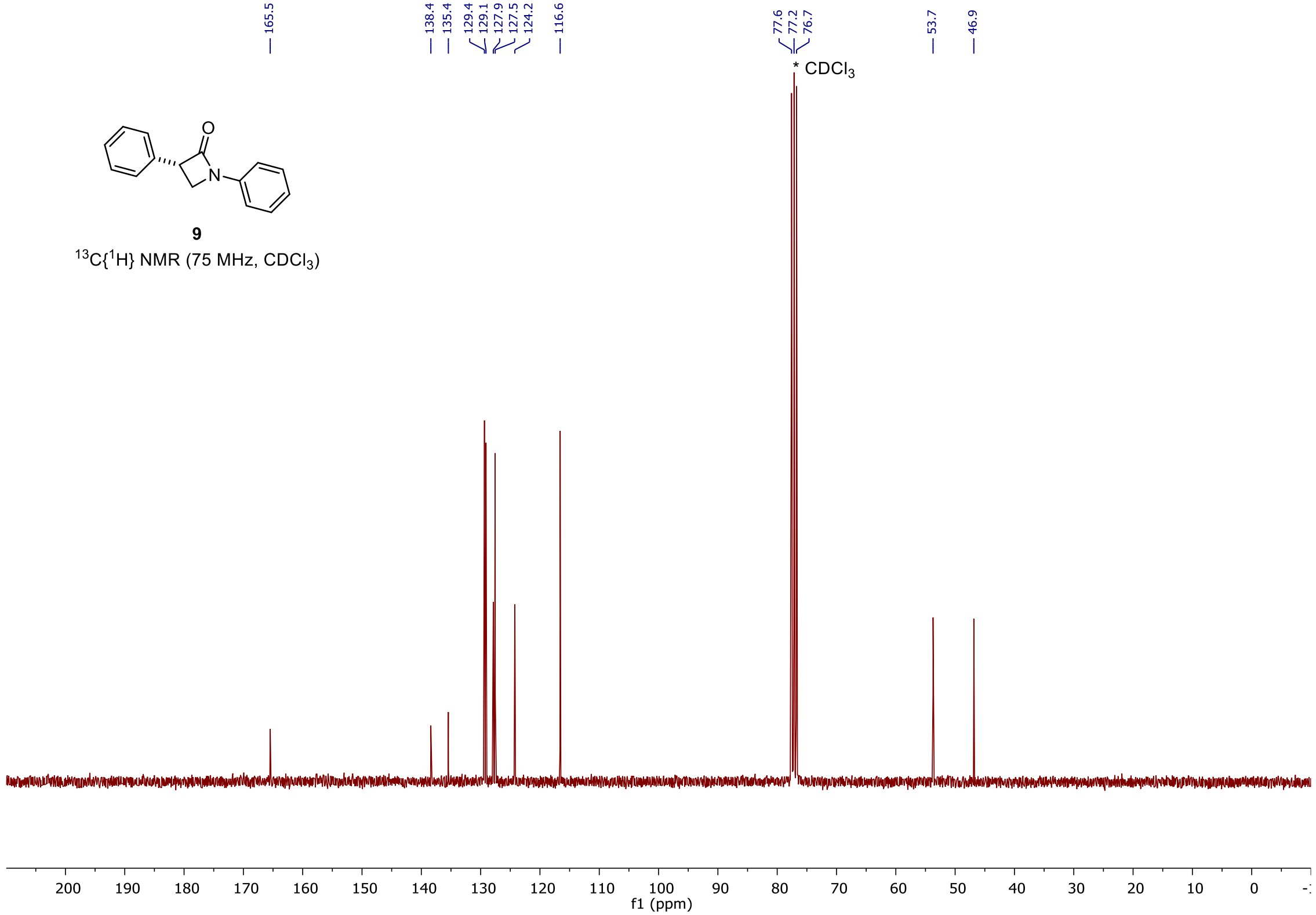


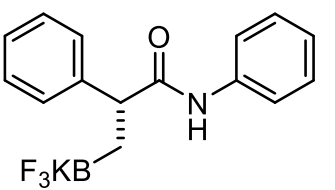

10

${ }^{1} \mathrm{H}$ NMR (400 MHz, Acetone-d6)

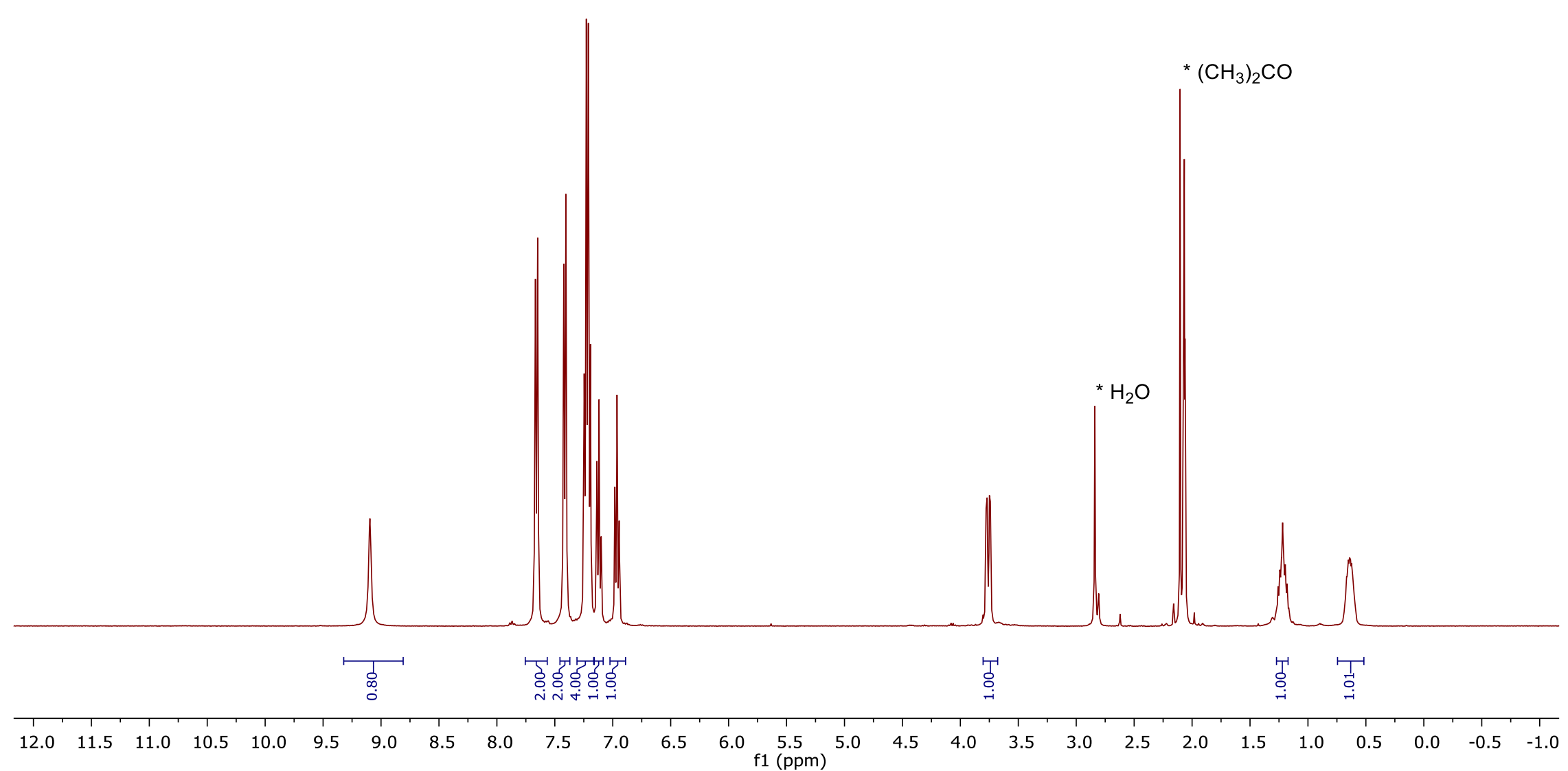




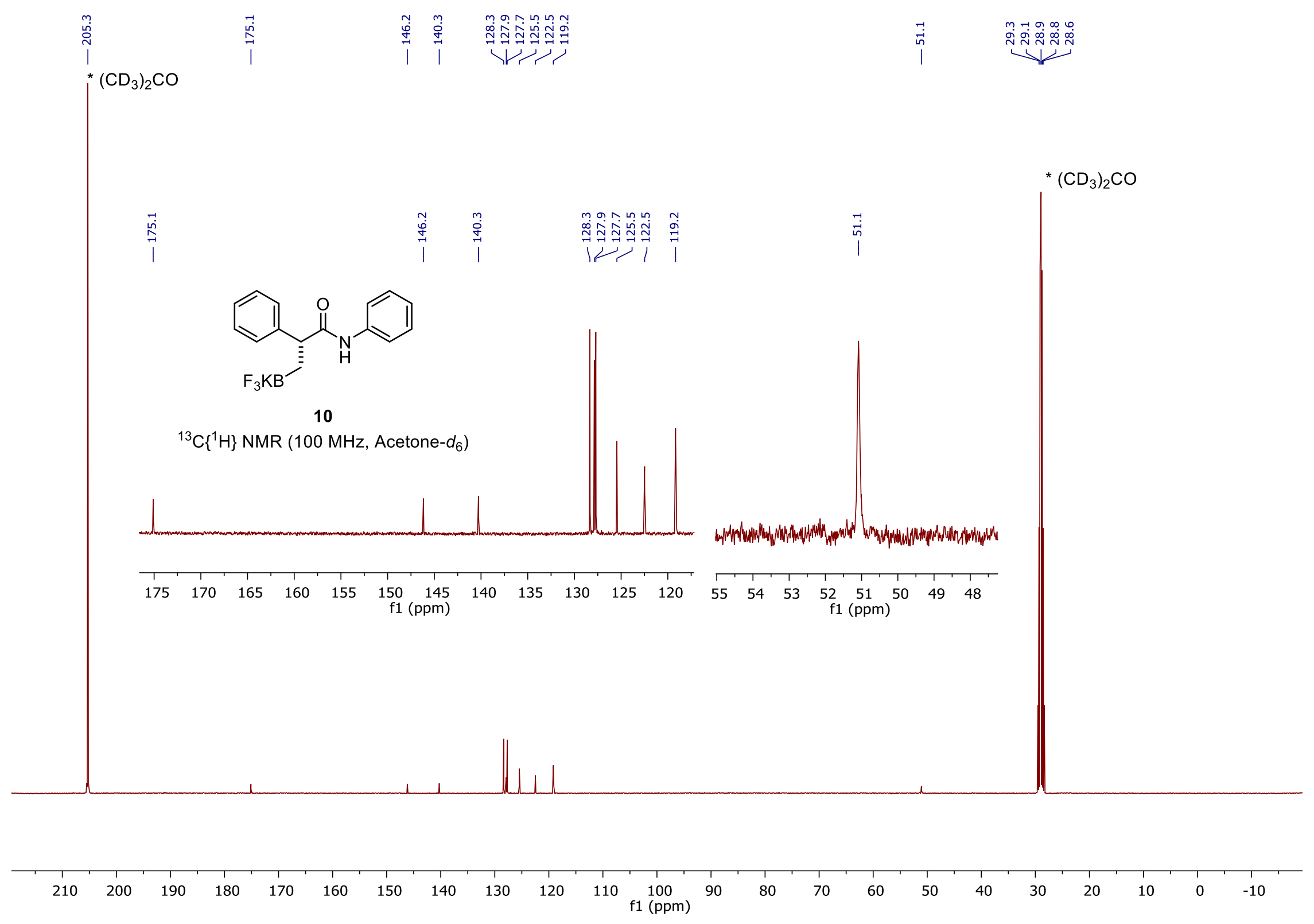




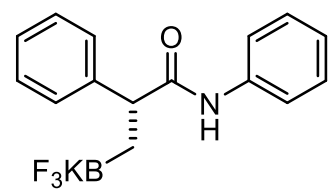

10

${ }^{11} \mathrm{~B}$ NMR $\left(128 \mathrm{MHz}\right.$, Acetone- $\left.d_{6}\right)$

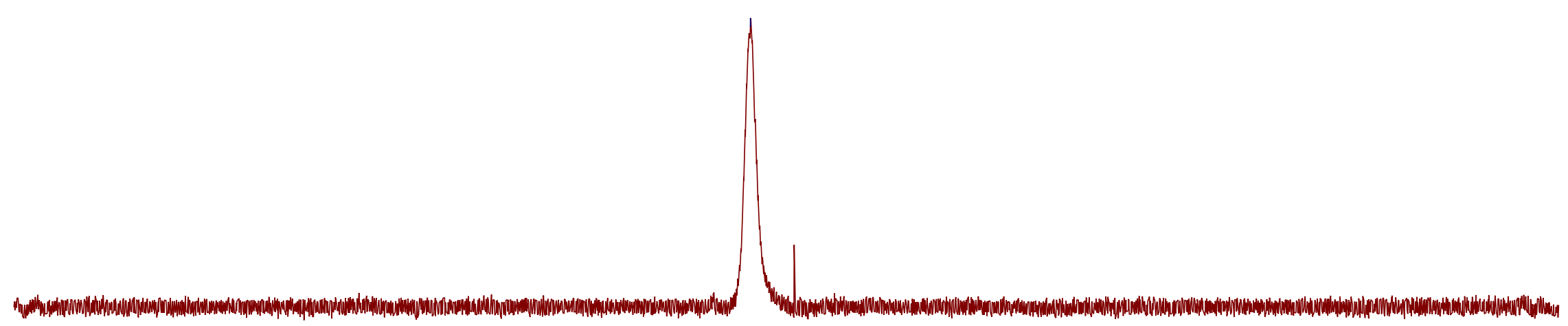

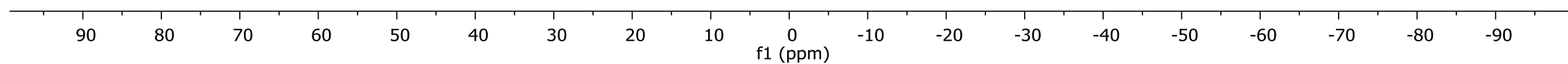




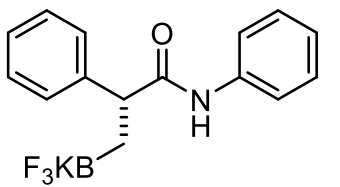

10

${ }^{19} \mathrm{~F}$ NMR (282 MHz, Acetone- $\left.d_{6}\right)$

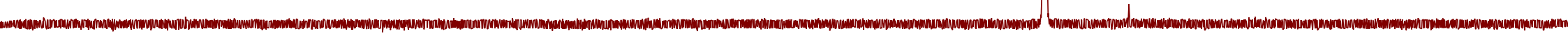

\begin{tabular}{rllllllllllllllllllllllll}
\hline 1 & 1 & 0 & -10 & -20 & -30 & -40 & -50 & -60 & -70 & -80 & -90 & -100 & -110 & -120 & -130 & -140 & -150 & -160 & -170 & -180 & -190 & -200 & -210 & 1
\end{tabular}




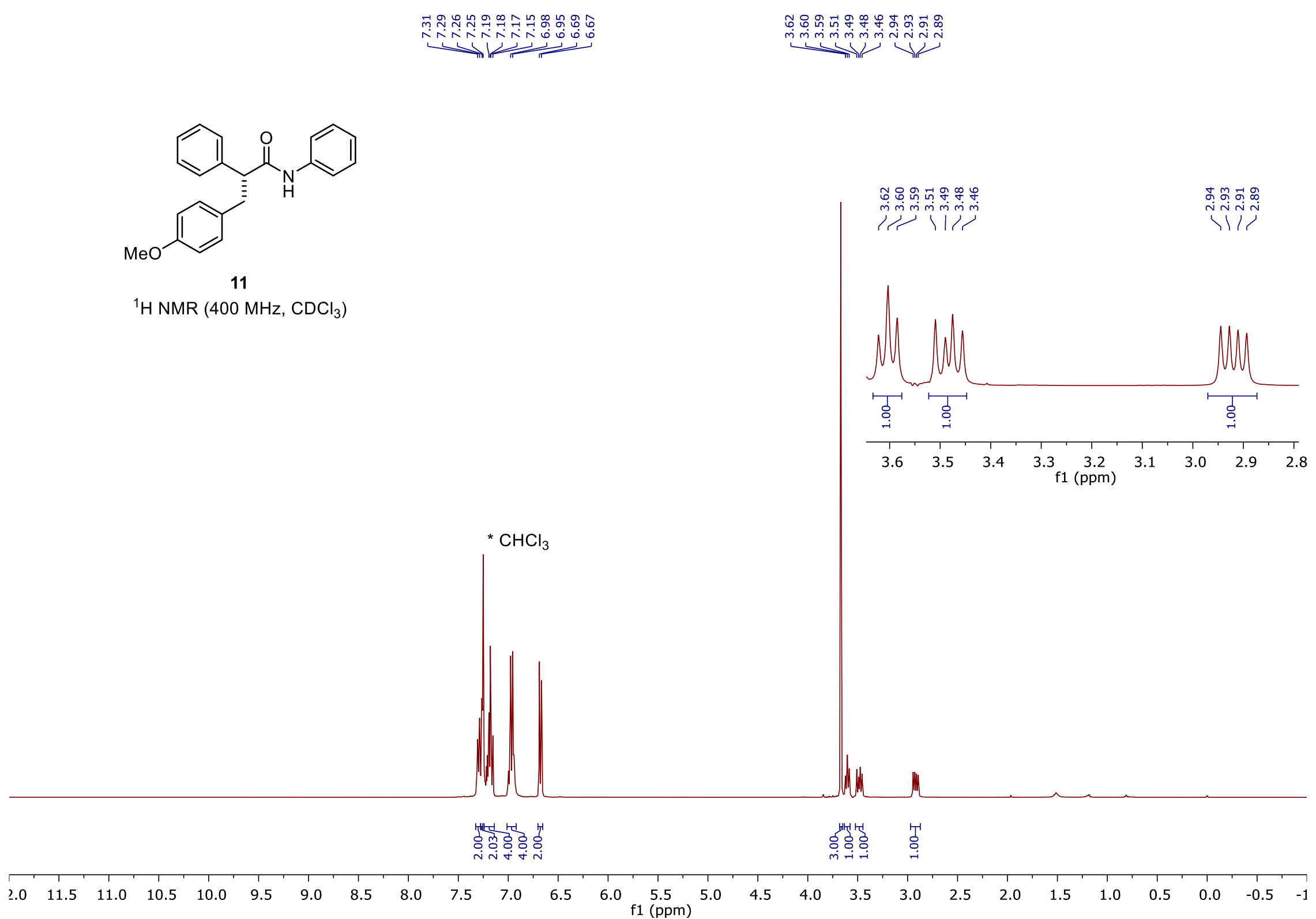




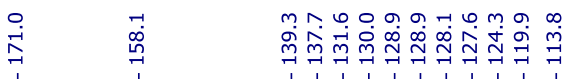

।

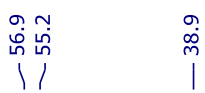

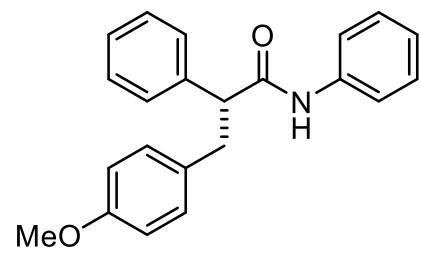

11

${ }^{*} \mathrm{CDCl}_{3}$

${ }^{13} \mathrm{C}\left\{{ }^{1} \mathrm{H}\right\} \mathrm{NMR}\left(101 \mathrm{MHz}, \mathrm{CDCl}_{3}\right)$

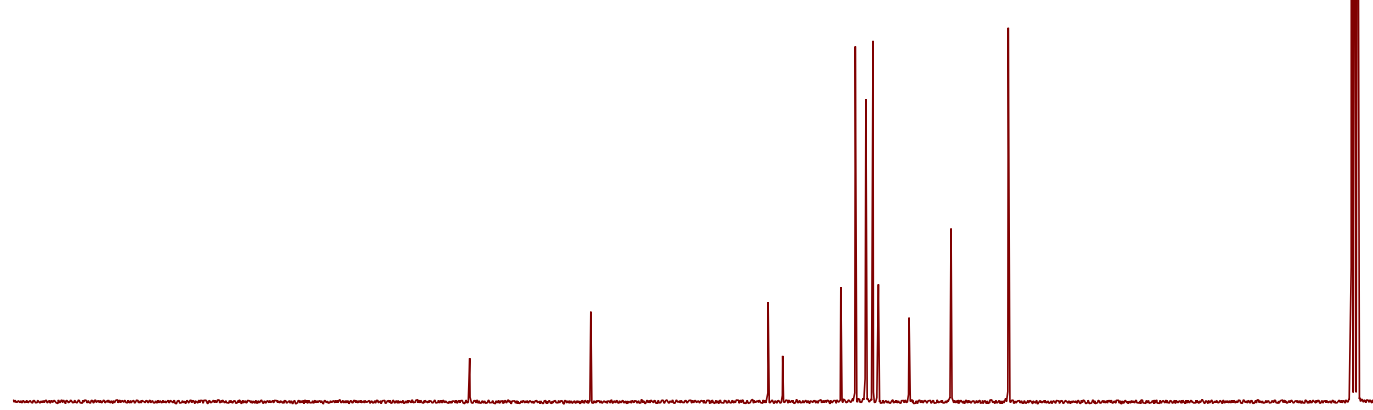

$210 \quad 200$

$170 \quad 160$

$150 \quad 140$

$\begin{array}{llll}130 & 120 & 110 & 100\end{array}$

$\stackrel{100}{\mathrm{f} 1(\mathrm{ppm})}$

80

60

40

30

2010

$0-10$ 


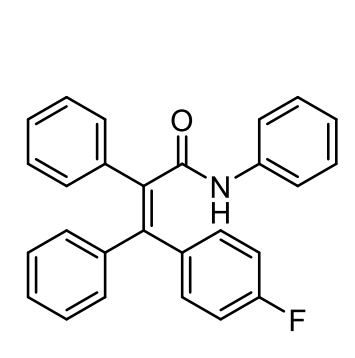

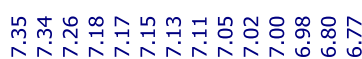

$\stackrel{\substack{n \\ j}}{i}$

${ }^{1} \mathrm{H}$ NMR (400 MHz, $\mathrm{CDCl}_{3}$ )

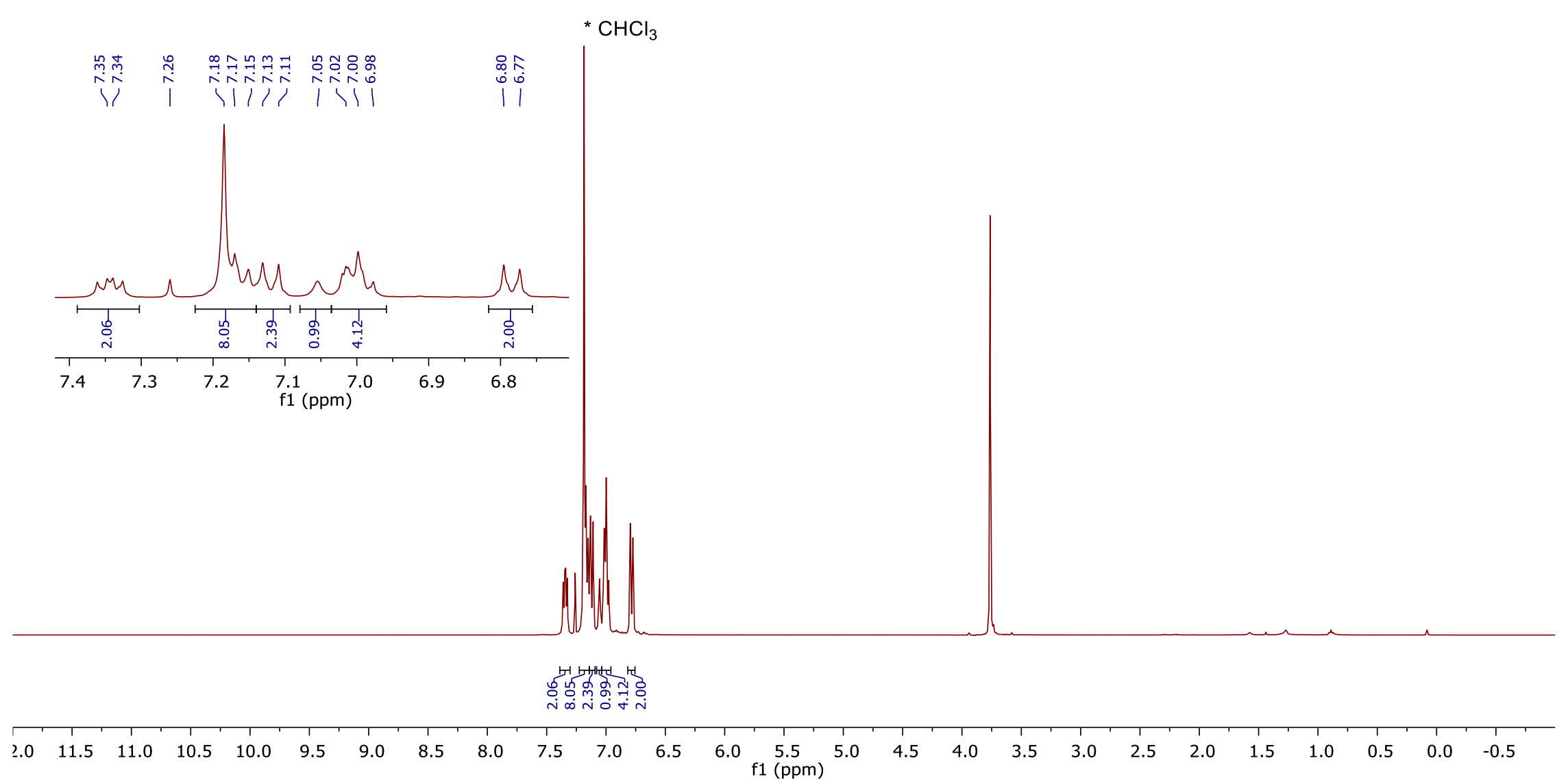




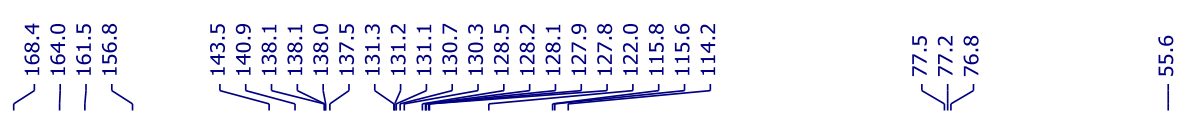

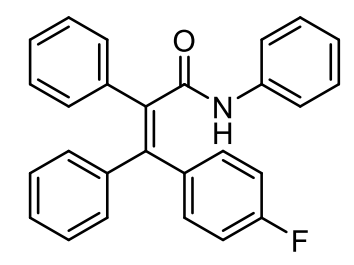

(Z)-12

${ }^{13} \mathrm{C}\left\{{ }^{1} \mathrm{H}\right\}$ NMR $\left(101 \mathrm{MHz}, \mathrm{CDCl}_{3}\right)$

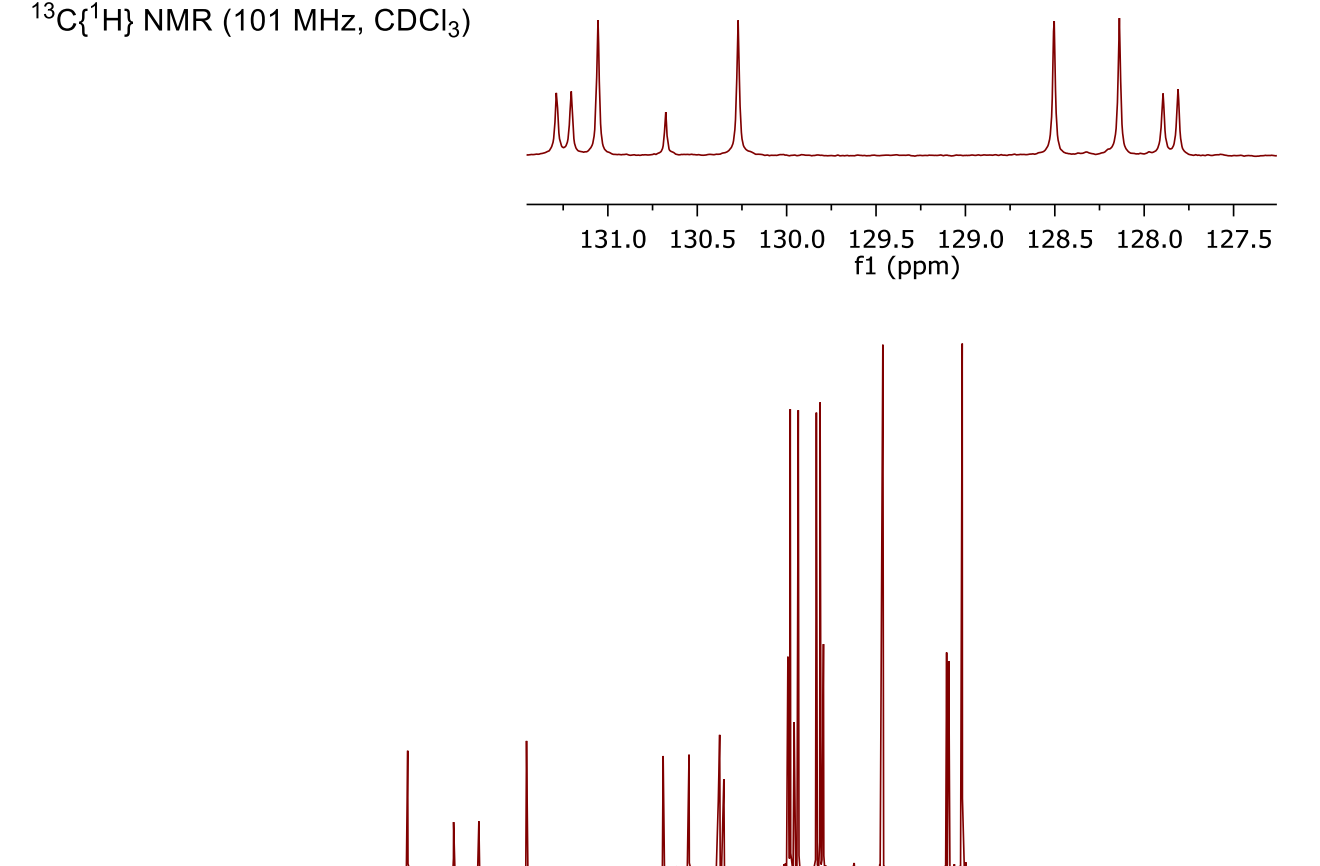

$\begin{array}{llllllll}131.0 & 130.5 & 130.0 & 129.5 & 129.0 & 128.5 & 128.0 & 127.5\end{array}$ f1 (ppm)

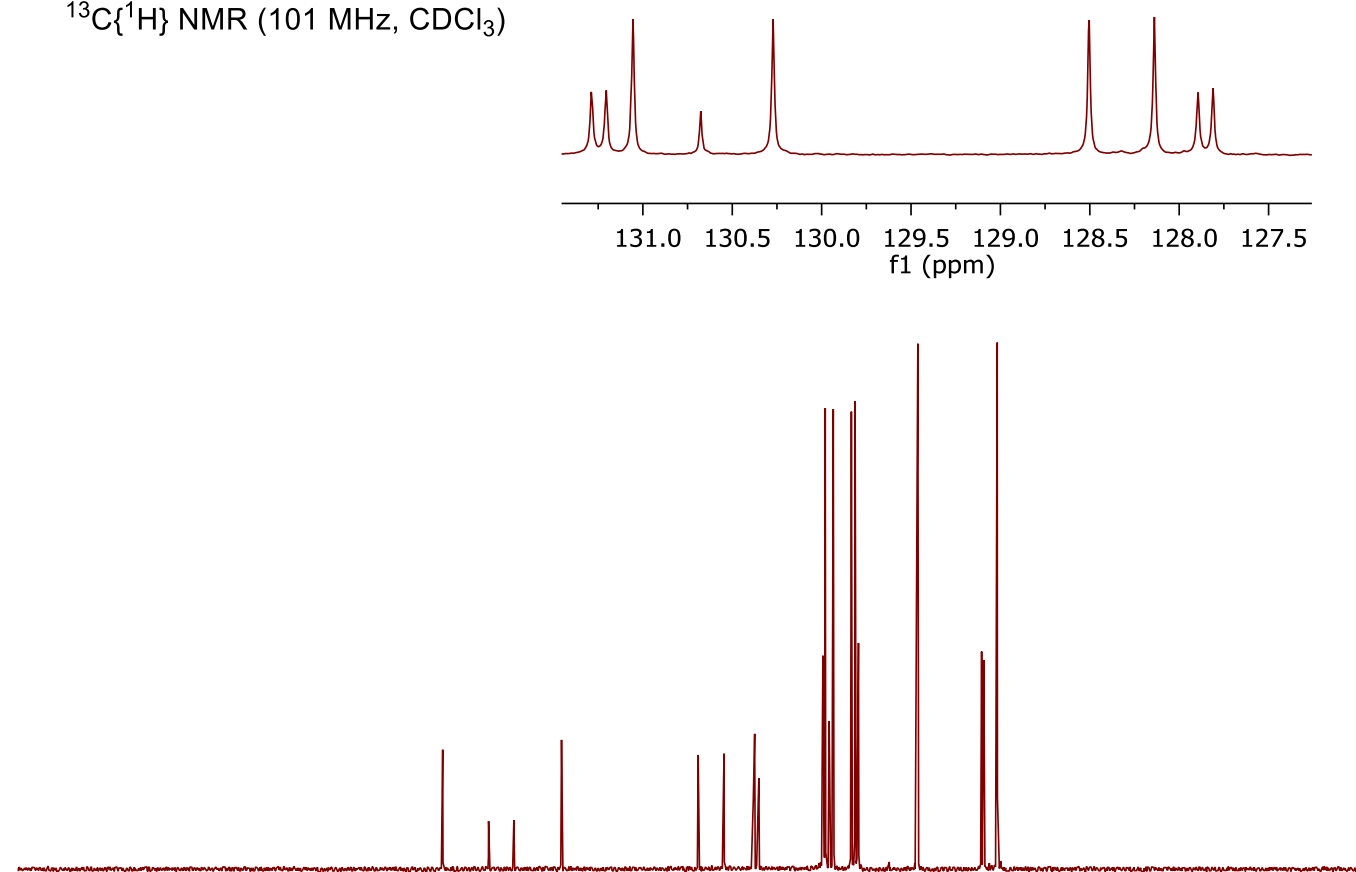

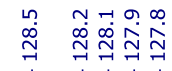

1) 1/1

${ }^{*} \mathrm{CDCl}_{3}$

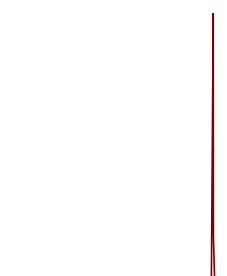

$\begin{array}{lllllllllllllllllllllllll}200 & 190 & 180 & 170 & 160 & 150 & 140 & 130 & 120 & 110 & 100 & 90 & 80 & 70 & 60 & 50 & 40 & 30 & 20 & 10 & 0 & -\therefore\end{array}$ 


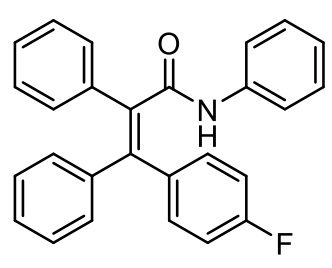

(Z)-12

${ }^{19} \mathrm{~F}\left\{{ }^{1} \mathrm{H}\right\}$ NMR $\left(282 \mathrm{MHz}, \mathrm{CDCl}_{3}\right)$

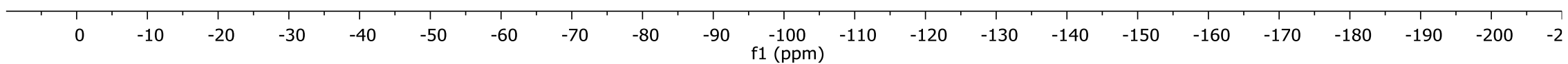




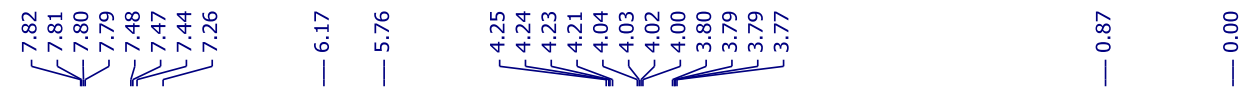

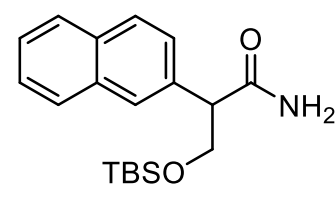

13

${ }^{1} \mathrm{H} \mathrm{NMR}\left(400 \mathrm{MHz}, \mathrm{CDCl}_{3}\right)$

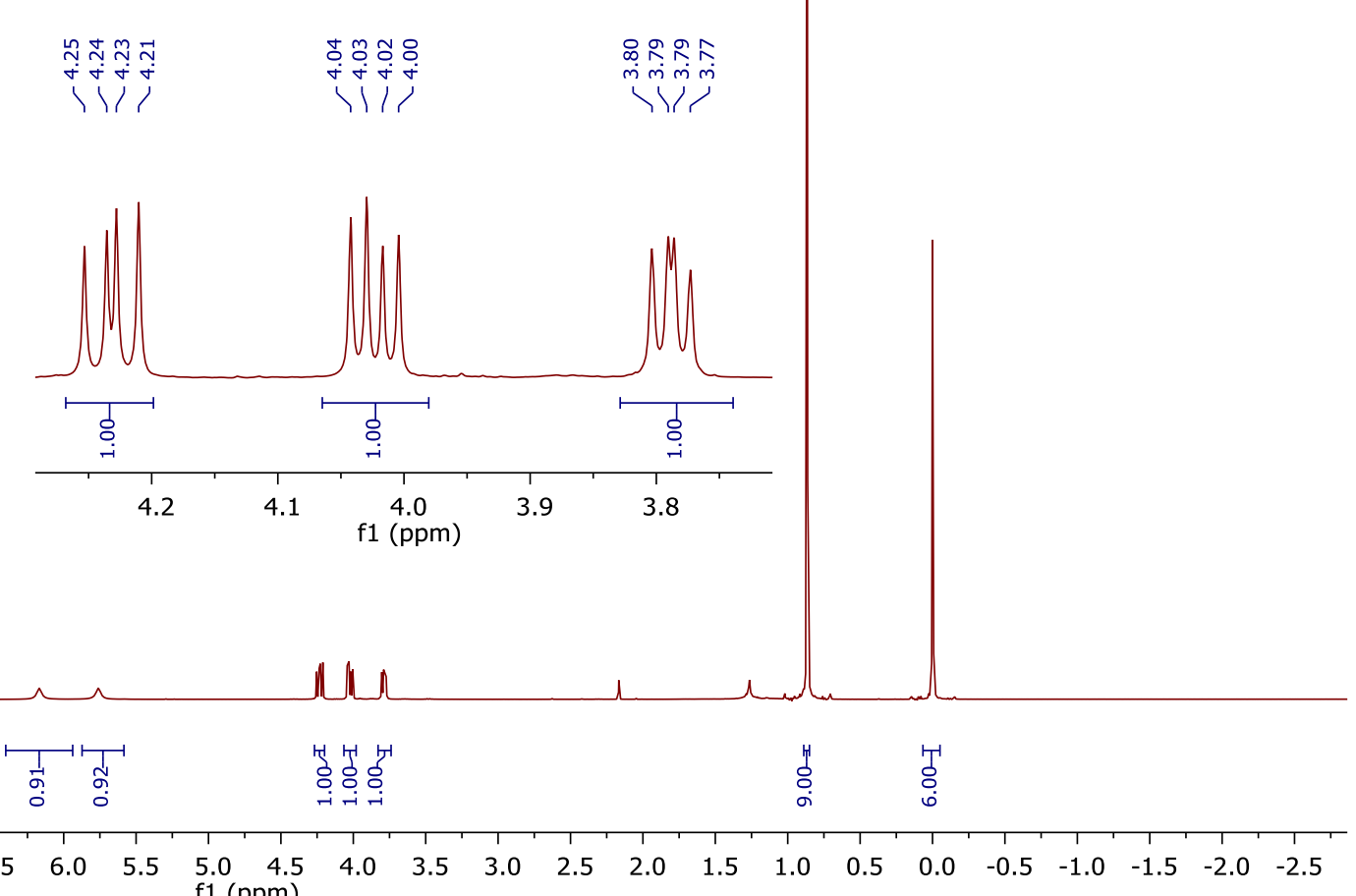

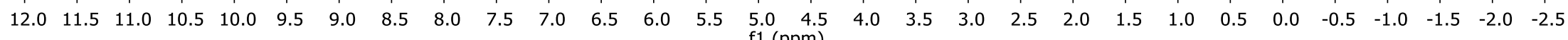




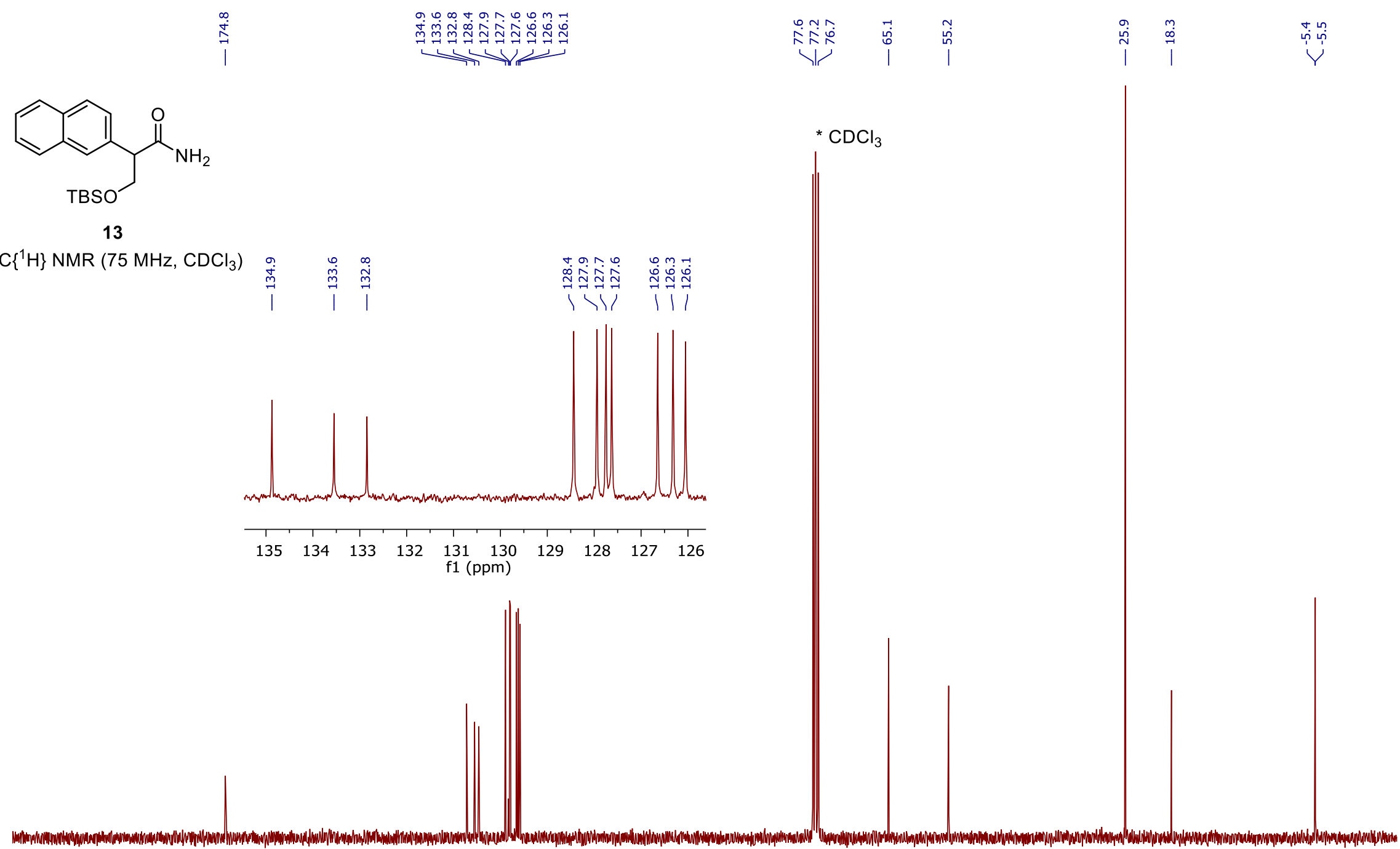

$10+200+190$

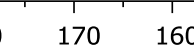

150

$140 \quad 130$

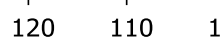

$100 \quad 90$

80

$70 \quad 60$

50

40

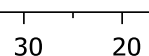

$10+\frac{1}{10}, \frac{1}{2}$ 


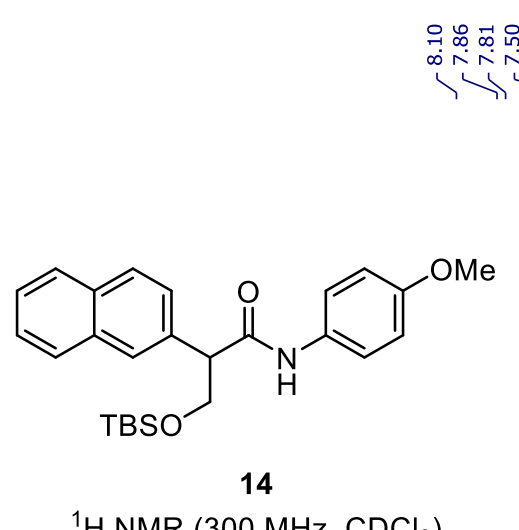

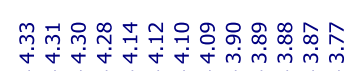



i

${ }^{1} \mathrm{H} \mathrm{NMR}\left(300 \mathrm{MHz}, \mathrm{CDCl}_{3}\right)$
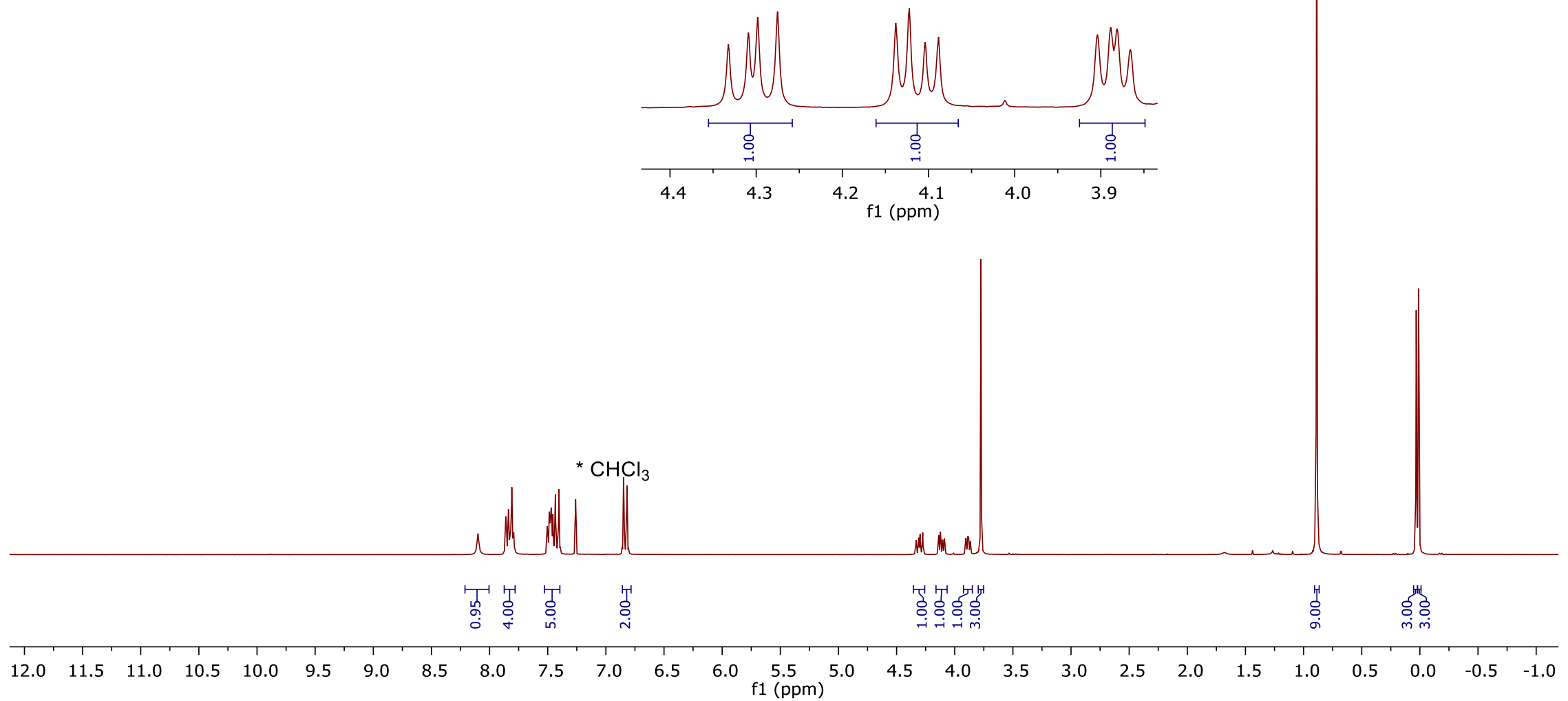

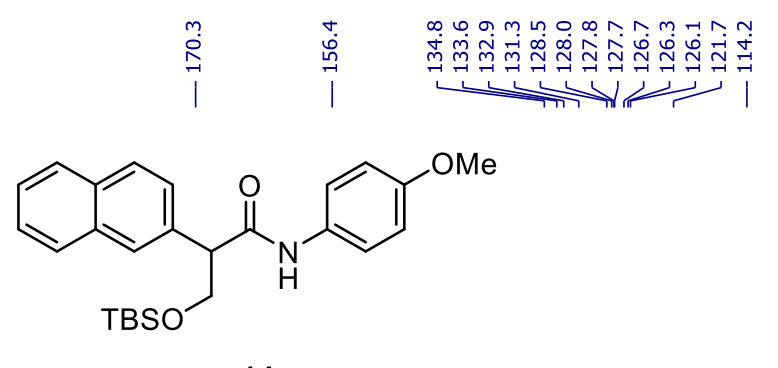

14

${ }^{13} \mathrm{C}\left\{{ }^{1} \mathrm{H}\right\}$ NMR $\left(75 \mathrm{MHz}, \mathrm{CDCl}_{3}\right)$

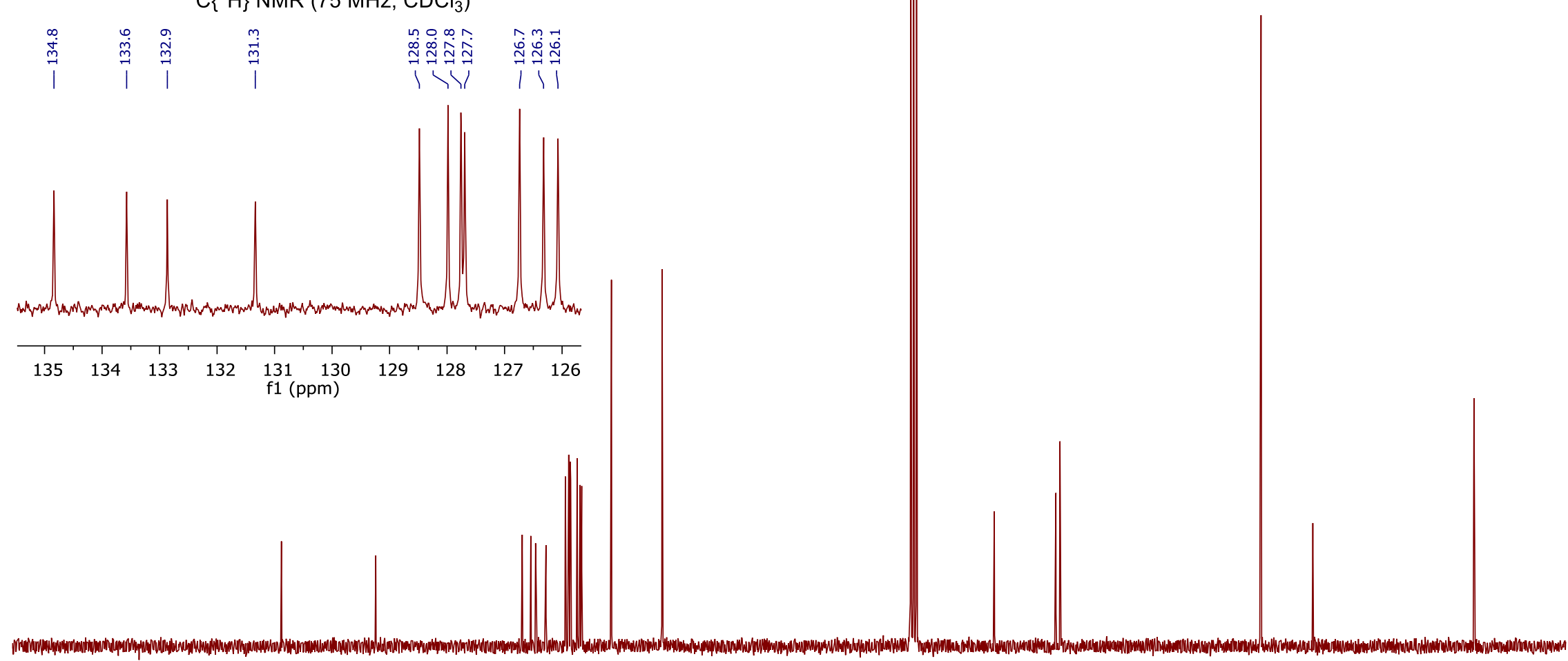

\begin{tabular}{llllllllllllllllllllllllll}
\hline 0 & 200 & 190 & 180 & 170 & 160 & 150 & 140 & 130 & 120 & 110 & 100 & 90 & 80 & 70 & 60 & 50 & 40 & 30 & 20 & 10 & 0 & -10 & 10
\end{tabular} 


\section{mָm \\ 411 \\ I}

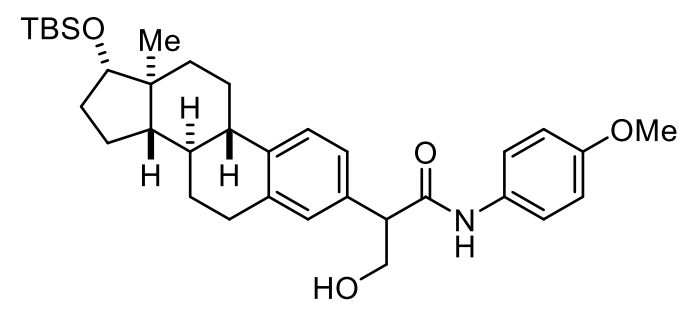

15

${ }^{1} \mathrm{H}$ NMR $\left(500 \mathrm{MHz}, \mathrm{CDCl}_{3}\right)$

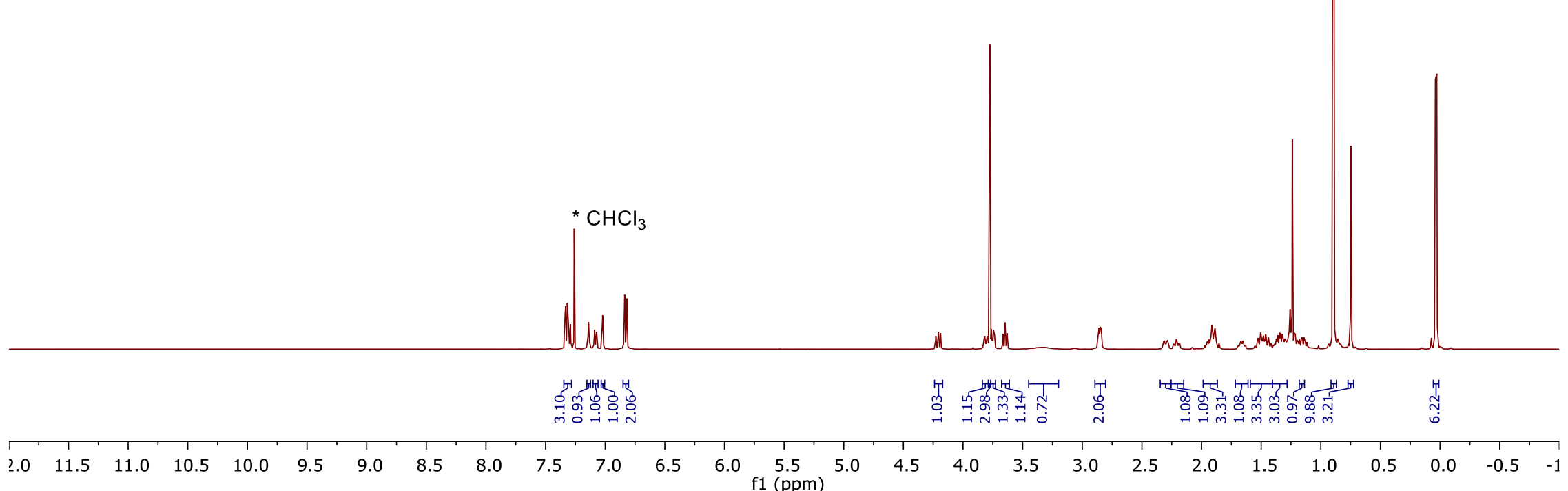




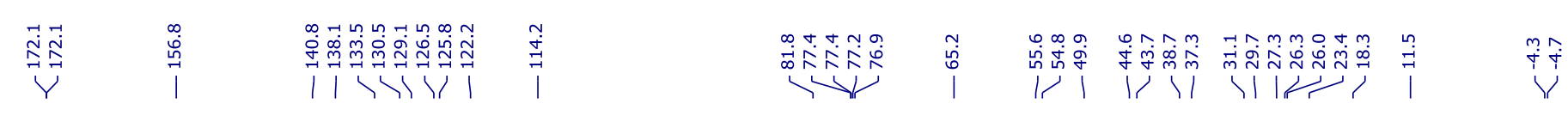

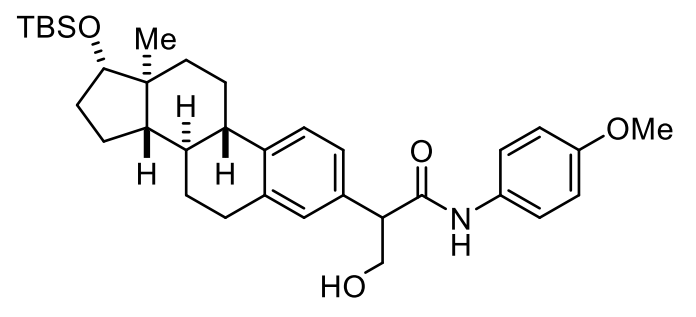

15

${ }^{13} \mathrm{C}\left\{{ }^{1} \mathrm{H}\right\}$ NMR $\left(126 \mathrm{MHz}, \mathrm{CDCl}_{3}\right)$
${ }^{*} \mathrm{CDCl}_{3}$

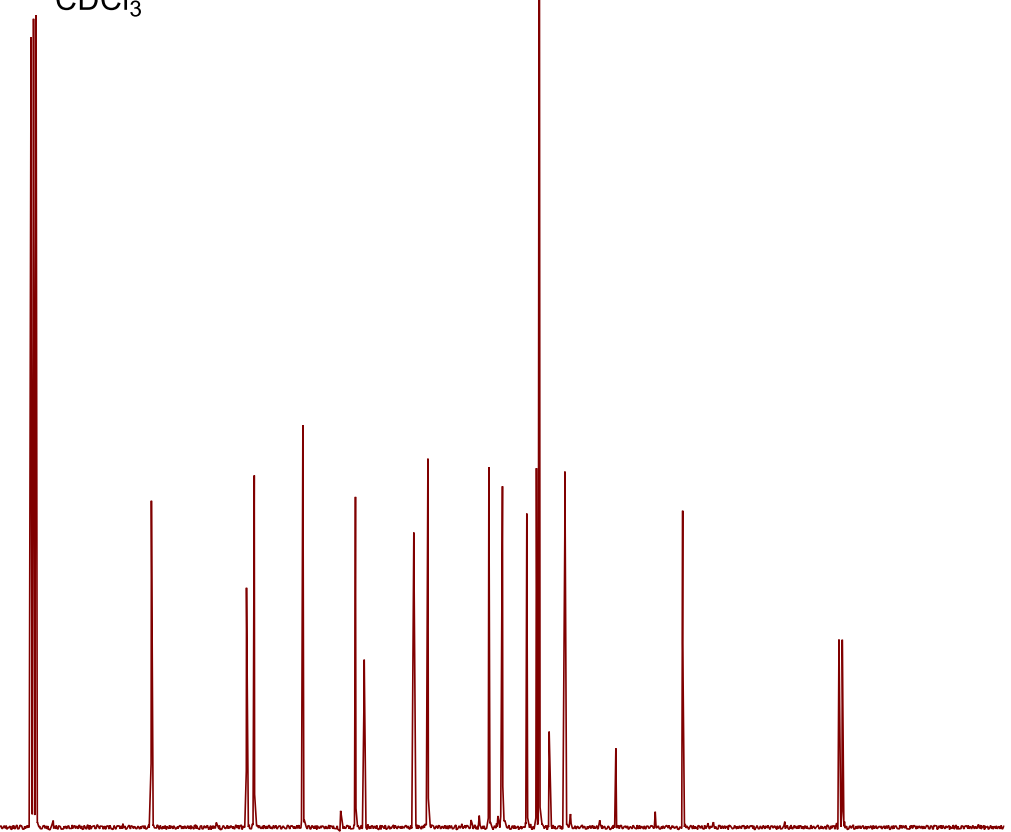




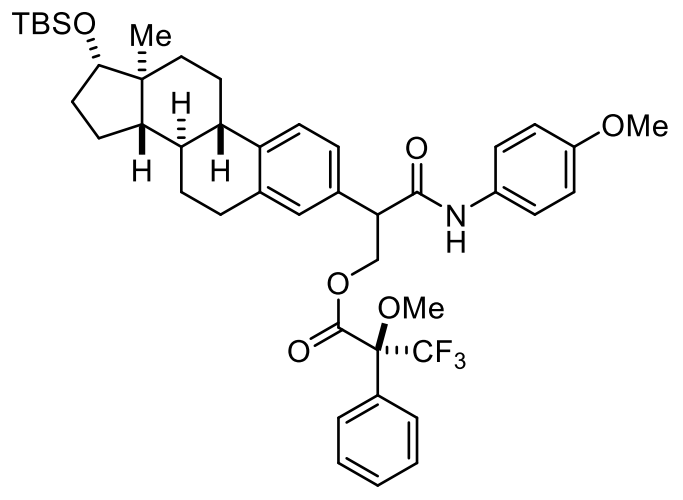

(S)-MTPA-15

${ }^{1} \mathrm{H}$ NMR $\left(500 \mathrm{MHz}, \mathrm{CDCl}_{3}\right.$ )

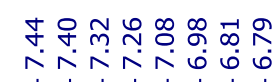

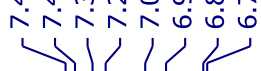

तill

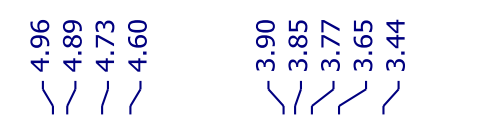

|

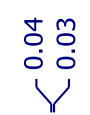
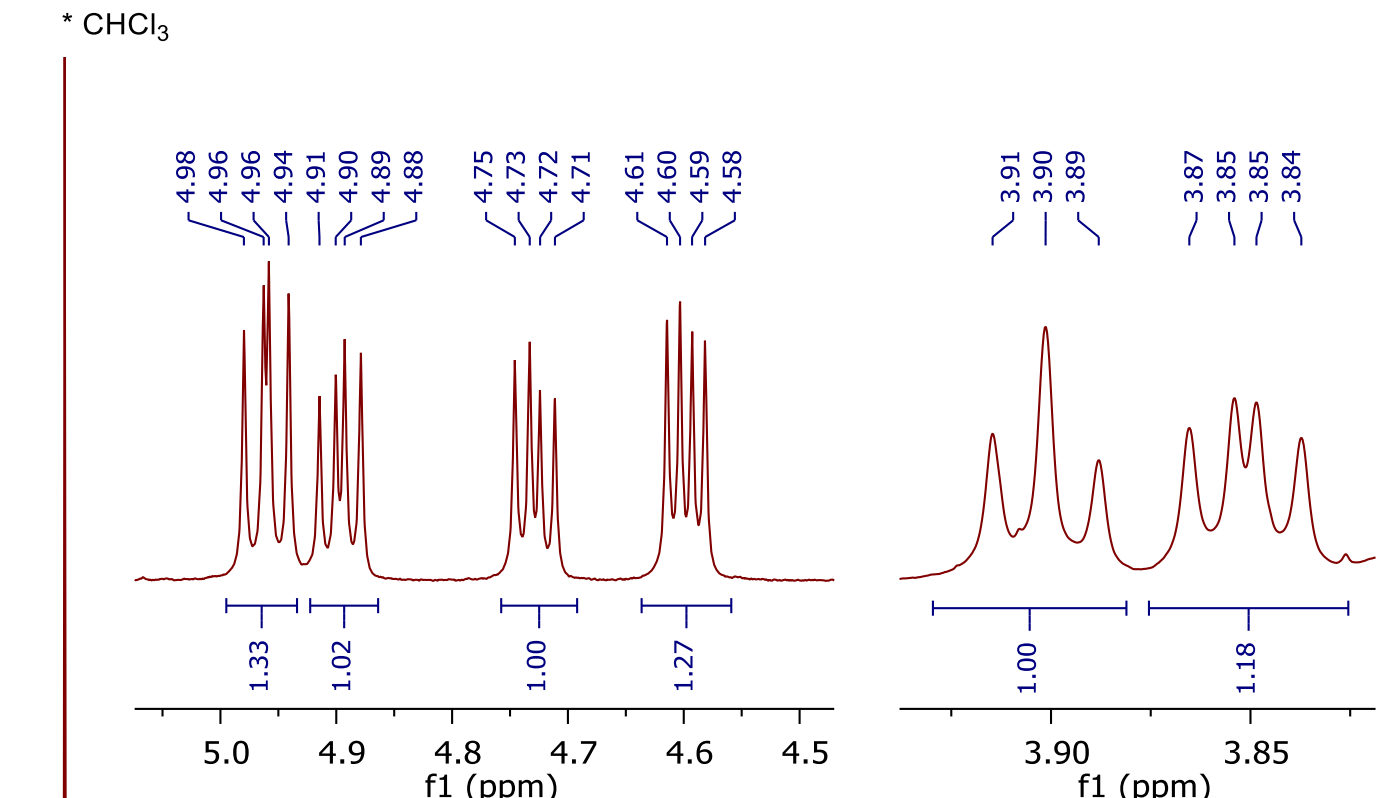

MIl

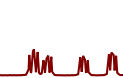

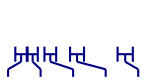

$\Lambda$

$T$

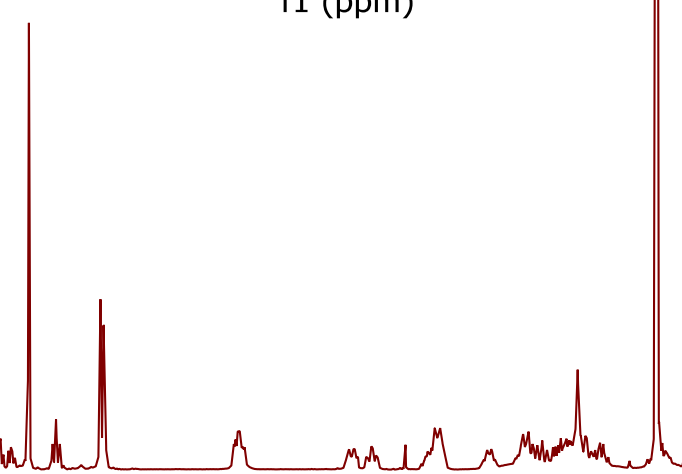




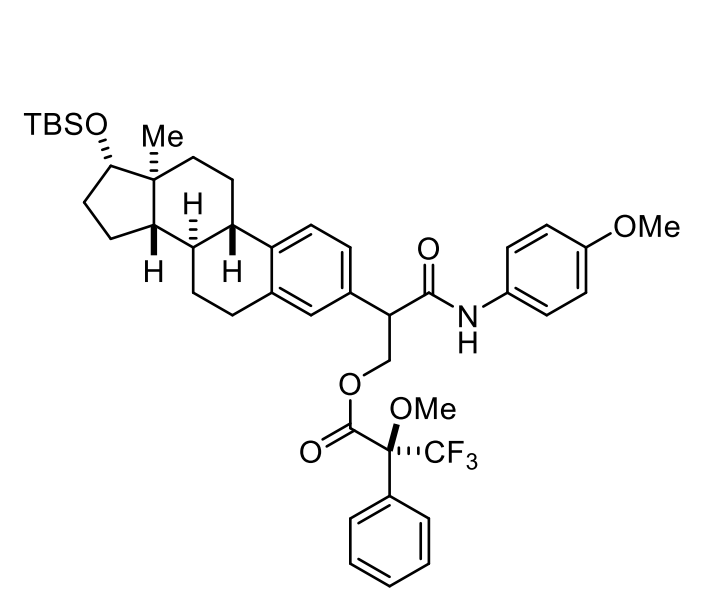

(S)-MTPA-15

${ }^{19} \mathrm{~F} \mathrm{NMR}\left(282 \mathrm{MHz}, \mathrm{CDCl}_{3}\right)$ î
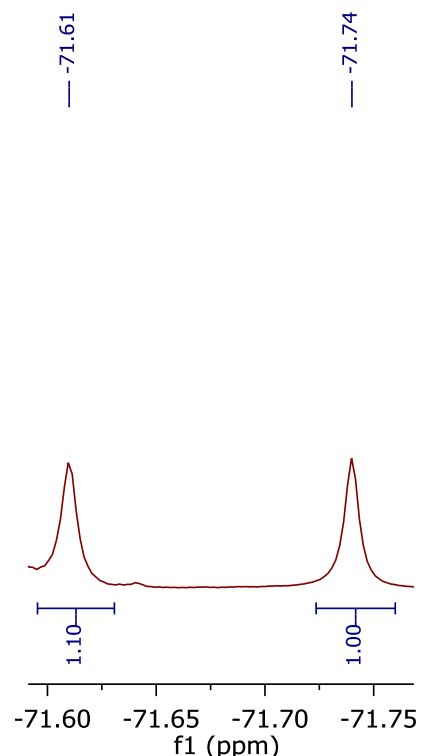